\title{
3 Traumatologie und gewaltsamer Tod
}

3.1 Rechtsgrundlagen -88

3.1.1 Vorbemerkung -88

3.1.2 Strafgesetzbuch (StGB) - Allgemeiner Teil -89

3.1.3 Strafgesetzbuch - Ausgewählte Tatbestände $\quad-89$

3.2 Kriminologie -91

3.2.1 Einführung -91

3.2.2 Kriminalstatistik und ihre Kritik -92

3.2.3 Operative Fallanalyse (OFA) $\quad-94$

3.3 Allgemeine Traumatomechanik - 95

3.3.1 Zusammenhangstrennung durch Zugspannungseinwirkung -95

3.3.2 Zusammenhangstrennungen durch Schubspannungseinwirkung -98

3.4 Sekundärfolgen mechanischer Gewalteinwirkungen, Todesursachen -99

3.4.1 Infektionen -100

3.4.2 Embolien - 101

3.4.3 Schock -102

3.5 Vitale Reaktionen und Zeitschätzungen - 103

3.5.1 Ereignisort und Spurenbild -103

3.5.2 Organsysteme - 103

3.5.3 Biochemische Veränderungen im Blut - Pharmakokinetik - 105

3.5.4 Histologische Untersuchungen - 105

3.6 Mechanische Insulte -111

3.6.1 Stumpfe Gewalt -111

3.6.2 Scharfe Gewalt -125

3.7 Schussverletzungen -134

3.7.1 Waffen und Munition - 134

3.7.2 Wundballistik -137

3.7.3 Einschuss und Ausschuss - 141

3.7.4 Entfernungsabhängigkeit der Einschussbefunde -143

3.7.5 Innere Befunde - 147

3.7.6 Viehbetäubungsapparate, Bolzensetzwerkzeuge $\quad-147$

3.7.7 Kriminalistische Aspekte -148

3.7.8 Explosionsverletzungen -149 
3.8 Gewaltsame Erstickung - 149

3.8.1 Strangulation -155

3.8.2 Die einzelnen Strangulationsarten -163

3.8.3 Tod im Wasser -169

3.9 Thermische Energie - 175

3.9.1 Hitze: lokale Hitzeschäden, Verbrennungen und Verbrühungen - 175

3.9.2 Kälte -185

3.10 Elektrotraumen, Blitzschlag - 190

3.10.1 Stromwirkungen im Niederspannungsbereich - 191

3.10.2 Stromwirkungen im Hochspannungsbereich -194

3.10.3 Blitzschlag -195

3.11 Verhungern -196

3.12 Kindestötungen -200

3.12.1 Rechtsmedizinische Untersuchung und Begutachtung - 200

3.13 Abtreibung -204

3.14 Tödliche Unfälle bei autoerotischer (autosexueller) Betätigung - 208

3.15 Tod in abnormer Körperposition - Physical restraint - 211

3.16 Spezialfragen bei der Begutachtung nichtnatürlicher Todesfälle $\quad-214$

3.16.1 Unterscheidung vitaler und postmortaler Verletzungen - 214

3.16.2 Konkurrenz und Koinzidenz von Todesursachen -214

3.16.3 Priorität von Verletzungen - 215

3.16.4 Handlungsfähigkeit nach Verletzungen - 215

3.16.5 Leichenbeseitigung und Leichenzerstückelung -217

3.16.6 Tötungsdelikte durch Tritte -220

Literatur -223 


\section{$>$ Einleitung}

\section{Tötungsdelikt oder suizidale Vergiftung}

Vorgeschichte: Eine 39 Jahre alt gewordene, alkoholabhängige Frau habe von ihrem Hausarzt eine Packung Distraneurinkapseln (25 Stück) verschrieben bekommen; sie solle morgens und mittags je 1, abends 2 Kapseln einnehmen. Aus der Apotheke zurückgekehrt, begann sie zu Hause, mit ihrem Ehemann Alkohol zu trinken. Es sei zu einem Streit gekommen, in dessen Verlauf die Frau die Polizei angerufen habe. Der Ehemann sei daraufhin gegen 17.00 Uhr in Gewahrsam genommen und zur Wache verbracht worden. Ein Atemalkoholtest habe einen Atemalkoholwert von 1,29 mg/l ergeben. Gegen 04.00 Uhr morgens sei der Ehemann wieder entlassen worden (!). Er selbst habe angegeben, seine Ehefrau schlafend auf dem Sofa angetroffen zu haben; er habe sie geweckt, sie habe u.a. zu ihm gesagt, dass sie die Distraneurintabletten eingenommen habe. Ihr Zustand habe sich deutlich von dem bei sonstiger Alkoholisierung unterschieden. Gegen 05.30 Uhr habe er den Notarzt verständigt, der nur noch den Tod feststellen konnte.

Aus der gegenüber der Polizei gemachten Strafanzeige der Frau geht hervor, dass es am Nachmittag zuvor in der gemeinsamen Wohnung zu einem verbalen Wortgefecht gekommen sei, es seien dann vom Ehemann auch einige Einrichtungsgegenstände zerstört worden. Der Ehemann habe seiner Frau an den Hals gefasst und dabei »leicht« zugedrückt und gesagt: »Wenn ich hier gleich wieder erscheine, mache ich weiter!« Zur Klärung der Todesursache, insbesondere zum Ausschluss eines Tötungsdeliktes wurde eine Obduktion angeordnet.

Sektionsergebnis: Hirnödem, ausgeprägtes hämorrhagisches Lungenödem, prallvolle Harnblase und überwiegend flüssiges Leichenblut. Diskrete punktförmige Hauteinblutung der linken Halsseite, teils strichförmige und teils fleckförmige, gering ausgeprägte Hautvertrocknungen der rechten Halsseite. Bis auf eine kleine Einblutung in das Unterhautfettgewebe der linken Halsseite keine weiteren Verletzungen der Halsweichteile und der Halsorgane. Keine Dunsung oder Zyanose im Kopfbereich, keine Stauungsblutungen. Prellung am linken Unterarm mit sulzig braunroter Einblutung in das Unterhautfettgewebe. Keine Zeichen einer inneren Erkrankung von todesursächlicher Dignität. BAK 0,99\%o, UAK 1,86 \%o. Chemisch-toxikologisch konnten im Femoralblut nachgewiesen werden: Diazepam $418 \mathrm{ng} /$ ml, Nordiazepam 258 ng/ml, Temazepam 65 ng/ml, Chlomethiazol $13,09 \mathrm{mg} / \mathrm{l}$. Während Diazepam und Metabolite in therapeutischen Konzentrationsbereichen vorliegen, liegt die Chlomethiazolkonzentration deutlich oberhalb des therapeutischen Bereiches, so dass als Todesursache eine Intoxikation mit Distraneurin in Kombination mit Alkohol und Diazepam vorliegt.

- Differentialdiagnose bei nichtnatürlichem Todesfall manchmal nur unter Ausschöpfung aller Untersuchungsmöglichkeiten erfolgreich.

\section{Leichenzerstückelung}

Vorgeschichte: Der als Eigenbrötler bekannte, 57 Jahre alt gewordene Mann konsumierte am Tattag zusammen mit zwei weiteren Männern erhebliche Mengen Alkohol. Im Rahmen eines Streites erhielt er zunächst heftige Schläge und Tritte gegen Gesicht, Hals und Oberkörper, dann Stichverletzungen durch ein Messer. Bewusstlos und blutend wurde das Opfer auf ein Bett gelegt, nach Feststellung des Todes wurde der Leichnam mittels einer Kettensäge horizontal in der Mitte zerteilt sowie in Kopf, Thorax und rechten Oberarm eingesägt. Zur Leichenbeseitigung wurde der Unterkörper in einen Keller verbracht, der Oberkörper in eine Mülltonne gesteckt und mit einer Plastikplane abgedeckt.

Sektionsergebnis: Bei der Obduktion fanden sich Zeichen maßgeblicher vitaler Gewalteinwirkung mit flächenhaften Hämatomen von der Kopfschwarte über Gesicht und Hals bis auf Thorax und Oberarme übergreifend, multiple Platzwunden und Trümmerfrakturen des Gesichtes, Zungenbein- und Schildknorpelfrakturen sowie Frakturen des 6. Halswirbelkörpers, des 11. Brustwirbelkörpers und der 2.-4. Rippe rechts. Zeichen der Fesselung an den Handgelenken, zudem 10 Stichverletzungen an den Extremitäten. Als postmortale Leichenzerstörung lag eine vollständige horizontale Durchtrennung des Rumpfes $9 \mathrm{~cm}$ oberhalb des Nabels vor, der Brustkorb war rechtsseitig großflächig eröffnet, der rechte Lungenoberlappen zerfetzt. Zudem horizontale Durchtrennung des Gesichtsschädels von den Mundwinkeln bis zum Hinterhauptsbein verlaufend und parasagittale Durchtrennung des Hirnschädels durch die linke Orbita mit Exenteration großer Anteile der linken Großhirnhemisphäre. Todesursächlich war die gröbste komprimierende Gewalteinwirkung gegen den Hals (Fußtritte) in Kombination mit Blutverlust bei mehrfachen Stichverletzungen.

- vitale, postmortale Verletzungen

- Leichenbeseitigung

\section{Tierfraßverletzungen}

Vorgeschichte: Die 75 Jahre alt gewordene Frau wurde 2 Tage nach dem letzten Lebenszeichen tot in ihrer regelrecht verschlossenen Wohnung aufgefunden. Bekannter Alkoholabusus. In der Wohnung zudem ein Pudel. Die Obduktion erfolgte zum Ausschluss eines Fremdverschuldens, da eine große Oberschenkelwunde bei der polizeilichen Leichenschau aufgefallen sei.

Sektionsergebnis: Hochgradige allgemeine Arteriosklerose mit Aneurysma des absteigenden Aortenbogens und subtotal lichtungsverschließendem Thrombus, mittelgradige Koronararteriosklerose mit hochgradiger Einengung des Abganges der rechten Koronararterie bei Rechtsversorgungstyp des Herzens. Ausgedehnter postmortaler Hautweichteildefekt an der Vorder- und Innenseite des linken Oberschenkels mit Eröffnung der linken Becken-/Beinschlagader. Keine Zeichen eines Blutverlustes, keine zu Lebzeiten entstandene, grobe äußere Gewalteinwirkung. Todesursache war hier die akute Koronarinsuffizienz bei hochgradiger Einengung der rechten Koronararterie und Rechtsversorgungstyp des Herzens sowie subtotaler Thrombose des Aortenbogens.

- Postmortale Verletzungen durch Tierfraß bei natürlichem Tod. 


\section{Agonale Sturzverletzung}

Vorgeschichte: Eine 62 Jahre alt gewordene Frau sei in den frühen Morgenstunden eines Januartages in eine neurochirurgische Klinik aufgenommen worden, dort, soweit durch die Polizei ermittelt, wegen einer Hirnblutung behandelt worden und zwei Tage später im Koma verstorben. Der Ehemann der Verstorbenen habe gegenüber der Polizei angegeben, dass seine Frau am Vorabend der Kliniksaufnahme gegen 18.00 Uhr mit einem Wäschekorb in den Keller gegangen sei. Als sie $1 \frac{1}{2}$ Stunden später noch nicht wieder zurückgekehrt sei, habe er aus Sorge nach ihr geschaut und sie im Keller in Rückenlage auf dem Fußboden vor der Kellertreppe liegend aufgefunden, wobei ihr Kopf unmittelbar vor der Kante der untersten Stufe gelegen habe. Da seine Frau alkoholabhängig und in berauschtem Zustand bereits wiederholt gestürzt sei, habe er einen erneuten Sturz in betrunkenem Zustand vermutet, seiner Frau ein Kissen unter den Kopf geschoben, sie mit einer Decke zugedeckt und keine weiteren Maßnahmen ergriffen. Als er sie am folgenden Morgen immer noch vor der Kellertreppe liegend aufgefunden habe, habe er sofort den Notarzt alarmiert. Bei der kriminalpolizeilichen Leichenschau wurden eine vernähte Platzwunde am Hinterkopf und eine Hautabschürfung an der Streckseite beider Ellenbogengelenke festgestellt. Zur sicheren Beweisführung wurde eine Obduktion angeordnet.

Sektionsergebnis: Die Sektion erbrachte folgenden Befund: $3 \mathrm{~cm}$ lange, chirurgisch vernähte Schädelschwartendurchtrennung des Hinterkopfes in der Mittellinie und in der Hutkrempenlinie, keine höhergradige lokale Umblutung, keine Verletzung von Schädelknochen und Hirnhäuten, keine kuppenständige Hirnrindenkontusion. Ausgedehnte, nahezu die gesamte linke Hemisphäre einnehmende Hirnmassenblutung mit Einbruch in die Ventrikel. Tiefe Aspiration von Mageninhalt. Schwere, fibrinös-eitrige, teilweise abszedierende Pneumonie beider Unterlappen. Feinknotige Leberzirrhose, portale Stauungsmilz. Geringe Prellungen an der Streckseite beider Ellenbogengelenke.

Eine Blutalkoholbestimmung an einer Blutprobe, die bei Kliniksaufnahme gesichert worden war, ergab eine Blutalkoholkonzentration von im Mittel 0,22 \%o.

- Was ist die Todesursache?

- Sind die Obduktionsbefunde auf eine Verursachung durch eine Gewalteinwirkung von außen, möglicherweise durch fremde Hand zu beziehen oder handelt es sich um vorbestehende innere Erkrankungen?

- Hätte das Leben der Verstorbenen bei frühzeitiger ärztlicher Hilfe gerettet werden können?

\section{Differentialdiagnose: Strangmarke - Drosselfurche}

Vorgeschichte: In einer Aprilnacht kurz nach Mitternacht brachte ein Kosovare bei der Polizei den Todesfall seiner Nichte zur Anzeige. Zum Sachverhalt gab er an, dass seine Schwester das zehnjährige Kind seit den Nachmittagsstunden des Vortages vermisst und bei Nachsuche auf einem nahe gelegenen Spielplatz leblos in einer Spielröhre liegend aufgefunden habe. Aus Ratlosigkeit habe sie das Kind zunächst in die elterliche Wohnung getragen und die Verwandtschaft benach- richtigt. In einer ersten Befragung äußerte die Mutter, ihre Tochter müsse einem Verrückten begegnet sein, sie hoffe, dass die Polizei den Täter rasch finden würde. Nachdem bei der kriminalpolizeilichen Leichenschau Verletzungen am Hals festgestellt wurden, wurde das Ermittlungsverfahren unter dem Verdacht eines Tötungsdeliktes geführt.

Sektionsergebnis: Die Obduktion erbrachte folgende Befunde: von der Halsvorderseite symmetrisch zum Nacken ansteigende und dort auslaufende Strangmarke, korrespondierende Einblutung des Unterhautfettgewebes über dem rechten $M$. sternocleidomastoideus und flächenhafte subperiostale Zerrungsblutungen des rechten Schlüsselbeins, flächenhafte Unterblutungen der Vorderflächen der Zwischenwirbelscheiben im tiefen Brustwirbelsäulen- und Lendenwirbelsäulenbereich.

Petechien der Haut hinter den Ohren, in der Haut der Augenlider, in den Bindehäuten der Augenlider und Augäpfel, der Mundvorhofschleimhaut sowie der Haut von Mundboden und Hals oberhalb der Strangmarke.

Zahlreiche doppelt konturierte Hämatome mit einer Breite von 2-4 mm und zum Teil bogenförmigem Verlauf, Lokalisationsschwerpunkt an Rücken, Gesäß, Streckseiten der Arme sowie Vorderseiten der Beine.

Todesursache war eine komprimierende Gewalteinwirkung gegen den Hals, wobei Erscheinungsbild und Verlauf der Strangmarke differentialdiagnostisch an erster Stelle einem Erhängen zuzuordnen waren. Die multiplen konturierten Hämatome mit charakteristischer »Doppelstriemenstruktur« sprachen nach Art und anatomischer Verteilung für eine Züchtigung.

Mit den Schlussfolgerungen aus dem Sektionsbefund konfrontiert, räumten Mutter und Familie ein, dass die Mutter das Kind wegen eines Gelddiebstahls in der Verwandtschaft zur Rede gestellt und im Rahmen eines eskalierenden Streits mit Schlägen gezüchtigt habe. Als Werkzeug habe sie ein zu einer Schlaufe zusammengelegtes Computerkabel verwendet. Am späteren Abend habe sie das Kind mit einem Bademantelgürtel erhängt auf dem Dachboden aufgefunden. Aus einem kurzen Abschiedsbrief habe sich als Suizidmotiv Reue über den Gelddiebstahl ergeben. Aus Angst vor der Reaktion des Ehemannes habe die Mutter gemeinsam mit ihrer Verwandtschaft beschlossen, gegenüber der Polizei ein Tötungsdelikt vorzutäuschen. Bei den anschließenden polizeilichen Ermittlungen habe sich auf dem Speicher an der Oberfläche eines giebelfernen Dachbalkens eine schmalstreifige Aussparung der bedeckenden Staubschicht gefunden, die als Aufhängestelle des Strangwerkzeugs in Betracht gekommen sei. Darüber habe auf dem Fußboden ein Koffer gestanden, der als Steighilfe habe genutzt werden können, so dass der Balken für das Kind erreichbar gewesen sei. Durch ein graphologisches Gutachten sei das Schriftbild des Abschiedsschreibens im Vergleich mit Schulheften dem verstorbenen Mädchen zugeordnet worden.

- Die stringente Deutung charakteristischer Obduktionsbefunde (hier Differentialdiagnose Strangmarke - Drosselfurche) ermöglicht in enger Kooperation mit den Ermittlungsbehörden eine frühzeitige richtungsweisende Fokussierung der Untersuchungen. 


\section{Handlungsfähigkeit}

Vorgeschichte: Ein 53 Jahre alt gewordener Mann wird von dem Sohn im Ehebett des von ihm alleine bewohnten Wohnhauses liegend aufgefunden. Neben dem Kopf liegt ein Revolver, vor dem Bett eine Patronenhülse. Zwischen Bett und einem im Keller befindlichen Waffenschrank zahlreiche Blutspuren. Langjährige Depressionen bekannt, deshalb zweimal in stationärer Behandlung und von Frau und Kindern verlassen.

Sektionsergebnis: Als maßgebliche Verletzungen zwei Durchschussverletzungen des Schädels: Einschuss des Mundbodens, hier Schussdefekt, Abstreifring, Schürfsaum und Kontusionshof, Schusskanalverlauf in Längsrichtung nach oben mit Durchschuss der Zunge, des harten Gaumens in Körpermittellinie, der Nasenhaupthöhle und beider Stirnhöhlen. Ausschuss oberhalb der Nasenwurzel, das Gehirn im Spitzenbereich durch kleine Knochensplitter marginal verletzt. Zweiter Einschuss in der rechten Schläfenscheitelregion mit trichterartig nach innen verbreitertem Knochendefekt, Durchschuss der Schläfenhirne beidseits mit Abtrennung von Brücke und verlängertem Mark, Zertrümmerung des linken Felsenbeins und Ausschuss oberhalb des linken Gehörganges. Zeichen des mäßiggradigen Blutverlustes, keine schussfremde Gewalteinwirkung.

- Schussverletzungen, Handlungsfähigkeit, Reihenfolge der Schussverletzungen.

\section{Posttraumatischer Todesfall}

Vorgeschichte: Nach einem Sturz auf den Kopf stellte sich der 47 Jahre alte Mann in der chirurgischen Ambulanz eines Krankenhauses vor, in der seine Kopfplatzwunde chirurgisch versorgt wurde. Eine Röntgenaufnahme wurde seitens des Behandelten abgelehnt. Zwei Tage später kam es zu Somnolenz und Kopfschmerzen, es trat mehrfaches Erbrechen auf. Zudem klagte der Patient über Herzschmerzen, sodass letztendlich der Notarzt alarmiert und eine Einweisung ins Krankenhaus erfolgte. Dort wurde der Patient reanimationspflichtig und verstarb.

Sektionsergebnis: Obduktion aufgrund eines Behandlungsfehlervorwurfes mit übersehener Schädelhirnblutung. Bei der Obduktion konnte ein faustgroßer Tumor im linken Lungenunterlappen, Befall der Hiluslymphknoten sowie eine fibrinöse Perikarditis mit $650 \mathrm{ml}$ Erguss im Herzbeutel festgestellt werden. Zeichen der groben äußeren Gewalteinwirkung lagen nicht vor. Die 3,5 cm lange Kopfplatzwunde des Hinterhauptes in Hutkrempenlinie war regelrecht chirurgisch versorgt, Verletzung von Schädelknochen, Hirnhäuten oder Gehirn lagen nicht vor. Todesursächlich war die (paraneoplastische) Perikarditis mit Herzbeuteltamponade, eine übersehene Schädelhirnblutung konnte ausgeschlossen werden.

- Differentialdiagnose: natürlicher - nichtnatürlicher Tod.

\section{Wasserimmersionszeit bei Ertrinken}

Vorgeschichte: Ein 12 Jahre alt gewordenes Mädchen besuchte bei warmem Sommerwetter gemeinsam mit seiner Cousine ein Freibad. Die beiden Kinder spielten Wetttauchen, wobei sie sowohl lange Stre- cken tauchten als auch Gegenstände vom Grund des Beckenbodens hoch holten. Nach einiger Zeit habe das jüngere Kind die ältere Cousine vermisst, mit ihrer Taucherbrille unter Wasser geschaut und dabei ihre Cousine gekrümmt mit dem Gesicht auf dem Beckenboden liegend gesehen. Sie habe weinend drei ältere Mädchen um Hilfe gebeten. Diese hätten in Mitte des Beckens, an der Schräge vom Nichtschwimmer- in den Schwimmerbereich, am Beckenboden ein bewegungsloses Kind in einer Art Hockstellung vorgefunden, mit auf dem Boden aufliegendem Gesicht. Nach der Bergung sei ihnen aufgefallen, dass das Mädchen bereits am gesamten Körper blau angelaufen gewesen sei, einen aufgeblähten Bauch und weit aufgerissene Augen gehabt habe. Aus Mund und Nase sei eine leicht bräunliche Flüssigkeit gelaufen. Durch das Schwimmbadpersonal, zwei zufällig anwesende Ärzte und den sofort herbeigerufenen Notarzt habe das Kind zunächst reanimiert werden können. Bei der Klinikaufnahme hätten sich eine livide Hautverfärbung, maximal weite, beginnend entrundete, nicht reagible Pupillen, minimale Kreislauftätigkeit unter Reanimationsbedingungen sowie eine Unterkühlung auf eine Temperatur von $34,7^{\circ} \mathrm{C}$ gezeigt. Das Kind sei bei schwerem hypoxischem Hirnschaden einen Tag später im Koma verstorben. Klinisch sei als Todesursache ein Ertrinkungsunfall im Schwimmbad angegeben worden. Autoptisch fanden sich die Zeichen des protrahierten Schocks, während Befunde des akuten Ertrinkungstodes, wie Emphysema aquosum, Paltauf'sche Flecke oder wässriger Inhalt in Magen und Keilbeinhöhlen, nicht nachzuweisen waren. Dies stand bei eintägiger intensivmedizinischer Behandlung im Anschluss an den Unfall der Annahme eines initialen Beinaheertrinkens mit hypoxischem Hirnschaden nicht entgegen.

Sektionsergebnis: Da der eigentliche Unfall nicht durch Zeugen beobachtet worden war, sollte als Grundlage für die strafrechtlich Würdigung aus rechtsmedizinischer Sicht u.a. zu der Frage Stellung genommen werden, nach welcher Zeitspanne unter Wasser eine erfolgreiche Reanimation grundsätzlich und im konkreten Fall noch möglich gewesen wäre. Hierzu belegen retrospektive Analysen von Ertrinkungsunfällen in der Badewanne, dass überlebende Kinder über einen Zeitraum von 3-5 Minuten (median 4 Minuten) mit den Atemöffnungen unter der Wasseroberfläche gewesen waren, während die zu Tode gekommenen Kinder sich über einen Zeitraum von 3-20 Minuten (median 5 Minuten) unter Wasser befunden hatten. Bezogen auf den konkreten Fall legt die Tatsache, dass die Wiederbelebungsbehandlung bei dem Kind im Endeffekt nicht erfolgreich war, die Schlussfolgerung nahe, dass es zumindest drei Minuten, mit großer Wahrscheinlichkeit mehr als 4 Minuten unter Wasser gelegen hatte, wobei auch deutlich längere Immersionszeiten möglich, jedoch nicht sicher zu beweisen waren.

- Ertrinken, Aufsichtspflichtverletzung, Begutachtung der Immersionsdauer. 


\subsection{Rechtsgrundlagen}

\author{
R. Wegener
}

\subsubsection{Vorbemerkung}

Forensisch-traumatologische Untersuchungen dienen der Befunderhebung, Dokumentation und Asservierung. Bei lebenden Geschädigten sollte auch die verletzungsbezogene klinische Symptomatik eingeschlossen sein. Derartige Befunde - als Beweismittel in ein Straf- (Meyer-Goßner 2005) oder Zivilverfahren (Baumbach 2005) eingeführt - können eine prozessentscheidende Rolle spielen. Eine sorgfältige Befunddokumentation mit metrischen Angaben zur Größe und Lokalisation von Verletzungen ist deshalb unerlässlich.

( Wichtig

Ärztliche Befundberichte fokussieren sich auf den behandlungspflichtigen Befund. Die Dokumentation von Nebenverletzungen mit forensischer Relevanz ist nicht selten unvollständig. Angaben von geschädigten Patienten sind oft von subjektiven Vorstellungen geprägt. Bei der Übernahme von Informationen gilt deshalb für den Sachverständigen das Prinzip des begrenzten Vertrauens.

In einer ersten gutachterlichen Stellungnahme oder im vorläufigen Gutachten kann nach dem Prinzip der befundnahen Interpretation über die Befundbeschreibung hinaus nur zu eindeutigen Verletzungsmustern Stellung genommen werden. Der Vorbehalt einer ausführlichen Bewertung bei Vorliegen detaillierter Kenntnisse eines Ereignisablaufes sollte deutlich ausgeführt werden.

Weitergehende Interpretationen eines Verletzungsmusters mit Versionsbildungen zu einer Tathandlung oder einem Ereignisablauf sowie die Stellungnahmen zur Kausalität erfolgen erst in einem zweiten Schritt. Sie werden durch den Untersuchungsauftrag bestimmt oder bleiben der gerichtlichen Hauptverhandlung vorbehalten. Befunde können durchaus auch zur Entlastung von Beschuldigten führen.

\section{F) Fallbeispiel}

Ein 15-jähriger Jugendlicher wird in einem Abrissgebäude von einem Bauarbeiter angetroffen. Bei einer sich entwickelnden Rangelei zieht der Beschuldigte ein Messer und versetzt dem 50jährigen Geschädigten einen Thoraxstich mit der Folge einer letalen Massivblutung. Bei der körperlichen Untersuchung durch den Rechtsmediziner stellte sich an der rechten Halsseite eine parallelstreifige Hautverfärbung mit drei in Reihe angeordneten randständigen Oberhautdefekten dar. In der Verhandlung sagte der Angeklagte aus, dass er einen von schräg hinten geführten Angriff des Bauarbeiters abwehren wollte. Der Geschädigte habe inn »am Hals gepackt«. Daraufhin habe er das Messer gezogen und aus einer ausholenden Drehbewegung heraus den Angreifer abwehren wollen. Dabei sei es zur tödlichen Stichverletzung ge$\nabla$ kommen. Der Vorwurf einer Körperverletzung mit Todesfolge konnte nicht aufrecht erhalten werden. Der Angeklagte wurde nach Anhörung des Sachverständigen aufgrund einer Notwehrlage freigesprochen (• Abb. 3.1).

Die Beweisregeln im Strafverfahren unterscheiden sich von denen im Zivilverfahren ( $\triangleright$ Kap. 10. u. Kap. 11). Weiterhin sind die gesetzlichen Regelungen zu beachten, die sich aus dem Versicherungsrecht und dem Sozialrecht ergeben ( $\triangleright$ Kap. 11).

Bei der Beurteilung von Verletzungen sind folgende Ereigniskategorien in Betracht zu ziehen:

- Suizide, Suizidversuche, Selbstschädigung ( Kap. 11 u. Kap. 5.3)

- Unfälle, Unglücksfälle

- Körperverletzungs- und Tötungsdelikte

Der Unfall ist in den Allgemeinen Unfallversicherungsbedingungen (AUB 2000) definiert.

\section{§ 1 Versicherte Gefahren, Geltungsbereich}

Ein Unfall liegt vor, wenn der Versicherte durch ein plötzlich von außen auf seinen Körper wirkendes Ereignis (Unfallereignis) eine Gesundheitsschädigung erleidet (...)

Diese Definition schließt eine Krankheit bzw. eine suizidale Handlung als Unfallereignis aus. Weiterhin finden Berufs- und Gewerbekrankheiten nach $₫ 1$ Abs. IV der AUB keine Berücksichtigung. Hingegen können indirekte Verletzungen anerkannt werden. Das betrifft Luxationen oder Muskelfaserrisse bei erhöhter Kraftanstrengung ebenso wie bei Schlägereien erlittene Verletzungen, wenn der Versicherte ohne Vorsatz teilgenommen hat.

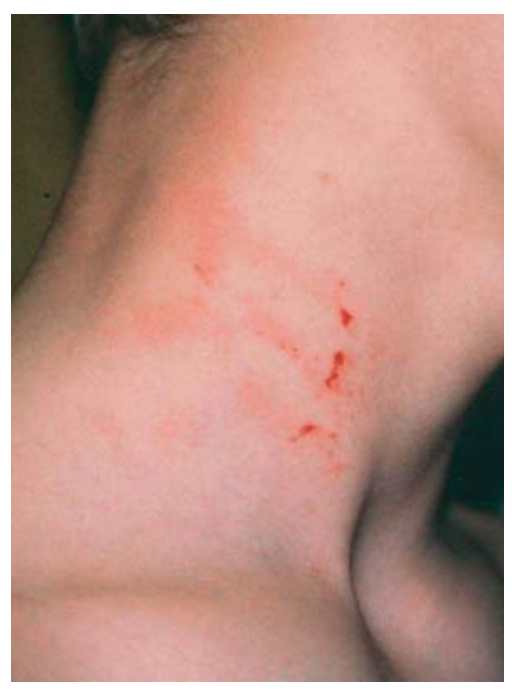

- Abb. 3.1. 15-jähriger Täter mit Verletzungsmuster der rechten Halsregion 
Im strafrechtlichen Kontext $(\$ 323 c$ StGB - Unterlassene Hilfeleistung) wird der Begriff Unglücksfall wesentlich weiter gefasst, weil es bei der Hilfspflicht in einer Notsituation nicht auf die Ursache einer hilflosen Lage ankommt. Danach kann auch eine sich plötzlich verschlimmernde Krankheit oder ein Suizidversuch ( $\triangleright$ Kap. 11) ein Unglücksfall sein.

\subsubsection{Strafgesetzbuch (StGB) - Allgemeiner Teil}

Ein einheitliches deutsches Strafgesetzbuch liegt seit 1871 vor, nachdem das erste deutsche Strafgesetz, die Peinliche Halsgerichtsordnung Kaiser Karls V. aus dem Jahre 1532, in zahlreiche Partikulargesetze übergegangen war. Mit den 1933 vorgenommenen Änderungen des StGB wurde der Weg frei für die Einführung eines dualistischen Systems des Strafrechtes: Strafe und/oder Maßregel (-Abb. 3.2). Das Sechste Gesetz zur Reform des Strafrechtes aus dem Jahre 1998 hat dem StGB - insbesondere für jene Tatbestände, die für die rechtsmedizinische Begutachtung von Bedeutung sind - das heutige Nummerierungssystem gegeben (Fischer 2006).

In dem Allgemeinen Teil des StGB sind die Vorschriften enthalten, die die gemeinsamen Wesenszüge von strafbaren Handlungen umfassen (Geltungsbereich, Sprachgebrauch, Grundlagen der Strafbarkeit, Begehungsformen und Rechtsfolgen):

- Bindung des Strafrechts an geschriebene Gesetze - keine Strafe ohne Gesetz, zeitliche Geltung, Verbot der Anwendung des Rechtes nach dem Zeitpunkt der Begehung ( $\$ \$ 1,2$ StGB)

- Jugendgerichtsgesetz (JGG) als eigenständiges Gesetz für Jugendliche und Heranwachsende

\section{Dualistisches System des Strafrechts}

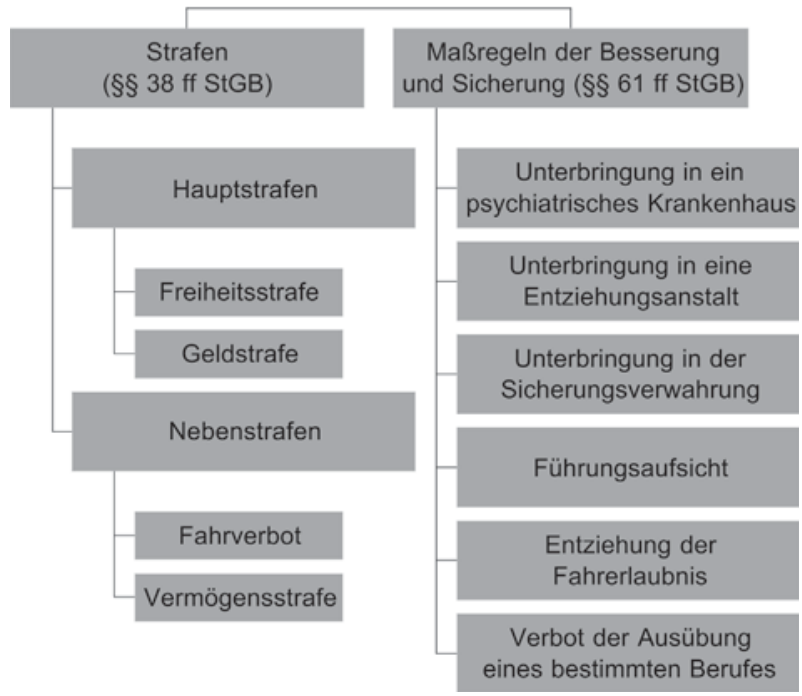

- Abb. 3.2. Dualistisches System des Strafrechts
- Verbrechen als rechtswidrige Tat im Mindestmaß mit Freiheitsstrafe $\geq 1$ Jahr; Vergehen als rechtswidrige Tat im Mindestmaß mit Freiheitsstrafe $\leq 1$ Jahr oder Geldstrafe ( $\$ 12$ StGB)

- Straftat als tatbestandsmäßige, rechtswidrige und schuldhafte Handlung; Handlung als jedes menschliche Verhalten (Tun und Unterlassen), Kausalzusammenhang (\$13 StGB)

- Vorsatz bei Wissen und Wollen der Tatbestandsverwirklichung; bedingter Vorsatz bei gewollter und billigender Inkaufnahme einer möglichen Tatbestandsverwirklichung

- Fahrlässigkeit bei rechtswidriger und vorwerfbarer Verwirklichung eines Tatbestandes ohne gewollte oder erkannte Herbeiführung; bewusste Fahrlässigkeit bei nicht gewollter Verwirklichung und (pflichtwidrigem) Vertrauen auf den Nichteintritt; unbewusste Fahrlässigkeit bei erkennbarer, aber fehlerhaft nicht erkannter Vorhersehbarkeit des verwirklichten Tatbestandes ( $\$ 15$ StGB)

- Strafrechtlich relevante Altersklassen (Eisenberg 2000):

- Kinder unter 14 Jahre: nicht verantwortlich, strafunmündig ( $\$ 19$ StGB)

- Jugendliche 14-18 Jahre: bedingt verantwortlich ( $\$$ 1-3 JGG)

- Heranwachsende 18-21 Jahre: grundsätzlich voll verantwortlich, Jugendstrafrecht nach $\$ 105$ JGG möglich (Ausnahme $\$ \$ 20,21$ StGB)

- Erwachsene $>21$ Jahre: grundsätzlich voll verantwortlich (Ausnahme $\$ \$ 20,21$ StGB)

- Versuch als begonnene, aber nicht vollendete Tat, Zurückbleiben der Handlung ( $\$ 22$ StGB)

\subsubsection{Strafgesetzbuch - Ausgewählte Tatbestände}

In dem Besonderen Teil des StGB sind die einzelnen Tatbestände und Deliktsgruppen in Abschnitten systematisch geordnet. Bei Straftaten gegen das Leben (16. Abschnitt StGB $\$ \$ 211-222$ ) und Straftaten gegen die körperliche Unversehrtheit (17. Abschnitt des StGB $\$ \$ 223-231$ ) ist der Gutachter gefordert, dem Gericht anhand von Verletzungsmustern Unterscheidungskriterien für die Tatbestände darzulegen. Die korrekte Zuordnung forensischtraumatologischer Befunde setzt die Kenntnis der Straftatsbestände in ihrer Differenziertheit voraus (•Tabelle 3.1).

\section{$\S 211$ StGB Mord}

(2) Mörder ist, wer aus Mordlust, zur Befriedigung des Geschlechtstriebs, aus Habgier oder sonst aus niedrigen Beweggründen, heimtückisch oder grausam oder mit gemeingefährlichen Mitteln oder um eine andere Straftat zu ermöglichen oder zu verdecken, einen Menschen tötet.

Die Befundinterpretation des medizinischen Sachverständigen dient der rechtlichen Würdigung jener Tatmerkmale, die auf eine 
- Tabelle 3.1. Straftaten gegen das Leben und die körperliche Unversehrtheit - Freiheitsstrafen (Auswahl)

\begin{tabular}{|c|c|c|c|}
\hline Kategorie & \multicolumn{2}{|l|}{ Delikt } & Freiheitsstrafe (Jahre) \\
\hline Tötung & $\begin{array}{l}\S 211 \\
\S 212 \\
\S 213 \\
\S 221 \\
\S 222\end{array}$ & $\begin{array}{l}\text { Mord } \\
\text { Totschlag } \\
\text { Besonders schwerer Fall } \\
\text { Minder schwerer Fall des Totschlags } \\
\text { Aussetzung } \\
\text { Todesfolge } \\
\text { Kind oder zur Betreuung anvertraute Person } \\
\text { Fahrlässige Tötung }\end{array}$ & $\begin{array}{l}\text { lebenslang } \\
\geq 5 \\
\text { lebenslang } \\
1-10 \\
0,25-5 \\
\geq 3 \\
1-10 \\
0-5\end{array}$ \\
\hline Körperverletzung & $\begin{array}{l}\S 227 \\
\S 229\end{array}$ & $\begin{array}{l}\text { Körperverletzung } \\
\text { Gefährliche Körperverletzung } \\
\text { Minder schwerer Fall } \\
\text { Misshandlung von Schutzbefohlenen } \\
\text { Minder schwerer Fall } \\
\text { Absichtlich oder Wissentlich } \\
\text { Minder schwerer Fall } \\
\text { Körperverletzung mit Todesfolge ( } \S \S 223-226) \\
\text { Minder schwerer Fall } \\
\text { Fahrlässige Körperverletzung }\end{array}$ & $\begin{array}{l}0-5 \\
0,5-10 \\
0,25-5 \\
0,5-10 \\
0,25-5 \\
\geq 3 \\
1-10 \\
\geq 3 \\
1-10 \\
0-3\end{array}$ \\
\hline
\end{tabular}

gefährliche unmenschliche Tatausführung (Mordmerkmale der 2. Gruppe) schließen lassen. So kann das Fehlen von Abwehrverletzungen bei ausgeprägten Drosselmarken darauf hindeuten, dass Heimtücke vorgelegen haben könnte (z.B. überraschender Angriff auf das wehrlose, schlafende Opfer). Zeichen der körperlichen Misshandlung unabhängig von den zum Tode führenden Verletzungen können ein Indiz für die Grausamkeit sein. Oft ist gerade der klinisch irrelevante Befund von besonderer forensischer Bedeutung.

\section{§ 212 StGB Totschlag}

(1) Wer einen Menschen tötet, ohne Mörder zu sein (...)

Bereits die Lokalisation von Verletzungen kann einen Hinweis auf den Tatvorsatz geben. Das Übermaß von Verletzungen kann den richterlichen Verdacht erhärten, dass der Täter besonders brutal mit starkem Vernichtungswillen vorgegangen ist und mithin ein besonders schwerer Fall des Totschlages - ohne Vorliegen von Mordmerkmalen - vorgelegen hat. Verletzungen sind deshalb immer auch unter Berücksichtigung der Konstitution von Täter und Opfer sowie der biomechanischen Parameter eines Tatwerkzeuges zu begutachten ( $\$$ auch Kap. 3.3).

\section{$\S 221$ Aussetzung}

(1) Wer einen Menschen

1. in eine hilflose Lage versetzt oder

2. in einer hilflosen Lage im Stich lässt, obwohl er ihn in Obhut hat oder ihm sonst beizustehen verpflichtet ist, und ihn dadurch der Gefahr des Todes oder einer schweren Gesundheitsschädigung aussetzt (...)
Das Versetzen in eine hilflose Lage, das begrifflich viel weiter $\mathrm{zu}$ fassen ist als das Aussetzen, und das Im-Stich-Lassen spielen in der Gutachtenpraxis durchaus eine Rolle. Das betrifft den Mitbürger im schweren Rauschzustand nach Alkohol oder Drogen ebenso wie den hilflosen Patienten oder den zur Betreuung Anvertrauten. Im medizinischen Bereich geht es bevorzugt bei später tot aufgefundenen Personen um die Beweisfrage, ob eine ambulant behandelte alkoholisierte Person hätte entlassen werden dürfen. Eine Strafbarkeit setzt den Vorsatz voraus.

\section{§ 223 StGB Körperverletzung}

(1) Wer eine andere Person körperlich misshandelt oder an der Gesundheit schädigt (...)

Rechtsgut ist die körperliche Unversehrtheit. Solange jede, in die körperliche Unversehrtheit eingreifende ärztliche Behandlungsmaßnahme den Tatbestand der (vorsätzlichen) Körperverletzung erfüllt - die Rechtsprechung hält an einem Reichsgerichtsurteil des Jahres 1894 fest - ist dieses Gesetz Richtschnur für die Begutachtung von Behandlungsfehlervorwürfen. Dabei ist zu beachten, dass das körperliche Wohlbefinden als Zustand, der vor der Einwirkung vorhanden war, und eine Gesundheitsschädigung als Hervorrufen oder Steigern eines auch vorübergehenden pathologischen Zustandes verstanden wird. Folglich können, wie nach einer körperlichen Misshandlung, über das therapeutisch unvermeidbare Maß hinaus zugefügte Schmerzzustände den Tatbestand einer Körperverletzung begründen, wenn pflichtwidriges Handeln festgestellt ist. Zur Problematik der Rechtfertigung eines 
ärztlichen Eingriffes durch Einholen der Einwilligung wird in Kapitel 10.5.2 Stellung genommen.

\section{§ 224 StGB Gefährliche Körperverletzung}
(1) Wer die Körperverletzung
1. durch Beibringung von Gift oder anderen gesundheitsschädlichen Stoffen
2. mittels einer Waffe oder eines anderen gefährlichen Werkzeuges
3. mittels eines hinterlistigen Überfalls
4. mit einem anderen Beteiligten gemeinschaftlich oder
5. mittels einer das Leben gefährdenden Behandlung begeht (...)

Die Gefahr ist ein Rechtsbegriff; bereits eine einfache Körperverletzung durch gemeinschaftliches Handeln per se, wie bei jugendlichen Gewalttätern nicht selten zu beobachten, begründet den Tatbestand einer gefährlichen Körperverletzung. Eine das Leben gefährdende Behandlung bedeutet keineswegs immer das Vorliegen einer realen Lebensgefahr im medizinischen Sinne. Die abstrakte Gefährdung hängt nach zahlreichen Gerichtsentscheidungen sehr von den Umständen des Einzelfalles ab. So kann das Hetzen eines Hundes auf den Menschen oder die Bedrohung durch eine vorgehaltene Waffe eine derartige Wertung erfahren. Der Gutachter sollte bereits bei Beauftragung auf das Vorhandensein von gutachtenrelevanten Anknüpfungstatsachen achten.

\section{§ 226 Schwere Körperverletzung}

(1) Hat die Körperverletzung zur Folge, dass die verletzte Person 1. das Sehvermögen auf einem Auge oder beiden Augen, das Gehör, das Sprechvermögen oder die Fortpflanzungsfähigkeit verliert,

2. ein wichtiges Glied des Körpers verliert oder dauernd nicht mehr gebrauchen kann oder

3. in erheblicher Weise entstellt wird oder in Siechtum, Lähmung oder geistige Krankheit oder Behinderung verfällt (...)

Die Tatbestandsmäßigkeit der schweren Körperverletzung ist durch die Rechtsprechung katalogartig festgeschrieben, sodass sich der Sachverständige über die hierzu vorliegenden Einzelentscheidungen informieren sollte. Die im $\$ 226$ StGB definierten schweren Folgen der (zumindest bedingt) vorsätzlichen Körperverletzung müssen fahrlässig verursacht worden sein. Zu beachten ist, dass bereits der Versuch einer Körperverletzung, wenn er die schwere Schädigung ausgelöst hat, strafbar sein kann. Die Erschwernisgründe absichtlich oder wissentlich ermöglichen dem Richter eine starke Abstufung des Strafrahmens.

Weitere Tatbestände $(\$ \$ 216,218,222,225,229 \mathrm{StGB})$ sind in anderen Kapiteln im Sachzusammenhang abgehandelt.

\subsection{Kriminologie}

\author{
R. Wegener
}

\subsubsection{Einführung}

\section{- Definition}

Das Forschungsgebiet Kriminologie (lat. crimen $=$ Verbrechen) untersucht Ursachen, Umfang und Erscheinungsformen der Kriminalität. Kriminologie befasst sich darüber hinaus mit Behandlungsmöglichkeiten für Straftäter und mit den Auswirkungen von Strafe und Maßregel.

Die Kriminologie wird zusammen mit der Kriminalistik den nichtjuristischen Kriminalwissenschaften zugerechnet. Sie versteht sich als eigenständige empirische Wissenschaft, die Erkenntnisse der Strafrechtswissenschaften, Soziologie, Demogra-

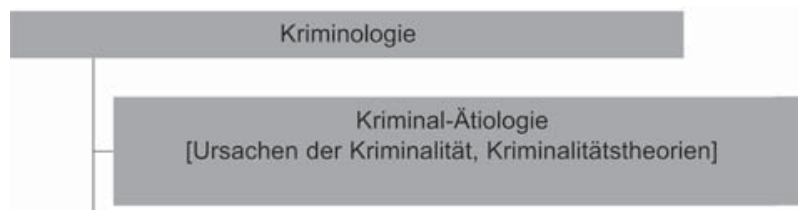

Kriminalphänomenologie

[Arten der Kriminalität, z.B. Gewalt-, Umweltkriminalität]

Kriminalstatistik

[Hell- und Dunkelfeld]

\section{Viktimologie}

[Opfer, Opferverhalten]

Poenologie und Kriminaltherapie

[Wirkungen der Strafe, Behandlungskonzepte]

Institutionenforschung

[Etikettierung abweichenden Verhaltens - labeling approach] [Strafverfolgungsbehörden u.a.]

Kriminalpsychiatrie, Forensische Psychiatrie [Psychische Erkrankungen als Kriminalitätsursache] [Schuldfähigkeit]

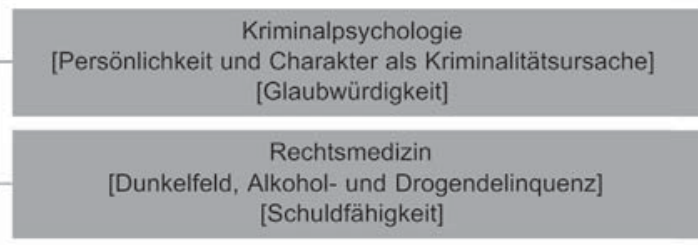

nach Schwind 2005

- Abb. 3.3. Forschungsbereiche der Kriminologie (Auswahl) 
phie, Psychiatrie, Psychologie und Ethologie in ihr Fachgebiet integriert. Da eine kurz gefasste, schlüssige Definition der Kriminologie nicht existiert, lässt sie sich am besten über ihre Arbeitsbereiche erschließen (• Abb. 3.3, Eisenberg 2005, Schwind 2005).

\section{- Definition \\ Die Kriminologie als Lehre von den Ursachen des Verbre- chens wird häufig mit der Kriminalistik als Lehre von der Be- kämpfung der Kriminalität verwechselt. Der Kriminalist be- fasst sich mit der Aufdeckung der Tat und der Überführung des Täters.}

Die Rechtsmedizin ist an kriminologischen Fragestellungen u.a. durch Untersuchungen zum Dunkelfeld der Gewaltkriminalität, zur Alkohol- und Drogendelinquenz und ihren Beitrag zur Schuldfähigkeitsbegutachtung unter Alkohol- und Drogeneinfluss beteiligt.

\section{(i) Vorgeschichte der Kriminologie}

Die wechselvolle Geschichte der Kriminologie beginnt mit einer 1764 von Cesare di Beccaria vorgelegten Schrift mit dem Titel »Über Verbrechen und Strafen«. Die Publikation des Strafrechtsreformers Beccaria setzt sich unter dem Einfluss der Aufklärung für ein faires Strafprozessrecht, gegen die Willkür der Polizei und für das Primat einer vorbeugenden Kriminalpolitik ein. Weitere Impulse kamen durch Cesare Lombroso, der mit seinen Hypothesen zur Erkennung des Verbrechers an äußeren Merkmalen (stigmata) in seinem Werk »Der Verbrecher« (1878) großes Aufsehen erregte. Die verkürzte Interpretation seiner Untersuchungen unter dem Schlagwort des "geborenen Verbrechers" (il nato delinquente) förderte eine Kriminalpolitik, die letztendlich die Unschädlichkeitmachung des Straftäters anstrebte. Obwohl Lombroso frühzeitig widerlegt wurde, ist der damalige Erfolg seiner italienischen kriminal-anthropologischen Schule durch die weite Verbreitung des Sozialdarwinismus erklärbar. Wortführer der französisch geprägten kriminal-soziologischen Schule wurde der Mediziner Alexander Lacassagne (1843-1924), der übrigens mit seinen forensisch-osteologischen Untersuchungen auch einen Meilenstein der Gerichtsmedizin gesetzt hat. Der soziologische Ansatz versteht den Täter als Spielball seiner Umwelt. Der deutsche Jurist Franz von Liszt (18511919) hat den Versuch unternommen, die Kriminalhypothesen der Französischen und Deutschen Schule zu vereinigen, indem er sowohl die Eigenart des Täters als auch die äußeren Tatumstände als kriminogene Faktoren anerkannte (Marburger Schule: Vereinigungstheorie). Er hat die Zusammenhänge von Sozial- und Kriminalpolitik erkannt und mit seiner Forderung nach spezialpräventiver Ausrichtung des Strafrech$\nabla$ tes neben der Prävention auch das Ziel der Besserung (Resozialisierung) verfolgt. Ausgehend von den kriminologischen Schulen entwickelten sich zahlreiche Kriminalitätstheorien, in denen biologisch-genetische, psychologischsozialpsychologische oder soziologische Erklärungsansätze favorisiert wurden.

\subsubsection{Kriminalstatistik und ihre Kritik}

Die vom Bundeskriminalamt Wiesbaden jährlich herausgegebene Polizeiliche Kriminalstatistik (PKS) registriert alle der Polizei bekannt gewordenen Straftaten einschließlich der mit Strafe bedrohten Versuche. Erfasst wird in der PKS nur das aufgeklärte Hellfeld. Da in Deutschland eine statistikbegleitende Dunkelfeldforschung nicht stattfindet, ist unklar, wie Hell- und Dunkelfeld miteinander korreliert sind. Veröffentlichte Zahlen der PKS können nur unter der Annahme annähernd konstanter Verhältnisse in definierten geographischen Räumen, bei vergleichbaren politischen und sozialen Rahmenbedingungen sowie bei Betrachtung über lange Zeiträume interpretiert werden. Relevante Einflussgrößen auf die Ergebnisse der PKS sind u.a.

- erhöhte Verfolgungsintensität durch die Polizei,

- Änderungen der Erfassungsstrategie der statistikführenden Institutionen,

- verstärkte Anzeigebereitschaft bei einem anwachsenden Gefühl des Bedrohtseins (fear of crime),

- wandelnde Auffassungen der Rechtsprechung und

- veränderte demographische Datenlage (Altersstruktur, Zuwanderung).

\section{Wichtig}

Trotz ihrer Fehlerquellen ist die PKS das beste Instrument zur Abschätzung von Daten zur Kriminalität. Sie ist dem delinquenten Verhalten sachlich und zeitlich am nächsten.

Die Strafverfolgungsstatistik (SVS) wird jährlich durch das Statistische Bundesamt Wiesbaden vorgelegt. Sie bezieht sich auf die von den Gerichten abgeurteilten Personen und liegt um Dimensionen unter den Tatverdächtigenzahlen. Von mehr als 50 Millionen begangenen Straftaten, die jährlich in Deutschland vermutet werden, kommen etwa 1 Million zur Aburteilung. Nur etwa 25 Prozent der wegen Mord und Totschlag polizeilich registrierten Tatverdächtigen werden verurteilt (•Tabelle 3.2).

Das Dunkelfeld der Delikte vorsätzlicher Tötung wird sehr kontrovers diskutiert und aus kriminologischer Sicht eher gering veranschlagt (Eisenberg 2005). Nach Schätzungen rechtsmedizinischer Fachvertreter wird eine Dunkelzifferrelation von etwa 1:1 angenommen.

Die Statistiken zur Gewaltkriminalität der vergangenen Jahrzehnte erlauben die Aussage, dass die Frequenz der registrierten Tötungsverbrechen, insbesondere nach sexueller Gewalt, nicht 
- Tabelle 3.2. Ausgewählte Straftaten (Fallzahlen) gegen das Leben und die körperliche Unversehrtheit. Polizeichliche Kriminalstatistik (PKS) versus Strafverfolgungsstatistik (SVS). Früheres Bundesgebiet (2000 einschl. Berlin-Ost)

\begin{tabular}{|l|l|l|l|l|l|l|l|}
\hline & \multicolumn{3}{|l|}{1970} & 1980 & 1990 & 2000 \\
\cline { 2 - 6 } & PKS & SVS & PKS & SVS & PKS & SVS & PKS \\
\hline Mord und Totschlag & 2.403 & 414 & 2.705 & 681 & 2.387 & 565 & 2.770 \\
\hline $\begin{array}{l}\text { Vergewaltigung und } \\
\text { sexuelle Nötigung }\end{array}$ & 6.889 & 1.159 & 6.904 & 1.876 & 5.112 & 1.554 & 7.499 \\
\hline $\begin{array}{l}\text { Sexueller Missbrauch } \\
\text { von Kindern }\end{array}$ & 16.468 & 2.511 & 13.165 & 1.790 & 12.741 & 1.687 & 15.581 \\
\hline $\begin{array}{l}\text { Gefährliche/Schwere } \\
\text { Körperverletzung }\end{array}$ & 37.895 & 9.692 & 65.479 & 13.738 & 67.095 & 12.737 & 116.912 \\
\hline $\begin{array}{l}\text { Vorsätzliche Körper- } \\
\text { verletzung }\end{array}$ & 47.919 & 12.260 & 112.021 & 15.132 & 128.880 & 16.199 \\
\hline
\end{tabular}

zugenommen hat. Hingegen zeigen die Häufigkeitszahlen - Anzahl der bekannt gewordenen Fälle auf 100.000 Einwohner - einen deutlichen Anstieg der Gewaltkriminalität. Überproportional beteiligt sind durch jugendliche und heranwachsende Täter begangene Raubtaten sowie Delikte der Körperverletzung bei vergleichsweise hoher Kriminalitätsbelastung in den neuen Bundesländern.

(i) Gewalt - Ursachen und Erscheinungsformen Gewalt entsteht in dynamischen Wechselwirkungsprozessen zwischen Täter, Opfer und Gesellschaft. Eine Handlung wird dann als »gewaltsam« interpretiert, wenn sie als zielgerichtet und sozial inadäquat wahrgenommen wird. Die große Medienpräsenz weniger spektakulärer Gewalttaten vermittelt den falschen Eindruck, dass Gewalt in der Öffentlichkeit stattfindet.

- Alltägliche Gewalt findet bevorzugt in der Familie, am Arbeitsplatz und im Bekanntenkreis statt. Aggressive Verhaltensmuster werden im sozialen Nahbereich erlernt und erprobt. - Institutionelle Gewalt wird durch die Institutionen selbst verursacht (Strafanstalten, Polizei, Schulen, Krankenhäuser und Seniorenheime).

- Strukturelle Gewalt ergibt sich aus ungleich verteilten Ressourcen, ungleichen Machtverhältnissen, Lebens- und Bildungschancen. Das Konzept der strukturellen Gewalt ist umstritten, da es die individuelle Komponente einer Gewalthandlung stark relativiert.

Eine Auswahl viel diskutierter Gewalttheorien zeigt, dass monokausale Erklärungsansätze der Komplexität von Gewalt nicht gerecht werden. Sie können allerdings einander ergänzen:

- Das psychoanalytische Konzept sieht nach Sigmund Freud die Aggression als Ausdruck des Todestriebes (Thanatos), der durch das Freisetzen von aggressiven Gefühlen (Katharsis) die Aggressivität vermindern kann. Das Konzept ist widerlegt, weil das Ausüben von Aggressionen die Aggressivität verstärkt.

- Die ethologische Aggressionstheorie kommt zu dem Ergebnis, dass der angeborene Aggressionstrieb des Menschen in der modernen, entwickelten Gesellschaft nicht entladen werden könne und das biosoziale Wesen »Mensch« keinen Hemm-Mechanismus für die Nichtanwendung von Aggressionen besitze. Die von Konrad Lorenz im Ergebnis von Tierversuchen entwickelte Instinkttheorie ist nicht auf den Menschen übertragbar.

- Die Frustrations-Aggressions-Theorie versteht Frustration als Verhinderung eines Handlungsentwurfes, wodurch Aggressionen ausgelöst werden können.

- Die soziostrukturellen Theorien gehen davon aus, dass gesellschaftlich-politische Verhältnisse und Wertvorstellungen die Gewaltentstehung begünstigen können. In Zeiten eines starken gesellschaftlichen Wandels kann es bei benachteiligten Bevölkerungsgruppen zu Orientierungslosigkeit kommen (Kulturkonflikttheorie, Anomietheorie). Der beobachtete starke Anstieg der Gewalt in den neuen Bundesländern in den ersten Jahren nach der Wende scheint das Konzept zu stützen.

- Das Subkultur-Modell aggressiven Verhaltens macht sich die Erkenntnis zunutze, dass in Umgebungen, bei denen Ag- 
gressivität eine hoch bewertete Eigenschaft ist, die Gewalt von Angehörigen derartiger Gruppierungen auch angewandt wird, um soziale Konflikte zu lösen. Subkulturen unterscheiden sich signifikant von übergeordneten Wertesystemen.

- Die sozial-kognitive Lerntheorie der Aggression geht von der Hypothese aus, dass die Aggression durch Anerkennung oder Belohnung erlernt und befördert wird. Dabei spielt die gegebene soziale Umwelt eine wichtige Rolle.

- Die Sozialisationstheorie wird durch die Erfahrung gestützt, dass autoritäre Erziehungspraktiken mit Ausübung von Gewalt und Aggressivität in der Familie auch bei den Kindern Aggressionen auslösen.

- Die Theorie der symbolischen Interaktion zielt auf die Situation zwischen Täter und Opfer ab. Nach Fehlinterpretation von Verhaltensweisen des Gegenüber kann es zu einer eskalierenden Auseinandersetzung kommen (nach Schneider 1994).

\subsubsection{Operative Fallanalyse (OFA)}

Die rechtsmedizinische Befunderhebung am Tatort und bei der Obduktion ist eine kriminalistische Aufgabe, wenn es um die Identifizierung von Personen und um die Individualisierung von Spuren geht. Kann ein Täter jedoch nicht zeitnah zur Tat und/ oder Auffindung einer Leiche ermittelt werden, ist eine umfassende Überschau erforderlich, um den Kriminalfall aus kriminalistischer und kriminologischer Sicht zu verstehen (Hoffmann und Musolff 2002). Der methodische Ansatz der operativen Fallanalyse (OFA) wurde von der US-amerikanischen Bundespolizei FBI zunächst zur Ermittlung von sexuell motivierten Gewaltdelikten und Tötungsverbrechen von Serientätern ermittelt. Die OFA soll über die Rekonstruktion eines Tatablaufes und die Interpretation der Begehungsweise zu Hypothesen führen, die ein Persönlichkeitsbild des gesuchten Täters (offender profiling) antizipieren. Es geht nicht nur um Hinweise zur Identität sondern auch um die Gefährlichkeitseinstufung des Täters. Das Täterprofil kann auch Handreichung für die Vernehmungsstrategie eines Tatverdächtigen sein. Bei Tatserien kann eine Typologie von Handlungsmustern - eine Handschrift des Täters (signature) erkennbar werden, die vom konkreten Tatverhalten, dem Modus operandi, abzugrenzen ist. Zunächst ist allerdings zu klären, ob eine Auffindungssituation überhaupt direkte Rückschlüsse auf das Tatverhalten zulässt.

\section{F) Fallbeispiel}

Eine 15-jährige Frau wurde als Opfer eines Gewaltverbrechens nahe einer Fußgängerbrücke in dichtem Gesträuch aufgefunden. Die Tötung erfolgte durch massive komprimierende Gewalteinwirkung gegen den Hals mit Mehrfachfrakturierung von Kehlkopf

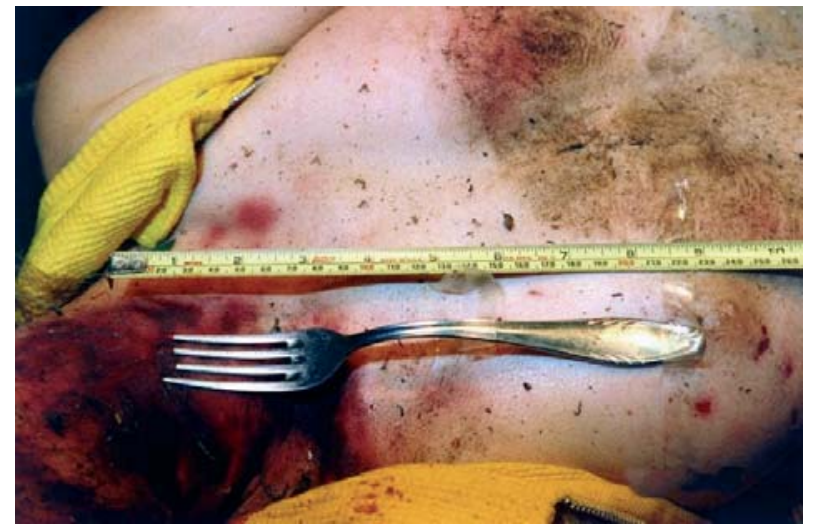

- Abb. 3.4. Avitale Stichverletzungen am Hals des Opfers, Nachtatverhalten mit psychopathologischer Komponente

und Zungenbein. Der Fall blieb zunächst unaufgeklärt. In der Folge verdichteten sich Hinweise, dass der mutmaßliche Täter zwischenzeitlich verstorben sein könnte.

Das Opfer war teilweise entkleidet. Auffällig war, dass der Täter die Kleidungsstücke der jungen Frau nach der eigentlichen Tötungshandlung am Tatort im Strauchwerk »platziert« hat. Post mortem übte der Täter mehrfache stumpfe Gewalteinwirkungen aus, wobei Gesicht und Oberkörper durch Erdreich verunreinigt wurden. Die Genitalregion war mit einem abgebrochenen Ast mehrfach perforiert worden. Offenbar gegen Ende der Handlungen applizierte der Täter eine Gabel in den Hals des Opfers (- Abb. 3.4). Das Nachtatverhalten deutet auf eine Erniedrigung und Verächtlichmachung des Opfers hin.

Die Verzahnung der operativen Fallanalyse mit einem Datenbanksystem erfolgte in Deutschland 1999 durch Einführung des Recherchesystems ViCLAS (Violent Crime Linkage System), das in Kanada entwickelt wurde. Erfasst werden in den ViCLAS-Erhebungsbögen neben den rechtsmedizinischen Befunden zur Leiche, wobei außergewöhnliche Begehungsweisen bzw. über die Tötungshandlung hinausgehende Handlungen besondere Beachtung finden, auch weitergehende Opferinformationen (Kleidung, Lebensstil, etc.). Die Trennung der Zuständigkeiten von Untersuchern bzw. Ermittlern einerseits und Fallanalytikern andererseits ist gewollt. Im Ergebnis der OFA wird eine Hypothese zur Chronologie der Ereignisse und eine Darstellung des Verbrechens mit einem bestimmten Plausibilitätsgrad angeboten. Mit dem Geständnis des Einzeltäters ist die Fallanalyse in aller Regel beendet. Die Zusammenarbeit der Rechtsmediziner mit den Täterprofilern ist ein neuer kriminalistischer Ansatz rechtsmedizinischer Untersuchungstätigkeit. 


\subsection{Allgemeine Traumatomechanik}

\section{H.-D. Wehner}

Fasst man aus physikalischer Sicht gesehen geeignet abgegrenzte räumliche Gebiete des menschlichen Körpers (z.B. Knochen, Haut, innere Organe, Gefäße, Sehnen, Bänder, usw.) je für sich oder auch in Kombination als Kontinua im mechanischen Sinne auf, so ist die Traumatomechanik als eine schädigende Krafteinwirkung zu definieren, die über eine Deformation eine Kontinuumsunterbrechung, also eine Gefügetrennung des Gewebes, nach sich zieht. Derartige Zusammenhangstrennungen werden als Verletzungen bezeichnet. Sie können mit Substanzverlust (z.B. Zahn-, Glieder-, Extremitätenverlust oder auch einschussbedingte Haut- und Knochendefekte) und ohne Substanzverlust einhergehen. Da mechanisch bedingte Verletzungen sehr häufig nicht durch statische Deformation, sondern durch sich relativ auf den Körper zu bewegende Massen (genannt: Werkzeuge) ausgelöst werden, bedarf es für deren Verständnis prinzipiell der kinetischdynamischen Betrachtungsweise. Dennoch führt auch die statische Betrachtungsweise zu heuristisch-plausiblen Erklärungen der Verletzungsmorphologie. Im Folgenden werden daher für die wichtigsten traumatomechanischen Phänomene derartige Plausibilitätsbetrachtungen angeboten:

In einem Kontinuum ist jedes Volumenelement gegenüber seinem Nachbarvolumenelement inneren Spannungen ausgesetzt (Man denke sich als Beispiel eine dreidimensionale Sprungfedermatratze). Für ein würfelförmiges Volumenelement der Kantenlänge Eins (• Abb. 3.5a) könnten diese Spannungen für jede der sechs Flächen in solche Komponenten zerlegt werden, deren Richtung senkrecht zur Flächenebene liegen (sog. Normal- spannungen), und in solche, deren Richtung tangential zur Fläche liegen (sog. Tangentialspannungen). In der $\bullet$ Abbildung 3.5a sind die Spannungen für die Grenzflächen eines Schnittes durch den Würfel eingezeichnet. Sie heben sich sowohl in den tangentialen Richtungen als auch in orthogonaler Richtung paarweise auf. Es ist hier ein Vertikalschnitt gewählt. Die Überlegungen gelten jedoch für jede andere Schnittrichtung in derselben Weise. Sind die Summen der Spannungen Null, findet weder eine Deformation noch eine Bewegung der Grenzflächen gegeneinander statt. Sind die Summen der Spannungen durch Einleitung äußerer Kräfte nicht Null, so vermögen sich die Schwerpunkte der Volumenelemente gegeneinander zu bewegen; gleichzeitig werden die Volumenelemente selbst deformiert. Sind derartig eingeleitete Spannungen in ihrer Resultierenden in Richtung der Normalspannungen wirksam, so heißen sie Zug(spannungen); wirken sie hingegen den Normalspannungen entgegen, werden sie Druck genannt. Wirken die von außen eingeleiteten Kräfte in Richtung der Tangentialspannungen, so spricht man von Schubspannungen. Wie im Folgenden beispielhaft gezeigt wird, kann jede traumatomechanische Zusammenhangstrennung plausibel durch die Einwirkung von Zug, Druck und Schub erklärt werden.

\subsubsection{Zusammenhangstrennung durch Zugspannungseinwirkung}

Überschreitet die Zugspannung die Festigkeitsgrenze eines Materials, so kommt es zu einer Zusammenhangstrennung, die vorwiegend senkrecht zur Zugrichtung gerichtet ist, nämlich zu einem Riss (-Abb. 3.5b). Derartige Risse findet man bei Überbe-
- Abb. 3.5a-d. Prinzip der durch Normalund Tangentialkräfte bewirkten Zusammenhangstrennungen. a Gleichgewicht von Normal- und Tangentialspannungen; b Zusammenhangstrennung durch Zug; c Zusammenhangstrennung durch Schub, c1 Grenzfall: Exkoriation, c2 Grenzfall: parallele Kratzer- und Schürfbildung (entsprechend der Struktur der verletzenden Oberfläche des Werkzeuges); d Zusammenhangstrennung durch die Kombination von Schub (Ablösung von der Unterlage) und Zug (Rissbildung). Zugbedingte Dehnungsstreifen bewirkt durch oberflächliche Rissbildungen, die quer zur Dehnungsrichtung verlaufen

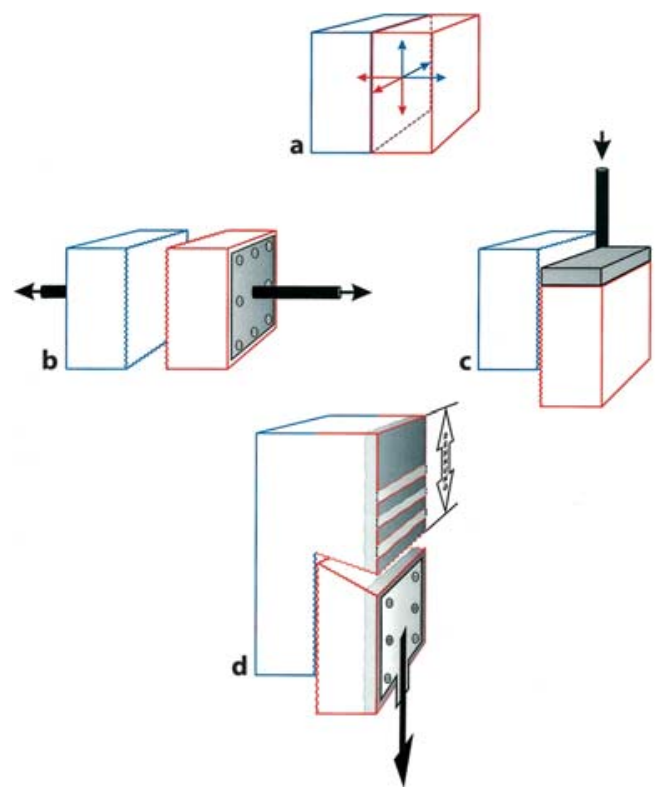

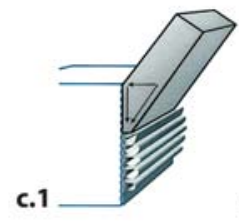

c.2

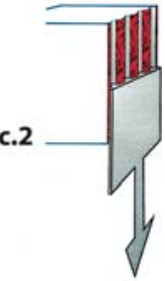


anspruchung durch Längendehnung z.B. von Bändern, Sehnen, Nerven, Gefäßen etc. Durch Gefäßrisse ergießt sich das Blut in das umgebende Gewebe oder in die Körperhöhlen (Hämatom). Die sich nach Gefäßzerreißung einstellende rein blutdruckbedingte Einspülung von Blut in umgebendes Gewebe (Suffusion, Sugillation) und damit die Form des Hämatoms hängt in erster Linie von der Beschaffenheit der Gewebedichte ab, sodass nach gefäßrisserzeugender Gewalteinwirkung aus der Hämatomform nicht oder nur sehr vage auf die Form des gewaltbringenden Schlagzeuges geschlossen werden kann. Erzeugt jedoch das Werkzeug den das Blut treibenden Druckgradienten aufgrund seiner Form und Profilierung selbst, so schlägt sich seine prägende Wirkung in der Form bzw. Textur des Hämatoms nieder. Beispiele hierfür sind die Hämatombildung nach Stock- und Peitschenschlag, das Reifenprofilhämatom (- Abb. 8.31a), aber auch die Textilabdruckspur. Ist unter der Zugwirkung lediglich die Intima eines Gefäßes gerissen, so liegt ein gedeckter Riss vor, der nicht selten zu einem Aneurysma führen kann.

Zugbedingte Zusammenhangstrennungen treten auch bei Überlastung von knöchernen Strukturen auf. Man spricht dann
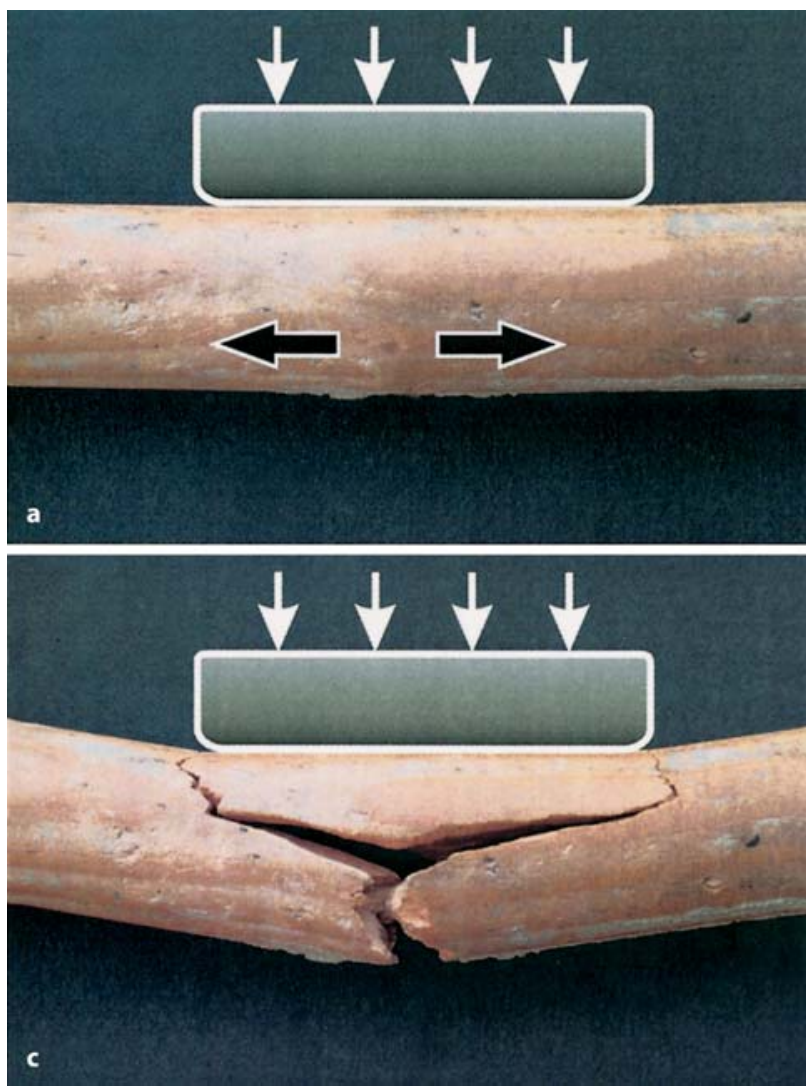

- Abb. 3.6a-d. Rissbruchentstehung am Stab. a Auftreten von achsenparallelen Spannungen gegenüber der Gewalteinwirkung; b Rissbildung gegenüber der Gewalteinwirkung und Auftreten neuer nicht mehr ach- seltener von einem Rissphänomen, sondern eher von Frakturen oder Berstungsphänomenen. Wird ein langer Röhrenknochen, der physikalisch idealisiert als Stab (Charakteristikum: Querschnitt klein gegenüber der dritten Dimension der Länge) angesehen werden kann (• Abb. 3.6a-d), durch eine quer zur Längsachse einwirkende Kraft (z.B. Stoßstange eines Personenkraftwagens) verbogen, so entwickelt sich auf der konvexen, also der der Krafteinwirkung fernen Seite, aufgrund der hier stattfindenden Dehnung eine vorwiegend achsenparallele Spannung, die bei Überdehnung zu einem infinitesimalen »Anfangsriss« führt. Dieser infinitesimale »Anfangsriss« durchsetzt nicht sofort den gesamten Querschnitt, denn er verändert im Kontinuum abrupt die anfänglichen vor der Rissentstehung herrschenden lokalen inneren Spannungsverhältnisse und setzt somit für die weitere infinitesimale Rissausbreitung eine erneute Anfangsbedingung, unter der sich das nächste infinitesimale Rissstück entwickelt und wiederum eine erneute Anfangsbedingung setzt. Die Aneinanderreihung dieser infinitesimalen Risse ergibt schließlich den Riss in seiner Gesamtheit. Man beobachtet, dass die rissbedingte Gefügetrennung auf zwei Flächen vonstatten geht, sodass letzt-
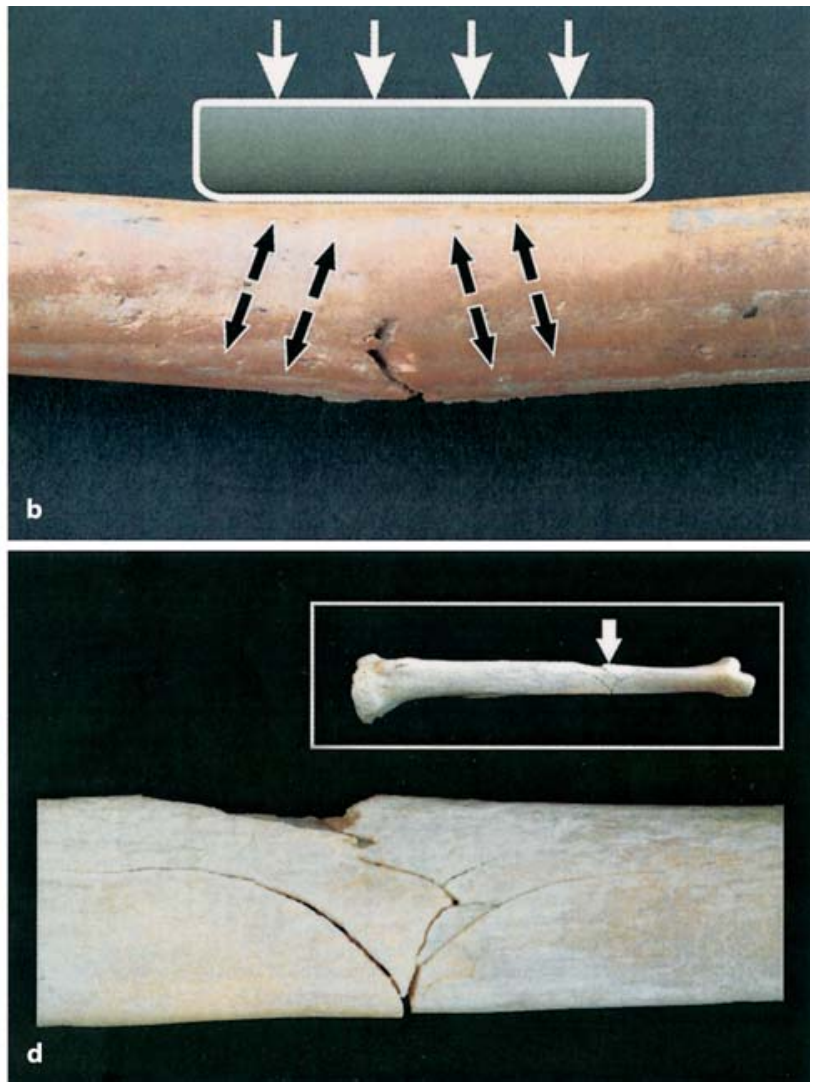

senparalleler Spannungen; c Keilbruch am Stab; d Keilbruch der Tibia (Messerer-Bruch) 
lich die für diese Gewalteinwirkung typische Keilform, der sog. Messerer-Bruch, entsteht. Da dieser durch Biegung entsteht, wird er Biegungsbruch genannt. Zugspannungsinduzierte Kontinuumsunterbrechungen treten nicht nur an stabförmigen Gebilden, sondern auch an Flächen auf. Wird z.B. die Haut extrem überdehnt (Beispiel: in der Leistenregion eines Fußgängers beim Auffahren von rückenwärts) so bilden sich streifenförmige parallel angeordnete Hautdehnungsrisse (• Abb. 3.16). Besondere flächenförmige Gebilde sind Schalen. Für die Zusammenhangstrennung von Schalen ist der Globusbruch des Schädels ein typisches Beispiel (• Abb. 3.7a-d und - Abb. 3.29):

Durch Schlag oder Sturz erzeugter Druck auf die Kuppel führt zu einer Umfangsvergrößerung der Breitengrade und damit zur Zugspannungsentwicklung. Diese führt zu Berstungen entlang der Longitudinalen (Berstungsbrüche). Dieser Vorgang ist nicht statisch $\mathrm{zu}$ verstehen, sondern Beobachtungen mit der High-speed-camera zeigen, dass sich die Bruchlinien vom Zentrum der Verformung mit hoher Geschwindigkeit nach peripher
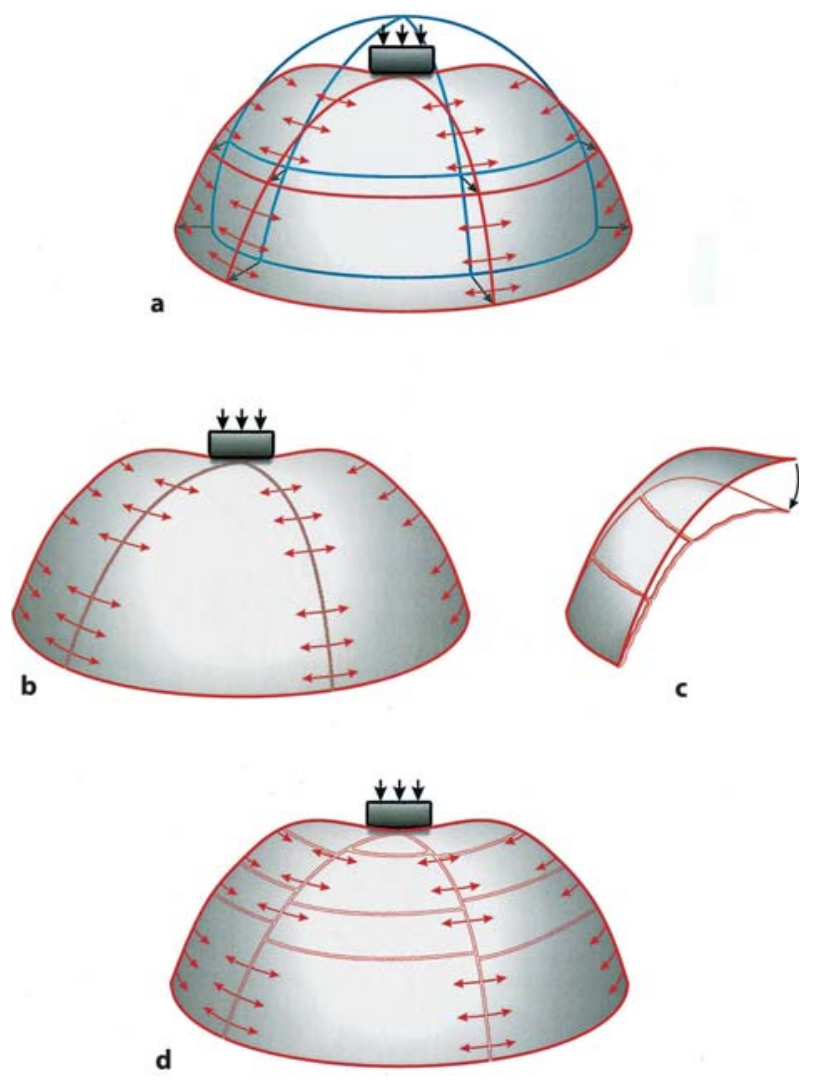

- Abb. 3.7a-d. Schalenbrüche (z.B. Globusbruch). a Umfangsvergrößerung der Breitengrade mit konsekutiver Spannungsentwicklung entlang der Longitudinalen; b Teilaspekt: longitudinale Berstungsbrüche; c Teilaspekt: Biegungsbrüche entlang der Breitengrade; d prinzipielles Muster des Globusbruches fortsetzen. Simultan mit den Berstungsbrüchen entstehen Biegungen der durch die Berstung entstandenen segmentalen Bruchschuppen, die ebenfalls zu Brüchen führen. Auf diese Weise entsteht das typische Globusbruchmuster des Schädels. Ein mit der Bruchbildung einhergehendes Einsinken der Kuppel führt auf der der Gewalteinwirkung fernen Schalenseite zu einer Bruchpyramidenbildung.

Da es zur Bruchfortpflanzung eines intakten Mediums, also eines intakten Knochens bedarf, überkreuzt eine Bruchfortpflanzungslinie niemals eine andere. So ist die Puppe'sche Regel zu verstehen, dass die peripheren Ausläufer des zeitlich nachfolgenden zweiten Bruches spätestens in den Bruchauslauflinien des ersten Bruches enden.

Schon bei der Erläuterung des Biegungsbruches, aber auch bei der Erklärung der Schalenberstung beim Globusbruch sieht man, dass die auslösenden Zugspannungen senkrecht zur Druckeinwirkungsrichtung gerichtet sind, dass die Zugspannung also erst indirekt über die druckbedingte Verformung des Kontinuums zustande kommt. Man muss daher, wenn man physikalisch ganz korrekt sein will, bereits bei diesen Zusammenhangstrennungen von einer indirekten Kraft- oder Gewalteinwirkung sprechen. Klar wird das Prinzip der indirekten Gewalteinwirkung am Beispiel der elastischen Kreis-Scheibe (• Abb. 3.8a). Wird auf diese in Richtung eines Durchmessers Druck und Gegendruck ausgeübt, so verformt sie sich unter Verlängerung des senkrecht zum Druckverlauf stehenden Durchmessers zu einer Ellipse. Der Zugspannungsaufbau in der größten Längsachse der Ellipse kann zum Riss bzw. zur Berstung führen. Berstungsbrüche der Schädelbasis unterliegen einer derartigen (oft durch einen Sturz bedingten) Traumatomechanik (• Abb. 3.28).

Allgemein spricht man von einer indirekten Gewalteinwirkung dann, wenn der Ort der Kontinuumstrennung nicht mit dem Ort der Krafteinleitung übereinstimmt.

Druckbedingte Zugspannungserzeugungen sind auch die Ursache der Riss-Quetsch-, besser Quetsch-Risswunde ( $\bullet$ Abb. 3.8b). Der Druck des Werkzeuges auf die auf ein Gegenlager (z.B. Schädelkalotte) gelagerte Haut führt zur Verformung senkrecht zur Krafteinleitungsrichtung und damit zu einem Zugspannungsaufbau, der bei Überschreitung der Festigkeitsgrenze entlang den Orten der geringsten Spaltbarkeit zum Riss führt. Da man es über den Hautquerschnitt verteilt mit unterschiedlich elastischen Gewebselementen zu tun hat, werden nicht alle $\mathrm{Ge}$ webselemente zerreißen; diese imponieren dann als Gewebsbrücken (• Abb. 3.15). - Abbildung 3.8b lässt erkennen, dass die werkzeugbedeckte Grenze $\overline{\mathrm{AB}}$ auf $\overline{\mathrm{A}^{\prime} \mathrm{B}}$ gedehnt wird. Dies ist mit einer Tangentialbewegung der Hautoberfläche verbunden und führt, da die Haut wegen des Quetschvorganges an die Werkzeugfläche gepresst ist, zu Schürfungen (Exkoriationen), die postmortal als Vertrocknungen imponieren. Kommt der Hautkontakt mit einem Prägemuster (z.B. Stoß durch Kühlergrill) zustande und sind die Verschiebungen nur minimal, so entsprechen die Hautabschürfungen dem Prägemuster und können dann als postmortale Vertrocknungen imponieren. Auch die Distanz $\overline{\mathrm{C}^{\prime} \mathrm{D}}$. 
- Abb. 3.8a-c. Durch Druck bedingte Verformungen und Zusammenhangstrennungen. a Druckbedingte Verformung einer elastischen Scheibe und dadurch induzierte Spannungsbildung $(\leftrightarrow)$ im Material; b Traumatogenese der Quetsch-Risswunde, c Traumatogenese der Stauchungsfraktur
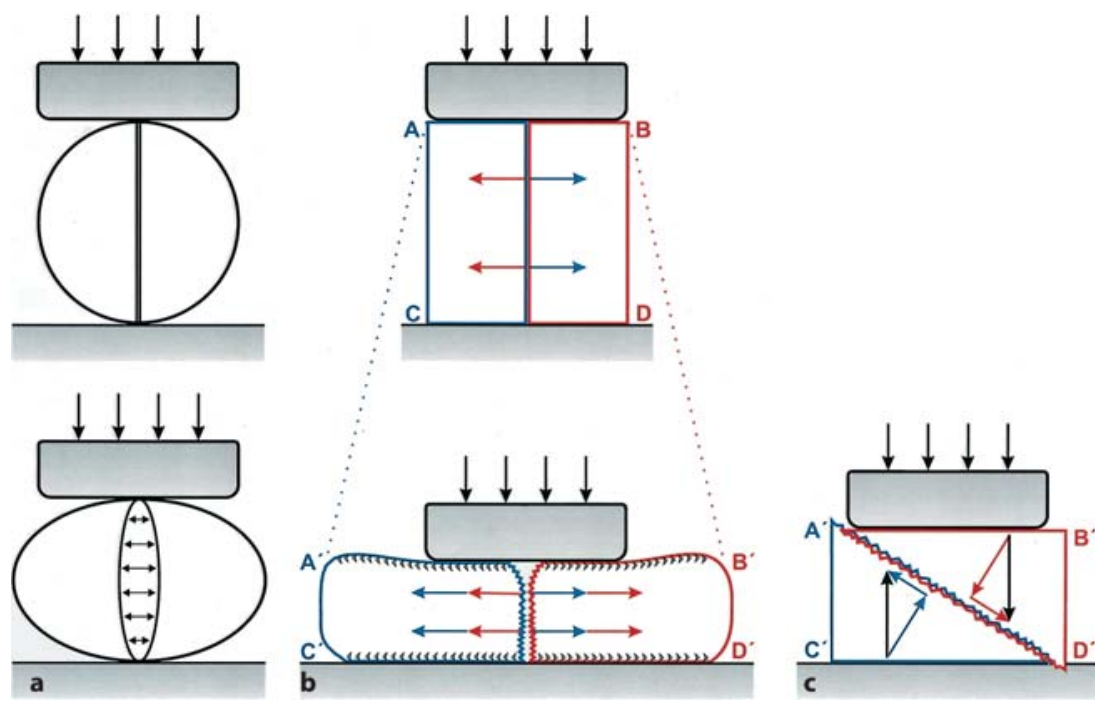

(- Abb. 3.8b) unterliegt infolge der traumatomechanischen Einwirkung einer Dehnung auf die Länge $\overline{C^{\prime} D^{\prime}}$. Konsekutiv mit der dehnungsbedingten Tangentialverschiebung geht hier eine Verankerung im Gegenlager verloren, so entsteht die häufig bei Quetsch-Risswunden zu beobachtende Unterminierung.

Ist das Kontinuum nicht so verformbar wie die Haut und ist es nicht auf einer Unterlage verschiebbar, so spricht man nicht von Quetschung, sondern von Stauchung. Während bei der Quetschung der Druck nahezu vollständig in eine Normalspannung umgelenkt wird und daher Schubspannungen vernachlässigt werden können, müssen Schubspannungen bei der Stauchung ganz unbedingt berücksichtigt werden (• Abb. 3.8c). Für jede Ebene in einem Volumenelement lässt sich der Druck in zwei zueinander senkrechte Komponenten zerlegen, von denen die einen gegeneinander und die anderen antiparallel gerichtet sind. Es gibt eine Ebene, in der die antiparallelen Kräfte bei gegebenem Druck maximal sind. Sind die antiparallelen Komponenten groß genug, so führen sie als Folge einer Scherung zu einer Gefügetrennung mit Dislokation, einem sog. stauchungsbedingten Gleitbruch oder kurz einer Stauchungsfraktur. Stauchungsfrakturen treten typischerweise an Wirbelkörpern auf. Auch die distale Radiusfraktur ist eine Stauchungsfraktur, sei es, dass sie durch das Abstützen einer Fallbewegung oder durch die Abstützhaltung am Lenkrad bei einem Aufprall zustande kommt. Auch die Dashboardfraktur proximal der Femurkondylen ist eine typische Stauchungsfraktur.

\subsubsection{Zusammenhangstrennungen durch Schubspannungseinwirkung}

Die Schubfestigkeit überwindende von außen eingeleitete Kräfte führen zu Scherverletzungen (• Abb. 3.5c). Der Scherbruch der
Schädelkalotte ist ein typisches Beispiel für eine solche wie gestanzt aussehende (daher auch Lochbruch genannte) Zusammenhangstrennung. Da (mit der nötigen Vorsicht!) die Konturen solcher Brüche der Werkzeugform zugeordnet werden können, spricht man von geformter Gewalt - im Gegensatz zu einer Einwirkung ungeformter Gewalt, für die eine Zuordnung zu einem formgebenden Werkzeug nicht möglich ist. Nicht nur an schalenförmigen Knochen, sondern auch an (im physikalischen Sinne stabförmigen) langen Knochen können Scherbrüche auftreten, nämlich dann, wenn die auftretende Gewalt so schnell (rasant) ist, dass es zu einer Biegungsverformung nicht kommen kann. Ein Grenzfall einer schubbedingten Verletzung ist die Exkoriation (• Abb. 3.5c1), in deren Entstehungsverlauf die oberflächlichen Hautabtragungen in Schubrichtung bewegt und oft ziehharmonikaartig zusammengestaucht werden, sodass aus diesem morphologischen Bild die Schubrichtung der einwirkenden Kraft abgelesen werden kann. Wie durch Deformation des Kontinuums Druckkräfte in Zugkräfte verwandelt werden können, so kann es auch durch Schubkräfte zu einer Zugbeanspruchung des Gewebes kommen (• Abb. 3.5d). Die großflächige Tangentialverschiebung der Haut z.B. kann einerseits zu deren Ablösung vom Untergrund (Décollement) führen und andererseits zusätzlich einen Riss bewirken (• Abb. 8.30). Auf diese Weise kommt es zu einem über den Riss erreichbaren zugänglichen Raum zwischen Untergrund und Haut, der sog. Wundtaschenbildung. Diese Taschen können wegen eines bogenförmigen Risses oft aufgeklappt werden. Es kommt zur Lappenbildung. Durch die Dehnung muss es nicht zwingend zu einem die ganze Hautschicht durchsetzenden Riss kommen, vielmehr ist es auch möglich, dass sich unter Einwirkung einer Tangentialkraft mehrere streifenförmig und parallel angeordnete oberflächliche Läsionen, sog. Dehnungsrisse (- Abb. 3.16), entwickeln. Wirkt die Tangentialkraft ausschließlich auf der Oberfläche, so spricht man von Schürfung. Die par- 
allel gerichteten Schürfspuren lassen zusammen mit den zusätzlich zu beobachtenden Exkoriationen auf die Richtung der Tangentialkraft schließen (• Abb. 3.5c2).

\subsection{Sekundärfolgen mechanischer Gewalteinwirkungen, Todesursachen}

\section{Oehmichen, S. Banaschak, B. Madea}

Definition
Neben den primären oder unmittelbaren Todesursachen
nach mechanischer Gewalteinwirkung können trotz medizi-
nischer Behandlung Folgen der primären Verletzungen mit
einer gewissen Latenzzeit zum Tode führen (Spättodesfälle
bzw. postintervalläre Todesfälle). Die Feststellung eines Todes
in Folge einer sog. sekundären Todesursache erfordert Kennt-
nisse der Krankengeschichte einschließlich des primären Ver-
letzungsmusters. Weiterhin zählen Komplikationen der not-
wendigen medizinischen Behandlung zu den sekundären
Verletzungsfolgen.

Primäre Todesursachen ( $\bullet$ Tabelle 3.3) sind zumeist bei einer Obduktion eindeutig festzustellen (Folgen grober äußerer Gewalteinwirkung) oder zumindest als Verdachtsdiagnose zu postulieren (Fettembolie, $>$ unten). Reflektorische Todesfälle, deren Existenz bzw. Genese nicht unumstritten ist, sind im Gegensatz dazu Ausschlussdiagnosen. Postintervalläre Todesfälle (•Tabelle 3.4) finden sich häufig nach Verbrennungen ( $\triangleright$ Kap. 3.9.1) und Verkehrsunfällen ( $\triangleright$ Kap. 8.4). Die Bestätigung oder der Ausschluss der Kausalität ergibt sich entweder aus dem morphologischen Befund bei der Obduktion allein oder erst nach Durchführung weiterer Untersuchungen. Die sekundären Todesursachen werden im weiteren erläutert.

\section{( Wichtig}

Ein mehr oder weniger langes Überleben nach einem Trauma muss daher die Kausalkette zwischen Gewalteinwirkung und Todeseintritt nicht unterbrechen. Bei durchgängiger medizinischer Behandlung (z.B. auf einer Intensivstation) ist dieser Zusammenhang offensichtlich. Verstirbt der Patient, handelt es sich um eine nichtnatürliche Todesart. Schwierigkeiten bei der Kausalitätsbeurteilung, z.B. durch vorbestehende Erkrankun-

$\nabla$

- Tabelle 3.3. Primäre oder unmittelbare Todesursachen nach mechanischer Gewalteinwirkung

Zertrümmerung lebenswichtiger Organe (Gehirn, Rückenmark, Lunge, Herz) Mechanische Behinderung der Funktionsfähigkeit lebenswichtiger Organe

Verbluten

Reflektorische Todesfälle

Luftembolie

Fettembolie der Lunge

Gewaltsames Ersticken
Einzeln oder kombiniert als Polytrauma nach z.B. (Verkehrs-) Unfällen, Stürzen aus der Höhe etc.

Raumforderung im Schädelinnenraum durch Blutungen beidseitiger Pneumothorax

(z.B. bei Rippenserienfrakturen mit Anspießungsverletzungen)

Thoraxkompression bei Verschüttung

Herzbeuteltamponade

Nach Innen oder Außen

Bolustod, Karotissinusreflex und/oder Reizung von Vagusbahnen bei Gewalteinwirkung gegen den Hals

Bei Eröffnung großer herznaher Venen und Eindringen von $>70 \mathrm{ml} \mathrm{Luft}$ bei ausgeprägten Weichteilverletzungen oder multiplen Knochenbrüchen komprimierende Gewalteinwirkung gegen den Hals, Thoraxkompression

- Tabelle 3.4. Ätiologie sekundärer Todesursachen

Infektionen

Embolien

Kreislaufschock

Verbrennungskrankheit
Wundinfektionen (Cave: Verschmutzungen), Pneumonien (nach Aspiration, hypostatische Pneumonie), bei Generalisierung: Sepsis

Thrombembolien bei Thrombosen (überwiegend der tiefen Beinvenen); verzögerte Fettembolien (aus Frakturen oder Weichteilverletzungen)

Die Kreislaufzentralisation (Reduktion der peripheren Durchblutung) kann zu unterschiedlichen Organbeteiligungen führen; sekundär kann ein sog. Multiorganversagen auftreten (Nierenversagen, Leberversagen, Lungenversagen bei ARDS)

Nach ausgedehnten Verbrennungen ( Kap. 3.9.1) 
gen, Fehler bei der Behandlung et cetera, können zumeist nur nach Durchführung einer Obduktion, eventuell histologischen Untersuchungen und unter Einbeziehung der Behandlungsunterlagen geklärt werden. Hierbei sollte zumindest durch die Bescheinigung einer ungeklärten Todesart eine Klärung herbeigeführt werden.

Die Wahrscheinlichkeit, ein Trauma zu überleben, hängt neben dem primären Verletzungsausmaß von zahlreichen weiteren Faktoren ab:

- Alter, Geschlecht,

- Vorerkrankungen (insbesondere des Herz-Kreislauf-Systems, Diabetes mellitus, Leberzirrhose) und Suchterkrankungen;

- Medikation: Kortikosteroide und Zytostatika verzögern die Wundheilung.

Die nach einer Verletzung erforderlichen Behandlungsmaßnahmen können ebenfalls mit spezifischen Risiken verbunden sein (-Tabelle 3.5); das Auftreten von Komplikationen darf dabei keinesfalls mit einem Behandlungsfehler gleichgesetzt werden.

\section{F) Fallbeispiel}

Eine 82-jährige Frau wurde nach einem Sturz mit einer Schenkelhalsfraktur ins Krankenhaus eingeliefert. Der Sturz hatte sich im Rahmen eines Handtaschenraubes ereignet. Die primäre Verletzungsbehandlung mit einer dynamischen Hüftschraube erwies sich als instabil, sodass eine Hüftprothese erforderlich wurde. Die Patientin hatte jeweils perioperativ Antibiotika erhalten. In Folge dieser Behandlung trat eine pseudomembranöse Kolitis auf, die trotz rechtzeitiger Diagnose und Therapie zu einer Sepsis führte. Die Patientin verstarb an den Folgen eines septischen Multiorganversagens. Es wurde eine nichtnatürliche Todesart bescheinigt und eine rechtsmedizinische Obduktion durchgeführt. Im abschließenden Gutachten wurde der kausale Zusammenhang zwischen dem Sturz und dem Todeseintritt bejaht. Die Täterin wurde nach Jugendstrafrecht zu einer 5-jährigen Haftstrafe wegen Raubes und Körperverletzung mit Todesfolge verurteilt.

\subsubsection{Infektionen}

Das Spektrum von Infektionen nach einem Trauma reicht von lokal beherrschbaren Wundinfektionen bis hin zu Allgemeininfektionen (Sepsis).

\section{Wundinfektionen}

Wunden beinhalten durch die Integritätsverletzung der Haut eine potentielle Infektionspforte, sowohl durch äußere Verunreinigungen als auch für Infektionen durch Keime der physiologischen Flora des Menschen. Bissverletzungen weisen aufgrund der Eintragung von Keimen durch den Speichel eine vergleichsweise hohe Infektionsrate auf. Im Allgemeinen sind Wundinfektionen heute durch entsprechende Desinfektion, Antibiotikabehandlung und/oder prophylaktische Impfungen selten geworden. Gefürchtet waren insbesondere:

- der Gasbrand (hervorgerufen durch Erreger der Gattung Clostridium, z.B. C. perfringens, C. novyi, C. septicum und C. histolyticum), anaerobe Wundinfektion und

- der Wundstarrkrampf (Tetanus, Erreger C. tetani), anaerobe Wundinfektion, auch bei kleinen Verletzungen (durch Holzsplitter, bei der Gartenarbeit); Impfung möglich.

\section{Infektionen bei besonderen Verletzungslokalisationen}

Je nach betroffener Körperregion können sich besondere Infektionsrisiken verwirklichen:

- Offene Schädel-Hirn-Verletzungen: Penetrierende Verletzungen des Hirnschädels mit freiliegender Dura mater oder von Hirngewebe, Schädelbasisfrakturen mit Austritt von Liquor und Mittelgesichtsverletzungen bergen die Gefahr einer Durchwanderungsmeningitis beziehungsweise -meningoenzephalitis.

- Penetrierende Darmverletzungen mit Austritt von Darminhalt in die freie Bauchhöhle können über eine kotige Peritonitis zum Tode führen.

\section{Pneumonien}

Bei Bewusstlosigkeit und Erbrechen besteht das Risiko einer Aspiration von Speisebrei durch Ausfall des Hustenreflexes. Ein primäres Ersticken aufgrund der vollständigen Verlegung der

- Tabelle 3.5. Behandlungsmaßnahmen und ihre Komplikationen

Reanimation

Langzeitbeatmung

Katheter

Antibiotikagaben, besonders bei Mehrfachbehandlung

Thrombosen
Frakturen von Sternum und Rippen (sollten keine todesursächliche Relevanz erlangen!)

Adult respiratory distress syndrome (ARDS), Pneumonien

Erhöhtes Infektionsrisiko (insbesondere zentralvenöse Zugänge)

Nosokomiale Infektionen, besonders mit resistenten Keimen

Können trotz adäquater Therapie (die zu überprüfen ist) auftreten und zu Lungenembolien führen 
Atemwege ist dabei selten. Häufiger wird ein gewisser Teil des Aspirates im Rahmen der Reanimation entfernt. Die in den tieferen Atemwegen verbliebenen Anteile führen durch die Andauung des Gewebes durch die enthaltene Magensäure zu einer sog. Aspirationspneumonie, die trotz adäquater Therapie mit einer hohen Letalität behaftet ist.

Die verletzungsbedingte Immobilisation kann zur Ausbildung hypostatischer Pneumonien führen. Dabei führt die Minderbelüftung der dorsalen Lungenabschnitte zu einer Begünstigung entzündlicher Veränderungen.

\section{Sepsis}

\section{Definition}

Sepsis ist eine Allgemeininfektion des Körpers durch Zirkulation von Bakterien beziehungsweise deren Wandbestandteilen oder Pilzen in der Blutbahn. Das klinische Bild ist von intermittierenden Fieberschüben gekennzeichnet. Foudroyante Verläufe können innerhalb weniger Stunden zum Tod führen.

Unabhängig vom Ausgangspunkt der Sepsis und dem Erreger im konkreten Fall wird das pathologisch-anatomische Bild bei der Sektion durch die Ausbildung von Schockorganen bestimmt (s. dort). Histologisch können bei bakteriellen Infektionen septische Streuherde in quasi allen Organen nachweisbar sein.

\subsubsection{Embolien}

Definition
Die Verschleppung körpereigener oder -fremder Substanzen
mit dem Blutstrom, die zum Verschluss von Gefäßen führt, ist
eine Embolie.

Direkt posttraumatisch kann es zur Verschleppung von Fremdmaterialien (z.B. Geschossen beziehungsweise Geschossbestandteilen) oder Gewebebestandteilen bei Organzertrümmerungen kommen. Diese sind zumeist nicht die eigentliche Todesursache, sondern als Vitalitätszeichen zu sehen (s. dort). Luftembolien bei Eröffnung großer, herznaher Venen führen ab einem Volumen von $>70 \mathrm{ml}$ zum sofortigen Tod.

\section{Fettembolie}

Fettembolien sind eine direkte Traumafolge. Diese entstehen aus zertrümmertem subkutanem Fettgewebe, seltener einer Fettleber oder durch Freisetzung aus dem Knochenmark bei Frakturen. Gelegentlich können im Rahmen eines Schocks Blutfette emulgieren. Auch postoperativ können Fettembolien auftreten, insbesondere nach Knocheneingriffen. 20-30 g Fett sollen für eine tödliche Fettembolie erforderlich sein. Todesursächlich können bei Fettembolien sein:
- Ein Rechtsherzversagen bei Verlegung der Lungenstrombahn (Angaben zwischen 1/3-3/4 aller Lungenkapillaren zur todesursächlichen Relevanz), bei Vorschädigungen können auch geringergradige Fettembolien zum Tode führen.

- Fettembolien des Gehirns (entweder direkt bei offenem Foramen ovale im Sinne einer gekreuzten Embolie oder verzögert durch Herauslösung des Fettes aus der Lungenstrombahn, 3-14 Tage nach dem Ereignis); diese imponieren klinisch durch auftretenden Schwindel, Erbrechen, Krämpfe und Bewusstseinseintrübungen.

\section{(i) Infobox}

Der Nachweis einer Fettembolie erfolgt immer durch histologische Untersuchungen (Fettfärbung). Die Graduierung der pulmonalen Fettembolie kann wie folgt vorgenommen werden:

0 . Keine Fettembolie oder ganz vereinzelt embolische Fetttropfen bei systematischer Suche,

1. leichte Fettembolie - einzelne bis mehrere embolische Fetttropfen in jedem Gesichtsfeld,

2. deutliche Fettembolie - zahlreiche Embolien in jedem Blickfeld und

3. massive Fettembolie - massenhaft Fetttropfen in jedem Gesichtsfeld. Mehr als die Hälfte der Lungenkapillaren enthält Embolien.

Im Gehirn können zusätzlich - eventuell schon makroskopisch erkennbare - Ring- oder Kugelblutungen (Mark des Mittelhirns, Pons, Capsula interna und Corpus callosum) nachweisbar sein.

\section{Thrombembolie}

Die posttraumatische Immobilisation des Patienten durch Bettruhe oder Anlage eines Gipsverbandes kann - trotz lege artis durchgeführter Prophylaxe - zur Venenthrombose, zumeist in den tiefen Beinvenen führen. Wird der Patient dann erneut mobilisiert, können sich Anteile der Thromben lösen und die Lungenstrombahn erreichen. Je nach Größe führen sie dort zu peripheren oder zentralen Lungenembolien. Der plötzliche Verschluss eines Pulmonalarterienhauptastes führt zu einem akuten Rechtsherzversagen. Zusätzliche Risikofaktoren für die Entwicklung einer Thrombose (Adipositas, weibliches Geschlecht, Krampfadern, Rauchen, umstritten: Einnahme von oralen Kontrazeptiva) erfordern eine erhöhte Aufmerksamkeit. Seltener embolisieren Thromben aus anderer Lokalisation (Armvenen, von Katheterspitzen). Einen Sonderfall stellt die sog. gekreuzte Embolie dar. Durch das offene Foramen ovale gelangen dabei die Thromben in den großen Kreislauf und können zu Infarkten (Gehirn, Extremitäten, Nieren, Darm) führen. 


\subsubsection{Schock}

\section{Definition}

Zahlreiche unterschiedliche Ereignisse können zu einem Schockzustand führen, der durch eine Dekompensation des peripheren Kreislaufs mit Mangeldurchblutung in der kapillären Strombahn gekennzeichnet ist.

Schockursachen sind in $\bullet$ Tabelle 3.6 zusammengefasst. Die primären Organveränderungen werden durch die Hypoxie bedingt und manifestieren sich klinisch durch einen zunehmenden Organausfall (•Tabelle 3.7+3.8), der letztendlich in ein Multiorganversagen münden kann. Bei den auftretenden Gerinnungsstörungen handelt es sich zumeist um eine Verbrauchskoagulopathie (disseminierte intravasale Gerinnung). Tritt der Tod innerhalb kurzer Zeit nach dem Trauma ein, besteht an der Kausalität kein Zweifel. Wird das primäre Trauma längere Zeit überlebt, können vorbestehende Erkrankungen den Ausgang (mit)bestimmen, wobei für den Kausalitätsnachweis dann weitergehende Untersuchungen erforderlich sind ( $\bullet$ oben).

- Tabelle 3.6. Ätiologie verschiedener Schockzustände nach einem Trauma

\begin{tabular}{|l|l|}
\hline Bezeichnung & Ätiologie \\
\hline Hypovolämischer Schock & $\begin{array}{l}\text { Ab ca. } 30 \% \text { venös bedingten Blutverlustes, bei arteriellem Blutverlust eventuell schneller; Plasmaver- } \\
\text { lust; Verlust extrazellulärer Flüssigkeit }\end{array}$ \\
\hline Kardiogener Schock & $\begin{array}{l}\text { Akute Herzinsuffizienz; Cave: vorbestehende Herzerkrankungen, dabei können auch geringere Blut- } \\
\text { verluste zu einem Schock führen }\end{array}$ \\
\hline Septischer Schock & Ausgelöst durch Endotoxine bei Infektionen mit gramnegativen Bakterien im Rahmen einer Sepsis \\
\hline Neurogener oder spinaler Schock & Bei Rückenmarksverletzungen \\
\hline Pankreatischer Schock & Akute Pankreatitis \\
\hline
\end{tabular}

- Tabelle 3.7. Spezifische Organveränderungen bei Schock

\begin{tabular}{|c|c|c|}
\hline Organ & Makroskopische Veränderungen & Mikroskopische Veränderungen \\
\hline Herz & Erweichung des Myokards & Disseminierte Myokardnekrosen \\
\hline Niere (sog. Schocknieren) & $\begin{array}{l}\text { Sog. trübe Schwellung; blasse } \\
\text { Nierenrinde, betonte Markkegel }\end{array}$ & $\begin{array}{l}\text { Tubulusektasie, Epitheldegeneration, intravasale Zellansamm- } \\
\text { lungen in den Vasa recta; Tubulusnekrosen }\end{array}$ \\
\hline Magen und Dünndarm & Akute Ulzera oder hämorrhagische Erosionen & \\
\hline
\end{tabular}

- Tabelle 3.8. Allgemeine Organveränderungen bei Schock 


\subsection{Vitale Reaktionen und Zeitschätzungen}

\section{Oehmichen, S. Banaschak, B. Madea}

Vitalen Reaktionen kommt eine herausragende Bedeutung bei der Beurteilung gewaltsamer Todesfälle zu. In der Alternative vital oder postmortal kulminieren praktisch alle Fragen zum Beweiswert von Befunden bei gewaltsamen Todesursachen. Aber nicht nur die Differenzierung zu Lebzeiten erlittener, vitaler Verletzungen von postmortalen ist von Bedeutung, sondern ebenso die Bestimmung der Überlebenszeit, unter Umständen die der Priorität, der Reihenfolge von Verletzungen.

\section{- Definition \\ Als vitale Reaktionen bezeichnet man Folgen am Organismus auf eine Traumatisierung, die einen sicheren Rückschluss da- rauf zulassen, dass das Trauma zu Lebzeiten - vital - einge- wirkt hat. \\ Zu differenzieren sind im Einzelnen: \\ - Vitale Reaktionen: nach Schädigung auftretende örtliche Veränderungen, Anzeichen der Gegenwirkung (Reaktion) des lebenden Gewebes \\ - Vitale Prozesse: Zusammengesetzte physiologische Vor- gänge, deren Vorbedingung das Bestehen der Funktion des Nervensystems, Atmungsapparates, Gefäß- und Lymphsys- tems, der Darmbewegung und Harnausscheidung ist. Funk- tionieren des ganzen Organismus, nicht nur von Zellen und Geweben \\ - Vitale Zeichen: Zustandsbilder, von welchen auf vitale Entstehung geschlossen werden kann (arterielle Spritzspur, Bluteinatmungsherde, Blutverschlucken)}

Vitale Reaktionen sind abzugrenzen von agonalen, supravitalen und postmortalen Veränderungen, wobei dies anhand des morphologischen Befundes allein nicht immer möglich ist. Die Abgrenzung vital entstandener Verletzungen ist wichtig für die Kausalitätsbeurteilung nach Gewalteinwirkung, da sich erhebliche rechtsrelevante Konsequenzen ergeben können.

Als allgemeine Vitalreaktionen bezeichnet man z.B. die durch Kreislauf und Atmung vermittelten vitalen Reaktionen, lokale Vitalreaktionen bilden sich am Ort der Gewalteinwirkung aus (Blutung, Blutunterlaufen, Entzündung usw.).

Als Teilaspekt der vitalen Reaktion dient die Wundaltersbestimmung wesentlich der Beantwortung der Frage nach der Überlebenszeit nach Traumatisierung. Die Agoniedauer bei rasch zum Tode führenden Verletzungen unterschreitet zumeist die Manifestationszeit lokaler Vitalreaktionen, die im Bereich von zumindest ca. 10-20 Minuten liegt, während die durch Kreislauf und Ventilation vermittelten, relativ einfachen, allgemeinen vitalen Reaktionen sich noch ausbilden können, da die großen Funktionssysteme noch kurze Zeit den Individualtod überdauern.
(D) Wichtig

Der Nachweis einer vitalen Reaktion bedeutet nicht, dass der Mensch zum Zeitpunkt der Ausbildung der Vitalreaktion noch gelebt hat, sondern dass die Funktionssysteme, auf denen die vitale Reaktion beruht, zum Zeitpunkt der Verletzungsentstehung intakt waren. Deutlich wird dies bei einer Dekapitation als Verletzung mit momentanem Todeseintritt. Der Nachweis einer Blutaspiration nach Dekapitation beweist, dass (a) der Kreislauf und (b) die Atemmotorik bei Verletzungsentstehung intakt waren. Umgekehrt werden bei stehendem Kreislauf im Gehirn, z.B. beim sog. intravitalen Hirntod, reaktive Veränderungen von Seiten hämatogener Zellen im Hirngewebe nicht mehr auftreten, während sie in allen übrigen Organen nachweisbar sein können.

\subsubsection{Ereignisort und Spurenbild}

Liegen Blutspuren (nicht Blutlachen) vor, so können diese Hinweise auf eine vitale Verletzungsentstehung geben (vitaler Blutverlust versus passives Ausbluten). Dies ist bei der Obduktion durch morphologische Zeichen des vitalen Blutverlustes zu bestätigen. Finden sich weitere Spuren, lassen diese eventuell Rückschlüsse auf die Handlungsfähigkeit bzw. Position des Opfers $\mathrm{zu}:$

- Trittspuren des Opfers in Blutlachen (mit entsprechenden Blutantragungen an den Fußsohlen) beweisen eine erhaltene Handlungsfähigkeit.

- Arterielle Blutspritzspuren beweisen einen funktionsfähigen Kreislauf bei Verletzungsentstehung.

- Abrinnspuren (nicht nur am Leichnam selbst) können Informationen über die Haltung des Opfers (senkrecht stehend, sitzend oder liegend usw.) geben.

- Abwehrverletzungen weisen auf eine Auseinandersetzung hin.

\subsubsection{Organsysteme}

Die in - Tabelle 3.9 aufgeführten Vitalreaktionen unterschiedlicher Organsysteme sind teilweise nur bei einer Obduktion zu erheben. Sie lassen Rückschlüsse darauf zu, dass das entsprechende Organsystem bei Traumatisierung intakt war. Zeitliche Rückschlüsse sind nur sehr begrenzt möglich.

\section{(8) Wichtig}

Die agonale Speisebreiaspiration ist ein häufiges Phänomen, das an sich keine todesursächliche Relevanz hat und allenfalls den Rückschluss auf eine Bewusstlosigkeit zulässt (Ausfall des Hustenreflexes). Bei todesursächlich relevanter Aspiration (Ersticken) muss die Frage geklärt werden, warum eine Bewusstlosigkeit vorgelegen hat. 
- Tabelle 3.9. Vitale Reaktionen unterschiedlicher Organsysteme

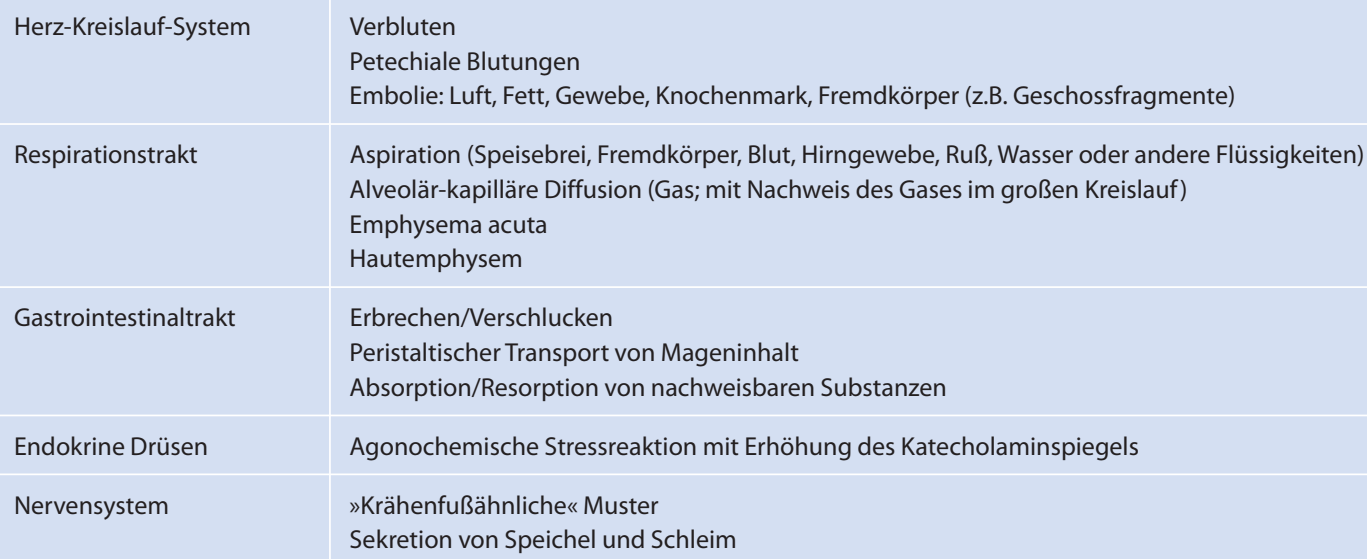

\section{(3) Fallbeispiele}

Eine 46-jährige Frau wurde mit insgesamt 6 Schüssen getötet. Todesursächlich waren mehrere Kopfschüsse mit schussbedingter Abtrennung des verlängerten Marks. Bei der histologischen Untersuchung zeigte sich eine Embolisation von Lebergewebe in einer Lungenschlagader. Zum Zeitpunkt des den Leberlappen zerreißenden Schusses muss demnach ein funktionierender Kreislauf vorgelegen haben.

Eine 76-jährige Frau wurde von ihrer Tochter, die an einer paranoid-halluzinatorischen Psychose litt, massiv geschlagen und getreten. Todesursächlich waren beidseitige Rippenserienfrakturen in Kombination mit einem Verbluten nach innen und außen. An Vitalitätszeichen lagen bei der histologischen Untersuchung Knochenmarks- und Fettembolien aus den Rippenfrakturen vor.

Weitere typische Kombinationen von Verletzungsursachen und Vitalitätsnachweis sind:

- Zustand nach Verkehrsunfall mit knöchernen Verletzungen und/oder Décollement von Fettgewebe (z.B. bei Überrollung):

- Nachweis von Fettembolien ( Kap. 3.4 »Sekundäre Todesursachen «),

- Kompression der Halsweichteile (bei primärem Verschluss der venösen Gefäße):

- Petechiale Einblutungen (an Augenlidern, -bindehäuten, hinter den Ohren; - Kap. 3.8.1 »Ersticken«,

- Todesursächliche Blutverluste (nach innen oder außen):

- Zeichen des Verblutens (geringe Ausdehnung und Intensität der Totenflecke, Hervortreten der Organeigenfarbe der inneren Organe, Milzkapselrunzelung, subendokardiale Blutungen),

- Blutaspiration bei Verletzungen der Halsweichteile (Dekapitation bei sog. Bahnleichen), Traumata des Gesichtsschädels oder der Schädelbasis:
- Blut in kleineren Bronchien und subpleurale Bluteinatmungsherde

\section{Wichtig}

Von einer vitalen Aspiration abzugrenzen ist die passive Verlagerung von Blut oder Speisebrei in die Trachea und großen Bronchien als postmortales Artefakt (zum Beispiel beim Leichentransport) oder als Epiphänomen nach Intubation und Beatmung.

\section{Zeitverlauf}

Bei Abschätzung der Zeitdauer zwischen Verletzungsbeibringung und Todeseintritt anhand systemischer Reaktionen muss die Dauer der Agoniephase berücksichtigt werden. Aussagen zur Verletzungsreihenfolge können bei Verletzungsbeibringung durch mehrere Täter oder zur Bestimmung eines Handlungsablaufes erforderlich sein. Sie sind jedoch nur mit großer Zurückhaltung unter Berücksichtigung aller Begleitumstände möglich. Gelingen kann sie dann, wenn verschiedene Arten von Gewalteinwirkungen gegen unterschiedliche Funktionssysteme gerichtet sind.

\section{F) Fallbeispiel}

Eine 35-jährige Frau wird gefesselt im Wasser in Ufernähe aufgefunden. Bei der Sektion finden sich schwere Schädel-Hirn-Verletzungen mit grobscherbiger Zertrümmerung des Scheitelbeins links und Austritt von Hirngewebe. Als Vitalitätszeichen der Kopfverletzungen zeigten sich zum einen massive Unterblutungen der Kopfschwarte, zum anderen Bluteinatmungsherde in beiden Lungen. Diese Verletzungen wären bereits für sich genommen geeignet gewesen, den Tod zu erklären. Es konnten jedoch zusätzlich ein Schaumpilz vor der Nase sowie Schaum in den tieferen Atemwegen nachgewiesen werden. Dies erlaubte die Feststellung, dass zum Zeitpunkt der Verbringung der sicherlich bewusstlosen Frau in das Wasser zumindest die Atmungsfunktion erhalten war und sie letztendlich ertrunken ist. 


\subsubsection{Biochemische Veränderungen im Blut - Pharmakokinetik}

Rein postmortale Veränderungen klinisch-chemischer Parameter können zur Todeszeitbestimmung herangezogen werden (etwa Anstieg der Kaliumkonzentration in der extrazellulären Flüssigkeit). Der Nachweis posttraumatischer biochemischer Veränderungen (z.B. Katecholamine, Serotonin, Histamin) zur Vitalitätsdiagnostik (sowohl systemisch als auch lokal an Wunden) wurde von zahlreichen Autoren untersucht. Die Beurteilung der Befunde ist jedoch schwierig, da es postmortal zum enzymatischen Abbau, Diffusion aus einem Kompartiment in das andere oder Zellabbau mit Freisetzung primär intrazellulärer Bestandteile kommt.

\section{(. Wichtig}

Die Reaktionen können bei sofortigem Todeseintritt negativ ausfallen, da sie zur Ausbildung die Leistungsbereitschaft verschiedener Funktionssysteme beanspruchen.

Sie könnten daher zur Differenzierung von Reflextodesfällen von solchen mit kurzer oder längerer Agoniephase (Stressreaktion mit Erhöhung der Katecholamine) eingesetzt werden. In der Praxis haben sich derartige Untersuchungen bisher nicht durchgesetzt.

Eher lassen pharmakokinetische Daten Rückschlüsse auf Fragen der Vitalität und den posttraumatischen Zeitablauf zu, wenn ausreichend Anschlussdaten zur Verfügung stehen:

- Fehlende Verstoffwechselung von Medikamenten oder Drogen (wie z.B. Morphin)

- agonale Applikation oder die Einnahme einer hohen, sofort tödlichen Dosis

- Hohe Urin- bei vergleichsweise niedriger Blutalkoholkonzentration

- längere Überlebenszeit nach Trinkende
- Nachweis von hohen Alkoholkonzentrationen im Hämatomblut bei subduralem Hämatom und Vergleich mit der Alkoholkonzentration im peripheren (intravasalen) Blut

- Informationen über die Dauer des posttraumatischen Intervalls und Anhaltspunkt für die Alkoholisierung zum Zeitpunkt der Hämatomentstehung

\subsubsection{Histologische Untersuchungen}

Nach Schädigung von Organen und Geweben - sei es aus innerer krankhafter Ursache (Infarkte, Embolien) oder traumatisch (penetrierende Hautverletzungen) - setzen regelhaft ablaufende Reparaturprozesse ein, die man als Wundheilung bezeichnet. Die Reparaturprozesse dienen neben der Blutstillung dem Umund Wiederaufbau des geschädigten Gewebes (• Abb. 3.9, Tabelle 3.10).

Die Reaktionen verschiedener Gewebe auf Schädigungen durch direkte mechanische Gewalteinwirkung verlaufen dabei für alle Gewebe gleichartig. Die körpereigenen Reparations- und Regenerationsmechanismen laufen phasenweise ab, wobei sich die einzelnen Phasen überlappen können. Die relative Konstanz des phasenhaften Ablaufes erlaubt eine Altersbestimmung, d.h. Bestimmung des Intervalls zwischen Zeitpunkt des Traumas und Tod. Mögliche Einflussfaktoren auf den Reaktionsablauf sind in - Tabelle 3.11 zusammengefasst.

\section{Phasen der Gewebereaktion}

- Phase der Gewebedestruktion: Gewebenekrosen, Einblutungen; Freisetzung biogener Amine, Proteoglykane, Proteasen, Lipidmediatoren, Zytokine.

- Phase der hämatogenen Zellreaktion: Emigration von Thrombozyten, neutrophilen Granulozyten, Monozyten/
- Abb. 3.9. Phasen der Wundheilung

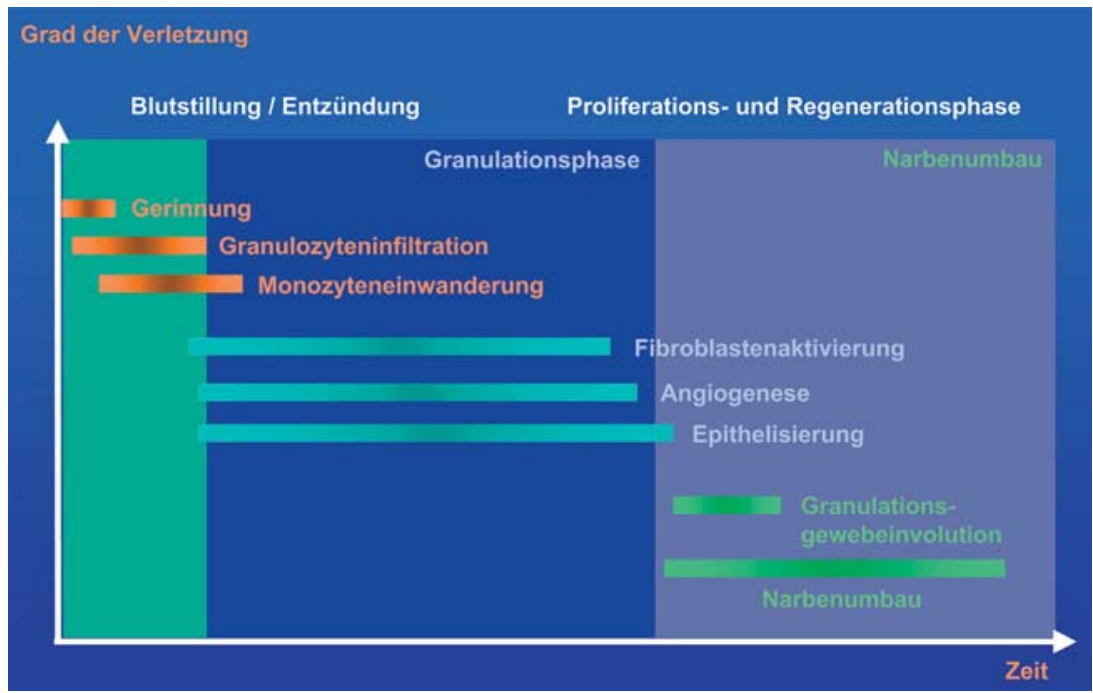


- Tabelle 3.10. Phasen der Reparation von Geweben (nach Oberholzer 2001)

\begin{tabular}{|c|c|c|c|}
\hline Phase & Schritt & Intervall & $\begin{array}{l}\text { Hauptakteure (zelluläre oder molekulare Mediato- } \\
\text { ren) }\end{array}$ \\
\hline 1 & Hämostase & Minuten & Fibrin, Thrombozyten \\
\hline 2 & $\begin{array}{l}\text { Aufbau einer provisorischen } \mathrm{ECM}^{1} \\
\text { durch Vernetzungen } \\
\text { Wundödem } \\
\text { Enzymaktivierung }\end{array}$ & Stunden & $\begin{array}{l}\text { Fibronektin, Fibrin, Kollagen, Moleküle, die von Thrombozy- } \\
\text { ten und neutrophilen Granulozyten abgegeben werden } \\
\text { Histamin-, Serotoninfreisetzung }\end{array}$ \\
\hline 3 & Entzündungsreaktion & Stunden & \\
\hline 3.1 & $\begin{array}{l}\text { Auftreten von Chemotaxinen für neurophile Granulo- } \\
\text { zyten }\end{array}$ & & $\begin{array}{l}\text { Komponenten des Komplementsystems und Gewebe- } \\
\text { fibronektin } \\
\text { Plasmafibronektin, neutrophile Granulozyten und Makro- } \\
\text { phagen }{ }^{2}\end{array}$ \\
\hline 3.4 & $\begin{array}{l}\text { Resorption von Fibrinabbauprodukten, Zelltrümmern } \\
\text { und Bestandteilen der ECM durch Phagozytose }{ }^{3} \\
\text { Hämosiderin }\end{array}$ & & Makrophagen und neutrophile Granulozyten, Siderophagen \\
\hline 4 & $\begin{array}{l}\text { Operative Phase } \\
\text { Faserneubildung }\end{array}$ & Tage & \\
\hline 4.1 & $\begin{array}{l}\text { Aufbau einer temporären ECM in Form von Granula- } \\
\text { tionsgewebe } \\
\text { Beginn der Epithelregeneration } \\
\text { Gefäßneubildung }\end{array}$ & & $\begin{array}{l}\text { Fibroblasten, Myofibroblasten, Endothelzellen, Makropha- } \\
\text { gen }\end{array}$ \\
\hline
\end{tabular}

Makrophagen (- Abb. 3.10a) aus der Blutbahn in das Gewebe. Die Erythrozyten werden dabei inkorporiert und zu intrazellulärem Siderin und/oder Hämatoidin abgebaut (- Abb. 3.10b, Abb. 3.11).

- Reaktive Phase lokaler Zellen: narbige Abheilung, wenn eine Regeneration des Ursprungsgewebes nicht möglich ist; Proliferation von Fibroblasten, Endothelzellen, Mastzellen, Produktion extrazellulären Materials durch Fibroblasten (Kollagen).

\section{(i) Infobox}

Auch für die körpereigenen Abräumreaktionen nach Herzoder Hirninfarkten liegen in der klinisch-pathologischen Literatur Daten zum Zeitablauf vor, die zum Beispiel eine Altersschätzung eines Herz- oder Hirninfarktes erlauben. Ebenso ist eine zeitliche Abschätzung des Thrombusalters anhand histologischer Untersuchungen möglich. Pneumonien können ebenfalls phasenweise ablaufen, was ungefähre zeitliche Rückschlüsse auf ihr Alter zulässt. 
- Tabelle 3.11. Mögliche Einflussfaktoren auf den Reaktionsablauf nach Gewebeschädigung

\begin{tabular}{|l|l}
\hline Lokale Faktoren & $\begin{array}{l}\text { Art und Intensität des auslösenden Reizes } \\
\text { Schweregrad und Ausdehnung der Alteration } \\
\text { Art des betroffenen Gewebes } \\
\text { Temperatur } \\
\text { Durchblutung (z.B. Verminderung der peripheren Durchblutung bei Schock) }\end{array}$ \\
\hline Allgemeine Faktoren & $\begin{array}{l}\text { Hereditäre Faktoren, Alter, Geschlecht } \\
\text { Ernährungszustand, Begleiterkrankungen } \\
\text { Endokrine Einflüsse } \\
\text { Vegetative Einflüsse } \\
\text { Stoffwechselstörungen }\end{array}$ \\
\hline Exogene Faktoren & $\begin{array}{l}\text { Pharmaka } \\
\text { Vitale Unterkühlung/Erwärmung }\end{array}$
\end{tabular}
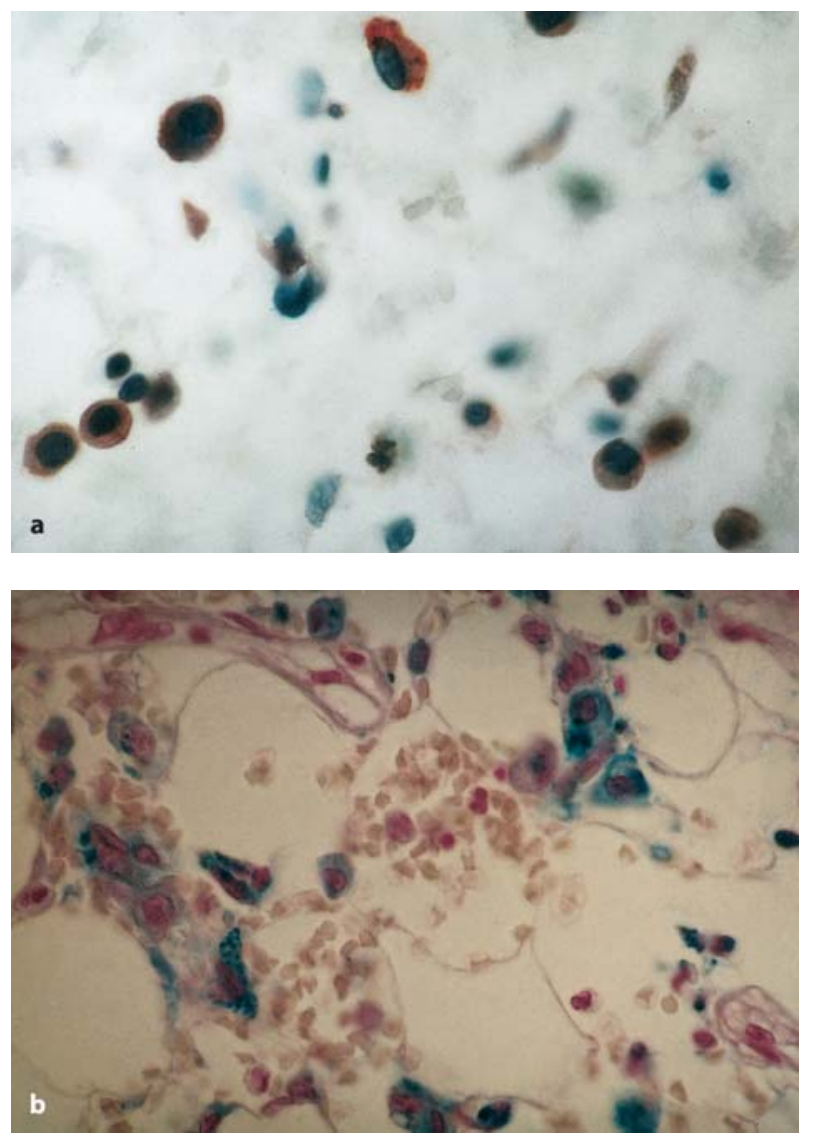

- Abb. 3.10. a Makrophagen in einer Hautwunde (brauner Farbstoffniederschlag = Expression von CD68; x 1.000); b Siderophagen im Lungengewebe nach Lungenblutung, zusammen mit Erythrozyten (blauer Farbstoffniederschlag $=$ Berliner Blaureaktion; $\times 1.000$ )

\section{Wundaltersbestimmung}

Bei der forensischen Wundaltersbestimmung von Hautwunden empfiehlt sich die Einteilung in drei unterschiedliche Stadien:

- Phänomene der Vitalität (Überlebenszeit bis ca. 30 Minuten; - Abb. 3.12),

- Phänomene der kurzen Überlebenszeit (30 Minuten-24 Stunden) und

- Phänomene der längeren Überlebenszeit (über 24 Stunden).

In - Tabelle 3.12 sind die den jeweiligen Zeitphasen zuzuordnenden Befunde zusammengefasst. Die Darstellung der genannten Marker erfolgt überwiegend immunhistochemisch. Im Gegensatz dazu sind in - Tabelle 3.13 die Befunde einzelner Marker in Bezug auf die früheste (entspricht der minimalen Überlebenszeit), regelmäßige und längste Nachweisbarkeit wiedergegeben, was die großen Spannbreiten aufzeigt. Die Beurteilung erhobener Befunde hat entsprechend vorsichtig zu erfolgen und muss sämtliche Erkenntnisse zu dem konkreten Fall einschließen.

\section{(. Wichtig}

Wundaltersbestimmungen sind von Bedeutung zur Einschätzung der Überlebenszeit nach Gewalteinwirkung und spielen z.B. bei wiederholter Misshandlung eine große Rolle.

Ähnliche zeitabhängige Raster lassen sich auch für andere Formen der traumatischen Schädigung erstellen, u.a. für Kontusionsblutungen am Gehirn. Dabei können hier weitere Kriterien Berücksichtigung finden, da u.a. Nervenzellen, Axone, Astroglia und Mikroglia jeweils spezifische Reaktionen aufweisen, die eine zusätzliche zeitliche Eingrenzung ermöglichen. Zu Details sei auf die weiterführende Literatur verwiesen.

\section{(3) Fallbeispiel zur Vitalitäts- und Wundaltersbestimmung} Kurz vor der geplanten Trennung von ihrem Ehemann wurde eine Frau ertrunken in der Badewanne aufgefunden. Bei der Obduk- 
- Abb. 3.11. Darstellung einzelner Phasen des Blutabbaues in Form einer »Adhäsion « von Erythrozyten an der Makrophagenoberfläche (links oben), einer »Ingestion« von Erythrozyten durch Makrophagen (rechts oben), eines Abbaues zu Siderin (blauer Farbstoffniederschlag; links unten) und zu Hämatoidin (brauner Farbstoffniederschlag, kristallin; rechts unten)
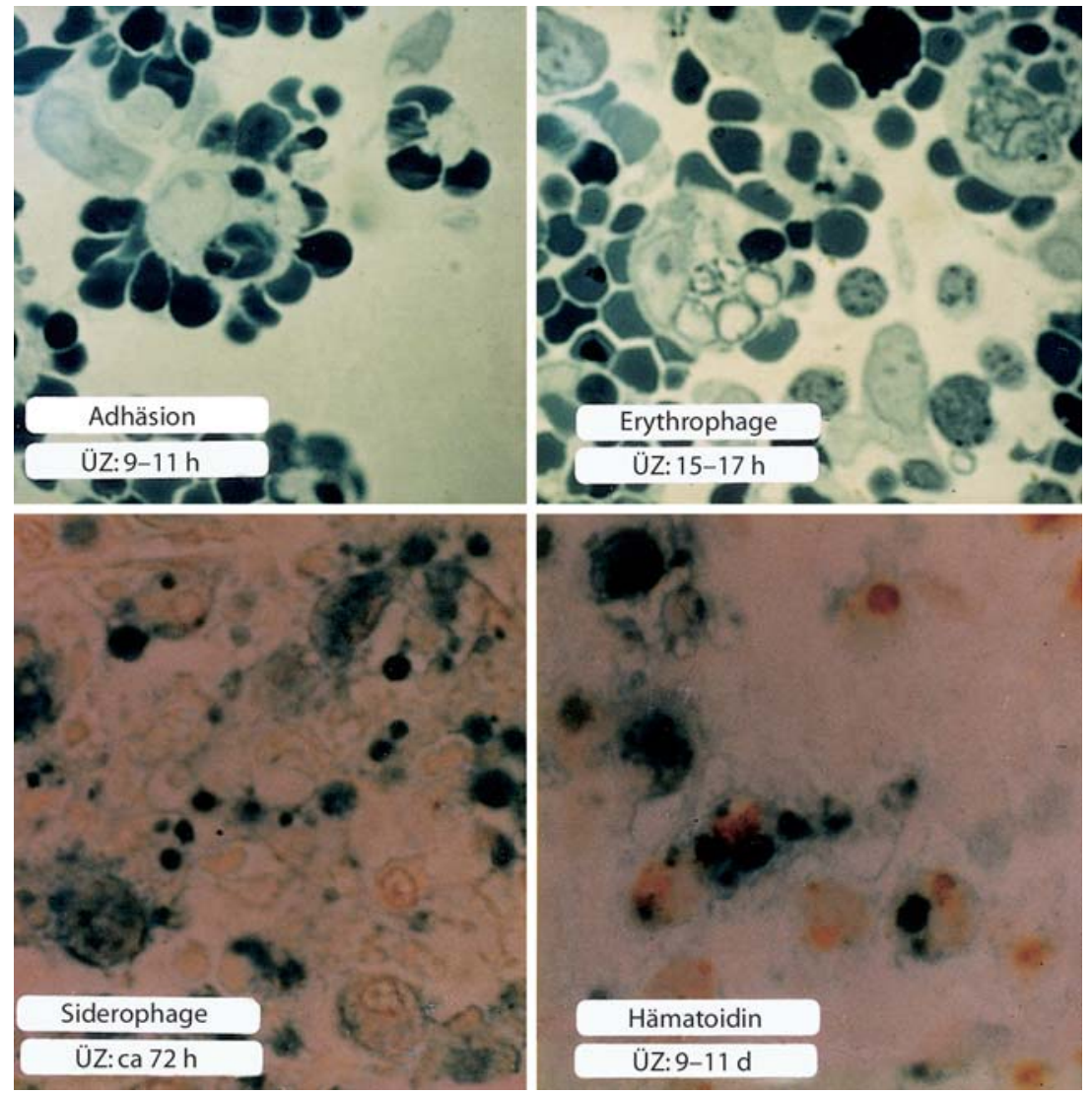

- Abb. 3.12. TNF- $\alpha$ : Epidermaler Reaktivitätsanstieg. Hautwunde mit einem Alter von 27 Minuten (rechts) gegenüber Normalhaut (links; APAAP, $\times 500$ )
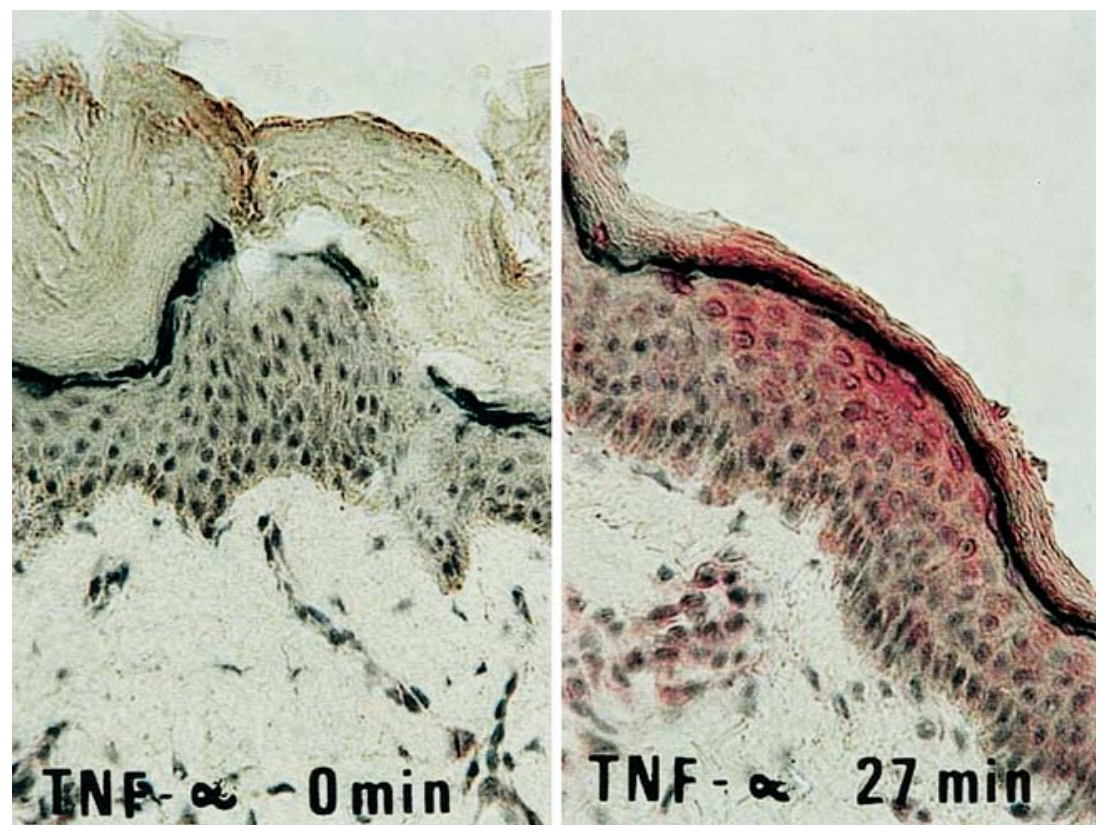
tion wurde ein massives Hämatom der Kopfschwarte festgestellt, sodass vermutet wurde, sie habe einen Schlag auf den Kopf bekommen, sei bewusstlos geworden und ertrunken. Bei der mikroskopischen Untersuchung wurden in dem Hämatom mehrere Siderophagen (Siderin enthaltende Makrophagen) beobachtet, die frühestens drei Tage nach einer Traumatisierung nachzuweisen sind. Das Hämatom konnte somit nicht mit dem Ertrinkungstod in Verbindung gebracht werden. Eine aktuelle Blutalkoholkonzentration von knapp $3 \%$ erlaubte die Schlussfolgerung eines Ertrinkens in der Badewanne unter Alkoholeinfluss.

\section{(i) Infobox}

Häufig unterschreitet die Agoniedauer die Manifestationszeit lokaler vitaler Reaktionen. Die frühesten Reaktionen erfordern eine Überlebenszeit von 10-20 Minuten (immunhistochemischer Nachweis von Fibronektin) bzw. 20-30 Minuten (Nachweis immigrierter neutrophiler Granulozyten, nicht zu verwechseln mit durch eine Einblutung eingeschwemmten Zellen). Zusätzlich besteht eine große interindividuelle Variabilität des frühesten Auftretens verschiedener Vitalitätsparameter an der Wunde. Jede Beurteilung ist unter Berücksichtigung von Fallkonstellation und sämtlichen Untersuchungsergebnissen vorzunehmen.

- Tabelle 3.12. Zusammenfassung immunhistochemischer Untersuchungsbefunde von Hautwunden

Phänomene der Vitalität Überlebenszeit

Phänomene der kurzen

Überlebenszeit

(30 Minuten bis 24 Stunden) bis ca. 30 Minuten)

- TGF- $\beta 1 \geq$ Minuten

- TGF- $\alpha$, IL-1 $\beta$, TNF- $\alpha$, IL- $6 \geq 10-20$ Minuten

- Fibronektin (ab ca. 10-20 Minuten)

- Neutrophile Granulozyten (ab ca. 20-30 Minuten)

- VCAM-1, E-Selektin, L-Selektin $\geq 30$ Minuten

- ICAM-1 $\geq 50-60$ Minuten

- Deutliche Ganulozyten-Infiltration (wenige Stunden)

- Makrophagen-Vermehrung (einige Stunden)

- Aktivitätssteigerung der Fibroblasten-Enzyme Unspezifische Esterase (ab ca. 1 Stunde) Saure Phosphatase (ab ca. 2 Stunden) ATPase, Aminopeptidase, alkalische Phosphatase (ab ca. 4 Stunden)

Phänomene der längeren Überlebenszeit (über 24 Stunden)

- Laminin- und HSPG-positive Myofibroblasten (ab ca. 1,5 Tagen)

- Tenascin (ab 2 Tagen)

- Kollagen III (ab 2-3 Tagen)
- Kollagen V (ab 3 Tagen)

- Kollagen VI (ab 3 Tagen)

- Erythrophagen (ab ca. (2-)3 Tagen)

- Lipophagen, Siderophagen, Hämosiderin (ab ca. 3 Tagen)

- Kollagen I (ab 4 bzw. 5-6 Tagen)

- Kollagen-IV-positive Myofibroblasten (ab ca. 4 Tagen)

- $\alpha$-SMC-Aktin-positive Myofibroblasten (ab ca. 5 Tagen)

- Hämatoidin (ab ca. 1 Woche)

- Fleckförmige Lymphozyten-Infiltrate (ab ca. 1 Woche)

- Vollständige Reepithelialisation (ab ca. 5 Tagen)*

- Basalmembran-Fragmente (Kollagen IV, VII, Laminin, HSPG) (ab 4 Tagen)

- Positive Reaktion mit Makrophagen-Marker RM 3/1 (ab 7 Tagen)

- Vollständig wiederhergestellte Basalmembran (ab ca. 8 Tagen)*

- Positive Reaktion mit Makrophagen-Marker 25 F 9 bzw. G 16/1 (ab 11 bzw. 12 Tagen)

- Vollständige Anfärbbarkeit der Basalzellschicht der neu gebildeten Epidermis für Keratin 5 (ab ca. 13 Tagen) *

- Fehlen von Basalmembran-Fragmenten (Wundalter $<13$ Tage)

- Noch nicht vollständige Reepithelialisation (Wundalter $<21$ Tage) *

- Noch nicht vollständige Basalmembran (Wundalter $<22$ Tage)*

- Noch keine vollständige Anfärbbarkeit der Basalzellschicht für Keratin 5 (Wundalter < 23 Tage) *

* gilt nur für chirurgisch versorgte und primär heilende Wunden 
- Tabelle 3.13. Zeitpunkt der Nachweisbarkeit immunhistochemisch fassbarer Parameter in Abhängigkeit von der Überlebenszeit in menschlichen Hautwunden

\begin{tabular}{|c|c|c|c|}
\hline Antigen/Marker & Früheste Nachweisbarkeit & $\begin{array}{l}\text { Regelmäßige Nachweisbarkeit } \\
\text { bzw. deutliche Reaktivität }\end{array}$ & Längste Nachweisbarkeit \\
\hline $\begin{array}{l}\text { TGF- } \alpha \\
\text { TGF- } \beta 1 \\
\text { Fibronektin } \\
\text { IL-1 } \beta \\
\text { IL-6 } \\
\text { TNF- } \alpha \\
\text { ICAM-1 } \\
\text { VCAM-1 } \\
\text { E-Selektin } \\
\text { L-Selektin } \\
\text { Tenascin } \\
\text { Kollagen III } \\
\text { Kollagen V } \\
\text { Kollagen VI } \\
\text { Kollagen I } \\
\text { Myofibroblasten } \\
\text { Laminin } \\
\text { HSPG } \\
\text { Kollagen IV } \\
\text { SMC-Aktin } \\
\text { Makrophagen-Marker } \\
\text { RM 3/1 } \\
25 \text { F 9 } \\
\text { G 16/1 } \\
\text { Epitheliale Basalmembran } \\
\text { (Lm, HSPG, K IV, K VII) } \\
\text { Fragmente } \\
\text { Vollständig } \\
\text { Keratin } 5 \\
\text { vollständige Anfärbung der } \\
\text { Basalzellschicht } \\
\text { Len }\end{array}$ & $\begin{array}{l}\text { ca. } 10 \text { min. } \\
\text { Minuten } \\
\text { ca. 10-20 Minuten } \\
15 \text { min. } \\
20 \text { min. } \\
15 \text { min. } \\
50 \text { min. } \\
30 \text { min. } \\
30 \text { min, } \\
30 \text { min. } \\
\text { 2-3 Tage } \\
\text { 2-3 Tage } \\
3 \text { Tage } \\
3 \text { Tage } \\
\text { 4-6 Tage } \\
\text { ca. 1,5 Tage } \\
\text { ca. 1,5 Tage } \\
\text { 4 Tage } \\
\text { 5 Tage } \\
\text { 7 Tage } \\
\text { 11 Tage } \\
\text { 12 Tage } \\
\text { 13 Tage } \\
\text { 4 Tage }\end{array}$ & $\begin{array}{l}\text { 30-60 min. } \\
30-60 \mathrm{~min} . \\
\text { über } 4 \text { Stunden } \\
30-60 \mathrm{~min} . \\
60-90 \mathrm{~min} . \\
60-90 \mathrm{~min} . \\
>2 \mathrm{~h} \\
>1,5 \mathrm{~h} \\
>1-1,5 \mathrm{~h} \\
>1,5 \mathrm{~h} \\
\text { ab } 5 \text { Tagen } \\
\text { ab 6 Tagen } \\
\text { ab 6-7 Tagen } \\
\text { ab 6 Tagen } \\
\text { ab 7 Tagen }\end{array}$ & $\begin{array}{l}\text { Monate } \\
\text { Monate } \\
\text { Monate } \\
\text { Monate } \\
\text { Monate } \\
\text { Monate } \\
\text { Monate } \\
\text { Monate } \\
\text { Monate } \\
\text { Monate } \\
\text { Monate } \\
\text { Monate } \\
\text { ca. } 21 \text { Tage }\end{array}$ \\
\hline
\end{tabular}

\section{Hämatomalter bei Lebenden}

Häufige Folgen stumpfer Gewalteinwirkung sind Hämatome, die auch in der Begutachtung (Hämatomalter) von Bedeutung sind. Anhand der Verfärbung des Hämatoms ist nur eine ungefähre Alterabschätzung möglich. Bereits die primäre Färbung des Hämatoms hängt von zahlreichen Faktoren ab: Stärke, Tiefe und Lokalisation. Der typische Farbverlauf und die Altersschätzungen sind in - Tabelle 3.14 angegeben. - Abbildung 3.13 zeigt beispielhaft den zeitlichen Farbverlauf an künstlich gesetzten Hämatomen. Kleine Hämatome sind selbstverständlich schneller vollständig resorbiert als sehr große Hämatome, die teilweise erst Stunden nach der Gewalteinwirkung ihre volle Ausprägung zeigen ( $\downarrow$ s. Kap. 3.6.1) und in den inneren Anteilen noch "frisch « wirken können, während in der Peripherie bereits grünliche oder gelbliche Verfärbungen erkennbar sind. Bei der Beurteilung ist also Zurückhaltung geboten.

\section{F) Fallbeispiel}

Eine 40-jährige Frau wurde von ihrem Ex-Liebhaber tätlich angegriffen. Eine körperliche Untersuchung durch einen Rechtsmediziner erfolgte nicht. Im nachfolgenden Gerichtsverfahren kam tatnah von einer Freundin angefertigten Fotos von Hämatomen an der Oberschenkelinnenseite wesentliche Bedeutung zu. Die rechtsmedizinische Aussage, dass aufgrund der (auch klinisch beschriebenen) Färbung der Hämatome eine Assoziation zum Tatgeschehen möglich ist und die Lokalisation zu den Angaben der Geschädigten zum Handlungsablauf korrespondierte, ergab eine für das Gericht zur Urteilsfindung wesentliche Nachvollziehbarkeit der Angaben der Geschädigten. 
- Abb. 3.13. Zeitverlauf der Farbveränderungen an künstlich gesetzten Hämatomen (Tutsch-Bauer et al.)

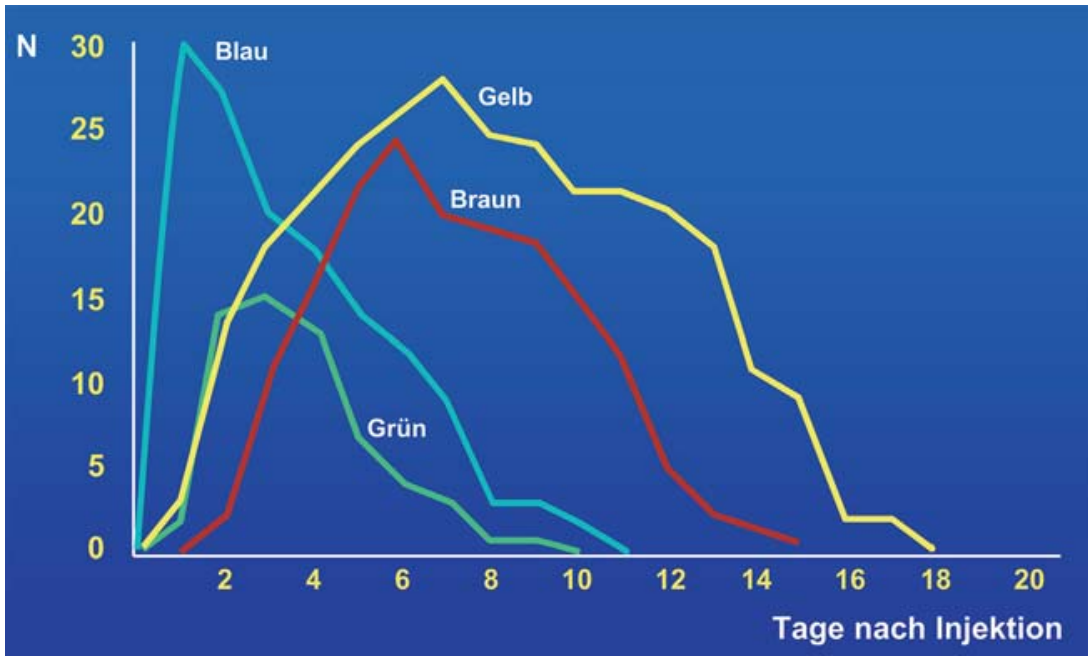

- Tabelle 3.14. Farbverlauf bei Hämatomen

\begin{tabular}{|l|l|}
\hline Färbung & Hämatomalter \\
\hline $\begin{array}{l}\text { graublau } \\
\text { blauviolett } \\
\text { grünlich }\end{array}$ & $\begin{array}{l}\text { frisch } \\
\text { maximal wenige Tage } \\
\text { gelblich } \\
\text { braunrot }\end{array}$ \\
$\begin{array}{l}\text { mindestens 4-5 Tage; in der Regel 6-8 Tage } \\
\text { ca.8 Tage } \\
\text { keine Einschätzung möglich }\end{array}$ \\
\hline
\end{tabular}

3.6 Mechanische Insulte

\subsubsection{Stumpfe Gewalt}

\section{H. Bratzke}

\section{Einleitung}

Stumpfe Gewalteinwirkungen treffen jeden Menschen mehr oder minder häufig bei Verrichtungen des täglichen Lebens, ohne dass es einer ärztlichen Behandlung bedarf oder Folgeschäden auftreten. Dabei sind die kleineren Prellungen und Quetschungen durchaus schmerzhaft, aber sie werden in Abhängigkeit von der Schmerzempfindlichkeit toleriert und lassen in ihrer Intensität schnell nach.

Erst wenn die Gewalteinwirkung ein gewisses Maß überschreitet, kommt es zu Wunden, Gefäßzerreißungen mit Blutungen oder Knochenbrüchen sowie Verletzungen innerer Organe.

Bei der ärztlichen Versorgung stehen zunächst kurative Gesichtspunkte im Vordergrund. Es gehört aber auch zu den ärztlichen Pflichten, im Interesse des Patienten für eine ausreichende Befunddokumentation zu sorgen, um spätere Fragestellungen und Ansprüche (z.B. von Seiten der Kriminalpolizei oder Versicherungen) aus einer gesicherten Dokumentation heraus beantworten zu können. Die Fragen stellen sich nach den Ursachen des Geschehensablaufes, Art und Anzahl der Gewalteinwirkung(en), Schmerzhaftigkeit, etwaigen Folgeschäden, dem Anteil vorbestehender Erkrankungen sowie Kausalität zwischen Einwirkung und festgestellter Erkrankung.

Aus forensischer Sicht ist über die klinischen Fragestellungen hinaus bei Verstorbenen eine Einschätzung der Vitalität bzw. des Wundalters vorzunehmen; es stellt sich die Frage des verwendeten Werkzeuges und schließlich auch, ob es sich um eine Fremdeinwirkung oder Selbstbeibringung handelt.

In Abhängigkeit von der Überlebenszeit kommt es zu charakteristischen Veränderungen im Wundbereich. Bei Blutunterlaufungen (die bei Lebenden nicht selten erst mit einer gewissen Verzögerung an der Oberfläche erkennbar sind) ist der fortschreitende Abbau der Blutung durch eine zeitliche Abfolge der Farben von blaurot bis gelbgrün (nach ca. 4-5 Tagen) gekennzeichnet.

Bei Verstorbenen werden Wunden und Blutunterlaufungen histologisch mit Routinefärbungen (HE, Azan, Berliner Blau) untersucht, zusätzliche Informationen liefern immunhistologische Methoden. In der perimortalen Phase (plus/minus 10-15 Minuten vor bzw. nach dem Tod) ist eine Einschätzung der Vitalität derzeit nicht möglich.

Die Abheilungsvorgänge an den Wunden erfolgen beim Lebenden ebenfalls mit einer gewissen Gesetzmäßigkeit, wobei biologische Faktoren (z.B. Diabetes) oder sekundäre Faktoren (Infektion, Manipulation) die Abläufe modifizieren können.

Von juristischer Seite sind exakte Diagnosen und Prognosen von Bedeutung, wenn es um die rechtliche Einordnung geht (gefährliche Körperverletzung z.B. bei Benutzung eines Werkzeuges, schwere Körperverletzung z.B. bei Verlust eines Organs oder der Zeugungsfähigkeit). 
Bei der Verletzungsbeurteilung sind Kenntnisse biomechanischer Belastungsgrenzen und physikalischer Gesetzmäßigkeiten unerlässlich, wie sie im $>$ Kapitel 3.3 beschrieben worden sind.

\section{Definition \\ Unter stumpfer Gewalt versteht man die mechanische Einwir- kung einer mehr oder minder begrenzten Fläche gegen den menschlichen Körper, wobei jedes Körperteil (Kopf, Rumpf, Gliedmaßen) gleichermaßen betroffen sein kann.}

\section{Allgemeine Traumatologie}

Stumpfe Gewalteinwirkungen führen in Abhängigkeit von Art, Ort und Intensität sowie von fakultativen Faktoren (z.B. Bekleidung) meist zu äußerlich wahrnehmbaren Spuren an der Körperoberfläche (Haut), die aber trotz schwerster innerer Verletzungen auch fehlen können. In der Regel trifft man auf Abschürfungen oder Blutunterlaufungen (wenig präzise als »Prellmarken « bezeichnet). Bei Wunden ist zwischen Risswunden, Quetschwunden und Quetsch-Risswunden zu unterscheiden (nicht ganz korrekt als »Platzwunden« bezeichnet).

Am häufigsten treten Quetsch-Risswunden auf, wenn Gewebe durch einen von außen kommenden Druck zusammengepresst und nach maximaler Kompression durch Zugkräfte auseinander gerissen wird. An der Kontaktstelle entstehen Abschürfungen der Oberhaut, die nach dem Tode vertrocknen. Vom Maximum der Gewalteinwirkung aus ziehen einzelne Hautrisse in das Gewebe hinein, z.T. auch sternförmig, und werden mit zunehmender Entfernung seichter (• Abb. 3.14). Dadurch erklären sich die Gewebsbrücken am Wundgrund, vor allem aber in den Wundwinkeln, die zur Unterscheidung gegenüber Stich- und Schnittwunden dienen (• Abb. 3.15).

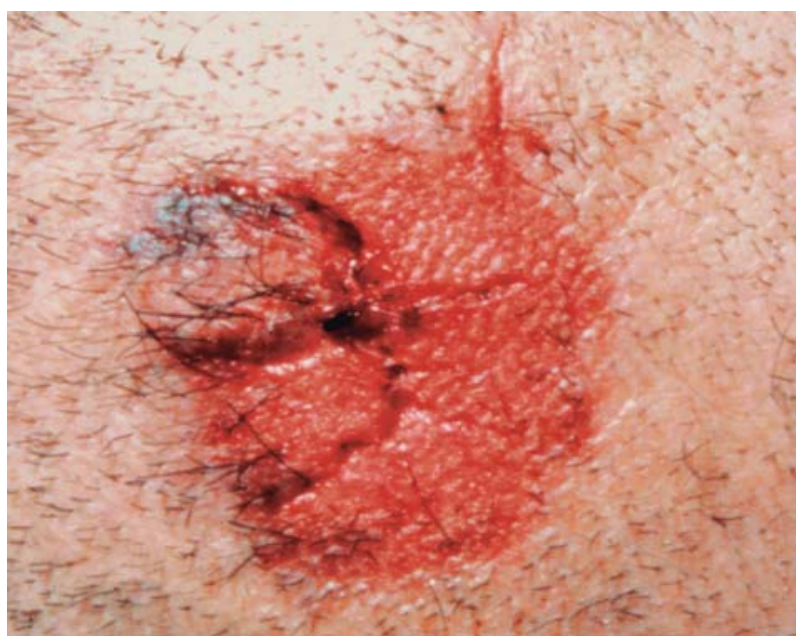

- Abb. 3.14. Quetsch-Risswunde
Isolierte Risswunden sind selten, sie treten vor allem bei Überstreckungen von Gliedmaßen und Körperteilen auf (z.B. bei Pkw-Fußgänger-Unfällen mit hohen Anprallgeschwindigkeiten; - Abb. 3.16).

Tangentiale Einwirkungen führen zur Ablederung (Lazeration), bei Walkvorgängen können ausgedehnte Wundtaschen zwischen den Gewebsschichten entstehen (»Décollement «).

Die Form der Vertrocknungen und Wunden hängt vom einwirkenden Werkzeug und der betroffenen Körperregion ab. An frischen Schürfwunden lässt sich bei genauer Betrachtung (Lupe) durch die am Ende der Schürfung liegenden Oberhautfetzen die Schürfrichtung bestimmen (- Abb. 3.17). Diese Wunden bluten

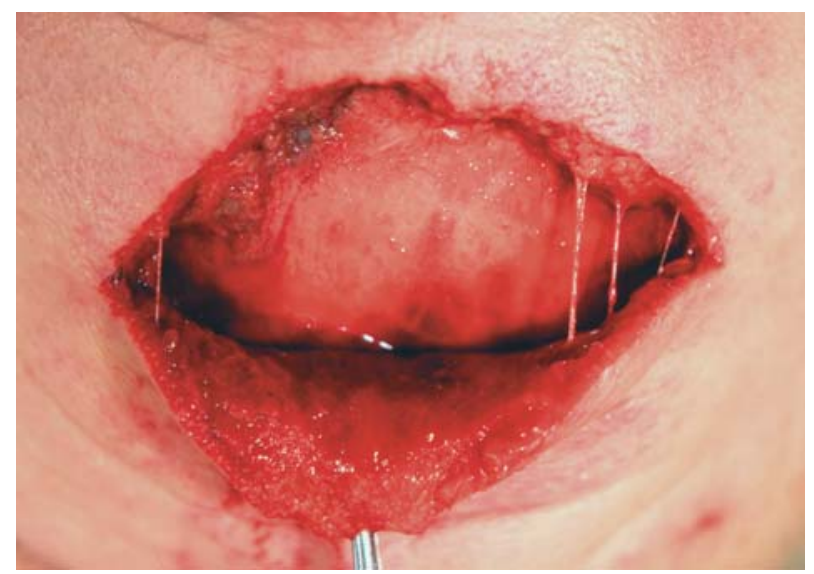

- Abb. 3.15. Gewebsbrücken

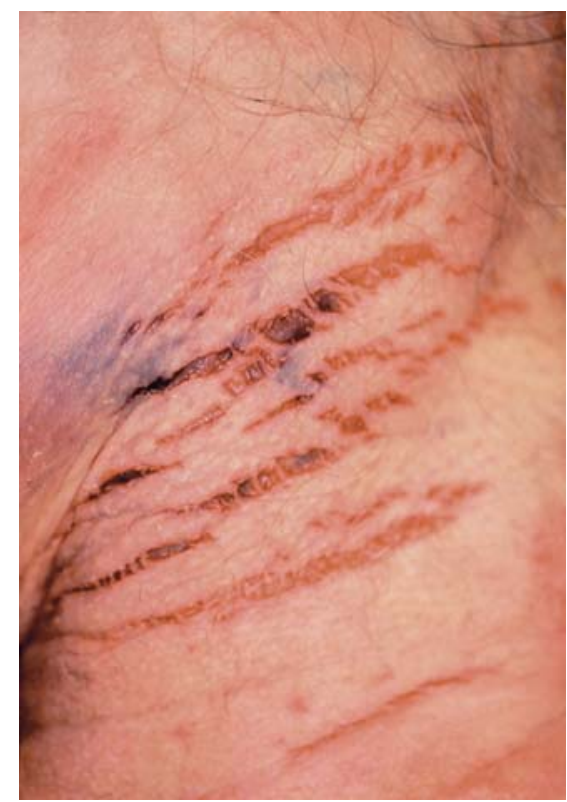

- Abb. 3.16. Überdehnung der Haut 


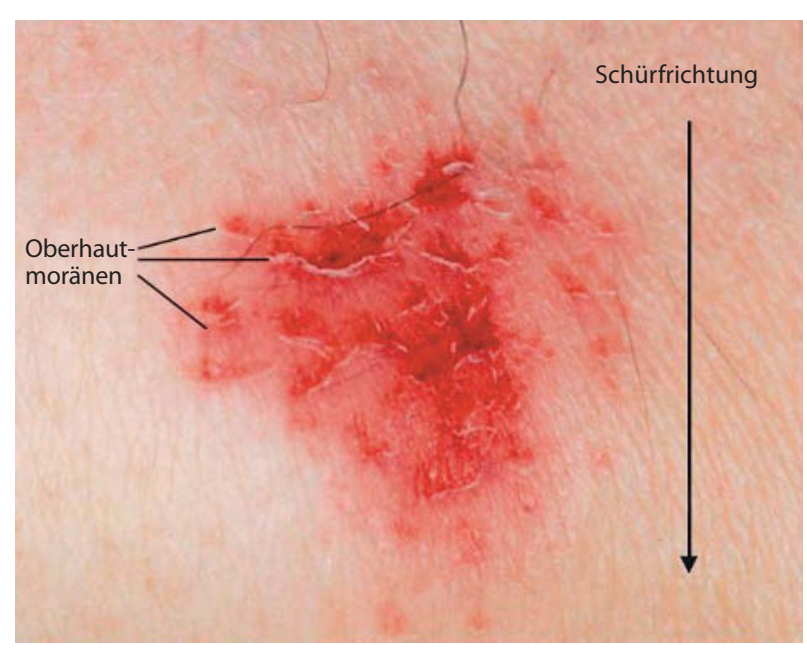

- Abb. 3.17. Schürfung mit Oberhautmoränen

in der Regel nicht. Bei Lebenden sind sie durch Lymphaustritt gekennzeichnet (leicht nässende Wunde), mit der Tendenz zur Superinfektion. Nach dem Tode kommt es zur Vertrocknung der freiliegenden Lederhaut, wobei sich mit fortschreitender Leichenliegezeit die Farbintensität von gelblich-bräunlich bis hin zu schwarzbraun verändert.

Bei der Verwendung von Werkzeugen können charakteristische Wunden auftreten, wie doppelt konturierte Blutunterlaufungen bei Schlägen mit stabförmigen Gegenständen (z.B. Stock, - Abb. 3.18a, b) oder gradlinige Wunden ohne erkennbare Wundrandschürfung bei Einwirkungen von stumpfkantigen bzw. kantigen Gewalten (z.B. Möbelkante, Stuhlbein oder Eisenstab).

Bei massivem Druck geformter Oberflächen auf die Haut entstehen als »Negativbild« Blutungen (»konturierte Hämatome ) an den Belastungsrändern. Sie kommen zum Beispiel beim Überrollen durch ein Kraftfahrzeug mit grobem Reifenprofil (LKW) vor (• Abb. 3.19), kaum aber bei gewöhnlichen PKWReifen, auch bei Zutreten mit profilierter Schuhsohle (»Schuhsohlenabdruckprofile«).

Umschriebene Flächen (z.B. Hammer) führen zu scharf begrenzten Vertrocknungen, die je nach Auftreffwinkel zu unterschiedlichen Bildern führen können (Quadrat, Rechteck, Raute). Lappenförmige Wunden lassen auf die Richtung der tangentialen Gewalteinwirkung schließen, der Hautlappen lässt sich in Richtung der einwirkenden Gewalt aufklappen. Breitflächige stumpfe Gewalteinwirkungen auf den Kopf führen wegen dessen Rundung meist zu rundlichen Vertrocknungen mit teils gradlinigen, teils sternförmigen Quetsch-Risswunden ( $\bullet$ Abb. 3.14).

Bei mechanischen Einwirkungen können Blutungen auch isoliert in den tieferen Hautschichten auftreten, vor allem wenn es sich um geformte Gegenstände handelt und Relativverschiebungen zwischen den Hautschichten auftreten. So kommt nicht selten eine »innere Gurtmarke« erst nach Fettgewebspräparation
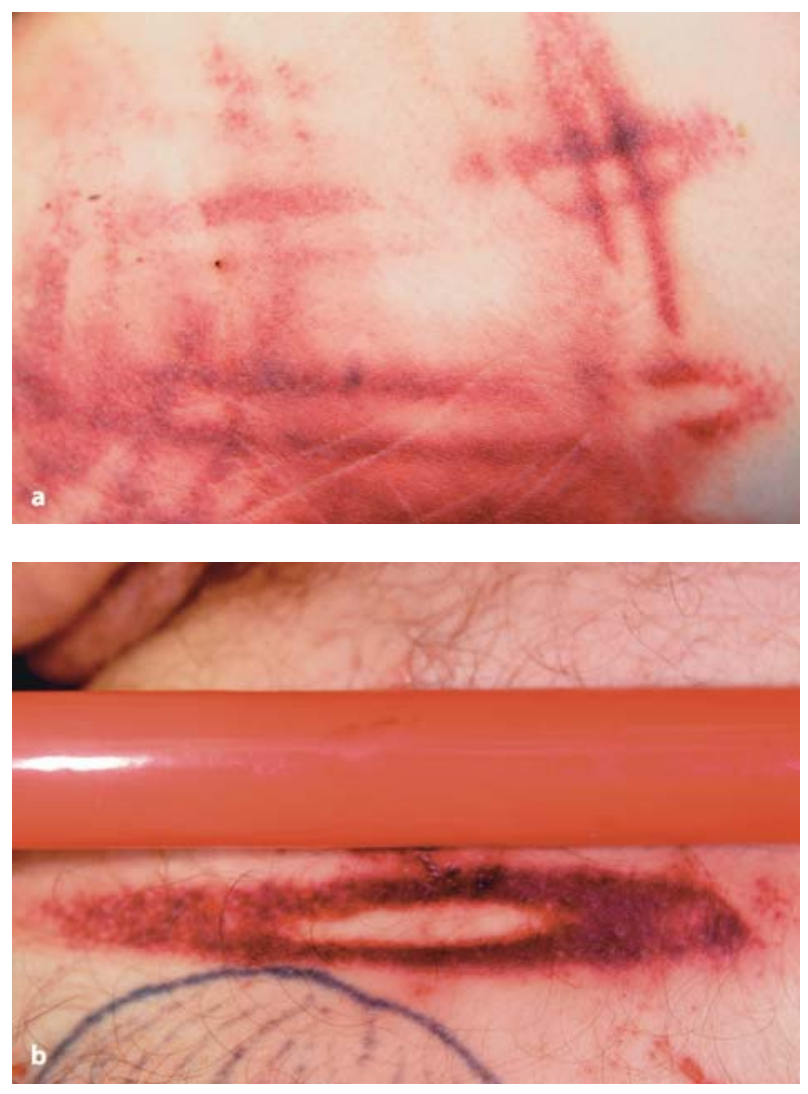

- Abb. 3.18a, b. Doppelt konturierte Blutung

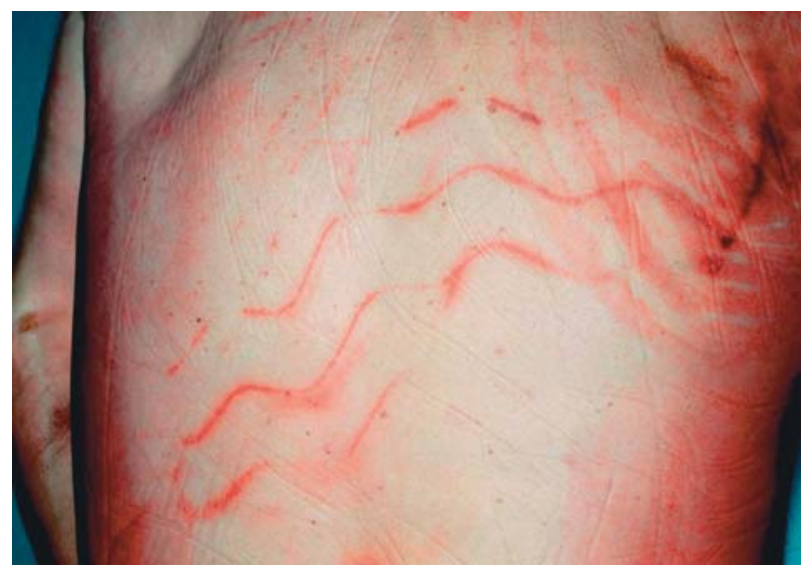

- Abb. 3.19. Reifenspuren 


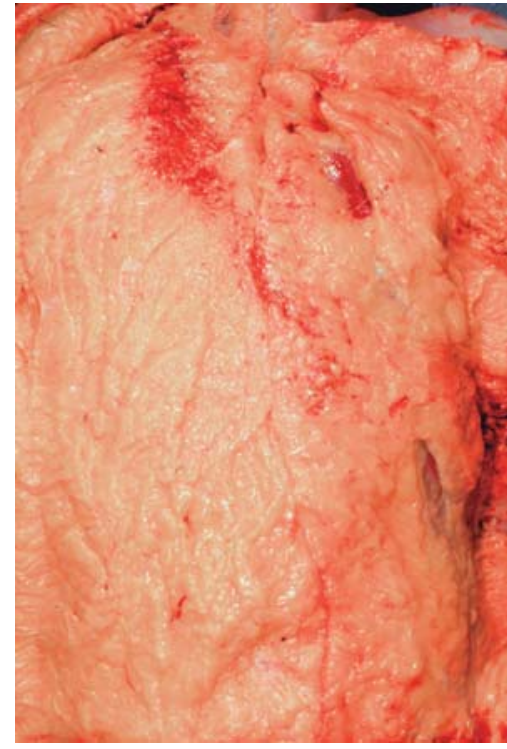

- Abb. 3.20. Innere Gurtmarke

zum Vorschein, obwohl äußerlich keinerlei Spuren an der Haut sichtbar waren (• Abb. 3.20).

\section{Todesursachen}

Bei Gewalteinwirkungen gegen den Kopf kommt es bei schwersten offenen Verletzungen mit teilweiser oder vollständiger Enthirnung zum sofortigen Zusammenbruch der Regelsysteme mit Erlöschen der Handlungsfähigkeit. Bei intrakraniellen Raumforderungen bricht das Regulationssystem durch Hirnstammeinklemmung oder sekundäre Hirnstammblutungen zusammen. Zerreißungen der Gefäße an der Schädelbasis oder des Sinus cavernosus führen zur Verblutung nach außen oder zur tödlichen Bluteinatmung.

Rupturen parenchymatöser Organe führen zur inneren Verblutung, wenn beim erwachsenen, gesunden Menschen eine Blutmenge von ca. 1,5 Liter erreicht ist (bei Kindern und kranken Menschen bedeutend weniger, 0,5-1 Liter!). Traumatische Amputationen von Gliedmaßen führen zur Verblutung aus zerrissenen Arterien und Venen, Gefäßrupturen zur inneren und äußeren Verblutung.

Als Todesursache kommen auch eine Lungenfett- und Knochenmarks- sowie eine Luftembolie in Frage, die gleichzeitig ein Vitalitätszeichen darstellen können (• Abb. 3.21a, b).

Bei der Fettembolie wird bei ausgedehnteren Weichteilquetschungen aus dem zermalmtem Fettgewebe durch die eröffneten Venen Fett aufgenommen, das in den Lungenkapillaren stecken bleibt und durch Rechtsherzüberlastung sowie Verminderung der Diffusionsfläche in der Lunge zum Tode führt. Knochenmarksembolien treten bei Frakturen der Röhrenknochen, aber auch nach Rippen(serien)frakturen gehäuft auf.
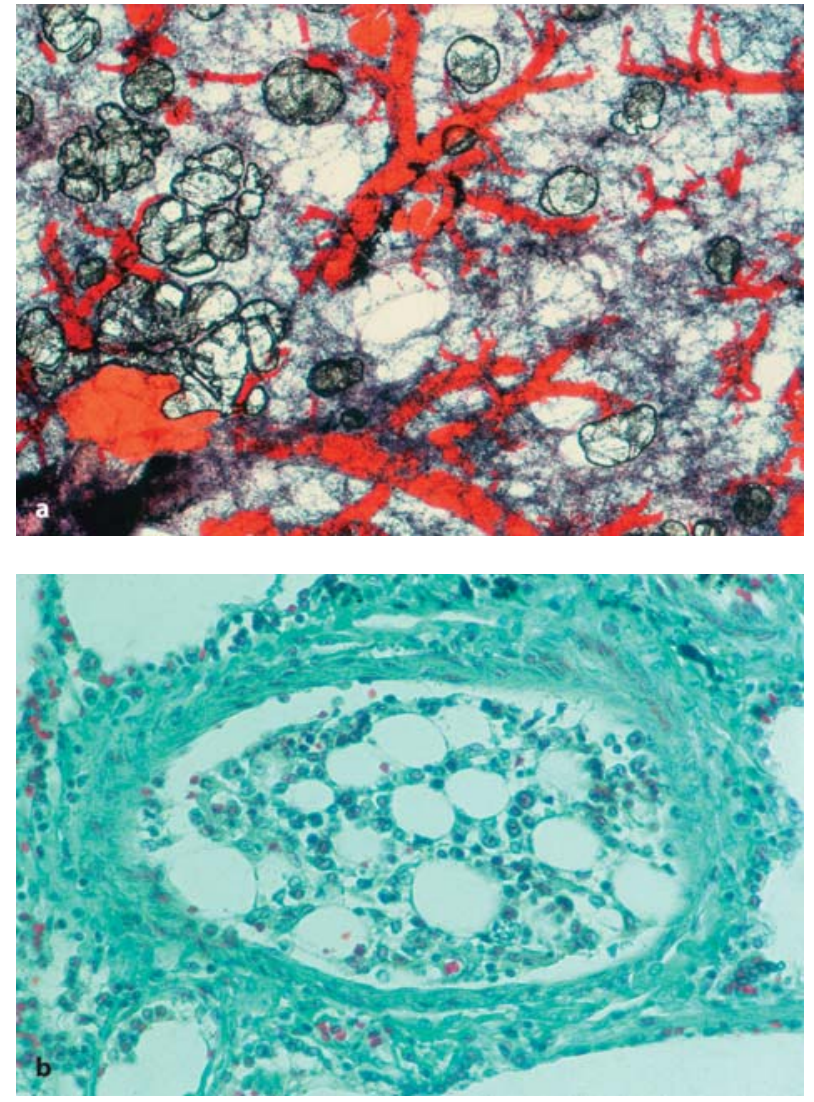

- Abb. 3.21. a Fettembolie; b Knochenmarksembolie

Durch intensivmedizinische Maßnahmen (Schockbekämpfung) hat die Lungenfettembolie als Todesursache an Bedeutung verloren. Bei jedem sonst nicht erklärbaren Todesfall ist aber daran zu denken und durch Fettfärbung der Lungen der Nachweis zu führen. Die Hirnfettembolie (»Purpura cerebri«) wird kaum mehr beobachtet.

Extremitätenfrakturen, vor allem aber die Immobilisation, führen zu Bein- und Beckenvenenthrombosen, die bei herzkranken Menschen auch durch adäquate Thromboseprophylaxe nicht immer verhindert werden können. Bei Todesfällen nach äußeren Gewalteinwirkungen (Sturz, Verkehrsunfall u.a.) handelt es sich ungeachtet der Vorerkrankungen und des Alters um einen nichtnatürlichen Tod.

( Wichtig

Äquivalenztheorie: Jede Bedingung ist im strafrechtlichen Sinn als kausal anzusehen, die nicht hinweggedacht werden kann, ohne dass nicht auch der Erfolg entfiele. Dabei spielt es keine Rolle, auf welchen vorbestehenden Zustand eine Gewalteinwirkung trifft! 
Pneumonien sind dank moderner Antibiotikatherapie selten geworden, häufiger werden mit Kreislaufschock und Beatmung verbundene Schäden der Lunge gesehen (»Schocklunge« oder Adult Respiratory Distress Syndrom = ARDS).

Wundinfektionen mit tödlicher Blutvergiftung werden im forensischen Bereich kaum mehr beobachtet, bei auffallend schnell eintretender Leichenfäulnis, Hautknistern sowie "Schaumorganen « ist eine Gasbrandinfektion durch histologische und bakteriologische Untersuchungen auszuschließen.

Stumpfe Gewalteinwirkungen gegen den Brustkorb können zu Herzkontusionen mit Herzrhythmusstörungen führen (Commotio bzw. Contusio cordis). Ausgedehnte Lazerationen (z.B. beim Fußgängeranstoß) gehen mit nicht unerheblichen Blutungen einher (bis zu einem Liter!), Weichteilquetschungen führen zur Lungenfettembolie.

\section{Spezielle Traumatologie: Kopf}

Leichte Gewalteinwirkungen gegen den Kopf (Bagatelltraumen) bleiben meist folgenlos und werden als »Kopfprellung « bezeichnet. An der Kontaktstelle kann es zu Blutunterlaufungen oder Wunden kommen, wobei Kopfschwartenwunden mitunter außerordentlich stark bluten (in seltenen Fällen bis hin zur tödlichen Verblutung). Die Einwirkungen gehen mit erheblichen, aber vorübergehenden Schmerzen in der mit Nerven gut versorgten Kopfschwarte, bisweilen auch mit leichten vegetativen Störungen (Kopfschmerzen) einher, haben aber keine Folgen für das Bewusstsein.

Die folgenlose »Kopfprellung « ist (auch wegen der rechtlichen Aspekte) von dem leichtesten Grad einer Hirnschädigung, der »Commotio«, abzugrenzen. Diese ist in der Regel durch kurze Bewusstseinsstörungen bzw. Bewusstlosigkeit gekennzeichnet, die dem Betreffenden nicht immer bekannt ist. In der Folgezeit treten Übelkeit, Erbrechen und Kopfschmerzen auf, die Erinnerung an die Zeit kurz vor dem Ereignis und danach ist gestört (retro- und anterograde Amnesie). In sehr seltenen Fällen kommt es zu posttraumatischen Dämmerzuständen, die an zusätzliche Bedingungen geknüpft sind (z.B. situationsinadäquates Verhalten, finale Schlafphase). Am Gehirn selbst zeigen sich keinerlei Verletzungsspuren, es handelt sich um zentralnervöse Störungen.

Gewalteinwirkungen mit fassbaren Schädigungen am Gehirn (Subarachnoidalblutung, Rindenprellung, Gewebs- oder Gefäßschäden), die klinisch mit z.T. längerdauernder Bewusstlosigkeit einhergehen und nicht folgenlos abheilen, bezeichnet man als »Contusio cerebri« (Hirnprellung).

Bei der »Compressio cerebri« kommt es sekundär durch Blutung und Ödem zur intrakraniellen Volumenzunahme mit Einklemmung der Medulla oblongata in das Foramen magnum und letztlich zum Versagen der zentralen Regulationen (Atmung, Kreislauf, Temperatur, Endokrinium) mit Eintritt des Hirntodes. Bei Kindern beobachtet man häufig ein massives, therapieresistentes traumatisches Hirnödem ohne größere Blutungen.

Neben dieser morphologisch orientierten Einteilung, die trotz aller Unzulänglichkeiten immer noch bei der Einteilung der
Schweregrade eines Schädelhirntraumas (SHT) benutzt wird, sollten aus klinischer Sicht andere Bewertungsschemata herangezogen werden. So kann je nach Dauer oder Ausmaß der posttraumatischen Bewusstseinsstörung zwischen einem leichten, mittelschweren und schweren SHT unterschieden werden. Die Differenzierung zwischen einem offenen/penetrierendem SHT (bei Verletzung der harten Hirnhaut) zum geschlossenen SHT ist wegen der Infektionsgefahr von Bedeutung (Liquorrhoe, posttraumatische Meningitis).

Die modernste Einteilung orientiert sich an der Glasgow coma scale (GCS) (•Tabelle 3.15) mit folgender Einteilung:
- Leichtes Schädelhirntrauma
13-15 Punkte
- Mittelschweres Schädelhirntrauma
- Schweres Schädelhirntrauma
8 oder weniger Punkte.

Die schwersten Verläufe mit Einklemmung des Hirnstammes und Sistieren der Blutzirkulation führen unter intensivmedizinischen Bedingungen zum Hirntod, der mit dem Individualtod gleichzusetzen ist. Die Kriterien sind durch »Richtlinien der Bundesärztekammer« geregelt.

Heftige stumpfe Gewalteinwirkungen, wie sie bei Schlägen mit der Faust oder Werkzeugen, Sturz zu ebener Erde oder aus der Höhe, beim Auftreffen von herabfallenden Gegenständen aus einiger Höhe sowie Beschleunigungsunfällen (Verkehrs- und Flugunfall) entstehen, führen neben der Kompression zu Translations- und Rotationsbeschleunigungen des Gehirnes (in der Regel zu Kombinationen) mit Gefäßzerreißungen und Rindenprellungsherden und davon ausgehenden raumfordernden intrakraniellen Blutungen.

Intrakranielle Blutungen. Die Blutungen im Schädelinneren werden je nach ihrer Lokalisation in epidurale, subdurale, sub-

Tabelle 3.15. Glasgow-Komaskala

\begin{tabular}{|c|c|c|}
\hline $\begin{array}{l}\text { zu bewertende } \\
\text { Reaktion }\end{array}$ & $\begin{array}{l}\text { beobachtete } \\
\text { Reaktion }\end{array}$ & $\begin{array}{l}\text { Punkt- } \\
\text { zahl }\end{array}$ \\
\hline Augenöffnen & $\begin{array}{l}\text { spontan } \\
\text { auf Aufforderung } \\
\text { auf Schmerzreiz } \\
\text { kein Augenöffnen }\end{array}$ & $\begin{array}{l}4 \\
3 \\
2 \\
1\end{array}$ \\
\hline $\begin{array}{l}\text { beste sprachliche } \\
\text { Antwort }\end{array}$ & $\begin{array}{l}\text { voll orientiert } \\
\text { unvollständig orientiert } \\
\text { verworren } \\
\text { unverständlich } \\
\text { keine }\end{array}$ & $\begin{array}{l}5 \\
4 \\
3 \\
2 \\
1\end{array}$ \\
\hline $\begin{array}{l}\text { beste motorische } \\
\text { Reaktion }\end{array}$ & $\begin{array}{l}\text { adäquate } \\
\text { gezielte Abwehr } \\
\text { unvollständige Abwehr } \\
\text { Beugesynergismen } \\
\text { Strecksynergismen } \\
\text { keine Bewegung }\end{array}$ & $\begin{array}{l}6 \\
5 \\
4 \\
3 \\
2 \\
1\end{array}$ \\
\hline
\end{tabular}


arachnoidale, intrazerebrale, intrazerebelläre und Hirnstammblutungen unterschieden (• Abb. 3.22).

Epidurale Blutungen sind nahezu immer traumatischen Ursprungs und meist auf eine Verletzung der A. meningea media unterhalb eines (meist parietal gelegenen) Schädelbruchs zurückzuführen. Andere Blutungsquellen sind ein- oder doppelseitige Sinusrupturen. Zu beachten ist das sog. »Freie Intervall«, das zwischen der Gewalteinwirkung (meist mit initialer kurzer Bewusstlosigkeit) und dem Einsetzen von Hirndrucksymptomen (neurologische Defizite, Erweiterung der zur Blutung ipsilateral gelegenen Pupille durch Einklemmung bzw. Zug am N. oculomotorius) liegt. Tödliche Verläufe mit Verkennung der Blutung kommen dank moderner diagnostischer Verfahren (CT) kaum mehr vor und haben in der Regel ein strafrechtliches Ermittlungsverfahren mit der Frage mangelnder ärztliche Sorgfalt zur Folge. Eine strafrechtliche Verurteilung erfolgt aber in der Regel nicht, weil die Kausalität zwischen der Sorgfaltspflichtverletzung und dem Tod (bei einer Letalität von ca. 5-10 \%) nicht zu belegen ist. Zivilrechtlich ist bei "grobem Behandlungsfehler« und »Beweislastumkehr» dagegen eine Haftung mit Schadensersatz sehr wahrscheinlich.
- Abb. 3.22a-d. Blutungen Gehirn a Bikonvex (linsenförmige) epidurale Blutung mit Schädelbruch und Ruptur der A. meningea media. Ausgeprägte Mittellinienverlagerung (»shifting "), sekundäre Blutung vor dem Balkenknie. Verkehrsunfall Mofa ./. PKW, 1 Tag überlebt. b Sichelförmige subdurale Blutung nach Rotationstrauma und Brückenvenenruptur (Sturz? Schlag?), kein Schädelbruch. Überlebenszeit unbekannt (Wohnungstod). Alkoholismus. c Traumatische Subarachnoidalblutung. Polytrauma, Schädelbasisfraktur, Bluteinatmung. Arbeitsunfall, Sturz aus der Höhe, kurz überlebt. d Traumatische zentrale Blutung (»Hirnruptur «) nach massivem Rotationstrauma (PKW-Fahrerin, seitlich von LKW erfasst), kein Schädelbruch. Ipsilaterale Hippocampusbltg., kontralaterale Balkenblutung.

4 Tage überlebt
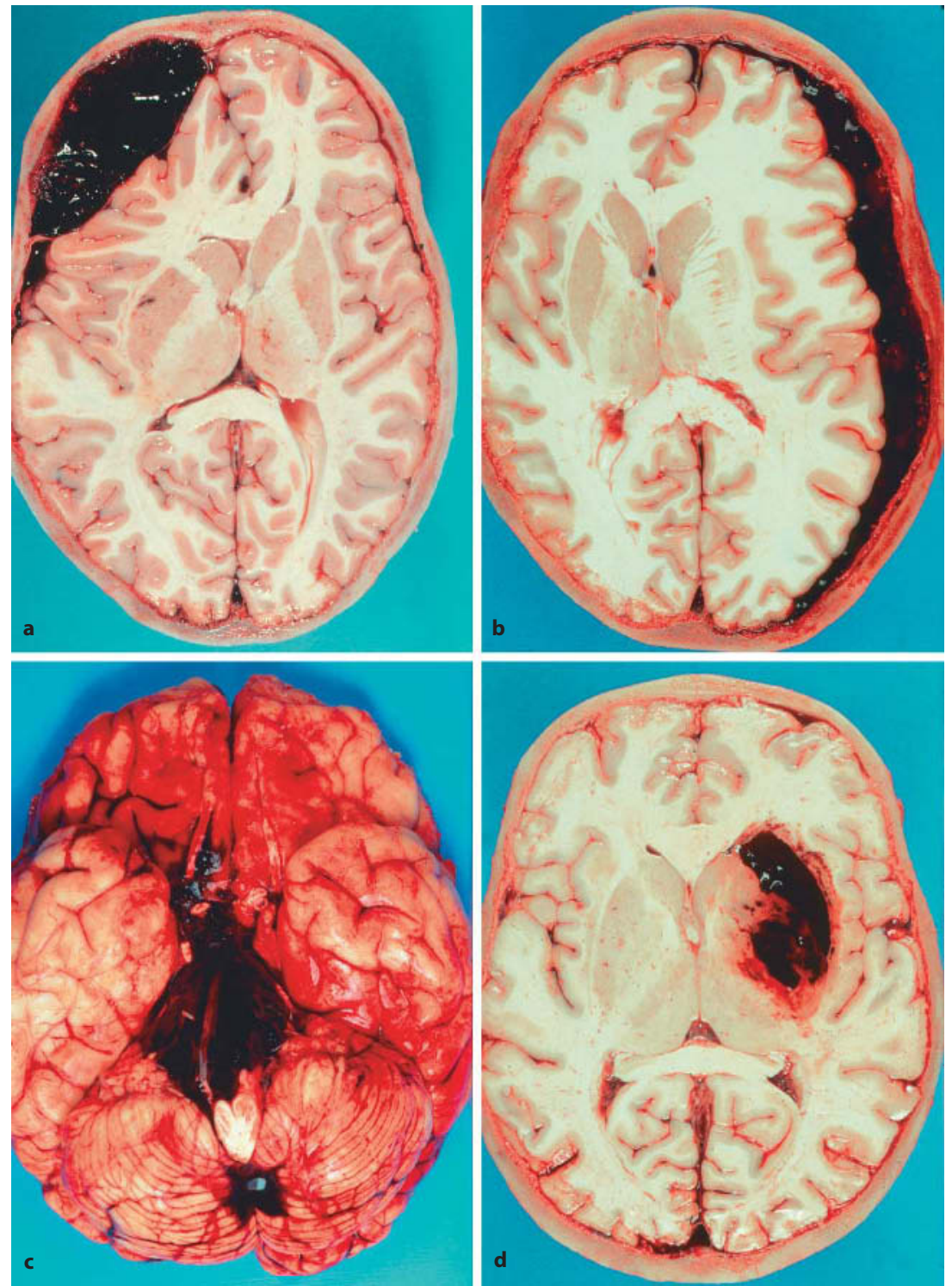


\section{(5) Fallbeispiel}

Ein 18 Jahre alter Bundeswehrsoldat wird zusammen mit zwei Bekannten von einem älteren Mann auf der Straße angepöbelt und fällt durch einen »Renner« gegen die Brust mit dem Hinterkopf auf die Straße. Er erwacht nach kurzer Bewusstlosigkeit und gibt gegenüber der vorsorglich alarmierten Notärztin einen »falschen Namen« an, was von ihr als Folge einer stärkergradigen Alkoholisierung gedeutet wird (tatsächliche Blutalkoholkonzentration zu dieser Zeit ca. 1 Promille). Bei der Krankenhauseinlieferung ist zwar auf dem angefertigten Röntgenbild ein Schädelbruch erkennbar, der aber als »abnormer Gefäßverlauf « missdeutet wird. Der Soldat wird »zur Ausnüchterung« in die Kaserne entlassen, wo es einige Stunden später zum Erbrechen und schließlich zur Bewusstlosigkeit kommt. Bei der neuerlichen Krankenhauseinlieferung zeigt sich im CT eine epidurale Blutung, die ausgeräumt wird. Auf Grund der Hirnschädigung war aber der tödliche Ausgang nicht mehr abzuwenden. Bei der Sektion fand sich der Schädelbruch an entsprechender Stelle, weitere schwerwiegende Verletzungen lagen nicht vor. Strafrechtlich wurde der Täter wegen Körperverletzung mit Todesfolge verurteilt, die Ärzte blieben straffrei, weil die Kausalität nicht zu beweisen war (- Abb. 3.23a, b).

Subdurale Blutung: Die Blutungen unter der harten Hirnhaut, die sich im CT und bei der Sektion sichelförmig darstellen, sind die häufigste schwerwiegende Komplikation nach schweren stumpfen Schädelhirntraumen, sie sind meist auf Blutungen aus sog. »Gegenstoßprellungsherden « (Contrecoup) zurückzuführen (- Abb. 3.24).

Beim Contrecoup handelt es sich um das Phänomen, dass bei okzipitalen und parietalen (nicht aber bei frontalen) Gewalteinwirkungen die der Einwirkung diametral gegenüberliegende Seite bedeutend massivere Hirnschädigungen aufweist als die Einwirkungsstelle selbst. Nach Sellier und Unterharnscheid sind die Gegenstoßprellungsherde durch innere Kavitation (»Unterdruck«) mit Herausreißen der kuppenständigen Gefäße zu erklären. Gegen Druck reagiert das Gehirn viel unempfindlicher (- Abb. 3.25a, b).

Von den Rindenprellungsherden aus kann es (gerade bei älteren Menschen) auch zu massiven Blutungen in das Marklager kommen, die klinisch nicht mit intrazerebralen Blutungen aus natürlicher Ursache verwechselt werden dürfen.

Kommt es unterhalb des Schädelbruchs zu einer Zerreißung der Dura und rinnenförmiger Einklemmung der Hirnoberfläche, können auch im Anstoßbereich ausgedehnte Schädigungen (meist hämorrhagische Infarzierungen, Nekrosen) auftreten.

Eine Besonderheit stellen subdurale Blutungen ohne Schädelbruch dar, die meist auf Rotationstraumen (z.B. heftiger Faustschlag) zurückzuführen sind und durch Abscherungen von Gefäßen (Schlagadern, Brückenvenen) an der Hirnoberfläche bedingt sind (Krauland).

Eine Sonderform stellt das »Schütteltrauma« des Säuglings oder Kleinkindes dar (»baby-shaken-syndrome«), bei dem durch
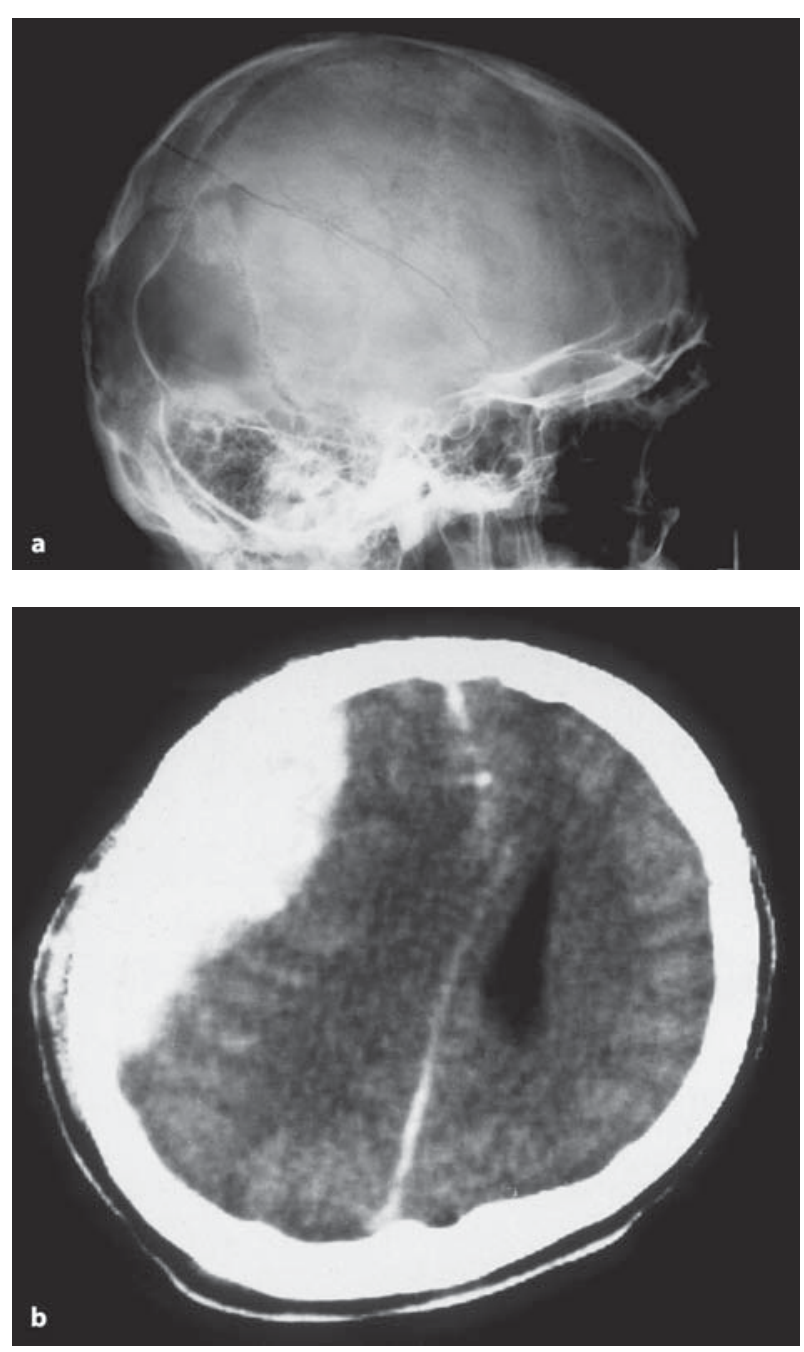

- Abb. 3.23. a Schädelbruch; b epidurale Blutung $C T$

heftige Schüttelbewegungen des Körpers und Hin- und Herpendeln des Kopfes ein- oder beidseitig Brückenvenen zerreißen, die zur subduralen Blutung führen. Auch eine massive Hirnschwellung kann Folge solcher mechanischer Belastungen sein. Werden die Gewalteinwirkungen überlebt, sollen die Kinder nicht selten erhebliche neurologische Defizite (Retardierung) aufweisen.

Intrazerebrale Blutungen: Blutungen in das Gehirn ohne Verbindung zur Hirnoberfläche sind als Folge traumatischer Einwirkungen außerordentlich selten und werden als Folge massiver Rotationsbeschleunigungen des Kopfes (auch ohne Schädelbruch!) beobachtet (»zentrale Hirnruptur«). Intrazerebrale Massenblutungen in engerem zeitlichen Zusammenhang mit traumatischen Einwirkungen (früher als »Bollingersche Spätapoplexie « heute als »Delayed Traumatic Intracerebral Hematoma = DTICH bezeichnet) werfen erhebliche gutachterliche Probleme auf (Mas- 


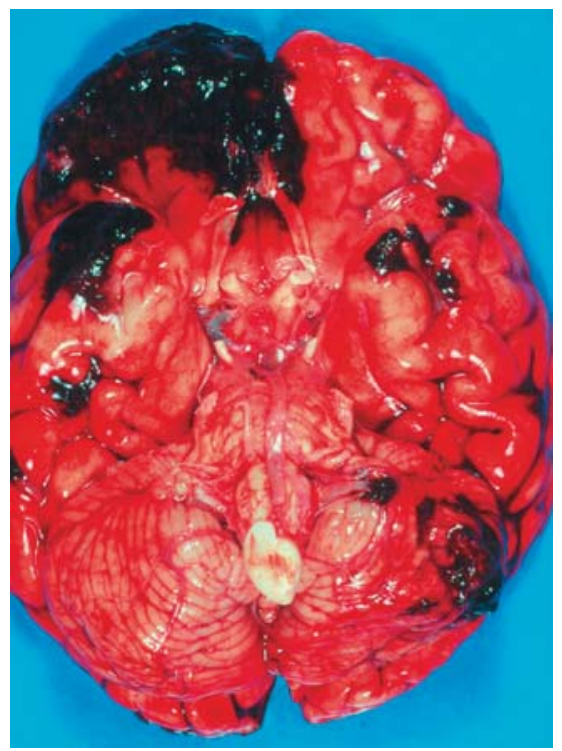

- Abb. 3.24. Coup, Contrecoup

senblutung mit nachfolgendem Unfall?, traumatische Intrazerebralblutung bei zweizeitiger Gefäßruptur?). Sie sind im Todesfall nur durch Sektion und kompetente Hirnuntersuchung zu klären.

Subarachnoidalblutung: Bei Rotationstraumen (Faustschlag) kann es durch Zerrung an der A. basilaris zu Längsrissen mit nachfolgender Subarachnoidalblutung kommen. Auch Gefäßeinrisse unterhalb von Schädelbrüchen können eine solche Blutung hervorrufen, wobei es zweizeitige Verläufe gibt. Ein besonderes Problem stellen Rupturen von vorbestehenden Aneurysmen dar, die bevorzugt an den Gefäßverzweigungen der Aa. communicantes ant., cerebri mediae, carotis internae und Aa. vertebrales liegen (sog. Forbus'sche Aneurysmen oder »berry aneurysms «). Im Todesfall kann nur die eingehende morphologische Untersuchung die Zusammenhänge zwischen Gewalteinwirkung und Aneurysmaruptur klären. Auch ist eine Abgrenzung gegenüber traumatischen Pseudoaneurysmen mit zweizeitigem Verlauf vorzunehmen.

\section{(3) Fallbeispiel}

Ein 28 Jahre alter Kaufmann wird nach Verlassen eines Lokals von Jugendlichen angepöbelt und schließlich mit der Faust in das Gesicht geschlagen, wobei er nach der zweiten Salve leblos zusammenbricht und durch den alarmierten Notarzt nicht mehr wiederbelebt werden kann. Todesursächlich ist eine Subarachnoidalblutung aus einem rupturierten Aneurysma der linken A. cerebri media, das sich auf Grund der histologischen Untersuchungen schon in den Tagen zuvor gedehnt hatte, sodass eine »Rupturbereitschaft« bestand. An der Rupturstelle waren inten-
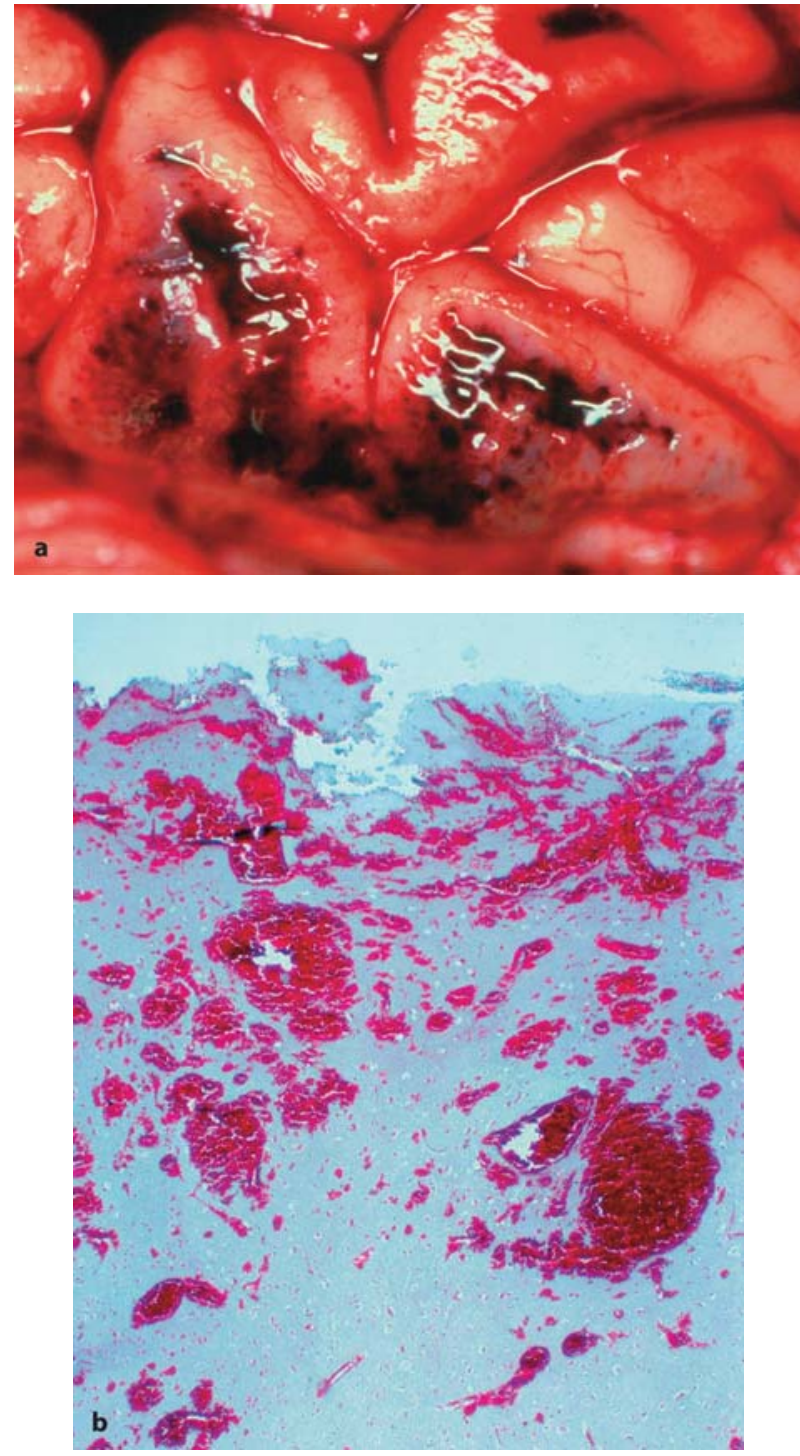

- Abb. 3.25a, b. Rindenprellungsherde. a makro; b mikro

sive leukozytäre Reaktionen mit Fibrin und Thrombozytenaggregaten nachzuweisen. Als Nebenbefund fand sich ein kleines nicht rupturiertes Aneurysma an der gegenüberliegenden Mediagabel. Im Gesicht fanden sich Blutunterlaufungen an den Lippen, aber keine knöchernen Verletzungen. Da strafrechtlich die Kausalität zwischen Gewalteinwirkung und Aneurysmaruptur nicht mit an Sicherheit grenzender Wahrscheinlichkeit zu belegen war (Es bestand die Möglichkeit, dass es allein durch die physische Belastungssituation beim Streit mit Blutdruckerhöhung schon vor den Schlägen zur Ruptur gekommen war), erfolgte strafrechtlich eine 
Verurteilung wegen Körperverletzung. Zivilrechtlich (es ging um Unterhaltsansprüche der minderjährigen Kinder) wurde in einem höchstrichterlichen Urteil (BGH) auf Grund der zeitlichen Koinzidenz ein kausaler Zusammenhang bejaht (• Abb. 3.26a, b).

Intrazerebelläre Blutung: Traumatische Blutungen in das Kleinhirn sind fast ausnahmslos Folge eines Schädelbruches in der hinteren Schädelgrube mit Durariss und Quetschung der Kleinhirnoberfläche mit nachfolgender Blutung und Nekrose.

Hirnstamm: Blutungen in Mesenzephalon, Pons und Medulla oblongata (»Hirnstamm«) können primär als Folge einer mas-
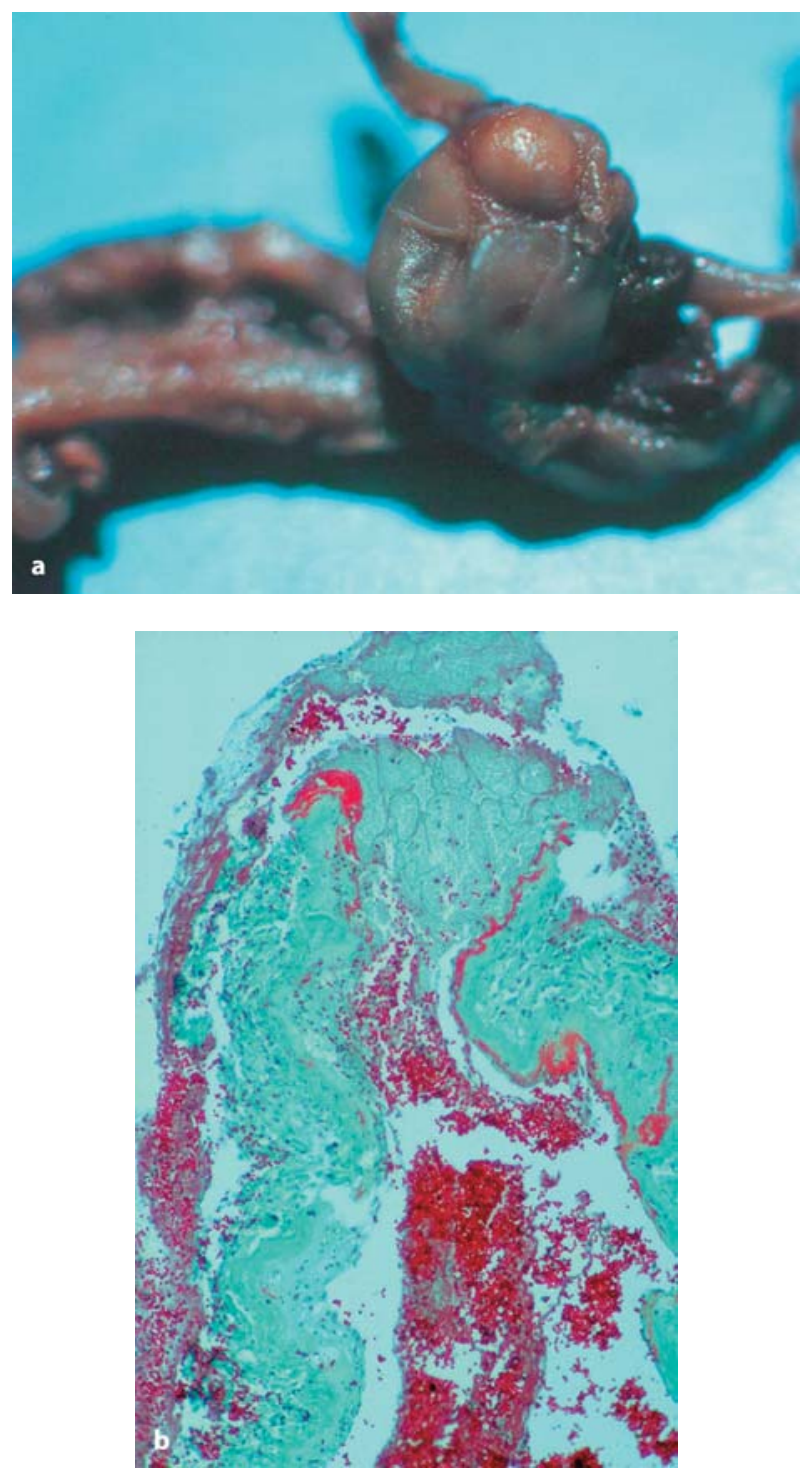

- Abb. 3.26. a Aneurysma linke A. cerebri media; b Histologie des Aneurysmas mit Rupturstelle siven Gewalteinwirkung (vor allem Rotationstrauma, auch ohne Schädelbruch!) auftreten oder (häufiger) sekundär durch intrakranielle Raumforderung (Blutung, Ödem) mit venöser Abflussbehinderung bzw. Verlagerung des Hirnstammes (»shifting«). Die sekundären Blutungen betreffen nie die kaudale Pons und die Medulla oblongata, weil deren venöser Abfluss infratentoriell liegt (Sinus petrosus).

Primäre Hirnstammblutungen müssen nicht unmittelbar tödlich sein, werden aber in der Regel nicht überlebt.

Zerreißungen des Hirnstammes (Hirnstammruptur) führen unmittelbar zum Tod; sie sind meist indirekt durch Zug (bei Kopfluxation oder Halswirbelsäulenfraktur) bedingt oder Folge massiver direkter Gewalteinwirkung auf den Schädel mit Frakturen.

Schädelfraktur. Schädelfrakturen haben für den tödlichen Ausgang keine eigenständige Bedeutung, auch wenn in der Internationalen Klassifikation der Todesursachen (ICD 10: S 02) ein »Schädelbruch« als mögliche Todesursache angegeben ist.

Die Ausprägung unterliegt Gesetzmäßigkeiten, wobei zwischen Berstungs- und Biegungsbrüchen sowie Impressionsfrakturen (Spezialfall: Loch- oder Terrassenbrüche, bei Kindern: Zelluloid- oder »Pingpongballfraktur«) unterschieden wird.

Berstungsbrüche (• Abb. 3.27) kommen durch Verkürzung des Schädeldurchmessers in Richtung der Gewalteinwirkung bei gleichzeitiger Verbreiterung im Querdurchmesser durch Zugspannungen zustande, sie folgen in ihrer Ausprägung den Trajektorien des Schädels. In der Regel ziehen sie auch in die Schädelbasis, wo es bei Beteiligung der vorderen und mittleren Schädelgruben zu Gefäßzerreißungen mit tödlichen Verblutungen kommen kann (unter dem Kopf des Verunglückten findet man eine große Blutlache mit Blutaustritt aus Mund, Nase und auch Ohren). Die Schlagadern am Hirngrund können unterhalb von Schädelbrüchen ein- oder zerreißen und zu tödlichen intrakraniellen Blutungen (meist subarachnoidal) führen. Bei massiver Einwirkung auf das Hinterhaupt kann es neben dem Berstungsbruch an der Einwirkungsstelle durch die Stauchung der Schädelbasis zu Impressionsfrakturen in den Orbitadächern, nachfol-

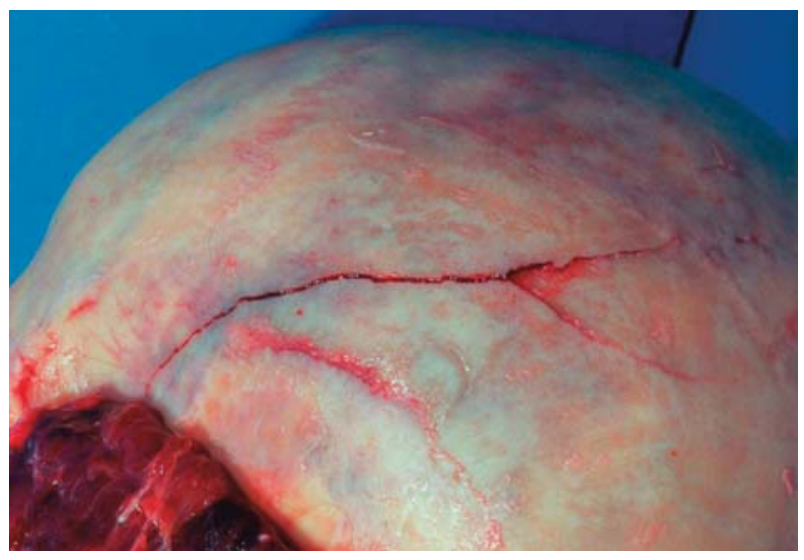

- Abb. 3.27. Schädelberstungsfraktur 


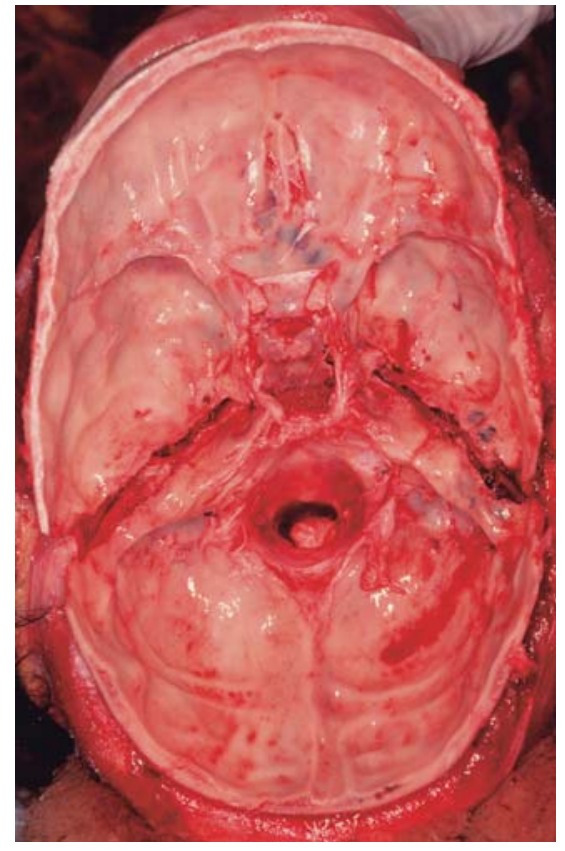

- Abb. 3.28. Schädelbasisfraktur (Scharnierbruch)

gend mit Ausprägung eines Monokel- oder Brillenhämatoms, führen.

Scharnierbrüche der Schädelbasis (• Abb. 3.28) stellen eine Sonderform der Berstungsbrüche dar, die in der Regel bei beidseitiger Einwirkung gegen den Kopf auftreten (z.B. Einklemmen des Kopfes zwischen Ladebord eines rückwärts fahrenden LKWs und der Wand oder Überrollen durch ein Kraftfahrzeug beim auf dem Boden liegenden Kopf). Unvollständige Scharnierbrüche sind bei massiver einseitiger Belastung zu beobachten.

Biegungsbrüche entstehen kreisförmig um die Einwirkungsstelle herum, sie gehen nahezu immer mit Berstungsbrüchen einher (»Globusbruch «) und setzen höhere Gewaltintensitäten als ein Berstungsbruch voraus ( $\bullet$ Abb. 3.29). Bei mehreren hintereinander folgenden, massiven Gewalteinwirkungen kann aus dem Abbruch der Bruchlinien an der vorausgegangenen Einwirkungsstelle auf die Reihenfolge geschlossen werden (»Puppe'sche Regel«).

Loch- oder Terrassenbrüche entstehen bei senkrechtem bzw. schrägem Auftreffen von geformten, kleinen Flächen (z.B. Hammerbahn), wobei die maximale Kantenlänge bei $4 \times 4 \mathrm{~cm}$ liegt.

Ringbrüche der Schädelbasis kommen selten vor. Sie sind entweder auf eine Stauchung des Kopfes in die Wirbelsäule zurückzuführen (z.B. Motorradunfall mit Aufprall des helmgeschützten Kopfes gegen eine Fläche) oder durch Traktion bedingt (Festhalten des Kopfes bei kaudaler Bewegung des Körpers). Meist kommt es dabei zur Hirnstammruptur und massiven Blutungen aus den intrakraniellen Schlagadern (Carotiden) mit schnellem Todeseintritt.

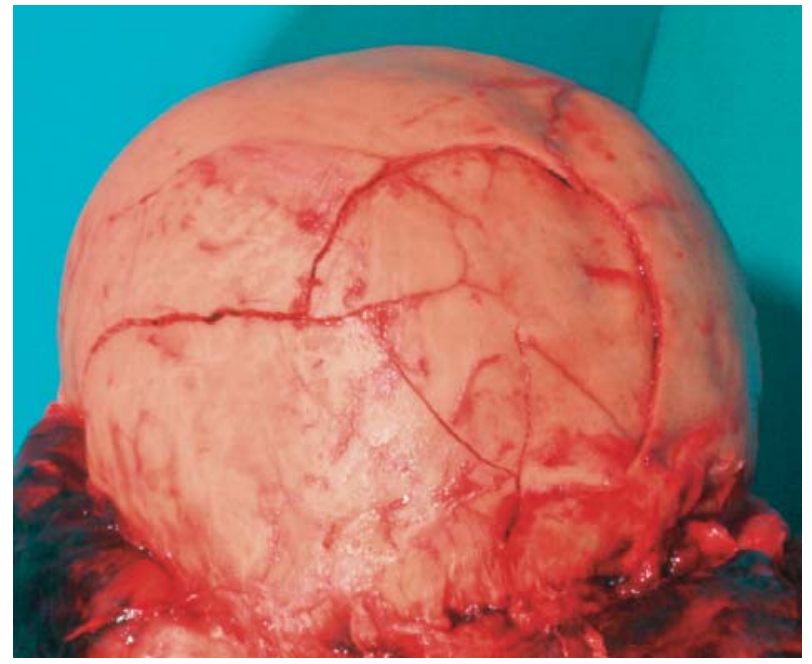

- Abb. 3.29. Berstungs-Biegungsfraktur (»Globusbruch«)

Wunden und Schädelbrüche im oberen Stirn-, Scheitel- und Hinterhauptsbereich, oberhalb der »Hutkrempenlinie« (= größter Kopfumfang), sprechen eher für von oben kommende Gewalteinwirkungen (z.B. Schlag mit einem Gegenstand auf den Kopf), während bei Sturz zu ebener Erde oder Anstoß in der Regel die prominenten Kopfpartien (Hinterhauptshöcker, NaseStirn-Partie) betroffen sind.

Ist die harte Hirnhaut eingerissen, spricht man von einem offenen Schädelhirntrauma mit der Gefahr von posttraumatischen Infektionen (vor allem bei frontobasalen Verletzungen!). Klinisch kennzeichnend (und bei Persistieren unbedingt therapiebedürftig!) ist eine Liquorrhoe aus Ohr oder Nase.

Kommt es bei Gewalteinwirkungen zur unvollständigen Zerreißung intrazerebraler Gefäße, kann sich ein traumatisches Pseudoaneurysma ausbilden, das auch noch nach längerer Zeit rupturieren kann. Selten werden traumatische Sinus-cavernosusFisteln beobachtet.

\section{Spezielle Traumatologie: Gesicht}

Stumpfe Gewalteinwirkungen gegen das Gesicht gehen häufig mit Nasenbeinfrakturen einher oder mit Gesichtsschädelbrüchen (Einteilung nach Le Fort I-III; - Tabelle 3.16 + - Abb. 3.30).

Bei Einwirkungen auf die Augen (z.B. durch Faustschlag, Tennisball) kann es zu Verletzungen des Augapfels und der Orbita kommen (Blow-out-Fraktur).

Nach Schlägen auf den Mund sind Verletzungen häufig nur an der Schleimhautinnenseite erkennbar (Widerlagerverletzungen durch die Zähne).

\section{Spezielle Traumatologie: Hals}

Direkte (Druck-) oder indirekte (Zug-) Kräfte am Hals führen vor allem zu partiellen oder vollständigen Zerreißungen der A. 
- Tabelle 3.16. Mittelgesichtsfrakturen nach Le Fort

Le Fort I (Guérin-Fraktur)

Le Fort II

Le Fort III
Durchsetzung der Apertura piriformis, Fossa canina, Kieferhöhlen und Keilbeinflügelfortsätze, im Wesentlichen mit Abtrennung des harten Gaumens

Heraussprengung des mittleren Gesichtsskelettes mit Brüchen der Nasenwurzel und der Jochbeinfortsätze. Orbitafraktur in der Fossa orbitalis inferior

Aussprengung des Gesichtsskelettes mit Jochbeinen bds., quer über die Nasenwurzel zu den Stirnfortsätzen der Oberkiefer, durch Augenhöhlen und Jochbeinbogen

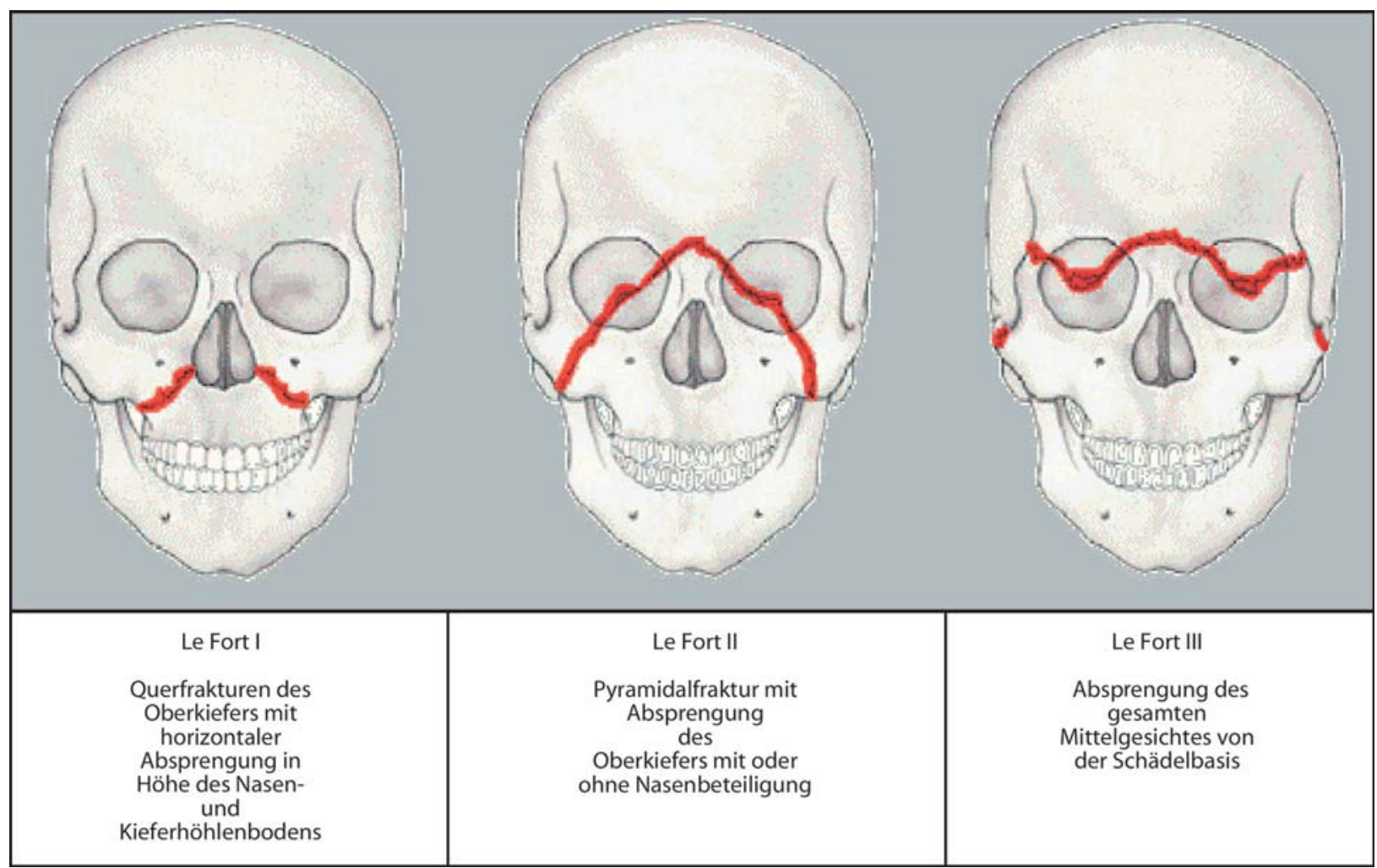

- Abb. 3.30. Mittelgesichtsfrakturen (Einteilung nach Le Fort)

carotis com. oder A. carotis int. Prädisponierend sind umschriebene stumpfe Gewalteinwirkungen auf den Hals (z.B. der Lenker eines Motorrades beim Fußgängerzusammenstoß) oder massive Überstreckungen (z.B. bei PKW-Frontalaufprall und falsch anliegendem Diagonalgurt). Während die (nur bei massivsten Kräften vorkommenden) Totalabrisse unmittelbar zum Tode führen, sind die inkompletten Rupturen mit einer wechselnden Symptomatik verbunden, die nicht selten in der Klinik verkannt wird.

\section{(3) Fallbeispiel}

Eine 21 Jahre alte Frau wird als Beifahrerin bei einem frontalen Auffahrunfall (hohe Kollisionsgeschwindigkeit) scheinbar ohne schwerere Verletzungen bei vollem Bewusstsein in ein Krankenhaus eingeliefert, wo eine LWK-Fraktur festgestellt und operativ stabilisiert wird. Sie entwickelt eine rechtsseitige Parese, das primäre CT ist unauffällig, das Kontroll-CT zeigt einen Mediainfarkt links. Dopplersonographisch ist ein Verschluss der linken A. carotis com. nachzuweisen. Tod nach $4 \frac{1}{2}$ Tagen infolge Hirnstammeinklemmung. Todesursächlich ist eine traumatische Karo- 
tisthrombose (- Abb. 3.31) bei ansonsten unauffälligen Gefäßen. Die Frau war angegurtet (innere Gurtmarke von re. oben nach li. unten), zusätzlich fand sich eine Zerrung am rechten Lungenhilus.

Bei Tötungsdelikten können durch stumpfe Gewalt (z.B. Tritte auf den Hals) Zertrümmerungen des Kehlkopfskelettes, vor allem aber Schwellungen und Blutungen auftreten, die durch Verlegung der Atemwege durch Ersticken zum Tode führen. Werden derartige Einwirkungen überlebt, ist eine fachärztliche (HNO-) Untersuchung unerlässlich.

Auch Ösophagus und Trachea sowie (sehr selten) der Ductus thoracicus können bei schweren Gewalteinwirkungen zerreißen, meist in Kombination mit anderen Verletzungen.

\section{Spezielle Traumatologie: Wirbelsäule}

Verletzungen der Wirbelsäule setzen in der Regel massive Gewalteinwirkungen voraus (Verkehrsunfall, Sturz aus der Höhe, Treppensturz), können aber bei vorbestehenden Erkrankungen auch schon bei vergleichsweise geringer Krafteinleitung (z.B. Sturz aus dem Stand) vorkommen. Die Gefährlichkeit liegt in einer Quetschung oder Zerreißung des Rückenmarkes, das je nach Höhe der Läsion zu Lähmungen bis hin zur Tetraplegie führt. Bei hohen Halswirbelsäulenfrakturen (C1/C2) und Kopfluxationen treten direkte Verletzungen am Hirnstamm und Halsmark mit schnellem Todeseintritt auf (»Genickbruch»).

Als Sonderfall ist das HWS-«Schleudertrauma« (Whiplash) zu betrachten, das vor allem bei Fahrzeuginsassen beim Heck-

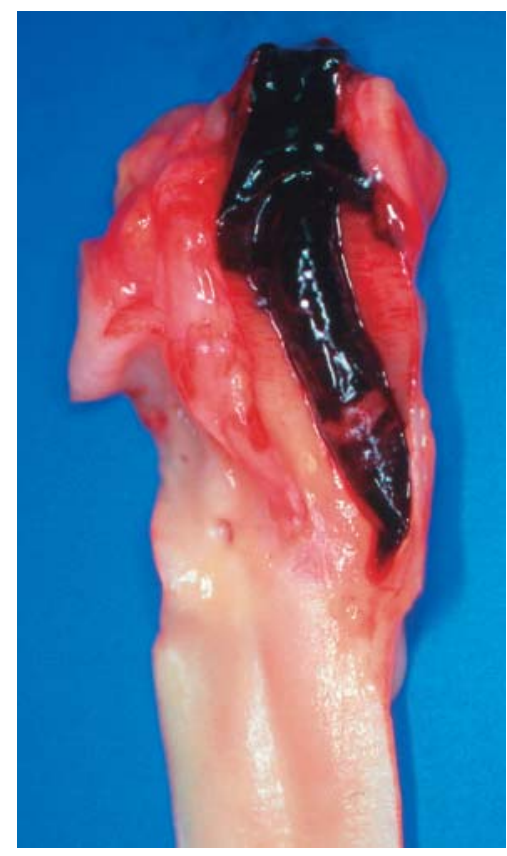

- Abb. 3.31. Karotisthrombose aufprall auftritt und häufig keine morphologischen Korrelate aufweist. Eine gutachterliche Befassung mit diesem Bereich empfiehlt sich nur bei intensiver Kenntnis der umfangreichen wissenschaftlich z.T. umstrittenen Materie.

Wenig Beachtung finden allgemein Verletzungen der Aa. vertebrales, die in den Querfortsatzlöchern der Halswirbelsäule (meist ab C7) über die Atlasschleife (C1/C2) in das Schädelinnere führen, wo sie sich zur A. basilaris vereinigen. Durch inkomplette Zerreißungen kann es zur Thrombose mit Gefäßverschluss, aber auch zur Embolisation in die intrazerebralen Arterien kommen, wobei häufig Gefäße des Hirnstammes betroffen sind (Ausprägung eines »Wallenberg-Syndroms«).

\section{Spezielle Traumatologie: Rumpf}

Bei stumpfen Gewalteinwirkungen gegen den Rumpf sind neben den Hautdecken knöcherne Strukturen betroffen (Rippen, Wirbelsäule, Becken, Extremitäten), aber auch innere Organe, die durch Druck und Zugspannung rupturieren.

Thorax. Die Brustorgane (Herz, Lungen, Aorta) sind zwar durch den Brustkorb und die Wirbelsäule vor direkten Gewalteinwirkungen weitgehend geschützt, doch kommt es nach Überschreiten der Belastungsfähigkeit zu Rippenserienbrüchen und (beim juvenilen elastischen Thorax auch ohne knöcherne Verletzungen) durch massive Brustkorbkompression zu Prellungen und Quetschungen von Herz und Lungen. Das Herz rupturiert bei ausreichender Gewalt vor allem im Bereich der rechten Kammer, massive Kräfte führen zu Zerfetzung und Abriss des Herzens mit schnellem Todeseintritt.

Indirekte Verletzungen sind bei Beschleunigungstraumen zu erwarten (Liftunfall, Sturz aus der Höhe, Verkehrsunfall, Sturz auf den Rücken), wobei durch vertikalen bzw. horizontalen Zug des Herzens Aortenrupturen entstehen, typischerweise am Ende des Bogens in Höhe des Aortenisthmus (»Botalli-Narbe«) oder (viel seltener) über den Aortenklappen.

In den Lungen kommt es bei massiven Kontusionen zu Gewebszerreißungen, ebenso auch durch Anspießung zur Ausbildung eines Hämatothorax und/oder Pneumothorax.

Der instabile Thorax bei Rippenserienfrakturen geht vor allem beim älteren Menschen mit schweren, bisweilen tödlichen Atemstörungen einher.

Bei Einklemmung des Thorax (z.B. Verschüttung, Verkehrsunfall mit Kompression der Fahrgastzelle) kommt es zur Einflussstauung vor dem rechten Herzen mit zumeist massiven petechialen Blutaustritten in der Haut oberhalb der Kompressionsebene (»Perthes' Druckstauung «). Einflussstauung und Behinderung der Atemexkursion führen letztlich zum Tode.

Bei sog. "Tottreten« als Folge schwerster Misshandlungen (»Stiefeln«) oder bei überstürzter Massenflucht (Panik) führt die Kombination von Kompression, Rippenbrüchen mit instabilem Thorax sowie Lungenfett- und Knochenmarksembolie zum Tode.

Abdomen. Bei Gewalteinwirkungen gegen den Bauch kommt es vor allem zu Milz-, Leber- und Darmrupturen, seltener sind 
Pankreas, Zwerchfell und Harnblase betroffen, bei rückwärtigen Einwirkungen auch die Nieren. Klinisch bedeutsam sind zweizeitige Rupturen (Milz!), die durch frühzeitige Ultraschalluntersuchungen und Lavage erkannt werden können.

Außerordentlich selten kommt es zur traumatischen Ruptur der Bauchaorta, meist bei arteriosklerotischer Vorschädigung.

Bei Schwangeren können massive stumpfe Gewalteinwirkungen zu einem Abort führen, der auf Loslösung der Plazenta beruhen kann, in seinen Ursachen aber nicht immer zu klären ist. Bei Unglücksfällen (z.B. Autounfall) handelt es sich strafrechtlich um eine straffreie fahrlässige Abtreibung (geahndet wird die Körperverletzung). Tritte in den Bauch bei Kenntnis der Schwangerschaft können neben der Körperverletzung auch zu einer Verurteilung wegen Abtreibung führen ( $\$ 218 \mathrm{StGB}$ ).

Becken. Knöcherne Verletzungen des Beckenringes treten meist als Folge von Verkehrsunfällen (Fußgänger/PKW), Stürzen aus der Höhe oder auch Treppenstürzen auf. Klinisch gehört die Untersuchung des Beckenringes (»Krepitation«) zu den unerlässlichen Grundmaßnahmen. Autoptisch lässt sich aus der Lokalisation und Art der Brüche die Anstoßstelle näher eingrenzen.

Genitale. Bei Beckenfrakturen (Symphysensprengung, Bruch der Schambeinäste) können Verletzungen der Urethra beim Mann auftreten, selten sind Verletzungen der Hoden durch direkte Gewalteinwirkung. Sehr selten sind auch bei Frauen Pfählungsverletzungen oder traumatische Uterusrupturen.

Extremitäten. Gewalteinwirkungen gegen die langen Röhrenknochen führen durch Kompression und Biegung zu Frakturen, die je nach Einwirkung zu unterschiedlichen Frakturmustern führen. Eine der häufigsten Verletzungen ist die Schenkelhalsfraktur des älteren Menschen durch Sturz auf die Hüfte. Unbeachtet der Ursachen eines solchen Sturzes (z.B. Bewusstseinsstörung durch zerebrale Durchblutungsstörungen) handelt es sich im Todesfall (meist durch Pneumonie, Lungenembolie) um einen (meldepflichtigen) »nichtnatürlichen Tod«, da dieser nicht allein aus innerer krankhafter Ursache erfolgte. Eine andere $\mathrm{Zu}$ ordnung wäre nur bei einem pathologischen Bruch (z.B. durch eine Knochenmetastase) zu vertreten.

Eine Sonderform stellt der Keilbruch (»Messererbruch «) nach umschriebener Gewalteinwirkung vor allem gegen den Unterschenkel dar, der früher bei Fußgänger-PKW-Kollisionen zu beobachten war (»Stoßstangenverletzung«). Bei dem Keil stellt die Basis den Ort der Gewalteinwirkung dar, die Keilspitze weist in Richtung der Gewalteinwirkung (• Abb. 3.32).

Bei Gefäßverletzungen (Aorta!) kommt es nicht selten erst mit einer gewissen Latenz zur vollständigen Ruptur (zweizeitige Rupturen), die mit erheblicher klinischer und gutachterlicher Problematik einhergehen (Frage der Erkennbarkeit, Unterlassung ausreichender Diagnostik und Therapie, kausaler Zusammenhang mit dem längere Zeit zurückliegenden Unfallereignis). Die gewissenhafte Begutachtung erfordert die Beiziehung sämtlicher ärztlicher Befunde, die Erhebung der Fremd- und Eigenanamnese (»Brückensymptome«) sowie im Todesfall der subtilen feingeweblichen Untersuchung der Rupturstelle zum Ausschluss

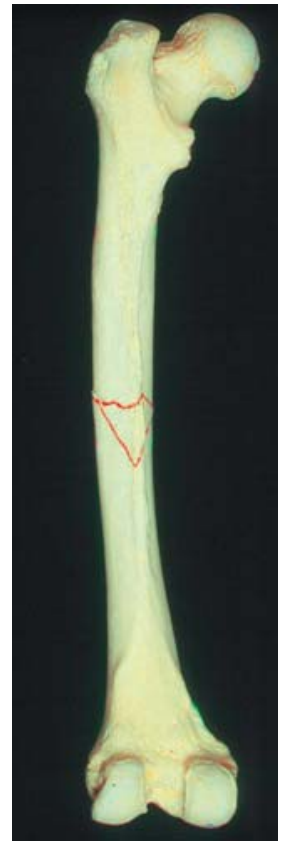

- Abb. 3.32. Keilfraktur (»Messerer-Bruch«)

einer Vorerkrankung (z.B. Medianecrosis Erdheim-Gsell; • Abb. $3.33 a, b)$.

\section{(3) Fallbeispiel}

Ein 50 Jahre alter Chemiearbeiter hatte bei einer Verpuffung am Arbeitsplatz Verbrennungen im Gesicht davongetragen, die ambulant ärztlich versorgt wurden. Zu Hause wurde er ca. 7 Stunden später auf der Toilette bewusstlos aufgefunden und verstarb kurz danach im Krankenhaus. Todesursächlich war eine $3,5 \mathrm{~cm}$ breite Ruptur oberhalb der Aortenklappen mit disseziierendem retround anterogradem Aneurysma und Herzbeuteltamponade. Nebenbefunde: Herzhypertrophie $(500 \mathrm{~g})$, weite Aortenklappen $(8,5 \mathrm{~cm})$. Zweitgradige Gesichtsverbrennungen. Kein Inhalationstrauma, keine knöchernen Verletzungen.

Histologie: Medianecrosis Erdheim-Gsell. Gutachterlich war kein direkter oder indirekter Zusammenhang erkennbar, die psychophysische Belastung mit Blutdrucksteigerung zwar denkbar, aber nicht mit der notwendigen Wahrscheinlichkeit zu belegen.

Für den klinisch tätigen Arzt und bei der Leichenschau ist besonders zu bedenken, dass bei stumpfen Gewalteinwirkungen zwischen äußerlich sichtbaren Verletzungen und dem Ausmaß der inneren Verletzungen keinerlei Zusammenhang bestehen muss. Besonders bei Kindern kommt es zu Leber-, Pankreas-, Darmund Milzrupturen, ohne dass äußerlich oder bei der Präparation der Hautschichten auch nur die geringste Verletzung erkennbar wäre. 

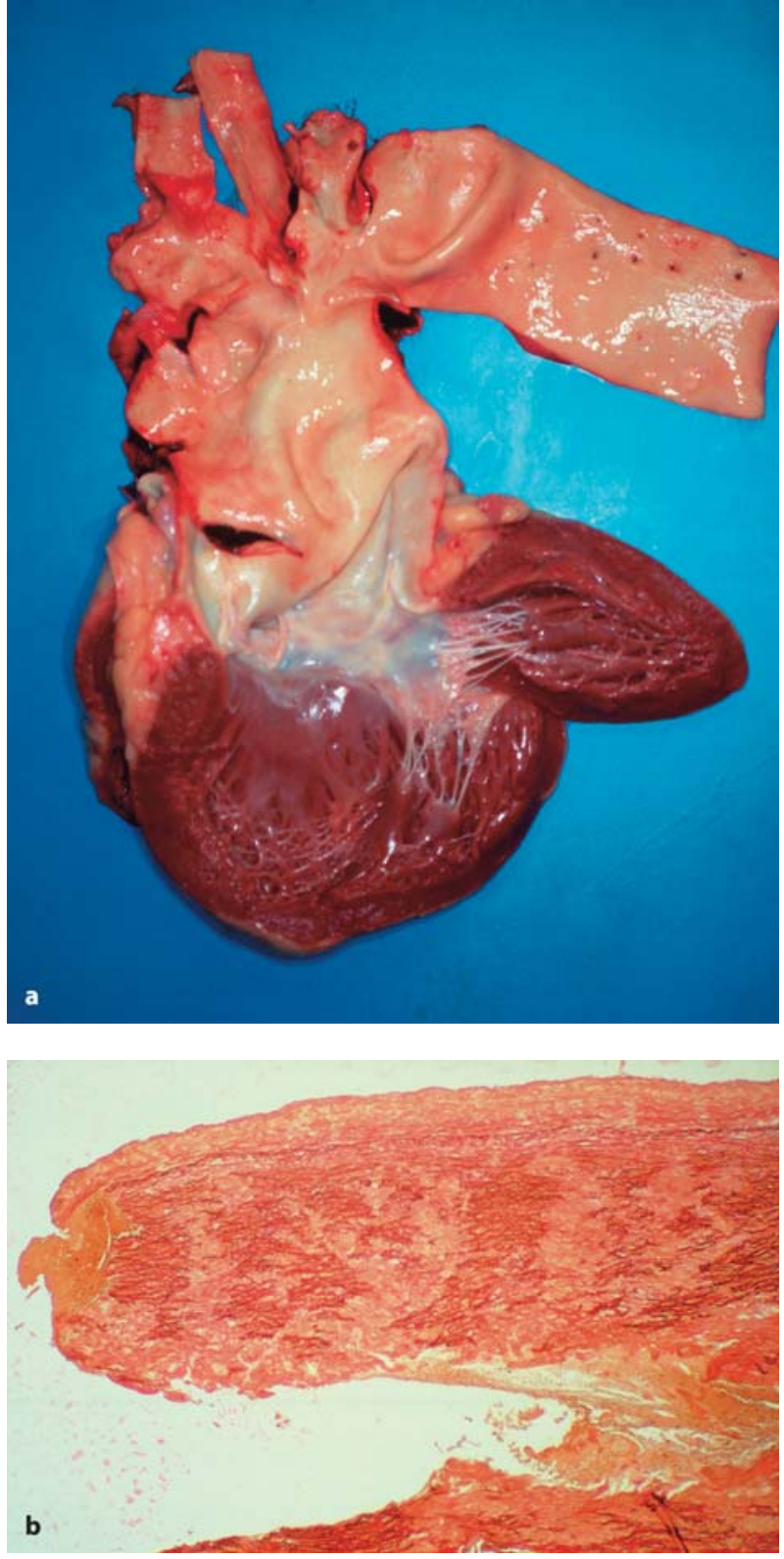

- Abb. 3.33. a Aortenruptur; b Medianecrosis Erdheim-Gsell

\section{Fallbeispiel}

Ein 10 Monate alter Säugling wird nach Angabe der Mutter während des Badens plötzlich leblos, der alarmierte Notarzt führt vergebliche Wiederbelebungsversuche durch und attestiert schließlich eine ungeklärte Todesart mit Hinweis auf plötzlichen Kindstod (SIDS). Äußerlich waren keinerlei Verletzungsspuren festzustellen. Zur Vorgeschichte gab die Mutter an, dass das Kind
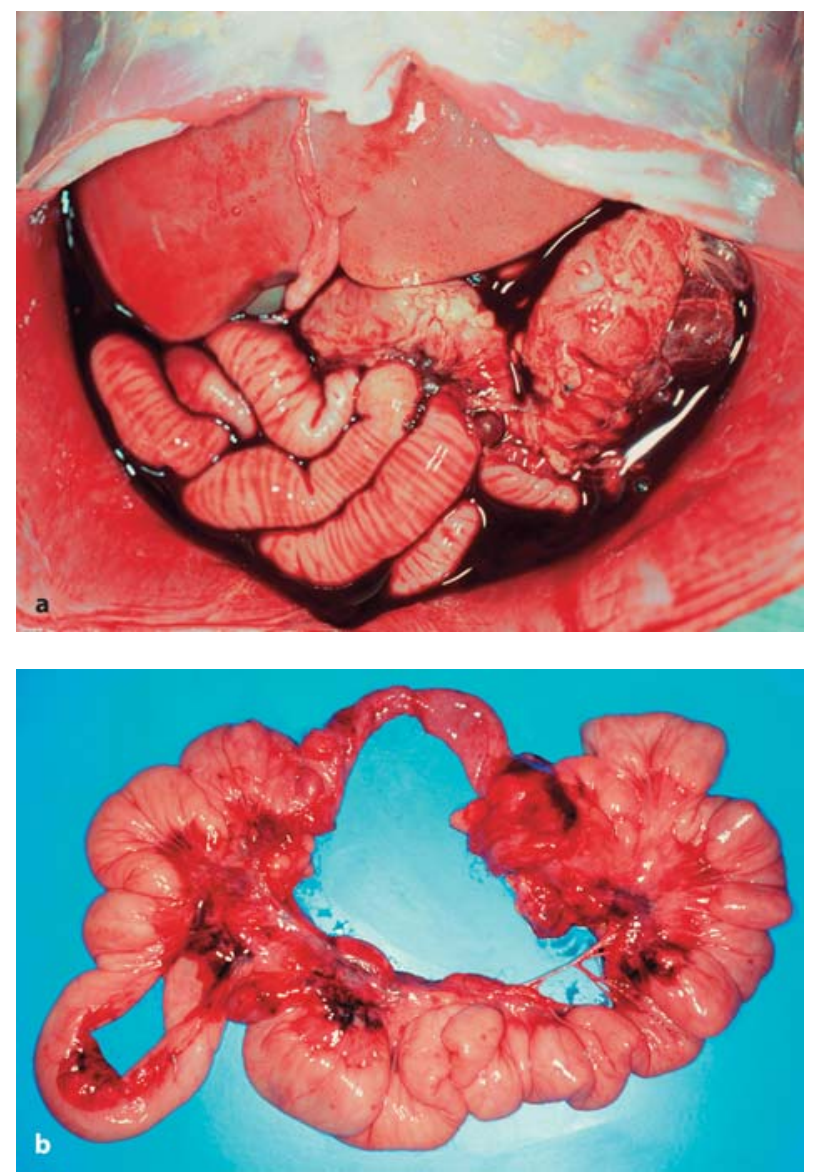

- Abb. 3.34a, b. Kindesmisshandlung. a Bauchsitus, innere Verblutung; b Duodenal-, Pankreas- u. Leberruptur

in den Tagen vorher einen Darminfekt gehabt habe. Nachdem bei der Sektion drei verschieden alte Schädelbrüche festgestellt worden waren (aber keine akute Hirnschädigung), erfolgte eine sorgsame Präparation sämtlicher Hautdecken, wobei sich auch hier nirgendwo Verletzungsspuren nachweisen ließen. Bei der Eröffnung der Bauchhöhle fanden sich ca. 500 ml Blut, eine Leber- und subtotale Pankreasruptur sowie subtotale Mesenterial-und Darmeinrisse. In einem späteren Geständnis gab die Mutter an, dem auf dem Boden liegenden Kind mit dem nackten Fuß den Bauch mit kreisenden Bewegungen tief eingepresst zu haben, wobei es dann durch die Quetschungen gegen die Wirbelsäule (Widerlager) zu den Rupturen kam (• Abb. 3.34a, b).

\section{Kriminalistik}

Die Verteilung und Lokalisation von Blutunterlaufungen, Schwellungen, Schürfungen und Wunden können Hinweise für eine Fremdeinwirkung liefern. So sprechen, wie bereits oben dargestellt, Verletzungen oberhalb der »Hutkrempenlinie« des Kopfes 
eher für Fremdeinwirkung und gegen einen Sturz auf das Hinterhaupt.

Einwirkungsspuren an den Streckseiten der Unterarme lassen an die aktive Abwehr der Einwirkungen denken (»Parierverletzungen «), ebenso auch Verletzungen an der Oberfläche der Hand, wenn diese schützend vor den eigenen Körper gehalten werden (insbesondere bei Einwirkungen gegen den Kopf und auf das Gesäß).

Verletzungen wie ein Monokelhämatom, Nasenbeinbruch oder Blutunterlaufungen der Lippen weisen bei gleichzeitigen sturzbedingten Verletzungen auf Schlag oder Schläge in das Gesicht hin, wobei die Möglichkeit einer sturz- oder anstoßbedingten Verletzung gerade bei stärkergradiger Alkoholisierung, Drogeneinfluss oder krankheitsbedingten Bewusstseinsstörungen in Erwägung zu ziehen ist.

Beim festen Anpacken an den Armen im Verlaufe einer tätlichen Auseinandersetzung sieht man nicht selten Blutunterlaufungen an deren Innen- und Außenseiten (»Griffspuren«), die sich aber (im Überlebensfall) bisweilen erst mit einiger Latenz zeigen. Im Todesfall kommen sie bisweilen erst bei der Präparation der Hautschichten zum Vorschein.

Liegt der Körper bei massiven Einwirkungen auf einer festen Unterlage (z.B. Fußboden) kann es zu Widerlagerverletzungen kommen, wie z.B. Blutunterlaufungen über den Schulterblättern beim Knien auf dem Brustkorb.

Wegen der weitgehenden Uniformität der Verletzungsfolgen nach stumpfen Gewalteinwirkungen sind sichere Rückschlüsse auf die Verletzungsursache und die Art der einwirkenden Gewalt häufig nicht oder nur mit Einschränkungen möglich. Eine Kombination direkter und indirekter Untersuchungstechniken (körperliche Untersuchung, Röntgen, CT, MRT, Ultraschall) und konsiliarische Beratung durch Rechtsmediziner, Traumatologen, Röntgenologen schafft aber häufig eine ausreichend sichere Bewertungsgrundlage für die Begutachtungen in den verschiedenen Rechtsgebieten.

\subsubsection{Scharfe Gewalt}

\section{Oehmichen, B. Madea}

\section{Grundlagen}

Begriffe und Definitionen. Verletzungen durch scharfe Gewalt werden verursacht durch spitz zulaufende oder schneidende Werkzeuge (Messer, Eispickel, Kugelschreiber, Schere, Nadeln, Spieße, Gabeln, Schraubenzieher, Glasscherben).

Penetrierende Hautverletzungen als Folge »scharfer Gewalteinwirkung « sind durch folgende gemeinsame morphologische Kriterien gekennzeichnet:

- durch eine Gewebedurchtrennung unterschiedlicher Tiefe,

- durch einen in der Regel geradlinigen und glattrandigen Wundrand,

- durch das Fehlen eines Vertrocknungs-, Schürf-, Quetschungssaumes (Ausnahme: Einstich bis zum Messerheft),
- durch das Fehlen von Gewebebrücken in der Tiefe der Verletzung, da alle Gewebsschichten gleichmäßig durchtrennt werden.

\section{Definition}

Grundsätzlich sind zu unterscheiden:

Stichverletzungen als Folge einer Gewebedurchtrennung mittels spitz zulaufendem Werkzeug (z.B. Messer), das überwiegend senkrecht zur Körperoberfläche geführt wird, mit einem in die Tiefe reichenden Stichkanal;

Schnittverletzungen mit der Folge einer längs verlaufenden scharfen Gewebedurchtrennung bei überwiegend parallel und/oder tangential zur Körperoberfläche geführtem Werkzeug und

Hiebverletzungen als Folge einer Schlagverletzung mittels scharfem Werkzeug (schwere Werkzeuge mit zumindest einer schneidenden Seite: Äxte, Beile, Säbel, Macheten, Propeller, Schiffsschrauben).

Stichwunden stellen heute nach Verletzungen durch stumpfe Gewalt die häufigsten Traumafolgen dar, bedingt u.a. dadurch, dass zunehmend Stichwaffen mitgeführt werden.

Medizinische und ermittlungstechnische Fragen bei Tod und Gesundheitsbeschädigung durch scharfe Gewalt. Dies sind

- Entstehungsart: Selbstbeibringung (Suizid/Selbstverstümmelung) versus Fremdbeibringung (Tötung, gefährliche Körperverletzung) versus Unfall

- Todesursache: inneres/äußeres Verbluten, Herzbeuteltamponade, Aspiration, Pneumothorax, Luftembolie

- tätliche Auseinandersetzung versus verletzt/getötet in einem arglosen oder handlungsunfähigen Zustand

- Agoniedauer und Fragen der Handlungsfähigkeit

- Kennzeichen des Tatwerkzeuges (Klingenbreite, Klingenlänge, einschneidiges, zweischneidiges Werkzeug usw.)

- Rekonstruktion des Handlungs- und Geschehensablaufs

- Zuordnung des Spurenbildes (Bluttropf- und -spritzspuren, Wischspuren) zum geschilderten Tatablauf.

Dokumentation. Hierzu muss insbesondere bei forensisch relevanten Fällen eine detaillierte Beschreibung aller Einzelverletzungen sowie eine umfangreiche Fotodokumentation erfolgen:

- Morphologie der Einzelverletzungen

- Handelt es sich um ein oder um mehrere Tatwerkzeuge?

- Zahl und Lokalisation der Verletzungen

- Verteilung der Verletzungen (gruppiert, disseminiert-verstreut)

- Verlauf der Verletzungen (oberflächlich, tangential, senkrecht in die Tiefe, schräg von oben nach unten, seicht auslaufend, unterminierend usw.) 


\section{Stichverletzungen}

Morphologie. Auch mit runden oder konischen Werkzeugen beigebrachte Stichverletzungen weisen in der Regel eine mandelförmige oder elliptische Gestalt auf, die je nachdem, wie die elastischen Fasern der Haut durchtrennt werden, auf einer unterschiedlichen Breite klaffen (• Abb. 3.35).

\section{(I) Infobox}

Werden die elastischen Fasern der Haut quer durchtrennt, klafft die Wunde naturgemäß breiter als bei parallelem Verlauf von Wunde und elastischen Fasern. Plastische Chirurgen legen daher, um kosmetisch unauffällige Narben zu erzielen, Hautschnitte parallel zu den Langer'schen Hautspaltlinien.

Drei- oder vierkantige Werkzeuge verursachen charakteristische Stichverletzungen korrespondierend zu ihrer Konfiguration (• Abb. 3.36).

Der Wundrand von Stichverletzungen ist in der Regel geradlinig und glattrandig, ohne Riffelung und Zähnelung; bei Messern mit Wellenschliff oder Scharten können hingegen charakteristische Zähnelungen auftreten.

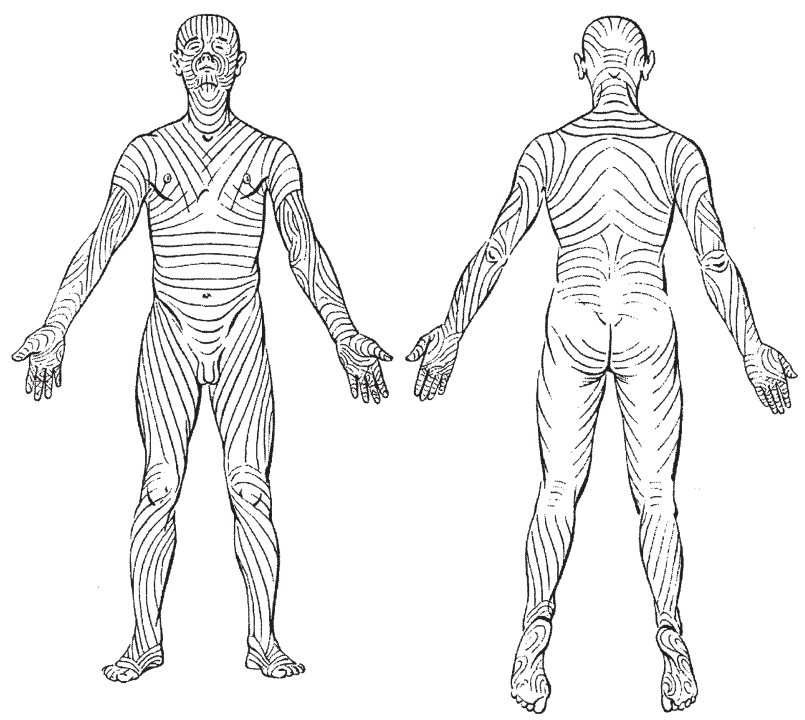

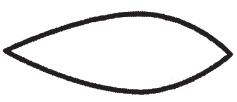

A

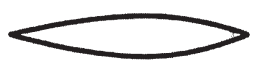

B
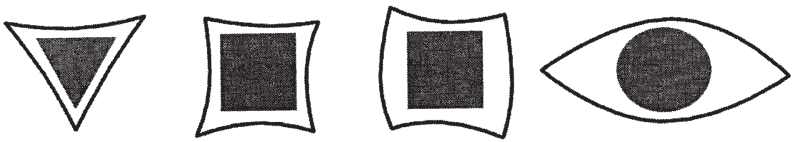

- Abb. 3.36. Stichwunden an der Haut durch drei-, vierkantige oder runde Werkzeuge

In der Regel fehlt bei Stichverletzungen angrenzend an die Kontinuitätsdurchtrennung ein Vertrocknungs-, Schürf- oder Quetschsaum, der allerdings bei homizidalen Stichverletzungen (Einstich der Messerschneide bis ans Heft) ein wichtiges differentialdiagnostisches Kriterium der Fremdtötung darstellen kann.

Der Wundwinkel ist abhängig von der Art des Werkzeuges und lässt sich bei Stichverletzungen in der Haut (nach Adaptation der Wunde) meist annähernd sicher erfassen: spitz zulaufend, korrespondierend zur Schneideseite der Klinge; dementsprechend zeigen sich bei zweischneidigen Werkzeugen zwei spitz zulaufende Wundwinkel. Bei einem einschneidigen Werkzeug kann der dem Messerrücken entsprechende Wundwinkel kantig, rund oder klein-schwalbenschwanzförmig konfiguriert sein (• Abb. 3.37). Insbesondere wenn die Kanten des Messerrückens sehr scharf sind, zeigt sich der zum Messerrücken korrespondierende Wundwinkel schwalbenschwanzförmig aufgegabelt.

Der spitz zulaufende Wundwinkel ist häufig zusätzlich gekennzeichnet durch einen so genannten Auszieher, eine seicht auslaufende Oberhautanritzung, die bei nicht ganz senkrechtem Herausziehen des Tatwerkzeuges an der Hautoberfläche entsteht und die der Schneideseite des Messers entspricht.

Schließlich können durch Drehung - Drehen des Messers in der Wunde oder Ausweichbewegung des Opfers nach Erhalt der Stichverletzung - mehrachsige Wundformen entstehen, weil die Lage des Messers sich im Stichkanal beim Herausziehen geändert hat - bei Vergleich mit dem Einstechen (• Abb. 3.38).

Häufig wird bei Einstich das Messer nicht nur senkrecht durch die Haut gestochen, sondern es kommt gleichzeitig zu einer Bewegung des Messers in Richtung Schneide, wodurch die Einstichwunde größer wird, als es der Klingenbreite entsprechen würde (• Abb. 3.39a).

In vielen Fällen lässt sich bei derartigen kombinierten StichSchnitt-Verletzungen bei genauer Inspektion des Wundrandes auf der einen Seite eine Stufen- oder Zipfelbildung, auf der anderen Seite eine Einkerbung feststellen, die bei Adaptation der Wundränder miteinander korrespondieren.

Stichkanallänge: Wird mit einem Messer heftig bis zum Heft eingestochen, kann es durch Kompression der Weichteile (zum Beispiel der Bauchdecken) zu einem Stichkanal kommen, der länger ist, als die Messerklinge (• Abb. 3.39b).

Weisen Messerrücken oder auch Schneide über der Klingenlänge eine unterschiedliche Konfiguration auf, kann dementsprechend - korrespondierend zur Einstichtiefe - auch die Morphologie der Stichverletzung variieren (• Abb. 3.40).
- Abb. 3.35. Verlauf der elastischen Fasern in der Haut, sog. Langer'sche Hautspaltlinien (oben); mit dem gleichen Stichwerkzeug beigebrachte Stichwunden, die mehr oder weniger klaffen, je nachdem, ob die Stichverletzung parallel (C) oder quer (A) zum Verlauf der elastischen Fasern liegt (unten) 

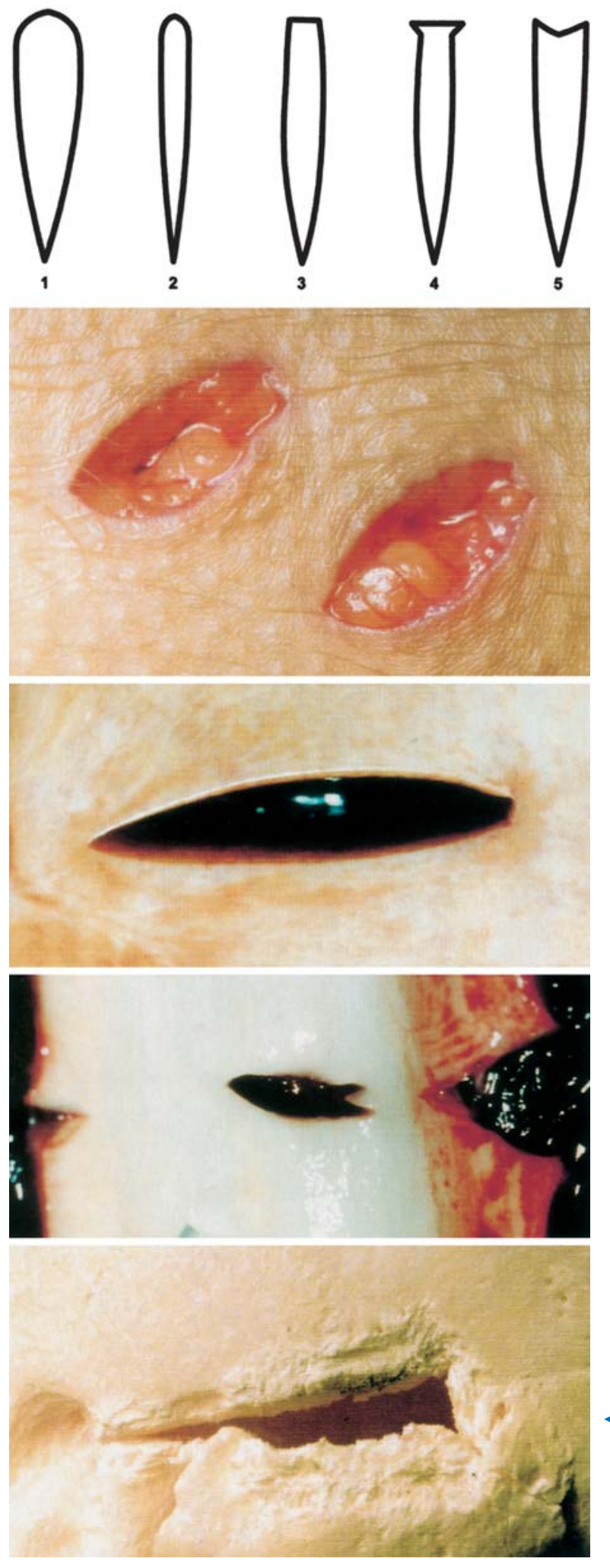
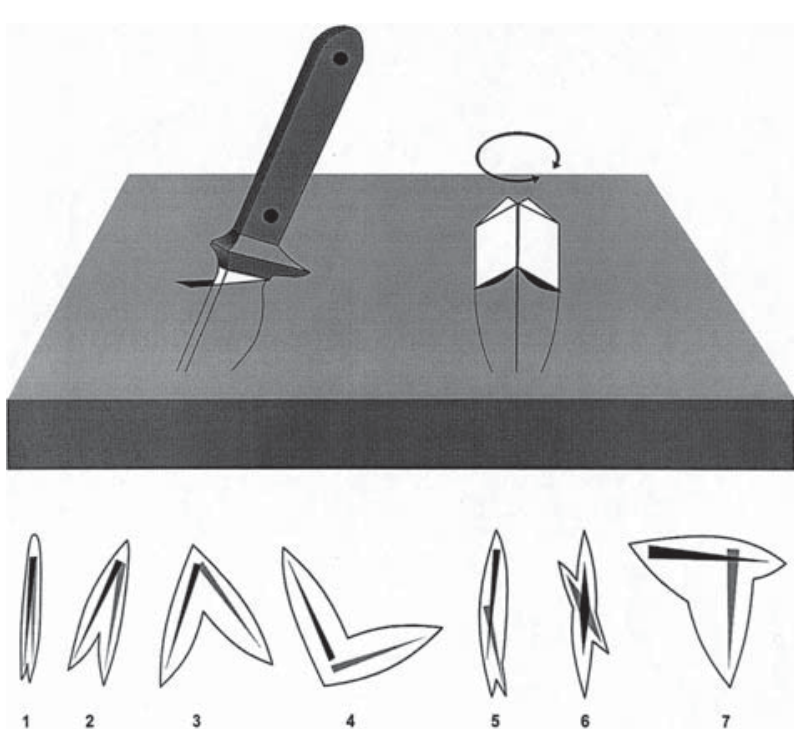

- Abb. 3.38. Kleiner Schwalbenschwanz an der Seite des Messerrückens, großer Schwalbenschwanz durch Relativ-Bewegung zwischen Messer und durchstochenem Objekt, so dass die Lage des Messers im Stichkanal beim Herausziehen anders ist, als beim Einstechen (oben). Verschiedene Formen des großen Schwalbenschwanzes (unten): 1-4 durch Drehen des Messers oder Ausweichbewegung des Opfers. 5-7 bei einer gleichzeitigen gröberen Schnittbewegung, die Wundform 6 mit zwei »großen« Schwalbenschwänzen ist nur bei zweischneidigem Messer möglich

\section{(8) Wichtig}

Aus den exakt zu erhebenden Maßen einer Stichverletzung können daher nur mit Zurückhaltung Rückschlüsse auf die Abmessungen des verursachenden Werkzeuges gezogen werden.

Grundsätzlich kann die Stichwunde größer, gleich groß oder kleiner sein als die Messerschneide, der Stichkanal länger, gleich lang oder kürzer als die Klingenlänge.

Tragfähig sind in der Regel Aussagen zur Schneidigkeit (einoder zweischneidig) sowie bei mehrfachen Stichverletzungen zu wiederholt gemessenen Längen der Stichverletzung (korrespondierend zur Breite der Klinge) und zu Stichkanallängen. Auf der Basis dieser Werte kann ein in Betracht kommendes Messer als zur Verursachung der Stichverletzungen geeignet angesprochen bzw. ausgeschlossen werden. Bei schrägem Einstich sind auch bei Stich mit einschneidigem Messer häufig beide Wundwinkel spitzwinkelig, wobei der in Stichrichtung

- Abb. 3.37. Form der Stichwunde in der Haut bei Stich mit einschneidigem Messer. Teils ist der Wundwinkel abgerundet, teils kantig, bei sehr scharfen Kanten des Messerrückens zeigt sich eine typische sog. kleine Schwalbenschwanzform. Darunter durch ein einschneidiges Werkzeug beigebrachte Stichverletzung in Haut, Leber, Aorta und Schädel. Der Messerrücken weist jeweils nach rechts 
- Abb. 3.39. a Beim Einstechen wird das Messer zuweilen in Richtung Schneide gezogen; entsprechend ist die Stich-/Schnittverletzung bezogen auf die Hautoberfläche größer als der Klingenbreite entsprechen würde. b Bei Einstich bis ans Messerheft und Kompression der Weichteile resultiert ein die Klingenlänge überschreitender Stichkanal
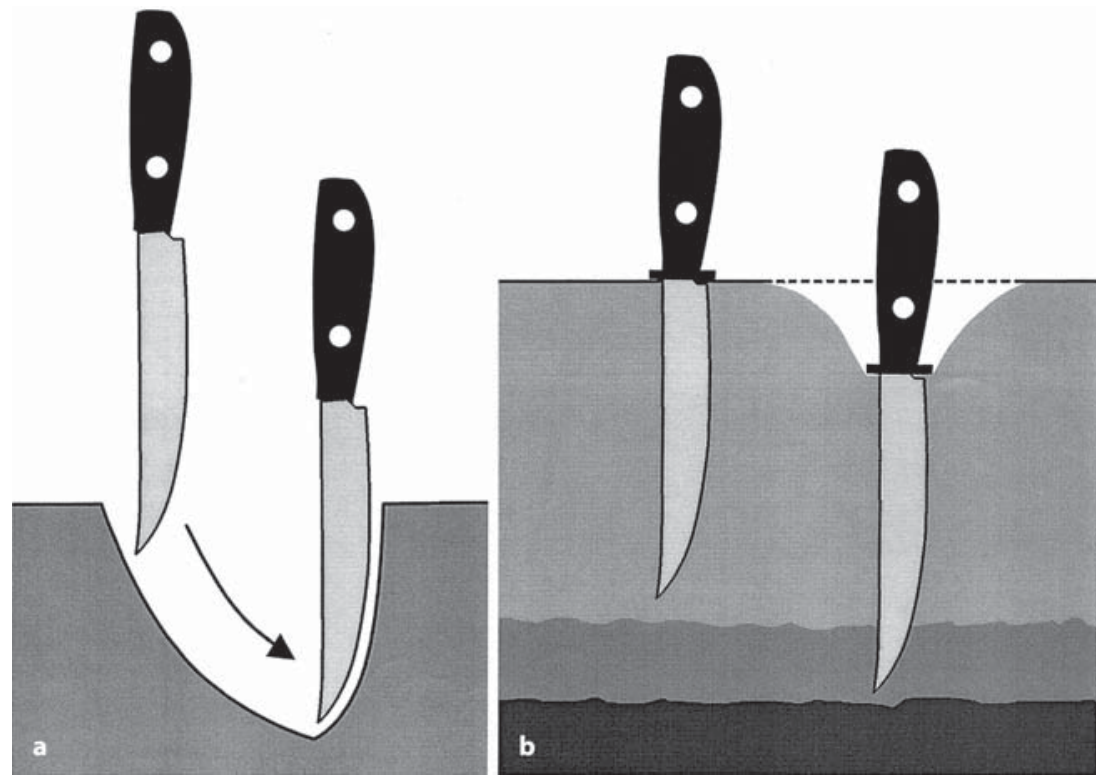

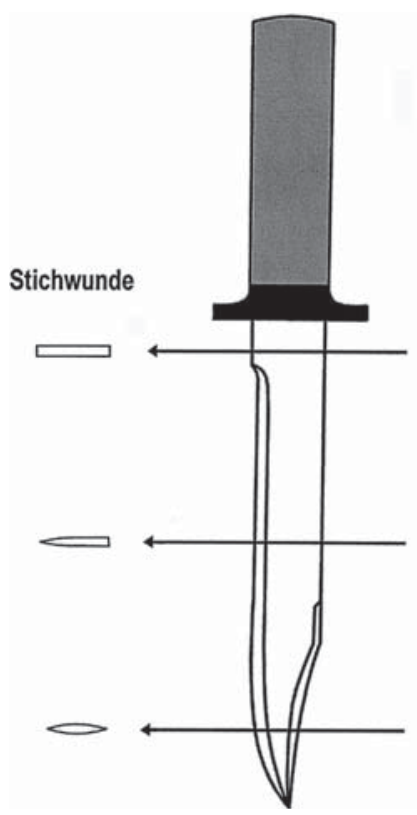

- Abb. 3.40. Variierende Morphologie der Stichverletzung korrespondierend zu unterschiedlichen Einstichtiefen

gelegene Wundrand unterminiert, während der andere abgeschrägt ist.

Morphologische Varianten: Es existiert eine Vielzahl an Stichwerkzeugen mit extremen Modifikationen, insbesondere der Klingen. Entsprechende Modifikationen treten auch in der Morphologie der Stichwunde auf.
Dies gilt auch für anders geartete Stichwerkzeuge, die nicht ganz spitz oder scharf zulaufen (• Abb. 3.41a-c). Findet zum Beispiel ein Kugelschreiber, Bratspieß oder Schraubenzieher $(\bullet$ Abb. 3.41a) Verwendung als Stichwerkzeug, entsteht oft ein unter Umständen nur diskreter Schürfsaum um die Einstichverletzung herum. Bei Scheren als Stichwerkzeugen variiert die Morphologie der Einstichverletzung je nachdem, ob mit einer oder mit beiden Branchen der Schere zugestochen wurde (offene oder geschlossene Schere). Bei Zustechen mit Gabeln erlaubt der Abstand der Hautperforationen unter Umständen einen Rückschluss darauf, ob senkrecht oder schräg zugestochen wurde (• Abb. $3.41 b, c)$.

Werden abgebrochene Flaschenhälse als Stichwerkzeug benutzt, entstehen Kontinuitätsdurchtrennungen der Haut, die die Konfiguration des Flaschenhalses wiedergeben können (• Abb. 3.42).

Forensische Schlussfolgerungen. Die häufigste Todesursache nach Stichverletzungen ist der akute VolumenmangelSchock, verursacht durch ein inneres und/oder äußeres Verbluten. Ursächlich sind in der Regel Eröffnungen von Schlagadern (oder auch größeren Venen) beziehungsweise die Eröffnung einer Herzkammer. Durch Bruststiche kann es einerseits zu einem akuten Blutverlust kommen, aber auch zur Ausbildung einer Herzbeuteltamponade, und damit zum Herzstillstand ohne wesentlichen Blutverlust. Eine akute Einblutung von ca. $150 \mathrm{ml}$ Blut in den Herzbeutel führt zur tödlichen Tamponade. Verbluten als Todesursache ergibt sich durch einen höhergradigen Blutverlust nach außen (Blutmenge am Auffindungsort, blutdurchfeuchtete Bekleidung) und/oder nach innen (Blutmenge in den Körperhöhlen, Weichteilen). 
- Abb. 3.41a-c. Einstichmorphologie, a bei Stich mit Schraubenzieher (Schürfung); b Gabel und c Schere
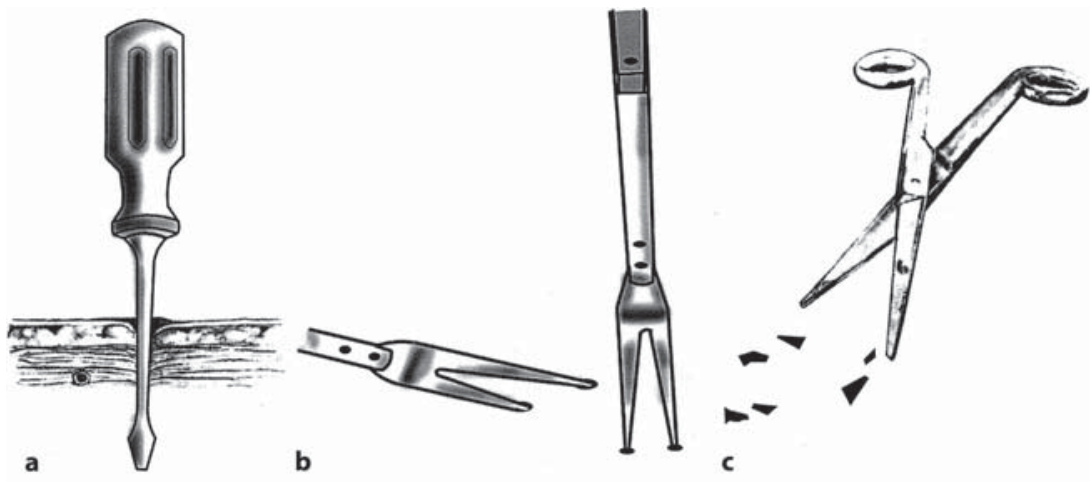

\section{Infobox}

Pathologisch-anatomische Zeichen des Verblutungstodes sind: geringe Ausdehnung und Intensität der Totenflecke, die aber kaum je vollständig fehlen, eine Ausblutungsblässe der inneren Organe mit Hervortreten der Organeigenfarbe von Leber, Nieren und Schilddrüse, eine Milzkapselrunzelung und streifige Unterblutungen des Endokards in der Ausflussbahn der linken Herzkammer (sog. Verblutungsblutungen).

Bei innerem Verbluten finden sich in den Brusthöhlen durchaus Blutvolumina von 1,5-2,5 Litern. Ein Blutverlust von 1/3 des Gesamtblutvolumens ist lebensgefährlich, von $2 / 3$ generell tödlich. Bei entsprechender Vorschädigung können auch geringere Blutverluste letal enden.

Das Blutvolumen des erwachsenen Menschen beträgt etwa 6-8 \% seines Körpergewichtes. Es lässt sich folgendermaßen errechnen:

- Männer: 0,041 × kg Körpergewicht + 1,53

- Frauen: 0,047 × kg Körpergewicht + 0,86

Prognoseentscheidend ist jedoch nicht nur das Ausmaß, sondern auch die Schnelligkeit des Blutaustritts.

Bei überlebten Stichverletzungen wird in foro die Lebensgefährlichkeit der Verletzungen zu erörtern sein. Hilfreich sind ärztliche Dokumentationen der Kreislaufsituation (Blutdruck, Puls, Schockindex - Puls/systolischer Blutdruck) neben anderen Verletzungsfolgen (z.B. Pneumothorax). Der Schockindex korreliert mit dem Volumenverlust (bei 1 ca. $30 \%$, bei 2 ca. $70 \%$ Volumenverlust).

Durch Eröffnung einer oder beider Brusthöhlen entsteht ein Pneumothorax und/oder Hämatothorax, die beide - falls nicht behandelt - den Tod zur Folge haben können.

Bei Halsstich kann über eine Eröffnung der Trachea eine Blutaspiration eintreten - und damit ein Erstickungstod; durch eine Eröffnung der großen Halsvenen besteht zudem die Gefahr der Ausbildung einer Luftembolie. Zur Entstehung einer tödlichen
Luftembolie sind Gasvolumina von 70-150 ml ausreichend. Bei Eröffnung zervikaler Venen wird bei Ansaugen von Luft teilweise ein typisch saugendes Geräusch wahrgenommen; durch Verlegung der pulmonalen Strombahn und Durchmischung von Blut mit Luft im rechten Ventrikel entsteht das auskultatorisch wahr-
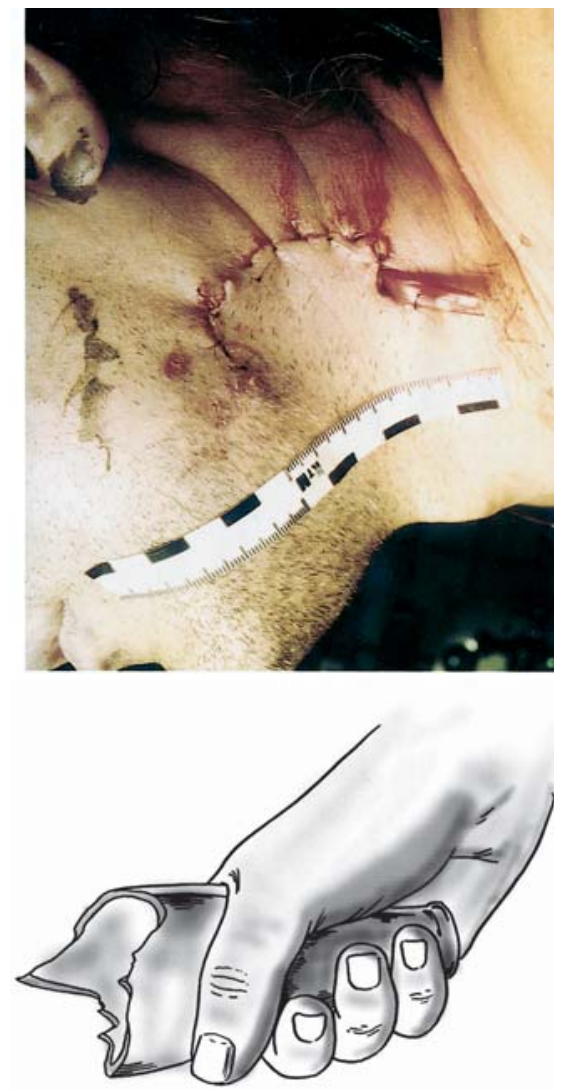

- Abb. 3.42. Abgebrochener Flaschenhals als Stichwerkzeug. Chirurgisch adaptierte, aber nicht exzidierte Wunde des Halses links 
nehmbare Mühlengeräusch, wobei die Betroffenen selbst ein brodelndes Geräusch wahrnehmen. Der postmortale Nachweis der Luftembolie erfolgt einerseits radiologisch, und/oder durch eine spezielle Sektionstechnik (Luftembolieprobe nach Richter).

\section{(I) Infobox}

Luftembolieprobe: Nach Eröffnung des Brustkorbs, unter Schonung der 1. Rippe zur Vermeidung von Verletzungen der V. subclavia, wird der Herzbeutel eröffnet und mit Wasser gefüllt. Bei Luftembolie zeigt sich typischerweise ein Gasauftrieb des Herzens. Bei Einstich in die rechte Herzkammer unter Wasser entweichen Luftblasen. Die akut dilatierte rechte Herzkammer weist in der Regel nur wenig schaumiges Blut auf. Gegebenenfalls ist die Luft mittels Aspirometer für gasanalytische Untersuchungen zu asservieren.

Ein Stich in das Genick oder den Nacken mit Verletzung des Halsmarks kann akut zu einem zentralen Atem- und Kreislaufstillstand führen. Ein Stich in den Schädel, besonders die Eröffnung des Sinus sagittalis, kann im Einzelfall eine akute intrakranielle Blutung mit Raumverdrängung zur Folge haben.

Bei überlebten Stichverletzungen ist mit Spätfolgen zu rechnen, wozu unter anderem Entzündungen, Verwachsungen, Querschnittslähmungen, Ausbildung einer Pneumonie oder Thrombembolie usw. gehören ( $\triangleright$ Kap. 3.4).

Entstehungsart: Stichverletzungen können durch fremde Hand willkürlich oder auch unwillkürlich (Unfall/Fahrlässigkeit) beigebracht werden bzw. durch eigene Hand entstehen (Selbstbeibringung bei Selbstverstümmelung, Suizid oder als Unfall).

In der Regel sind die tödlichen Stichverletzungen von fremder Hand beigebracht.

Unter den suizidalen Einstichlokalisationen prävaliert eindeutig die Herzgegend. Suizidtypische Befundmuster sind:

- mehrere dicht nebeneinander liegende, topographisch eng begrenzte Stichverletzungen,

- Herzgegend,

- Entkleidung der Einstichlokalisation,

- gleiche, überwiegend horizontale Verlaufsrichtung, insbesondere bei Herzstichverletzungen,

- Zauderverletzungen,

- Kombination mit Schnittverletzungen an anderen Körperregionen,

- Probierstiche, die bereits in der Epidermis, Dermis, nur selten in der Subkutis enden und

- neben vielen oberflächlichen nur wenige tiefe Stichverletzungen.

Abweichungen vom suizidtypischen Muster kommen jedoch immer wieder vor: Durchstechen der Bekleidung, mehrfache tiefe Stichverletzungen, atypische Werkzeuge, große Schwalben- schwanzbildungen, atypische Einstichlokalisation wie singuläre Bauchstiche.

Wenn die klassischen Kriterien für die Differenzierung zwischen Homizid und Suizid versagen, sind folgende Befunde wichtig:

- Verletzungslokalisation, die topographisch einer Selbstbeibringung zugänglich ist.

- Die Letalität ergibt sich entweder aus der Summe der einzelnen Verletzungen oder es liegen nur wenige tödliche Einzelverletzungen vor.

- Bei einer Vielzahl von Verletzungen muss die Gesamtzahl mit länger andauernder Handlungsfähigkeit vereinbar sein.

Für Fremdeinwirkung typisch sind multiple Stichverletzungen, die überwiegend tief sind. Betroffen sind neben dem Hals vorwiegend der Brustkorb links (• Abb. 3.43) sowie auch der Rücken, der unter Umständen das primäre Angriffsziel darstellt (für das Opfer überraschender Angriff). Die Fremdbeibringung der
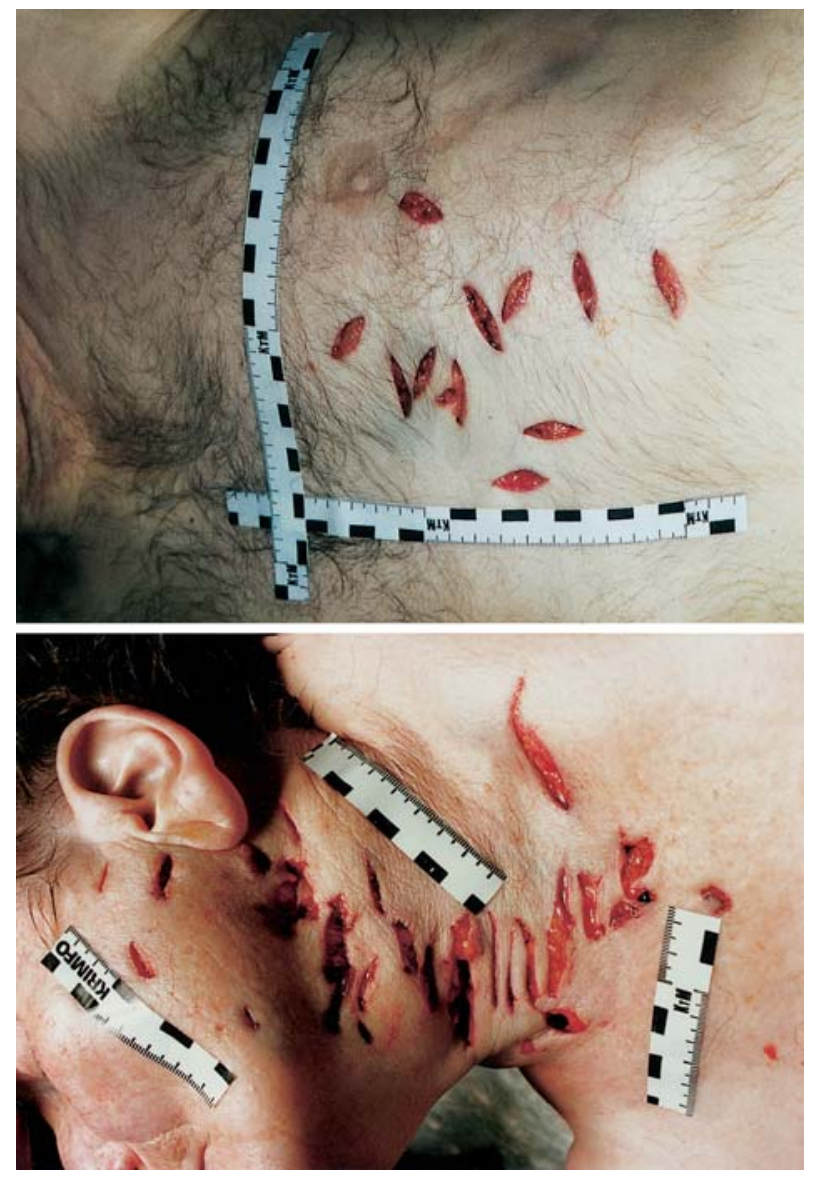

- Abb. 3.43. Homizidale Stichverletzung des Brustkorbes mit Herzstich sowie des Halses 
Stichverletzungen ergibt sich neben der Verteilung am Körper, der Intensität auch aus dem Vorliegen von aktiven und passiven Abwehrverletzungen:

\section{- Definition}

- Aktive Abwehrverletzung: Stich- und Schnittverletzungen lokalisiert an den Beugeseiten der Finger und den Hohlhänden durch Hineingreifen in die Messerschneide.

- Passive Abwehrverletzung: Stich- und Schnittverletzungen lokalisiert an der Außenseite der Oberarme bzw. Streckseite/Kleinfingerseite der Unterarme und Streckseite der Hände, die schützend vor das Gesicht gehalten werden.

Bei den Abwehrverletzungen kann es zu Durchstichen von Armen und Händen kommen.

Einlassungen. Bei durch dritte Hand beigebrachten Stichverletzungen wird zuweilen ein unfallmäßiger Geschehensablauf behauptet (»das Opfer sei in das Messer gerannt/gestürzt«). Eine Rekonstruktion unter Berücksichtigung der Messerhaltung in der Hand des Tatverdächtigen (Händigkeit des Täters), der Lokalisation und morphologischen Charakteristika der Einstichverletzung und des Stichkanalverlaufs kann eine derartige Schutzbehauptung in der Regel ausschließen. Für homizidal beigebrachte Stiche wird unter Umständen auch das berufliche Wissen eines Täters genutzt: gezielte Durchtrennung der Karotis bzw. Stich in den Nacken mit Durchtrennung des Halsmarkes bei Metzgern und Jägern.

In foro steht häufig der notwendige Kraftaufwand zur Erzielung einer Stichverletzung zur Diskussion. Die notwendige Kraft, um eine Hautperforation zu erzeugen, ist abhängig von der Konfiguration der Messerspitze und der Schärfe der Schneide. Ist die Haut durchtrennt, stellen die weiteren Weichgewebe dem Vordringen des Stichs keinen wesentlichen Widerstand entgegen. Die Haut selbst weist intraindividuell und interindividuell topische Unterschiede des Widerstandes auf. Größerer Kraftaufwand ist beim Durchstoßen von Knorpel und Knochen, aber auch Bekleidung (z.B. Lederjacke) erforderlich.

Unfallmäßige, unter Umständen letale Stichverletzungen kommen immer wieder bei Sprung oder Sturz in Glasscheiben vor. Teilweise liegen nur einzelne, auch an den Extremitäten lokalisierte Stichverletzungen mit Durchtrennung großer Gefäße vor.

Handlungsfähigkeit nach Stichverletzungen. Die Handlungsfähigkeit nach Stichverletzungen hängt ganz von den Umständen des Einzelfalles ab, sodass sich generelle Aussagen verbieten. Retrospektive Analysen von überlebten und letalen Stichverletzungen ergaben, dass eine Vielzahl von Verletzten auf Stichverletzungen des Abdomens mit Eröffnung der Bauchhöhle, zum Teil mit Organverletzungen, zunächst besonnen reagierten. Auch lebensgefährliche Schnitt- oder Stichverletzungen, die erfahrungsgemäß schnell zu Handlungsunfähigkeit oder Tod führen, müssen dabei subjektiv nicht unmittelbar bemerkt werden.
Die Handlungsfähigkeit lässt sich dabei folgendermaßen differenzieren:

- Fähigkeit zu schwierigen, zielgerichteten und vom Bewusstsein getragenen Handlungen,

- instinktive und situationsentsprechende Handlungen (z.B. Abwehrverletzungen),

- bei Bewusstlosen ablaufende, zusammenhängende und gleichförmige Bewegungsabläufe (z.B. Automatismen, Reflexabläufe),

- unzusammenhängende und schnell erschöpfbare Bewegungsabläufe, z.B. Streckkrämpfe.

Als handlungsfähig wird man nur Personen mit vom Bewusstsein getragenen Handlungen entsprechend den ersten zwei Kategorien einstufen können.

Oftmals tritt bei Schwerverletzten Handlungsunfähigkeit erst ein, wenn der Verletzte - unabhängig von den Verletzungsfolgen (z.B. Blutverlust) - die Schwere der Verletzung wahrnimmt. Sekundär kann Handlungsunfähigkeit durch den hämorrhagischen Schock eintreten. Für die Frage der Handlungsfähigkeit von Bedeutung ist oftmals nicht die Anzahl der Verletzungen, sondern die Größe der Verletzungen, die wiederum von Bedeutung ist für die Schnelligkeit und das Ausmaß des Blutverlustes. Bedeutender als das Ausmaß ist für die Handlungsfähigkeit die Schnelligkeit des Blutverlustes. Relativ rasche bis unmittelbare Handlungsunfähigkeit liegt vor bei breiter Eröffnung einer Herzkammer, der Aorta und A. pulmonalis und Durchtrennung von Koronararterien.

\section{Schnittverletzungen}

Schnittverletzungen werden durch ähnliche Werkzeuge verursacht wie Stichverletzungen. Sie können von ganz dezenten Oberhautanritzungen bis zu tiefen Weichteilwunden variieren.

Morphologie/Wundrand. Wirkt das schneidende Werkzeug nicht senkrecht auf die Haut ein, sondern schräg, ist wie bei Stichverletzungen ein Wundrand abgeschrägt, der andere unterminiert. In der Regel sind die Wundränder geradlinig glattrandig, naturgemäß fehlen Gewebsbrücken im Wundgrund. Bei stumpfen Schnittwerkzeugen, Wellenschliff oder Scharten der Schneide finden sich Variationen des Wundrandes wie bereits bei den Stichverletzungen beschrieben. Akzidentelle Schnittverletzungen werden durch Scherben, Blechkanten oder Ähnliches verursacht.

Teilweise sind beide Wundwinkel seicht auslaufend, wobei die Schnittwunde in dezenten Oberhautanritzungen beginnt und endet. Sowohl bei Suiziden als auch bei Homiziden findet man korrespondierend zu einem kräftigen Zuschneiden auch gleich tiefe Weichteildurchtrennungen.

Entstehen beim Schneiden in Schnitt- und damit auch Zugrichtung Hautfalten, wird die Haut nur auf den Faltenkämmen und nicht in den Faltentälern durchtrennt: Es resultiert eine mehrfach unterbrochene, jedoch einer Schnittbeibringung zuzuordnende Hautdurchtrennung.

Schnittverletzungen enden meistens nicht tödlich, sondern werden klinisch behandelt. 

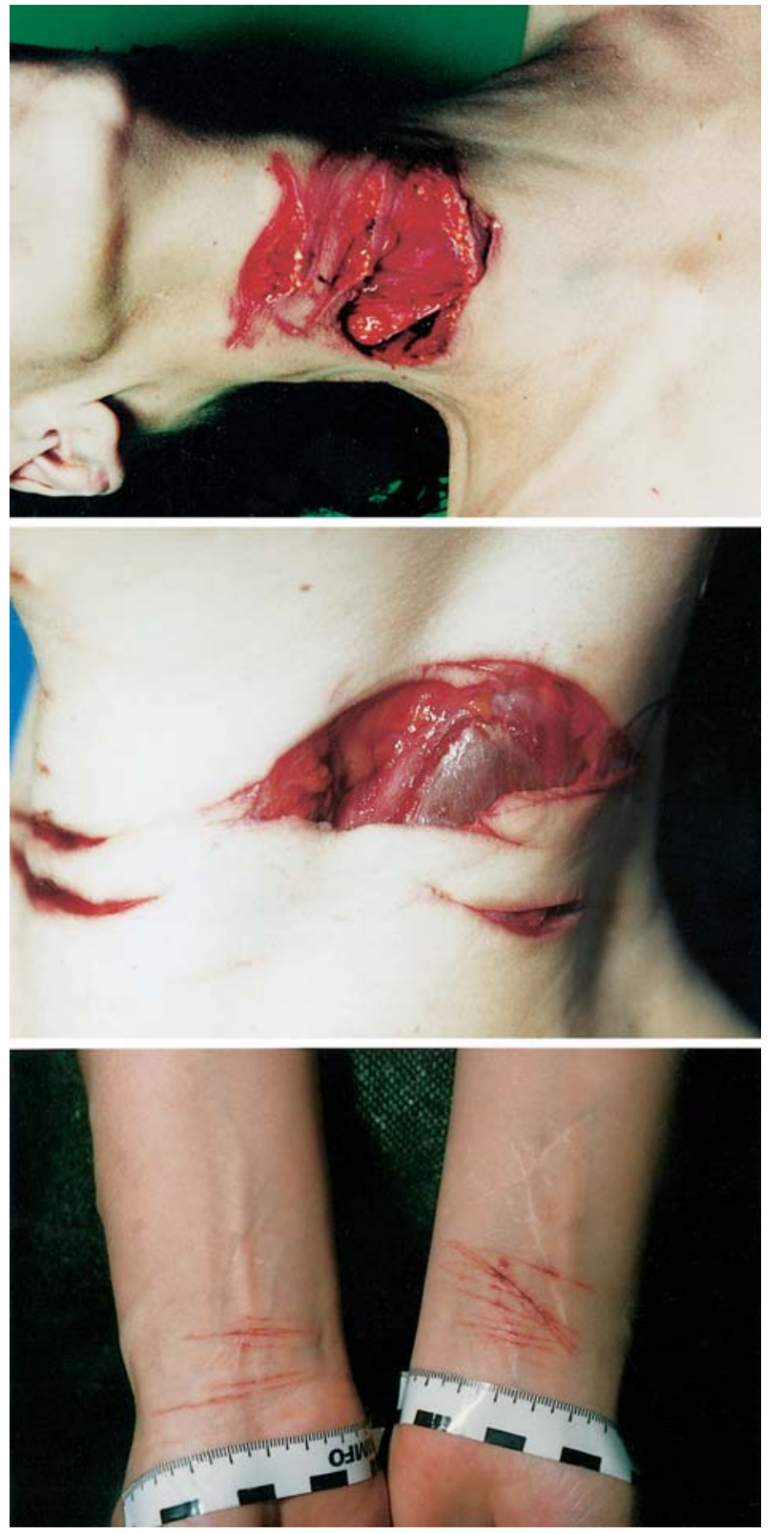

- Abb. 3.44. In suizidaler Absicht beigebrachte Schnittverletzungen des Halses und der Handgelenksbeugen

Lokalisation der Verletzungen bei Suiziden: Schnittverletzungen werden häufig in suizidaler Absicht beigebracht. Typische Prädilektionsstellen sind die Handgelenksbeugen, die Ellenbeugen und der Hals, jeweils an der der Gebrauchshand kontralateralen Seite lokalisiert (• Abb. 3.44). Hier sollen größere Blutgefäße eröffnet werden. Typischerweise finden sich zahlreiche - etwa an der Handgelenksbeuge - quer zur Armlängsachse beigebrach- te, unterschiedlich tiefe Haut-Unterhautfettgewebsdurchtrennungen, in der Regel neben zahlreichen nur ganz oberflächlichen oder allenfalls bis in die Dermis reichenden Hautanritzungen. In Armlängsachse verlaufende Probierschnitte und Schnittverletzungen kommen seltener vor. Selbst bei Verletzung arterieller Gefäße kommt es durch Einrollung der Gefäßintima zur spontanen Blutstillung. Suizidanleitungen folgend begeben sich Suizidenten daher in mit heißem Wasser gefüllte Badewannen, um eine gute Durchblutung der Verletzung zu gewährleisten.

Am Hals ist die für den Rechtshänder typische Verlaufsrichtung selbst beigebrachter Schnittverletzungen von links oben nach rechts unten (•Tabelle 3.17). Oftmals finden sich aus einer tieferreichenden Hautdurchtrennung mehrfache seicht auslaufende Wundwinkel, die mehrfaches Ansetzen des Werkzeuges und die Anzahl der Schnittführungen beweisen. Doch auch bei suizidalen Halsschnittverletzungen finden sich zuweilen ausschließlich tiefe Weichteildurchtrennungen, die die gesamte Halsvorderseite umfassen können. Für die Abgrenzung gegenüber homizidalen Verletzungen ist die Analyse des Blutspurenbildes am Leichnam und am Fundort sowie der Sitz der Bekleidung u.a. maßgeblich.

Bei selbst beigebrachten Schnittverletzungen, vor allem mit Rasierklingen, Glasscherben, selbstgebastelten Schnittwerkzeugen usw., finden sich teilweise feine Oberhautanritzungen von Daumen und Zeigefinger der Schnitthand.

Die Auffindung des Leichnams neben einem Spiegel kann einen Hinweis auf einen Siuzid darstellen (Kontrolle der Schnittführung im Spiegel).

Lokalisation der Verletzungen bei Homiziden: In homizidaler Absicht beigebrachte Schnittverletzungen finden sich überwiegend am Hals. Von hinten beigebrachte Halsschnittverletzungen umfassen in der Regel den gesamten Hals vorderseitig. Der Beginn der Schnittverletzung liegt in der Regel etwas weiter kranial als das Ende. Zauderverletzungen fehlen. Mehrfache bis auf die Halswirbelsäule geführte Schnittverletzungen beweisen Fremdbeibringung, insbesondere, wenn großkalibrige Gefäße wie die Karotis mehrfach getroffen wurden. Tiefe Halsschnittverletzungen können auch Trachea und Larynx vollständig durchtrennen.

Todesursachen. Todesursächlich bei Schnittverletzungen ist in der Regel der höhergradige Blutverlust nach außen, bei tiefen Halsschnittverletzungen unter Umständen in Kombination mit Blutaspiration und Luftembolie des Herzens.

Auch klinisch sind selbst beigebrachte Schnittverletzungen unterschiedlichster Motivation (Betrug einer Unfallversicherung, Vortäuschung eines Unfalls, appellativer Charakter) relevant: Die teilweise außerordentlich charakteristischen Befunde, die oftmals bereits eine prima facie Diagnose zulassen, werden in $>$ Kapitel 5.3 dargestellt.

\section{Hiebverletzungen}

Hiebwerkzeuge sind gekennzeichnet durch das relativ große Eigengewicht mit zumindest einer schneidenden Seite (Äxte, Beile, Säbel, Macheten, Propeller, Schiffsschrauben). 
- Tabelle 3.17. Kriterien der Selbst- und Fremdbeibringung bei Halsschnittverletzungen

\begin{tabular}{|c|c|c|}
\hline & Suizide & Homizide \\
\hline $\begin{array}{l}\text { Halswunde } \\
\text { - Lokalisation } \\
\text { - Schnittverlauf } \\
\text { - Zauderverletzungen }\end{array}$ & $\begin{array}{l}\text { Regio sternocleidomastoidea } \\
\text { Geradlinig zur Schnitthand absteigend } \\
\text { Vorhanden }\end{array}$ & $\begin{array}{l}\text { Regio thyreoidea } \\
\text { Horizontal, zirkulär »Halsabschneiden« } \\
\text { Keine }\end{array}$ \\
\hline $\begin{array}{l}\text { Begleitverletzungen } \\
\text { - Lokalisation } \\
\text { - Anordnung } \\
\text { - Verletzungsintensität } \\
\text { - Probierschnitte } \\
\text { - Abwehrverletzungen }\end{array}$ & $\begin{array}{l}\text { Gut erreichbare Stellen } \\
\text { Empfindliche Stellen ausgespart } \\
\text { Gruppiert, teilweise parallel } \\
\text { Konstant } \\
\text { Kontralaterale Halsseite, Ellenbeuge, Handgelenk } \\
\text { Keine }\end{array}$ & $\begin{array}{l}\text { Überall, auch am Rücken } \\
\text { Regellos } \\
\text { Wechselnd } \\
\text { Keine } \\
\text { Hände u. Unterarme }\end{array}$ \\
\hline Blutablaufstraßen & Regelmäßig, lotrecht & Unregelmäßig \\
\hline Bekleidung & Unversehrt & Evtl. beschädigt \\
\hline
\end{tabular}

Morphologie. Hiebverletzungen führen in der Regel zu relativ geradlinigen, glattrandigen Kontinuitätsdurchtrennungen der Weichteile, wobei - je nach Schärfe der Schneide - die Wundränder bzw. das an die Wundränder angrenzende Gewebe Schürfungen und Quetschungen aufweisen können. Bei tangentialer Einwirkung resultieren Skalpierungsverletzungen (• Abb. 3.45).

Knöcherne Verletzungen: Wird auch der darunter liegende Knochen getroffen, weist dieser unter Umständen Einkerbungen auf, Impressionsfrakturen, Biegungsbrüche oder aber eine Spaltung von Anteilen des Schädeldaches. Das Hiebwerkzeug kann im Bruchspalt stecken bleiben. Bei tangentialer Einwirkung können Anteile des Schädeldaches abgekappt werden. Wird neben der Schneideseite auch die stumpfe Seite eines Beils als Schlagwerkzeug benutzt, finden sich alle morphologischen Variationen auf scharfe und stumpfe Gewalteinwirkung zu beziehender Verletzungsfolgen.

Todesursachen. Todesursache bei Hiebverletzungen sind in der Regel schwere Schädel-Hirn-Zertrümmerungen bzw. Verbluten. Hiebverletzungen werden nahezu regelhaft durch fremde Hand beigebracht. Eigenhändig beigebrachte Hiebverletzungen
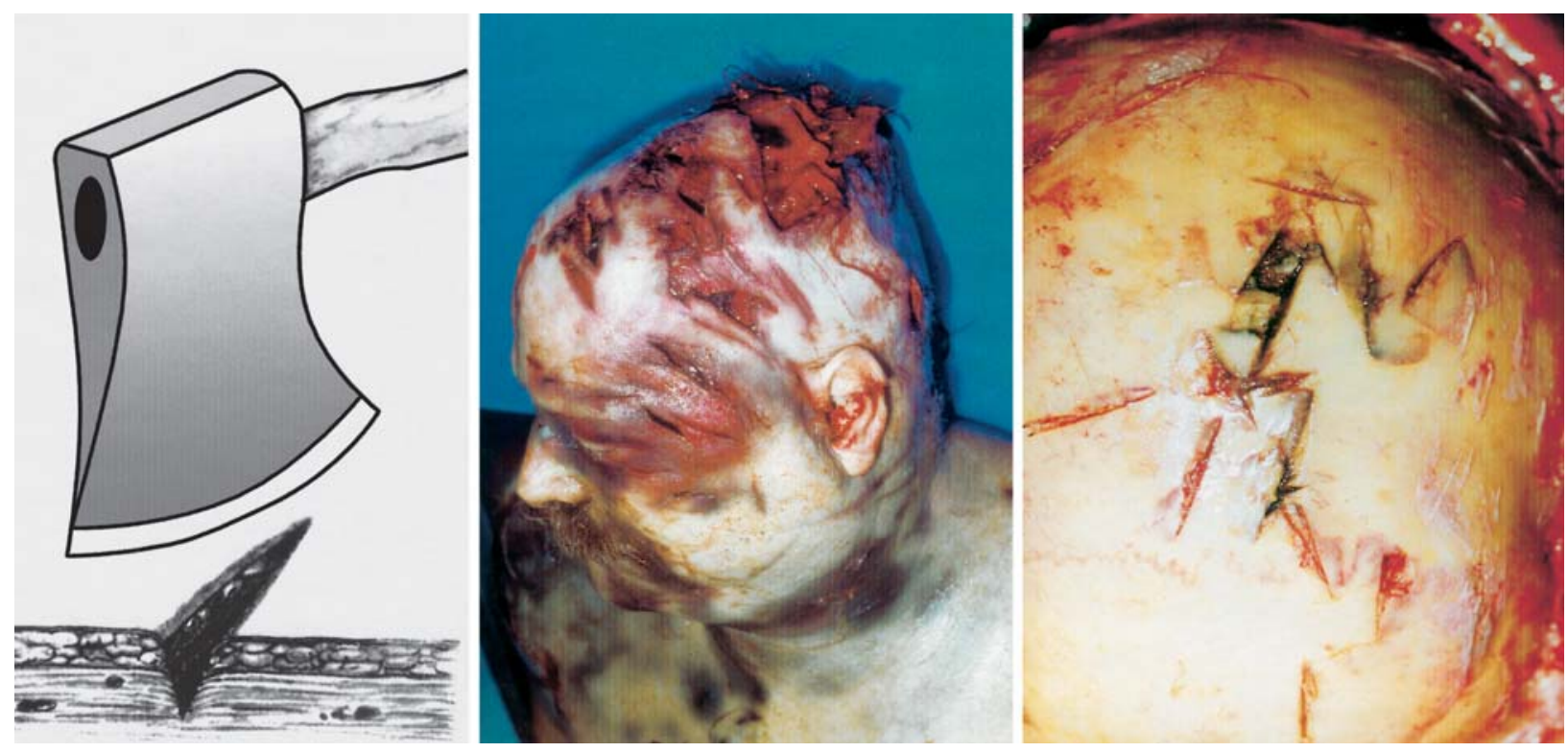

- Abb. 3.45. Hiebverletzungen des Schädels mit zahlreichen Kerbenbildungen des Schädeldaches 
des Schädels finden sich allenfalls bei psychisch Kranken. Das Verletzungsmuster ist in der Regel charakterisiert durch zahlreiche, parallel dicht nebeneinander stehende, gradlinige Kontinuitätsdurchtrennungen der Haut mit Ankerbungen des Schädeldaches über den Scheitelbeinen (Zuschlagen mit einem mit beiden Händen gehaltenem Beil).

Schläge mit Glasflaschen. Bei Verwendung von Glasflaschen als Schlagwerkzeugen liegen oftmals neben Platz- auch Schnittwunden vor, da es bei Schlageinwirkung von Glasflaschen auf den Schädel, der das bevorzugte Angriffsziel darstellt, nahezu regelhaft zum Zerbersten von Flaschen kommt, während Schädelfrakturen eher selten sind. Schlageinwirkungen durch Flaschen können folgenlos überstanden werden, oder zu einem tödlichen Blutverlust der Kopfplatzwunde bzw. einer Schädel-Hirn-Zertrümmerung führen.

\subsection{Schussverletzungen}

\section{S. Pollak}

\section{Einleitung}

Die Schussverletzung wird als eine Sonderform des stumpfen Traumas aufgefasst. Die Schädigung des Organismus beruht auf der Einwirkung eines Geschosses (Projektils), das durch hochgespannte (Verbrennungs-)Gase aus einem Waffenlauf getrieben wird und mit hoher Geschwindigkeit auf den Körper trifft. Zu den Schussverletzungen im weiteren Sinn zählt man auch Läsionen durch Schreckschusswaffen - aus diesen werden Knallkartuschen (»Platzpatronen«) ohne Projektile verschossen - sowie Verletzungen durch Viehbetäubungsapparate, baugewerbliche Bolzensetzwerkzeuge und ähnliche Geräte.

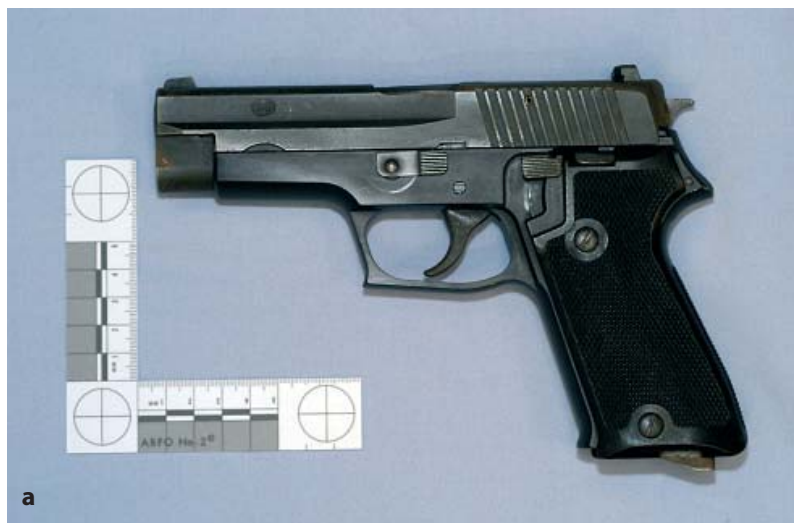

- Abb. 3.46. Beispiele für Faustfeuerwaffen. a Pistole (SIG-Sauer P 220, Kal. 9 mm Parabellum). b Revolver (Smith \& Wesson, Mod. 66, Kaliber 357

\subsubsection{Waffen und Munition}

\section{Waffenarten}

Nach der Waffenart unterscheidet man Faustfeuerwaffen (kurzläufige Schusswaffen für den einhändigen Gebrauch: Pistolen, Revolver; - Abb. 3.46) und Handfeuerwaffen (tragbare, langläufige Schusswaffen für den zweihändigen Gebrauch: Gewehre).

Moderne Pistolen verfügen über ein Magazin, das im Griffstück untergebracht ist; nach dem Abfeuern einer Patrone wird deren Hülse vom zurückgleitenden Verschluss ausgeworfen. Bei Revolvern stecken die Patronen in einer drehbaren Trommel; die Hülsen bleiben nach der Schussabgabe in der Waffe.

Gewehre haben entweder einen gezogenen Lauf ( $\bullet$ unten) - z.B. jagdlich verwendete Büchsen (zum Verschießen von Einzelgeschossen) und militärisch verwendete Sturmgewehre - oder sie besitzen einen glatten Lauf; dazu gehören jagdlich verwendete Flinten (zum Verfeuern von Schrot und Flintenlaufgeschossen), aber auch so genannte Vorderschaftrepetierflinten (»Pumpguns«), die in manchen Ländern als Polizei- oder Selbstverteidigungswaffen in Gebrauch sind.

\section{Gezogene und glatte Läufe}

Ein gezogener Lauf ( $\bullet$ Abb. 3.46b) zeigt an seiner inneren Oberfläche spiralig verlaufende Erhabenheiten (Felder) und dazwischen liegende Vertiefungen (Züge). Die Felder schneiden sich in den zylindrischen Teil des Geschosses ein, wodurch dieses in eine Rotationsbewegung um seine Längsachse (»Drall«) versetzt wird. Die kreiselartige Rotation stabilisiert das Geschoss auf seiner Flugbahn.

Glatte Läufe ohne Züge und Felder finden sich bei Flinten und manchen Flobert-Waffen. Sie sind zum Verfeuern von Schrot- oder Flintenlaufgeschosspatronen bestimmt. Der vordere Anteil des zylindrischen Laufes kann geringfügig konisch verjüngt sein (Würge- oder Choke-Bohrung zur Beeinflussung der Streuung einer Schrotgarbe).

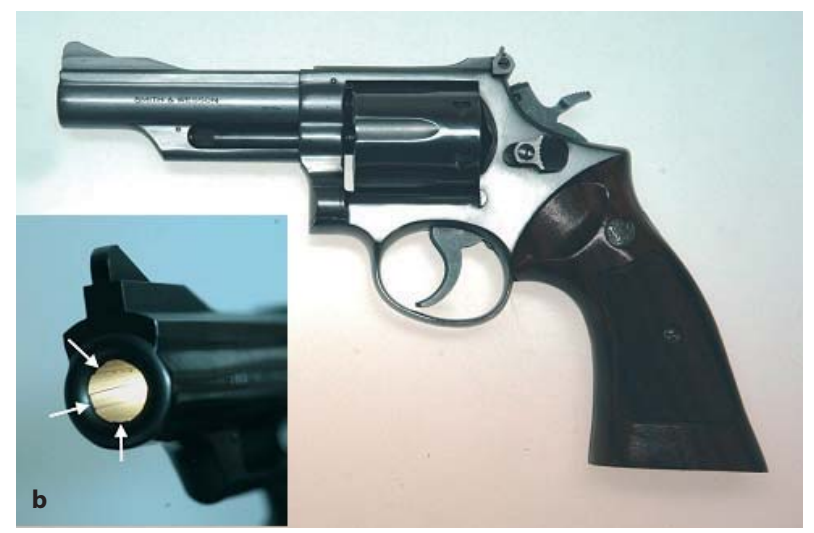

Magnum). Bildausschnitt: Blick in den mündungsnahen Teil des Revolverlaufes mit erhabenen Feldern (Pfeile) und tiefer liegenden Zügen 


\section{Kaliber}

Der Ausdruck »Kaliber" bezeichnet einerseits die Laufweite (Durchmesser der Laufbohrung), andererseits den Querdurchmesser des Geschosses. Die Kalibermaße sind Nominalangaben, die bei metrischen Kalibern in der Regel vom Felddurchmesser (maximale diametrale Entfernung zwischen den Feldern im Laufinneren) abgeleitet sind. Die Geschosse haben zumeist einen etwas größeren Durchmesser (Beispiel: beim Kal. 7,65 mm Browning beträgt der Felddurchmesser 7,63 mm, der Geschossdurchmesser 7,85 mm). Die angloamerikanischen Kaliberangaben (in Zoll bzw. inches, 1 in. $=25,4 \mathrm{~mm}$ ) lehnen sich eher an den Geschossdurchmessern an; während man früher vor die Kaliberzahl einen Punkt gesetzt hat (.357 in. entsprechend $9 \mathrm{~mm}$ ), um auszudrücken, dass es sich eigentlich um die Stellen hinter dem Komma handelt, wird heute dieser Punkt - dem Sprachgebrauch folgend - weggelassen (»Kaliber 357 «).

Bei Pistolen sind die Kaliber 6,35 mm, 7,65 mm, 9 mm und 45 vorherrschend, bei Revolvern 32, 357, 38 und 44.

Kleinkaliber(KK)-Patronen im Kaliber 5,6 mm lfB (»lang für Büchsen«, entspricht 22 L.R. = long rifle) können nicht nur aus Faustfeuerwaffen, sondern auch aus Langwaffen verschossen werden.

Bei Jagdpatronen für Büchsen ist es üblich, im Rahmen der Kaliberbezeichnung auch die Hülsenlängen in Millimetern anzugeben (z.B. 7 x 64 mm, 8 x 57 mm). Gleiches gilt für die Patronen der Armeehandfeuerwaffen (z.B. 5,56x45 mm, 7,62x51 mm).

Das Kaliber von Schrotläufen ist nicht mit dem Innendurchmesser identisch, sondern eine historisch begründete Maßangabe: Das Schrotkaliber gibt die Anzahl von gleich großen Kugeln aus Blei an, die zusammen genommen die Masse eines englischen
Pfunds (453,6 g) haben; der Durchmesser dieser Bleikugeln entspricht dem Innendurchmesser des Laufes (z.B. 18,2 mm bei Schrotkaliber 12 und 16,8 $\mathrm{mm}$ bei Schrotkaliber 16).

\section{Patronen}

Die Patronen von Faustfeuerwaffen (• Abb. 3.47) und Büchsen bestehen aus einer Hülse und einem Geschoss (Projektil).

Die Hülse ist normalerweise aus Messing gefertigt. Patronen für Pistolen und Büchsen haben knapp über dem Hülsenboden eine Rille, um nach der Schussabgabe das Auswerfen (mit Hilfe der Auszieherkralle) zu ermöglichen. Revolverpatronen haben einen überstehenden Hülsenbodenrand.

Im Boden der Hülse befindet sich der Zündsatz. Ausgelöst durch die Betätigung des Abzugs, bewirkt der mittels Federkraft vorschnellende Schlagbolzen die Zündung. Wenn die Auftreffstelle der Schlagbolzenspitze in der Mitte des Hülsenbodens liegt, spricht man von Zentralfeuerpatronen. Bei Randfeuerpatronen ist der Zündsatz in einem rundum laufenden Wulst über dem Hülsenboden eingebracht; der Schlagbolzen trifft bei solchen Patronen (z.B. Kaliber 22 L.R.) im Randbereich auf.

Das Zündelement beinhaltet einen schlagempfindlichen Sprengstoff, dessen Wärme und Flamme zur Zündung der eigentlichen Treibladung führt. Anfänglich bestand der Zündsatz aus Knallquecksilber, später aus einer Kombination chemischer Verbindungen (»Sinoxid « mit Bleitrinitroresorcinat als Hauptkomponente). Daher spielt Blei - so wie Antimon und Barium - als Schmauchelement beim kriminaltechnischen Nachweis von Schussspuren eine wichtige Rolle. Allerdings kommen heute auch bleifreie Zündsätze zur Anwendung (z.B. »Sintox« mit den Leitelementen Zink und Titan).
- Abb. 3.47. Beispiele für gängige Pistolen- und Revolverpatronen mit Reinblei-, Vollmantel- und Teilmantelgeschossen

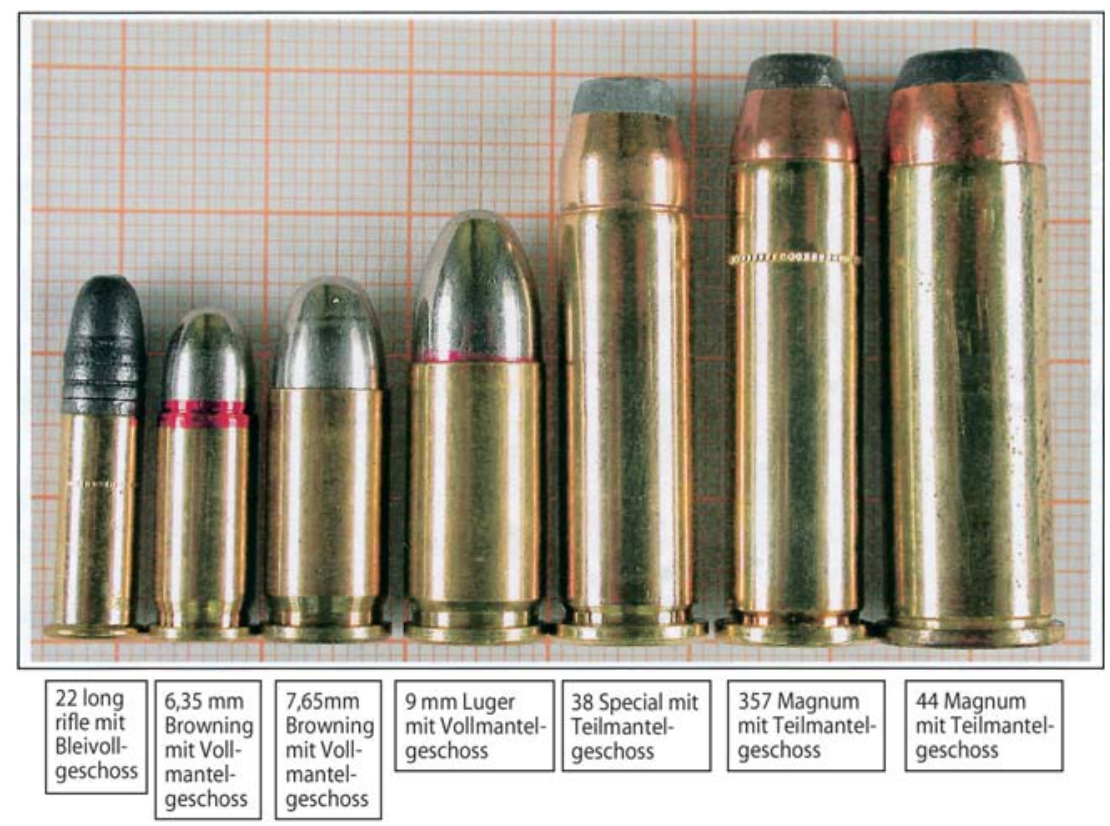


Über dem Zündsatz ist die Hülse mit dem Treibsatz (»Pulver«) gefüllt. Das seit Jahrhunderten bekannte Schwarzpulver ein Gemisch aus 75\% Kaliumnitrat, 15\% Kohlenstoff und 10\% Schwefel - wird heute nur noch sehr selten als Treibmittel benutzt (für historische oder nachgebaute Vorderladerwaffen oder in manchen Knallkartuschen). Die Rauchbildung ist bei Schwarzpulver wegen des hohen Anteils fester Verbrennungsrückstände sehr stark.

Die heute ganz überwiegend verwendeten Treibmittel sind rauchschwache Pulver: entweder aus Nitrozellulose (NC) alleine (»single base powder «) oder aus Nitrozellulose, die in Nitroglycerin gelöst wurde (»double base powder«). Die blassgrünlichen Pulverteilchen haben die Form dünner Plättchen (flake, disk powder), kleiner Kügelchen (ball powder) oder kurzer Röhrchen (tubular powder). Die Durchmesser der einzelnen Pulverpartikel variieren von einigen Zehntelmillimetern bis $>1 \mathrm{~mm}$. Bei Flobert-Patronen (Kaliber $6 \mathrm{~mm}$ oder $9 \mathrm{~mm}$ ) fungiert der Zündsatz zugleich als Treibmittel.

Durch den Abbrand der Treibladung bilden sich reichlich Gase $\left(\mathrm{CO}_{2}, \mathrm{CO}, \mathrm{H}_{2}, \mathrm{~N}_{2}\right.$, nitrose Gase, Wasserdampf). Diese stehen unter hohem Druck und erteilen dem Geschoss eine entsprechende Beschleunigung. Die Vorgänge innerhalb der Waffe werden als »Innenballistik« bezeichnet.

Die Mündungsgeschwindigkeit liegt bei Patronen für Faustfeuerwaffen in der Größenordnung von 300-400 m/s, bei Jagdund Militärwaffen wesentlich höher (ca. 700-1.000 m/s).

Aus Pistolen und Militärgewehren werden meist Patronen mit Vollmantelgeschossen verfeuert. Mantelgeschosse haben in der Regel einen Bleikern, der vorne und seitlich (also unter Aussparung des Geschossbodens) mit einem Mantel aus Stahl oder aus einer Kupferlegierung umhüllt ist.

Bei Jagdmunition sind Teilmantelgeschosse üblich: Das im Spitzenbereich nicht ummantelte Projektil besitzt eine höhere Deformationsbereitschaft und gibt daher mehr Energie an den Tierkörper ab.
Standard-Kleinkaliberpatronen haben normalerweise mantellose Vollbleigeschosse. Aus Revolvern werden überwiegend Patronen mit Vollblei-, aber auch solche mit Teilmantelgeschossen verfeuert.

Nach der Form des Geschosskopfes unterscheidet man Rundkopf-, Flachkopf-, Spitz-, Zylinder- und Kegelstumpfgeschosse; nach dem Geschossaufbau wird zwischen Voll-, Vollmantel-, Teilmantel- und Hohlspitzgeschossen differenziert. Die meisten Teilmantel- und Hohlspitzgeschosse deformieren sich bereits nach Zurücklegung einer kurzen Eindringstrecke.

Für das Wirkungspotential sind neben Form und Art des Projektils vor allem dessen Masse und Geschwindigkeit von Bedeutung. Aus der Masse $m$ und der Geschwindigkeit v errechnet sich die Geschossenergie $\mathrm{E}\left(\mathrm{E}=\mathrm{m} / 2 \cdot \mathrm{v}^{2}\right)$; sie wird in Joule $(\mathrm{J})$ angegeben. Exemplarisch seien die Massen eines 22 L.R.-Geschosses (2,55 g) und eines $9 \mathrm{~mm}$ Parabellum-Geschosses $(8,0 \mathrm{~g})$ genannt; die Geschossenergie liegt im ersten Fall (22 L.R.) bei etwa $140 \mathrm{~J}$, im zweiten Fall (9 mm Para) bei etwa 430 J. Die Geschossenergie von Militär- und Jagdpatronen ist etwa 10-mal so hoch.

\section{Flintenmunition}

Übliche Schrotpatronen werden aus (glatten) Flintenläufen verschossen. Anstelle eines Einzelgeschosses enthalten sie meist zahlreiche (200-500) kugelige Schrotkörner aus Hartblei (• Abb. 3.48a). Der Durchmesser der Schrotkörner variiert zwischen 2 und $4,5 \mathrm{~mm}$, die Schrotgeschwindigkeit beträgt beim Verlassen des Laufes etwa $300 \mathrm{~m} / \mathrm{s}$.

Die Patronenhülsen bestehen aus Pappe oder Kunststoff, der Boden (meist aus Messing) enthält das Zündhütchen. Darüber befindet sich (rauchschwaches) Nitropulver. Schrot und Pulver sind durch Zwischenmittel getrennt (Filzpfropfen oder Plastikpfropfen mit Schrotbecher).

Neben üblicher Flintenmunition gibt es auch Patronen mit sehr kleinen Schrotkörnern (»Vogeldunst«) oder besonders gro-
- Abb. 3.48a, b. Beispiele für den Aufbau von Flintenmunition. a Patrone mit Schrotladung; b Patrone mit BrennekeFlintenlaufgeschoss

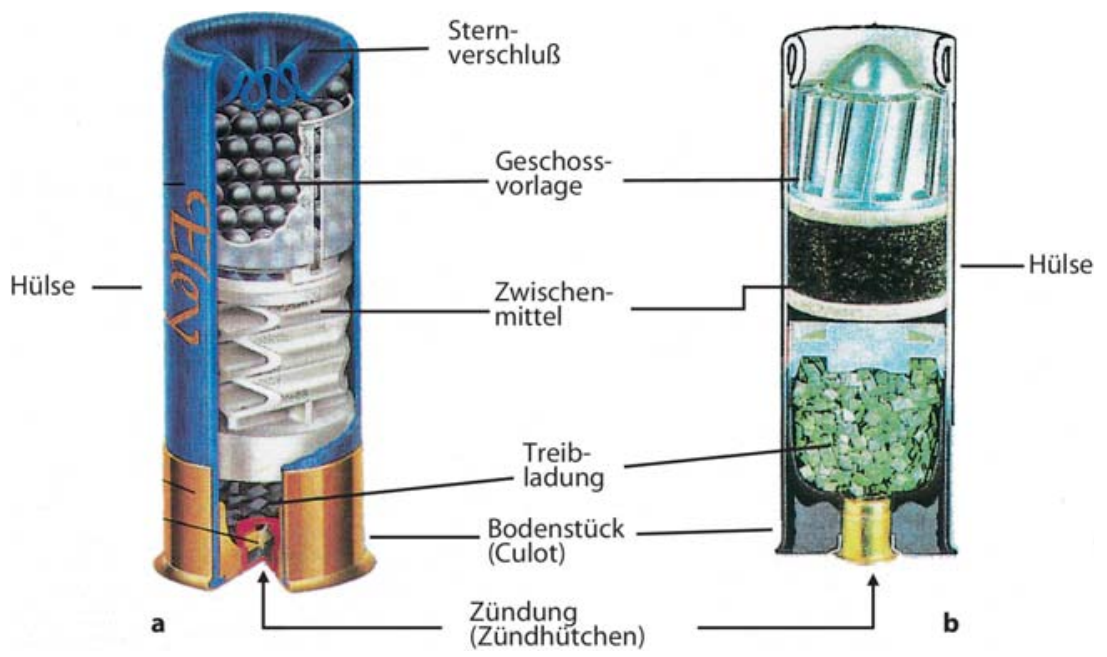


ßen Schrotkörnern (»Rehposten«), mit Einzelgeschossen (z.B. Flintenlaufgeschoss vom Typ Brenneke; • Abb. 3.48b) und mit Gummigeschossen.

\subsubsection{Wundballistik}

Die Außenballistik beschäftigt sich mit dem Verhalten des Geschosses nach dem Verlassen des Laufes (Flugbahn und -geschwindigkeit etc.), die Zielballistik mit der Wechselwirkung zwischen Geschoss und Zielobjekt. Wenn es sich dabei um einen menschlichen oder tierischen Körper handelt, spricht man von Wundballistik.

\section{Grundlagen der Verletzungswirkung}

Die verletzende Wirkung von Geschossen beruht einerseits auf direkter Zerstörung von anatomischen Strukturen im Verlauf des Schusskanals, andererseits auf Läsionen, die abseits davon durch Druckschwankungen und Gewebsdislokationen (mit Dehnung und Scherung) zustande kommen. Das Ausmaß der mechanischen Geschosswirkung ist davon abhängig, wie viel kinetische Energie im Gewebe abgegeben wird.

Beim Durchdringen des Gewebes wird dieses vom Projektil nach seitlich (radiär) - also im rechten Winkel zum Schusskanal - verdrängt und zentrifugal beschleunigt, so dass kurzzeitig eine »temporäre Wundhöhle« entsteht, deren Durchmesser um ein Vielfaches größer sein kann als jener des Geschosses. Sobald die Bewegungsenergie des radiär verlagerten Gewebes vollständig umgesetzt ist, erfolgt eine gegenläufige (zentripetale) Rückverlagerung zum geometrischen Schusskanal hin. Der dabei neuerlich entstehende Überdruck führt wieder zu einer (reibungsbedingt geringeren) Zentrifugalbewegung, so dass die Höhle noch "pulsieren" kann, wenn das Geschoss den Körper bereits verlassen hat.

Der skizzierte Vorgang ist bei Geschossen mit hoher Energie, wie sie aus Militär- und Jagdwaffen verschossen werden, besonders ausgeprägt. An flüssigkeitsgefüllten Organen (Herz, Harnblase) oder im Bereich des Gehirnschädels kann die radiäre Expansion sogar zu einer "hydrodynamischen Sprengwirkung " mit Berstung der Hüllstrukturen führen. Wenn in solchen Fällen das Gehirn in toto aus der Schädelkapsel herausgeschleudert wird, spricht man von einem (nach dem Erstbeschreiber so benannten) Krönlein-Schuss. Auch bei geringerer Energieabgabe können - abseits des eigentlichen Wundkanals - indirekte Verletzungen, z.B. Schussbrüche der Schädelkapsel, Hirnkontusionen und Dehnungsrisse der Gesichtsweichteile entstehen.

Der »bleibende« Schusskanal, also die eingeblutete Zerstörungszone im Verlauf der Geschossbahn, ist von einer mehr oder weniger breiten Zone umgeben, in der das Gewebe temporär gedehnt und dadurch (ultra)strukturell geschädigt wurde (»Zone der Extravasation «).

Durch den Beschuss von "Simulanzien « wie Gelatine oder Glyzerinseife kann die Energieabgabe in biologischen Weichge- weben modellhaft dargestellt werden, da die Dichte der genannten Materialien gut mit jener von Muskulatur übereinstimmt. Im Gegensatz zur elastischen Gelatine verformt sich Seife nahezu plastisch. Der nach dem Beschuss in der Seife zurückbleibende Schusskanal bzw. das Volumen der Kavitation ist proportional zur abgegebenen Energie (• Abb. 3.49a-d).

Ein stabiles Geschoss mit geringer Deformationsbereitschaft (Vollmantelgeschoss) erzeugt im Simulanzmedium zunächst einen relativ engen Schusskanal (»narrow channel«), der sich abrupt - bei Querstellung des Geschosses - durch verstärkte Energieabgabe zur temporären Höhle vergrößert (• Abb. 3.49c); auch eine hinzutretende Geschossverformung führt zur Volumenzunahme der Kavitation. Bei Deformationsgeschossen (z.B. Teilmantel-Hohlspitzgeschoss) beginnt die Höhlenbildung unmittelbar nach dem Eindringen (• Abb. 3.49b, d).

Wenn Geschosse von gleicher Bauart, Kopfform und Masse verschossen werden, dann hängt die Energieabgabe in einem dichten Medium und damit auch die Größe der temporären Höhlenbildung im Wesentlichen von der Geschossgeschwindigkeit ab. Dieser Zusammenhang lässt ich durch experimentellen Beschuss von Simulanzien gut demonstrieren (• Abb. 3.50).

\section{Schusskanal}

Körpertreffer werden in Steckschüsse, Durchschüsse, Streifschüsse und Prellschüsse unterteilt. Im Fall eines Steckschusses verbleibt das Projektil im Körper; dementsprechend liegt eine Einschusswunde, aber kein Ausschuss vor. Nicht selten bleiben matte Geschosse an der dem Einschuss gegenüberliegenden Körperseite unter der Haut stecken, wo eine Hämatomverfärbung und/oder eine tastbare Resistenz die Endlage anzeigen (• Abb. 3.51). Zur Auffindung und zur lagemäßigen Dokumentation der im Körper verbliebenen Geschosse und Geschossteile empfiehlt sich in jedem Fall eine Röntgenuntersuchung.

Zur Bestimmung des Schusswinkels (bezogen auf die Horizontal-, Sagittal- und Frontalebene des Körpers) müssen die Länge des Wundkanals sowie die Lokalisation der Ein- und Ausschusslücke bzw. bei Steckschüssen die Endlage des Geschosses genau vermessen und dokumentiert werden (Höhe über der Fußsohlenebene, Seitenabstand von der Medianebene). Aussagen über die Schussrichtung im Raum sind nur möglich, wenn zusätzliche Angaben (z.B. die Körperhaltung des Opfers und/ oder die Position des Schützen) bekannt sind; im Falle einer Durchschussverletzung ist die Kenntnis etwaiger sekundärer Geschossaufprall/-eindringstellen (im Boden, an der Wand, in Möbeln etc.) für die Rekonstruktion der Flugbahn wichtig.

Meist verläuft der Schusskanal im Körperinneren geradlinig. Vollmantel-Gewehrgeschosse verursachen allerdings einen geknickten Schusskanal, wenn dessen Länge im Körper größer als 20-30 cm ist. Die Schussrichtung darf in solchen Fällen nicht durch geradlinige Verbindung von Ein- und Ausschusslücke bestimmt werden. Die Ablenkung von der geradlinigen Bahn erfolgt beim ersten Querstellen des Geschosses, also im Bereich der ersten Kavitation, wenn die Druckverteilung längs des Geschos- 

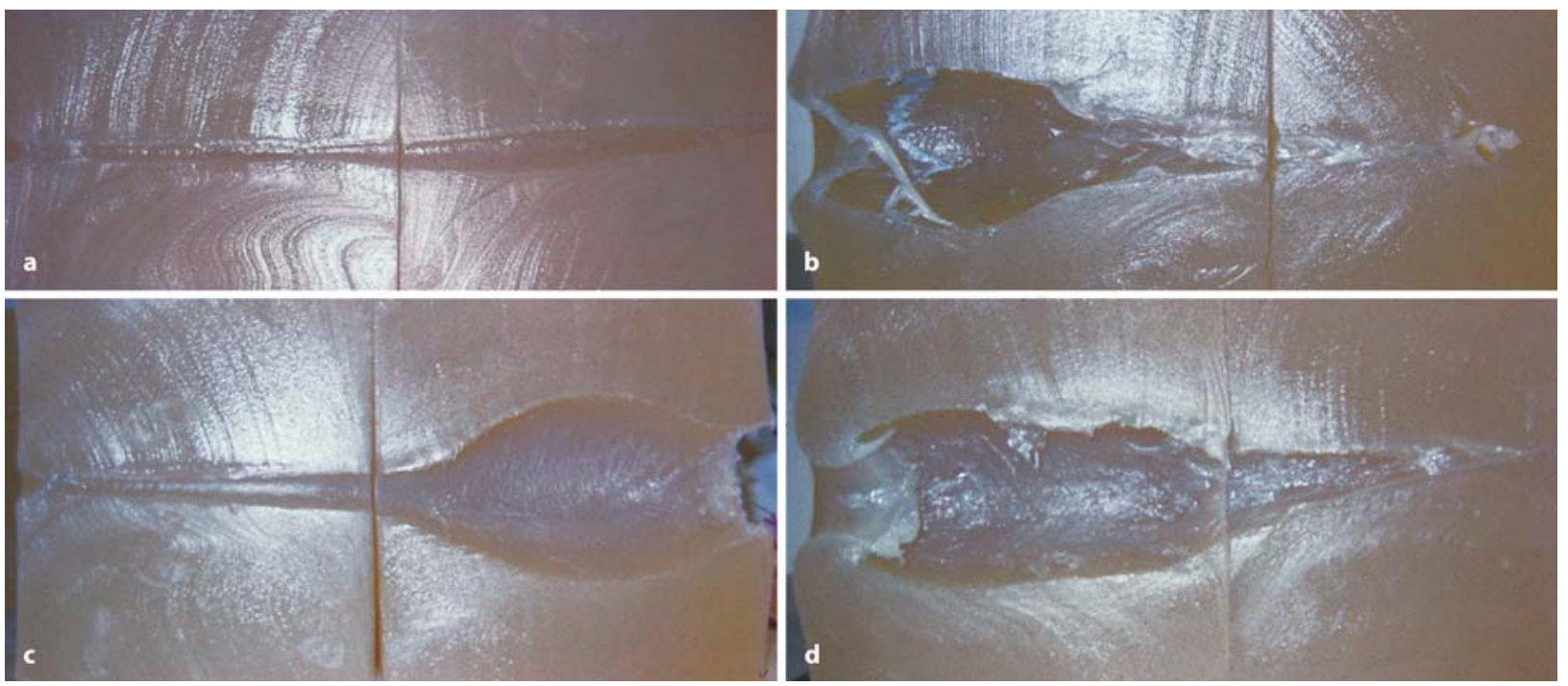

- Abb. 3.49a-d. Experimenteller Beschuss von Glyzerinseife zur Sichtbarmachung der Energieabgabe im Verlauf des Schusskanals. Die Einschüsse sind jeweils an der linken Seite der Seifenblöcke gelegen. a Kurzwaffe, 9 mm Luger, Vollmantel-Rundkopfgeschoss; b Kurzwaffe,

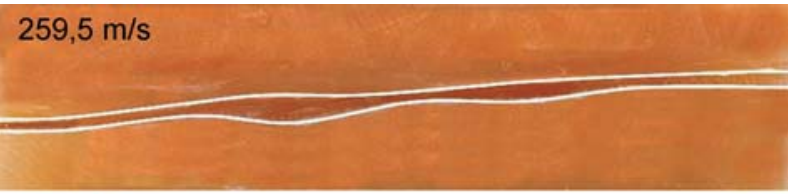

\section{$371,0 \mathrm{~m} / \mathrm{s}$}

44 Remington Magnum, Teilmantel-Flachkopfgeschoss; c Langwaffe, 7,62 x 51 mm, Vollmantel; d Langwaffe, 7,62 × 51 mm (entspricht Kal. 308 Win.), Teilmantel

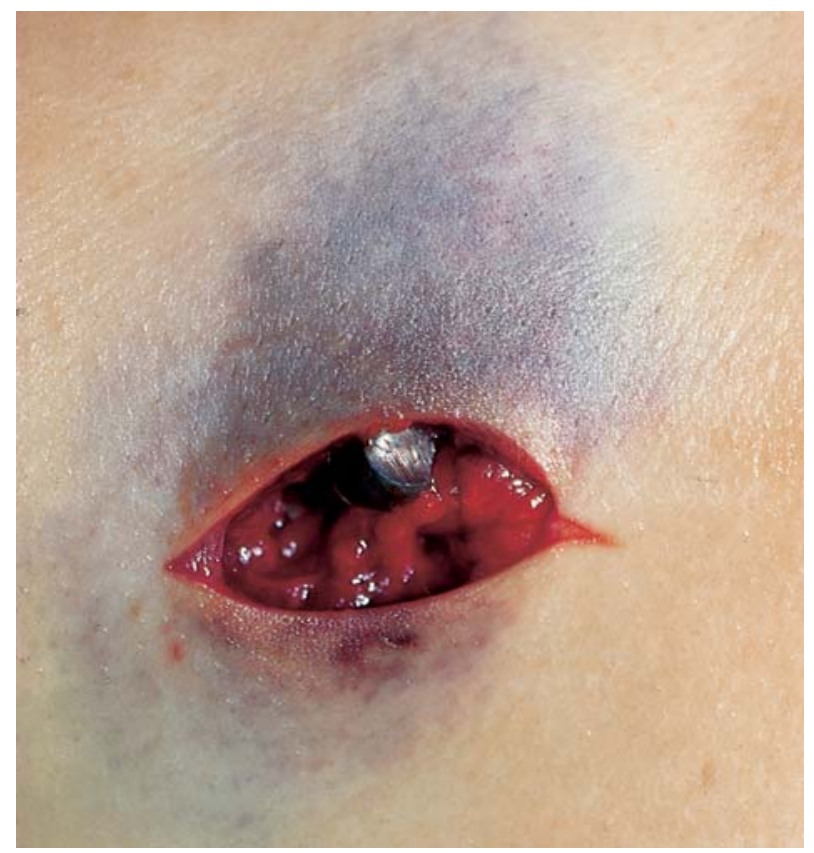

ses asymmetrisch wird und eine Kraftkomponente quer zur Bewegungsrichtung entsteht.

Weitere Beispiele für geknickte Schusskanäle sind die Winkel- und Ringelschüsse, die typischerweise innerhalb der Schädelkapsel vorkommen. Die Häufigkeit des intrakraniellen »Rikoschettierens « (ricocher [fr.]: abprallen) wird mit 10-25\% beziffert. Voraussetzung ist ein schräger sekundärer Geschossaufprall mit nur mehr geringer Restenergie.

- Abb. 3.51. Postmortale Inzision in ein hämatomverfärbtes Hautareal (seitliche Brustwand) zur Darstellung eines subkutan stecken gebliebenen Geschosses (Vollblei-Rundkopfgeschoss im Kaliber 38 Spl., verschossen aus einem umgebauten Schreckschussrevolver) 
Beim intrakraniellen Winkelschuss trifft ein in den Schädel eingedrungenes Projektil auf die Tabula interna der Gegenseite, von wo es winkelig in das Gehirn zurückgeworfen (»reflektiert«) wird.

Die Ringelschüsse werden auch als Kontur- oder Bogenschüsse bezeichnet. Sie kommen nicht nur an der Konkavität des Gehirnschädels, sondern auch an den Innenflächen der Rippen vor. Es handelt sich um eine bogige Geschossablenkung an der inneren Oberfläche von knöchern begrenzten Körperhöhlen. Die konkave Grenzfläche erteilt dabei dem Geschoss eine kontinuierliche Richtungsänderung (in der Schädelkapsel bogenförmiger Verlauf zwischen Dura und Hirnoberfläche).

Im Fall eines Durchschusses erzeugt das Projektil eine Ausschusswunde und verlässt dort den Körper. Matte Geschosse sind mitunter nicht mehr in der Lage, über der Ausschusswunde befindliche Kleidungsstücke zu durchschlagen. Von einem 2-Segmenttreffer spricht man, wenn das Geschoss zunächst einen Körperteil (z.B. Arm) durchschlägt und nach dem Austreten in einen weiteren Körperteil derselben Person (z.B. Thorax) eindringt (»re-entry«).

Streifschüsse erzeugen rinnenförmige Verletzungen an der Körperoberfläche, die manchmal von kurzen Wundrandeinrissen begleitet sind. Ein Prellschuss liegt dann vor, wenn ein mattes Geschoss auf den Körper trifft, aber nicht eindringt.

\section{Zwischenziele, Geschossablenkung}

Für die Interpretation einer Schussverletzung kann es wichtig sein, ob das Geschoss primär den menschlichen Körper getroffen hat, oder ob zuvor eine Interaktion mit einem Zwischenziel stattgefunden hat. So hat die Durchdringung eines Zwischenziels (z.B. einer Tür) zur Folge, dass am Kleider- bzw. Körpereinschuss der ansonsten typische Abstreifring (s. unten) fehlt, weil die an der Geschossoberfläche haftenden Verunreinigungen bereits auf das Primärziel übertragen wurden.

Nach der Kontaktnahme mit einem dichten Medium kommt es zum Taumeln des Geschosses (Rotation der Figurenachse um die Flugbahnachse). Aus einer Schräg- oder Querstellung des Projektils beim sekundären Körperaufprall resultiert eine längliche Einschussöffnung, aber auch eine erhöhte Energieabgabe im Anfangsteil des Wundkanals. Analoge Auswirkungen kann das Auftreffen eines schon am Primärziel deformierten Geschosses haben.

Wenn ein Geschoss an der Oberfläche eines Intermediärzieles abgelenkt wird, spricht man von einem Rikoschettschuss oder Geller. Als Ursachen einer solchen Richtungsänderung kommen beispielsweise Geschosskontakte mit Stein, Beton oder Asphalt in Betracht. Die Projektile können in solchen Fällen einseitige Abplattungen mit abgeschliffen erscheinender Oberfläche oder Einlagerungen von Fremdmaterial aufweisen. Als Folge der ablenkungsbedingten Geschossdeformation oder -fragmentierung entstehen oft atypische Einschüsse mit fehlendem oder unvollständigem Abstreifring ( $>$ unten). Wegen des Geschwindigkeitsverlustes und der Instabilität des abgeprallten Geschosses ist die Eindringtiefe im Körper geringer als bei primären Treffern nach ungestörtem Freiflug.

\section{Tödliche Schussverletzungen}

Nach statistischen Untersuchungen verlaufen etwa 20\% der Schussverletzungen "primär tödlich«; d.h. diese Opfer versterben, noch bevor sie einer ärztlichen Versorgung zugeführt werden können.

Grundsätzlich muss mit tödlichen Folgen einer Schussverletzung auch dann gerechnet werden, wenn Waffen zum Einsatz kommen, die in Laienkreisen als wenig gefährlich angesehen werden (z.B. Luftdruck-, Flobert-, Schreckschuss- und Kleinkaliberwaffen). So können übliche Luftgewehrkugeln eine dünne Schläfenbeinschuppe perforieren oder durch die Orbita in die Schädelhöhle eindringen. Der Gasstrahl von Schreckschusswaffen hat bei Schüssen aus unmittelbarer Nähe wiederholt zu penetrierenden Verletzungen der Haut, zu Knochenfrakturen und tödlichen Gefäß-/Organläsionen geführt. Sofern wichtige Organe oder große Gefäße im Verlauf des Schusskanals liegen, können selbstverständlich auch energiearme Geschosse aus Kleinkaliber- oder Flobert-Waffen tödliche Verletzungen erzeugen.

Bei letal verlaufenden Schussverletzungen kann die unmittelbar tödliche Funktionsstörung auf verschiedenen Ursachen beruhen. Ein Sonderfall ist die schussbedingte »Exenteration « des Gehirns aus der Schädelkapsel, der bereits erwähnte KrönleinSchuss. Eine direkte Zerstörung von lebenswichtigen Zentren des Hirnstamms ist u.a. bei Genickschüssen zu erwarten. Häufiger führt nicht die zerebrale Läsion als solche, sondern die nachfolgende intrakranielle Drucksteigerung (durch intrazerebrale, subarachnoidale und subdurale Blutung, evtl. in Verbindung mit einem Hirnödem) zum Tode.

Schussfrakturen der knöchernen Schädelbasis gehen oft mit einer Blutung in den Nasenrachenraum einher, woraus im Zustand der Bewusstlosigkeit eine tödliche Blutaspiration resultieren kann. Schussbedingte Zerreißungen der Sinus durae matris kommen als Eintrittspforte einer venösen Luftembolie in Betracht.

Verletzungen des Herzens, großer Gefäße oder parenchymatöser Organe sind Quellen massiver innerer Blutverluste mit konsekutivem Blutungsschock. Lungenschüsse mit traumatischem Pneumothorax stellen - besonders bei bilateralen Verletzungen - wegen der damit verbundenen Atmungsbehinderung eine akute Bedrohung dar. Entzündliche Komplikationen, wie sie besonders nach Bauchschüssen und nach Kopfschüssen mit Viehbetäubungsapparaten auftreten können, sind mögliche Ursachen von Spättodesfällen.

\section{Handlungsfähigkeit}

Bei medizinischen Laien besteht häufig die irrige Vorstellung, dass eine Schussverletzung des Kopfes oder Rumpfes zwingend eine sofortige Aktionsunfähigkeit nach sich ziehe. Dieser Auffassung stehen reale Fälle gegenüber, in denen Schussverletzte, trotz schwerster Traumatisierung lebenswichtiger Organe, noch überraschend differenzierte Handlungen verrichtet haben. 
Handlungsunfähigkeit beruht in aller Regel auf einer Funktionsstörung des zentralen Nervensystems, die entweder durch direkte Gewebsläsionen oder indirekt durch unzureichende Sauerstoffversorgung verursacht sein kann. Unmittelbare Aktionsunfähigkeit ist jedenfalls dann zu erwarten, wenn die für physische Aktivität essentiellen Hirnteile durch die Schusswirkung zerstört wurden - im Extremfall durch Exenteration des ganzen Organs. $\mathrm{Zu}$ den »targets of immediate incapacitation « zählen das obere Halsmark, der Hirnstamm, das Kleinhirn sowie große Teile des Zwischen- und Mittelhirns einschließlich der Basalganglien, die motorischen Hirnrindenareale und die großen motorischen Nervenbahnen. Dabei muss das Geschoss nicht notwendigerweise die genannten Regionen des Zentralnervensystems direkt passieren, da die hohen Drucksteigerungen innerhalb der knöchernen Schädelkapsel auch schusskanalferne Nervengewebsbezirke strukturell und funktionell schädigen können.

Eine zerebrale Hypoxie mit konsekutiver Bewusstlosigkeit ist im Zusammenhang mit Brustschüssen meist durch massiven Blutverlust bedingt. Allerdings tritt auch bei Treffern des Herzens, der Aorta oder anderer großer Arterien kaum jemals ein unmittelbarer Kreislaufstillstand ein und selbst in einem solchen Fall kann die Sauerstoffreserve des Gehirns ausreichen, noch einfache/kurze Handlungen auszuführen. Dementsprechend ist bei Schussverletzungen des Herzens, der Aorta und der Pulmonalarterie zwar mit einer raschen, nicht aber mit einer sofortigen Aktionsunfähigkeit zu rechnen.

Aus den skizzierten pathophysiologischen Überlegungen ergeben sich wichtige Implikationen für die forensische Beurteilung von Suizidfällen mit wiederholter Schussbeibringung. Gründe für das Erhaltenbleiben der Handlungsfähigkeit nach einer Gehirnschussverletzung sind vor allem die Verwendung energiearmer Munition und/oder ein Schusskanalverlauf, der die o.g. Strukturen (oberes Halsmark, Hirnstamm, Mittel- und Zwischenhirn, motorische Rindenregionen, große motorische Leitungsbahnen) verschont. Meist ist in solchen Fällen entweder nur das Stirnhirn oder nur einer der Schläfenlappen betroffen. Wesentlich häufiger als multiple suizidale Gehirnschädelschüsse sind eigenhändige Mehrfachschussverletzungen der Herzregion.

\section{Stopping power}

Der Ausdruck »Stopping Power« (sog. Aufhaltekraft) soll das biologische Wirkungspotential eines Geschosses charakterisieren, speziell seine Fähigkeit, eine Person an der Fortbewegung oder an einem Angriff zu hindern. Die durch Kino- und Fernsehfilme suggerierte Vorstellung, dass ein Geschoss beim Auftreffen auf den menschlichen Körper diesen aufhalten (»stoppen«) oder gar umwerfen würde, ist jedoch aus physikalischer Sicht unzutreffend. Tatsächlich resultiert die Geschosswirksamkeit aus der an den Körper abgegebenen Energie mit der Folge einer lokalen Gewebsverlagerung und -zerstörung. Außerdem hängt die Wirksamkeit wesentlich von der Form des Projektils ab: Bei »stumpfem« Geschosskopf sind Verzögerung und Energieübertragung größer. Die tatsächliche Wirkung eines Geschosses ergibt sich aber nicht nur aus dessen Wirkungspotential, sondern ganz entscheidend aus der Treffpunktlage, also aus der verletzten Region und den dort gelegenen anatomischen Strukturen.

\section{Geschossembolie}

Die selten vorkommende Verschleppung von eingedrungenen Projektilen oder Schrotkörnern innerhalb des Blutgefäßsystems wird als Geschossembolie bezeichnet. Meist handelt es sich um Geschosse mit kleinem Durchmesser und geringer Restenergie: Diese reicht nur noch zum Eindringen in die Arterie oder Vene, nicht aber zum Verlassen des Gefäßes aus, so dass der nun intravasale Fremdkörper in eine Körperregion abseits des Schusskanals embolisiert werden kann, wo er sich radiologisch leicht darstellen lässt.

Geschossembolien werden mehrheitlich im arteriellen System beobachtet (Eintritt des Projektils durch das Herz oder die Aorta, Verschleppung z.B. in die Beinarterien). Seltener gelangen die Geschosse in Venen des Körperkreislaufes und von dort in das (rechte) Herz oder in die Pulmonalarterienäste. Vereinzelt wurden auch paradoxe (gekreuzte) Embolien beschrieben.

\section{Spätfolgen}

Bei überlebten Schussverletzungen mit Verbleib von Geschossen/ Schrotkörnern im Körper stellt sich die Frage nach der Möglichkeit einer chronischen Bleivergiftung. Im Allgemeinen ist die Wahrscheinlichkeit als sehr gering einzuschätzen. Die meisten Fälle, die in der Literatur beschrieben wurden, betrafen Patienten, bei denen die Geschosse in Gelenken oder Knochen steckten. Die Latenzzeit bis zum Manifestwerden einer Intoxikation variiert zwischen wenigen Monaten bis zu einigen Jahrzehnten.

\section{Kriminalistische Aspekte}

Die klinische oder autoptische Untersuchung von schussverletzten Personen dient u.a. der Klärung folgender Fragen:

- Lässt sich die Annahme einer Schussverletzung befundmäßig objektivieren?

- Wie viele Treffer liegen vor?

- Handelt es sich um Steckschüsse, Durchschüsse oder Streifschüsse?

- Wie war die Schussrichtung?

- Sind Rückschlüsse auf die Art der Tatwaffe und der Tatmunition möglich?

- Aus welcher Entfernung wurde geschossen (absoluter Nahschuss, relativer Nahschuss, Fernschuss; $>$ Kap. 3.7.4)?

- Spricht das Verletzungs- und Spurenbild für Selbst- oder Fremdbeibringung?

- Hat die Schussverletzung zu sofortiger Handlungsunfähigkeit geführt? 


\subsubsection{Einschuss und Ausschuss}

Die Bestimmung der Schussrichtung setzt voraus, dass Ein- und Ausschussöffnung richtig erkannt werden.

\section{Einschusszeichen}

Der Einschuss in der Haut weist in typischen Fällen folgende Merkmale auf:

- zentraler, nicht adaptierbarer Substanzdefekt,

- epidermisfreie Randzone (Kontusionsring, »Schürfsaum«),

- grauschwarzer Abstreifring (wenn das Projektil zuvor kein anderes Primärziel durchschlagen hat) und

- etwaige Nahschusszeichen (bei Schüssen aus geringer Di$\operatorname{stanz})$.

\section{Einschussdefekt}

Der zentrale Einschussdefekt ist rundlich (bei orthogonalem Auftreffen des Projektils) oder oval (bei Schrägschüssen). Der Durchmesser ist meist kleiner als jener des Geschosses.

Die Diskrepanz zwischen Geschosskaliber und Größe der Einschusslücke lässt sich durch das elastische Verhalten der jeweiligen Hautregion erklären: Beim Aufprall des Geschosskopfes führen die radial wirkenden Kräfte zu einem kurzzeitigen, zentrifugalen Auseinanderweichen des Defektrandes (temporäre, reversible Erweiterung der Durchtrittsstelle). Mit dem Aufhören der Verformungskräfte nimmt die elastische Haut wieder ihre frühere Gestalt ein, so dass die bleibende und nicht adaptierbare Einschussöffnung wesentlich kleiner sein kann als der Geschossquerschnitt (besonders ausgeprägt ist dieses Missverhältnis an den stark verhornten Handflächen und Fußsohlen).

\section{(C) Wichtig}

Die Abmessungen der Hautwunde erlauben keine exakten Rückschlüsse auf das Geschosskaliber.

Der Hautdefekt am Einschussort kommt im Wesentlichen dadurch zustande, dass Gewebsteilchen durch das Geschoss in die Tiefe des Schusskanals verlagert werden. Außerdem werden kleine Hauptpartikel beim Auftreffen des Geschosses entgegen der Schussrichtung zurückgeschleudert.

\section{Kontusionsring („Schürfsaum»)}

Die Einschusslücke ist in der Regel von einer epidermisfreien Zone umgeben, die sich in frischem Zustand als feuchter, rötlicher Saum darstellt und postmortal infolge Vertrocknung einen bräunlichen Farbton annimmt (• Abb. 3.52). Dieser Bezirk wurde früher allgemein als Schürfsaum bezeichnet, neuerdings bevorzugt man den Begriff Kontusionssaum oder Kontusionsring.

Die Entstehungsweise dieses Einschussbefundes wurde durch die hochfrequenzkinematographischen Untersuchungen von Sellier geklärt: Beim Auftreffen des Geschosskopfes führt die örtliche Drucksteigerung zum kegelförmigen Zurückspritzen oberflächlicher Gewebspartikel. Die frühere Vorstellung, dass der

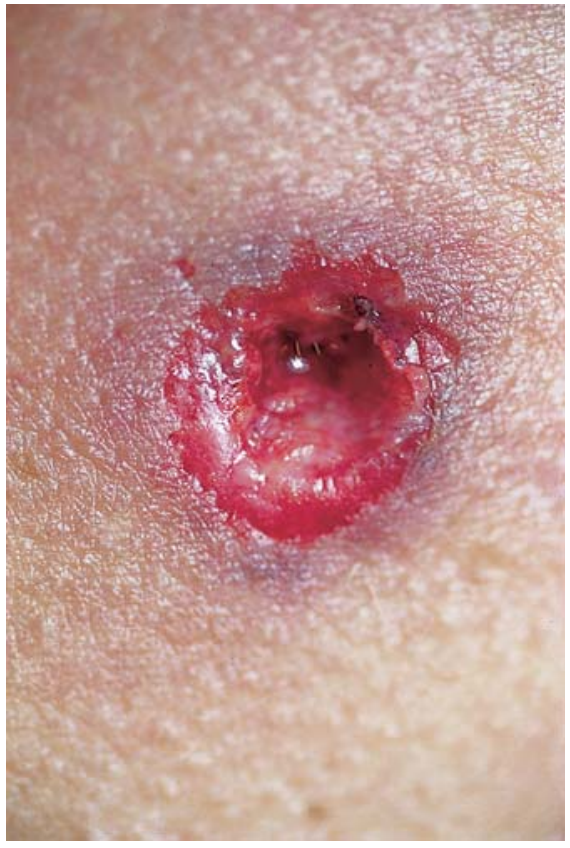

- Abb. 3.52. Einschussöffnung in einer bekleideten Körperregion (obere Rückenpartie): rundlicher Substanzdefekt, zirkulärer Kontusionsring (»Schürfsaum«), aber kein Abstreifring; die umgebende Haut ist zart rotviolett verfärbt (angedeuteter »Dehnungssaum«); Tatwaffe: Revolver Kaliber 357 Magnum

Geschosskopf vor dem Eindringen die Haut einstülpen und im Randbereich schürfen würde, ist unzutreffend.

Am Rand des Kontusionsringes ist die Epidermis abgehoben, aufgeworfen und z.T. radiär eingerissen. Das Ausmaß der sekundären Vertrocknung kann daher über die Zone des echten Epidermisverlustes hinausgehen. Bei Schrägschüssen sind die Kontusionsringe elliptisch begrenzt und zum Schützen hin verbreitert. Wenn sich die Haut des Einschussbereiches unter Wasser befindet, unterbleibt die Ausbildung eines Kontusionsringes.

\section{Abstreifring ("Schmutzring")}

Der Abstreifring verdankt seine kriminalistische Bedeutung vor allem der Tatsache, dass er - zumindest am Primärziel - ein verlässliches Indiz für den Einschuss ist. Die früher gebräuchlich gewesenen Synonyme ("Schmutzring", "Schmauchring ") geben deskriptiv den optischen Eindruck wieder, indem sie eine saumartige Schwärzung des Einschussrandes beschreiben.

Der Ausdruck »Abstreifring « bezieht sich auf den Entstehungsmechanismus: Im Augenblick des Hautkontaktes überträgt das Projektil die an ihm haftenden Verunreinigungen (Rückstände des Zünd- und Treibsatzes, eventuell auch des Waffenöles). Au- 


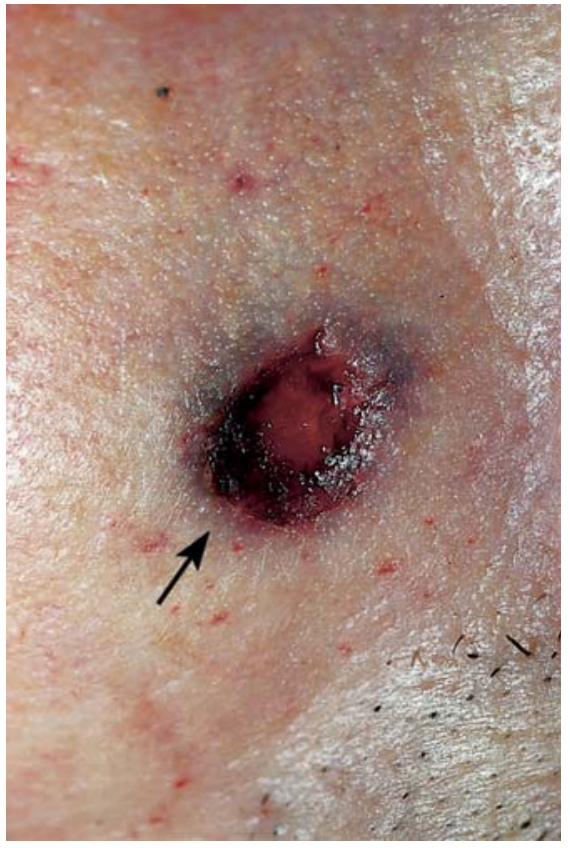

- Abb. 3.53. Einschussöffnung mit exzentrischem Abstreifring bei Schrägschuss (KK-Gewehr, Vollbleigeschoss). Die Wundrandschwärzung ist schützenseitig verbreitert, der Pfeil zeigt die Schussrichtung an. In der Umgebung des Einschusses vereinzelte Pulvereinsprengungen als Nahschusszeichen

ßerdem können vom Geschoss selbst bzw. vom Mantel Metallspuren an der Eintrittsstelle deponiert werden.

Der Abstreifring bedeckt bei primären Hauteinschüssen (• Abb. 3.53) die epidermisfreie Zone des Kontusionsringes (Schürfsaumes). Wird eine bekleidete Körperregion getroffen, dann findet sich der Abstreifring auf der obersten Textillage (- Abb. 3.54), aber nicht (oder nur angedeutet) am Rand der Einschusswunde. Bei Schrägschüssen ist der Abstreifring schützenseitig exzentrisch verbreitert. Der Abstreifring ist kein Nahschusszeichen.

\section{Weitere (fakultative) Einschusszeichen}

Manche Autoren erwähnen als weiteres, allerdings nicht konstant auftretendes und nicht für einen Einschuss beweisendes Zeichen den so genannten Dehnungssaum, der durch die kurzzeitige Radialverlagerung des einschussnahen Gewebes infolge zirkulärer Überdehnung (mit konsekutiver Gefäßzerreißung) auftreten kann. Er manifestiert sich als mehr oder weniger breite, rotviolette Unterblutung, deren Durchmesser meist deutlich größer ist als jener des Kontusionsringes (Schürfsaumes).

Als weiteres fakultatives und nicht verlässliches Einschusszeichen wird von manchen Autoren die Anwesenheit von Textilfasern in der Tiefe der Schusswunde genannt (bei Treffern in bekleideten Körperregionen); durch den Unterdruck in der

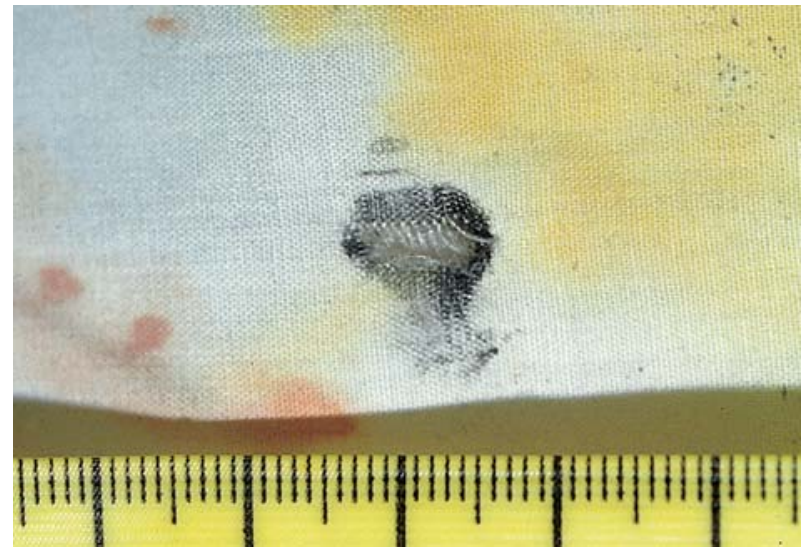

- Abb. 3.54. Baumwollgewebe einer Bluse mit Einschusslücke und schwarzem Abstreifring. Tatmunition: Revolverpatrone Kal. 38 Spl

temporären Wundhöhle können Fasern auch retrograd in den Ausschuss verlagert werden.

\section{Ausschuss}

Der Ausschuss stellt sich als schlitzförmige oder mehrstrahlige Zusammenhangstrennung dar. In typischen Fällen ist - anders als beim Einschuss - keine echte Lückenbildung, also kein Gewebsdefekt vorhanden, d.h. die Wunde kann durch Aneinanderlegen der Ränder völlig verschlossen werden (»Adaptierbarkeit«; - Abb. 3.55).

Die Größe übertrifft mehrheitlich, aber nicht immer die des Einschusses. Die unkritische Anwendung dieser unverlässlichen »Regel « führt in der Praxis oft zu Fehlbeurteilungen. So entstehen bei Kopfschüssen mit aufgesetzter Waffe oft »Einschussplatzwunden « mit langen Rissstrahlen; der Ausschuss kann in solchen Fällen wesentlich kleiner sein, wenn das Geschoss mit nur noch geringer Restgeschwindigkeit austritt und dabei einen kurzen, rissartigen Wundschlitz erzeugt. In Fällen von Splitterverletzungen (z.B. durch Fragmente von Explosivwaffen) ist der Einschuss immer größer als der Ausschuss.

Die Größe der Ausschussöffnung hängt vor allem davon ab, welchen Durchmesser die temporäre Höhle an der Austrittsstelle des Geschosses hatte. Bei langsamen Projektilen kann auch das »Mitführen« von Knochensplittern zur Vergrößerung des Ausschusses beitragen. Viele Geschosse verlassen den Körper in deformiertem Zustand und/oder als »Querschläger «, was bei geringer Austrittsgeschwindigkeit die Form der Ausschusswunde mitbeeinflusst.

Ein Abstreifring ist am Ausschuss naturgemäß niemals vorhanden. Bisweilen ist auch der Ausschuss von einem schürfsaumartigen Epidermisdefekt umgeben. Voraussetzung dafür ist das Vorhandensein eines Widerlagers über der Geschossaustrittsstelle (z.B. eng anliegendes Kleidungsstück wie Hosenträger oder BH; flächiger Kontakt der Ausschussregion mit dem Boden, der Wand oder einer Stuhllehne; - Abb. 3.56). Im Unterschied zum »ech- 

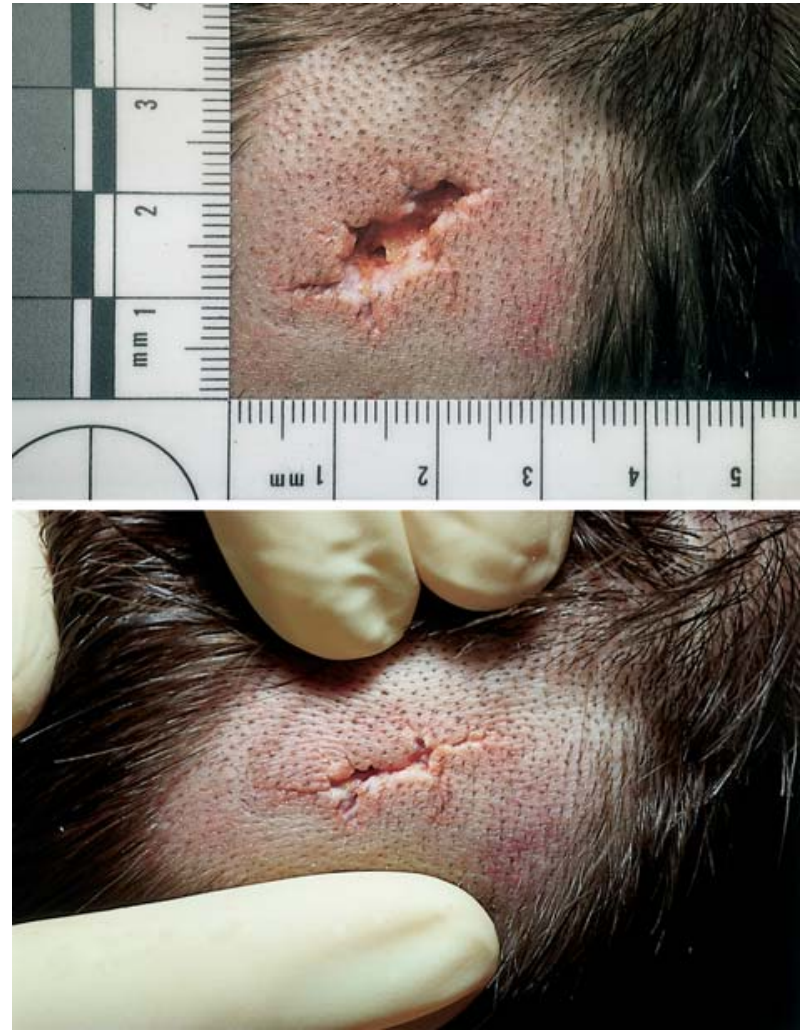

- Abb. 3.55. Ausschusswunde in der Hinterhauptsregion nach suizidalem Mundschuss mit einer Pistole 9 mm Luger. Rissförmige, adaptierbare Zusammenhangstrennung ohne Abstreifring und ohne Schürfungssaum. Das Kopfhaar wurde im Zuge der Obduktion teilweise rasiert

ten « Schürfsaum (Kontusionsring) am Einschuss ist ein etwaiger Oberhautverlust am Rand des Ausschusses meist unregelmäßig begrenzt. Die Epidermis wird bei Vorliegen eines Widerlagers am Ausschuss tatsächlich abgeschürft, während der Epidermisverlust am Einschuss durch »Zurückspritzen« kleiner Gewebspartikel zustande kommt.

\subsubsection{Entfernungsabhängigkeit der Einschussbefunde}

\section{Vorgänge beim Schuss}

Zum Verständnis der unterschiedlichen Schussbilder ist es notwendig, die wichtigsten Vorgänge nach Auslösung eines Schusses zu kennen. Zunächst bringt die Schlagbolzenspitze den Zündsatz zur Detonation; durch den anschließenden Abbrand des Pulvers entsteht ein großes Gasvolumen, das unter hohem Druck steht und das Geschoss aus dem Lauf treibt.

Schon bevor das Projektil den Lauf verlässt, tritt aus der Mündung eine Schmauchwolke aus. Der Terminus »Schmauch« be-

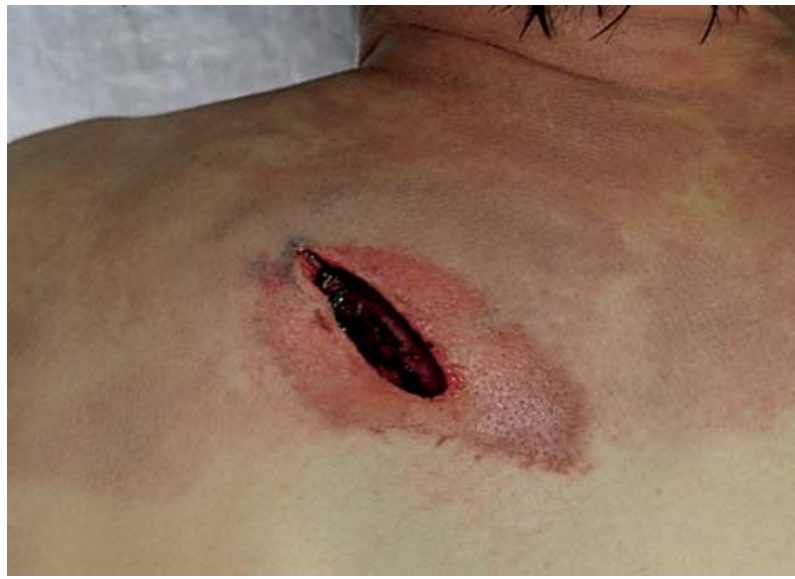

- Abb. 3.56. Spaltförmige, $5 \mathrm{~cm}$ lange Ausschussöffnung in der linken oberen Rückenpartie einer 45-jährigen Frau. Der dazugehörige Einschuss befand sich an der rechten Brustseite. Tatwaffe war ein alter Militärkarabiner Kal. 7,5 × 55 mm; verschossen wurde ein VollmantelSpitzgeschoss. Da die Frau bei Erhalt der Schussverletzung mit dem Rücken auf dem Boden lag, ist es rund um den Ausschuss atypischerweise zu einer Exkoriation gekommen, die später infolge Luftzutritts braunrot vertrocknet ist

zeichnet die grau(schwarz) gefärbten Verbrennungsrückstände des Pulvers, das nicht zur Gänze in Gase umgesetzt wird. Im Wesentlichen handelt es sich beim Schmauch um unverbrannten Kohlenstoff, der in Form feinster (ultravisibler) Partikel - also in Form von Ruß - vorliegt.

Neben den Treibgasen, neben feindispersem Ruß und Schmauchelementen verlassen stets auch unverbrannte und partiell verbrannte Pulverteilchen den Lauf. Die Wolke aus winzigen Rußpartikeln wird rasch abgebremst, so dass Schmauchauflagerungen nur in relativ geringer Entfernung von der Mündung zu erwarten sind. Die größeren Pulverkörnchen (Durchmesser mehrere Zehntelmillimeter) gelangen auch auf weiter entfernte Zielobjekte.

Intensität und Flächenverteilung der genannten Nahschusszeichen (Schmauch und Pulverteilchen) auf einem Zielobjekt hängen nicht nur von der Schussentfernung, sondern ebenso von der Patronenart und der Waffe ab. Eine hinreichend genaue Eingrenzung der Schussdistanz ist daher nur nach Vergleichsschüssen mit Tatwaffe und Tatmunition möglich.

Eine makroskopisch sichtbare Beschmauchung ist bei Faustfeuerwaffen bis zu einer Schussentfernung von etwa $10-15 \mathrm{~cm} \mathrm{zu}$ erwarten. Pulverteilchen können in Abhängigkeit von Waffe und Munition auf Ziele gelangen, die einige bis mehrere Dezimeter entfernt sind (bei Langwaffen auch $>1 \mathrm{~m}$ ). Die Verwendung von Schalldämpfern schwächt die Beschmauchung stark ab, so dass fälschlich der Eindruck einer größeren Schussentfernung entsteht.

An der Laufmündung treten zwei verschiedene Lichtphänomene auf: erstens das Feuer aus der Mündung (»flame«) - ein kurzer und meist dunkelrot gefärbter Feuerstrahl, verursacht 
durch die noch nicht ganz abgeschlossene Verbrennung der Treibsatzpartikel; zweitens das Mündungsfeuer (»muzzle flash «) - ein greller Feuerball in einiger Entfernung vom Laufende, hervorgerufen durch die Reaktion der unvollständig oxidierten Pulvergase mit dem Sauerstoff der Luft.

Bei Nitropulver reicht die extrem kurze Einwirkungszeit der Mündungsflamme trotz deren hoher Temperatur üblicherweise nicht aus, im Nahschussbereich substantielle Verbrennungen an der Kleidung oder Haut hervorzurufen. Allenfalls kann man an den einschussnahen Haaren eine Kräuselung erkennen. Eine thermische Schädigung ist bei Schüssen mit Nitromunition im Entfernungsbereich von wenigen Zentimetern dann möglich, wenn thermolabile Kunstfasertextilien durch die hohen Temperaturen auf der Haut schmelzen. Bei Verwendung von Schwarzpulvermunition können Nahschüsse im Einwirkungsbereich der Mündungsflamme zu eindrucksvollen, flächenhaften Verbrennungen führen (»Brandhof«, • Abb. 3.57).

In der Rechtsmedizin werden nach morphologischen Kriterien drei Schussentfernungsbereiche unterschieden:

- der absolute Nahschuss,

- der relative Nahschuss und

- der Fernschuss.

\section{Absoluter Nahschuss}

Definition -

Der Begriff »absoluter Nahschuss« bedeutet, dass die Laufmündung bei der Schussabgabe »aufgesetzt« war (Syn: Kontaktschuss). Beim absoluten Nahschuss gelangen schmauch$\checkmark$

- Abb. 3.57. Unterhemd und linke vordere Brustwand eines jungen Mannes mit präkordialem Einschuss, der von einem Brandhof und Schmauchablagerungen umgeben ist. Suizidaler Herzschuss (durch die Kleidung) mit einer großkalibrigen Vorderladerpistole (Schwarzpulver-Munition) haltige Pulvergase durch die Einschusslücke in die Tiefe. Sie expandieren unter der Haut und verfärben den Anfangsteil des Wundkanals schwarzgrau (»Schmauchhöhle«). Wegen des hohen Kohlenmonoxidgehaltes der Verbrennungsgase (bis zu $50 \%$ ) hat das umliegende Gewebe oft einen hellroten Farbton.

Der Einschussbereich wird durch den Druck der eingedrungenen Pulvergase aufgetrieben und ballonartig gegen die Waffenmündung vorgewölbt; dabei kommt es an der Haut zu einer Abprägung des Waffengesichtes (also jener Konstruktionsteile, die in der Mündungsebene oder knapp dahinter liegen). Dieser Befund wird als »Stanzmarke« bezeichnet. Verletzungsmechanisch stellt die Stanzmarke eine geformte Exkoriation (»patterned abrasion«) durch orthogonale Druckwirkung dar. Nach ausreichend langem Luftzutritt imponiert die Stanzmarke als braune Hautvertrocknung. Im Einzelnen können sich neben der Stirnfläche (bzw. den kantigen Konturen) des Laufes auch andere mündungsnahe Waffenteile abbilden: z.B. das Korn, der Vorholfederführungsstift oder die Mündungsebene eines nicht abgeschossenen Laufes (bei mehrläufigen Waffen; • Abb. 3.58).

Aus der Anwesenheit, Form und Ausrichtung einer Stanzmarke lassen sich kriminalistisch bedeutsame Schlüsse ziehen:

- Die Waffe war zum Zeitpunkt der Schussabgabe aufgesetzt,

- die Konfiguration der Stanzmarke korrespondiert mit den mündungsnahen Strukturen (• Abb. 3.59) und

- die Lagebeziehung zwischen charakteristischen Stanzmarkenteilen und der Einschussöffnung gibt Auskunft über
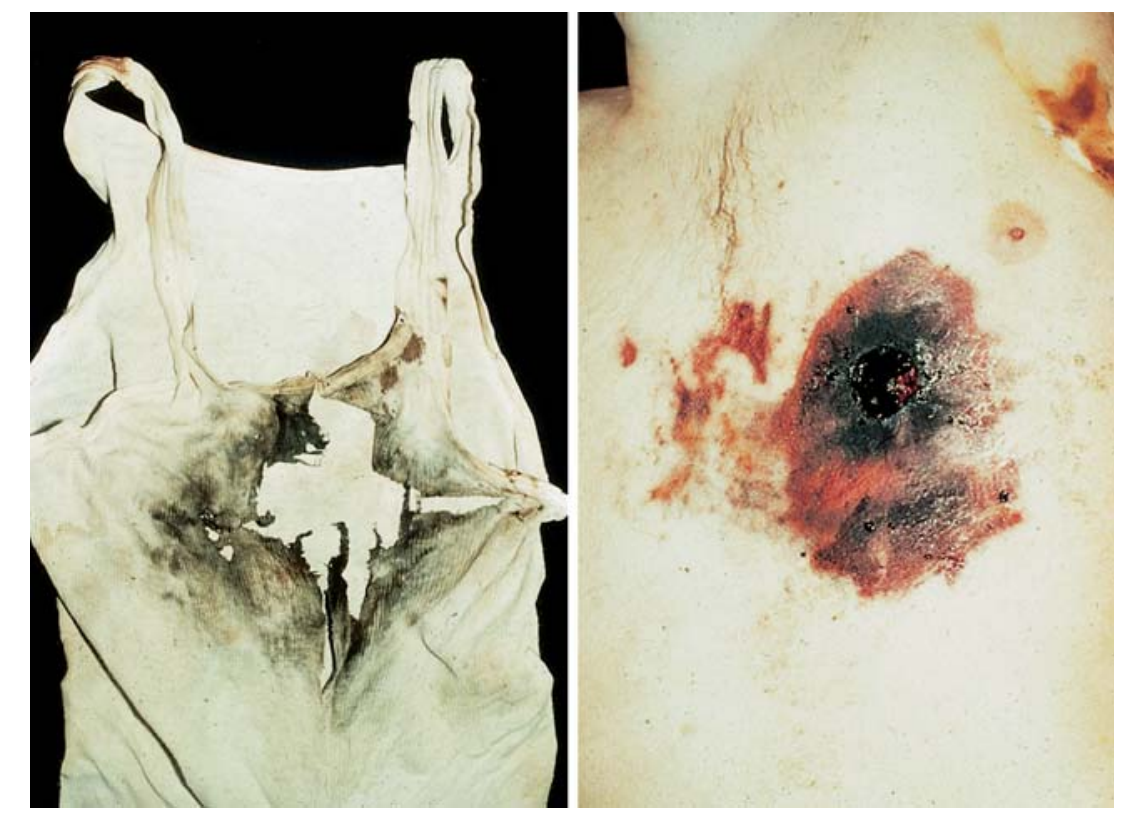


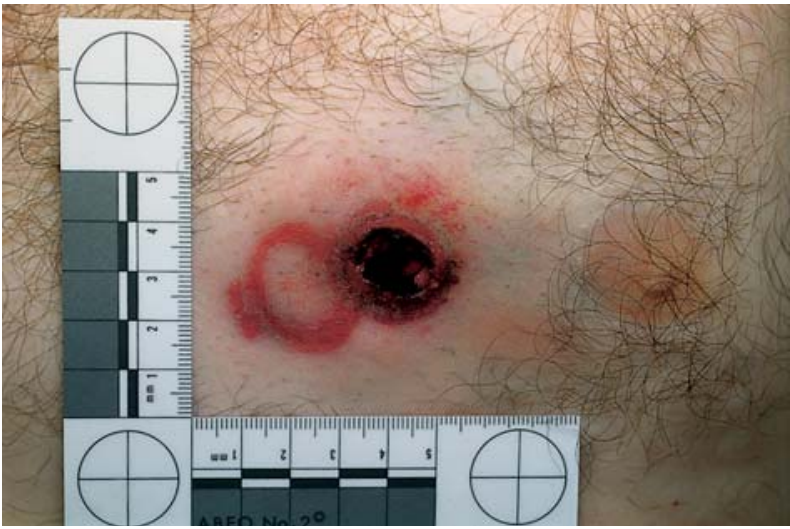

- Abb. 3.58. Suizid mit einer Bockbüchsflinte (Kipplauf-Jagdgewehr mit 2 übereinander angeordneten Läufen). Präkordiale Einschusswunde mit charakteristischer Stanzmarke. Neben der eigentlichen Einschussöffnung hat sich die Mündung des nicht abgeschossenen (oberen) Flintenlaufs mit dem Kornsattel als braun vertrocknete Stanzmarke abgeprägt. Aus dem unten liegenden Büchsenlauf wurde eine Patrone Kaliber 7 x 65 $\mathrm{mm}$ mit Teilmantel-Rundkopfgeschoss verfeuert. Keine sternförmige Hautaufplatzung, aber Schmauchschwärzungen in der Tiefe des Einschusses. Zum Zeitpunkt der Schussabgabe war der Oberkörper mit Hemd und Unterhemd bekleidet (keine Entblößung der Einschussregion!)

die Waffenhaltung: So bedeutet ein Kornabdruck an der Unterseite, dass die Waffe verdreht (mit dem Griffstück nach oben) gehalten wurde.

Wenn sich unter dem Einschuss ein knöchernes Widerlager befindet (z.B. in der Stirn- und Schläfenregion), kann die von eingedrungenen Verbrennungsgasen vorgewölbte Haut infolge Überdehnung radiär einreißen (»Einschussplatzwunde«). Dieses dritte Zeichen eines absoluten Nahschusses ist fakultativ: Schüsse mit energiearmer Munition, z.B. 22 L.R., brauchen auch an den Prädilektionsstellen mit knöchernem Widerlager keine sternförmige Hautaufplatzung zu verursachen.

\section{Relativer Nahschuss}

\section{- Definition \\ Beim relativen Nahschuss lassen sich Schussrückstände (Schmauch und/oder Pulverteilchen) in der Umgebung des \\ Einschusses nachweisen. Man unterscheidet den näheren und den weiteren relativen Nahschuss.}

Der nähere relative Nahschuss ist definiert durch die Anwesenheit eines Schmauchhofes (meist in Verbindung mit zusätzlich vorhandenen Pulverauflagerungen/-einsprengungen). Der grauschwarze Schmauch führt zu einer flächenhaften, wolkig strukturierten Haut- oder Textilverfärbung (• Abb. 3.60), deren Intensität mit größer werdender Schussentfernung abnimmt. Schrägschüsse führen zu einem asymmetrischen Schmauchbild mit Ausziehung zur schützenfernen oder schützennahen Seite (je nach Schusswinkel und Schussentfernung). Durch Interposition von Kleidungsstücken oder Körperteilen (Hand) kann der Schmauchstrahl teilweise »abgeschattet« sein. Mündungsstücke, die bei Armeegewehren häufig zur Dämpfung des Mündungsfeuers und zur Verminderung des Rückstoßes am Laufende montiert sind, verfügen über seitliche Gasauslassschlitze, wodurch mehrstrahlige Schmauchbilder entstehen können.

Vom weiteren relativen Nahschuss spricht man, wenn rund um den Einschuss keine Schmauchablagerungen mehr sichtbar sind, wohl aber unverbrannte bzw. teilverbrannte Treibsatzpartikel, die der Haut bzw. der Kleidung aufgelagert oder in diese eingesprengt sind. Das Eindringvermögen der Pulverkörnchen hängt von der Munitionsart, der Waffe, der Schussentfernung und den Oberflächeneigenschaften des Zielobjektes ab. An der Haut verursachen die Pulverteilchen entweder oberflächliche Epidermisbeschädigungen (mit nachfolgender Vertrocknung, - Abb. 3.61) oder - bei subepithelialer Lagerung - punktförmige
- Abb. 3.59. Absoluter Nahschuss in der rechten Schläfenregion (Suizid eines 28-jährigen Mannes mit einer Pistole Glock Kal. 9 $\mathrm{mm}$ Luger). Die braun vertrocknete Stanzmarke gibt markante Strukturen des Waffengesichtes (rechts) wieder. Der Einschussrand ist im Sinne einer Einschussplatzwunde radiär eingerissen. In der Tiefe des Einschusses schwarzgraue Verfärbung (»Schmauchhöhle«)
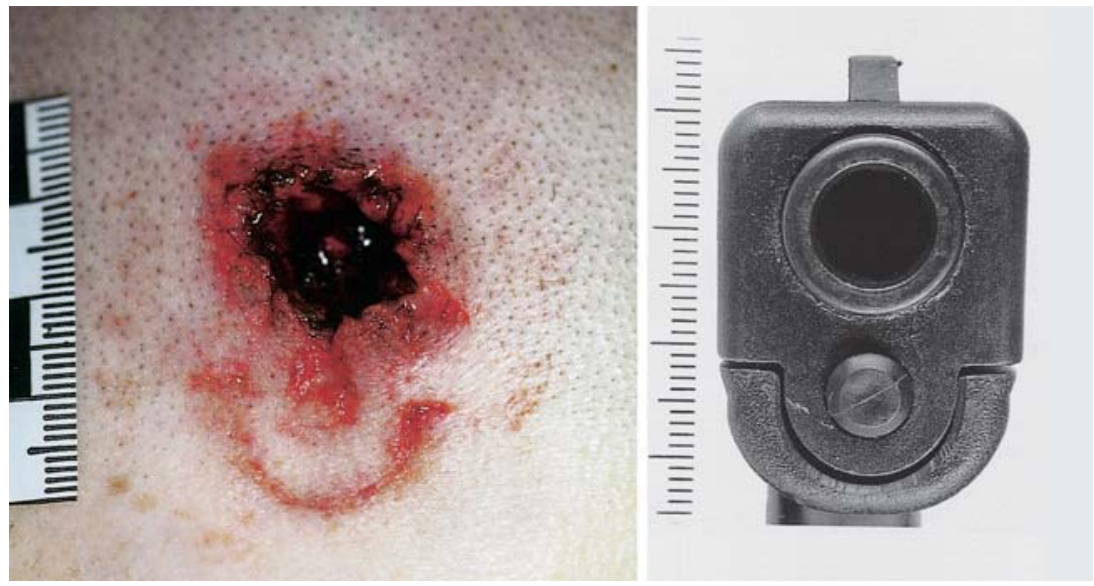


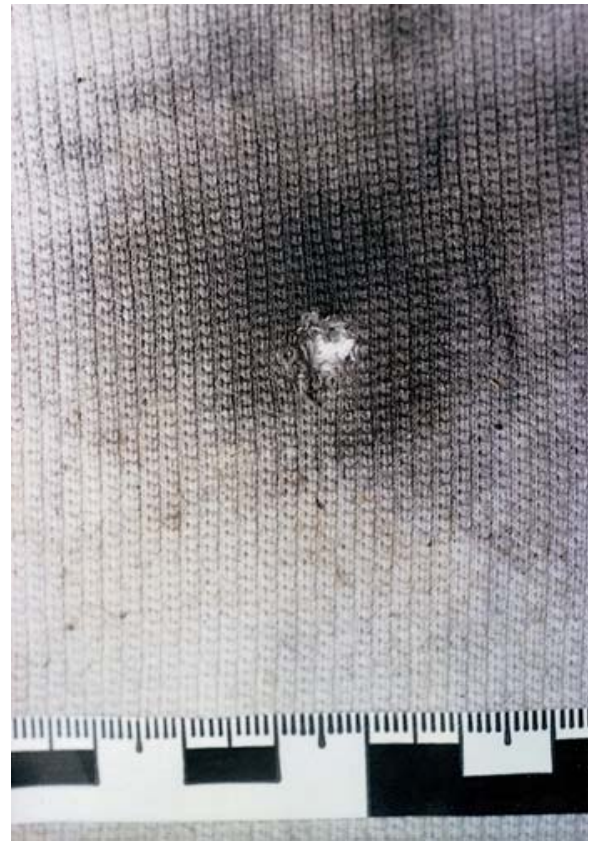

- Abb. 3.60. Einschuss im Baumwollgewebe eines T-Shirts, umgeben von einem dichten Schmauchhof (näherer relativer Nahschuss). Tatwaffe: Pistole Walther P 5 Kal. 9 mm Luger

Lederhautblutungen. Das Verteilungsmuster der Pulvereinsprengungen variiert mit dem Schusswinkel: Nur bei rechtwinkelig auftreffendem Geschoss entsteht ein radial-symmetrisches Bild; in den meisten anderen Fällen ist das betroffene Hautareal elliptisch begrenzt. Die Einschusswunde kann außerhalb der Ein- sprengungen liegen, wenn ein Teil der Pulverkörnchen von Kleidern oder anderen Primärzielen abgefangen wird.

\section{Fernschuss}

\section{- Definition}

Der Begriff »Fernschuss« bedeutet im rechtsmedizinischen Sprachgebrauch das Fehlen von Nahschusszeichen (kein Schmauchhof, keine Pulvereinsprengungen oder -auflagerungen). Die Untergrenze dieses Entfernungsbereiches variiert nicht nur in Abhängigkeit von Waffe und Munition, sondern auch von der Empfindlichkeit der angewandten Untersuchungsmethode.

Auf die speziellen Verfahren der Schussentfernungsbestimmung kann hier nicht eingegangen werden. Bei der Sicherung und sachgerechten Asservierung von etwaigen Schussrückständen an der Kleidung und in der Umgebung von Einschusswunden ist eine enge Zusammenarbeit mit den kriminaltechnischen Sachbearbeitern erforderlich. In jedem Fall sollte eine fotografische Dokumentation erfolgen; Hautexzisate mit Schusswunden und die zur Tatzeit getragene Kleidung sind wichtige Beweisstücke, die für eine objektive Schussentfernungsbestimmung unbedingt benötigt werden.

\section{Schrotschüsse}

Das Schussbild von Schrotmunition ändert sich in Abhängigkeit von der Entfernung zwischen Laufmündung und Zielobjekt: Mit zunehmender Schussdistanz wird die zunächst einheitliche Schusslücke im Randbereich girlandenartig eingekerbt; ab einer Entfernung von ca. 2 m erzeugen randständige Schrotkörner satellitenartige Einschläge außerhalb des zentralen Einschussdefek-
- Abb. 3.61. Brustregion einer 45-jährigen Frau mit Einschuss am medialen Rand der rechten Mamma. Tatwaffe war ein alter Militärkarabiner Kaliber 7,5 x 55 mm, verschossen wurde ein Vollmantel-Spitzgeschoss. Der Oberkörper war zurzeit der Schussabgabe unbekleidet. Der Einschuss ist auffallend klein (unterkalibergroß) und im Randbereich geschwärzt (Abstreifring). Neben der Einschusslücke zarte Beschmauchung. Nahezu die gesamte vordere Brustwand weist punktförmige, braun vertrocknete Pulvereinsprengungen auf (relativer Nahschuss). Durchschuss mit Geschossaustritt im linken Rückenbereich. Fremdtötung im Rahmen eines Familienstreites

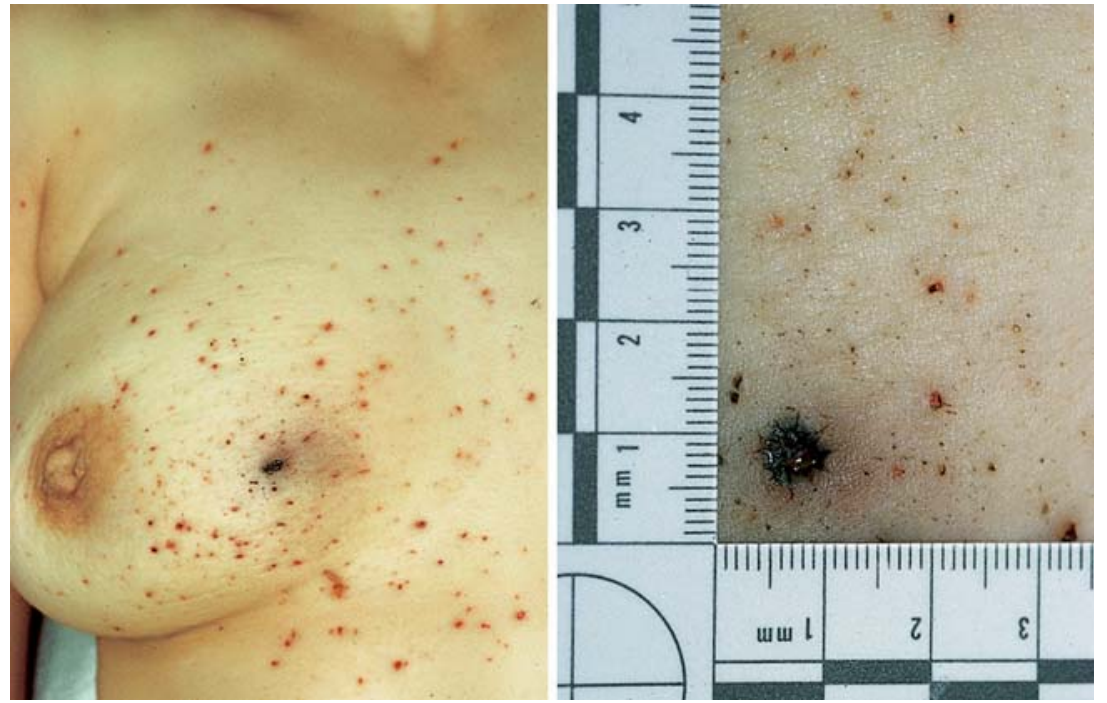




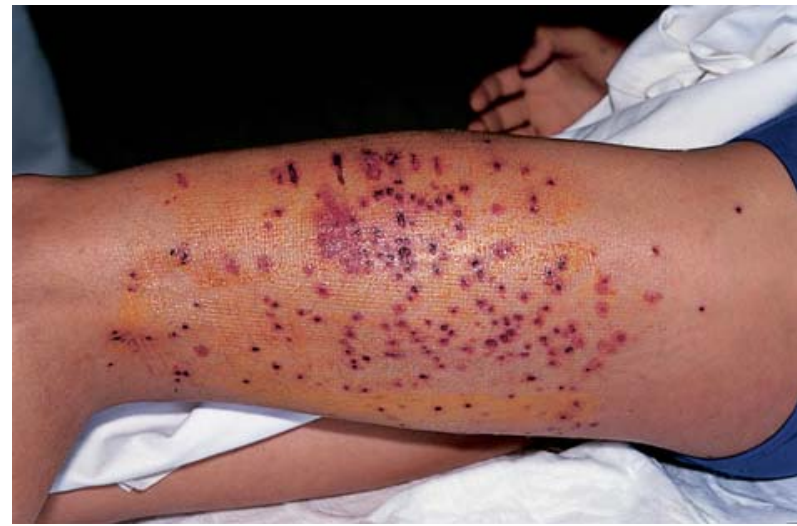

- Abb. 3.62. Überlebte Schrotschussverletzungen des linken Oberschenkels. Der Schrotkorndurchmesser betrug 2,5 mm. Tatwaffe war eine Vorderschaftrepetierflinte mit gekürztem Lauf. Die Schussabgabe erfolgte aus einer Entfernung von einigen Metern durch eine verglaste Tür auf das dahinter befindliche Opfer

tes. Schussentfernungen von einigen bis mehreren Metern sind durch ein siebartiges Trefferbild (• Abb. 3.62) charakterisiert. Bei Schüssen aus sehr geringen Entfernungen können Pfropfen und/ oder Plastikbecher ( Kap. 3.7.1) mit dem Schrot in den Körper gelangen oder an der Haut charakteristisch geformte Exkoriationen verursachen.

Flintenlaufgeschosse (engl.: shotgun slugs) sind Einzelgeschosse, die für glatte Schrotläufe - auch für solche mit Würgebohrung - bestimmt sind. Die Treffgenauigkeit ist wesentlich geringer als bei Büchsengeschossen, weshalb die Einsatzschussweite bei der Jagd auf 35-50 m beschränkt bleiben sollte. Die meisten Flintenlaufgeschosse sind nach dem Pfeilprinzip konstruiert (vorne schwer, hinten leicht). Im deutschsprachigen Raum hat das Brenneke-Flintenlaufgeschoss weite Verbreitung gefunden.

\subsubsection{Innere Befunde}

Die schussbedingten Verletzungsbefunde im Körperinneren können hier nur gestreift werden. Besondere Erwähnung verdient die Form des Schussloches in den platten Knochen des Schädeldaches. Die Schussdefekte haben dort das Aussehen von rundlichen Lochfrakturen. Der einschussseitige Durchmesser stimmt größenordnungsmäßig mit dem Geschosskaliber überein. Die Knochenlücke erweitert sich trichterförmig in Schussrichtung, ist also ausschussseitig "kraterförmig ausgesprengt « (• Abb. 3.63). Aus diesem Befund lässt sich selbst am isolierten, weichteilfreien Knochen noch die Schussrichtung bestimmen. Ausschusslücken verhalten sich umgekehrt: Die Tabula externa ist kraterförmig ausgebrochen. Spitzwinkelig auftreffende Geschosse erzeugen schlüssellochförmige Knochenlücken mit schützenferner Randabsprengung der Tabula externa.

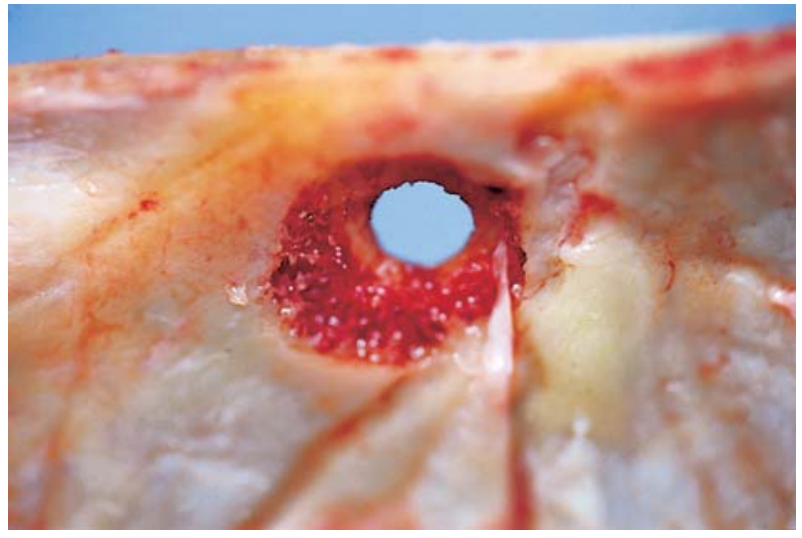

- Abb. 3.63. Knocheneinschuss in der rechten Schläfenregion (Innenansicht). Die Einschusslücke in der Tabula externa ist etwa kalibergroß und rund, die Tabula interna ist trichterförmig in Schussrichtung ausgesprengt. Suizidaler Schläfenschuss mit einer Pistole Kal. 6,35 mm Browning

Bei absoluten Nahschüssen gegen den Gehirnschädel (Stirn-, Schläfen-, Scheitel- und Hinterhauptregion) finden sich Schmauchantragungen auch im Randbereich der Knocheneinschusslücke (ringförmig an der Tabula ext. und an der Unterseite des abgehobenen Periosts, oft sogar an der knochenseitigen Oberfläche der Dura mater).

Häufig gehen von den Ein- und Ausschusslücken radiäre Schussbrüche aus. Nach der Puppe'schen Regel enden sekundär entstandene Bruchlinien an vorbestehenden Frakturen, woraus in manchen Fällen eine Aussage über die Priorität der einen oder anderen Verletzung abgeleitet werden kann.

In den Weichgeweben ist der Wundkanal nicht röhrenförmig, sondern kollabiert und/oder blutgefüllt. Da sich die Gewebsschichten kulissenartig gegeneinander verschieben können, ist eine postmortale Sondierung des Schusskanals mit der Gefahr von Artefaktbildungen verbunden und sollte daher unterbleiben. Parenchymatöse Bauchorgane wie die Leber und die Milz können an den Geschossdurchtrittsstellen große sternförmige Wunden zeigen (• Abb. 3.64).

Chirurgisch oder autoptisch aus dem Körper entfernte Geschosse oder Geschossteile müssen für spätere kriminaltechnische Untersuchungen sichergestellt werden. Ein verfeuertes Tatgeschoss, das nicht allzu stark verformt ist, gibt Auskunft über Kaliber, Drallrichtung, Zahl und Breite der Felder und Züge sowie Individualspuren vom Lauf der Tatwaffe.

\subsubsection{Viehbetäubungsapparate, Bolzensetzwerkzeuge}

Viehbetäubungsgeräte sind Schussapparate, mit deren Hilfe ein zylindrischer Stahlbolzen ca. $10 \mathrm{~cm}$ tief in den Gehirnschädel 


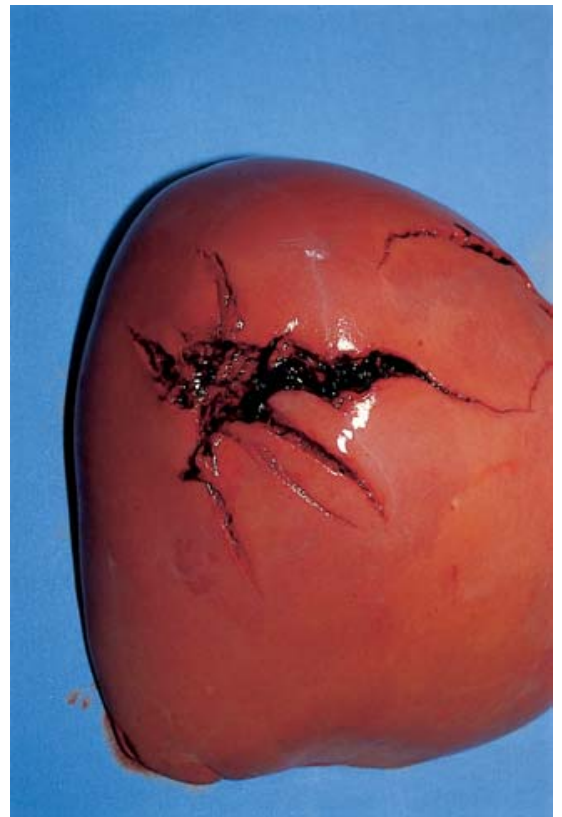

- Abb. 3.64. Sternförmige Geschossdurchtrittsstelle im rechten Leberlappen (homizidale Durchschussverletzung des Rumpfes mit einer Pistole Kal. $9 \mathrm{~mm}$ Luger)

eines Schlachttieres getrieben wird, was in aller Regel zu schlagartig einsetzender Bewusstlosigkeit führt. Die »Schlachtschussapparate " werden mit Kartuschen (projektillosen Patronen) geladen. Nach der Schussabgabe kehrt der Schussbolzen durch Federkraft in seine Ausgangsstellung zurück.

In der rechtsmedizinischen Praxis werden Bolzenschussverletzungen überwiegend nach Suizidhandlungen beobachtet; Fremdtötungen und letal verlaufende Unfälle gehören zu den Raritäten. Die Selbsttötungen sind mehrheitlich »berufsbezogen«:
Sie werden von Personen verübt, die beruflichen Umgang mit Viehbetäubungsapparaten haben (Landwirte, Schlachthausarbeiter, Metzger).

Der Schussbolzen ist an seinem Vorderende scharfrandig und gekehlt; er verursacht daher Stanzverletzungen. Da der Bolzen nach jeder Schussabgabe in das Gerät zurückkehrt, bleibt in der Tiefe des Wundkanals kein Projektil zurück, wohl aber imprimiertes Material (Haut, Knochen). Wegen der begrenzten Vorschnellstrecke des Bolzens sind bei Kopfschüssen keine Ausschüsse zu erwarten. Das Einschussbild ist bei manchen Gerätetypen durch zwei (seltener vier) Schmauchhöfchen charakterisiert, die einander paarig gegenüberliegen und von den in gleicher Weise angeordneten Gasabzugskanälchen herrühren (• Abb. 3.65).

Baugewerbliche Bolzensetzgeräte und Nagelschussapparate dienen zum Einschießen von (meist gewindetragenden) Setzbolzen in Wände, Holz, Beton etc. Anders als bei Viehbetäubungsapparaten verlässt der abgeschossene Setzbolzen das Gerät, so dass unfallmäßig ein Freiflug des Geschosses möglich ist. Aus Sicherheitsgründen kann ein Schuss nur dann abgegeben werden, wenn die Stirnplatte des Gerätes fest an das beschossene Objekt angepresst wird.

\subsubsection{Kriminalistische Aspekte}

Die Klassifikation einer Schussverletzung als suizidal, homizidal oder akzidentell erfordert die synoptische Auswertung der Verletzungs- und Kleiderbefunde, der kriminaltechnischen Untersuchungsergebnisse und der übrigen Umstände des Falles.

Aus rechtsmedizinischer Sicht stellt sich die Frage, ob der Einschuss in einer für Suizide typischen Region lokalisiert ist (Schläfe, Mund, Herzgegend, Stirn, Submentalregion). In fast allen Suizidfällen wird die Laufmündung aufgesetzt oder in die Mundhöhle eingeführt; bei Schüssen in die Brust erfolgt nur selten eine vorherige Entblößung der Haut. Faustfeuerwaffen ver-

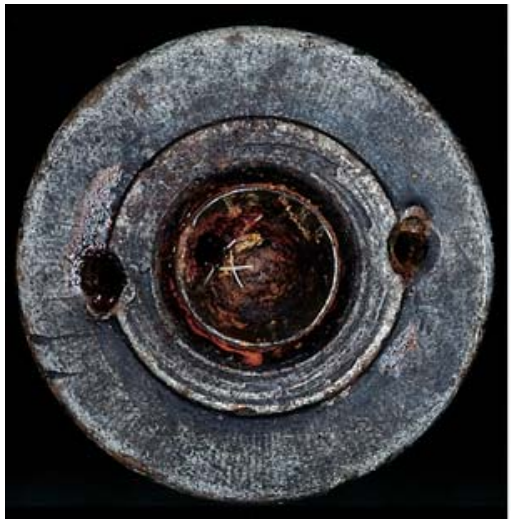

- Abb. 3.65. Scheitelregion eines 63-jährigen Landwirtes, der sich mit einem Viehbetäubungsapparat suizidierte. In Bildmitte die Bolzen-Stanzverletzung, flankiert von zwei einander gegenüberliegenden Schmauch-
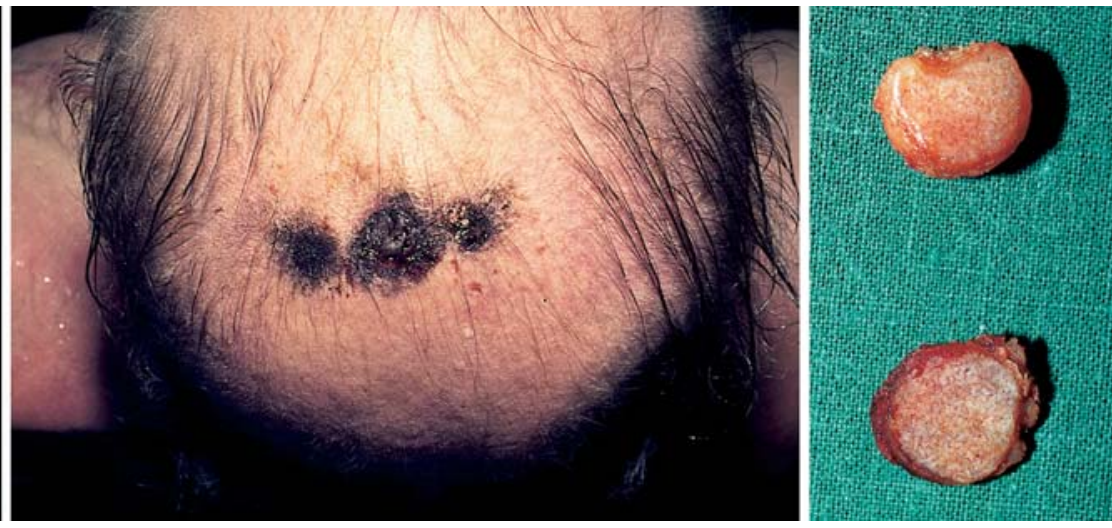

höfchen (lagemäßig korrespondierend mit den Öffnungen der Gasabzugskanälchen an der Kopfplatte des verwendeten Viehbetäubungsapparates; links). Rechts im Bild imprimiertes Haut- und Knochenstück 


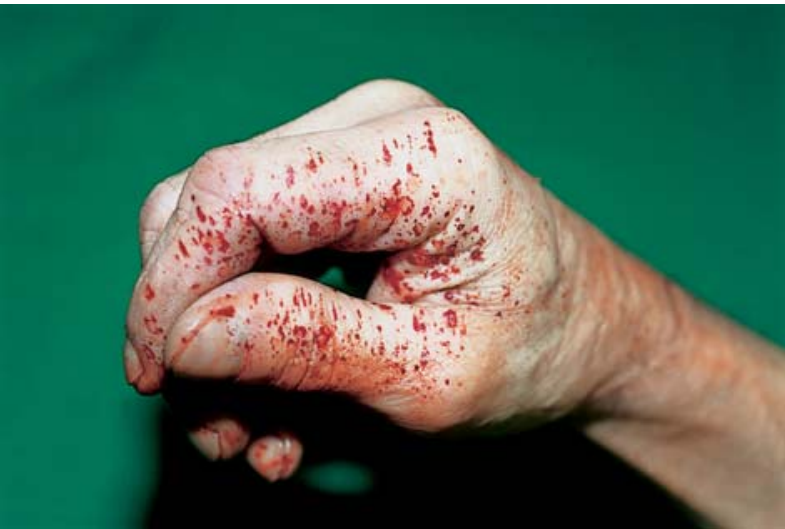

- Abb. 3.66. Rechte Hand eines 59-jährigen Mannes, der sich durch einen Mundschuss (mit einer Pistole Kaliber 7,65 mm Brown.) suizidiert hat. An der Schusshand zahlreiche spritzerartige Blutantragungen (»backspatter«)

bleiben in etwa einem Fünftel der Suizidfälle in der Hand des Schützen.

Auf die Schusshanduntersuchung zum Nachweis von Schmauchbestandteilen (vor allem Blei, Antimon und Barium) kann an dieser Stelle nur hingewiesen werden. Mitunter findet man bereits makroskopisch fassbare Hinweiszeichen für eine eigenhändige Schussabgabe: z.B. spritzerartige Blut- oder Gewebsantragungen an der Schusshand (»backspatter" aus der Einschusswunde, - Abb. 3.66), Beschmauchung am Daumen und Zeigefinger der "Haltehand « (wenn die Laufmündung mit der zweiten Hand umfasst und an die Einschussstelle gehalten wurde, - Abb. 3.67) oder so genannte Schlittenverletzungen zwischen Daumen und Zeigefinger (verursacht durch die Kanten des zurückgleitenden Verschlussstückes einer Pistole). Direkter und konstanter Hautkontakt mit Stahlteilen der Waffe in feuchtem Milieu begünstigt die Bildung von bräunlichen »Rostmarken « (• Abb. 3.68); ein solcher Befund kommt besonders bei Suizidenten vor, beweist aber nicht die eigenhändige Schussabgabe.

\subsubsection{Explosionsverletzungen}

In Friedenszeiten werden Verletzungen und Todesfälle durch Sprengstoffexplosionen meist in Zusammenhang mit politisch motivierten oder terroristischen Anschlägen gegen Personen (Brief- und Paketbomben!), gegen Fahrzeuge und Gebäude beobachtet. Unfallereignisse sind auf unsachgemäßen Umgang mit Sprengmitteln, Feuerwerkskörpern etc. zurückzuführen. Selbsttötungen unter Verwendung von Sprengstoffen sind in Mitteleuropa selten und üblicherweise auf Personen beschränkt, die einschlägige berufliche Erfahrungen haben.

Das Verletzungsbild ist oft durch ein komplexes Nebeneinander von verschiedenartigen Schädigungen charakterisiert:

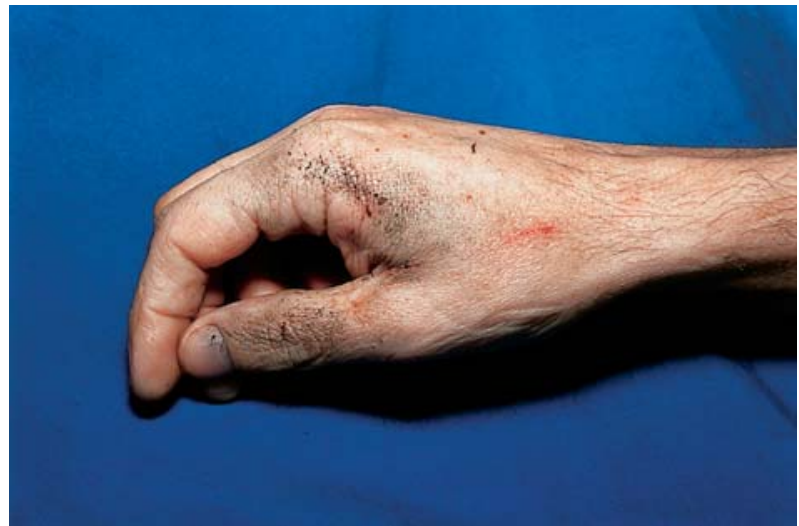

- Abb. 3.67. Rechte Hand mit intensiven Schmauchantragungen am Daumen sowie an der Haut zwischen Daumen und Zeigefinger. Die Hand umfasste zum Zeitpunkt der Schussabgabe die Laufmündung (»Haltehand«). Der Abzug wurde mit dem linken Daumen betätigt. Suizidaler Schuss in die Submentalregion mit einem alten Repetiergewehr

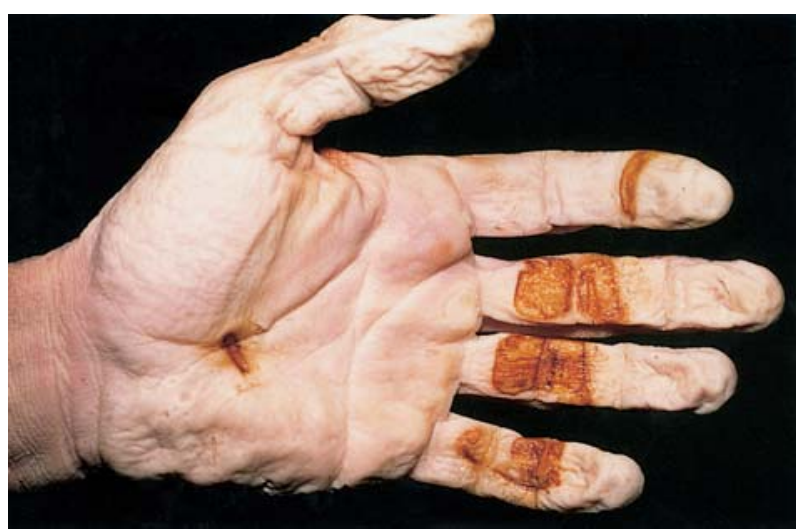

- Abb. 3.68. Linke Hand eines 25 -jährigen Suizidenten (Mundschuss mit einer Pistole Kaliber 9 mm Luger). Der Mann lag im Freien unter einer Schneedecke; in der linken Hand befand sich die Tatwaffe. Ausgedehnte gelbbraune Rostmarken an den Kontaktstellen des Waffenstahls

mechanische Gewebszerstörungen bis zu traumatischen Amputationen, penetrierende Verletzungen durch geschossartig eingedrungene Fremdkörper, Verbrennung und Rußschwärzung der Haut, druckbedingte Überblähung und Rupturen der Lungen.

\subsection{Gewaltsame Erstickung}

\section{H. Maxeiner}

Ersticken im weitesten Sinne ist ein mit jedem Sterben verbundener Vorgang: der Herz-Kreislauf-Stillstand führt über den Ausfall 
der Blut- und damit Sauerstoffversorgung zu einer sekundären »Erstickungs«-Situation der Organe. Im rechtsmedizinischen Sinne spricht man vom Erstickungstod, wenn die Erstickung der primäre todesursächliche Faktor ist. Hierzu gehören alle Situationen, die an irgendeiner Stelle die Bereitstellung oder den Transport des Sauerstoffes zwischen der Umgebung und den Organen unterbrechen, z.B. durch Beeinträchtigung der Atemwege oder der Atmung ganz allgemein, des Sauerstoff-Transportmediums »Blut«, der Blutzirkulation und des Umgebungsmilieus.

\section{- Definition \\ Im Zusammenhang mit dem Erstickungstod finden folgende Begriffe Verwendung: \\ - Hypoxie/Anoxie:Verminderung/Fehlen von Sauerstoff (im Blut, Gewebe, Organ oder im ganzen Körper) \\ - Asphyxie: Lebensbedrohliche Verminderung, Aussetzen bzw. Unterbrechung der Atmung. Kombination von Hypoxie/ Anoxie und Hyperkapnie (= Anstieg der Kohlendioxidbela- dung des Blutes) \\ - Zyanose (»Blausucht«): als bläuliche Verfärbung von Schleimhäuten und Haut sichtbare Verminderung der Sauer- stoffbeladung der roten Blutkörperchen. Erkennbar ab einer Konzentration von $5 \mathrm{~g} / 100 \mathrm{ml}$ reduzierten (nicht sauerstoff- haltigen) Hämoglobins \\ - Ischämie: Blutleere/unterbrochene arterielle Blutversor- gung (eines Organs bzw. eines Gefäßversorgungsgebietes) \\ - Dyspnoe/Apnoe: erschwerte Atmung, Atemnot/Atemstill- stand}

\section{Pathophysiologie des Erstickungstodes}

Die schädigenden Auswirkungen einer solchen Situation - und damit der Ablauf des Erstickungstodes im Allgemeinen - sind eng mit einer von Organsystem zu Organsystem unterschiedlichen Toleranz gegenüber einem Sauerstoffmangel verbunden. Während es Gewebe gibt, die eine vollständige Unterbrechung der Blutversorgung verhältnismäßig lange ohne irreversible Schäden tolerieren können, kommt es an anderen (insbesondere am Herzen und am Zentralnervensystem) bereits nach einer kurzen Unterbrechung der $\mathrm{O}_{2}$-Versorgung (Sekunden) zu zunächst noch reversiblen Funktionsstörungen, die bei anhaltender Unterbrechung innerhalb weniger Minuten in einen irreversiblen Strukturschaden übergehen.

\section{(i) Infobox}

Basierend auf Beobachtungen am Menschen, insbesondere beim Erhängen, sowie nach den Ergebnissen von Tierversuchen dauert der Sterbevorgang bei einer sofort und anhaltend vollständigen Erstickungssituation etwa 3-5 Minuten. $\nabla$
Folgende Phasen werden durchlaufen, die jeweils etwa 1-2 Minuten dauern:

- Phase der Atemnot: verstärkte Atemtätigkeit, inspiratorische Dyspnoe, Zyanose, Bewusstseinsverlust;

- Erstickungskrämpfe (Folge des zerebralen $\mathrm{O}_{2}$-Mangels): Pulsschlag meist beschleunigt, Blutdruck erhöht; Urin- und Kotabgang kommen vor.

- Präterminale Atempause: Atemstillstand, Blutdruckabfall, Tachykardie;

- Phase der terminalen Atembewegungen: schnappende Atembewegungen, gefolgt vom endgültigen Atemstillstand. Kreislauf kann den Atemstillstand minutenlang überdauern; elektrische Herzaktivität ist mitunter bis zu 20 Minuten nachweisbar.

Von der Symptomatik her zu unterscheiden sind Abläufe, bei denen es schnell zum $\mathrm{O}_{2}$-Mangel des Gehirnes und damit zum Bewusstseinsverlust in sehr kurzer Zeit kommt (z.B. Erhängen), von solchen, bei denen der $\mathrm{O}_{2}$-Mangel sich auch am Gehirn erst allmählich entwickelt und das Bewusstsein länger erhalten bleibt (obstruktive Asphyxie, z.B. gewaltsamer Verschluss der Atemöffnungen, Erwürgen). Solange es neben dem $\mathrm{O}_{2}$-Mangel nicht zu einem deutlichen Anstieg von Kohlendioxid im Blut kommt (bei ungehinderter Atmung; Beispiel: allmähliches Ersticken unter einer über den Kopf gezogenen Plastiktüte), kann es ohne schwerere, quälend erlebte und äußerlich sichtbare Erstickungserscheinungen zum Eintreten der Bewusstlosigkeit kommen. Das Atemzentrum wird vor allem durch einen erhöhten $\mathrm{CO}_{2}$-Gehalt des Blutes stimuliert. Modifikationen der experimentell beobachteten Agonie sind in vielerlei Hinsicht möglich. So wird jede Unterbrechung der äußeren Einwirkung zu einer gewissen Regeneration und damit zu einer zeitlichen Verlängerung führen. Andererseits dürfte die Intensität und Dauer der Agonie auch von der individuellen Konstitution bzw. "Abwehrkraft« des Opfers abhängen, obwohl es hierzu natürlich keine Vergleichsstudien gibt. Außerdem gibt es in der Art der Einwirkung begründete Möglichkeiten einer Abkürzung der Strangulationsagonie. Hier ist vor allem an reflektorische Mechanismen zu denken, wie sie beim Ertrinken oder bei Strangulation vorkommen können.

\section{Allgemeine Autopsiebefunde beim Erstickungstod}

Regelhaft werden die folgenden Veränderungen genannt, die zwar bei Erstickungsfällen mehr oder weniger ausgeprägt sind, jedoch auch bei anderen Todesursachen vorkommen - also unspezifisch sind. Noch nicht einmal ihr Fehlen kann ohne weiteres als Beleg gegen einen Erstickungstod verwendet werden:

Zyanose. Zyanose ist beim Lebenden ein deutlich sichtbares Zeichen für eine mangelhafte $\mathrm{O}_{2}$-Beladung des Blutes. Die Verwendung dieses Begriffes an der Leiche ist aber problematisch, da die $\mathrm{O}_{2}$-Beladung des Blutes nach dem Tode abfällt, und eine Differenzierung zwischen der »üblichen« Leichenveränderung und 
einem ggf. vorbestehenden $\mathrm{O}_{2}$-Mangel rasch nach dem Tode unmöglich wird.

Blutstauung. Blutstauung ist ein klassischerweise mit dem Erstickungstod in Verbindung gebrachter Obduktionsbefund. Hierzu gehört zum einen eine akute allgemeine Blutüberfüllung der Organe des großen und kleinen Kreislaufes, oft verbunden mit einer weiten (erweiterten) rechten Herzhälfte, zum anderen eine auf den Kopf- und Halsbereich beschränkte, äußerlich sichtbare "obere Einflussstauung". Eine solche kann auf eine Halskompression deuten, jedoch auch Folge verschiedener (krankhafter) Rückflussbehinderungen des Blutes in die rechte Herzhälfte sein. Man hat sich hier auch immer zu fragen, inwieweit man es überhaupt mit einem zu Lebzeiten entstandenen Phänomen zu tun hat; Auswirkungen der Hypostase sind zu beachten, möglicherweise auch Blutverschiebungen infolge der Wirkung der Totenstarre am Herzen. Seit jeher wird darauf verwiesen, dass in den Gefäßen einer Leiche eines Erstickten flüssiges Blut vorgefunden wird, was erfahrungsmäßig meist zutrifft (eine Ausnahme: starke Alkoholisierung).

Lungenveränderungen. Da ein Erstickungsvorgang in besonderer Weise die Atmungsorgane betrifft, liegen zahlreiche Berichte über Lungenveränderungen vor. Je nach den Gegebenheiten kann es zu Störungen des Luftgehaltes (diffuse oder mit Minderbelüftungen wechselnde Überblähung = akutes Lungenemphysem), zu einem Flüssigkeitsübertritt in den Alveolarraum (alveoläres Lungenödem), insbesondere mit Blutbeimengung (hämorrhagisches Ödem), sowie zu einer Leukozytose und/oder einer Ausschwemmung von Knochenmarkszellen bis hin zu einem Mikroemboliesyndrom kommen. In Einzelfällen können solche Befunde hilfreich sein, soweit nicht die Situation durch Fäulnis oder künstliche Beatmung verändert bzw. überlagert ist.

Petechiale (punktförmige) Blutungen. Sie kommen als Stauungsblutungen im Gesicht und als Blutungen unmittelbar unter der Oberfläche von Organen (subseröse Extravasate) vor - auffälligerweise nur an den Brustorganen, d.h. unter dem Lungenoder Herzfell oder an der inneren Brustdrüse. Man hat diese Petechien daher mit der erschwerten Atemtätigkeit unter einer Erstickungssituation in Verbindung gebracht und lange von (Tardieu'schen) Erstickungsblutungen gesprochen, jedoch hat dies allenfalls historische Bedeutung. In letzter Zeit wurden das Auftreten und die Verteilung von Petechien vor allem im Thymus bei plötzlichen Kindstodesfällen als »nützliches Kriterium« für diese Diagnose herausgearbeitet. Petechien in der Haut und den Schleimhäuten des Kopfes gehen auf eine so starke obere Einflussstauung zurück, dass es zum Platzen kleiner Blutgefäße kommt; es handelt sich um Stauungsblutungen; bei Erstickungstodesfällen ohne Auftreten einer Blutstauung des Kopfes fehlen sie meist. Andererseits kann man sie aber als Folge ganz verschiedener Einwirkungen bzw. Erkrankungen bei Lebenden und Toten beobachten (-Tabelle 3.18). Eine besonders schwierige Situation ergibt sich, wenn ernsthaft die Möglichkeit einer allein postmortalen Entstehung in Betracht zu ziehen ist, wie dies z.B. bei Bauchlage recht oft vorkommt.

Angesicht dieser wenig befriedigenden Situation wird seit langem versucht, andere diagnostische Kriterien zu etablieren, z.B. mittels histologischer Untersuchung innerer Organe, an denen als Folge eines $\mathrm{O}_{2}$-Mangels z.B. Bläschenbildungen (Vakuolen) oder feinsttropfige Verfettungen vorkommen kön-

- Tabelle 3.18. Häufigkeit petechialer Lid- bzw. Bindehautblutungen bei verschiedenen Ursachen im eigenen Obduktionsgut sowie aus der Literatur (hier auch Beobachtungen an Lebenden enthalten)

\begin{tabular}{|c|c|c|}
\hline & $\begin{array}{l}\text { Eigenes Material } \\
\text { Fallzahl (Petechien in \%) }\end{array}$ & $\begin{array}{l}\text { Daten aus der Literatur } \\
\text { Fallzahlen (Petechien von-bis \%) }\end{array}$ \\
\hline $\begin{array}{l}\text { Eigenes Material, alle Todesursachen außer } \\
\text { Strangulation } \\
\text { Herztod } \\
\text { Erkrankungen des ZNS } \\
\text { Infektionen } \\
\text { SIDS } \\
\text { Lungenembolie } \\
\text { Lebende Neugeborene } \\
\text { Stumpfe Gewalt } \\
\text { Thoraxkompression } \\
\text { Ertrinken } \\
\text { Intoxikation } \\
\text { CO-Intoxikation } \\
\text { Feuer, Hitze, Rauchgas } \\
\text { Schussverletzungen } \\
\text { Scharfe Gewalt } \\
\text { Sonstige }\end{array}$ & $\begin{array}{l}450(14 \%) \\
84(19 \%) \\
25(12 \%) \\
64(17 \%) \\
10(0 \%) \\
14(0 \%) \\
75(11 \%) \\
19(16 \%) \\
48(13 \%) \\
13(31 \%) \\
20(0 \%) \\
13(0 \%) \\
65(17 \%)\end{array}$ & $\begin{array}{l}200-518(4,3-12 \%) \\
79(7,6 \%) \\
28(3,5 \%) \\
45-250(0-2,4 \%) \\
45(0 \%) \\
78(39,7 \%) \\
13-14(25-100 \%) \\
79-171(3,8-13 \%) \\
436(2,3 \%)\end{array}$ \\
\hline
\end{tabular}


nen. $\mathrm{Zu}$ deren Entwicklung bedarf es aber einer gewissen Dauer des $\mathrm{O}_{2}$-Mangels, sodass sie bei akuten Todesfällen meist noch nicht gefunden werden können. Insgesamt ist die Diagnose des Erstickungstodes ein schwieriges Unterfangen, das mitunter nur per exclusionem gelingt. Seit langem gilt auch in der gerichtlichen Medizin der Leitsatz, dass in solchen Fällen - wenn schon die tatsächlichen Auswirkungen nicht zuverlässig diagnostizierbar sind - das »erstickende Agens « sichtbar oder wenigstens hinlänglich plausibel gemacht werden muss, wozu es oft entscheidend auf die Umstände bzw. das Ermittlungsergebnis ankommt.

\section{Forensisch wichtige Erstickungsformen}

Verlegung der Atemöffnungen. Bei Tathandlungen mit unmittelbarem Kontakt zwischen Opfer und Täter kommt es nicht selten zu einem Angriff gegen die Atemöffnungen (Zuhalten des Mundes oder von Mund und Nase), um das Opfer am Schreien zu hindern. Folgen können am Mittelgesicht kleinfleckige Abschürfungen oder Fingernagelkratzer sein, weiterhin Blutunterlaufungen, insbesondere an der Innenseite der Lippen ( $\bullet$ Abb. 3.69). Todesfälle durch isolierten Verschluss der äußeren Atemöffnungen sind bei Erwachsenen sehr selten, was an der lange erhaltenen Handlungsfähigkeit und intensiven Gegenwehr liegen dürfte - soweit der Betroffene hierzu fähig ist. Wir beobachteten allerdings massives Erbrechen unter einem solchen Fremdan-

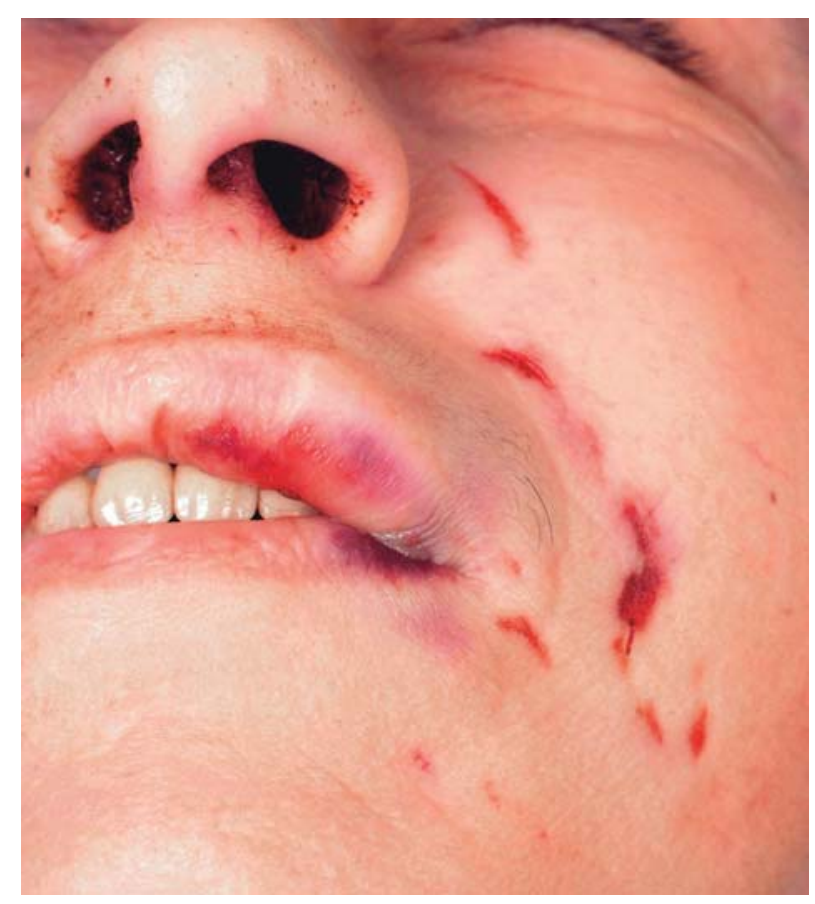

- Abb. 3.69. Schmale, halbmondförmige Kratzer (= Fingernageleindrücke) und kleinfleckige Abschürfungen um Mund und Nase sowie eine angeschwollene Lippenquetschung als Folge eines »Angriffes gegen die Atemöffnungen« griff, das infolge des anhaltenden Druckes auf das Gesicht zur tödlichen Aspiration führte. Bei schwer kranken, abwehrgeschwächten oder bewusstlosen Patienten dürfte ein »reines" Ersticken auch ohne das Hinterlassen relevanter Verletzungen möglich sein. Die Gefahr eines Übersehens im Rahmen der klinischen Leichenschau ist daher gegeben, und angesichts bekannter Erkrankungen mag die Attestierung eines natürlichen Todes nahe liegen; man wird daher mit einem gewissen Dunkelfeld zu rechnen haben. Bei Säuglingen ist ein Tod durch Verschluss der Atemöffnungen ohne weiteres möglich und muss keine hierauf hinweisenden Verletzungen hinterlassen, da der erforderliche Kraftaufwand gering ist. Tötungen durch Aufdrücken der Hand oder Bedeckung mit dichten Materialien sind lange bekannt. Nach Auffassung einiger Autoren kann auch allein das Gewicht des Kopfes bei auf einer ungünstigen (z.B. durchfeuchteten) Unterlage aufliegendem Gesicht ausreichen (weiteres hierzu - Kap.4.16). Die Auffassungen in der Literatur zur Häufigkeit von Erstickungstodesfällen bei Säuglingen differieren sehr, was nicht verwundert, da man einen solchen Vorgang im konkreten Fall oft weder beweisen noch widerlegen kann.

Wenn sich eine Person (absichtlich oder bei Kindern versehentlich) eine Plastiktüte über den Kopf zieht, sodass ein ausreichender Gasaustausch zwischen dem in dem Beutel befindlichen Gasvolumen und der Umgebungsluft nicht möglich ist, kann ein tödlicher Sauerstoffmangel resultieren; eine feste Abdichtung am Hals ist dazu nicht unbedingt notwendig. Aufgrund der anfangs noch möglichen $\mathrm{CO}_{2}$-Abatmung kommt es nicht zu einer dramatischen Erstickungssymptomatik; entsprechend gering bis fehlend sind die Befunde. Solche Fälle wurden vor Jahren immer wieder einmal als Unfallereignisse im Zusammenhang mit einer Inhalation von Lösungsmitteln (zum Ziele einer Berauschung) beobachtet, sind zumindest bei uns inzwischen aber sehr selten geworden. Suizide durch Ersticken unter einer Plastiktüte kommen gelegentlich zur Beobachtung. Der Nachweis einer solchen Todesursache ist extrem schwierig bis unmöglich, wenn die ursprüngliche Situation durch Wegnahme des Beutels verändert wurde.

D Wichtig

Finden sich im Rahmen einer Leichenschau auch nur diskrete kleine Vertrocknungen oder gar Abschürfungen im Mittelgesicht (perioral, perinasal), deren »harmlose« Verursachung nicht primär klar ist, so sollte an einen Angriff von fremder Hand gegen die Atemöffnungen gedacht werden; entsprechendes gilt für alle sichtbaren Verletzungsspuren am Hals, die Folge einer stattgefundenen Strangulation sein könnten.

Knebelung. Bei uns fanden sich Knebelungen bei 2,9 \% aller Tötungen; bei Strangulation lag die Rate bei immerhin $10 \%$.

Definition
Unter Knebelung versteht man das Einbringen von Fremd-
materialien (am häufigsten: Textilien) in den Mund. Dies dient
meist dem Zweck, ein Opfer am Schreien zu hindern.


Da die Opfer meist auch noch anderweitig misshandelt werden, kann eine Knebelung eigenständig zum Erstickungstod führen, insbesondere bei Bewusstlosigkeit. Ein Volumen eines Knebels von etwa $100 \mathrm{ml}$ kann noch (ohne aktiven Druck auf den Knebel) tolerabel sein; bei 150-200 ml wird es auch im Rachenraum sehr eng (Maxeiner 1996). Ein innerer Atemwegsverschluss kann dann durch den Knebel selbst oder die durch ihn verdrängte Zunge erfolgen. Eine zusätzliche Behinderung der Nasenatmung (durch Aufliegen, durch Umschnürung oder Klebeband) macht eine Knebelung rasch unmittelbar lebensbedrohlich. Nicht selten wurden nach Tathandlungen Knebelungen wieder entfernt. Kommt eine solche den Umständen oder Schleimhautverletzungen im Mund nach in Betracht, empfiehlt es sich, Ausstriche aus der Mundhöhle auf Fasern zu untersuchen. In der Literatur finden sich zahlreiche Fallbeschreibungen selbst beigebrachter Knebelungen, meist im Zusammenhang mit Suiziden oder autoerotischen Praktiken. Selbstknebelungen bei simulierten Verbrechen (etwa um eine Mitwirkung des angeblichen Opfers zu verschleiern) wurden ebenfalls beschrieben.

Bolustod. Gelegentlich kommt es vor, dass in den Mund aufgenommene, unzerkaute Nahrungsbrocken (beobachtet wurden allerdings auch schon die absonderlichsten anderen Materialien wiez.B. eine Maus) aufgrund ihrer Größe nichtheruntergeschluckt - und auch nicht wieder herausgewürgt - werden können und im Schlundbereich stecken bleiben. Der Großteil solcher Fälle ereignet sich bei hastiger Nahrungsaufnahme, also unfallmäßig; sehr selten wird man auch ein absichtliches Einstopfen eines Bolus zu diskutieren haben (wie - Abbildung 3.70 in einem Suizidfall zeigt). Auch Todesfälle infolge eines Verrutschens schlecht fixier- ter Zahnprothesen im Schlaf, mit Einklemmung der Prothese im Schlundbereich wurden als Unfallereignisse beobachtet; wir sahen auch bei Tötungsdelikten Prothesen infolge eines äußeren Druckes gegen den Mund tief in den Schlund eingepresst. Beim "typischen « Bolustod sind die Betroffenen entweder (meist hochgradig) alkoholisiert oder weisen eine sonstige zentralnervöse Störung auf. Anwesende Zeugen berichten z.T., die Betroffenen seien reaktionslos und ohne Erstickungssymptomatik kollabiert und alsbald verstorben. Dies führte schon vor langer Zeit zu der Annahme, es würde sich nicht um einen Erstickungs-, sondern um einen Reflextod handeln: Infolge der akuten Überdehnung des mit vegetativen Nervenfasern reich versorgten Schlund- und Kehlkopfbereiches komme es zur fehlerhaften Stimulierung des parasympathischen Nervensystems (Vagus), was infolge der Verbindungen dieses Systems mit der Steuerung der Herztätigkeit einen unmittelbaren Herzstillstand auslösen könne. Der »Vagustodtheorie « wurde aber verschiedentlich widersprochen (Mallach und Oehmichen 1982, Bratzke et al. 1990), da auch Fälle mit einer Erstickungssymptomatik beobachtet werden.

\section{F) Fallbeispiel}

Ein 59-jähriger, stark alkoholisierter Mann (BAK 2,8 \%o) würzt sich ein ca. $20 \mathrm{~cm}$ langes Stück Leber und fängt an zu essen. Sein Gegenüber bemerkt ein Blauwerden der Lippen; der Mann versucht zu husten und würgt, kann aber nur wenig Schleim von sich geben und nicht sprechen. Das ganze Gesicht wird erst blau, dann blass, worauf der Mann vom Stuhl fällt und sich nicht mehr regt. Der alarmierte Notarzt kann ca. 30 Minuten später den (praktisch $\nabla$
- Abb. 3.70a, b. Bolustod infolge suizidalen Hineinstopfens eines Knäuels aus Toilettenpapier (Volumen ca. $70 \mathrm{ml}$ ) in den Schlund; typische Lage des Bolus mit verlegtem Kehlkopfeingang. a Einblick in die Tiefe der Mundhöhle und den Rachen während der Obduktion; $\mathbf{b}$ die entnommenen Halsorgane. 82-jährige psychotische Krankenhauspatientin, wurde kurz nach einen Toilettengang tot vorgefunden. Es fanden sich minimale Petechien der Konjunktiven
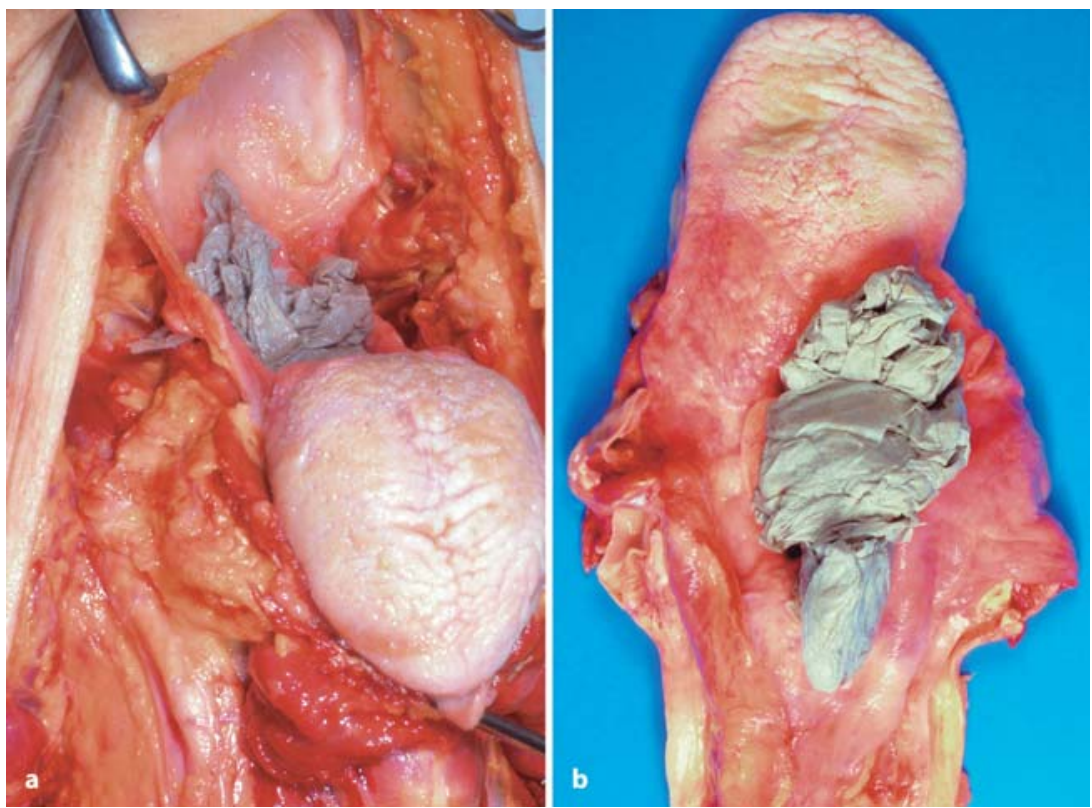
unzerkauten) Bolus aus dem Mund entfernen, den Mann aber nicht wieder beleben. Autoptisch findet sich lediglich ein hochgradiger akuter Blutstau im Lungen- und Körperkreislauf.

In manchen Fällen kommt es neben einer Zyanose auch zu Petechien im Gesicht oder einem beträchtlichen Lungenödem. Befunde, die bei einem reflektorischen Herzstillstand kaum zu erwarten wären. Diskutiert wird auch das Hinzutreten einer Verkrampfung der Kehlkopf- oder Bronchialmuskulatur (Laryngospasmus, Bronchospasmus) - insgesamt also eine tödliche Atemstörung. Soweit es auf eine solche Differenzierung in einem konkreten Fall praktisch ankommt (was gutachtlich die Ausnahme ist), wird man die Umstände und die Obduktionsbefunde jeweils kritisch bewerten müssen.

Verlegung der Atemwege, Aspiration. Die Atemwege können durch äußerlichen Druck auf den Hals komprimiert werden (s. Strangulation). Eine innerliche Verlegung der Atemwege ist krankheitsbedingt möglich. So kommen gelegentlich lebensbedrohliche Schleimhautschwellungen am Kehlkopf im Rahmen von Atemwegsinfektionen (vorwiegend bei Kindern) vor; weitere Beispiele sind allergische Zustände mit krampfhaftem Verschluss (Spasmus) der Kehlkopflichtung oder Schleimhautschwellung, Anomalien an den Atemwegen (z.B. Zysten) oder auch mit innerer Raumforderung verbundene Erkrankungen wie bösartige Tumoren. Hiervon zu trennen ist die Verlegung anatomisch intakter Atemwege durch eingeatmetes oder sonst dorthin gelangtes Fremdmaterial. Von Aspiration spricht man, wenn solches durch aktive Atemtätigkeit in die Atemwege gelangte. Leider ist dies jedoch auch artifiziell möglich, etwa infolge von passiven Bewegungen des Brustkorbes. Das Vorhandensein von Mageninhalt in den Atemwegen nach Wiederbelebungsversuchen (solche sind in unserem Obduktionsgut bei etwa 1/4-1/3 aller Todesfälle erfolgt) ist daher schwierig zu beurteilen. Eine Häufigkeit von $21 \%$ gegenüber $9 \%$ bei Fällen ohne Reanimation (eigene Daten an je 150 Herztodesfällen) verdeutlicht die Problematik. Auch agonale und sogar postmortale Einflüsse können zum Eindringen von Mageninhalt in die Atemwege führen. Bei vielen Todesfällen kommt es in der Phase des Sterbens zum Erlöschen von Schutzreflexen und Erbrechen oder auch nur zum retrograden Auslaufen von Mageninhalt, mit nachfolgender Einatmung. Schon wenige (letzte) Atemzüge können für eine Aspiration ausreichen, die sich aber möglicherweise gar nicht mehr auf den bereits in seiner Endphase befindlichen Sterbevorgang auswirkte (agonale Aspiration). Hierbei würde es sich allerdings immer noch um einen an Lebensäußerungen gebunden Vorgang handeln. Im Leichenexperiment (s. bei Knight 1996) konnte aber gezeigt werden, dass allein Umlagerung oder Transport von Leichen zum Auslaufen von Mageninhalt und Eindringen bis in die Atemwege führen kann; Bestandteile des Mageninhaltes in den Atemwegen finden sich daher bei vielen Obduktionen. Berechtigterweise wird vor einer unkritischen Heranziehung eines solchen Befundes als Todesursache dringend gewarnt. Je mehr Fremdmaterial je peripherer in den Atemwegen ist, umso eher dürfte noch Atemtätigkeit vorgelegen haben und eine Mitwirkung am Todesgeschehen in Betracht kommen. Neben Mageninhalt und - deutlich seltener - noch nicht im Magen gewesener Nahrung wird häufig Blut aspiriert. Bei primärer Blutung in die Atemwege (etwa aus einem Tumor, einer Kaverne) oder bei gleichzeitiger Verletzung von Atemwegen und Blutgefäßen am Hals (Schuss-, Stich-/Schnittverletzungen) kann eine tödliche Blutaspiration resultieren, die sich auch durch an der Lungenober- und Schnittfläche scharf begrenzte rote Bluteinatmungsherde darstellt. Die Vitalität einer stärkeren Blutaspiration ist meist offensichtlich.

Beim wachen und gesunden Menschen dürfte ein Aspirationstod ohne eine schwere Erstickungssymptomatik kaum möglich sein. Allerdings beobachteten wir mehrfach förmliche Austamponierungen von Luftröhre und Bronchien durch Speisebestandteile bei alten, pflegebedürftigen und hirngeschädigten Patienten. Eine bedrohliche Erstickungssymptomatik war den die Nahrungsaufnahme z.T. unterstützenden bzw. die Patienten (offenbar manchmal nicht ganz behutsam) fütternden Pflegepersonen nicht aufgefallen. Eine Bewusstseinsstörung (z.B. Kopftrauma, Intoxikation) ist auch sonst eine Voraussetzung für das Zustandekommen einer gravierenden Aspiration. Seltene Ausnahmen betreffen Unfälle, bei denen Personen vollständig in Massen einer Substanz geraten, deren Teilchen aufgrund ihrer Größe eingeatmet werden können (z.B. Verschüttung in einem Getreidesilo).

Thoraxkompression, traumatische Asphyxie, Druckstauung. Unter einer Thoraxkompression versteht man eine Situation, bei der der Rumpf von außen so komprimiert wird, dass ausreichende Atembewegungen nicht mehr möglich sind. Dies kommt bei Verschüttungen (Arbeitsunfälle: Baugruben) oder Einklemmungen vor. Beispiele für Einklemmungen sind vielfältig, z.B. unter einem Fahrzeugrad, Insassen bei Fahrzeugdeformationen, Eisenbahnkatastrophen, Erdbeben; Todesfälle wurden beim Ausbrechen einer Panik bei großen Menschenansammlungen beobachtet. Ein Sitzen oder Knien auf einem überwältigten Opfer einer Gewalttat kann über eine Behinderung der Atemexkursionen zum Erstickungstod führen (»Burking»); allmähliches »Erdrücken« durch eine Riesenschlange wurde beschrieben (DiMaio 1989).

\section{(8) Fallbeispiel}

Ein 52-jähriger Mann kniet in einer $2 \mathrm{~m}$ breiten und 2,2 $\mathrm{m}$ tiefen Baugrube und arbeitet am Anschluss einer Rohrleitung, ein zweiter steht daneben. Plötzlich bricht die unsachgemäße Verschalung durch und der Aushub stürzt in die Grube. Der Kniende habe einmal aufgeschrien, dann »sei Ruhe« gewesen, der zweite Mann wurde bis in Brusthöhe verschüttet. Er konnte rasch befreit werden und blieb praktisch unverletzt. Die Bergung der Leiche des gänzlich Verschütteten gelang erst etwa 1/2 Stunde später. Die Obduktion ergab - von Reanimationsfolgen und Abschürfungen abgesehen - keine Verletzungen, intensive petechiale Blutaus$\nabla$ 
tritte an Gesicht und Oberkörper sowie einen starken akuten Blutstau der Organe. Nur wenige Sandkörnchen in den Atemwegen, keine Überblähung oder stärkere Ödembildung der Lungen. Der Tod war unmittelbare Folge der Behinderung der Atmung infolge des äußeren Druckes.

Neben einer »isolierten « Thoraxkompression gibt es Situationen, bei denen es unmittelbar vor einer Einklemmung durch stumpfe Gewalt (deshalb der Begriff traumatische Asphyxie) zu einem Bemerken der drohenden Gefahr durch das Opfer mit starker Einatmung (und u.U. reflektorischem Verschluss der Stimmritze) kommt. Infolge dieser dem Aufprall vorangehenden Druckerhöhung im Brustkorb kommt es dann zu einer massiven Blutverschiebung in Richtung auf Kopf und Hals. Die Opfer können schwere stumpfe Verletzungen erleiden, jedoch auch weitgehend unverletzt bleiben. Hält die Einklemmung an, entsteht eine Druckstauung, und der Tod erfolgt durch die starke Beeinträchtigung der Kreislaufsituation (Lungenstauung) oder durch eigentliches Ersticken. Bei der Obduktion imponiert eine massive, auch die Haut des Oberkörpers umfassende obere Einflussstauung; mikroskopisch findet sich ein starkes Mikroemboliesyndrom der Lungen (Brinkmann 1978).

\subsubsection{Strangulation}

Neben dem Ertrinkungstod ist Strangulation (»Halskompression«, »Kompression der Halsweichteile«, »Angriff gegen den Hals«) die häufigste Art des gewaltsamen Erstickungstodes. 3 Hauptformen werden unterschieden (• Abb. 3.71):

Erhängen. Beim Erhängen liegt ein Strangwerkzeug (Seil, Schnur, Schal, Stoffstreifen o.Ä.) um den Hals. Bei nur einer Umschlingung mit nicht eng zugezogener Schlinge liegt es der Haut meist nicht vollständig zirkulär fest an, sondern weicht von dieser am höchsten Schlingen- bzw. Knotenpunkt etwas ab, bei mehreren Umschlingungen liegt der Strang der Halshaut teilweise auch vollständig zirkulär an. Auch ohne vollständig um den Hals reichende Tour ist bei ausreichendem Druck nur gegen den Vorderhals Erhängen möglich (z.B. in quer ausgespannter Wäscheleine). Mindestens ein Ende oder Teil des Strangwerkzeuges ist oberhalb des Niveaus des Halses befestigt (z.B. an einem Baum, Wandhaken, Fenstergriff). Der Druck auf den Hals wird durch das Eigengewicht des (eines Teils des) Körpers bewirkt.

Erdrosseln. Beim Erdrosseln wird ebenfalls ein Gegenstand als Strangwerkzeug um den Hals geschlungen, ohne außerhalb des Körpers fixiert zu sein. Der Druck auf den Hals wird durch Zug an den Enden des Stranges - und somit Zuziehen der Schlinge - bewirkt; durch Verknoten kann der Druck aufrecht erhalten bleiben.

Erwürgen. Unter Erwürgen versteht man die Kompression der vorderen Halsweichteile mittels einer oder beider Hände, die gegen den Hals gedrückt werden. Bei Drossel- und mehr noch bei Würgeangriffen kommt es zwangsläufig zu einem intensiven körperlichen Kontakt zwischen Opfer und Täter. Bei Tätern, die früh

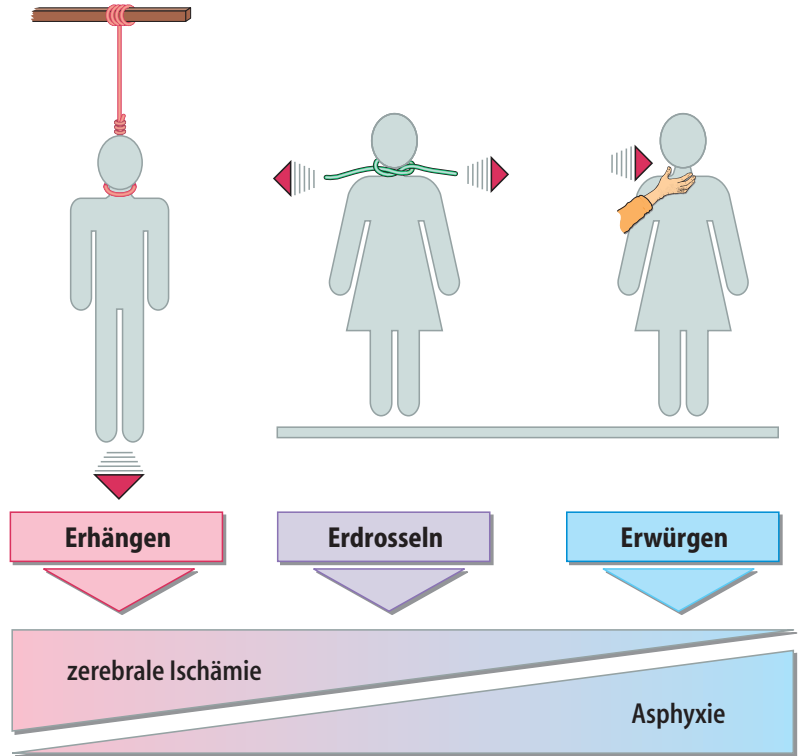

venöse Stauung

- Abb. 3.71. Schematische Darstellung der 3 Hauptformen des Strangulationstodes sowie der beteiligten pathophysiologischen Letalfaktoren (modifiziert nach einer Abbildung von Püschel 1982)

nach dem Geschehen rechtsmedizinisch untersucht werden, sind oft körperliche Spuren nachweisbar, insbesondere Kratzer und/ oder Abschürfungen an den Handrücken, Unterarmen, im Gesicht und am oberen Rücken (Härm und Rajs 1981). Soweit es die Situation ermöglicht, sollte also nicht nur eine Besichtigung des Opfers, ggf. des Tatortes, sondern auch eine körperliche Untersuchung eines Tatverdächtigen erfolgen.

\section{Pathophysiologie des Strangulationstodes}

Bei einer Kompression des Vorderhalses kommt es abhängig von der Intensität, Höhe und Form (breit komprimierend, schmal einschnürend, punktuell) der Einwirkung zu einer Einengung bis hin zum Verschluss lebenswichtiger Versorgungsleitungen. Bereits bei einem geringen, nicht nur punktuellen Druck auf den Hals (ca. $2 \mathrm{~kg}$ ), kommt es zur Behinderung des venösen Blutabstromes aus dem Kopfbereich. Ist die arterielle Blutzufuhr wenigstens teilweise noch erhalten, resultieren eine Blutfülle des Gesichtes bis hin zur Anschwellung (Dunsung), meist verbunden mit einer Zyanose, die von Tatbeteiligten mitunter berichtet wurde, sowie Petechien im Kopfbereich. Auch zur Abklemmung der Halsarterien bedarf es keiner großen Kraft - wenige kg ( $\mathrm{ab}$ ca. 3-5 kg) genügen bei "passender« Lokalisation der Druckwirkung. Für den Verschluss der Wirbelarterien sind höhere Drücke 
und vor allem eine Lage des Strangulationswerkzeuges hoch im Nacken nötig. Eine Verlegung der Atemwege ist durch Verlagerung der oberen Halsweichteile und des Mundbodens nach kopfwärts möglich, wodurch die verdrängte Zunge den Rachen verlegen kann, ferner durch Hochschieben des Kehlkopfes mit der Folge eines Kehldeckelverschlusses durch den Zungengrund, aber auch durch Zusammendrücken der Luftröhre oder des Kehlkopfes. Eine Krafteinwirkung von etwa 8-12 kg genügte bei (postmortalen) Würgeversuchen zur Unterbrechung der Atemwege. Begreiflicherweise spielt die Zeit einer Halskompression eine herausragende Rolle, da bei einem nur kurzdauernden Druck auf den Hals keiner dieser Faktoren in entscheidender Weise wirksam werden kann. Eine isolierte Unterbrechung der Atemwege kann vergleichsweise lange ohne Bewusstseinsverlust überstanden werden, selbst wenn es bereits zu einer starken Atemnotsymptomatik gekommen ist. Eine Unterbrechung der arteriellen Hirnversorgung hat demgegenüber bereits nach wenigen Sekunden den Verlust des Bewusstseins zur Folge: Bei über 100 Versuchspersonen wurde eine Druckmanschette um den Hals rasch stark aufgepumpt; alle verloren das Bewusstsein innerhalb von 6 bis 10 Sekunden (Rossen und Kabat 1943). Dieser Zustand ist aber verhältnismäßig lange reversibel: alle z.T. bis über eine Dauer von 100 Sekunden laufenden Experimente blieben ohne gesundheitliche Schäden. In einem konkreten Fall wird man natürlich auf keine vergleichbaren objektiven Beobachtungen zurückgreifen können, sodass nur indirekt auf solche $\mathrm{Zu}$ sammenhänge geschlossen werden kann. Der einzige Befund, der überhaupt retrospektiv Rückschlüsse auf zeitliche Bezüge des Strangulationsgeschehens zulassen kann, sind Stauungsblutaustritte (• Abb. 3.73). Konkrete Daten zur ihrer Entstehungszeit gibt es aber kaum, sodass die erforderliche Zeitspanne einer Halskompression bis zum Auftreten von Petechien in der Literatur auch nicht einheitlich angegeben wird: von etwa 20 Sekunden bis etwa 3 Minuten. An sich vermögen gesunde Blutkapillaren einen längeren Zeitraum eine Stauung infolge venöser Abflussbehinderung unbeschädigt zu überstehen, wie ein klinischer Test (Rumpel-Leede-Test) zur Überprüfung der Kapillarresistenz zeigt; hier wird am Arm eine solche Stauung über 5 Minuten angelegt. Aufgrund tierexperimenteller Untersuchung wurde von Jarosch (s. Prokop und Göhler 1976) berichtet, dass bei relativ geringem Druck auf den Hals ( $35 \mathrm{~mm} \mathrm{Hg}$ ) Petechien erst nach 15 Minuten, bei $90 \mathrm{~mm}$ Hg dagegen nach 3 Minuten auftraten; bei Saugglockenversuchen an menschlicher Gesichtshaut traten Petechien ebenfalls erst nach ca. 3 Minuten auf. Kompliziert wird die Situation allerdings dadurch, dass bei nicht allmählichem, sondern stoßartigem Anstieg des venösen Druckes im Kopfbereich (z.B. bei starkem Husten) Petechien nach sehr viel kürzerer Zeit beschrieben worden sind und gerade bei Tötungsdelikten durch Strangulation meist keine isolierte venöse Stauung vorliegt, sondern auch verstärkte Atemtätigkeit gegen einen Widerstand mit der Folge einer starken Druckerhöhung im Brustraum. Daher ist der Versuch, gutachtlich mit exakten »Erfolgszeiten arbeiten zu wollen, aufgrund der Komplexität und des Variantenreich- tums der pathophysiologischen Vorgänge unangemessen «(Henssge 1990). Verhältnismäßig einig sind sich allerdings fast alle Autoren darin, dass das Vorhandensein intensiverer Stauungsveränderungen zumindest einen reflektorischen Herzstillstand unmittelbar nach Einsetzen einer Druckwirkung auf den Hals ausschließt. Solche reflektorischen Mechanismen sollen prinzipiell bei allen Druckwirkungen auf den Hals eine Rolle spielen können. Hierbei handelt es sich um mechanisch ausgelöste Reizung des vegetativen (parasympathischen) Nervensystems, insbesondere an den Teilungsstellen der Halsschlagadern. Hier sitzen Rezeptoren, die Veränderungen des Blutdruckes registrieren und so an der Herz-Kreislauf-Regulation mitwirken. Bei einer äußeren (unphysiologischen) mechanischen Reizung kommt es zum Blutdruckabfall, zum Abfall der Herzfrequenz, u.U. zu Herzrhythmusstörungen, und es soll auch zum unmittelbaren Herzstillstand (Vagusreflex, Vagustod, Reflextod) kommen können. Diese Frage spielt praktisch nur bei Würgeangriffen (weiteres s. dort) eine forensische Rolle. 0 Abbildung 3.71 (modifiziert nach einer Abbildung von Püschel 1982) zeigt die Mitwirkung der vier beschriebenen schädigenden Hauptfaktoren bei den wesentlichen Strangulationstypen.

Eine Reihe älterer Selbstversuche bzw. Berichte von Überlebenden nach Beinahe-Erhängen findet sich bei Prokop und Göhler 1976 zitiert; hierbei wurde z.T. von Schmerzempfindungen, z.T. von angenehmen Gefühlen berichtet. Nach Beobachtungen bei Hinrichtungen durch den Strang erlischt das Bewusstsein hier offenbar augenblicklich. Im weiteren Ablauf wurden völlig »stille« Verläufe ohne Zuckungen oder Krämpfe registriert, manchmal unmittelbar einsetzende schnappartige Atembewegungen; oft kam es nach anfänglicher Bewegungslosigkeit des Körpers zu einer Krampfphase, als tetanische Starre und/oder Auftreten von Streck- oder Zitterkrämpfen. Nach etwa 5 Minuten wurden die ersten Herzstillstände registriert; Herzschlag kann jedoch mitunter noch deutlich bis etwa 20 Minuten erhalten bleiben. Einzelbeobachtungen (z.B. tödlich verlaufener autoerotischer Unfall vor laufender Videokamera) sprechen für eine prinzipielle Ähnlichkeit beim Erhängen in unterstützter Position. Über den Ablauf der Agonie bei Tötungen durch Würgen/Drosseln gibt es vereinzelt Täterangaben, die sich jedoch kaum als verlässliche Informationsquelle eignen.

\section{Autopsiebefunde beim Tod durch Strangulation}

Wie bei allen Erstickungsfällen ist auch hier der eigentlich tödliche Mechanismus an der Leiche nicht sichtbar. Zu nachweisbaren Gewebsuntergängen (z.B. im Gehirn) kommt es aufgrund der Kürze der zum Tode führenden Abläufe nicht. Alle sichtbaren, als Strangulationsfolgen imponierenden Befunde können je nach den Gegebenheiten auch andere Ursachen haben. Die an einem Verletzten oder Getöteten nachgewiesenen Lokalbefunde (z.B. eine Fraktur am Zungenbein oder Kehlkopf) sind zudem meist nicht als ernsthaft bedrohliche Verletzung anzusehen, sondern nur als Indikatoren einer Druckwirkung gegen den Hals, die eben nicht durch die eigentliche lokale Verletzung, sondern über die 
Beeinträchtigung der Durchblutung und/oder Atmung gefährlich oder tödlich wurde - oder nicht. Kompliziert wird die Situation dadurch, dass Todesfälle (auch Tötungsdelikte) durch Strangulation beobachtet werden können, die nur äußerst spärlich entwickelte oder gar ganz fehlende Lokalbefunde aufweisen - auf der anderen Seite kann auch ein den Befunden nach anscheinend schwerster Angriff gegen den Hals (wenn er denn "rechtzeitig" beendet worden war) zunächst überlebt worden sein. Umstände und der gesamte Obduktionsbefund samt Histologie und Toxikologie müssen daher sorgfältig bewertet werden. Da neben den Leichenbefunden die Details der Fundortsituation oft überaus aufschlussreich sein können, wäre eine Einbeziehung des Rechtsmediziners in die Untersuchung bereits am Fundort eigentlich immer wünschenswert. Den folgenden Einzeldarstellungen sei eine - natürlich vereinfachende - Übersicht über das typische Vorkommen der geläufigen äußeren und inneren Befunde bei den verschiedenen Strangulationsformen vorangestellt ( $\bullet$ Tabelle 3.19).

Biochemische Untersuchungen. Verschiedentlich wurde versucht, anhand biochemischer Analysen eine Auskunft über die Art und Intensität der Agonie zu gewinnen. So wurde nachgewiesen, dass es beim Erstickungstod zu einer Erhöhung der Konzentration von (agonal, vermutlich aus der Leber ausgeschütteten) Phospholipiden im Serum kommt. Lag zum Zeitpunkt des Todes infolge Erhängens eine suffiziente Unterbindung der arteriellen Blutzufuhr zum Gehirn vor, so resultiert eine Konzentrationsdifferenz zwischen dem Herzblut und Blut aus den Blutleitern der harten Hirnhaut innerhalb des Kopfes. Tierexperimentell wurde auch ein starker Anstieg von Katecholaminen (Nebennierenmarkhormone, "Stresshormone«: Adrenalin, Noradrenalin) beobachtet, wobei eine ausgesprochene Autolysean- fälligkeit bestand; widersprüchliche Daten liegen über einen Anstieg von Histamin vor. Eine weitere Methode basiert auf der Überlegung, dass es bei Druckwirkung auf den Hals zu einer Quetschung der Schilddrüse kommt, sodass dort gespeicherte Substanzen (Thyreoglobulin) in die Blutbahn übertreten, sich bei erhaltenem Kreislauf verteilen und im Blut erhöht nachgewiesen werden. Mit diesen Methoden konnten durchaus diagnostische Erfolge erzielt werden, allerdings ist die Streuung der Ergebnisse beträchtlich. In der forensischen Routine haben sich die Methoden bisher nicht allgemein durchgesetzt, und nach Eisenmenger et al. (1990) ist eine eindeutige Diagnose einer obstruktiven Asphyxie aufgrund pathobiochemischer Untersuchungen noch nicht möglich.

Stauungssyndrom des Kopfes. Bei Erhängungsfällen mit freier Suspension bleibt das Gesicht meist blass und wird so auch an der Leiche vorgefunden. Bei Tötungsdelikten wurde von Tätern mitunter berichtet, das Opfer sei unter der Strangulation blau angelaufen. Auch an der Leiche eines erwürgten oder erdrosselten Opfers kann das äußere Erscheinungsbild durch eine massive dunkelblaue Stauung des Kopfes so gekennzeichnet sein, dass man schon beim ersten Blick aus einiger Entfernung den dringenden Verdacht auf eine Strangulation gewinnen wird; ein Ablaufen feiner Blutspuren aus Mund, Nase oder Ohren infolge Platzens oberflächlicher Schleimhautgefäße wird dann öfter gesehen. Auch eine starke vitale Blutstauung wird aber postmortal modifiziert, was durch Umlagerungen der Leiche (z.B. im Rahmen der Untersuchung oder beim Transport) verstärkt werden kann. Eine zum Todeszeitpunkt vorhandene Blutstauung kann weitgehend verschwinden; noch problematischer ist aber, dass eine solche auch überhaupt erst postmortal entstehen kann (bei Tieflage des Gesichtes, z.B. in Bauchlage). Wie schwierig die Be-

- Tabelle 3.19. Auftreten ausgewählter Befunde bei den verschiedenen Strangulationsformen

\begin{tabular}{|c|c|c|c|c|c|}
\hline & $\begin{array}{l}\text { Erhängen } \\
\text { "typ." }\end{array}$ & $\begin{array}{l}\text { Erhängen } \\
\text { "atyp.» }\end{array}$ & $\begin{array}{l}\text { Erdrosseln } \\
\text { als Suizid }\end{array}$ & $\begin{array}{l}\text { Erdrosseln } \\
\text { als Tötung }\end{array}$ & Erwürgen \\
\hline Petechien im Kopfbereich & + & ++ & +++ & +++ & +++ \\
\hline Grobe Blutungen innerhalb der Zunge & $?$ & + & +++ & +++ & ++ \\
\hline $\begin{array}{l}\text { Einblutungen im Unterhautfettgewebe unter den } \\
\text { Hautmarken }\end{array}$ & $?$ & $?$ & $?$ & ++ & +++ \\
\hline Frakturen von großen Zungenbeinhörnern & ++ & ++ & + & + & +++ \\
\hline Frakturen von Schildknorpeloberhörnern & ++ & ++ & + & ++ & +++ \\
\hline Frakturen der Schildknorpelplatten & $?$ & $?$ & $?$ & + & ++ \\
\hline Ringknorpelfrakturen & + & $?$ & $?$ & + & ++ \\
\hline Einblutungen an den Kehlkopfgelenken & + & + & $?$ & + & +++ \\
\hline $\begin{array}{l}\text { Grobe Weichteilblutungen des Kehlkopfes } \\
\text { (insbesondere in den Stimmfalten) }\end{array}$ & $?$ & $?$ & $?$ & + & +++ \\
\hline
\end{tabular}



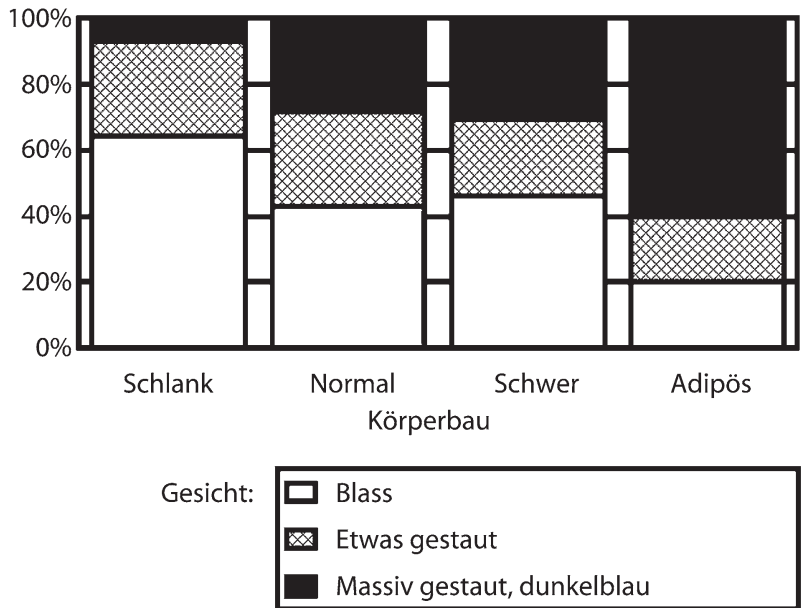

- Abb. 3.72. Beziehung zwischen Körperbau (Konstitution) und allgemeiner Stauung der Gesichtshaut bei 100 Opfern von Tötungsdelikten durch Strangulation

wertung letztlich sein kann, zeigt ein Vergleich einer an Leichen vorhandenen Blutfülle (»Stauung«) der Gesichtshaut mit dem Körperbau (Konstitution): Bei schlanken Personen in Rückenlage reichen die Totenflecke an Kopf und Hals meist weniger weit nach ventral als bei adipösen. Diese Ausdehnung der Totenflecke beeinflusst ihrerseits die Blutfülle des Gesichtes, die somit auch von Faktoren abhängt, die überhaupt nichts mit der Todesursache zu tun haben (- Abb. 3.72).

Der wichtigste Stauungsbefund sind petechiale Blutaustritte. Wenn vorhanden, finden sie sich praktisch immer in den Bindehäuten der Augenlider; bei stärkerer Intensität auch in der Haut der Augenlider, des Gesichtes sowie in den weiteren Schleimhäuten (z.B. der Mundschleimhaut; unlängst auch in den Nasennebenhöhlen beschrieben). Es handelt sich um winzige, "flohstichartige" Extravasate infolge stauungsbedingter Ruptur kleinster Blutgefäße (• Abb. 3.73). Bei Todesfällen durch Erwürgen oder Erdrosseln fehlen sie praktisch nie; beim Erhängen hängt ihr Auftreten sehr von den speziellen Gegebenheiten der Erhängungssituation ab. Auch das Lebensalter könnte eine gewisse Rolle bei ihrer Intensität spielen: Bei Personen in jüngerem und mittlerem Lebensalter sind sie meistens stärker entwickelt als bei alten Personen - eine Beobachtung, die auch bei Petechien aus innerer Ursache (»Herztod«) zu gewinnen war. Das Auftreten postmortaler Blutaustritte innerhalb hypostatischer Bezirke ist ein wohl bekanntes Phänomen. Solche Vibices sind als solche zwar meist unschwer zu erkennen (z.B. an der Lokalisation und Verteilung; sie sind auch größer als vital entstandene Petechien). Finden sich aber Petechien im Gesicht einer schon einige Zeit auf dem Bauch gelegenen Leiche, so wird die Situation sehr schwierig: In Betracht kommt u.U. eine allein postmortale Entstehung, aber auch eine postmortale Größenzunahme vitaler Petechien.
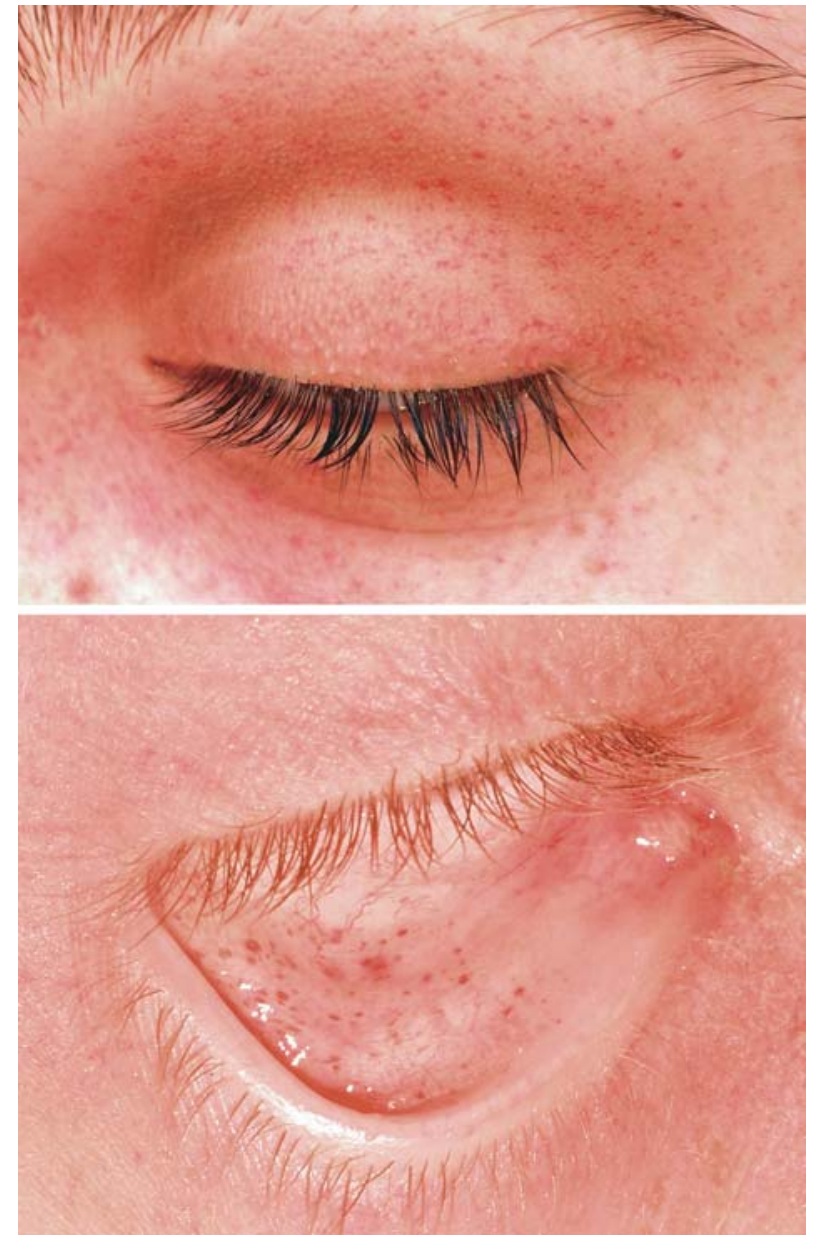

- Abb. 3.73. Augenlider und Augenbindehäute mit dichtstehenden punktförmigen Blutaustritten (Petechien) als Folge einer oberen Einflussstauung. Im vorliegenden Fall handelte es sich um einen Erhängungstod in sitzender Position

Derzeit nicht verbindlich zu beantworten ist die Frage, ob allein (frustrane) Wiederbelebungsversuche zur Entstehung von Stauungsblutaustritten im Kopfbereich führen können. Dies wurde in einigen rechtsmedizinischen Publikationen angenommen; die intensivmedizinische Literatur ist in dieser Hinsicht »leer«, und eigene Analysen sprechen eher gegen einen solchen Zusammenhang.

Halshautverletzungen. Durch mechanischen Druck und/ oder tangentiale Traumatisierung wird die Oberhaut des Halses so oberflächlich lädiert, dass dies oft erst infolge der postmortalen Austrocknung Stunden nach dem Tode deutlich sichtbar wird. Unterbleibt diese, bleiben diskrete Verletzungen u.U. unsichtbar; dies kann in feuchtem Milieu geschehen; sogar postmortale Salbenbehandlung zur Verdeckung des Sichtbarwerdens wurde beschrieben. Die resultierenden Spuren sind Strang- 
(ulations)marken bei Verwendung bandartiger Werkzeuge oder Würgemale bei Halskompression mit der Hand. Wie deutlich eine Strangmarke ausgeprägt ist, hängt im Wesentlichen von der Beschaffenheit (Breite, Festigkeit, Rauigkeit) des Strangmaterials, einer eventuellen Interposition von Kleidungsteilen oder Haaren sowie der Stärke der einwirkenden Kräfte ab. Handelt es sich um relativ schmale Gegenstände (Elektrokabel, Seil), so ist die Strangmarke oft über eine weite Strecke tief in die Halshaut eingeschnürt, klar abgegrenzt, und gibt z.T. Formmerkmale des Strangwerkzeuges wider; bei breiteren und weicheren Gegenständen sind die Marken flacher, unschärfer und mitunter überhaupt ganz unscheinbar. Liegt der Strang mehrmals um den Hals, so werden Streifen der Halshaut förmlich abgequetscht; an solchen Stellen ("Zwischenkamm») bilden sich mitunter Hautblutungen, die lange als Beleg für eine Entstehung dieser Strangmarke zu Lebzeiten angesehen wurden; auch dieser Befund erwies sich jedoch als postmortal erzeugbar. Die - bislang meist nicht eindeutig beantwortbare - Frage nach der Vitalität äußerer Strangulationsmarken ist nicht nur von akademischem Interesse, da auch an der Leiche auf vielfältige artifizielle Weise sehr ähnlich aussehende Spuren zustande kommen können, etwa im Rahmen von Bergungs-, Transport- oder auch Reanimationsmaßnahmen.

Beim Erhängen verläuft die Strangmarke in den meisten Fällen nicht horizontal um den Hals, sondern steigt in Richtung auf den höchsten Punkt der Schlingenführung deutlich an und ist dort auch oft unterbrochen. Da das Strangwerkzeug meistens infolge des Gewichtes des Körpers nach oben rutscht, bis es durch den Unterkiefer gehalten wird, liegt die Erhängungsmarke typischerweise auch mit ihren tiefsten Anteilen weit oben am Hals, meist oberhalb des Kehlkopfes. Kommt es aufgrund der speziellen Gegebenheiten während des Erhängungsvorganges zu einem Lagewechsel der Schlinge, so kann auch bei eintouriger Umschlingung u.U. eine zweite Strangmarke entstehen ( $\bullet$ Abb. 3.81). Fast immer fehlen Einblutungen unmittelbar unterhalb einer Erhängungsmarke. Beim Erdrosseln liegt die (Drossel-) Marke meist in Höhe des mittleren Halsdrittels und verläuft ziemlich horizontal um den Hals. Aufgrund der Dynamik von Tätlichkeiten ist die Variabilität besonders hoch: Selbst bei »nur « eintouriger Umschlingung kann es bei zunächst erfolgreicher Gegenwehr des Opfers zu mehrfachen Marken kommen, die auch an ungewöhnlicher Stelle (z.B. über das Gesicht) verlaufen können. Bei suizidalem Erdrosseln ist das gesamte äußere Erscheinungsbild weitaus weniger auffällig. Die Vitalität einer Drosselmarke lässt sich oft indirekt daran ablesen, dass Petechien an Kopf und Hals durch die Strangulationsebene nach kaudal hin scharf begrenzt sind (• Abb. 3.83), auch finden sich häufiger Einblutungen im Unterhautfettgewebe. Einer Drosselmarke ähnelnde Hautverletzungen können auch bei alleinigem Würgen auftreten, wenn sich zwischen der würgenden Hand und der Halshaut ein entsprechender Gegenstand (Halskette) befindet. Würgemale selbst entstehen durch den direkten Fingerdruck; besonders charakteristisch sind sichelmondförmige schmale Vertrocknungen von etwa 1-1,5 cm Länge, die den in die Haut eingepressten Rändern der Fingernägel entsprechen (- Abb. 3.84) und die so typisch angeordnet sein können, dass Rückschlüsse über die verwendete Hand des Täters möglich sein können. Andere Spuren sind längliche oberflächliche Kratzer durch die Nagelränder, kleinfleckige Abschürfungen oder auch lediglich Einblutungen in Haut und Unterhaut. Der starke lokale Druck bewirkt meist kleine Blutunterlaufungen unterhalb von Würgemalen in den tieferen Halsschichten. Bei deutlicher Ausprägung ist ein solches äußeres Verletzungsbild kaum zu übersehen oder zu missdeuten; bricht die Gegenwehr des Opfers rasch zusammen (z.B. infolge der Massivität und Effektivität des Angriffes oder einer gravierenden krankhaften oder toxischen Beeinträchtigung des Angegriffenen), so resultieren mitunter nur unscheinbare äußere Spuren, die bei einer nur oberflächlichen Leichenschau leicht übersehen werden können. Auf der anderen Seite kommen Abschürfungen an der Halshaut auch als unbedeutsame Gelegenheitsverletzungen vor. Eine Interpretation allein aufgrund der äußerlichen Untersuchung wird hier oft nicht möglich sein.

Befunde an den Halsweichteilen. Praktisch alle Weichteilstrukturen des Halses können im Einzelfall Spuren der äußeren Halskompression zeigen, als Folge einer von drei wesentlichen Ursachen:

- Blutstauung,

- direkter mechanischer Druck (Quetschung) oder

- indirekte mechanische Verletzung (Zerrung).

An den inneren Schleimhäuten, in den lockeren Verschiebeschichten (zwischen den Halsmuskeln) und in der Zunge führt eine starke Blutstauung häufig zu beträchtlichen Stauungsblutaustritten. Blutungen in den vorderen Halsmuskeln selbst sind z.T. ebenfalls Stauungsfolge, häufiger jedoch Ausdruck mechanischer Verletzung durch direkten Druck oder Zerrung. Druckund Zerrungsblutungen können mitunter an ihrer Form unterschieden werden: die Druckblutungen sind kräftiger, mehr rundlich bzw. fleckförmig und durchsetzen den Muskel bis in seine Tiefe. Zerrungsblutungen sind schmal, länglicher, streifenförmig und oberflächlicher. Druckblutungen zeichnen sich durch eine Beteiligung mehrerer Schichten aus (- Abb. 3.74). Als Stauungsfolge sind Blutungen deutlich oberhalb der Höhe der Strangulationsebene anzusehen. Blutaustritte als Folge einer Muskelzerrung sind beim Würgen und Drosseln ungewöhnlich, demgegenüber beim Erhängen häufig, vor allem im Ursprungsbereich der Kopfwendermuskeln am Schlüsselbein (intra- oder subperiostale Blutungen; - Abb. 3.75). Histologische Studien zeigten bei vitaler mechanischer Muskelzellschädigung u.a. einen Ausfall der Querstreifung, das Auftreten einer Längsstreifung, einen diskoiden oder segmentalen Zerfall; bei immunhistochemischen Spezialfärbungen (Myoglobin) fanden sich entfärbte (»opake«) Muskelzellen.

Schleimhautblutungen sind ein geläufiger Befund bei starker oberer Einflussstauung, insbesondere beim Erdrosseln. Sie 


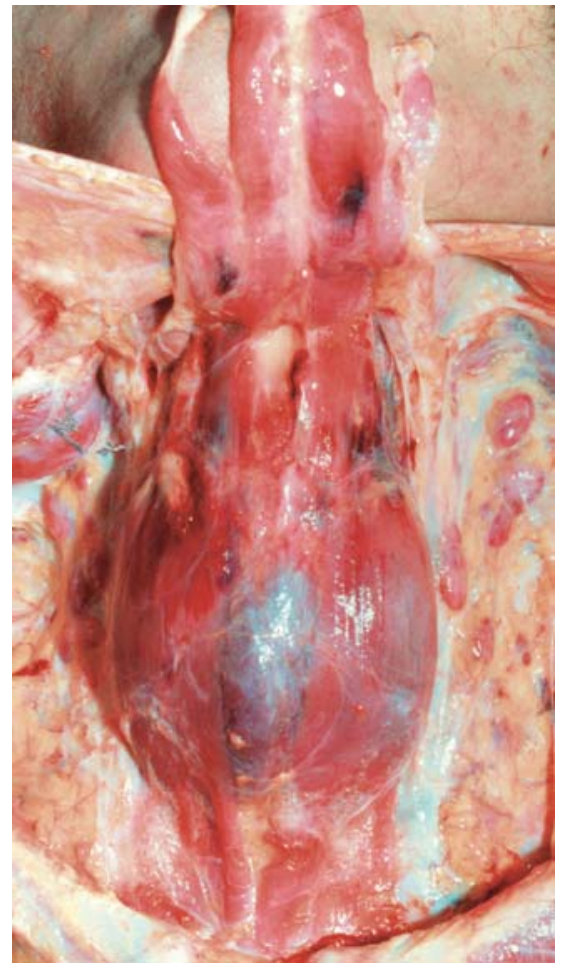

- Abb. 3.74. Obduktionssitus während der schichtweisen Präparation der vorderen Halsmuskeln. Kräftige fleckförmig Einblutungen an den Vorder- und Rückseiten der Muskeln in jeweils gleicher Position, als Ausdruck einer punktuellen starken Kompression. 21-jährige Frau, Tötung durch Würgen und Drosseln

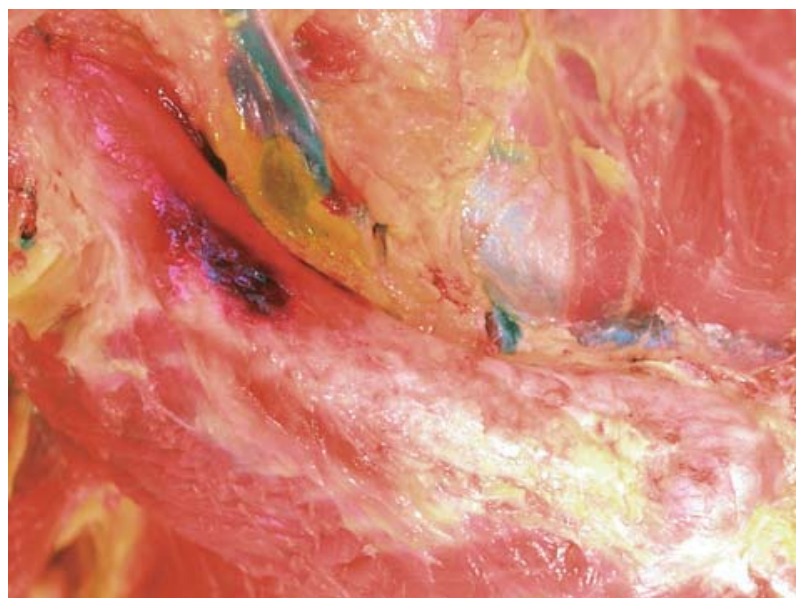

- Abb. 3.75. Rechtes Schlüsselbein, Knochenhaut noch belassen. Ausgedehnte Einblutung (Zerrungsblutung, typisch beim Erhängen) im und unter dem Periost liegen am Gaumen, Rachenring und Zungengrund. Häufig sind auch Petechien, die vor allem an der Rückfläche des Kehldeckels und am Ösophagusmund gut erkennbar sind. Die Zunge zeigt beim Erwürgen und Erdrosseln häufig (2/3 der Fälle) Einblutungen. $\mathrm{Zu}$ etwa gleichen Teilen handelt es sich um Bissspuren an den Rändern sowie disseminierte kleine oder ausgedehnte Blutungen in der Zungenmuskulatur. Neben einer wohl meist hinzutretenden Stauung wird für solche inneren Blutungen - je nach Lokalisation und Anordnung - eine Quetschung durch die vorderen Zahnbögen oder ein Einpressen des Zungenbeines infolge der äußeren Druckwirkung auf den Hals angenommen. Verletzungen der Luftröhre (ausgenommen Rupturen bei Erhängen mit Sturz in eine Schlinge) wurden erst in letzter Zeit intensiver untersucht; diskrete Knorpelverletzungen fanden sich beim Erdrosseln in fast einem Fünftel der Fälle. Kapselblutungen der Schilddrüse sind beim Würgen und Drosseln häufig (Vorkommen in unserem Material: 18\% bzw. 8\%); Parenchymverletzungen sind dagegen praktisch nicht beschrieben. Über die Beteiligung großer Halsvenen beim Strangulationstod gibt es nur sehr seltene Mitteilungen. An den Halsschlagadern kommen äußere und innere Gefäßwandblutungen sowie Einrisse der Intima (selten auch vollständige Rupturen) vor. Intimarisse der Halsschlagadern verlaufen beim Erhängen (Häufigkeit: 5\%-16\%) meistens quer und liegen mehr oder weniger dicht unterhalb der Gabelung (• Abb. 3.76). Mögliche Ursachen: direkter Druck des Stranges (oder beim Würgen auch des Fingers) auf das Gefäß, Zugbelastung in Längsachse beim Erhängen, Gefäßquetschung durch ein abgebrochenes und seitlich unter die Arterie disloziertes Zungenbein- oder Schildknorpeloberhorn. Spättodesfälle infolge sekundärer Halsschlagaderverschlüsse (Dissektionen, Thrombosen) bei zunächst überlebter Strangulation sind verschiedentlich beobachtet worden.

Verletzungen von Kehlkopf und Zungenbein. Kehlkopf und Zungenbein sind zunächst knorpelige, im Verlauf des Lebens durch Kalkeinlagerung und/oder Verknöcherung starr werdende Gerüststrukturen in den vorderen Halsweichteilen. Verletzungen kommen bei ganz verschiedenen Einwirkungen vor, jedoch ist Strangulation die bei weitem häufigste Ursache (- Abb. 3.77). Eine Verletzungsmöglichkeit durch notfallmäßige Intubation wird diskutiert, scheint nach eigenen Erfahrungen aber eher unwahrscheinlich.

Das Zungenbein ist eine etwa U-förmige, aus einem Körper (vorne) und zwei nach hinten gerichteten großen sowie zwei kleinen Hörnern aufgebaute Struktur. Große Hörner und Körper sind zunächst gelenkähnlich miteinander verbunden, wachsen später meist knöchern zusammen. Bei Krafteinwirkung gegen den Vorderhals kommt es zum Anstemmen gegen die Halswirbelsäule mit direkt bedingten Frakturen; auch indirekte Verletzungen infolge starker Zerrung bei Überstreckungstraumen sind möglich. Vereinzelt wurde auch über "spontane« Zungenbeinfrakturen allein infolge von Muskelkontraktionen berichtet (so beim Essen oder Erbrechen). Zur Fraktur eines verkalkten Zungenbeinhornes genügt bereits leichter Fingerdruck gegen den fi- 


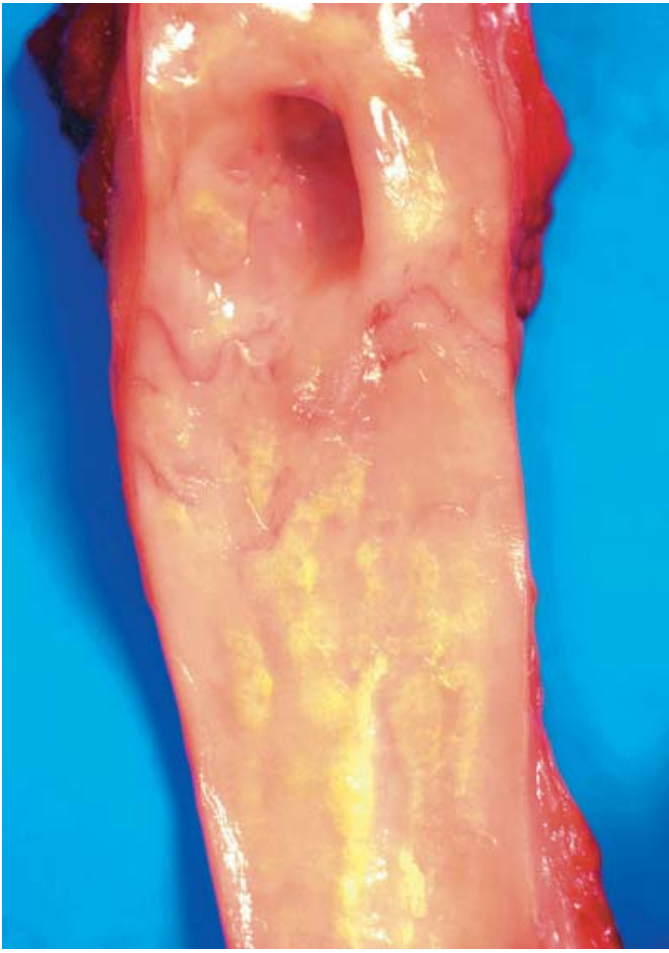

- Abb. 3.76. Längs bis in die innere Kopfschlagader eröffnete Halsschlagader. Flache arteriosklerotische Beete. Mehrere sehr schmale, nur die innersten Gefäßschichten durchsetzende, etwas geschwungen quer zur Längsrichtung des Gefäßes verlaufende Einrisse. 64-jähriger Mann, suizidales Erhängen (freie Suspension, höchster Schlingenpunkt am Nacken)

xierten Knochen; dass solche Frakturen dennoch nicht häufig vorkommen, liegt an der geschützten Lage und der Beweglichkeit. Zungenbeinfrakturen sind schmerzhaft, heilen jedoch (ggf. unter bleibender Fehlstellung) spontan aus.

Der Kehlkopf besteht aus dem Schild- und dem Ringknorpel, die durch hinten gelegene Gelenke verbunden sind, sowie den paarigen kleinen Stellknorpeln, die im Binnenraum des

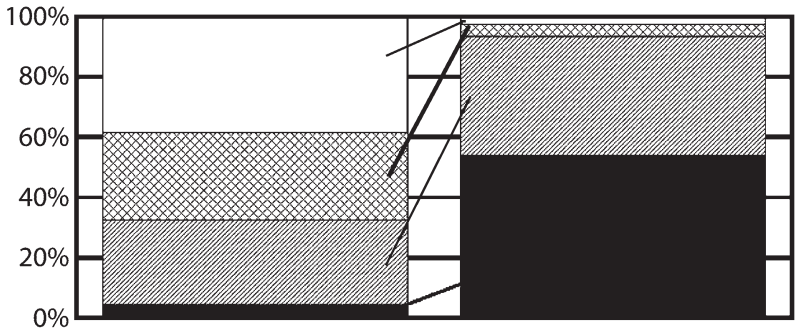

Todesart aller 6431 Obduktionen

Todesart der 309 Fälle mit Frakturen

- Abb. 3.77. Vorkommen von (frischen) Frakturen an Kehlkopf und Zungenbein in einem rechtsmedizinischen Obduktionsmaterial; Untersuchungszeitraum 11 Jahre. Strangulationsfälle (alle Arten) machen rund $5 \%$ des Obduktionsgutes aus, sind aber für mehr als die Hälfte aller beobachteten Frakturen verantwortlich. Bei atraumatischen Todesfällen werden solche Verletzungen kaum gesehen.

Kehlkopfes dem Ringknorpel hinten oben gelenkig aufsitzen. Im Verlauf des Lebens kommt es zu einer Versteifung aller Knorpel infolge Verkalkung und/oder Verknöcherung, was eine gewisse Altersabhängigkeit beobachteter Verletzungsraten erklärt (•Tabelle 3.20). Der Schildknorpel besteht aus zwei Platten, die vorne miteinander verbunden sind und nach rückwärts auseinander weichen. Ihre hinteren Kanten laufen je in einem Ober- und einem Unterhorn aus. Brüche der Oberhörner (• Abb. 3.78) sind die häufigste Kehlkopfverletzung; sie können bereits bei geringer lokaler Krafteinwirkung (Größenordnung: 2 kg) entstehen. Abgeheilte Frakturen als Zufallsbefund bei Obduktionen sind nicht ganz selten (4\%). Brüche der Unterhörner sind ungewöhnlich. Bricht die vordere Verbindung der Schildknorpelplatten (Folge einer groben Einwirkung), so kann die Stabilität des Kehlkopfes beeinträchtigt sein; Einblutungen der inneren Kehlkopfweich-

Tabelle 3.20. Häufigkeit (Angaben in Prozent) von Frakturen von Kehlkopf und Zungenbein in Beziehung zum Lebensalter bei 194 Tötungsfällen durch Strangulation

\begin{tabular}{|c|c|c|c|c|c|c|c|c|}
\hline $\begin{array}{l}\text { Lebensalter } \\
\text { (Fallzahl) }\end{array}$ & $\begin{array}{l}-20 \\
(13)\end{array}$ & $\begin{array}{l}21-30 \\
(23)\end{array}$ & $\begin{array}{l}31-40 \\
(27)\end{array}$ & $\begin{array}{l}41-50 \\
(26)\end{array}$ & $\begin{array}{l}51-60 \\
(31)\end{array}$ & $\begin{array}{l}61-70 \\
(19)\end{array}$ & $\begin{array}{l}71-80 \\
(29)\end{array}$ & $\begin{array}{l}>80 \\
(26)\end{array}$ \\
\hline Keine Fraktur & 77 & 49 & 33 & 16 & 19 & 26 & 14 & 8 \\
\hline Nur Hornfraktur(en) & 8 & 39 & 52 & 42 & 55 & 58 & 62 & 42 \\
\hline $\begin{array}{l}\text { Beteiligung von Schildknorpelplatte } \\
\text { oder Ringknorpel }\end{array}$ & 15 & 12 & 15 & 42 & 26 & 16 & 24 & 50 \\
\hline
\end{tabular}




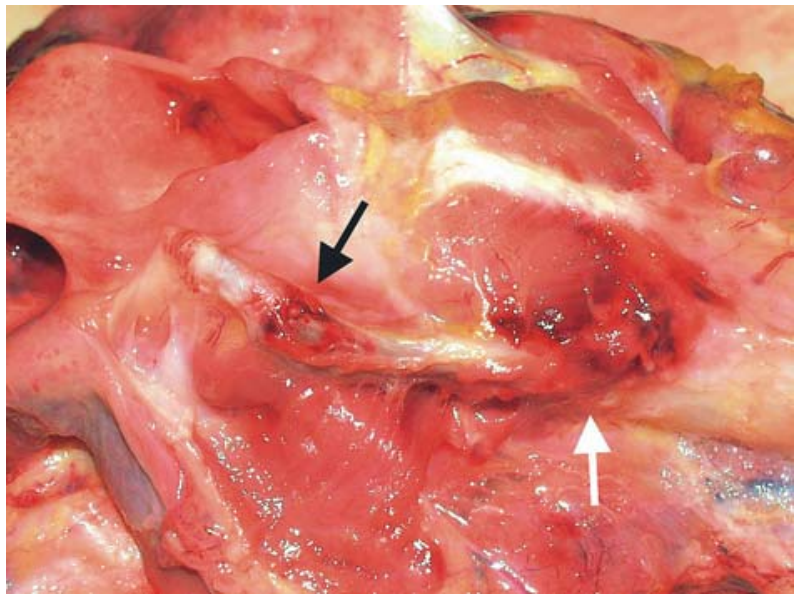

- Abb. 3.78. 51-jährige Frau, Tötung durch Erdrosseln mit festem Textilgürtel. Kehlkopf von links hinten. Fraktur des linken Schildknorpel-Oberhornes (schwarzer Pfeil), mit Riss des Periostes und Umblutung. Das Ringknorpel-Schildknorpel-Gelenk (weißer Pfeil) stark umblutet; Petechien am Kehldeckel

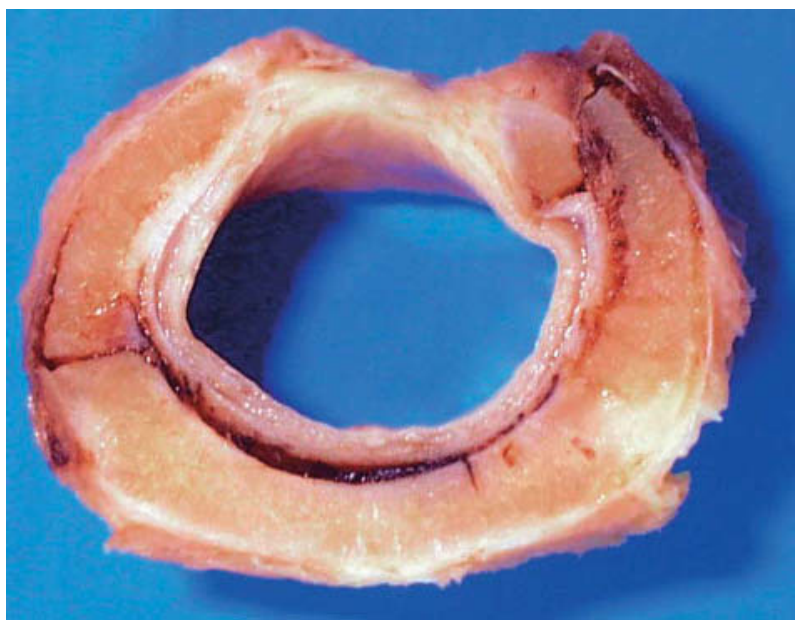

- Abb. 3.79. Horizontalschnitt durch den oberen Teil des Ringknorpels. Rechts die vordere Ringknorpelspange vollständig durchgebrochen und verschoben; links ein nichtdislozierter Bruch. Infolge der von vorne nach hinten gerichteten Kompression entstand auch eine nur die inneren Teile des Knorpels betreffende Spaltbildung (Fissur) hinten rechts in der Ringknorpelplatte. Rezidivierte Misshandlung, u.a. mit Leberverletzung und Blutung in die Bauchhöhle; nachhaltiges Würgen

teile mit Anschwellung können die Atemwege bedrohen. Der Ringknorpel schließt den Zungenbein-Kehlkopfapparat nach unten hin ab und leitet zur Luftröhre über. Er hat einen siegelringähnlichen Aufbau mit der breitesten Stelle an der Rückseite. Frakturen deuten auf ein schweres Halstrauma hin; sie liegen meistens vorne, im Bereich des dünneren Ringknorpelbogens

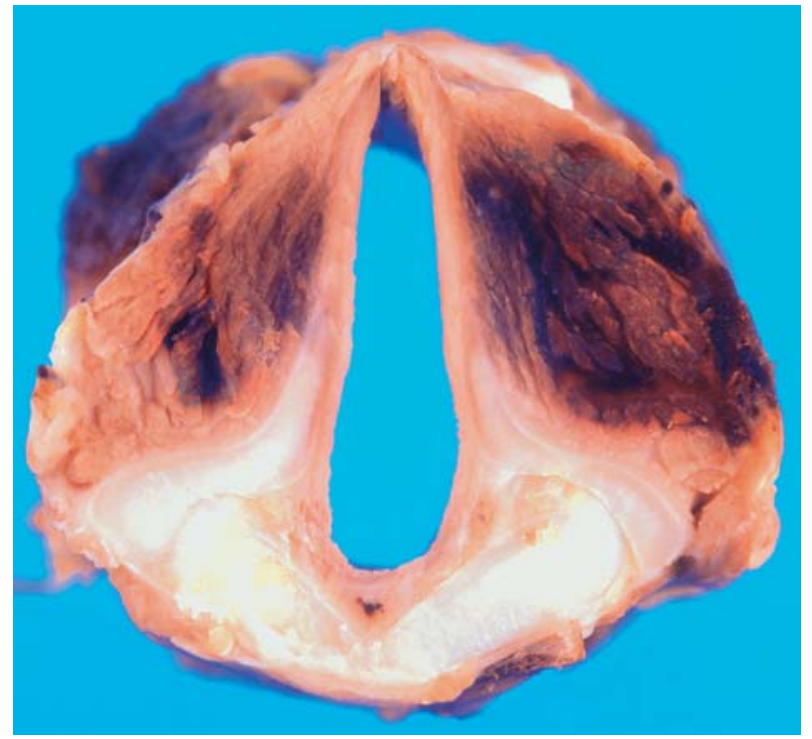

- Abb. 3.80. Horizontalschnitt durch den mittleren Teil des Kehlkopfes. Unten im Bild der kopfnahe Teil der Ringknorpelplatte sowie die beiden Stellknorpel. In beiden Stimmfalten ausgedehnte Einblutungen als Folge einer intensiven Kehlkopfkompression

(• Abb. 3.79). Bei Dislokation oder stärkeren Weichteilblutungen droht eine Einengung der Atemwege. Bei den meisten schwereren Kehlkopftraumen manifestiert sich die Belastung auch an Einblutungen der (Gelenkkapseln oder Gelenkräume der) Kehlkopfgelenke. Die inneren Weichteile des Kehlkopfes (- Abb. 3.80), vor allem die Stimmfalten im Kehlkopfinneren oder die kleinen Kehlkopfmuskeln an seiner Rückfläche, sind dann ebenfalls meistens eingeblutet. Auch ohne Frakturen am Kehlkopf kommen solche Blutungen vor; bei jüngeren Personen mit noch elastischen Knorpeln können sie die einzige Verletzungsfolge am Kehlkopf sein. Blutaustritte der Kehlkopfschleimhaut sind überhaupt häufig (5-6\% im allgemeinen Obduktionsgut); bei Todesfällen durch Würgen und Drosseln finden sie sich in über $50 \%$.

Beteiligung der Halswirbelsäule. Weichteilverletzungen an der Halswirbelsäule (Einblutungen in Muskeln, Bändern, Gelenken oder Bandscheiben, vereinzelt auch Umblutungen oder Intimarisse an Wirbelarterien, selten auch Bandzerreißungen) werden bei tödlicher Strangulation bei gezielter Untersuchung nicht ganz selten nachgewiesen, am häufigsten beim Erhängen (Saternus 1979, 1990). Knöcherne Verletzungen sind demgegenüber beim Würgen und Drosseln nur in ganz ungewöhnlichen Fällen zu erwarten; beim Erhängen sind Wirbelverletzungen allerdings durchaus möglich ( $\triangleright$ unten, »Erhängen«). 


\section{8 .2}

\section{Die einzelnen Strangulationsarten Erhängen}

\section{Infobox}

Erhängen ist eine sehr rasch zur Bewusstlosigkeit (praktische Unmöglichkeit einer Selbstrettung) und zum Tode führende, also sehr »effiziente« Strangulationsform, und mit 5.0006.000 Todesfällen pro Jahr die häufigste Suizidmethode (1999: 5.667 Erhängungsfälle bei 11.157 Suiziden in Deutschland). Nahezu alle tödlichen Erhängungen gehen auf Suizide zurück; vereinzelt handelt es sich auch um Unfälle. Tötung durch Erhängen ist möglich, wird jedoch äußerst selten beobachtet; eher noch spielen vorgetäuschte suizidale Erhängungssituationen eine Rolle, bei denen ein Opfer zuvor auf andere Weise (z.B. durch Erdrosseln) getötet worden war. Nur in einem Teil der Erhängungstodesfälle (weit weniger als die Hälfte) erfolgt eine rechtsmedizinische Untersuchung und ggf. Obduktion.

Die Aufklärung der Todesumstände eines Erhängungsfalles hängt naturgemäß von der Intensität der Untersuchung von Anfang an $\mathrm{ab}$; oft werden die Ermittlungsverfahren jedoch ohne rechtsmedizinische Beteiligung oder Obduktion eingestellt. Damit kommt dem Leichenschauarzt eine besondere Verantwortung zu. Hinweise für ein verdecktes Tötungsdelikt könnten sich aus der Umgebungssituation (z.B. Art der Aufhängung oder markante Schleifrinne durch Reibung des Seiles am Aufhängeort), aus dem äußeren Leichenbefund (z.B. intensive Kopfstauung und Petechien bei in typischer Erhängungssituation aufgefundener Leiche, Würgemale an der Halshaut, Abwehrverletzungen) oder auch erst bei der Obduktion (intensive Verletzungen der inneren Halsstrukturen) ergeben. Da aber auch bei tatsächlich suizidalem Erhängen »verdächtige« äußere Spuren entstehen können (z.B. zweite Strangmarke infolge Verrutschens des Stranges (• Abb. 3.81), Kratzer am Hals von Eigenmanipulation oder Hautverletzungen an Handrücken und Armen infolge Anschlagens an Heizkörpern o.Ä. in der Erhängungsagonie (Krampfstadium)), bedarf die abschließende Bewertung großer Erfahrung. Kriminalistisch kann u.U. der Nachweis von Fasern des Strangwerkzeuges an den Händen des Toten hilfreich sein; man kann sie mit Klebefolie von den Händen abnehmen und ggf. mit Fasern des Stranges mikroskopisch vergleichen. Diese Faserprobe wurde lange Zeit als zuverlässig angesehen, jedoch wurde dies inzwischen auch bezweifelt. Unfallmäßige Erhängungssituationen werden beobachtet, z.B. wenn intoxikierte oder anderweitig bewusstseinsgestörte Personen (Epilepsiekranke) in eine strangulationsähnliche Situation geraten. Eine bedrückende Fallgruppe sind Säuglinge und Kleinkinder, die sich im häuslichen Bereich mit dem Kopf in Haltegestängen oder Spielzeugbändern oder auf Spielplätzen mit zur Bekleidung gehörenden Schnüren in Spielgeräten verfangen; wir untersuchten auch Todesfälle etwas größerer Kinder, die sich auf Spielplätzen in Kletternetzen erhängt hatten. Eine besondere Untergruppe sind so genannte autoerotische Unfälle. Hierbei handelt es sich von den Betroffenen allein oder mit Einwilligung durch andere herbeigeführte, nicht selten technisch ausgeklügelte Strangulations-Fesselungs-Situationen, die zu einer »dosierten « Einschränkung der Hirndurchblutung mit dem Effekt einer sexuellen Stimulierung führen sollen. Bei geringfügigem Abweichen vom geplanten Verlauf kann eine solche Situation rasch in eine unbeabsichtigte tödliche Erhängung übergehen, aus der eine Selbstbefreiung nicht mehr möglich ist. Die rechtsme-
- Abb. 3.81. 71-jähriger Mann, der sich offenbar das doppelt und zur laufenden Schlinge genommene Kabel zunächst zirkulär eng um den Hals geschlungen hatte, wofür der rote, einem Zwischenkamm entsprechende zirkuläre Streifen spricht. Im Krampfstadium dürfte die Schlinge letztlich in die dargestellte Endposition gekommen sein. Die horizontale Marke führte - richtigerweise - zur Beiziehung eines Rechtsmediziners an den Fundort (Verdacht einer vorherigen Drosselung). Nach Vorliegen aller Befunde und Umstände war der Fall aber letztlich hinreichend sicher als Suizid zu deuten
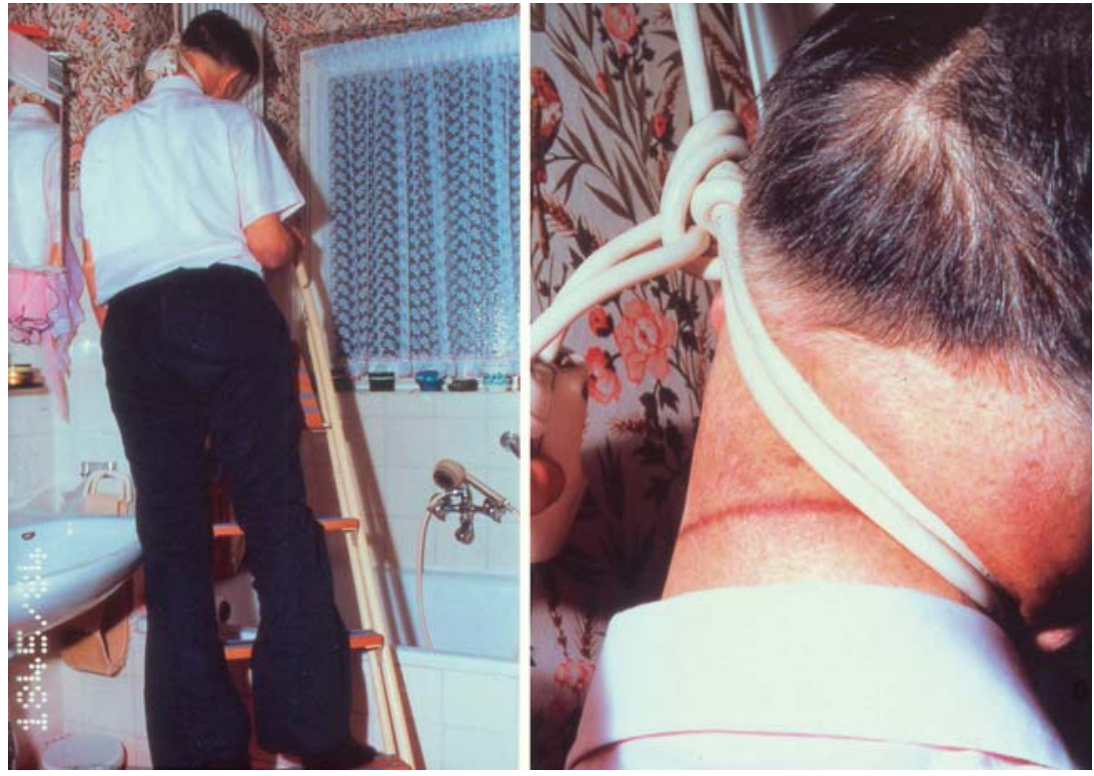
dizinische Literatur weist eine umfangreiche Kasuistik solcher Fälle auf. Die Umgebungssituation ist meist eindeutig; mitunter kommt allerdings auch eine Mitwirkung anderer in Betracht, denen u.U. vorgehalten werden könnte, den Ablauf nach Entgleisung der Situation nicht abgewendet zu haben.

Je nach dem Typ des verwendeten Strangwerkzeuges, der Art und Anzahl der Umschlingungen des Halses, der eventuellen Verknotungen oder Schlingenbildungen, der Höhe der Fixierung des Stranges über der letzten Position des Körpers und der Art des in die Schlinge Gelangens (Sturz, Sprung, Hineingleiten) sind unzählige Erhängungssituationen möglich. Entsprechend vielfältig sind die vorkommenden Befundmuster, die von einem völligen Fehlen jeder Verletzung bzw. sichtbaren Strangulationsfolge überhaupt bis zum vollständigen Abriss des Kopfes reichen. Wurde die Situation (verständlicherweise, z.B. von den den Erhängten auffindenden Angehörigen) unrekonstruierbar verändert, bleiben nicht selten Fragen offen. Sofern beim Eintreffen der Polizei oder des Notarztes bei einer noch hängend vorgefundenen Person bereits sichere Leichenerscheinungen vorhanden sind, sollte eine Abnahme der Leiche unter Schonung oder alsbaldiger Rekonstruktion (z.B. Fixierung durchtrennter Abschnitte mittels Aneinanderknoten mit einer Schnur) der Schlingensituation erfolgen. Der innere Lokalbefund am Hals ist oft diskret. Ursprungsblutungen der Kopfwendermuskeln (• Abb. 3.75) werden bei uns fast immer gefunden. Weitere Blutungen der vorderen Halsmuskeln fehlen oft, Innenhautrisse der Halsschlagadern (- Abb. 3.76) kommen nicht ganz selten vor. Verletzungen an Kehlkopf oder Zungenbein sind - je nach Quelle - eher selten (rund $20 \%$ ) oder sehr häufig (70-80\%); sie betreffen meistens lediglich die großen Zungenbein- und oberen Schildknorpelhörner. Ein für das Erhängen relativ charakteristischer und (bei frei hängendem Körper) häufiger Befund sind Einblutungen im/unter dem vorderen Längsband der Lendenwirbelsäule über den Zwischenwirbelscheiben (Simon'sche Blutungen; • Abb. 3.82) infolge einer Längsdehnung unter Hinzutreten durch krampfbedingte Bewegungen bedingter Querbelastungen an der LWS.

\footnotetext{
Definition

Von typischem Erhängen wird gesprochen, wenn der Körper in aufrechter Position, praktisch frei hängt und der höchste Schlingen- bzw. Knotenpunkt bei in etwa symmetrischem Verlauf des Stranges um den Hals am Nacken liegt. Dann ist ein rascher, vollständiger Verschluss aller 4 hirnversorgenden Arterien möglich, mit der Folge eines meistens völlig ausbleibenden Stauungssyndroms.

Als atypisch werden Erhängungssituationen bei nicht frei hängendem Körper und nicht zum Nacken hin ansteigender Strangulationsebene bezeichnet. Hier ist meist ein Teil der arteriellen Blutzufuhr zum Kopf erhalten, sodass recht ausgeprägte Stauungsveränderungen resultieren können. Der Eintritt der Bewusstlosigkeit scheint allerdings auch hier vergleichsweise rasch zu erfolgen.
}

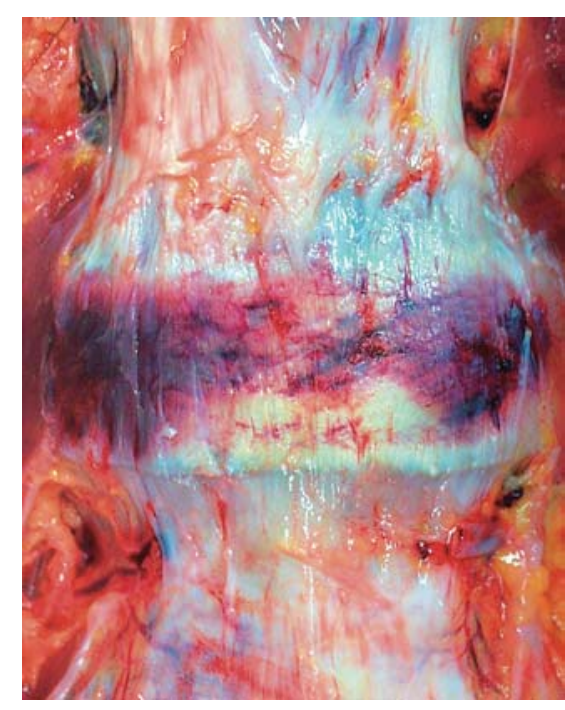

- Abb. 3.82. Aufsicht auf die mittlere Lendenwirbelsäule nach weitgehender Freilegung. Vor der Bandscheibe, unter dem straffen Längsband, eine quere, ausgedehnte Blutung, wie sie beim Erhängen (insbesondere bei freier Suspension, wie bei dieser 44-jährigen Frau) häufig beobachtet werden kann.

Der Unterscheidung in »typische« und »atypische« Erhängungsformen wurde z.B. durch Prokop (1976) ein praktischer Wert abgesprochen, da atypische Erhängungssituationen am häufigsten vorkommen (somit in anderem Sinne typisch sind). Gerade für weniger erfahrene Untersucher macht aber eine Unterscheidung Sinn, da nur vor diesem Hintergrund eine Bewertung der Plausibilität der vorhandenen äußerlichen Leichenbefunde möglich ist.

Tödliche Verletzungen der Halswirbelsäule kommen praktisch nur bei Hinrichtungen oder vergleichbaren Abläufen (d.h. Sturz über eine größere Höhe in eine Schlinge) vor. Nach älteren Darstellungen soll es dabei zu einem Abriss des Zahnfortsatzes des 2. Halswirbelkörpers oder einem Durchriss seines Haltebandes kommen, sodass der Dens das obere Halsmark quetscht; eine solche Verletzung konnte zwar (leichen)experimentell erzeugt werden (Saternus 1990), wurde aber bei konkreten Fällen kaum gefunden. Die Technik eines "good and proper hanging « soll einen raschen Tod infolge knöcherner Halswirbelverletzung mit Halsmarkschädigung bewirken und eine Dekapitation vermeiden, was durch eine geeignete Länge des Strickes (um etwa $2 \mathrm{~m}$ ) und Position des Henkersknotens (seitlich links am Unterkiefer oder unter dem Kinn) erreicht werden soll. Infolge der massiven Überstreckungsbelastung des Halses soll die typische »hangman's fracture « resultieren: Auseinanderreißen des zweiten Halswirbelkörpers. Untersuchungen der HWS von Hingerichteten zeigten häufig diese, aber auch vielfältige andere Verletzungen, jedoch auch ein gänzliches Fehlen von Frakturen. Für den bei Exekutionen typischerweise momentanen Bewusstseinsverlust und mit- 
unter raschen Herzstillstand werden z.T. reflektorische Mechanismen verantwortlich gemacht; z.T. wird ein akuter traumatischer Spinalschock mit Paralyse der lebenswichtigen Zentren im Hirnstamm angenommen.

Aufgrund der sehr schnell eintretenden Bewusstlosigkeit ist eine Selbstrettung beim Erhängen praktisch unmöglich, selbst wenn es die Situation zulassen würde (z.B. durch einfaches Aufrichten beim Hängen in hockender Position). Fälle von Überleben sind daher - im Hinblick auf die Häufigkeit des Erhängens überhaupt - sehr selten. Hierzu kann es kommen, wenn die Situation rasch genug durch andere erfasst wird und sofortige Wiederbelebungsmaßnahmen greifen. Im günstigsten Fall ist eine vollständige Restitutio möglich. Kam es noch nicht zur irreversiblen Hirnschädigung, so bedrohen vor allem pulmonale Komplikationen den Patienten (Aspirationspneumonie, acute respiratory distress syndrome), andernfalls gelingt u.U. die Wiederherstellung des Kreislaufes und sogar der Atemtätigkeit, der Patient bleibt jedoch im Koma. Mehrfach beschrieben wurden verzögert eingetretene Todesfälle z.B. infolge $\mathrm{O}_{2}$-Mangelschädigung des Gehirnes, Verschlüssen von beim Erhängungsvorgang verletzten Halsarterien oder durch Blutaspiration bei Trachealverletzung nach Reißen des Strangwerkzeugs oder Abgleiten vom Aufhängepunkt. In solchen Fällen wurden Opfer auch in einiger Entfernung vom primären Aufhängeort tot vorgefunden, die sie den Umständen nach noch selbst zurückgelegt haben mussten (erhaltene Handlungsfähigkeit trotz z.T. schwerer Verletzungen).

\section{Erdrosseln}

\section{(i) Infobox}

Der überwiegende Teil von Todesfällen durch Erdrosseln geht auf fremde Hand zurück; gelegentlich werden Suizide und ganz selten Unfälle (z.B. Verfangen eines Halstuches oder Schals in einer Maschine oder einem Rad) beobachtet. Die amtliche Todesursachenstatistik fasst alle Arten von Strangulation durch fremde Hand (also auch Erwürgen und Kombinationen) zusammen; hiernach kam es 1999 in Deutschland unter insgesamt 719 Tötungen in 112 Fällen durch Strangulation zum Tode des Opfers.

Ablauf und Befunde hängen sehr vom speziellen Hergang ab: Bei überraschender Umschlingung des Halses mit einem festen Strangwerkzeug und starker Zugwirkung kann es rasch zur Bewusstlosigkeit, fehlender Gegenwehr und damit spärlichen äußeren Verletzungen kommen. Andererseits kann es einem Opfer zunächst gelingen, eine vollständige Umschlingung z.B. mit den Händen zu verhindern, was zum erneuten Ansetzen bzw. zur Verlagerung der Schlinge führt, mit der Folge eines beträchtlichen äußeren Verletzungsbildes am Hals. Drosselangriffe sind nicht selten mit anderen Arten einer Gewalteinwirkung kombiniert, waren in unserem Material allerdings dennoch meistens die entscheidende Todesursache. Der Leichenbefund ist fast immer

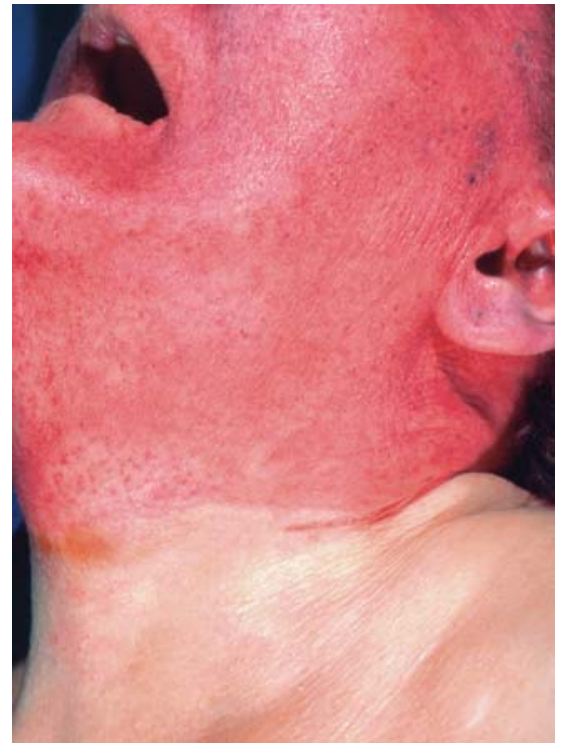

- Abb. 3.83. Intensives Stauungssyndrom mit massiven Petechien am ganzen Kopf und oberen Hals, in einer fast geraden horizontalen Linie quer über den Hals scharf gegen die blasse Haut unterhalb davon abgegrenzt. Vorne, über dem Kehlkopfbereich, eine diskrete quere gelbbraune Vertrocknung; unterhalb des Ohres eine feine, rote Linie, entstanden durch Zwischenkammblutungen. Die 76-jährige Frau war von ihrem Ehemann erdrosselt worden - den Umständen nach vermutlich mit ihrem Einverständnis. Das Strangwerkzeug (dünnes Elektrokabel) war wieder entfernt worden und lag neben der Leiche.

charakterisiert durch eine ausgeprägte obere Einflussstauung, die am Hals mit der Strangulationsebene abrupt endet (• Abb. 3.83); Stauungsblutaustritte fehlen praktisch nie. Je nach der Art des verwendeten Strangwerkzeuges und vor allem der Intensität der Drosselung kommen z.T. beträchtliche Weichteilblutungen in allen Halsschichten vor. Finden sie sich korrespondierend zur äußeren Drosselmarke, mitunter in gleicher Höhe in verschiedenen Schichten, so sind sie mechanisch bedingt (innere Strangmarke); oberhalb der Drosselungsebene sind meist stauungsbedingte Weichteilblutungen. Selten sind Verletzungen der Halsschlagadern, häufiger dagegen Brüche an Kehlkopf oder Zungenbein. Innere Verletzungen können aber auch fehlen. Mitunter sehr schwierig ist die Unterscheidung zwischen homizidalem und suizidalem Erdrosseln, falls sich nicht weitere richtungsweisende Verletzungen (etwa frische Probierschnitte beim Suizid oder Misshandlungsspuren bzw. Abwehrverletzungen bei einer Tötung) finden. Solche Suizidfälle sind sehr selten (bei uns etwa 1 Fall pro Jahr). Natürlich wird die Situation bereits primär klar erscheinen, wenn die Leiche ohne fest anliegendes, d.h. noch strangulierendes Drosselwerkzeug vorgefunden wird; ein Suizid setzt eine Fixierung der Druckwirkung z.B. durch Verknotung voraus. 
- Tabelle 3.21. Vergleich der Intensität der Drosselmarke und der inneren Halsverletzungen bei Todesfällen durch suizidales und homizidales Erdrosseln

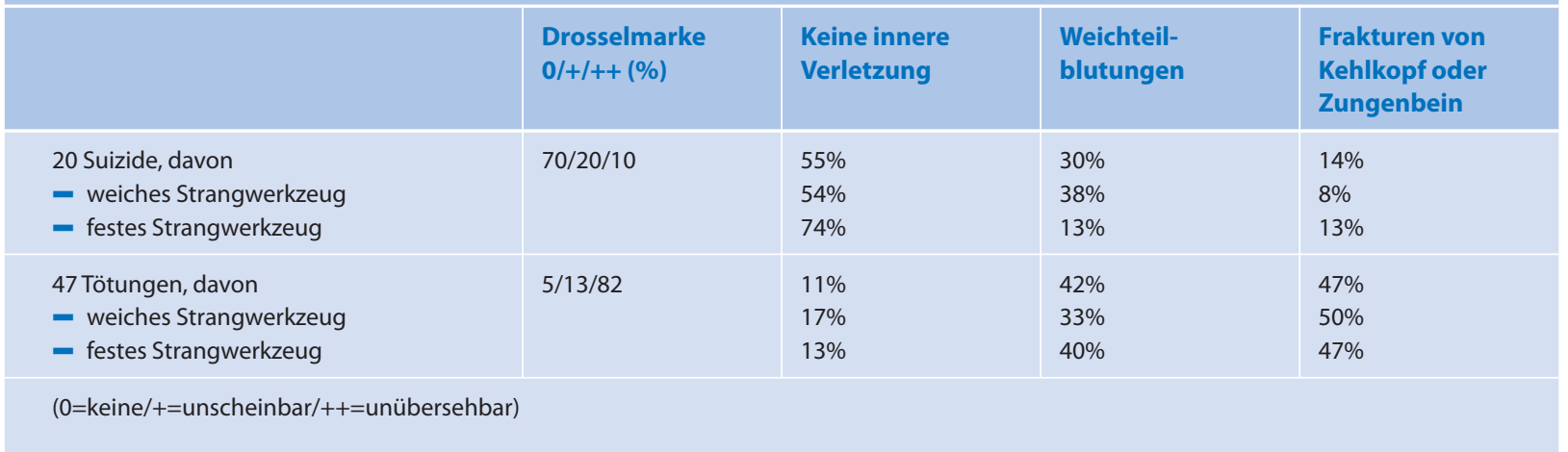

\section{(3) Fallbeispiel}

Allerdings untersuchten wir einen Fall, bei dem der seine Frau erdrosselt vorfindende Ehemann über die Situation derart in Panik geriet, dass es ihm anfänglich (beim Eintreffen der von ihm alarmierten Polizei) nicht möglich war, seine Handlungsweise ( $\mathrm{Er}$ hatte den einfach verknoteten Gürtel offensichtlich in Hilfeleistungsabsicht entfernt.) nach dem Auffinden wiederzugeben. Da der Strang jetzt nur locker über dem Hals lag, geriet er selbstverständlich in Tatverdacht und wurde festgenommen. Die rasche Aufklärung gelang glücklicherweise, da ein Rechtsmediziner sofort an den Ort gerufen wurde, und die aufgrund der Leichenerscheinungen einzuschätzende Todeszeit seine Täterschaft aufgrund der Umstände ausschloss, und die Mitwirkung einer anderen Person ermittlungsseitig nicht in Betracht kam. Der Obduktionsbefund allein hätte die Differenzierung Suizid - Tötung nicht mit Sicherheit erlaubt.

Bei suizidaler Drosselung wird meist keine grobe Kraft angewendet, was Auswirkungen auf die Ausprägung einer Drosselmarke, das innere Verletzungsbild, aber auch auf die Zeitspanne einer erhaltenen Handlungsfähigkeit hat. Daher ist es möglich, dass mehrere Strangwerkzeuge um den Hals geschlungen und auch mehrfach verknotet werden können oder andere komplexe Handlungen erfolgen.

\section{(3) Fallbeispiel}

Eine 53-jährige Frau sprang letztlich aus dem Fenster ihrer im 3. Stockwerk gelegenen Wohnung und erlitt eine geschlossene Schädelzertrümmerung. Die Leiche hatte einen Damenstrumpf $6 \mathrm{fach}$ eng um den Hals geschlungen und verknotet. Ein massiver Blutverlust (große Blutlachen in der Wohnung) war aus Schnittverletzungen an beiden Handgelenken mit Durchtrennung der Aa. radiales erfolgt. Aufgrund stark ausgebildeter Petechien an Gesicht und Hals (d.h. bei Drosselwirkung noch keine stärkere
Anämie), einem Fehlen von Beblutungen an der Halshaut unter dem Drosselwerkzeug und der weiteren Rekonstruktion war anzunehmen, dass die Frau sich zunächst die (durchaus effektive) Drosselung angelegt, dann die Pulsschlagadern aufgeschnitten hatte und schließlich in die Tiefe gesprungen war.

Der innere Leichenbefund kann eine Unterscheidung zwischen Suizid und Tötung erlauben, wenn beträchtliche Verletzungen der Halsweichteile oder des Kehlkopfes vorliegen; solche sind dem suizidalen Erdrosseln fremd. Art oder Anzahl der Strangwerkzeuge, die Anzahl der Umschlingungen oder mehrfache Verknotungen sind meist nicht für eine Differenzierung geeignet. Bei Suiziden liegen die Knoten zwar meist vorne oder seitlich, jedoch wurden auch Fälle mit Verknotung im Nacken berichtet. Eine Gegenüberstellung innerer und äußerer Halsverletzungen bei Tötungen und Selbsttötungen zeigt • Tabelle 3.21. Ein verletzungsprägender Einfluss der Art des Drosselwerkzeuges war bei unseren Fällen nicht deutlich; meistens ist die eingesetzte Kraft für die Schwere der Traumatisierung wichtiger als die Art des Strangmaterials. Der Nachweis einer Drosselung im Rahmen einer Tathandlung hat wegen ihrer besonderen Gefährlichkeit Folgen für die juristische Bewertung: In unserem Beobachtungsgut erfolgte die Verurteilung von Tätern in etwas mehr als der Hälfte wegen Mordes und etwas seltener wegen Totschlages, jedoch nie wegen Körperverletzung mit Todesfolge (Rothschild und Maxeiner 1990). Anders als bei Erhängen kommt Überleben nach Drosselangriffen nicht ganz selten vor; Opfer eines solchen Angriffes werden dann ärztlich zu untersuchen sein - medizinisch im Hinblick auf mögliche bedrohliche Verletzungsfolgen, forensisch im Hinblick auf eine eventuelle Lebensgefährlichkeit des erfolgten Angriffes (vgl. nächster Abschnitt u. • Tabelle 3.22). 
Tabelle 3.22. Stauungsblutungen und geklagte Symptome bei der rechtsmedizinischen Untersuchung von 82 überlebenden Opfern eines Angriffes gegen den Hals (überwiegend Würgen, seltener Drosseln). (Nach Strauch et al 1990)

\begin{tabular}{|l|c|c|}
\hline & Opfer ohne Bewusstlosigkeit (42) & Opfer mit Bewusstlosigkeit (32) \\
\hline Stauungsblutungen im Gesicht & $55 \%$ & $78 \%$ \\
Urinabgang & $7 \%$ & $31 \%$ \\
Kotabgang & 0 & $16 \%$ \\
Amnesie & 0 & $25 \%$ \\
Dyspnoe & $29 \%$ & $13 \%$ \\
Dysphagie & $71 \%$ & $78 \%$ \\
Dysphonie & $5 \%$ & $6 \%$ \\
\hline
\end{tabular}

\section{Erwürgen}

\begin{abstract}
(i) Infobox
Würgegriffe gegen den Hals kommen vermutlich bei vielen letztlich mit dem Tode endenden Auseinandersetzungen vor, ohne dass sie immer richtungsweisende Befunde hinterlassen müssen. Häufig sind Kombinationen mit Drosseln, mit einem gewaltsamen Verschluss der äußeren Atemöffnungen, mit stumpfer Gewalt und mit Stich- oder Schnittverletzungen. Je nach Lokalisation und Art des Griffes kann es neben dem meistens im Vordergrund stehenden Atemwegsverschluss auch zur Kompression von Halsschlagadern kommen. Die Frage eines sofortigen (reflektorischen, für den Täter nicht vorhersehbaren) Todeseintrittes infolge eines Griffes an den Hals wird sehr kontrovers diskutiert. Ob Selbsterwürgen unter exzeptionellen Umständen möglich ist, ist fraglich. Für praktische Belange ist es sicher zulässig, grundsätzlich von fremder Hand auszugehen. Im Gegensatz zum Erhängen oder Drosseln ist ein Überleben nach Würgeangriff nicht ungewöhnlich, sodass Überlebende auch zur klinischen Untersuchung kommen.
\end{abstract}

Eine Kompression der vorderen Halsweichteile ist durch unterschiedliche Handhaltungen möglich, die jeweils zu einer differenten (mehr breitflächigen oder mehr punktuell imprimierenden) Krafteinleitung und damit auch zu unterschiedlichen äußeren und inneren Spuren führen. Aufgrund von Schilderungen Überlebender (Härm und Rajs 1981) war das beidhändige Würgen von vorne die häufigste Angriffsform. Im Leichenexperiment kam es bei Würgegriffen gegen die mittlere Halsregion (Kehlkopfbereich) wie auch bei beidhändigem Würgen allenfalls zum Verschluss einer, jedoch nicht beider Halsarterien, und dies erforderte einen ziemlich hohen Kraftaufwand (18-20 kg), bei dem die Atemwege bereits verlegt waren. Dies deutet auf eine führende Rolle der Asphyxie, sodass meist ein längere Zeit erhaltenes Bewusstsein mit entsprechender Gegenwehr des Opfers anzunehmen ist. Entsprechend ausgeprägt sind auch in den meisten
Fällen die Lokalbefunde am Hals, aber auch weitere Verletzungen am Körper. Im Falle eines komplexen Tatgeschehens kann mitunter die Reihenfolge von Einwirkungen aufgrund des Vorhandenseins bzw. der Intensität eines Stauungssyndroms rekonstruiert werden; kam es z.B. aus Stichverletzungen zu einem massiven Blutverlust und findet sich an der Leiche dennoch ein Stauungssyndrom, so ging die Halskompression den Sticheinwirkungen voraus. Bei protrahierten Verläufen kann auch eine ggf. mikroskopisch nachweisbare, frühe, lokale Vitalreaktion eine zeitliche Reihung ermöglichen. Auf eine solche Untersuchung darf bei Tötungsdelikten dieser Art nicht verzichtet werden, um gravierende Fehlbeurteilungen zu vermeiden.

\section{F) Fallbeispiel}

Im Rahmen einer »Routineobduktion«, also ohne konkreten Verdacht eines Tötungsdeliktes, wurde die Leiche einer 54-jährigen Frau obduziert. Schürfungen über dem mittleren Halsbereich und kräftig umblutete Frakturen am Schildknorpel und Zungenbein wiesen auf einen Würgeangriff. Der daraufhin beschuldigte Lebensgefährte räumte ein, die Frau im Rahmen eines Streites abends am Hals gepackt zu haben, um sie »ruhig zu stellen«, was ihm auch gelungen sei. Die Frau habe dann aber erkennbar schlafend - also noch lebend - im Bett gelegen; am folgenden Morgen war sie tot. An den Halsverletzungen war bereits eine deutliche Zellemigration zustande gekommen, die tatsächlich eine Überlebenszeit anzeigte. Die toxikologische Analyse ergab eine tödliche Intoxikation durch Schlafmittel. Offenbar hatte die Frau zeitnah mit der Auseinandersetzung eine hohe Tablettenüberdosis in suizidaler Absicht eingenommen.

Weniger als $10 \%$ der bei uns untersuchten Opfer von Würgeangriffen zeigten keinerlei weitere Verletzungen. In je etwa 1/4 der Fälle bestand eine konkurrierende oder eine andere Todesursache, zumeist durch scharfe oder stumpfe Gewalt. Das äußere und innere Verletzungsmuster variiert in einem breiten Bereich - zwischen unübersehbaren (• Abb. 3.84) und fehlenden Griffspuren an der Halshaut, zwischen unverletztem und schwer deformiertem Kehlkopf. Es verwundert daher nicht, dass immer 


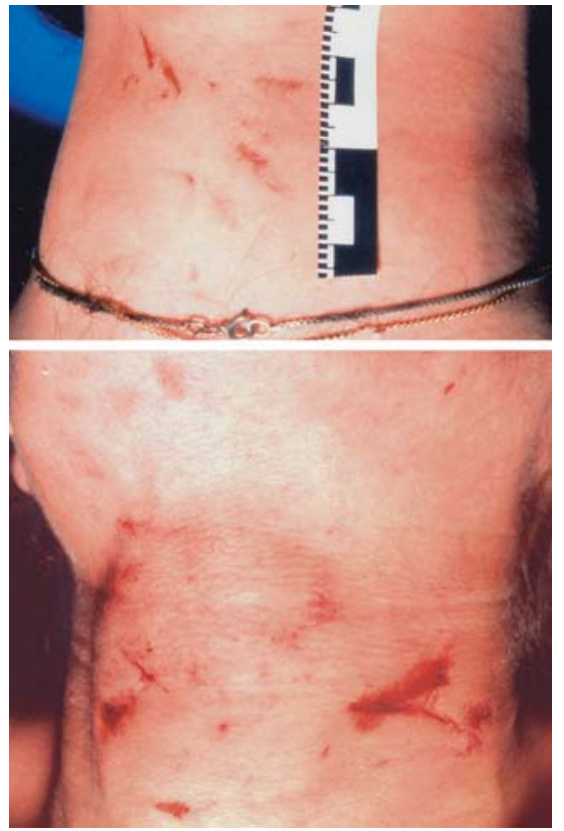

- Abb. 3.84. Folgen von Würgeangriffen an der Halshaut. In der oberen Bildhälfte schmale, z.T. bogenförmige Kratzer, konzentriert über dem Kehlkopfbereich. Die 20-jährige Frau wurde nach dem Geständnis des Täters rücklings am Boden liegend von vorne beidhändig erwürgt. In der unteren Bildhälfte (26-jährige Frau, Tötung durch Erwürgen, wohl von vorne) auch größere Abschürfungen (scharfkantiger Anhänger einer Halskette) und diskrete Hautblutungen. Verletzungen finden sich in diesem Fall auch weit seitlich bzw. hinten am Hals

wieder Fälle beschrieben werden, bei denen durch einen Leichenschauarzt ein natürlicher Tod attestiert wurde und die Tat erst nachträglich z.B. durch Anzeigen Dritter oder Geständnisse aufkam. Das Gesicht kann infolge einer u.U. trotz Hypostase erhalten gebliebenen Stauung düsterbläulich sein, muss es aber nicht. Stauungsblutaustritte sind dagegen praktisch immer vorhanden, jedoch variiert deren Intensität von Fall zu Fall stark.

\section{Akuter Reflextod}

Da wir gezwungen sind, von den Befunden Rückschlüsse auf die Art, Schwere und Zeitdauer der Gewalteinwirkung zu ziehen, stellt sich bei Minimalbefunden die Frage nach der Möglichkeit eines Todes trotz nur geringer und kurzdauernder Einwirkung gegen den Hals (akuter Reflextod). Auch in diesem Fall mag der Kausalzusammenhang zwischen Tat und Tod unzweideutig sein; für den Täter dürfte aber ein solcher Verlauf nicht vorhersehbar sein, sodass ihm eine Tötungsabsicht nicht unterstellt bzw. zumindest nicht nachgewiesen werden könnte. Die Unsicherheit in der Beurteilung solcher Fälle ist beträchtlich. In unserem Beobachtungsmaterial (das immerhin einen hohen Anteil an Fällen mit schweren inneren Halsverletzungen ent- hält) wurden Tötungen durch alleiniges Erwürgen nur in einem Viertel der Fälle juristisch als Mord, in mehr als der Hälfte der Fälle als Totschlag, und in immerhin 13\% »lediglich « als Körperverletzung mit Todesfolge qualifiziert. Nahezu alle Standardwerke des Faches verweisen darauf, dass die jeweils anderen Autoren in ihrer Mehrzahl »zugeben, dass es solche akuten reflektorischen Todesfälle gibt «, jedoch liegen bei kritischer Überprüfung überzeugende Falldarstellungen kaum vor. Sehr kompliziert wird die Situation, da eine durch ggf. nur kurz bedingte Druck- oder Schlagwirkung gegen den Hals ausgelöste vagale Irritation nicht nur zum sofortigen Herzstillstand führen soll, sondern auch zur Auslösung letztlich tödlicher Herzrhythmusstörungen. Dies würde eine den Angriff überdauernde Zeit einer Kreislauftätigkeit zur Folge haben, sodass es auch zu Blutunterlaufungen an Verletzungen und u.U. zu einem Lungenödem kommt, sodass deren Vorhandensein keinen Rückschluss auf eine Länge der Halskompression zulassen würde (ausführliche Erörterungen hierzu s. Polson and Gee 1973 und vor allem Knight 1996). Dieser Autor hält reflektorische Mechanismen in etwa der Hälfte seiner Fälle für den entscheidenden Mechanismus und führt als Beleg hierfür u.a. das häufige Vorhandensein eines blassen Gesichtes an. Gerade dieser Befund ist nach eigener Erfahrung ( $\bullet$ Abb. 3.72) aber ungeeignet für so weitreichende Rückschlüsse. Hinzu kommt, dass die Intensität von Verletzungen und weiteren Folgen (hier: Kopfstauung) nicht nur von der Stärke des Angriffes, sondern auch von der Konstitution des Opfers abhängen dürfte. So ist ein im Vergleich zu einem jungen, kräftigen Individuum spärlicherer Befundkomplex bei einem Greis auch ohne Annahme von den Sterbeverlauf abkürzenden reflektorischen Mechanismen durch eine Einschränkung seiner kardiopulmonalen Leistungsreserve erklärbar. Aufgrund einer Literaturauswertung kommen Kleemann et al. (1990) auch zu einer völlig gegensätzlichen Einschätzung, wonach die »Wahrscheinlichkeit eines Karotisreflextodes als äußerst gering anzusehen « und bei gesunden Personen praktisch auszuschließen sei (in dieser Deutlichkeit auch: DiMaio 1989). Obwohl die Angabe einer exakten Mindestzeit eines zum Tode führenden Würgens nach heutiger Kenntnis nicht möglich ist, weist doch die weit überwiegende Zahl an bekannt gewordenen experimentellen Daten, Befunden und Umständen nach hiesiger Interpretation auf einen weit eher nach Minuten denn nach Sekunden abzuschätzenden Zeitraum hin. Die Bewertung mancher Einzelfälle ist allerdings dennoch überaus schwierig, etwa wenn ernsthaft konkurrierende Letalfaktoren (z.B. hochgradige Alkoholintoxikation) vorliegen.

\section{"Selbsterwürgen«}

Allgemein wird davon ausgegangen, dass ein eigenhändiges Würgen zwar möglich, ein Selbsterwürgen jedoch nicht möglich ist, da mit Eintritt der Bewusstlosigkeit die aktiv würgende Kraft der Hand oder Hände zusammenbricht. In der Literatur gibt es ganz vereinzelte Beschreibungen, die eine solche Möglichkeit in beson- 
deren Fällen (z.B. auf Unterlage aufgestützte Hände, zwischen denen der Hals aufliegt) diskutieren.

\section{Überlebende}

Von allen Strangulationsarten kommen wache Überlebende eines Würgens am häufigsten zur medizinischen Untersuchung. Auch diese Erfahrungen belegen die besondere Bedeutung der Länge des für eine Todesfolge notwendigen Zeitintervalls einer anhaltenden Halskompression. Das äußerliche Verletzungsbild kann hier sehr intensiv sein und ähnelt dem bei Todesfällen ohne weiteres, insbesondere auch im Auftreten und der Intensität petechialer Stauungsblutungen im Gesicht (Härm und Rajs 1981, Strauch et al. 1990). Die am häufigsten geschilderten Symptome ergeben sich aus der - Tabelle 3.22. Bei Würgeangriffen kommt es nicht selten zu Frakturen, die einfache Abbrüche von Zungenbein- oder Schildknorpelhörnern weit übersteigen und eine Instabilität des Kehlkopfes bewirken können, sowie zu beträchtlichen Quetschungsblutungen im Kehlkopfinneren; häufig scheinen solche Folgen bei Überlebenden allerdings nicht zu sein. Hiervon abgesehen wird die Lunge bei einer massiven Halskompression u.U. beträchtlich in Mitleidenschaft gezogen, durch die stark intensivierte Atmung gegen den Widerstand mit Auftreten hoher negativer intrathorakaler Drücke. Die Entwicklung eines Lungenödems als Folge eines temporären Atemwegsverschlusses (meist aus innerer Ursache, jedoch vereinzelt auch als Strangulationsfolge) wurde klinisch wiederholt beschrieben. Patienten mit lokalen Halsbeschwerden sollten alsbald HNO-ärztlich untersucht werden; bestehen gar Atembeschwerden, so ist der Patient u.U. in akuter Lebensgefahr. Gutachtlich besteht Einhelligkeit darüber, dass beim Vorliegen intensiver Verletzungsspuren am Hals in Verbindung mit einem ausgeprägten Stauungssyndrom des Kopfes von einer konkreten Lebensgefährlichkeit des Angriffes ausgegangen werden kann.

\section{Seltene Strangulationsformen}

Neben Erdrosseln und Erwürgen kommen bei Tätlichkeiten auch Halskompressionen mit dem Fuß oder mit festen Gegenständen vor; eine systematische Zusammenstellung der inneren Befunde bei solchen seltenen Einzelberichten liegt nicht vor. Bei eigenen derartigen Fällen war das innere Verletzungsbild immer besonders stark ausgeprägt, was typisch sein dürfte. Die besondere »Wirksamkeit» eines Druckes auf den Vorderhals machen sich Armhaltegriffe (Unterarmwürgegriffe) zunutze, die ebenfalls bei Auseinandersetzungen, aber auch bei polizeilichen Maßnahmen oder bei bestimmten Sportarten angewendet werden; im weitesten Sinne handelt es sich um den allgemein gebräuchlichen Begriff des »Schwitzkastens «. Hierbei kann der Vorderhals entweder in den Winkel zwischen den Ober- und Unterarm des seinen einen Arm anwinkelnden Angreifers eingeklemmt werden, oder es wird der Unterarm quer direkt auf den Hals gedrückt, wobei die zweite Hand den auf den Hals drückenden Arm fixiert und den Druck kontrolliert. Hierbei ist nicht nur ein Verschluss der Hals- sondern auch der Wirbelarte- rien möglich (Denk et al. 1990). Die Folge ist die - gewollte innerhalb weniger Sekunden eintretende Handlungsunfähigkeit; wird der Druck (etwa bei selbst erregtem, nicht kontrolliert agierendem Angreifer) zu lange aufrecht erhalten, so kann es zu Todesfällen kommen. Über Jahrzehnte wurde lediglich ein einziger Todesfall (infolge den Hals betreffender Haltegriffe) beim Judo-Sport gemeldet. Der 21-jährige Mann wurde etwa $1 / 2$ Minute von hinten am Hals gehalten, gab auf und war zunächst symptomfrei. Am Ende des Matches kollabierte er; die Autopsie ergab »einige Spuren einer Verletzung von Halsgefäßen «; vermutet wurde ein etwas verspätet einsetzender Reflextod infolge dieser Halsgefäßschäden.

\subsubsection{Tod im Wasser}

\section{W. Keil}

Todesfälle im Wasser sind zumeist durch Unfälle bedingt. Relativ häufig sind Kinder betroffen, die nicht schwimmen bzw. sich nicht selbst retten können (z.B. Unfälle in Schwimmbädern, Swimmingpools oder Gartenteichen). Der Anteil älterer Menschen, die im Wasser versterben, hat zugenommen.

\section{(i) Infobox}

Im Jahr 2004 wurden in Deutschland 401 Unfälle durch Ertrinken oder Untergehen registriert. 47 Opfer waren Kinder unter 10 Jahren. 102 Personen waren älter als 70 Jahre. In 62 Fällen kam es zum Tod in der Badewanne.

Die Unfälle können durch Eigenschaften des Wassers und durch Konditionen des Verunfallten zustande kommen:

- Wasser: Tiefe, Temperatur (sehr kalt/sehr warm), Strömung, Brandung;

- Verunfallter: Schwimmer/Nichtschwimmer, überhitzt ins Wasser (z.B. nach stärkerer Sonneneinstrahlung), Sprung ins Wasser, Unterkühlung im Wasser, Erschöpfung, Panik, vorbestehende Erkrankungen, Kollapsneigung, Einnahme zentral wirksamer Substanzen. Alkoholische Beeinflussung und reichlicher Füllungszustand des Magens sollen den Todeseintritt im Wasser grundsätzlich begünstigen.

Suizide kommen wesentlich seltener vor (z.B. Sprung ins Wasser von hohen Brücken). Tötungsdelikte sind Raritäten (z.B. durch Ertränken in der Badewanne oder Ins-Wasser-Stoßen). Häufigste Todesursache ist das Ertrinken. Der so genannte Badetod oder spezielle Formen der Tauchunfälle sind selten zu beobachten. Vereinzelt kommt es im Wasser zu plötzlichen Todesfällen aus natürlicher Ursache. 


\section{Ertrinken}

\section{Definition}

Ertrinken ist eine Form des Erstickens aufgrund einer Flüssigkeitsaspiration, zumeist der Aspiration von Wasser, wobei das Individuum während der Aspiration zumindest mit dem Gesicht in die Flüssigkeit eingetaucht war.

Bei industriellen Unfällen kann es zum Ertrinken außer in Wasser auch in anderen Flüssigkeiten kommen (z.B. Benzin, Milch). Bewusstlosen kann Wasser in Mund und Nase eingebracht werden, sodass sie an der Flüssigkeitsaspiration sterben. In diesen seltenen Fällen handelt es sich per definitionem nicht um Ertrinken.

Pathophysiologie. Es sind theoretisch folgende Stadien abgegrenzt worden:

- Inspiration: Zumeist reflektorisch ausgelöst beim plötzlichen Hineinkommen ins kalte Wasser, vermittelt durch Rezeptoren an Rücken- und Brusthaut.

- Apnoe: Willkürliches Anhalten der Atmung, nur kurze Zeit möglich.

- Dyspnoe: Anstieg der $\mathrm{CO}_{2}$-Konzentration mit Reizung des Atemzentrums und unwillkürlichem Wiedereinsetzen der Atmung. Wasser gelangt in den Kehlkopf, wodurch es zum Husten kommt. Dabei, aber auch später, können nicht unerhebliche Flüssigkeitsmengen verschluckt werden. Erbrechen kann auftreten. Letztlich Eintritt von Bewusstlosigkeit durch den $\mathrm{O}_{2}$-Mangel, sodass Wasser in Luftröhre und Bronchien aspiriert wird. Durch die Erhöhung des Atemwiderstandes Ausbildung eines zumeist monströsen akuten Lungenemphysems (Emphysema aquosum). Während des krampfhaften Atmens Vermischung von Wasser, Luft und Atemwegsschleim, sodass ein weißlich-schaumiger Atemwegsinhalt resultiert.

- Krampfstadium: Tonisch-klonische Krämpfe infolge fortschreitender zerebraler Hypoxie.

- Atemlähmung: Zunächst präterminale Atempause, dann finale Schnappatmung, schließlich Atemstillstand, während der Herzschlag, wie bei den anderen Erstickungsformen auch, noch einige Zeit erhalten bleiben kann.

Ein Ertrinkungsvorgang soll vier bis fünf Minuten dauern. Anhand von Augenzeugenberichten ergibt sich zumeist kein Anhalt für einen relevanten Überlebenskampf im Wasser. Im Gegensatz zu früheren Meinungen ist das Volumen der aspirierten Flüssigkeit in der Regel relativ klein. Nach Erkenntnissen der Notfallmedizin hat die Osmolarität des Wassers nur einen geringen Einfluss auf den Pathomechanismus, obwohl das hypotone Süßwasser in den Alveolen schnell resorbiert und im Körper verteilt werden kann. Dennoch werden bei Beinahe-Ertrunkenen weder signifikante Zunahmen des Wasseranteils im Blut (Hydrämie) noch relevante Verschiebungen der Serumelektrolytkonzentrationen beobachtet. Früher wurde die Ansicht vertreten, dass es besonders beim Ertrinken im hypertonen Salzwasser durch Osmose zur Ausbildung eines Lungenödems komme (Oedema aquosum). Demgegenüber ist heute gesichert, dass unabhängig von der Osmolarität das eingeatmete Wasser, also auch Süßwasser, zu einem Lungenödem führen kann, welches neben dem akuten Emphysem häufig zu beobachten ist.

Leichenschau. Schaum vor Mund und/oder Nase, Schaumpilz genannt (• Abb. 3.85), kann der einzige äußere Hinweis auf einen Ertrinkungstod sein. Bei unmittelbar aus dem Wasser geborgenen Leichen kann der Schaumpilz noch fehlen und sich erst später ausbilden. An der Luft trocknet er rasch ein, sodass nur noch geringe weißliche Schleimspuren perioral sowie in und um die Nasenöffnungen auf ihn hindeuten.

Obduktionsbefunde. Befunde mit hohem Beweiswert für die Diagnose:

\section{- Lungen und Atemwege:}

- Weißlich-schaumiger, teils wässriger Inhalt in den Atemwegen, evtl. mit Schaumpilzbildung. Selten können zusätzlich feste Partikel (z.B. Pflanzenteile, Sand) aus dem Wasser in den Atemwegen aufgefunden werden.

- Akutes Lungenemphysem (Emphysema aquosum), häufig bei gleichzeitig bestehendem intraalveolären Ödem (Oedema aquosum). Dabei sind beide Lungen in der Regel so voluminös, dass sie sich vorn im Mediastinalraum berühren oder fast berühren (• Abb. 3.86). Mit dem Emphysem geht ein Elastizitätsverlust des Lungengewebes

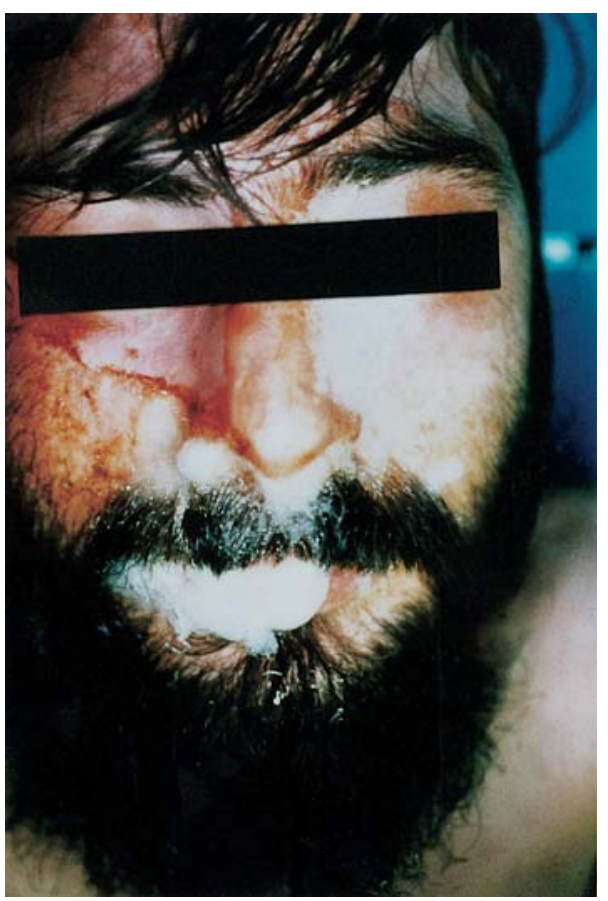

- Abb. 3.85. Schaumpilz, 7 Stunden postmortal beobachtet (31 Jahre alter Mann, Sportbootunfall) 


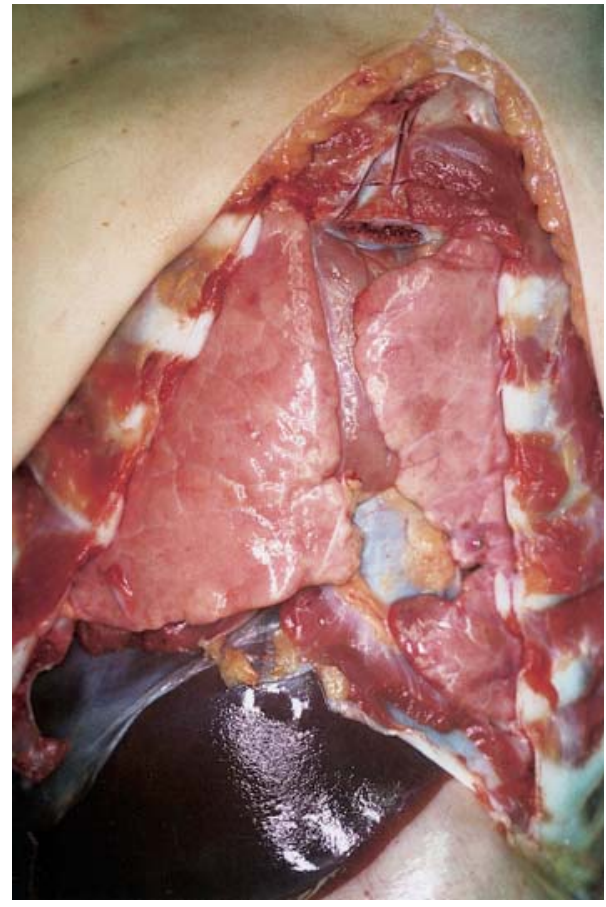

- Abb. 3.86. Emphysema aquosum. Die monströs geblähten Lungen berühren sich fast im Mediastinum (14 Monate altes Kind, Ertränken durch die Mutter in der Badewanne)

einher, d.h. durch die Finger bedingte Eindrücke auf der Lungenoberfläche bleiben als Eindellungen bestehen (aufgehobenes Retraktionsvermögen).

- Selten zusätzlich Aspiration von erbrochenem Speisebrei.

- Magen und Zwölffingerdarm:

- Verwässerter Mageninhalt, bei dem nach Umfüllen in durchsichtige Gefäße eine Dreischichtung beobachtet werden kann. Oben: weißer Schaum, offenbar durch Bauchpresse beim Ertrinken verursacht bzw. verschlucktes Material aus den Atemwegen. Mitte: Wasser. Unten: feste Nahrungsbestandteile (Wydler'sches Zeichen).

- Selten Schleimhautrisse, besonders am Mageneingang, wie beim Mallory-Weiß-Syndrom, offenbar durch Erbrechen während des Ertrinkungsgeschehens verursacht (Sehrt'sche Schleimhautrisse).

- Selten verwässerter Inhalt im Zwölffingerdarm.

Befunde mit niedrigem Beweiswert für die Diagnose:

\section{- Schädel:}

- Nachweis von vermehrter Flüssigkeit, d.h. Ertrinkungsflüssigkeit, in der Keilbeinhöhle (Svechnikov-Zeichen). Dieser Befund gilt bis zum Eintritt von Fäulnis als zusätzliches Indiz für die Sicherung der Diagnose Ertrinken.
- Einblutungen in die Cellulae mastoideae. Postmortale Entstehung durch Hypostase erscheint möglich.

- Lungen:

- Rötliche Flecken unter der Pleura pulmonalis, benannt nach ihrem Erstbeschreiber (Paltauf-Flecken). Paltauf'sche Flecken werden als durch die Ertrinkungsflüssigkeit hämolysierte Petechien angesehen (größer als Tardieu'sche Flecken), sie treten keineswegs konstant auf und sind häufig schwer zu beurteilen.

Unspezifische Befunde:

- Flüssiges Leichenblut

- Akute Blutfülle der inneren Organe, häufig nicht der Milz.

( Wichtig

Durch Reanimationsmaßnahmen (z.B. Absaugen der Atemwege, Beatmung) können die ursprünglichen Ertrinkungsbefunde erheblich verändert oder sogar beseitigt werden. Bei längerer Leichenliegezeit verändern Autolyse und Fäulnis die Befunde derart, dass der Ertrinkungstod morphologisch nicht mehr nachgewiesen werden kann.

Zusatzuntersuchungen mit niedrigem Beweiswert für die Diagnose:

- Diatomeennachweis: Der Nachweis kann aus Lebergewebe oder Knochenmark erfolgen. Diatomeen (Kieselalgen, von denen es einige tausend Arten gibt) können mit der Ertrinkungsflüssigkeit in den Blutkreislauf gelangen. Da Diatomeen jedoch ubiquitär sind, hat ihr Auffinden für den Nachweis eines Ertrinkungstodes häufig keine entscheidende Bedeutung.

- Mikroskopischer Nachweis lädierter elastischer Fasern in den Alveolarsepten.

\section{So genannter Badetod - Reflexmechanismen im Wasser}

\section{Definition}

Plötzliches Untertauchen mit Todeseintritt, dessen Ursache vor allem durch im Wasser ausgelöste vagale Reflexe erklärt wird. Demzufolge können die pathologisch-anatomischen Ertrinkungsbefunde nicht oder nur in verminderter Ausprägung beobachtet werden.

Pathophysiologie. Einige im Kopf-Halsbereich auslösbare Reflexe haben eine vagotone Wirkung und können schnell zu Kreislaufzentralisation und Asystolie führen, wodurch der plötzliche Todeseintritt beim Untertauchen erklärbar wird. So ist der Ebbecke- oder »dive«-Reflex durch kaltes Wasser an der Gesichtshaut auslösbar, im Kehlkopf kann der Vagusanteil des N. laryngeus superior gereizt werden. Außerdem wird der okulokardiale Reflex diskutiert. Darüber hinaus sind zu berücksichtigen: ValsalvaMechanismus, Kälteurtikaria, Laryngospasmus. Alkoholisierung, die bei derartigen Fällen nicht selten vorliegt, kann die Wasser- 
induzierten Vaguseffekte verstärken. Da die Ertrinkungsbefunde in diesen Fällen nicht oder nur gering ausgebildet sind, wurde von manchen Autoren dafür der Begriff des atypischen Ertrinkens verwendet.

\section{Leichenschau und Obduktionsbefunde}

Die Ertrinkungsbefunde fehlen oder haben nur geringe Intensität. Manchmal existieren vorbestehende körperlichen Leiden, z.B. Virusinfektionen (kardiale Infektion bis hin zur dezenten Myokarditis), die für sich allein den Tod keineswegs erklären würden, aber die reflexbedingte Asystolie begünstigen können. Insofern ist bei derartigen Fällen neben der histologischen auch eine virologische Diagnostik am Leichengewebe durchzuführen.

\section{Tod beim Tauchen}

Je nach Bedingungen spielen sehr unterschiedliche Mechanismen eine Rolle. Zwei praktisch häufigere Situationen sind:

Apnoe-Tauchen. Beim Tauchen ohne Hilfsmittel wird vorbereitend hyperventiliert, um die Apnoezeit zu verlängern. Die vermehrte Abatmung von $\mathrm{CO}_{2}$ führt zur Verminderung des $\mathrm{CO}_{2}$ Partialdruckes im Blut. Damit ist der Reiz auf das Atemzentrum herabgesetzt und eine einsetzende Hypoxie kann unbemerkt zu plötzlicher Bewusstlosigkeit führen (Schwimmbad-Blackout), sodass unter Wasser ein Ertrinken resultieren kann.

Tauchen mit Pressluft. Dabei sind beim Auftauchen die Dekompressionszeiten und -tiefen einzuhalten, da es sonst zur zu schnellen Stickstoffentsättigung mit Gasblasenbildung in den Kapillaren und den fettreichen Geweben kommt (Caisson-Krankheit). Die häufigste Folge bei Überlebenden sind Osteoarthropathien. Bei den seltenen Todesfällen sind immer Untersuchungen auf kardiale, pulmonale bzw. zerebrale Gasembolien durchzuführen. Allerdings sind die meisten tödlichen Unfälle beim Tauchen mit Pressluft durch Ertrinken bedingt, vor allem weil in Panik (z.B. Klaustrophobie) der Atemregler nicht mehr in den Mund genommen wird.

\section{Plötzlicher Tod im Wasser aus natürlicher Ursache}

\section{Definition}

Plötzlicher krankheitsbedingter Tod, zu dem es nur zufälligerweise beim Schwimmen oder Baden kommt.

Diese Todesfälle sind vor allem durch Erkrankungen des Herzkreislaufsystems bedingt, die den plötzlichen Todeseintritt zwanglos auch außerhalb des Wassers erklären könnten. Bei Kindern können angeborene, nicht erkannte Herzfehler oder Aortenisthmusstenosen eine Rolle spielen. Bei Erwachsenen kommen Myokardinfarkte oder rupturierte Aortenaneurysmen vor. In allen Altersgruppen ist an epileptische Anfälle zu denken. Bei der Obduktion können neben dem zum Tode führenden Leiden in der Regel auch Ertrinkungsbefunde unterschiedlicher Intensität beobachtet werden, die sich agonal ausgebildet haben.

\section{Nichtertrinkungsbedingter, nichtnatürlicher Tod im Wasser}

Es handelt sich vor allem um Suizide, die in der mit Wasser gefüllten Badewanne realisiert werden, z.B. Tablettenintoxikationen, Pulsaderschnitte oder Einwirkung von Elektrizität (Haartrockner).

\section{Besonderheiten von Wasserleichen}

Totenflecke. Leichen treiben häufig in Bauchlage, mit dem Kopf nach unten. Insofern sind die Totenflecke, die oft nur spärlich ausgeprägt sind, zumeist an Kopf, Hals sowie am vorderen Schultergürtel vorhanden.

Waschhaut. Mit zunehmender Liegezeit im Wasser quillt besonders die Haut an den Handflächen und Fußsohlen, beginnend an den Fingerbeeren, auf und verfärbt sich weiß - ein Vorgang, den man in geringerer Intensität auch vom Lebenden her kennt. Anschließend geht diese Erscheinung auch auf die Streckseite der Hände und Füße über. Die Nägel können sich lösen und die Oberhaut kann zumindest partiell handschuhartig abziehbar werden (• Abb. 3.87a, b). Je höher die Wassertemperatur ist, des-
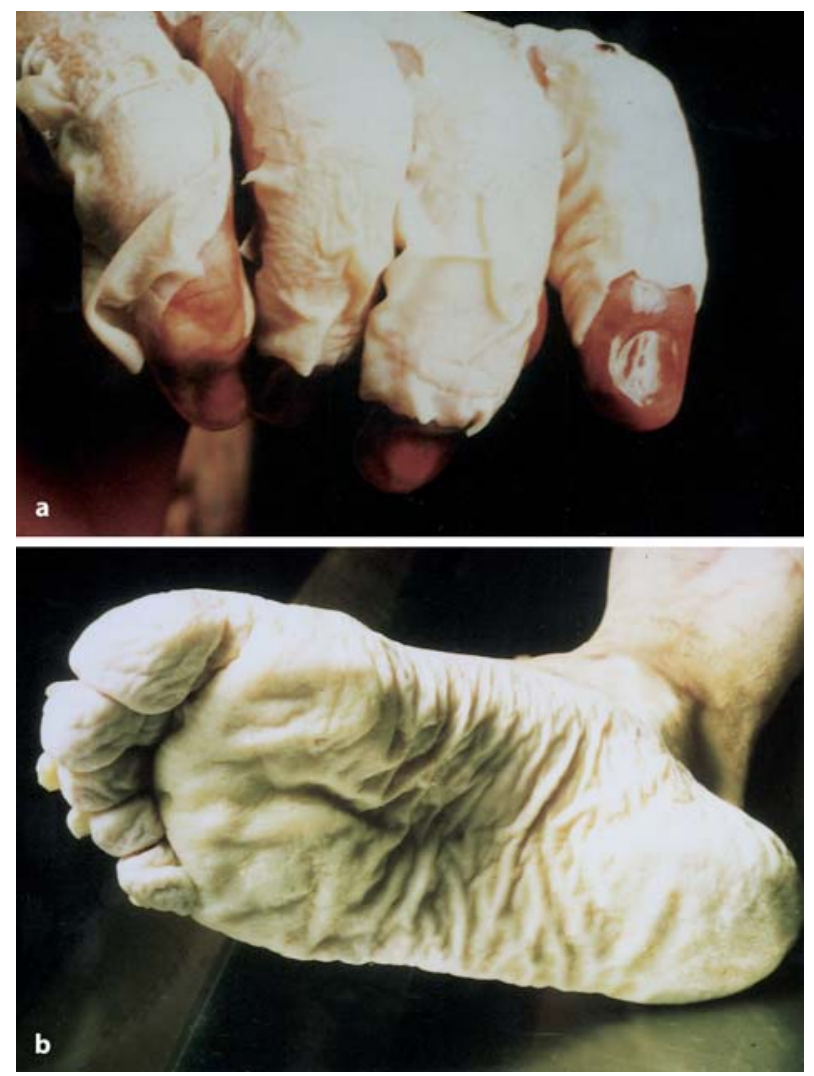

- Abb. 3.87a, b. Fortgeschrittene Waschhautbildung an a Hand mit handschuhartiger Ablösung der Epidermis sowie Verlust der Fingernägel und $\mathbf{b}$ Fuß 
- Tabelle 3.23. Mindes-Wasserzeiten (in Tagen) von Leichen, die nach der Bergung 2-3 Tage lang im Kühlraum bei $+4^{\circ} \mathrm{C}$ lagerten (nach Reh)

\begin{tabular}{|c|c|c|c|c|c|c|c|c|c|c|c|c|}
\hline & \multicolumn{12}{|c|}{ Monat der Bergung } \\
\hline & Jan. & Febr. & März & April & Mai & Juni & Juli & Aug. & Sept. & Okt. & Nov. & Dez. \\
\hline & \multicolumn{12}{|c|}{ Durchschnittliche Wassertemperatur $\left({ }^{\circ} \mathrm{C}\right)$} \\
\hline & 3,2 & 3,9 & 5,8 & 9,9 & 13,0 & 17,4 & 18,6 & 18,6 & 17,3 & 13,2 & 8,8 & 4,7 \\
\hline 1. Venenzeichnung & 35 & 25 & $16(23)$ & $9-10$ & $4-5$ & 2 & $1-2$ & 2 & 3 & $4-5$ & 10 & 17 \\
\hline 2. Leiche aufgebläht & 35 & 25 & $16(23)$ & 10 & $4-5$ & $2-3$ & 2 & 3 & $3-4$ & 7 & 10 & 17 \\
\hline 3. Leiche stark verfärbt & 35 & 25 & $16(23)$ & (14) & $4-5$ & 2 & 2 & 3 & $3-4$ & 7 & 10 & 17 \\
\hline 4. Oberhaut abgelöst & 35 & 25 & $16(23)$ & (16) & $4-5$ & 3 & 2 & 3 & $3-4$ & 7 & 10 & 17 \\
\hline 5. Haare abgelöst & 35 & 25 & $16(23)$ & $10-12$ & $4-5$ & $2-3$ & $2-3$ & 3 & $3-4$ & 7 & 10 & 17 \\
\hline $\begin{array}{l}\text { 6. Hände: Beginn der } \\
\text { Waschhautbildung }\end{array}$ & (1) & (1) & $\begin{array}{l}(12 \\
\text { Std. })\end{array}$ & & & (6 Std.) & & & 2 Std. & & 2 Std. & (1) \\
\hline 7. Nägel gelockert & 35 & $\begin{array}{l}28-30 \\
40)\end{array}$ & 23 & 16 & 5 & $2-3$ & 3 & 3 & $3-4$ & 11 & 17 & 28 \\
\hline 8. Waschhaut-Fetzen & $\begin{array}{l}\text { über } \\
35\end{array}$ & $\begin{array}{l}30-32 \\
(45)\end{array}$ & 23 & 16 & 10 & 3 & 3 & $3-4$ & 4 & 7 & 20 & 28 \\
\hline 9. Nägel abgelöst & $\begin{array}{l}\text { über } \\
35\end{array}$ & 45 & $30(40)$ & 21 & 14 & 8 & 3 & 4 & 10 & $\begin{array}{l}\text { über } \\
11\end{array}$ & 20 & $\begin{array}{l}\text { über } \\
35\end{array}$ \\
\hline $\begin{array}{l}\text { 10. Füße: Beginn der } \\
\text { Waschhautbildung }\end{array}$ & (1) & (1) & $\begin{array}{l}(12 \\
\text { Std. })\end{array}$ & (1) & & (6 Std.) & $1 / 2$ Std. & & 2 Std. & & 2 Std. & (1) \\
\hline 11. Nägel gelockert & $\begin{array}{l}\text { über } \\
53\end{array}$ & 40 & $26(35)$ & 17 & 10 & 5 & 3 & 4 & 8 & 12 & 17 & 28 \\
\hline 12. Waschhaut-Fetzen & $\begin{array}{l}\text { über } \\
53\end{array}$ & 60 & 35 & 16 & 10 & 5 & 3 & $5-6$ & $8-9$ & $\begin{array}{l}\text { über } \\
11(14)\end{array}$ & 20 & 28 \\
\hline 13. Nägel abgelöst & $\begin{array}{l}\text { über } \\
53\end{array}$ & $\begin{array}{l}\text { über } \\
60\end{array}$ & 53 & $\begin{array}{l}\text { über } \\
35\end{array}$ & $\begin{array}{l}\text { über } \\
28\end{array}$ & $\begin{array}{l}\text { über } \\
10\end{array}$ & 3 & $\begin{array}{l}\text { über } \\
10\end{array}$ & $\begin{array}{l}\text { über } \\
10\end{array}$ & $\begin{array}{l}\text { über } \\
11\end{array}$ & $\begin{array}{l}\text { über } \\
20\end{array}$ & $\begin{array}{l}\text { über } \\
35\end{array}$ \\
\hline 14. Pleura-Transsudat* & 35 & $25(40)$ & $18(35)$ & 10 & 5 & $3-4$ & 3 & 3 & 5 & 11 & $\begin{array}{l}\text { über } \\
20\end{array}$ & \\
\hline 15. Herz blutleer & $\begin{array}{l}\text { über } \\
39\end{array}$ & $\begin{array}{l}32-34 \\
(40)\end{array}$ & 23 & $14-15$ & 9 & 4 & 3 & 3 & 5 & 11 & 20 & 28 \\
\hline 16. Gehirn erweicht & 35 & $30(40)$ & (23) & $14-15$ & 5 & $3-4$ & 3 & 3 & 6 & 10 & 17 & 28 \\
\hline
\end{tabular}

to schneller kommt es zur Waschhautausbildung. Aufgrund der Intensität der Waschhautbildung kann bei bekannter Wassertemperatur grob auf die Liegezeit im Wasser geschlossen werden. Zur Einschätzung der Mindest-Wasserzeit bietet die Tabelle von Reh (- Tabelle 3.23) Anhaltspunkte, die nicht nur die Waschhautbildung, sondern auch die Progression der Fäulnisveränderungen in
Abhängigkeit von der aktuellen Wassertemperatur (zu messen $0,5-1 \mathrm{~m}$ unter dem Wasserspiegel) berücksichtigt. Die vermutliche maximale Liegezeit ergibt sich unter Berücksichtigung der noch nicht vorliegenden Leichenerscheinungen.

Weitere Oberhautablösungen. Außer an Händen und Füßen kommt es in allen anderen Körperregionen ebenfalls zu Ober- 


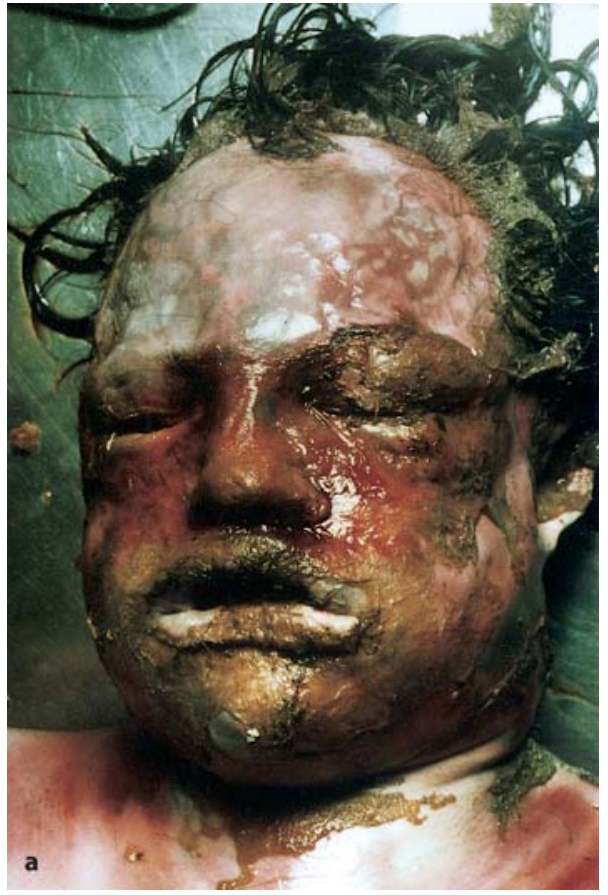

- Abb. 3.88. Algenrasen der Gesichtshaut mit Ausnahme der Stirn (Treibhaltung mit treibender Stirn über dem Wassergrund: fehlender Algenrasen und Oberhautverlust)

hautablösungen, die allerdings keine weißliche Verfärbung aufweisen. Auch dieser Befund ist stark von der Wassertemperatur abhängig. Es kann vor allem bei noch frischen Leichen beobachtet werden, die in sehr warmem Wasser $\left(\mathrm{ca} . \mathrm{ab} 45^{\circ} \mathrm{C}\right)$ gelegen haben, z.B. in der Badewanne. Das Phänomen sollte nicht mit der später einsetzenden allgemeinen Autolyse, die ebenfalls zu Oberhautablösungen führt, verwechselt werden.

Skelettmuskelverfärbungen. Durch die Einwirkung von heißem Wasser, mindestens ca. $50^{\circ} \mathrm{C}$, beginnt sich die Skelettmuskulatur zu verfärben. Sie zeigt eine blassbraune Farbe und wirkt hitzedenaturiert. Die Veränderung kann zugleich mit den erwähnten Oberhautablösungen beobachtet werden.

Algenrasen. Bei Leichen in Gewässern kann es schon nach wenigen Tagen zu einem Algenrasen auf der Haut kommen (- Abb. 3.88). Nach vorsichtiger Entfernung der Anhaftungen mit dem Messerrücken ist die Körperoberfläche oft gut beurteilbar.

Fettwachsbildung. Wasser begünstigt das Auftreten dieser Leichenerscheinung.

Postmortale Verletzungen. Treibverletzungen, bedingt durch Schleifen auf dem Gewässergrund, finden sich besonders an Hand- und Fußrücken sowie an den prominenten Gesichtspartien als avital imponierende Hautdefekte. Dabei können abgeschliffene Knochen freiliegen. Durch Anschlagen an Steine und Uferbefestigungen können sogar Schädelbrüche auftreten. Schiffsschraubenverletzungen sind durch tiefe glattrandige

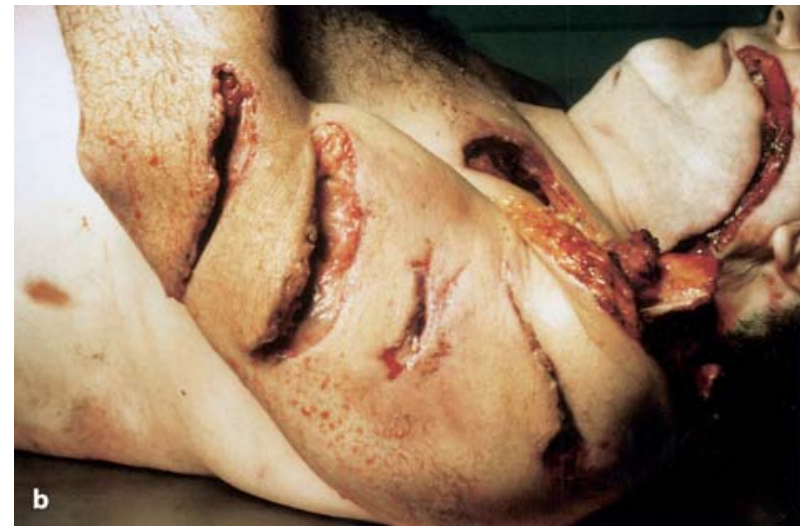

- Abb. 3.89. Schiffsschraubenverletzungen

Weichteilverletzungen mit Parallelität charakterisiert, teils mit Knochenbeteiligung (• Abb. 3.89). Bergeverletzungen, wie sie z.B. durch Reinigungsrechen an Staustufen von Flüssen entstehen, sind gelegentlich sehr gravierend und können zu ausgedehnten Knochenbrüchen und tiefen, meist glattrandigen Weichteilverletzungen führen. Unvermeidbarerweise wird dadurch die Diagnostik etwaiger vorbestehender Verletzungen (z.B. eine vorangehende Traumatisierung außerhalb des Wassers) erheblich erschwert. Bei Wasserleichen ist mit Tierfraßverletzungen $\mathrm{zu}$ rechnen, z.B. durch Ratten.

\section{Checkliste}

Bei der Untersuchung von Wasserleichen ist stets zu beachten:

- Kann der Ertrinkungstod bewiesen werden?

- Gibt es Befunde (morphologisch/toxikologisch) oder in der Vorgeschichte Anhaltspunkte (z.B. Abschiedsbrief), die das Zustandekommen des Ertrinkungstodes erklären?

- Ist beim Fehlen von Ertrinkungszeichen die Annahme eines Badetodes oder eines natürlichen Todes im Wasser gerechtfertigt?

- Finden sich Verletzungen (z.B. Würgemale) oder toxikologische Befunde, die durch fremde Hand entstanden sein können? 


\subsection{Thermische Energie}

\subsubsection{Hitze: lokale Hitzeschäden, Verbrennungen und Verbrühungen}

\section{B. Madea, P. Schmidt}

\begin{abstract}
Definition
Unter Verbrennungen versteht man die Verletzungen, die durch die Einwirkung von Wärme bzw. hohen Temperaturen auf den Körper verursacht werden. Verbrühungen entstehen bei Einwirken heißer Flüssigkeiten und Dämpfe.
\end{abstract}

Im Gegensatz zu Verbrennungen sind bei Verbrühungen die Haare nicht thermisch geschädigt.

Charakteristische Verletzungsbefunde entstehen bei chemischen Verbrennungen, Stromunfällen und - von herausragender forensischer Bedeutung - Kontaktverbrennungen (• Tabelle 3.24).

Rechtsmedizinisch relevante Ereignisse mit Brandschäden. Dies sind

- der Unfalltod im Brandherd,

- der Tod anlässlich einer Brandstiftung,

- die suizidale Selbstverbrennung,

- Leichenbeseitigung,

- Mordbrand und

- Brandmord.

Bei Verbrühungen sind Misshandlungen durch Übergießen mit beziehungsweise Eintauchen in heiße Flüssigkeiten von rechtsmedizinischer Relevanz, wie Verbrühungen pflegebedürftiger Patienten durch Verletzung von Obhuts- und Aufsichtspflichten.

\section{Korrelation zwischen Hitzeschädigung, einwirkender Temperatur, Einwirkungsdauer und Tiefenpenetration}

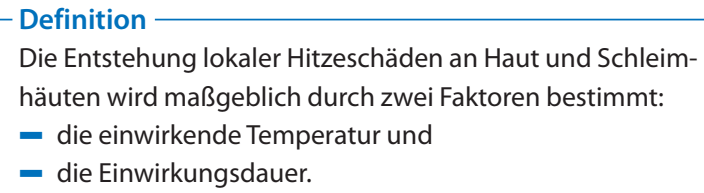

Für die Schädigungsfolgen ist dabei nicht nur die von außen einwirkende Wärme, sondern vielmehr die tatsächlich im Gewebe in der Tiefe erreichte Temperatur ausschlaggebend. Diese hängt wiederum von der Wärmekapazität und Leitfähigkeit der unterschiedlichen Gewebsschichten ab. Die im Gewebe erreichte Temperatur sinkt dabei mit zunehmender radialer Eindringtiefe sehr schnell ab (• Abb. 3.90).

Umfangreiche tierexperimentelle Untersuchungen und Versuche an freiwilligen Probanden zur Beziehung zwischen Einwirkungsdauer, Höhe der einwirkenden Temperatur und Ausmaß der thermischen Schädigung der Haut mit systematischer Variation von Expositionsdauer und Temperatur liegen vor und führten zu der in - Abb. 3.91 gezeigten Temperatur-Zeit-Kurve im Bereich der Schädigungsschwelle bei einwirkenden Temperaturen zwischen 44 und $70^{\circ} \mathrm{C}$ (Verbrühung). Die niedrigste Wassertemperatur, die zur Verbrühung führte, lag bei $44^{\circ} \mathrm{C}$, die Entstehung irreversibler Schädigungen erforderte dabei jedoch eine Einwirkungsdauer von 6 Stunden. Am oberen Rand der Skala wurden zwei Beobachtungen einer Exposition mit $60^{\circ} \mathrm{C}$ heißem Wasser mitgeteilt. Bei einer Expositionsdauer von 3 Sekunden resultierte eine transiente Hyperämie, also eine erstgradige Schädigung. Bei einer Expositionsdauer von 5 Sekunden entstand hingegen bereits eine komplette Nekrose der Epidermis.

Gleichartige Schwellenwerte der Hitzeschädigung wurden auch für die Einwirkung trockener Hitze ermittelt. Sie liegen deutlich höher als Ausdruck der unterschiedlichen Wärmeleitfähigkeit der einwirkenden Medien.

In - Abb. 3.91 sind die Temperaturen zusammengestellt, die bei Einwirkung von heißer Luft oder Dämpfen auf die Atemöffnungen im Tracheobronchialsystem entstehen.

- Tabelle 3.24. Klassifikation der Verbrennungsquellen

Verbrennungserscheinungen durch Kontakt mit der Hitzequelle bei

Verbrennungserscheinungen durch Strahlung bei

Verbrennungserscheinungen durch elektrischen Strom bei
- chemischer Verbrennung (z.B. ungelöschter Kalk),

- Verbrühung durch heiße Dämpfe, Gase,

- erhitzten Festkörpern (Kochplatte) und Flüssigkeiten (Hochofenunfall) und

- direkter Flammeneinwirkung.

- wärmeaussendenden Apparaturen (Höhensonne, Infrarotlicht) und

- atomarer Energiestrahlung.

- Berührung eines Stromleiters und

- Funkenentladungen (Blitz). 

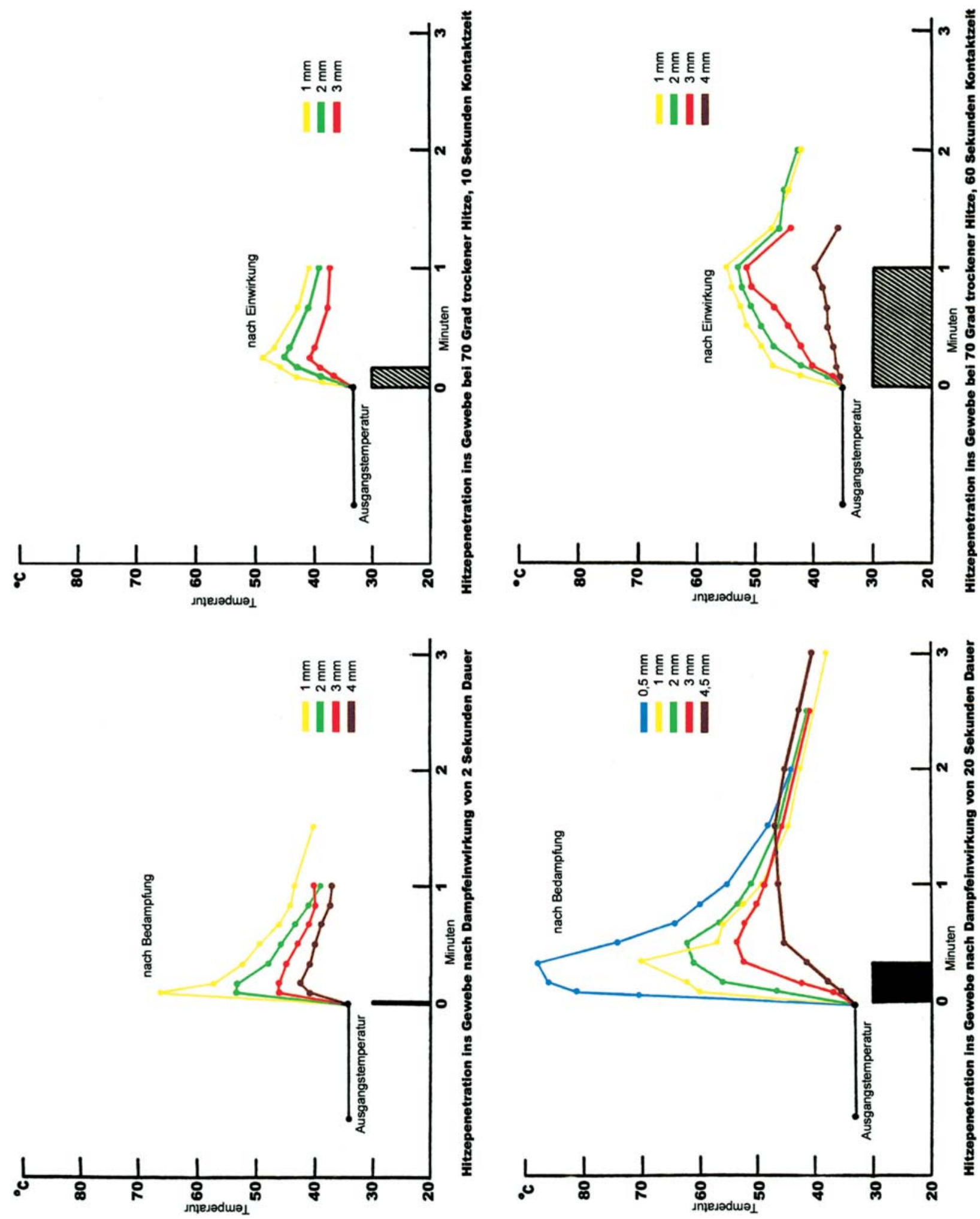

- Abb. 3.90. Hitzepenetration ins Gewebe nach Dampfeinwirkung (Verbrühung links) bzw. Kontaktverbrennung (rechts) unterschiedlicher Dauer (Dampfeinwirkung von 2 und 20 Sekunden Dauer, Kontaktver-

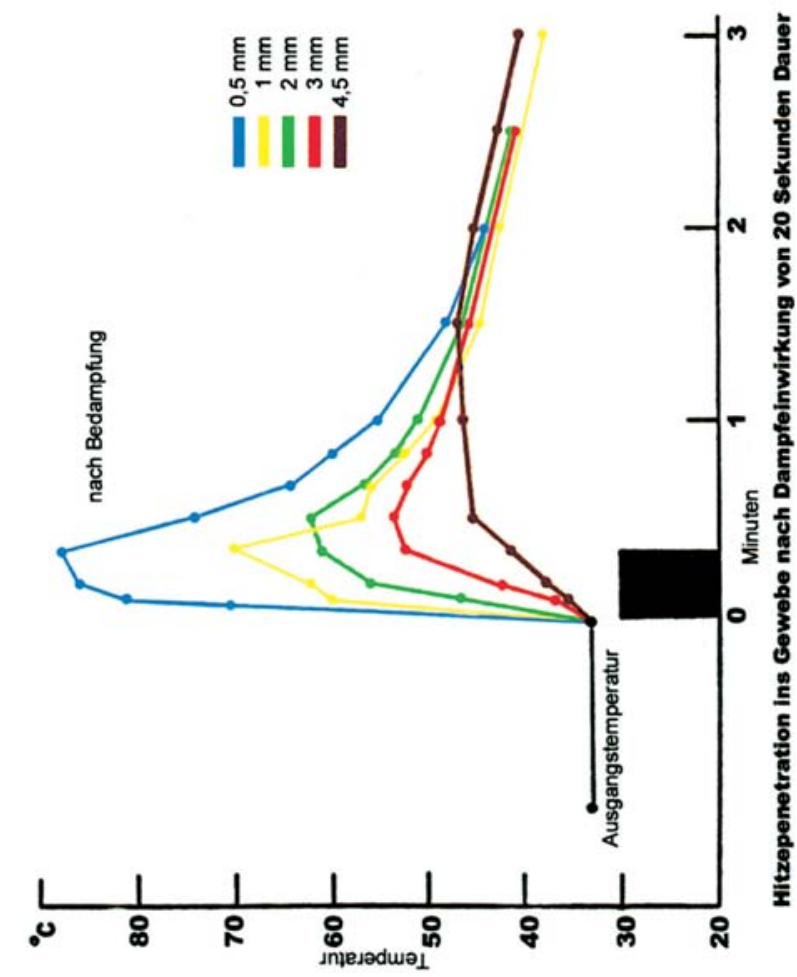

brennungen von 10 und 60 Sekunden Dauer). Darstellung des Temperatur-Zeitverlaufes in unterschiedlichen Gewebstiefen (blau 0,5 mm, gelb 1 $\mathrm{mm}$, grün $2 \mathrm{~mm}$, rot $3 \mathrm{~mm}$, braun $4 \mathrm{~mm}$ ) 


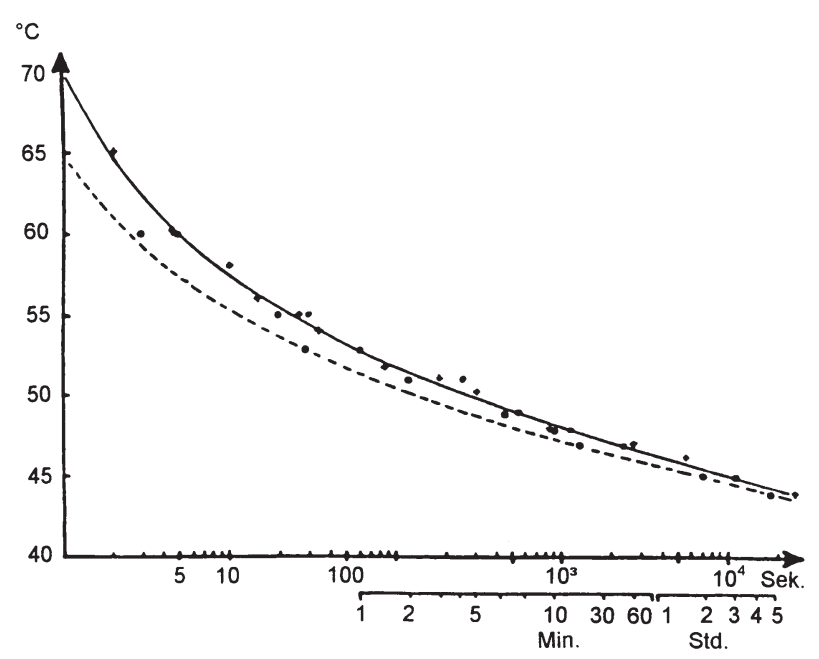

- Abb. 3.91. Verlauf der Temperatur-Zeit-Kurven im Bereich der Schädigungsschwelle bei einwirkenden Temperaturen zwischen 44 und $70^{\circ} \mathrm{C}$ (Verbrühung); gestrichelte Kurve: Schwelle zur reversiblen Hitzeschädigung; durchzogene Kurve: Schwelle zur irreversiblen Hitzeschädigung

Experimentell wurden bei Einwirken von trockener Luft mit einer Temperatur von $350^{\circ} \mathrm{C}$ auf die Atemöffnungen im Larynx Temperaturen von $159-182^{\circ} \mathrm{C}$ gemessen, an der Bifurkation war keine Temperaturerhöhung mehr festzustellen. Bei Einwirkung von trockener Luft von $550{ }^{\circ} \mathrm{C}$ auf die Respirationsöffnungen wurden im Larynx Temperaturen von $267-327^{\circ} \mathrm{C}$ festgestellt, an der Bifurkation von $50{ }^{\circ} \mathrm{C}$. Ferner sind in - Abb. 3.92 die bei Einwirkung von Feuer auf die Atemöffnungen im Larynx und an der Bifurkation erzielten Temperaturen dargestellt. Es lässt sich ablesen, dass Dampf aufgrund seiner größeren Wärmeleitfähigkeit bereits bei deutlich niedrigeren Temperaturen zu schädigenden Temperaturerhöhungen führt. Hierin liegt die physikalische Grundlage dafür, dass die Gefährlichkeit einer Dampf-

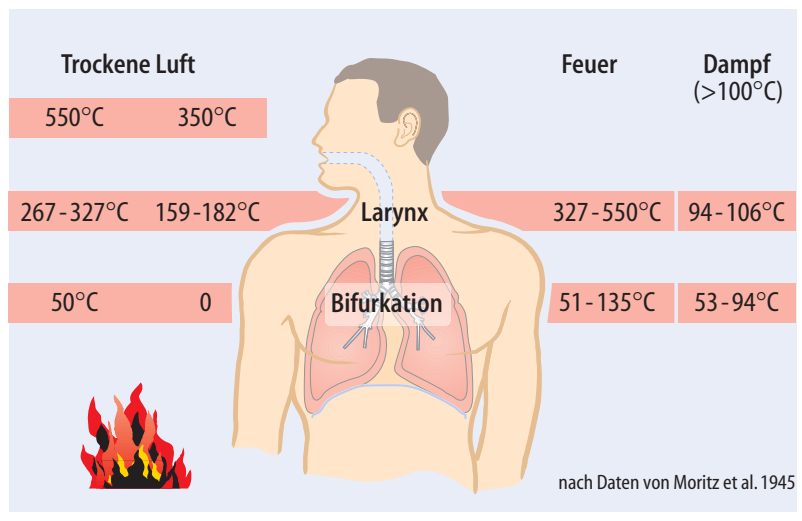

- Abb. 3.92. In Larynx und an der Bifurkation tierexperimentell gemessene Temperaturen bei Einwirken von erhitzter trockener Luft, Dampf oder Feuer auf die Atemöffnungen

kesselexplosion nicht nur aus einer Schädigung des Integuments, sondern insbesondere der Schleimhaut der Respirationswege resultiert.

\section{Verbrennungen - Graduierung der Tiefenausdehnung}

Definition

Verbrennungen werden eingeteilt in 1., 2., 3. oder 4. Grades

bzw. superficial, partial thickness und full thickness burns

oder in eine Kombination beider Nomenklaturen (• Abb.

$3.93+3.94, \bullet$ Tabelle 3.25 )

Verbrennungen 1. Grades oder superficial burns. Die charakteristischen Befunde bestehen in einer Hautrötung, d.h. einem Erythem ohne Blasenbildung. Die Epidermis ist intakt, einige geschädigte Zellen können wie kleieartige Schuppen desquamiert werden. Die

- Tabelle 3.25. Leitsymptome der Verbrennung

\begin{tabular}{|l|l|l|l|l|l|l|}
\hline Grade & $\begin{array}{l}\text { Verbrennungs- } \\
\text { tiefe }\end{array}$ & $\begin{array}{l}\text { Farbe } \\
\text { Aussehen }\end{array}$ & $\begin{array}{l}\text { Gewebe- } \\
\text { struktur }\end{array}$ & $\begin{array}{l}\text { Kapillar- } \\
\text { füllung }\end{array}$ & $\begin{array}{l}\text { Schmerz- } \\
\text { empfindung }\end{array}$ & \begin{tabular}{l} 
Abheilung \\
\hline $1^{\circ}$
\end{tabular} \\
\hline & $\begin{array}{l}\text { oberflächlich } \\
\text { epidermal }\end{array}$ & rot & normal & + & + \\
\hline $2^{\circ}$ & $\begin{array}{l}\text { oberflächlich } \\
\text { dermal }\end{array}$ & rot, Blasen & ödematös & + & + & $\begin{array}{l}\text { 5-10 Tage } \\
\text { geringe Narben }\end{array}$ \\
\hline & $\begin{array}{l}\text { tief } \\
\text { dermal }\end{array}$ & rosa oder weiß, Blasen & verdickt & $+/-$ & + Tage \\
\hline $3^{\circ}$ & transdermal & weiß, braun & lederartig & - & 25-60 Tage, narbig \\
\hline $4^{\circ}$ & subkutan & verkohlt & Haut fehlt & - & - & keine Spontanheilung \\
\hline
\end{tabular}




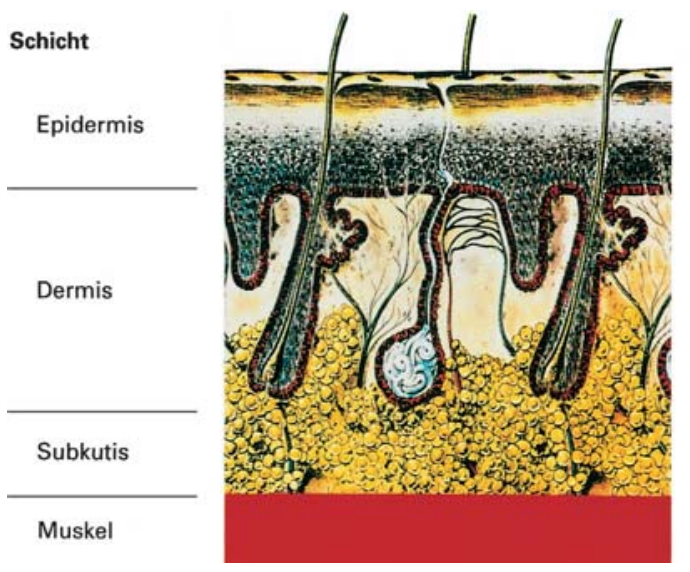

Grad

I.

llb.

III. D Wichtig

Bei der 2.-gradigen, oberflächlichen Verbrennung sind ledig-

IV. lich Stratum corneum und granulosum zerstört, die Basalzellschicht bleibt hingegen erhalten. Die Abheilung erfolgt innerhalb von 2-3 Wochen.

- Abb. 3.93. Verbrennungsgrade und betroffene Hautschichten

gerötete Haut ist schmerzhaft. Histologisch stellen sich 1.-gradige Verbrennungen als dilatierte, blutgefüllte dermale Gefäße dar. Eine komplette Abheilung erfolgt innerhalb von 8-10 Tagen.

Verbrennungen 2. Grades oder partial bzw. full thickness burns. Dieses Stadium ist durch Blasenbildung gekennzeichnet. Die Haut ist feucht, gerötet und schmerzhaft. Mikroskopisch können die den Blasen entsprechenden, mit seröser Flüssigkeit gefüllten Hohlräume zwischen Horn- und Keimschicht der Epidermis, innerhalb der Keimschicht oder an der Grenzfläche zwischen Epidermis und Dermis gelegen sein.

$\nabla$

- Abb. 3.94. Tiefenausdehnung der Hitzeschädigung, illustriert durch weggelassene Hautanteile
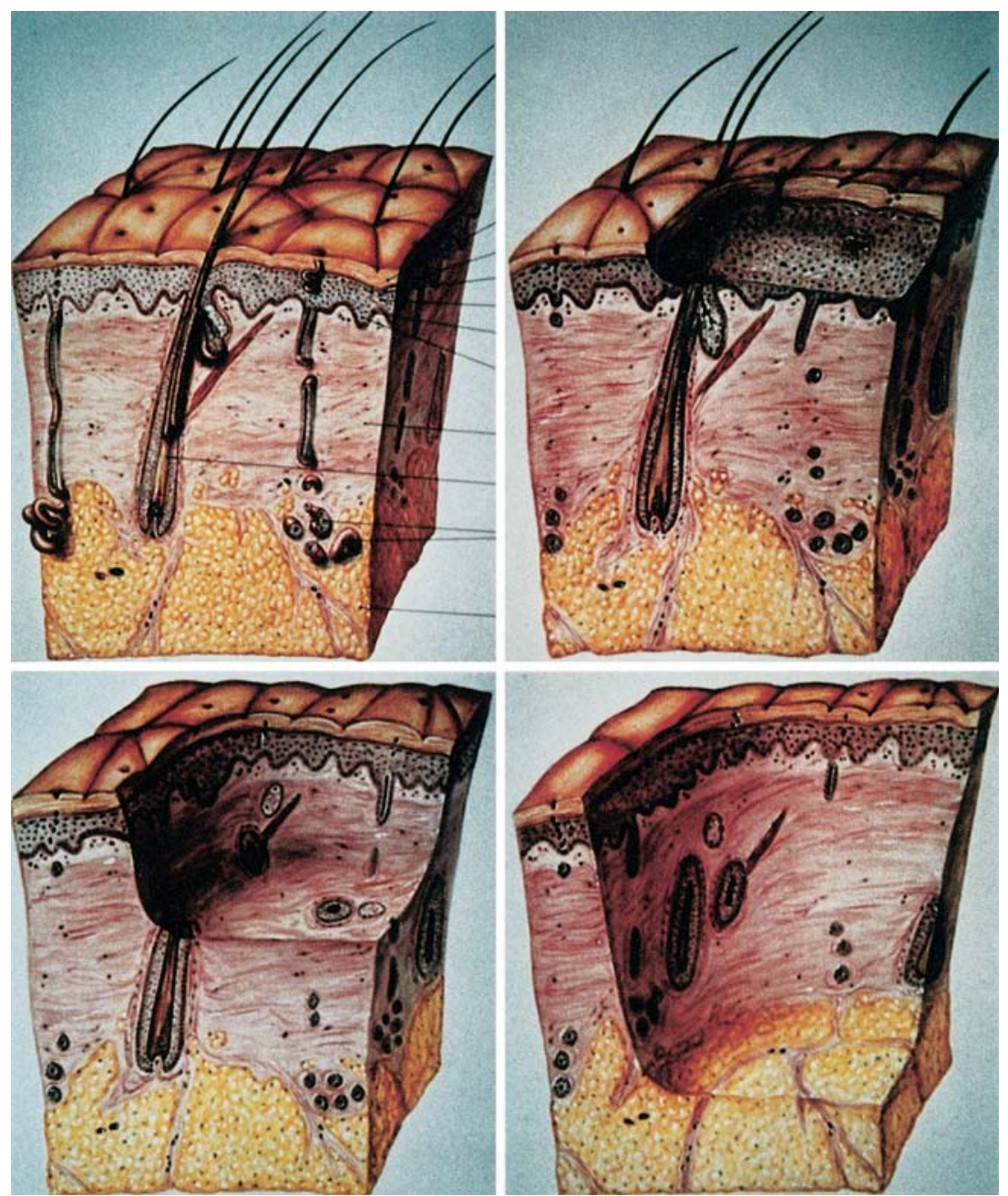
Bei der tiefen 2.-gradigen Verbrennung ist die komplette Epidermis inklusive Basalzellschicht zerstört, die Hautanhangsgebilde (Haare, Schweißdrüsen) sind jedoch ausgespart und bilden den Ausgangspunkt einer multizentrischen Reepithelialisierung. Die Wundheilung verläuft verzögert und kann mehr als 3 Wochen in Anspruch nehmen.

Verbrennungen 3. Grades. Hierbei handelt es sich um eine Koagulationsnekrose der gesamten Epidermis und Dermis mit Zerstörung auch der Hautanhangsgebilde. Die Haut sieht trocken, weiß und lederartig aus. Die Schmerzempfindung ist aufgehoben.

Verbrennungen 4. Grades. Bei weiterem Vordringen in die Tiefe greift die Verbrennung auf Haut und Unterhautfettgewebe über und betrifft auch Muskulatur und Knochen.

\section{Flächenausdehnung der Verbrennung}

Die Ausdehnung der Verbrennung wird nach der »Neuner-Regel « errechnet (- Abb. 3.95). Beim Kleinkind ist zu berücksichtigen, dass der Kopf einen größeren Anteil an der Gesamtoberfläche einnimmt als beim Erwachsenen.

Forensisch ist neben der Flächenausdehnung auch die Verteilung der Verbrennungen von Bedeutung, da sie wertvolle Hinweise für die Rekonstruktion von Geschehensabläufen geben kann.

\section{F) Fallbeispiel}

Hat zum Beispiel bei einer Frau ein locker sitzender Rock Feuer gefangen, so zeigt die Verbrennung eine »Gangart von unten nach aufwärts«. Die Verbrennungen sind schwerpunktmäßig schwimmhosenartig an den Oberschenkeln, in der Bauch- und Gesäßgegend lokalisiert und können den oberen Teil von Brust, Kopf, Hals und Armen aussparen.

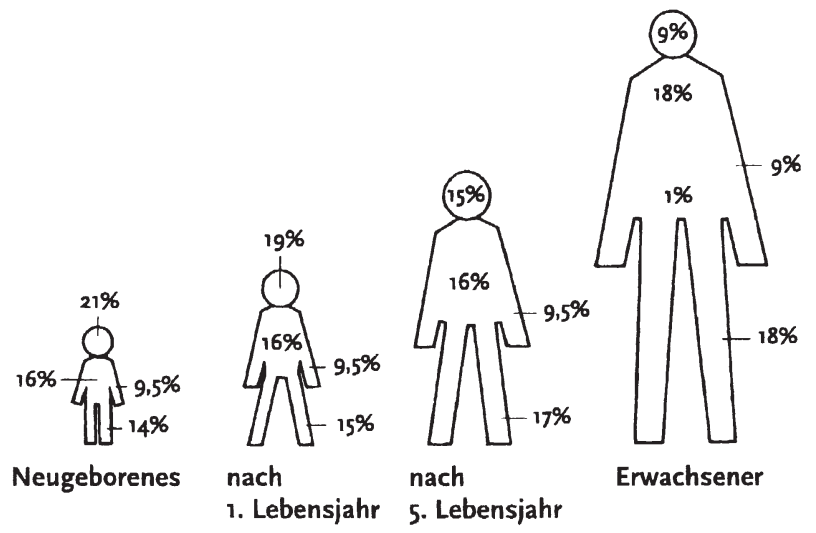

- Abb. 3.95. Schätzung der Ausdehnung der Verbrennungen nach der Neuner-Regel bzw. nach der Methode von Lund und Browler (Nach Herndon)
D Wichtig

Die Prognose thermischer Hautschäden ist abhängig von der verbrannten Körperoberfläche (Flächenausdehnung), dem Grad der Verbrennung (Verbrennungstiefe) sowie dem Lebensalter.

Mit zunehmendem Lebensalter (ab dem 40. Lebensjahr) sinkt die Prognose, aber auch Neugeborene und Kleinkinder sind besonders gefährdet.

\section{Wichtig}

Entsprechend klinischer Faustregeln gilt nach wie vor: Addieren sich Lebensalter und Ausdehnung der 2.- und 3.-gradigen Verbrennungen (Verbrennungsindex) zu 100, beträgt die Überlebenschance auch bei optimaler Therapie maximal $50 \%$.

Nach klinischen Erfahrungen besteht bei einem Verbrennungsindex $<80$ geringe Lebensgefahr, einem Index von 80-120 akute Lebensgefahr, bei einem Index $>120$ ist das Überleben unwahrscheinlich. Auch heute haben erwachsene Patienten mit tiefen Verbrennungen von über 30 \% Körperoberfläche eine ernste Prognose, Verbrennungen von mehr als 50 \% der Körperoberfläche werden nur in Ausnahmefällen überlebt.

D Wichtig

Bei zusätzlich vorliegendem Inhalationstrauma, auf das Verbrennungen des Gesichts und der Perioralregion bereits hinweisen, wird die Prognose schlechter.

\section{Postmortale Hitzeschäden}

Die Destruktion einer Leiche im Brandherd ist Folge direkter Brandzehrung sowie der Wasserdampfbildung mit Änderung des Kondensationszustandes extra- und intrazellulärer sowie transzellulärer Flüssigkeiten von der flüssigen in die gasförmige Phase. Die Körperflüssigkeiten werden durch Wärmezufuhr zum Sieden gebracht, wobei der Siedepunkt des Wassers bei etwa $100^{\circ} \mathrm{C}$ liegt. Sind die Körperflüssigkeiten einmal in Wasserdampf überführt, dehnt sich dieser bei weiterer Temperaturerhöhung aus. Die hitzebedingte Erwärmung des Körpers führt sowohl zur Hitzefixation von Geweben als auch zur fäulnisähnlichen temporären Aufblähung infolge der Gasbildung, denn unter Hitzewirkung kommt es zu einer Verkochung und später Verdampfung von Körper- und Gewebsflüssigkeiten mit gasbedingter Aufblähung von Lippen und Protrusion der Zunge sowie Auslaufen von Flüssigkeiten aus Mund und Nase. Weitere postmortale Hitzeschäden jenseits der Verdampfung führen zu hitzebedingter Schrumpfung, etwa der Haut, aber auch von Knochen, und direkter, flammenbedingter Aufzehrung. Als charakteristische Befunde postmortaler Hitzeschädigung gelten:

- Hitzerisse der Haut,

- Fechterstellung und

- Brandhämatom.

Hitzerisse der Haut. Als Folge hitzebedingter Schrumpfung kann die Haut einreißen, es kommt zum Aufplatzen der Bauchhöhle. Die Hauteinrisse imponieren teilweise als relativ geradlinig-glatt- 
randig und dürfen nicht mit vitalen Schnittverletzungen verwechselt werden.

Fechterstellung. Die charakteristische Stellung des Leichnams ist Folge einer hitzebedingten Beugekontraktur mit Schrumpfung von Muskulatur und Sehnen. Sie äußert sich gewöhnlich in Abduktion in den Schultergelenken, Flexion in den Ellenbogen- und Handgelenken, Streckung der Beine und Spitzfußstellung (• Abb. 3.96).

Brandhämatom. Bei direkter Flammeneinwirkung auf den Schädel kommt es schließlich zu einer zwischen Schädelknochen und Dura, also epidural, gelegenen Ansammlung von ziegelrotem oder bräunlichem, bröckeligem, trockenem oder lehmartigen Blut, das im Randbereich auch von flüssiger oder halbflüssiger Beschaffenheit sein kann. Diese postmortalen epiduralen Extravasate entstehen einerseits durch Verdrängung des im Schädelknochen vorhandenen Blutes, das von der Hitze nach innen gegen die harte Hirnhaut gepresst wird, zum anderen durch Schrumpfung und Ablösung der harten Hirnhaut von der Schädelinnenfläche, wobei kleine, aus dem Sichelblutleiter zum Knochen führende Venen rupturieren (• Abb. 3.97).

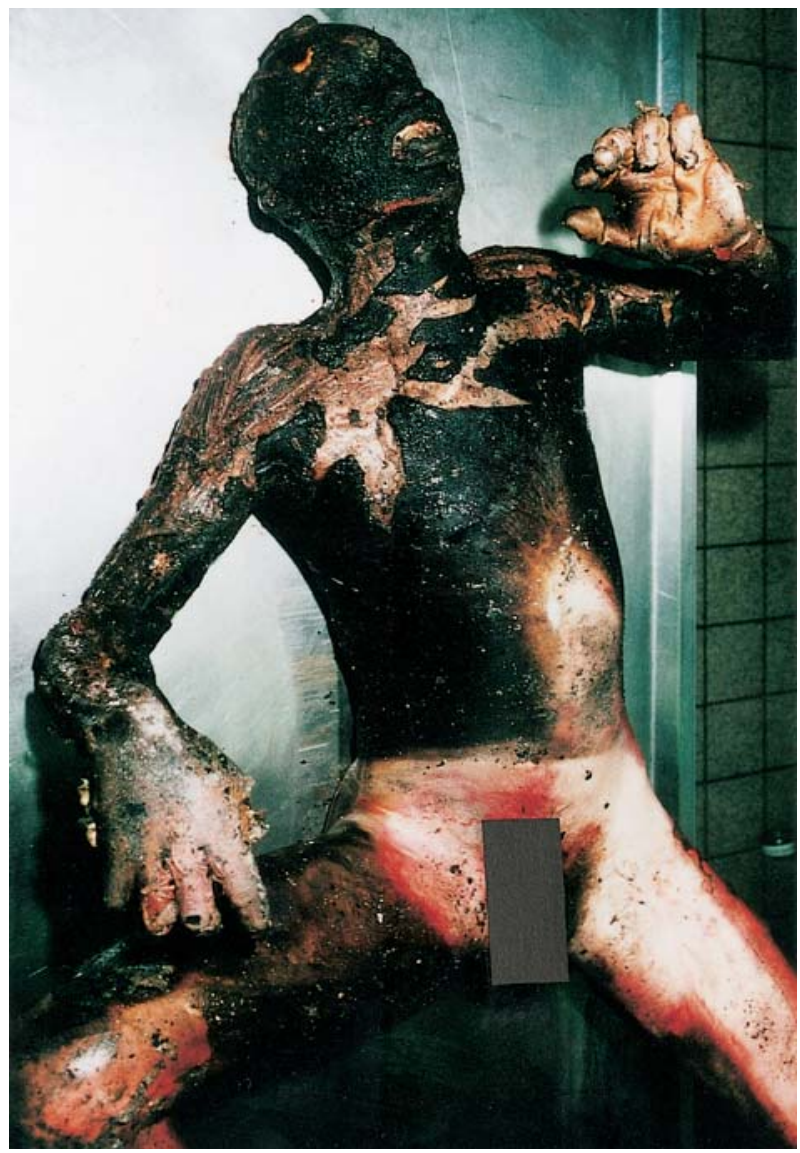

- Abb. 3.96. Fechterstellung eines Leichnams

\section{Wichtig}

Bei Bergung einer Leiche aus einem Brandherd besteht neben der Klärung von Identität, Grundleiden und Todesursache eine wesentliche Aufgabe in der Feststellung, ob der nunmehr Verstorbene lebend ins Feuer geriet oder zum Zeitpunkt der Hitzeeinwirkung bereits verstorben war.

Auch bei weitgehender Verkohlung von Weichteilen und Muskulatur können diese Fragen in der Regel eindeutig geklärt werden, da aufgrund des radial zum Körperkern gerichteten Temperaturabfalles die inneren Organe in der Regel relativ gut erhalten sind (Hitzefixierung durch Flüssigkeitsverlust).

\section{Vitale Zeichen und Reaktionen}

Rußaspiration. Die Einatmung von Rußbestandteilen bis in die tieferen Luftröhrenverzweigungen innerhalb der Lungen ist eine eindeutige vitale Reaktion. Autoptisch findet sich eine Verrußung der Respirationswege bis in die feinsten Bronchien mit reichlicher Schleimabsonderung (• Abb. 3.98).

Rußverschlucken. Parallel kann auch Ruß verschluckt werden, sodass Rußpartikel im Magen oder oberen Dünndarm nachweisbar sind.

Rauchgasinhalation. In der Regel finden sich bei Lebendverbrennungen positive CO-Befunde. Doch zeigen sich Abhängig-

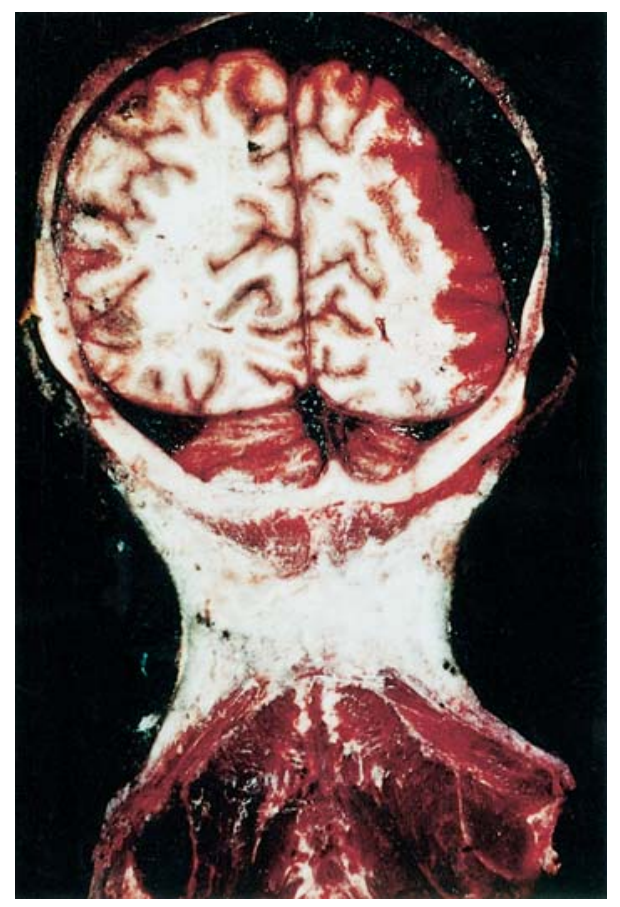

- Abb. 3.97. Brandhämatom vorwiegend über der rechten Hirnhalbkugel bei Einblick in den Schädel von okzipital (Aus dem Bildarchiv von Prof. Dr. G. Dotzauer) 
keiten der CO-Hb-Konzentration vom Lebensalter und vor allem von den Umständen und der Lokalität des Brandes. In höherem Lebensalter finden sich häufig deutlich geringere Konzentrationen als in jüngeren Jahren. Auch bei Zimmerbränden werden gelegentlich, trotz geschlossener Räume und Rußaspiration, negative CO-Befunde beobachtet. In gleicher Weise sind bei Brandfällen im Freien (häufig suizidale Selbstverbrennung) die COBefunde oftmals niedrig beziehungsweise negativ.

\section{(C) Wichtig}

Typische Sektionsbefunde der Kohlenmonoxidintoxikation sind hellrote Farbgebung des Blutes sowie lachsrote Verfärbung der Muskulatur.

Allerdings kann auch allein durch die Hitzeeinwirkung ohne erhöhte CO-Hb-Konzentration eine hellrote Farbe des Blutes hervorgerufen werden. Beim Brand stickstoffhaltiger Polymere, natürlicher oder synthetischer Herkunft, bilden sich in Abhängigkeit von der zur Verfügung stehenden Sauerstoffmenge entweder Stickoxyde oder bei Sauerstoffmangel Zyanwasserstoff. Letale inhalatorische Zyanidkonzentrationen im Rahmen einer Rauchgasvergiftung können bei längerer Einwirkung entstehen, wenn
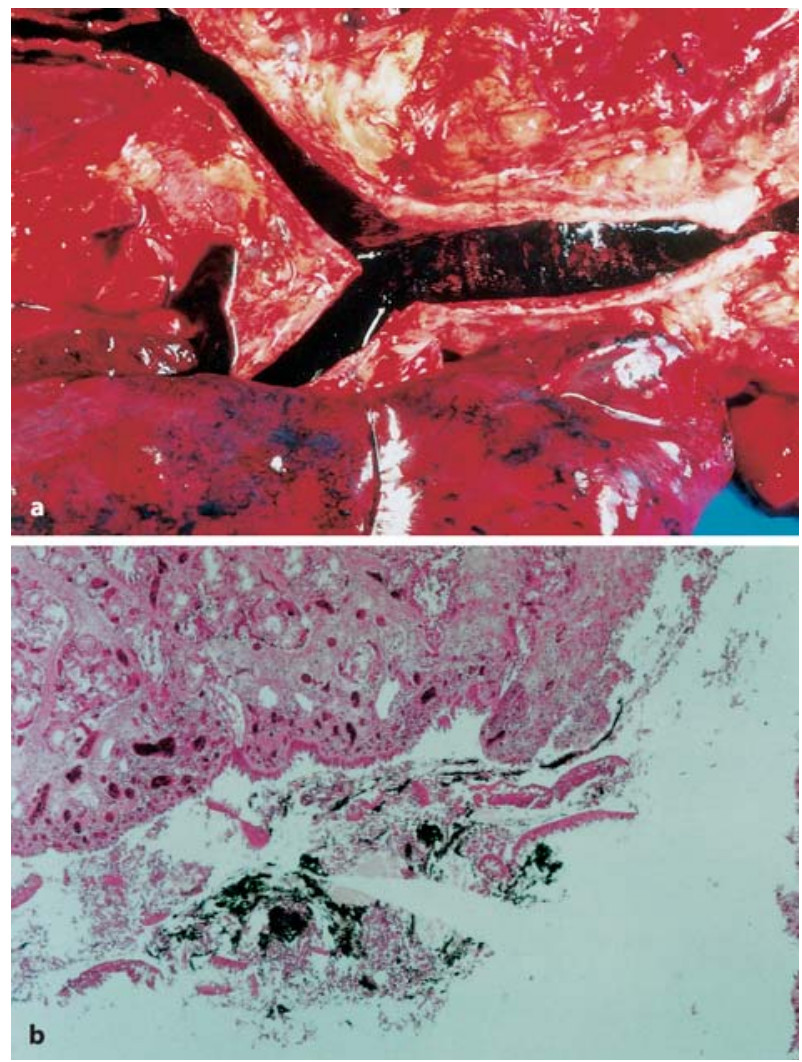

- Abb. 3.98. a Makroskopisches und b histologisches Bild der Rußaspiration die Einatemluft einen Gehalt von 90 ppm aufweist, bereits nach kurzer Einwirkung, wenn der HCN-Gehalt der Einatemluft 180270 ppm erreicht.

Thermische Atemwegsschädigung (Inhalationstrauma). Schließlich entstehen durch Heißluftinhalation charakteristische Schädigungen des Tracheobronchialsystems, bei Soforttodesfällen etwa eine fetzige und membranös deformierte Koagulationsnekrose der Schleimhaut in Nasenrachenraum sowie Kehlkopf und Trachea. Die Flimmerepithelien der Trachea weisen eine lumenwärts gerichtete Zell- und Kernelongation auf (• Abb. 3.99).

\section{(C) Wichtig}

Ein ausgeprägtes Schleimhautödem mit Ektasien der Lymphspalten und -gefäße, die kapilläre und venöse Hyperämie mit Mikrohämorrhagien sowie das intraalveoläre, interstitiell septale und perivaskuläre Lungenödem sind als vitale Reaktionen zu werten.

\section{Todesursachenspektrum}

Im Todesursachenspektrum können grundsätzlich unmittelbare Todesfälle am Ereignisort der Hitze- und Flammeneinwirkung auf der einen sowie primär überlebte Verbrennungen auf der anderen Seite unterschieden werden.

- Soforttodesfälle:

- Rauchgasvergiftung

- Lokale Hitzeschäden der Haut

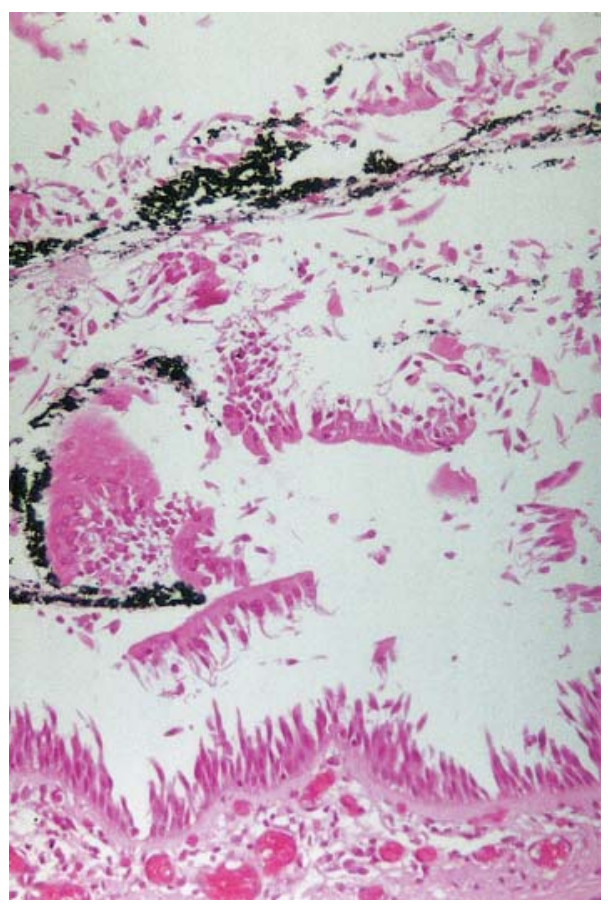

- Abb. 3.99. Weitgehende Abschilferung des Flimmerepithels, angedeutet lumenwärts gerichtete Zell- und Kernelongation der wandhaftenden Epithelien 
Spättodesfälle:

- Verbrennungskrankheit

- Infektiöse Komplikationen des Tracheobronchialsystems (bei Inhalationstrauma)

\section{i. Infobox}

Hitze- und Flammentod ohne Vitalitätszeichen

Die Häufigkeit derartiger Todesfälle wird mit knapp 3 bis gut $10 \%$ aller Verbrennungstodesfälle angegeben. Sind ein natürlicher Tod in zeitlichem Zusammenhang mit dem Brandausbruch und ein Mordbrand (s. unten) ausgeschlossen, sind folgende Kausalfaktoren in Betracht zu ziehen:

- Zyanidintoxikation: In Abhängigkeit vom verbrannten Material kann in Einzelfällen eine tödliche Zyanidintoxikation so rasch zustande kommen, dass sich erhöhte $\mathrm{CO}-\mathrm{Hb}$-Gehalte nicht mehr ausbilden

- Flashfire: Atemstillstand über einen Laryngospasmus/ Bronchospasmus, einen vagalen Reflex oder einen Inhalationshitzeschock

- Sauerstoffmangel: Sauerstoffzehrung im Brandherd

- Hitzeschock: Umverteilung des zirkulierenden Blutvolumens in Folge Hitzeeinwirkung auf die Haut

- Hitzestarre: Funktionelle Beeinträchtigung der Atemexkursionen durch schlagartig entstehende Hitzestarre des Brustkorbes

\section{Branddauer und Verkohlungsgrad einer Brandleiche}

Bei definierter Temperatur kommt es zu einer regelhaften Progression der Brandzehrung eines Leichnams, die in entsprechend gelagerten Fällen einen Rückschluss aus dem Brandzehrungsgrad auf die Branddauer erlaubt. Derartige Schätzungen können notwendig werden, wenn der Branddauer entscheidende Bedeutung für die Überprüfung des Alibis eines Tatverdächtigen beziehungsweise der Stichhaltigkeit seiner Brandwahrnehmungen zukommt.

In modernen Kremationsöfen ist eine vollständige Aufzehrung eines Leichnams bis auf kleinere Knochenstücke in der Regel innerhalb von 1-2 Stunden erreicht.

\section{Checkliste}

\section{Untersuchung einer Brandleiche}

\section{Antragungen von der Brandstelle}

- 1.1. Verteilung, Ausdehnung und Schichtdicke von Rußantragungen

- 1.2. Spuren von brandfördernden Mitteln wie zum Beispiel Kohleanzünder oder brennbare Flüssigkeiten

- 1.3. Art, Menge und Verteilung von Brandstoffen, heiße Materialien, insbesondere hoch erhitzte Metallteile, ge$\nabla$ schmolzenes Glas oder Aluminium, die Hinweise auf eine mögliche Position des Opfers an der Brandstelle geben können. Abtropfspuren von schmelzenden Baustoffen, wie zum Beispiel Deckenverkleidungen, brennende Materialien wie brennbare Flüssigkeiten bei Suiziden oder Zündbeschleuniger bei Unfällen

- 1.4. Reststücke von auf den Leichnam gefallenen Gegenständen, zum Beispiel abfallenden Bauteilen bei einstürzenden Gebäuden

- 1.5. Materialanhaftungen an den Händen

\section{Bekleidung}

- 2.1. Lokalisation, Ausdehnung, Form und Richtung thermischer Beschädigungen (Cave: Bekleidungsschichten).

- 2.2. Brandfremde Beschädigungen

- 2.3. Bei nicht oder nicht sicher identifizierten Brandleichen individuelle Merkmale wie die Konfektionsgröße, Material, Beschaffenheit, Farbe, Etiketten, Tascheninhalt, zum Beispiel persönliche Dokumente, sowie Schmuckstücke, zum Beispiel Armbanduhr (Uhrzeit).

\section{3. Äußere Leichenschau}

- 3.1. Hellrote Farbgebung von Totenflecken und Nagelbetten?

- 3.2. Thermische Schädigung von Kopfhaar, Augenbrauen und Barthaar

- 3.3. »Krähenfüße«

- 3.4. Rußpartikel in Nasenlöchern und Mundhöhle?

- 3.5. Lokalisation, Flächenausdehnung, Tiefe und Verteilungsmuster der Verbrennungen (Kongruenz mit der Bekleidung?), Übergangszonen zur nichtthermisch geschädigten Haut

- 3.6. Korrelation von Hautdurchtrennungen/Aufplatzungen, knöchernen Fissuren/Frakturen, Gelenkssprengungen, intrakraniellen Hämatomen mit Befunden der lokalen Hitzeund Flammeneinwirkung oder Antragungen von der Brandstelle ( $\triangleright$ oben)

- 3.7. Befunde der postmortalen Hitzeeinwirkung wie Verkohlung oder Fechterstellung der Gliedmaßen

- 3.8. Nicht auf eine Verursachung durch Hitze- oder Flammeneinwirkung beziehbare Verletzungen

- 3.9. Bei nicht oder nicht sicher identifizierten Brandleichen individualtypische Merkmale wie Körpergröße und Gewicht (geschätzt), Haarfarbe, Augenfarbe, Operationsnarben, äußere Geschlechtsmerkmale, Zustand nach Amputationen, Tätowierungen

\section{Innere Besichtigung}

- 4.1. Befunde zur Vitalität der Hitze- und Flammeneinwirkung, wie Rußverschlucken, Rußaspiration und thermische $\nabla$ 
Schädigung der Atemwegsepithelien im Rahmen des Inhalationstraumas

- 4.2. Postmortale Befunde der Hitze- und Flammeneinwirkung, wie Brandhämatom, Hitzesprengung von Gelenken, »Knochenbrüche« und Kalzinierung im Bereich lokaler Brandeinwirkung

- 4.3. Außerhalb der Hitze- und Flammeneinwirkung lokalisierte Verletzungen brandfremder Verursachung

- 4.4. Vorbestehende innere Erkrankungen, die als konkurrierende Todesursache in Betracht kommen oder die Empfindlichkeit gegenüber toxischen Rauchgasbestandteilen herabsetzen können

- 4.5. Bei nicht oder nicht sicher identifizierten Brandleichen individualtypische Merkmale wie operative Entfernung von Organen, innere Geschlechtsmerkmale, osteologische ( $\checkmark$ Kap. 2.5) und anderweitige Befunde (zum Beispiel degenerative Erkrankungen) zur Abschätzung des Lebensalters.

\section{Zusatzuntersuchungen}

- 5.1. Histologische Untersuchungen insbesondere der lokalen Hitzeschäden auf Vitalität und Tiefenausdehnung, der Atemwege und der Lungen auf Befunde des Inhalationstraumas - 5.2. (eventuell) Stereomikroskopische Untersuchungen von »Knochenbrüchen« zur Differenzierung zwischen mechanischer und thermischer Entstehung

- 5.3. Toxikologische Untersuchungen insbesondere auf Rauchgasbestandteile (CO, Zyanwasserstoff, Chlorwasserstoff und Schwefeldioxid) sowie auf zentral-nervös wirksame Pharmaka und Betäubungsmittel und eine Blutalkoholbestimmung (Herabsetzung der Handlungsfähigkeit bei Brandausbruch?)

\section{Wichtig}

Haaranalysen zur Verifizierung eines eventuellen chronischen Betäubungsmittelkonsums (möglicherweise auch Beitrag zur Individualisierung)

\section{Kriminologische Aspekte}

Unter kriminologischen Gesichtspunkten sind folgende Konstellationen zu unterscheiden:

- Unglücksfälle: zum Beispiel Einschlafen bei brennender Kerze oder Zigarette, zündelnde Kinder,

- Suizide: gelegentlich epidemieartig,

- Tötungsdelikte: Tötung durch direktes Inbrandsetzen (selten) und Verbrennen zur Verdeckung eines anderweitig begangenen Deliktes.

\section{F) Fallbeispiele}

In einem Waldstück wurde in einem vollkommen ausgebrannten Pkw der weitgehend verkohlte Leichnam eines Mannes gefunden, der aufgrund charakteristischen Osteosynthesematerials als
Halter des Fahrzeugs identifiziert werden konnte. Autoptisch zeigten sich eine Hitzesprengung des Schädels sowie weitgehende Brandzehrung der Halsweichteile, der Thoraxwand und der Gliedmaßen. Die erhaltenen Anteile der Trachea und der Bronchien waren bis in die feinen Verzweigungen mit einem bräunlichen, bröckeligen Inhalt ausgefüllt, der sich histologisch als hitzekoaguliertes Blut darstellte. Die Blutalkoholkonzentration betrug 0,81\%o. Das CO-Hb war negativ. Die aufgrund dieses Befundes intensivierten polizeilichen Ermittlungen erbrachten den Nachweis von Brandbeschleuniger im Fahrzeug, verdächtige Blutspuren auf dem Waldboden in der Nähe und Blutspuren in der ehelichen Wohnung des Verstorbenen, die nach Lokalisation und Konfiguration einem Kampfgeschehen zugeordnet werden konnten. In einem Indizienprozess wurden die Ehefrau des Verstorbenen und ihr Liebhaber überführt, den Verstorbenen durch einen Angriff gegen den Hals getötet und anschließend einen Suizid vorgetäuscht zu haben, um ein gemeinsames Leben beginnen zu können.

In einem Einfamilienhaus wurde nach einem Brand in der Waschküche im Keller im angrenzenden Treppenhaus der Leichnam des Hausbesitzers gefunden. Die Obduktion erbrachte zweit- bis drittgradige Verbrennungen von ca. 90 \% der Körperoberfläche, eine Knebelung, Fesselung von Händen und Unterschenkeln, Rußaspiration und Rußverschlucken. Die CO-Hb-Konzentration betrug $6 \%$. Bei Benzingeruch und der noch am Leichnam vorhandenen durchfeuchteten Textilfetzen, war in Proben von Blut, Lunge und Gehirn qualitativ Benzin nachzuweisen. Zwei Tage später wurde die Ehefrau des Verstorbenen bei dem Versuch, Deutschland zu verlassen, mit zweit- bis drittgradigen Brandverletzungen der Beugeseiten beider Unterarme, der Vorderseiten der Unterschenkel sowie des rechten Fußrückens aufgegriffen. Nach dem weiteren Ergebnis der kriminalpolizeilichen Ermittlungen hatte die krankhaft eifersüchtige Frau nach einem ehelichen Zerwürfnis den Plan gefasst, ihren Mann zu verbrennen. Mittels Pfefferspray versetzte sie ihr Opfer in einen wehrlosen Zustand, fesselte und knebelte es, übergoss es dann mit zuvor an einer Tankstelle besorgtem Benzin und zündete es an.

Bei Verbrühungen sind Obhuts- und Aufsichtspflichtverletzungen nicht selten (zu heißes Baden von Kindern und Pflegebedürftigen). In der Regel versuchen sich die Beschuldigten dahingehend zu entlasten, die Verletzten seien nur ganz kurze Zeit unbeaufsichtigt Wasser mit einer gering erhöhten Temperatur ausgesetzt gewesen. Derartige Einlassungen können an der in Abbildung 3.88 gezeigten Temperatur-Zeit-Kurve im Bereich der Schädigungsschwelle überprüft werden.

\section{Wichtig}

Verbrühungen sind insbesondere klinisch-rechtsmedizinisch von Bedeutung, da 5-14 \% der kindlichen Verbrühungen einer Verursachung durch Misshandlungen zuzuordnen sein sollen, wobei für Immersionsverbrühungen sogar Werte von 12-55\% mitgeteilt werden (•Tabelle 3.26). 
- Tabelle 3.26. Differentialdiagnose Unfall - Misshandlung bei Verbrühungen im Kindesalter

\section{Unfall}

Adäquate Anamnese

Geringe Verletzungsschwere

Unregelmäßige landkartenartige Konfiguration

Asymmetrisch

Keine begleitenden Verletzungen

\section{Misshandlung}

Diskrepanz zwischen Angaben zur Verursachung und Befund Höhergradige Verbrühung, Verbrennung

Linerare Begrenzung (Wasserstand)

Symmetrisch

Weitere Verletzungen

\section{Checkliste}

\section{Gründe für klinische Verdachtsschöpfung}

- Diskrepanz zwischen anamnestischen Angaben und Verletzungsmustern

- Inadäquate oder inkompatible Vorgeschichte

- Abstreiten der offensichtlich hitzebedingten Verursachung

- Benennung von Patient oder Geschwisterkind als Verursacher

- Bagatellisieren oder Abstreiten von Schmerzen (durch Eltern oder Kind)

\section{Allgemeine Hitzeschäden, Hyperthermie}

Der Mensch gehört zu den so genannten homoiothermen Lebewesen, deren Körperkerntemperatur durch körpereigene Thermoregulation auf einem Sollwert von rund $37^{\circ} \mathrm{C}$ mit Tagesschwankungen von etwa $+/-0,5^{\circ} \mathrm{C}$ konstant gehalten wird. Dies setzt voraus, dass Wärmeaufnahme und Wärmeproduktion auf der einen Seite mit der Wärmeabgabe des Organismus auf der anderen Seite im Gleichgewicht gehalten werden. Die Wärmeproduktion hängt vom Energieumsatz des Organismus ab und wird in Ruhe zu mehr als 50 \% von den inneren Organen und zu knapp 20 \% von der Muskulatur getragen. Bei körperlicher Arbeit kann die Wärmebildung um ein Mehrfaches ansteigen. An der Wärmeabgabe nach außen sind im Wesentlichen drei Mechanismen beteiligt:

- Strahlung,

- Leitung und Konvektion sowie

- Wasserverdunstung durch Haut und Lungen.

Missverhältnisse von Wärmeproduktion und Wärmeabgabe führen über verschiedene, sich teilweise überlappende pathophysiologische Wege - Salzverlust, Versagen der Kreislaufregulation, pathologische Erhöhung der Körperkerntemperatur, Wärmeeinstrahlung auf den Schädel mit lokaler Hyperthermie des Gehirns - zu systemischen Störungen des Wärmehaushaltes. Diese gliedern sich (• Tabelle 3.27) in:

- Hitzekrämpfe,

- Hitzeerschöpfung (Hitzekollaps),

- Hitzschlag und

- Sonnenstich.

\section{Hitzekrämpfe}

Definition

Hitzekrämpfe betreffen vor allem Personen, die strahlender Hitze ausgesetzt sind und gleichzeitig schwer körperlich arbeiten müssen.

Pathogenetisch entscheidende Faktoren sind die Dehydratation und der Natriumchloridverlust. Beim Vollbild bestehen neben tetanischen Krämpfen auch eine starke Gefäßerweiterung und eine Erhöhung der Pulsfrequenz. Eine vitale Gefährdung kann durch eine Störung der Erregungsleitung des Herzens gegeben sein.

\section{Hitzekollaps}

Definition

Beim Hitzekollaps kommt es zum Zusammenbruch der Kreislaufregulation bei zunächst erhaltener Temperaturregelung.

Man unterscheidet einen primären Kreislaufkollaps infolge stark erhöhter Hautdurchblutung (heat exhaustion) und einen sekundären Hitzekollaps aufgrund der Abnahme der zirkulierenden Blutmenge bei Wasserverlust (dehydration exhaustion).

D Wichtig

Prädisponierend wirken schwüle Witterung, schwere körperliche Arbeit und unzweckmäßige Kleidung (»Heizerohnmacht«, »Marschohnmacht«).

\section{Hitzschlag}

\section{Definition}

Die gefährlichste Form der Hitzeschädigung ist der Hitzschlag, gekennzeichnet durch einen Zusammenbruch der zentralen thermoregulatorischen Funktion infolge Zunahme der Körperkerntemperatur, insbesondere auch der Hirntemperatur.

Ein Hitzschlag entsteht bei einer Kombination von verhinderter Wärmeabgabe und abnorm großer Wärmezufuhr. Die Umgebungstemperatur liegt in der Regel höher als die Körperkerntemperatur, sodass die Wärmeabgabe nur noch durch Wasserverdunstung an der Körperoberfläche möglich ist. Prädisponierend 
Tabelle 3.27. Ursachen und Symptome systemischer Hitzeschäden

\begin{tabular}{|c|c|c|c|c|}
\hline & Hitzekrämpfe & Hitzeerschöpfung & Hitzschlag & Sonnenstich \\
\hline Ursache & $\begin{array}{l}\text { schwere körperliche } \\
\text { Arbeit bei strahlender } \\
\text { Hitze } \\
\text { Dehydration } \\
\text { NaCl-Verlust }\end{array}$ & $\begin{array}{l}\text { Versagen der Kreislaufregulation bei } \\
\text { Hitzebelastung } \\
\text { Vasodilatation } \\
\text { Dehydratation } \\
\text { bei Stehen Absacken des Blutes in } \\
\text { die Beine } \\
\text { Abnahme von HZV und RR }\end{array}$ & $\begin{array}{l}\text { abnorm große Wärmezufuhr von au- } \\
\text { ßen bei Behinderung der Wärmeab- } \\
\text { gabe } \\
\text { pathologische Erhöhung der Körper- } \\
\text { temperatur auf Werte bis } 43^{\circ} \mathrm{C} \\
\text { hohe Luftfeuchtigkeit begünstigend }\end{array}$ & $\begin{array}{l}\text { ungehinderte } \\
\text { Wärmeeinstrahlung } \\
\text { auf den Schädel }\end{array}$ \\
\hline Symptome & $\begin{array}{l}\text { Muskelkrämpfe } \\
\text { Mattigkeit } \\
\text { Brechneigung } \\
\text { Rückgang der Harn- } \\
\text { sekretion }\end{array}$ & $\begin{array}{l}\text { Haut gerötet, schweißbedeckt } \\
\text { Schleimhäute trocken, quälender } \\
\text { Durst } \\
\text { Kopfschmerzen, Schwindelgefühl } \\
\text { Flimmerskotome, Ohrensausen } \\
\text { Parästhesien, Kreislaufschock }\end{array}$ & $\begin{array}{l}\text { »rotes Stadium«, mit roter, trockener } \\
\text { Haut, solange Kreislaufregulation } \\
\text { noch nicht zusammengebrochen, } \\
\text { "graues Stadium« nach Zusammen- } \\
\text { bruch des Kreislaufs, myogene Herz- } \\
\text { insuffizienz, zerebrale Symptome mit } \\
\text { deliranten und Dämmerzuständen, } \\
\text { Bewusstlosigkeit, epileptiforme } \\
\text { Krämpfe, meningitische Symptome }\end{array}$ & $\begin{array}{l}\text { meningeale Reiz- } \\
\text { erscheinungen } \\
\text { meningeale Blutun- } \\
\text { gen } \\
\text { Purpura cerebri }\end{array}$ \\
\hline
\end{tabular}

wirken neben vorbestehenden Erkrankungen Medikamente und konstitutionelle Faktoren. Gefährdete Berufsgruppen sind Schiffsheizer, Arbeiter in Bergwerken, Tunneln, Heizanlagen, Eisengießereien, Glasbläsereien und Soldaten auf dem Marsch in tropischem beziehungsweise heißem Klima (•Tabelle 3.28a, b).

\section{Sonnenstich}

\section{- Definition}

Der Sonnenstich ist eine Sonderform der exogenen Hyperthermie, in deren Pathogenese die unmittelbare Sonneneinstrahlung auf den entblößten Schädel von zentraler Bedeutung ist.

Infolge der besonderen Wärmeempfindlichkeit des Gehirns können bedrohliche zerebrale Symptome auftreten, bevor die allgemeine Körpertemperatur so ausgeprägt wie beim Hitzschlag erhöht ist.

\section{(C) Wichtig}

Die morphologischen Befunde bei Tod durch allgemeine Hyperthermie sind in der Regel unspezifisch, neben subpialen Blutaustritten finden sich subseröse Blutungen. Die Diagnose wird in der Regel durch Ausschluss anderer Todesursachen unter Berücksichtigung von Umständen des Todeseintritts und Auffindungssituation gestellt. Morphologische und toxikologische Untersuchungen dienen darüber hinaus der Aufdeckung prädisponierender Faktoren.

\subsubsection{Kälte}

\section{E. Lignitz}

Andersen hat in seinem Märchen »Das Mädchen mit den Schwefelhölzchen « die Problematik der allgemeinen Unterkühlung in allen Details hervorragend beschrieben. Kälte ist ein ubiquitäres Gefahrenmoment, das allgemein unterschätzt wird. Plötzlich hereinbrechend kann sie unter bestimmten disponierenden Bedingungen deletär wirken. Die Kältewirkung entfaltet sich keineswegs erst bei Temperaturen von $0{ }^{\circ} \mathrm{C}$ und darunter. Eine Auskühlung kann sich selbst in tiefer Narkose bereits im Operationssaal bei Umgebungstemperaturen um $21^{\circ} \mathrm{C}$ entwickeln. Allein durch Kälte zu erklärende Todesfälle sind in den kalten Regionen eher seltener anzutreffen, weil die Menschen aus Erfahrung rechtzeitig geeignete Schutzmaßnahmen gegen die Kältewirkungen treffen.

(i) Infobox

Epidemiologische Angaben

Todesfälle durch Unterkühlung machen im Sektionsgut von Großstadtinstituten etwa $1 \%$ aus. Im Sektionsgut von Instituten in Regionen mit vorwiegend klein- und mittelstädtischer sowie ländlicher Struktur kommen sie häufiger (2\%) vor. Dabei standen $50 \%$ der Verstorbenen unter erheblichem Alkoholeinfluss, der durchschnittlich 1,3\%o erreichte. Bei den nichtalkoholisierten Kälteopfern handelt es sich um meist ältere, kranke oder geistig verwirrte, orientierungslose Menschen. 
- Tabelle 3.28a. Hitzschlag - klassische Form - prädisponierende Faktoren

\begin{tabular}{|l|l|l}
\hline Vorbestehende Krankheiten & Medikamente & Konstitutionelle Faktoren \\
\hline - Herzinsuffizienz & - Diuretika & - Kleinkinder \\
- Schilddrüsenkrankheit & $-\beta$-Blocker & - Übergewichtige \\
- Infektionskrankheiten & - Phenothiazine & - Ältere Menschen \\
- Alkohol- und Drogenkrankheit & - Amphetamine & - Noch nicht akklimatisierte Personen \\
- Parkinsonkrankheit & - Antiparkinson-Mittel \\
- Hautkrankheiten: & - Trizyklische Antidepressiva \\
& Angeborene ektodermale Dysplasie & - Antihistaminika \\
- Zystische Fibrose & \\
- Diabetes mellitus &
\end{tabular}

- Tabelle 3.28b. Formen des Hitzschlags

\begin{tabular}{|c|c|c|}
\hline & Anstrengungshitzschlag & Klassischer Hitzschlag \\
\hline $\begin{array}{l}\text { Betroffener Personen- } \\
\text { kreis }\end{array}$ & $\begin{array}{l}\text { - Sportler } \\
\text { - Soldaten } \\
\text { - Bergleute } \\
\text { - Hüttenarbeiter }\end{array}$ & $\begin{array}{l}\text { - Ältere mit chronischen Erkrankungen } \\
\text { - Alkoholiker } \\
\text { - Drogensüchtige } \\
\text { - Kleinkinder }\end{array}$ \\
\hline $\begin{array}{l}\text { Häufigkeit } \\
\text { Symptome }\end{array}$ & $\begin{array}{l}\text { - Sporadisch } \\
\text { - Hyperpyrexie } \\
\text { - Desorientiertheit } \\
\text { - Keine Anhidrosis } \\
\text { - Laktatazidose }\end{array}$ & $\begin{array}{l}\text { - Epidemisch } \\
\text { - Koma, Hyperpyrexie } \\
\text { - Anhidrosis } \\
\text { - Respiratorische Alkalose }\end{array}$ \\
\hline Komplikationen & $\begin{array}{l}\text { - Hyperdynamer Kreislauf bei Diagnosestellung } \\
\text { - Rhabdomyolyse } \\
\text { - Disseminierte intravaskuläre Gerinnung } \\
\text { - Akutes Nierenversagen } \\
\text { - Irreversible Schädigung des ZNS } \\
\text { - Hyper- und Hypokaliämie }\end{array}$ & $\begin{array}{l}\text { - Hyperdynamer Kreislauf bei Diagnosestellung } \\
\text { - Schädigung des ZNS, Komplikation seitens der } \\
\text { Grunderkrankung } \\
\text { - Myokardinfarkt } \\
\text { - Hypokaliämie }\end{array}$ \\
\hline
\end{tabular}

\section{- Definition}

Unterkühlungen sind der Ausdruck eines erheblichen Ungleichgewichts zwischen erhöhtem Wärmeverlust und unzureichender Wärmebildung. Als Unterkühlung werden alle Zustände bezeichnet, bei denen die Körperkerntemperatur auf unter $35^{\circ} \mathrm{C}$ abgesunken ist. Klinisch werden sie je nach Auskühlungszustand in leichte bis tiefe Grade der Hypothermie eingeteilt.

\section{Physiologie des Wärmehaushaltes und Pathophysiologie der Kältewirkung}

Dank einer autonomen Temperaturregelung, d.h. der gesteuerten Wärmeabgabe und der Fähigkeit zur regulatorischen Wärmebildung, gehört der Mensch zu den homoiothermen (endothermen) Lebewesen und kann seine hohe Körpertemperatur über dem Niveau der durchschnittlichen Temperatur seines Lebensraumes halten. Entscheidend ist dabei, dass im Falle der Auskühlung die Wärmeneubildung regulatorisch durch Steigerung des Energieumsatzes angehoben wird. Sichtbar wird das durch rhythmische Muskelkontraktionen, die als Kältezittern wahrzunehmen sind und sichtbar werden.

\section{Wichtig}

Neugeborene und Kleinkinder bilden Wärme »zitterfrei« mittels des mitochondrienreichen braunen Fettgewebes.

Kältezittern ist aber wegen der gleichzeitigen erhöhten Wärmeabgabe durch Konvektion nicht ausreichend effektiv. Beim Menschen besteht ein Temperaturgefälle vom Körperkern zur Körperoberfläche (Körperschale). In warmer Umgebung ist die Hautdurchblutung gesteigert, die Haut warm und demzufolge die Wärmetransportrate hoch. In kalter Umgebung wird die Hautdurchblutung reduziert, der Wärmetransport und der Wärmeverlust verringert, die Haut kühlt ab. Die sog. Körperschale nimmt zu 
und der praktisch isotherme Körperkern verkleinert sich. Das radiäre Temperaturgefälle vom Körperkern zur Peripherie wächst; an den Gliedmaßen entwickelt sich zusätzlich ein axiales Temperaturgefälle. Deshalb ändert sich die Temperatur an den Gliedmaßen mit abnehmender Umgebungstemperatur stärker.

\section{Wichtig}

Wegen der Unterschiede der Körperkern- und Körperoberflächentemperatur muss zur Erfassung realistischer Werte und zur Reduzierung von Messfehlern die Körperkerntemperatur bei Unterkühlung (und zur Todeszeitbestimmung, s. Kap. 2.1.4) tief im Rektum oder unter intensivmedizinischen Bedingungen im Ösophagus gemessen werden.

Wärmeabgabe. Bei Abnahme der Umgebungstemperatur und regulatorischer Minderung der Hautdurchblutung erreicht die Isolationskraft der Körperschale, zu der auch das Unterhautfettgewebe gehört, ihr Maximum. An der Wärmeabgabe beteiligen sich die Konvektion, d.h. Wärmeabgabe der wärmeren Haut zur niedrigeren Temperatur der Umgebung und in gewissem Umfang die Strahlung durch Emission langwelliger Infrarotstrahlung, die zusammen als »trockene« Wärmeabgabe beschrieben werden. Wärmeabgabe erfolgt weiter durch die Konduktion, d.h. die Wärmeleitung durch direkten Kontakt von Haut und kalter Umgebung, z.B. beim Aufenthalt im Wasser. Schließlich wird die Wärmeabgabe durch Verdunstung von Schweiß ergänzt, sie ist zugleich die effektivste Form der Wärmeabgabe (thermoregulatorisches Schwitzen).

Warm- und Kaltsensoren erfassen die Körpertemperatur. Durch einen Regelkreis mit paradoxer Rückkopplung kann der Organismus Änderungen der Temperatur entgegenwirken. Bei niedrigen Umgebungstemperaturen, die individuell unterschiedlich als Kälte empfunden werden, übersteigt trotz maximaler Engstellung der Hautgefäße die Wärmeabgabe die Wärmebildung.

Hypothermie. Wenn eine Wärmeneubildung durch Steigerung des Energieumsatzes nicht mehr gedeckt werden kann, setzt eine Auskühlung (Hypothermie) ggf. bis zum Kältetod ein. Eine prolongierte Auskühlung führt zu einer »kälteinduzierten« Diurese und damit zu einer Zunahme der Blutviskosität und Verringerung des Blutflusses. Bei Progression stellt sich eine Anurie ein. Bei tiefen Temperaturen entstehen Elektrolytverschiebungen. Kalium wird aus dem Extrazellular- in den Intrazellularraum verlagert bei weitgehend konstanten Natriumkonzentrationen. Diese Veränderung des Kalium/Natrium-Quotienten und die feste Bindung des Sauerstoffs an das Hämoglobin bei gesteigerter $\mathrm{CO}_{2-}$ Löslichkeit bewirken Kammerflimmern, Azidose und Hirnödem (Kälteschwellung des Gehirns).

Adaption an Kälte. Mehrmalige wiederkehrende Kältebelastungen führen zu einer gewissen Toleranzadaptation, d.h. die Schwelle zum Kältezittern ist zu niedrigeren Werten verschoben (Eisbaden!). Das gilt auch für die Schmerzempfindung bei lokaler Kältewirkung, die gewissermaßen trainiert werden kann. Wenn jedoch die Mechanismen der Kälteabwehr überfordert werden
(z.B. in Bergnot oder bei Seenotfällen, die zu langem Aufenthalt in kaltem Wasser führen), so folgt einer vorübergehenden Phase der Kompensation alsbald der Ausfall jeglicher Regulation. Diesen sog. akzidentellen Hypothermien kann auch eine zentrale Störung der Kälteabwehr (z.B. bei Alkoholintoxikationen) zugrunde liegen.

(i) Infobox

Der Sauerstoffbedarf der Gewebe wird bei der Chirurgie am offenen Herzen unter entsprechender Myokardprotektion durch künstliche Hibernation reduziert. Eine ausgeprägte Hypothermie $\left(28-18^{\circ} \mathrm{C}\right.$, in Ausnahmefällen $\left.18-4^{\circ} \mathrm{C}\right)$ erlaubt Kreislaufstillstandszeiten von 60-90 Minuten.

\section{Lokale Kältewirkung (Erfrierung)}

Örtliche Erfrierungen betreffen die Gefäßendstrombahn. So sind die Akren Finger, Zehen, Hände und Füße und am Kopf Nase und Ohren am meisten betroffen. Der Grad der Gewebeschädigung hängt von der Dauer der Kälteexposition ab, die zu einer Gefäßkonstriktion führt und eine Ischämie der nachgeschalteten Gewebe bewirkt. Die Ausschüttung histaminartiger Substanzen unterhält einen exsudativ-entzündlichen Prozess, der später zu einer Vasodilatation mit einer erhöhten kapillären Durchlässigkeit, Schmerzreaktionen und letztlich zu Nekrosen führt. Disponierend wirken bei der örtlichen Kältewirkung auch enge Kleidung, Hand- und Fußschweiß und ein reduzierter Allgemeinzustand.

Definition
Die lokale Erfrierung (Congelatio) wird in Stadien eingeteilt:
— Dermatitis congelationis erythematosa: Die betroffene
Körperstelle wird infolge der anfänglichen Gefäßkonstriktion
weiß, gefühllos oder schmerzhaft, später deutlich gerötet
und geschwollen, häufig stark juckend.
— Dermatitis congelationis bullosa: Nach Wiedererwär-
mung einer länger und tiefer kälteexponierten Region bilden
sich subepidermal seröse oder hämorrhagische Blasen.
- Dermatitis congelationis gangraenosa (escharotica): Die
betroffene Extremität verfärbt sich blauschwarz als Ausdruck
des Gewebstodes. Im günstigen Falle entwickelt sich ein tro-
ckener Gewebsbrand. Die Gliedmaße wirkt wie mumifiziert.
Bei bakterieller Besiedlung entsteht eine feuchte Gangrän. In
jedem Falle wird das betroffene Gewebe gegenüber dem ge-
sunden auffällig demarkiert.

Folgeschäden. Nach prolongierter und progressiver Kälteschädigung treten häufig thrombotische und obliterierende Gefäßwandschäden in Venen und Arterien oder nur in Arterien (Buerger'sche Erkrankung) auf. Man spricht auch von invisiblen Kälteschäden, die Neuritiden und Neuralgien im Gefolge haben.

Frostbeulen. Sie stellen ein eigenes Krankheitsbild dar. 


\begin{abstract}
- Definition
Als Frostbeulen (Perniones) werden blaurote, ödematöse, unscharf begrenzte, knötchen- oder kissenartig umschriebene Schwellungen beschrieben, die schon gelegentlich unterhalb normaler Zimmertemperaturen auftreten können.
\end{abstract}

Bevorzugt treten sie an Streckseiten von Fingern und Zehen auf. Sie setzen eine konstitutionelle Gefäßfunktionsstörung voraus und kommen meist bei jungen Menschen in den kühleren Jahreszeiten vor. Nässe, enge Kleidung und enges Schuhwerk disponieren.

In der forensischen Pathologie werden als Nebenbefund - gelegentlich auch bei Kältearbeitern - schwielige Verdickungen des Ohrmuschelrandes nach lokalen Erfrierungen gesehen (Chondrodermatitis helicis), die ggf. Identifizierungswert haben könnte.

\section{Allgemeine Kältewirkung (Unterkühlung, Hypothermie)}

Die Unterkühlung tritt vorzugsweise akzidentell auf, zumeist in Verbindung mit Alkoholgenuss, oder im Gefolge körperlicher Erschöpfung, konsumierender Krankheit und bei hohen Windgeschwindigkeiten (wind chill effect). Bei bestimmten Lufttemperaturen und hohen Windgeschwindigkeiten (auch durch Fahrtwind, z.B. auf Motorrädern und Rennbooten) ergeben sich Kältewerte, wie sie im Polargebiet vorkommen (• Tabelle 3.29). Bei durchnässter Kleidung entfällt deren isolierende Schutzfunktion und der Wärmeverlust wird unkontrollierbar. Die Dauer der Kälteexposition entscheidet wesentlich über die Folgen (•Tabel-

- Tabelle 3.29. Wirksame Temperatur bei unterschiedlichen Windgeschwindigkeiten

Bezugstemperaturen in ${ }^{\circ} \mathrm{C}$ bei Windstille

\begin{tabular}{l|l|l|l|l}
\hline $0 \mathrm{Bft}$ & +10 & +5 & 0 & -5 \\
\hline
\end{tabular}

wirksame Temperatur in ${ }^{\circ} \mathrm{C}$ bei unterschiedlichen Windstärken in Bft ( $\mathrm{m} / \mathrm{s})$

\begin{tabular}{|l|r|r|r|r}
\hline $3 \mathrm{Bft}$ & +4 & -2 & -9 & -15 \\
$5 \mathrm{Bft}$ & 0 & -8 & -15 & -22 \\
$7 \mathrm{Bft}$ & -3 & -10 & -18 & -26
\end{tabular}

- Tabelle 3.30. Zusammenhang zwischen Wassertemperatur und Überlebenszeit

\begin{tabular}{|c|l}
\hline Wassertemperatur & Überlebenszeit \\
\hline $20^{\circ} \mathrm{C}$ & Ca. 40 Stunden \\
$15^{\circ} \mathrm{C}$ & Ca. 5 Stunden \\
$10^{\circ} \mathrm{C}$ & Ca. 3 Stunden \\
$5^{\circ} \mathrm{C}$ & Ca. 2 Stunden \\
$0^{\circ} \mathrm{C}$ & nach 30 Minuten akute Lebensgefahr
\end{tabular}

le 3.30), sodass auch Wassertemperaturen um $20^{\circ} \mathrm{C}$ zu Unterkühlungen führen können. Insoweit sind Unterkühlungen bedeutungsvoll für den Tod im Wasser. Bei vielen Ertrinkungsfällen ist die Unterkühlung als initialer Prozess zu vermuten.

\section{Wichtig}

Unfälle im Wasser bei weit unter $20^{\circ} \mathrm{C}$ bedeuten eine schwere Gefahr durch übermäßigen Wärmeverlust und führen zwangsläufig zu einer Unterkühlung. Körperliche Aktivität (z.B. Schwimmen) im kalten Wasser erhöht die Abkühlungsgeschwindigkeit um etwa $30-50 \%$.

Alkoholeinfluss wirkt der an sich schützenden Vasokonstriktion entgegen, die anfangs den Wärmeverlust einzuschränken vermag, und täuscht infolge Gefäßdilatation subjektiv Wärmeempfinden vor. Bei längerer Unterkühlungsdauer treten Vigilanzeinbußen und damit einhergehend Kritiklosigkeit und Orientierungsverlust auf, immer verbunden mit zunehmender Handlungsunfähigkeit (• Tabelle 3.31). Beim unbeabsichtigten Sturz in kaltes Wasser (meist unter $10^{\circ} \mathrm{C}$ ) kann durch sofortige generalisierte Hemmung von Kortex und Subkortex ein reflektorischer Kälteschock eintreten.

\section{Wichtig}

Sauerstoffmangel und Kreislaufversagen bis zum Herzstillstand werden von stark unterkühlten Personen weitaus länger toleriert als von normothermen Personen. Bei Unterkühlten sind daher intensive und stundenlange Wiederbelebungsversuche auch nach längerem Kreislaufstillstand angebracht. Tiefe Unterkühlung bewirkt scheintodähnliche Zustände. Todesfeststellung in jedem Falle gründlich absichern!

Zentralnervöse Störungen. Bereits geringe Grade von Unterkühlung haben einen negativen Einfluss auf die Entscheidungsfindung und bewirken einen Leistungsabfall. Trotz Kälteeinfluss und sinkender Körpertemperatur wird subjektiv ein Wärmegefühl spürbar.

\section{- Definition}

Dieses Wärmegefühl führt zu einem Verhaltensmuster, das als »Kälteidiotie« bezeichnet wird. Es ist gekennzeichnet durch die völlig unlogische Entfernung der Kleidungsstücke bis zur völligen Nacktheit.
Kriminalistisch ergeben sich daraus beinahe regelmäßig Fehleinschätzungen und falsche Ermittlungsansätze. Wenn nicht schon die Leichenschau den Verdacht auf eine allgemeine Unterkühlung lenkt, finden sich bei der Sektion entsprechende Hinweise.

\section{Forensische Pathologie der allgemeinen Unterkühlung}

Im klassischen Falle lassen sich Befunde zusammentragen, die in ihrer Gesamtheit eine allgemeine Unterkühlung als Todesursache eindeutig gestatten, obwohl jede Einzelveränderung für sich unspezifisch ist. 
- Tabelle 3.31. Klinische Stadieneinteilung und Symptomatik der akzidentellen Hypothermie (nach Singer)

\begin{tabular}{|c|c|c|c|c|}
\hline $\begin{array}{l}1 \text { leicht } \\
36-33^{\circ} \mathrm{C}\end{array}$ & $\begin{array}{l}2 \text { mittel } \\
33-30^{\circ} \mathrm{C}\end{array}$ & & $\begin{array}{l}3 \text { tief } \\
30-27^{\circ} \mathrm{C}\end{array}$ & $\begin{array}{l}4 \\
\text { unter } 27^{\circ} \mathrm{C}\end{array}$ \\
\hline $\begin{array}{l}\text { maximale Kälte- } \\
\text { regulation }\end{array}$ & $\begin{array}{l}\text { Abnahme des aktiven } \\
\text { Muskeltonus }\end{array}$ & $\Rightarrow$ & Zunahme der passiven Muskelrigidität & $\begin{array}{l}\text { entweder weitere Dämpfung der } \\
\text { erhaltenen Vitalfunktionen }\end{array}$ \\
\hline Muskelzittern & Sinusbradykardie & $\Rightarrow$ & Bradyarrhythmie & \\
\hline $\begin{array}{l}\text { gesteigerte Vigilanz, Ver- } \\
\text { wirrtheit; Schmerzhaftig- } \\
\text { keit der Akren }\end{array}$ & $\begin{array}{l}\text { Desorientierung, Apa- } \\
\text { thie } \\
\text { Abklingen der Schmerzen }\end{array}$ & $\Rightarrow$ & $\begin{array}{l}\text { Bewusstlosigkeit, } \\
\text { Reflexverlust }\end{array}$ & \\
\hline
\end{tabular}

\section{Äußerliche Besichtigung:}

- Hell-rötlich gefärbte Totenflecke: Diese sind durchgehend hellrot und nicht nur an den Rändern, wie es an Leichen gesehen wird, die kühl oder auf kühler Unterlage gelagert werden.

- "Gänsehautbildung" (kann auch Ausdruck der Totenstarre sein!);

- blau-livide Hautverfärbungen (Akren, Handrücken, Kniegelenk-Streckseiten) sind von zumeist glänzender Beschaffenheit und weisen eine allgemeine teigige Schwellung auf (• Abb. 3.100).

\section{Innere Besichtigung:}

- Hellrotes Blut in den Blutgefäßen der Körperperipherie und dunkelrotes Blut in den Organen, die bei zentralisiertem Kreislauf noch durchblutet werden.

- Hämorrhagische Schleimhauterosionen des Magens, gelegentlich auch im Duodenum (sog. Wischnewski-Flecke), als Folge vasomotorischer Störungen (häufig; »leopardenfellartige Fleckung «; $\bullet$ Abb. 3.101).

Einer von beiden Befunden, Wischnewski-Flecke oder akrozyanotische Kälteschäden der Haut, werden bei Tod an Hypothermie mit großer Regelmäßigkeit gefunden! Beschrieben wurden auch Blutungen der inneren Hüftmuskeln (M. iliopsoas), die in der Exitationsphase entstehen dürften, aber eher seltener angetroffen werden. Beinahe regelmäßig fällt ein Kollapszustand der Milz mit feiner Fältelung ihrer Kapsel auf, eine morphologische Bestätigung der Blutvolumenverschiebung im protrahierten Schock bei nichtkompensierter Kältewirkung. Blutungen der Synovia bzw.

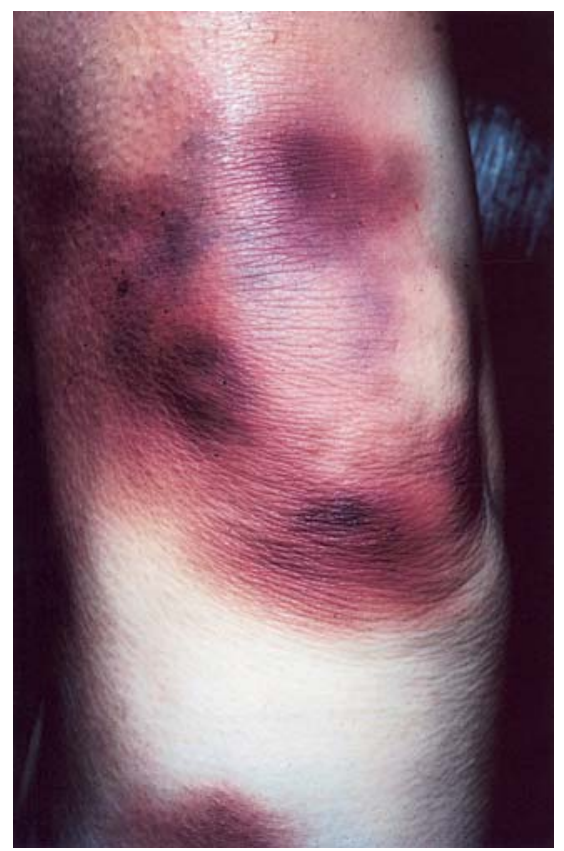

- Abb. 3.100. Blau-glänzende Hautschwellung der Kniegelenksstreckseite bei Unterkühlung

Blutbeimischungen zur Gelenkflüssigkeit wurden in den Kniegelenken bei gesicherten Unterkühlungsfällen immer wieder beobachtet, sodass sie das Mosaik der morphologisch fassbaren Kälteschädigung ergänzen dürften. Dazu gehören häufig Nieren- 


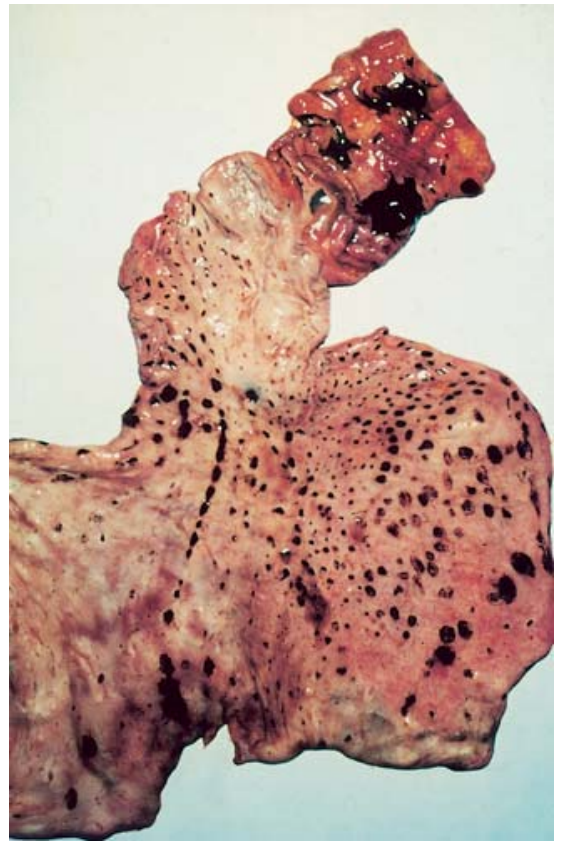

- Abb. 3.101. Hämorrhagisch-erosive Gastroduodentis (sog. Wischnewski-Flecke) bei allgemeiner Unterkühlung

epithelverfettung gelegentlich auch subendokardiale Blutungen. Histologisch lassen sich ein Lungenödem, intraalveoläre, intrabronchiale und interstitielle Hämorrhagien nachweisen. Im Herzmuskel bestehen Areale von Myozytolysen. Auch Glykogenverluste in Leberzellen, Herz- und Skelettmuskelzellen werden beschrieben. In Summation der Erkenntnisse aus der Pathophysiologie und Morphologie der Kältewirkung sehen Experten eine Hypoxidose und Hypoxämie (»inneres Ersticken«) als eigentlichen Todesmechanismus an. Man darf nicht erwarten, das Vollbild aller bekannten Kälteschäden zu finden. Viel eher ist ein Tod durch allgemeine Unterkühlung mindestens zu einem gewissen Teil auch eine Ausschlussdiagnose anderer todeswürdiger Faktoren. Die abschließende Diagnose sollte sich immer auf ein gutes Ermittlungsergebnis stützen dürfen. $\mathrm{Zu}$ beachten ist, dass bei Ertrinkungstodesfällen, denen eine initiale Auskühlung vorausgeht, offensichtlich wegen des raschen Ablaufes kaum makroskopisch fassbare kälteassoziierte Veränderungen zu erkennen sind!

\section{( Wichtig}

Bevor hellrot gefärbte Totenflecke als Zeichen der Unterkühlung angesehen werden, ist ihre Differentialdiagnose wegen der Möglichkeit von Vergiftungen zu prüfen.

Tod durch Unterkühlung ist wegen des Fehlens spezifischer beweiserheblicher Befunde wenigsten teilweise eine Ausschlussdiagnose.

\section{F) Fallbeispiel}

Ein zu Alkoholmissbrauch neigender Mann wurde mehrfach beobachtet, wie er unter erkennbarer Alkoholwirkung spät nachts seine Wohnung erreichte, sich im Vorgarten entkleidete und hinlegte. An dieses merkwürdige Verhaltensmuster hatte sich sein Umfeld bereits gewöhnt. In der kälteren, aber noch frostfreien Jahreszeit wurde er zur allgemeinen Verwunderung eines Morgens nackt inmitten seiner verstreut liegenden Kleidungsstücke tot aufgefunden.

Rechtliche Grundlage. Unter bestimmten Bedingungen kann sich bei Todesfällen durch allgemeine Unterkühlung der Tatbestand der unterlassenen Hilfeleistung erfüllen.

\section{$\S 323 c$ StGB}

Wer bei Unglücksfällen oder allgemeiner Gefahr oder Not nicht Hilfe leistet, obwohl dies erforderlich und ihm den Umständen nach zuzumuten, insbesondere ohne erhebliche eigene Gefahr und ohne Verletzung anderer wichtiger Pflichten möglich ist, wird mit Freiheitsstrafe ... oder mit Geldstrafe bestraft.

\section{Checkliste}

- Zur Sicherung der Diagnose »Tod durch Unterkühlung« sind konkurrierende Todesursachen auszuschließen.

- Zur Klärung der meteorologischen Situation sollten bei unklaren Todesfällen im Freien die rezenten klimatischen Bedingungen in regionalen Wetterstationen angefragt und in die Bewertung mit einbezogen werden.

- In jedem Falle sind toxikologische Untersuchungen durchzuführen (Alkohol, Drogen, Medikamente).

\subsection{Elektrotraumen, Blitzschlag}

\section{S. Pollak}

\section{Einleitung}

Trotz zunehmender Anwendung der elektrischen Energie weist die Statistik einen Rückgang der Elektrounfälle aus. Eine sinkende Tendenz ist in gleicher Weise bei überlebten und bei tödlichen Unfällen festzustellen. Dieser rückläufige Trend besteht im industriell-gewerblichen Bereich ebenso wie in den Haushalten. Das relativ seltene Vorkommen derartiger Ereignisse begünstigt die Verkennung spurenarmer Stromtodesfälle bei der Leichenschau: Wenn die Auffindungssituation nicht konkret auf ein Elektrotrauma hinweist, wird diese Todesursache kaum noch in die differentialdiagnostischen Erwägungen einbezogen.

Stromunfälle entstehen im Niederspannungsbereich $(<1.000$ V) vor allem durch Berührung von elektrisch leitenden Gegenständen/Oberflächen, die unter Spannung stehen. Wenn die elektrische Einwirkung keine gesundheitliche Schädigung nach sich zieht, dann spricht man von einer folgenlosen Durchströmung. 
Die Sonderstellung des Stromunfalls innerhalb der Traumatologie ist u.a. durch seine hohe Letalität bedingt.

\subsubsection{Stromwirkungen im Niederspannungsbereich}

Elektrizität kann den menschlichen Organismus auf zweierlei Weise beeinflussen und schädigen:

- durch spezifische (»physiologische«) Wirkung (Auslösung von Erregungsprozessen an Muskeln und Nerven) und

- durch unspezifische Wirkung (Umwandlung in Wärmeenergie, thermische Gewebsschädigung).

Bei Niederspannung resultiert die Gefährlichkeit ganz überwiegend aus der spezifischen Wirkung: Die Durchströmung führt entweder zum tödlichen Herzkammerflimmern oder sie wird meist ohne bleibende Beeinträchtigung - überlebt (Alles-oderNichts-Gesetz).

Art und Umfang der Gefährdung hängen von zahlreichen äußeren und inneren Faktoren ab; dazu zählen u.a:

- Der Zeitpunkt des Stromstoßes in Bezug auf die vulnerable Periode der Herzaktion (entspricht der relativen Refraktärzeit und fällt im EKG mit dem aufsteigenden Schenkel der T-Welle zusammen);

- die Durchströmungsdauer (Einwirkungszeiten unter $100 \mathrm{~ms}$ sind im Allgemeinen ungefährlich, mit zunehmender Durchströmungsdauer sinkt die Schwellenstromstärke für die Flimmerauslösung am Herzen bis gegen $50 \mathrm{~mA} \mathrm{ab}$ );

- der Stromweg durch den Körper (bei Querdurchströmung von Hand zu Hand fließt ein geringerer Anteil des Stromes über das Herz als bei Längsdurchströmung);

- das Lebensalter (mit zunehmendem Alter steigt die Letalität von Durchströmungsunfällen, offenbar in Abhängigkeit von der Inzidenz kardialer Vorschäden);

- die Stromstärke als Funktion der vorgegebenen Spannung (im Haushalt meist $230 \mathrm{~V}$ ) und des Widerstandes (Summe aus Körper- und Übergangswiderstand).

Zur Charakterisierung der Gefährdung durch technischen Wechselstrom werden verschiedene Stromstärkenbereiche unterschieden. Neuere Schwellenwertangaben hinsichtlich der physiologischen Effekte berücksichtigen nicht nur die Stromstärke, sondern auch die Einwirkungsdauer auf den Körper (»Effects of current on human beings and livestock«, Publikation IEC 479-1 der International Electrotechnical Commission, 1994).

Neben der oben erwähnten Flimmerschwelle des Herzmuskels, die v.a. von der Reizstromdauer und - im Falle eines Wechselstroms - von dessen Frequenz abhängt, ist auch die sog. Loslassstromstärke von Bedeutung: Noch bevor der Strom das Herz zum Flimmern bringt, erregt er bereits die Nerven mit der Folge einer anhaltenden Muskelkontraktion. Ein mit der Hand ergriffener, unter Spannung stehender Gegenstand kann dann nicht mehr losgelassen werden. Die »Loslassgrenze« wird ab etwa $15 \mathrm{~mA}$ überschritten.

Die lokale, im Gewebe freigesetzte Wärmeenergie steigt mit zunehmender Stromdichte (Stromstärke pro Fläche). Die Wahrscheinlichkeit einer lokalen thermischen Hautläsion ist daher bei kleiner Kontaktfläche höher als bei großflächiger Berührung des Leiters. Weitere Einflussgrößen sind der spezifische Widerstand des durchströmten Gewebes und die Stromflussdauer. Im Niederspannungsbereich ist die Strommarke der wichtigste elektrothermische Effekt. Der anfänglich hohe Widerstand der Haut sinkt schon in der ersten Sekunde nach Beginn des Stromflusses rasch ab. Wenn es dem Betroffenen nicht gelingt, sich vom spannungsführenden Gegenstand zu lösen, dann kommt es zum Durchschlag der Haut, die damit ihre Schutzfunktion weitgehend verliert.

Der elektrische Strom wurde als »anonyme Bedrohung « bezeichnet: Die Sinnesorgane des Menschen werden durch elektromagnetische Felder, die spannungsführende und stromdurchflossene Leiter umgeben, nicht erregt. Im Haushalt steht diese potentiell lebensbedrohliche Energieform auch dem elektrotechnischen Laien zur Verfügung. Die bekannte Tatsache, dass kurzzeitige Kontakte mit einem unter Spannung stehenden Leiter (»Wischer«) ohne Schaden überlebt werden können, verleitet dazu, die tödliche Gefahr eines elektrisch ausgelösten Herzkammerflimmerns zu unterschätzen. Der im Haushalt verfügbare Wechselstrom hat eine Frequenz von $50 \mathrm{~Hz}$; die Wahrscheinlichkeit der Flimmerauslösung ist bei einem Strom, dessen Richtung sich ständig ändert, wesentlich höher als bei Einwirkung von Gleichstrom. Bei sehr hohen Frequenzen ist die Eindringtiefe des Stromes gering, sodass die thermische Wirkung an der Grenzfläche zur Elektrode im Vordergrund steht (z. B. Hochfrequenzchirurgie im Frequenzbereich von $300-2000 \mathrm{kHz}$ ).

Die meisten Stromunfälle kommen durch Erdschluss zustande: Der Körper fungiert als Verbindung zwischen einer Phase (spannungsführender Leiter) und Erde. Wenn die Berührungsspannung vorgegeben ist (z.B. 230 V), wird der im Körper flieBende Strom durch den Gesamtwiderstand limitiert; dieser setzt sich aus dem Übergangswiderstand (zwischen Körper und Erde) und dem Körperwiderstand zusammen. Bei Durchströmungen gegen Erde hängt der Übergangswiderstand von der Kleidung (vor allem Schuhsohlen) und vom isolierenden Effekt der Unterlage (Bodenbeschaffenheit) ab. Der Körperwiderstand wird hauptsächlich durch den Hautwiderstand an den Kontaktstellen bestimmt; der Körperinnenwiderstand ist vergleichsweise gering.

Der Hautwiderstand steigt mit zunehmender Dicke der Hornschicht. Eine Befeuchtung (z.B. mit Schweiß, Waschlauge, Badewasser etc.) senkt den Hautwiderstand ganz wesentlich. Die Schweißsekretion ist bei körperlicher Arbeit und hoher Umgebungstemperatur besonders stark; eine zusätzlich vorhandene Wasserdampfsättigung der Luft (in Feuchträumen) verhindert die Schweißabdunstung und erhöht damit die Gefährlichkeit eines Leiterkontakts. 
Die Wahrscheinlichkeit des Auftretens von Strommarken, also von elektrothermischen Hautläsionen durch die lokal entstehende Joule'sche Wärme, wird mit steigender Stromdichte (an der Kontaktstelle) und mit zunehmender Stromflussdauer größer. Selbst nach einem tödlichen Elektrotrauma müssen jedoch keine Strommarken zurückbleiben, wenn die Kontaktfläche groß und der Hautwiderstand gering ist (z.B. beim Stromtod in der Badewanne). Man schätzt, dass in etwa $30 \%$ aller tödlichen Niederspannungsunfälle keine Strommarke an der Körperoberfläche erkennbar ist. Umgekehrt können aber überlebende Opfer deutliche Strommarken in Form von zweit- und drittgradigen Verbrennungen davontragen.

Im Niederspannungsbereich zählen Isolationsmängel an elektrischen Betriebsmitteln, Anschlüssen und Verlängerungskabeln zu den besonders häufigen Unfallursachen. Ein Vorkommensschwerpunkt sind Feuchträume (Badezimmer), wo auch intakte (gegen Berührung von spannungsführenden Teilen geschützte) Geräte zum Ausgangspunkt von Fehlerströmen werden, z.B. wenn ein Haarföhn, ein Heizstrahler oder ein Radioapparat in die mit Wasser gefüllte (und geerdete) Badewanne hineinfällt oder gewollterweise dorthin verbracht wird (bei Suiziden bzw. Fremdtötungen). Der Einbau eines Fehlerstrom-Schutzschalters (FI-Schalter mit einem Nennfehlerstrom von $30 \mathrm{~mA}$ und einer Abschaltzeit von $<0,2 s$ ) verhindert infolge Unterbrechung des Stromkreises im Allgemeinen eine tödliche Durchströmung des Körpers.

Suizide unter Anwendung von Elektrizität sind trotz der einfachen Verfügbarkeit von Stromquellen sehr selten, wenn man von der bereits erwähnten Begehung in einer wassergefüllten Badewanne absieht. In den übrigen Fällen erfolgt die Strombeibringung typischerweise über blanke Elektrokabel, die an verschiedenen Körperstellen (Handgelenke, Sprunggelenke, Finger, Zehen, Rumpf, Hals, Kopf) befestigt sein können, wobei manchmal Zeitschaltuhren zur Auslösung des Stromflusses eingesetzt werden. Wenn die Elektroden im Genital- und/oder Analbereich angebracht sind, muss an einen Unfall bei autoerotischer Betätigung gedacht werden.

Eine der wesentlichsten Voraussetzungen für die Erkennung letal verlaufener Niederspannungsunfälle ist die sorgfältige Vornahme der Leichenschau. Wenn nach dem Leichenbefund oder nach der Auffindesituation die Möglichkeit einer prämortalen Stromeinwirkung gegeben ist, sollte unbedingt eine autoptische Abklärung angestrebt werden. Schon die Symptome und Umstände, unter denen der Tod eingetreten ist, können auf einen Stromunfall hindeuten: tetanische Verkrampfung der Muskulatur, »Hängenbleiben« an einem von der Hand umfassten Leiter, plötzliches Aufschreien mit nachfolgendem Bewusstseinsverlust und Pulslosigkeit, terminales Zusammenbrechen bei Berührung eines Elektrogerätes oder -anschlusses, Totauffindung in der Nähe von Stromquellen etc.

Die Beurteilung von Todesfällen in der Badewanne ist besonders schwierig und verantwortungsvoll. Wenn autoptisch fassbare Residuen einer elektrischen Einwirkung (Strommarken) fehlen, kann die Diagnose »Stromtod « oft nur per exclusionem und unter Bezugnahme auf den elektrotechnischen Befund gestellt werden. Aus kriminalistischer Sicht stellt sich in solchen Fällen die Frage, ob die elektrische Durchströmung in der Badewanne als Unfall, Suizid oder Tötungsdelikt zu interpretieren ist.

Das wichtigste Kennzeichen eines stattgehabten Elektrotraumas ist zweifellos die Strommarke. Ihr Erscheinungsbild entspricht dem einer lokalen Verbrennung 2.-4. Grades, verursacht durch Joule'sche Wärme bei der Durchströmung der Haut, deren elektrischer Widerstand (bei vorgegebener Kontaktfläche) in trockenem Zustand und an stark verhornten Stellen relativ hoch ist $\left(40-100 \mathrm{k} \Omega \cdot \mathrm{cm}^{2}\right)$. Die Morphologie der Strommarke unterliegt zahlreichen modifizierenden Einflüssen: Form und Oberflächenrelief des elektrischen Leiters, Stromdichte, Kontaktdauer, Hautwiderstand (abhängig vom Ausmaß der Verhornung und von einer etwaigen Befeuchtung). Unter besonderen Umständen kann es zu einer formgetreuen Abprägung des elektrischen Leiters durch Ausbildung einer kongruenten Strommarke kommen (• Abb. $3.102+3.103)$. Die Strommarken sind grundsätzlich an den Kontaktstellen des elektrischen Leiters bzw. an den Stromübertrittsstellen gegen Erde lokalisiert. Am häufigsten sind die Hände betroffen (• Abb. 3.104).

An der stark verhornten Leistenhaut (Palma manus, Planta pedis) imponieren die Strommarken makroskopisch als grauweiße (»alabasterfarbene«, "porzellanfarbene«), wallartige Erhaben-

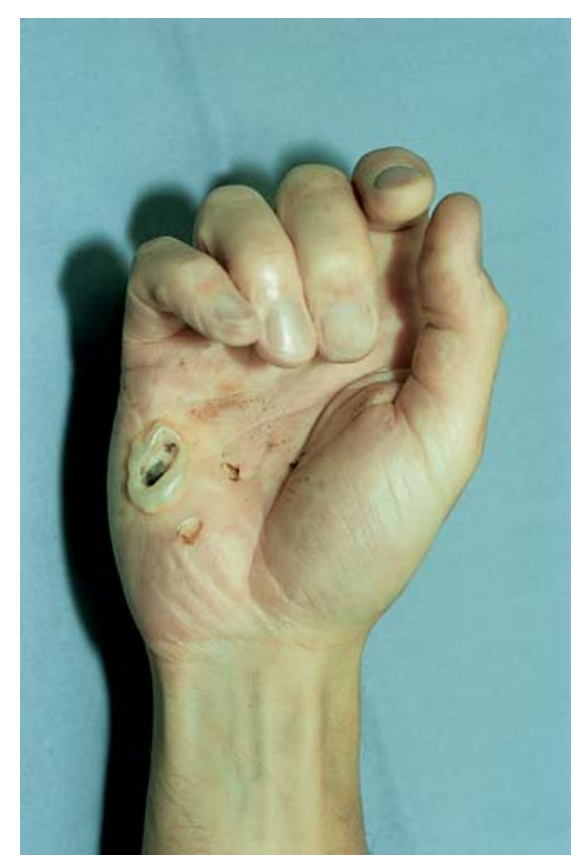

- Abb. 3.102. Rechte Hand eines 57-jährigen Mannes mit geformten Strommarken am Hypothenar (tödlicher Unfall infolge fehlerhafter Anbringung eines Kupplungssteckers am geräteseitigen Ende eines Verlängerungskabels) 


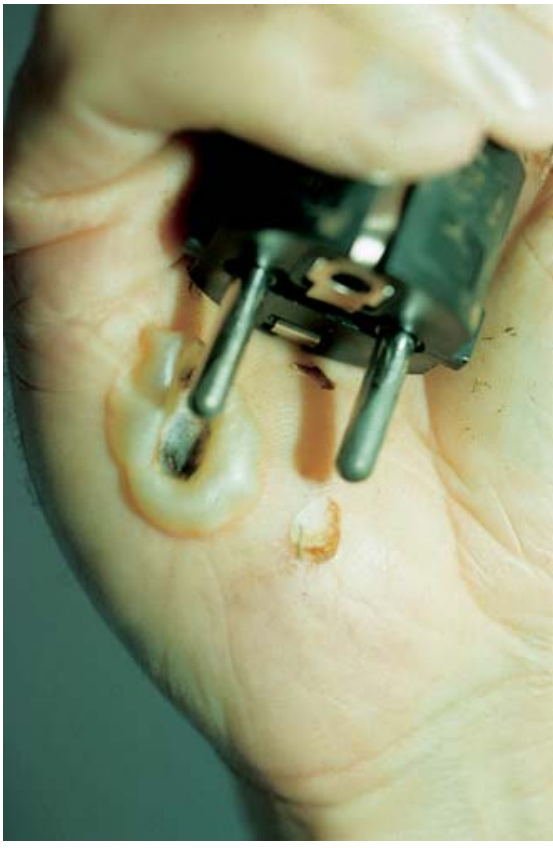

- Abb. 3.103. Rekonstruktion des Kontaktes zwischen Hand und Stecker

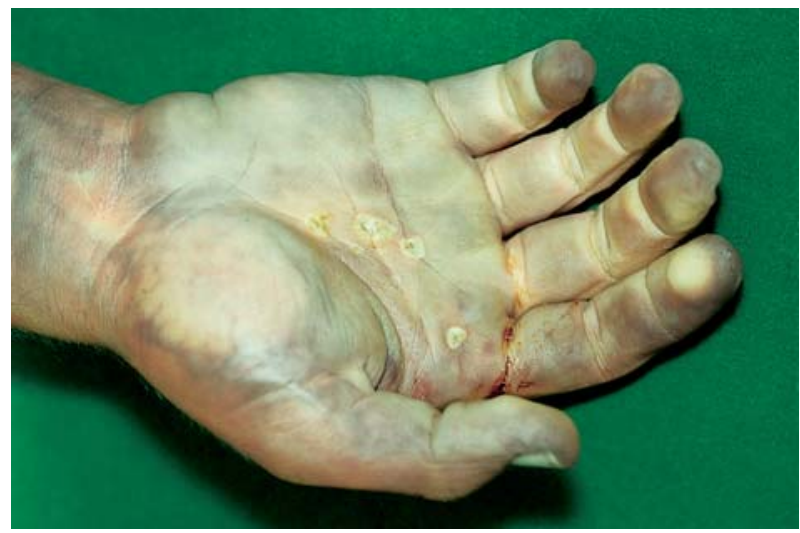

- Abb. 3.104. Linsengroße Strommarken an der Hohlhand eines 33jährigen Mannes (tödlicher Unfall beim Hantieren mit einer elektrischen Wasserpumpe; 220 V)

heiten - die örtliche Aufwerfung ist durch Gasblasen (»Hitzewaben «) in der Epidermis bedingt - mit gelbbraunen bis schwarz verkohlten Anteilen (• Abb. 3.105); bei überlebenden Opfern können sich schon nach kurzer Zeit flüssigkeitsgefüllte Brandblasen bilden. Die dünnschichtig verhornte Felderhaut zeigt demgegenüber im Kontaktbereich meist uncharakteristische Epidermisläsionen, die postmortal oft nur als braune Vertrocknungen imponieren.

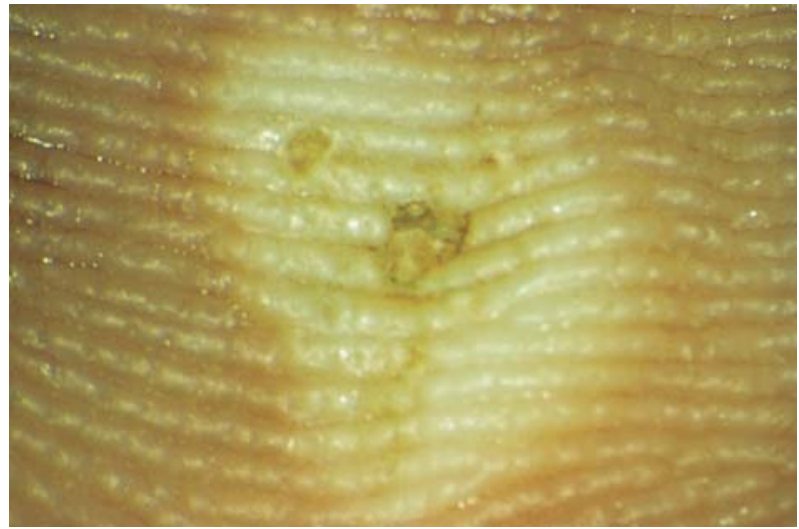

- Abb. 3.105. Detail einer porzellanfarbenen Strommarke (dickschichtig verhornte Haut der Palma manus)

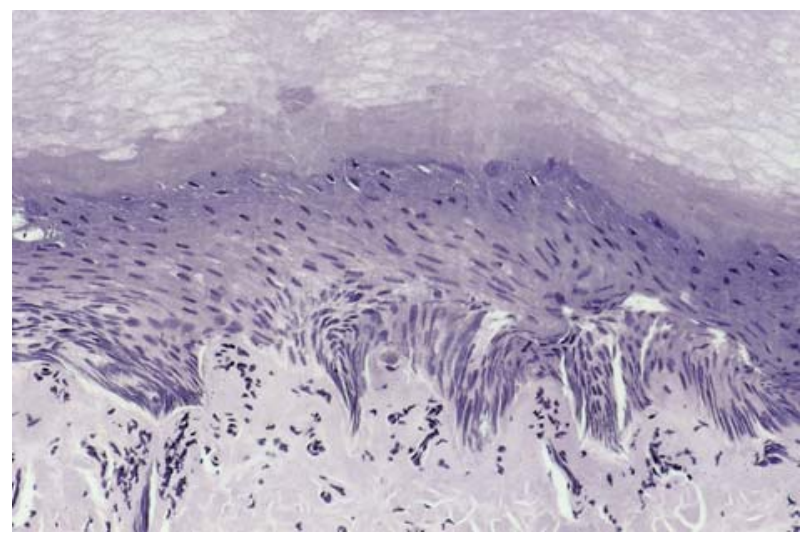

- Abb. 3.106. Epidermisveränderungen im Bereich einer Strommarke (Palmarhaut): büschelförmige Elongation und Palisadenstellung der Basalzellen (HE-Färbung)

Bei der histologischen Untersuchung von Strommarken findet man typischerweise eine büschelförmige Elongation der basalen Epidermiszellen (»Palisadenstellung«, »ziegenbartförmige Ausziehung «; - Abb. 3.106) in Kombination mit Hitzewaben (Gasblasen in der Epidermis, vor allem im Stratum corneum; - Abb. 3.107). Die Abgrenzung von nichtelektrisch verursachten Verbrennungen kann schwierig sein; bezeichnend für Strommarken ist die Inhomogenität der thermischen Gewebsschädigung (in Abhängigkeit vom strukturell unterschiedlichen Widerstandsverhalten: z.B. gute Leitfähigkeit entlang der Schweißdrüsenausführungsgänge). Ablagerungen des Leitermetalls im Strommarkenbereich (»Metallisation«) lassen sich histochemisch oder mittels Atomabsorptionsspektrometrie (AAS) nachweisen. Elektrothermische Verbrennungen können prinzipiell auch postmortal erzeugt werden, sind also - für sich allein betrachtet - kein sicherer Beweis für eine intravitale, todesursächliche Stromeinwirkung. 


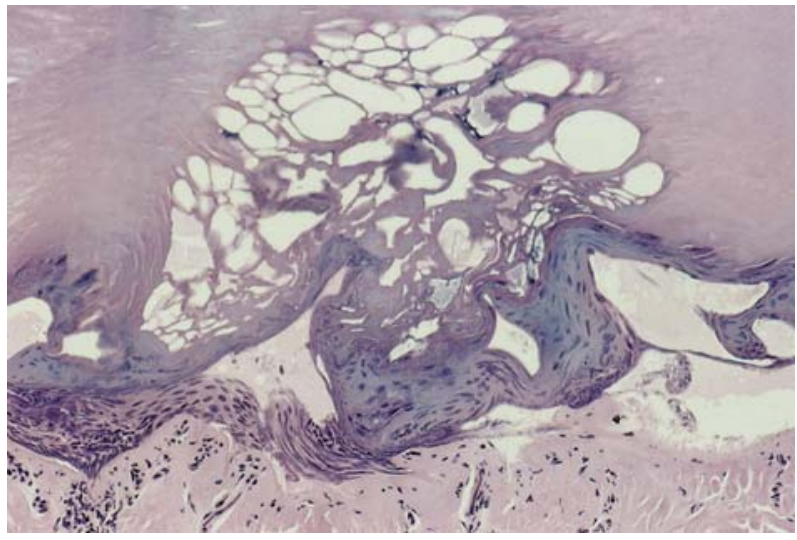

- Abb. 3.107. Epidermale »Hitzewaben« als histologisches Korrelat der Blasenbildung (Strommarke in der Palmarhaut, HE-Färbung)

Die inneren Befunde sind beim Stromtod im Allgemeinen unspezifisch. Wegen der aufgezeigten diagnostischen Schwierigkeiten sollte unbedingt in jedem Verdachtsfall eine sorgfältige elektrotechnische Untersuchung des Sterbeortes hinsichtlich möglicher Stromquellen und Leiterkontakte veranlasst werden.

\subsubsection{Stromwirkungen im Hochspannungsbereich}

Im Gegensatz zur Niederspannung ist bei Einwirkung hochgespannter Elektrizität auch mit Spättodesfällen zu rechnen. Allerdings versterben etwa 4/5 der Opfer noch an der Unfallstelle, zumeist an Herzrhythmusstörungen (Kammerflimmern). Die Überlebenszeit der Übrigen variiert in Abhängigkeit vom Grad und von der Ausdehnung der erlittenen Verbrennungen.

Mitunter wird die Meinung vertreten, dass die durch Hochspannung verursachten Todesfälle stets leicht zu erkennen seien. Die große Formenvielfalt der Strommarken und ihre multifaktorielle Bedingtheit können aber dazu führen, dass selbst die Spuren hochgespannter Elektrizität unerkannt bleiben oder falsch interpretiert werden. Bei kurzem, innigem Kontakt und niedrigem Hautwiderstand können die durch Hochspannung erzeugten Strommarken jenen im Niederspannungsbereich gleichen und sogar übersehen werden (besonders dann, wenn sie an den üblicherweise bekleideten Füßen lokalisiert sind). Typischerweise sind aber neben porzellanfarbenen und verkohlten Anteilen auch kraterförmige Durchschlagsstellen vorhanden (• Abb. 3.108). Häufig weichen die elektrothermischen Hautläsionen in Größe und Aussehen von den modellhaften Strommarken ab.

In etwa der Hälfte aller tödlich ausgehenden Hochspannungsunfälle bestimmen großflächige Verbrennungen das Er-

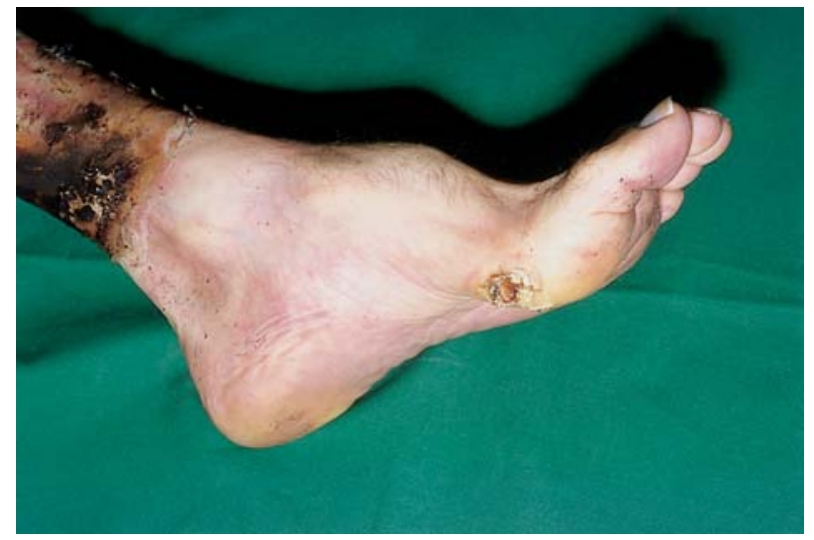

- Abb. 3.108. Distaler Unterschenkel und Fuß eines 44-jährigen Mannes, der bei Malerarbeiten in einer Trafostation tödlich verunglückte (Hochspannungsunfall, 20 kV). 3.- und 4.-gradige Verbrennung des Unterschenkels bis zum Oberrand der ursprünglich getragenen Schuhe, kraterförmige Strommarke am Großzehenballen

scheinungsbild. Meist beruhen diese auf der Einbeziehung des Körpers in einen Lichtbogen: Bei Annäherung an einen unter hoher Spannung stehenden elektrischen Leiter kann es auch ohne Berührung durch Überschlag zu einem Stromübergang mit Zünden eines Lichtbogens kommen. Im Kernbereich eines solchen Lichtbogens liegen Temperaturen von einigen tausend Grad Celsius vor. Bei einer Spannung von $20 \mathrm{kV}$ beträgt die Überschlagsentfernung etwa $6 \mathrm{~mm}$, bei $100 \mathrm{kV}$ bereits $35 \mathrm{~mm}$. Neben den im Einwirkungsbereich entstehenden, meist drittgradigen Verbrennungen findet man oft Versengungen der Kopfhaare sowie der Augenbrauen, Wimpern und Barthaare. Lichtbogenentladungen gehen manchmal mit einer makroskopisch sichtbaren, meist grauschwarzen Aufdampfung von Leitermaterial (»Metallisation«) an exponierten Körperpartien einher.

Die Häufigkeit mechanischer Folgetraumen ist bei Hochspannungsunfällen naturgemäß besonders groß. Die Kontaktmöglichkeiten sind - anders als bei Haushaltsspannung - im Wesentlichen auf Freileitungen, Fahrdrähte der Bahn, Transformatoren und Umspannwerke beschränkt. Der Stromübertritt findet daher oft mehrere Meter über dem Umgebungsniveau statt, so dass nachfolgende Stürze zu schweren Sekundärverletzungen führen können. Sie stellen mitunter sogar die unmittelbare Todesursache dar. Da Strommarken bei flüchtigem Leiterkontakt nur sehr gering ausgeprägt zu sein brauchen, sollten tödliche Arbeitsunfälle mit Sturzverletzungen stets den Verdacht einer vorherigen Stromeinwirkung nahe legen.

Im Gesicht sind Verbrennungen und etwaige Metallisationen nach Lichtbogeneinwirkung oft entlang der Hautfalten nach Art von »Krähenfüßen« ausgespart. Während man früher Krähenfüße als ein vitales Zeichen - verursacht durch Zusammenkneifen der mimischen Muskulatur - interpretierte, wird heute auch eine direkte (elektrisch induzierte) Muskelkontraktion und damit eine 
potentiell supravitale Entstehung diskutiert. Nicht selten findet man nach tödlichen Hoch- und Niederspannungseinwirkungen punktförmige Blutungen in den Konjunktiven, in der Gesichtshaut und in den Schleimhäuten des oberen Respirationstraktes, manchmal in Kombination mit subepikardialen und subpleuralen Blutungen. Einige Autoren haben diese Zeichen im Sinne einer »elektrischen Asphyxie» gedeutet. Als mögliche Ursachen werden eine tetanische Verkrampfung der Atemmuskulatur mit daraus resultierender intrathorakaler Drucksteigerung und ein starker Anstieg des arteriellen Blutdrucks in Verbindung mit massiver venöser Stauung diskutiert.

Bei lang anhaltendem Stromfluss durch den Körper stehen die direkten und indirekten Verbrennungsfolgen im Vordergrund. Die Erscheinungen reichen von ausgedehnten Verkohlungen über eine Verkochung der Muskulatur bis zur Aufsprengung von Gelenken. Bei Kontakt mit Leiterseilen wurden auch elektrothermische Gewebsdurchschneidungen mit Abtrennung von Kopf, Rumpf und Gliedmaßenteilen beobachtet. Im Einwirkungsbereich von Lichtbögen können sich an Knochen sog. Schmelzperlen aus geschmolzenem Kalziumphosphat bilden.

Bei den Todesfällen durch hochgespannte Elektrizität handelt es sich ganz überwiegend um Unfälle; die Opfer sind mehrheitlich Männer, die bei berufsbezogenen Arbeiten verunglücken (z.B. beim Bedienen von Kränen und anderen hochragenden Baumaschinen), in Umspannwerken oder Transformatorstationen sowie auf Bahnanlagen mit elektrischen Fahrleitungen. Auch eine zufällige Berührung der Freileitung mit einem langen, metallischen Gegenstand (z.B. bei der Obsternte) ist möglich. Mitunter erklettern Personen aus Leichtsinn oder im Rahmen von Mutproben abgestellte Eisenbahnwaggons oder Gittermaste von Hochspannungsleitungen. Sehr selten werden auch Suizide durch Besteigen eines Hochspannungsmastes und bewusste Annäherung an Leiterseile verübt; eine zweite Begehungsvariante besteht darin, dass vom Boden aus ein Draht, an dessen Ende ein Stein o. ̈̈. befestigt ist, über eine Fahr- oder Freileitung geworfen wird.

\subsubsection{Blitzschlag}

Der Blitz ist ein Ausdruck »kosmischer Elektrizität « und beruht auf einer stromstarken Entladung zwischen Wolken und Erdboden, wobei Spannungen von einigen Millionen Volt gemessen wurden. Im Verlauf der Hauptentladung mit der dort extrem hohen Stromstärke kommt es infolge Erhitzung zu einer explosionsartigen Ausdehnung der Luft; in feuchten Materialien wie Baumstämmen verdampft das Wasser entlang der Blitzbahn, so dass hochgespannter Dampf entsteht, der entsprechende mechanische Schädigungen verursacht. Rund um die Blitzeinschlagstelle bildet sich ein so genannter Spannungstrichter, in dessen Bereich die Spannung peripherwärts abnimmt. Dadurch kann - bei Schrittstellung der Beine - zwischen den Füßen eine gefährliche Potentialdifferenz bestehen (sog. Schrittspannung). Als weitere Formen der Blitzstrom-Übertragung sind zu nennen: der direkte Einschlag, der »Kontakteffekt» (das Opfer berührt einen Gegenstand, der vom Blitz getroffen wird), der »Überschlagseffekt« (Übersprung von einem getroffenen Objekt auf das benachbarte Opfer) sowie Blitzeinwirkungen, die über elektrische Leiter (Telefon!) vermittelt werden.

Die Letalität von Blitzunfällen beträgt 30-40\%. Bei überlebenden Opfern bestehen häufig Verbrennungen, Lähmungen oder Sensibilitätsstörungen. Bei tödlichen Verläufen stellt sich die Frage, welche der vitalen Funktionen als erste ausgesetzt hat. Diskutiert wird einerseits eine zentrale Atemlähmung mit sekundärem Kreislaufstillstand, andererseits ein primäres Herzkammerflimmern.

Die kriminalistische Bedeutung der Blitzbefunde wird durch mannigfaltige Möglichkeiten der Fehlinterpretation unterstrichen. So kann die Abwesenheit auffallender Verbrennungen zur irrtümlichen Annahme eines natürlichen Todes verleiten. Mitunter erweckt die Auffindungssituation den Anschein eines Tötungsdeliktes (zerrissene Kleidung, mechanische Begleitverletzungen).

Die Kleiderbefunde umfassen ein breites Spektrum mechanischer und thermischer Veränderungen. Gruppierte Lückenbildungen zählen zu den typischen Folgen einer Blitzeinwirkung (»schrotschussartige« Beschädigungen). An den metallischen Bestandteilen der Opferbekleidung (Schnallen, Druckknöpfe etc.) sowie an Uhren und Schmuckstücken können kleine Schmelzungen nachgewiesen werden; an den Schuhen zeigen sich punktförmige Durchlöcherungen oder umschriebene Zerreißungen in der Sohle ( Abb. 3.109) bzw. im benachbarten

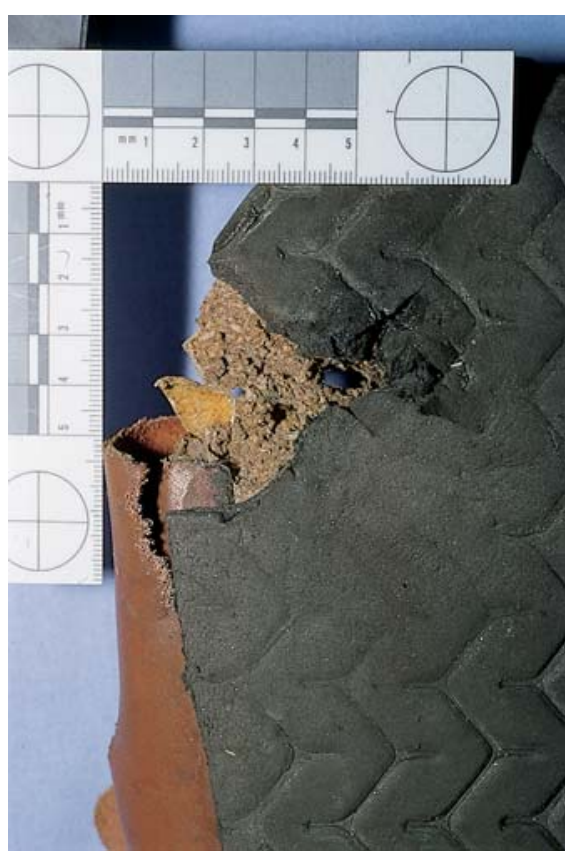

- Abb. 3.109. Schuh eines Blitzunfallopfers (Unterseite): punktförmige Durchlöcherung der Innensohle, Zerreißung und partieller Verlust der Gummisohle an der »Blitzabsprungstelle» 


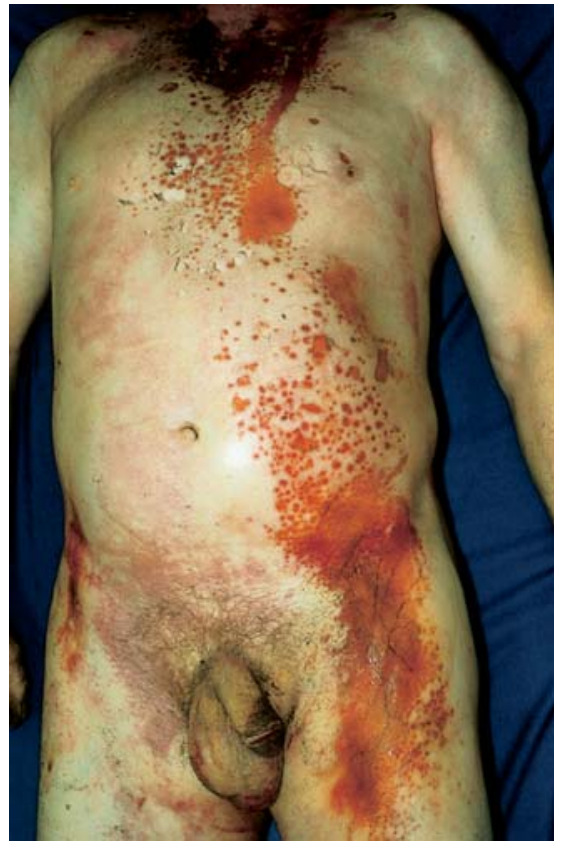

- Abb. 3.110. Diskontinuierliche, braun vertrocknete Verbrennung bei einem durch Blitzschlag getöteten Opfer

Oberleder (ähnlich wie bei Hochspannungsunfällen). An Kunstfasertextilien findet man neben uncharakteristischen Zerreißungen auch Hitzeveränderungen mit lokaler Verhärtung und Einrollung der Ränder sowie mikroskopisch fassbaren Schmelzeffekten (kolbige Auftreibungen der Faserenden).

Die Auftreffstelle des Blitzes liegt bei den tödlich verletzten Opfern typischerweise in der Scheitelregion; die Kopfhaare sind dort angesengt (»fuchsrote« Verfärbung, Kräuselung und kolbenartige Verdickung der Enden). Großflächige Verbrennungen der Körperoberfläche sind möglich, aber keineswegs obligat. Ihr Ausbleiben lässt sich damit erklären, dass der Blitzstrom überwiegend extrakorporal abgeleitet wird. Die Entladung hinterlässt häufig an der Körperoberfläche eine mehrfach unterbrochene »Blitzstraße« (• Abb. 3.110) mit Verbrennung der Haut und ihrer Anhangsgebilde (z.B. Augenbrauen, Wimpern, Scham- und Körperhaare).

An den bodennahen Absprungstellen finden sich häufig umschriebene Hautverbrennungen, die aspektmäßig den Stromübertrittsstellen bei Einwirkung technischer Elektrizität gleichen. Dort, wo metallische Gegenstände wie Halsketten, Uhren und Gürtelschnallen der Haut aufliegen, entstehen so genannte Kontaktverbrennungen, die häufig von Metallisationseffekten begleitet sind. Diese können auch in der Umgebung der Kontaktverbrennung zu einem flächenhaften Niederschlag (»Materialanflug «) auf ungeschädigter Haut führen.

Der bekannteste morphologische Befund bei Blitzschlagopfern ist die so genannte Blitzfigur (- Abb. 3.111): Es handelt sich

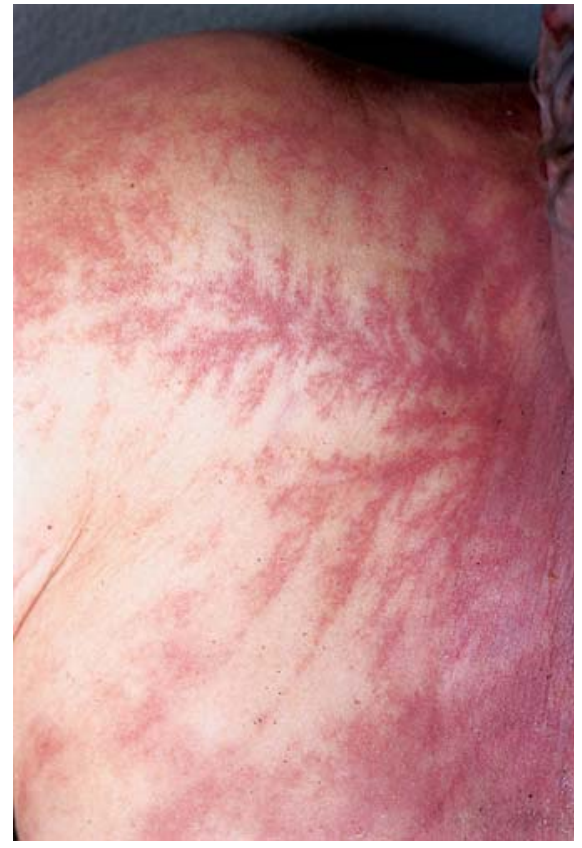

- Abb. 3.111. Arborisiertes Erythem (»Blitzfigur«) an der rechten vorderen Brustwand eines Blitzschlagopfers

um dendritisch verästelte Hautrötungen, die auf einer lokalen Hyperämie beruhen (nicht auf intra- oder subkutanen Blutextravasaten!) und mit zunehmender Leichenliegedauer abblassen bzw. verschwinden. Histologisch findet man im Verlauf der Blitzfiguren eine Erweiterung der kleinen Gefäße im Korium, manchmal in Kombination mit einer Elongation und einer Palisadenstellung der basalen Epidermiszellen.

\subsection{Verhungern}

\section{B. Madea, S. Banaschak}

Weltweit stellt das Verhungern nach wie vor eine häufige Todesursache dar. Dem Verhungern geht bei exogenem Nahrungsmangel das Bild der Kachexie voraus, das freilich auch endogene Ursachen haben kann, die differentialdiagnostisch abzugrenzen sind (• Abb. 3.112). Der tägliche Kalorienbedarf des Menschen über den Grundumsatz hinaus hängt insbesondere von der körperlichen Aktivität ab. Unterschreitet die Kalorienzufuhr den Kalorienbedarf, kommt es zu einer negativen Energiebilanz mit resultierendem Abbau von Körpersubstanz. Die entsprechenden somatischen Folgen sind in $\bullet$ Tabelle 3.32 zusammengefasst. 
Bezeichnung

morphologisch

Sichtbarer Ausdruck des Nahrungsmangels

Absoluter oder relativer exogener Nahrungsmangel oder falsche Nahrungs-

Pathogenetische Vorgänge bei Inanition

Entleerung der Speicher

zusammensetzung

Erschöpfung der Reserven

Biochemische Alteration

Funktionelle Störung

Störung der Regulationen

Zusammenbruch der Regulationen

Tod
Submikroskopische und mikroskopische Strukturänderungen der Zellen

Inanitionsatrophie

Alimentäre Dystrophie
Hungeratrophie

Hungerkrankheit

a) trockene Form

b) feuchte Form

Ödemkrankheit

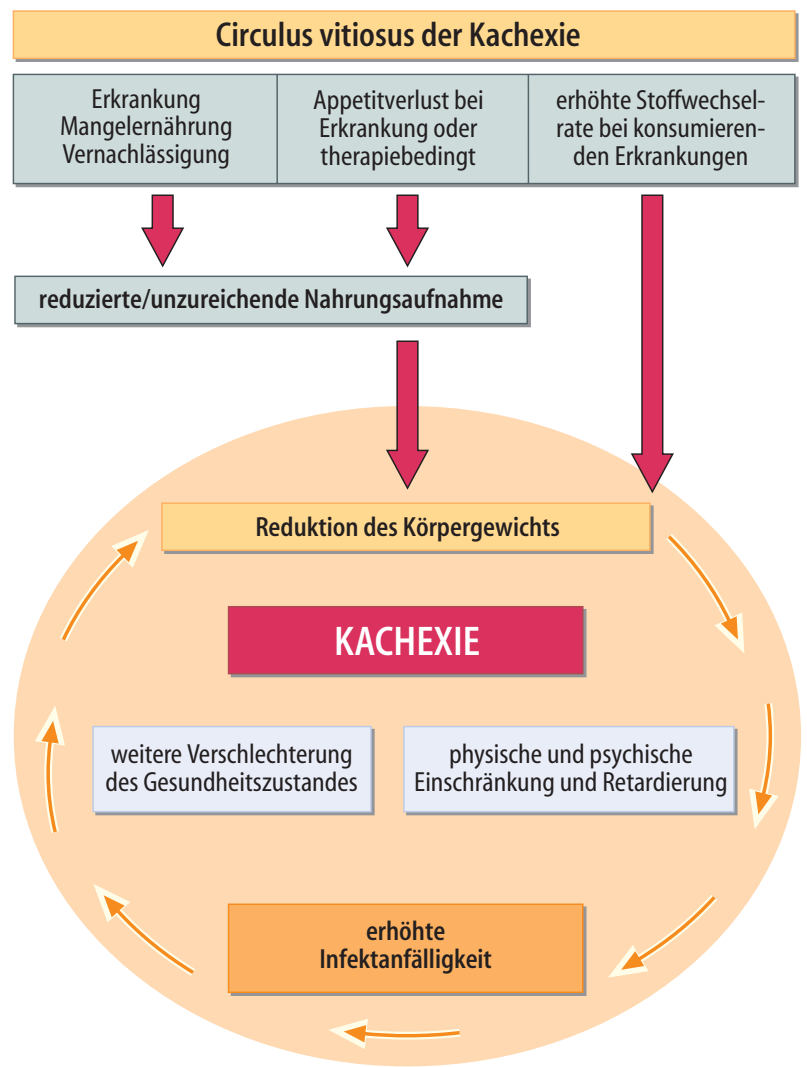

- Abb. 3.112. Circulus vitiosus der Kachexie (i) Infobox

Ursachen des Verhungerns

- Nahrungsverweigerung (z. B. Hungerstreik, Kranke, alte Menschen)

- Krankheitsbedingte Unmöglichkeit der Nahrungsaufnahme (zum Beispiel bei Ösophaguskarzinom)

- Konsumierende Erkrankungen beziehungsweise krankheitsbedingte Erhöhung des Stoffwechselumsatzes (maligne Tumoren, Infektionen - insbesondere Tuberkulose -, Schilddrüsenerkrankungen)

- Psychische Erkrankungen (Anorexia nervosa, Schizophrenie mit Angst vor Vergiftung)

- Unglücksfälle mit Verschüttung und Eingeschlossensein (zum Beispiel Bergwerksunfälle, Hauseinstürze, Erdbeben)

- Vorsätzliche oder fahrlässige Fehl- oder Mangelernährung

- Nahrungsentzug als Form der Sterbenachhilfe

\section{Rechtliche Grundlagen}

Juristisch und damit auch rechtsmedizinisch relevant sind vor allen Dingen die Fälle mit möglicher Verantwortlichkeit Dritter: Vorsätzliche oder fahrlässige Fehlernährung im Rahmen der Verletzung der Obhuts- und Aufsichtspflicht (Vernachlässigung von Kindern, Aufsichtspflichtverletzung bei psychisch Kranken, Patienten mit Anorexia nervosa, Pflegebedürftigen) von Seiten der Eltern beziehungsweise der Pflegepersonen ( $\$ 223 \mathrm{~b}$ StGB - Misshandlung Schutzbefohlener, $\$ 170 d$ StGB - Verletzung der Fürsorge- oder Erziehungspflicht), aber auch des Jugend- oder Gesundheitsamtes. Weiterhin können ärztliche, juristische und ethische Probleme im Zusammenhang mit Hungerstreiks sowie kalorisch unzureichender Ernährung als Form des Behandlungsabbruchs entstehen. 
Im Vordergrund stehen Fälle des Verhungerns von Kindern in den ersten zwei bis drei Lebensjahren (jenseits des 3. Lebensjahres begrenzte Selbstversorgungsmöglichkeiten), die von alkohol- und drogenabhängigen Eltern nicht versorgt, beziehungsweise längere Zeit unversorgt alleine gelassen werden, teilweise im Bewusstsein, sie einer tödlichen Gefahr auszusetzen.

\section{F) Fallbeispiel}

In einer ungeheizten und verwahrlosten Wohnung werden ein toter, 3 Monate alt gewordener Säugling und ein unterkühltes Kleinkind aufgefunden. Die Mutter habe zwei Wochen zuvor die Wohnung verlassen und in einem Hotel gewohnt. Die Körperlänge des Säuglings betrug 93 \% in Bezug auf den Erwartungswert, das Gewicht in Bezug auf das Erwartungsgewicht bei der aktuellen Größe 57 \% (nach der Waterlow-Klassifikation, > unten).

\section{Pathophysiologie des Hungerns}

Die pathophysiologischen Abläufe bei Hungerzuständen umfassen in der zeitlichen Abfolge folgende Phasen:

- Glykogenverbrauch, Blockade der Glukoseaufnahme durch Muskulatur und Fettgewebe; Ziel: Sicherstellung des Glukosebedarfs des Gehirns, Dauer ca. 18 Stunden

- Glukoneogenese durch Proteolyse (100 g Glukose erfordern 200 g Protein); negative Stickstoffbilanz; Dauer ca. 2-3 Wochen

- Nach 2-3 Wochen Lipolyse und Ketogenese

Der Gesamtstoffwechsel ist während des Hungerns herabgesetzt, der Grundumsatz erniedrigt, die Körpertemperatur $0,5^{\circ}-1^{\circ} \mathrm{Cel}-$ sius unterhalb des Normbereiches. Typischerweise kann beim Hungern Aceton im Urin nachgewiesen werden. Klinisch werden folgende Phasen des Hungerns unterschieden:

- 1. Phase: Verlust des subjektiven Wohlbefindens (Konzentrationsprobleme, Störungen des Gedächtnisses, Verlangsamung des Denkens, der Reaktion und der Bewegungen, schnelle Ermüdbarkeit, Hungergefühl, Schlafbedürfnis),

- 2. Phase: Verschwinden des Hungergefühls, starker Gewichtsverlust mit aggressiver Schwäche, Apathie, Hypotonie, Bradykardie, Hypothermie, Herabsetzung des Grundumsatzes,

- 3. Phase: extreme Lethargie, mentale Retardierung und Hungerödeme.

Die mögliche Zeitdauer des Hungerns bis zum Todeseintritt ist variabel und hängt von zahlreichen Faktoren ab: Alter, Ernährungszustand, Flüssigkeitszufuhr, Umgebungstemperatur. Bei vollständigem Nahrungs- und Flüssigkeitsentzug tritt der Tod nach 8-21 Tagen ein (bei Neugeborenen und Kindern unter Umständen wesentlich schneller), bei alleinigem Nahrungsentzug nach ca. 60 Tagen. Nach einem Bericht über letale Hungerstreiks organgesunder junger Männer trat der Tod im Mittel nach 61,6 $+/-2,5$ Tagen ein.

\section{Tod durch Verhungern}

Die Diagnose des Todes durch Verhungern ergibt sich prima facie aus Umständen und Befunden; sie wird beweiskräftig durch den Ausschluss anderer Todesursachen und durch umfangreiche histologische und toxikologische Untersuchungen. Insbesondere ist auf konsumierende Prozesse als Ursache oder Komplikation (Tbc) einer Kachexie zu achten, bei Kindern darüber hinaus auf kongenitale Vitien, Malabsorptions- und Malassimilationssyndrome.

Aufgrund der gestörten Resistenz können komplizierend interkurrente Erkrankungen (insbesondere Infektionen, Diarrhöen, Tuberkulose) auftreten, die bei Unterernährung häufig atypisch verlaufen und daher auch schwieriger zu erkennen sind.

(i) Infobox

Typische pathologisch-anatomische Befunde des Verhungerns

- Massiver Gewichtsverlust, bei Kindern bei chronischem Nahrungsmangel zusätzlich Wachstumsretardierung

(• Abb. 3.113)

- Vollständiger Schwund des Unterhautfettgewebes sowie des Fettgewebes des großen Netzes; eventuell gallertige Umwandlung von Fettgewebsresten

- Atrophie innerer Organe, endokriner Drüsen und des lymphatischen Gewebes

- Atrophie der Muskulatur

- Kontrahierter Magen-Darm-Trakt, bis auf galligen Schleim beziehungsweise wenig Kot (Schleim, Galle, Epithelien) leer, verdünnte Darmwände, zur Ernährung ungeeignete Substanzen im Darm, Kotsteine

- Prallgefüllte Gallenblase

Zum Zeitpunkt des Todeseintritts ist in der Regel ein Gewichtsverlust von 30-40\% des ursprünglichen Körpergewichtes eingetreten, die Organgewichte (mit Ausnahme des Gehirns) nehmen in der Regel in der gleichen Größenordnung ab.

\section{Chronische Unterernährung bei Kindern}

Zur Graduierung und Quantifizierung chronischer Mangelernährung wurden in der Pädiatrie verschiedene Klassifikationen entwickelt, die auf der Basis anthropometrischer Daten eine rasche Einschätzung des Zustandes eines unterernährten Kindes erlauben und, wenn klinische Daten vorliegen, neben einer Verlaufseinschätzung auch für die Differenzierung akute/chronische Mangelernährung hilfreich sind (• Tabelle 3.33). Eine ProteinEnergie-Malnutrition (PEM) umfasst Folgezustände unzureichender Energie- und meist auch Proteinzufuhr mit der Folge mangelnder Gewichtszunahme, konsekutivem Untergewicht, bei chronischem Bestehen mangelnder Längenentwicklung mit Kleinwuchs. Zur Einteilung einer PEM nach Schweregraden wurden verschiedene Klassifikationssysteme entwickelt, von de- 


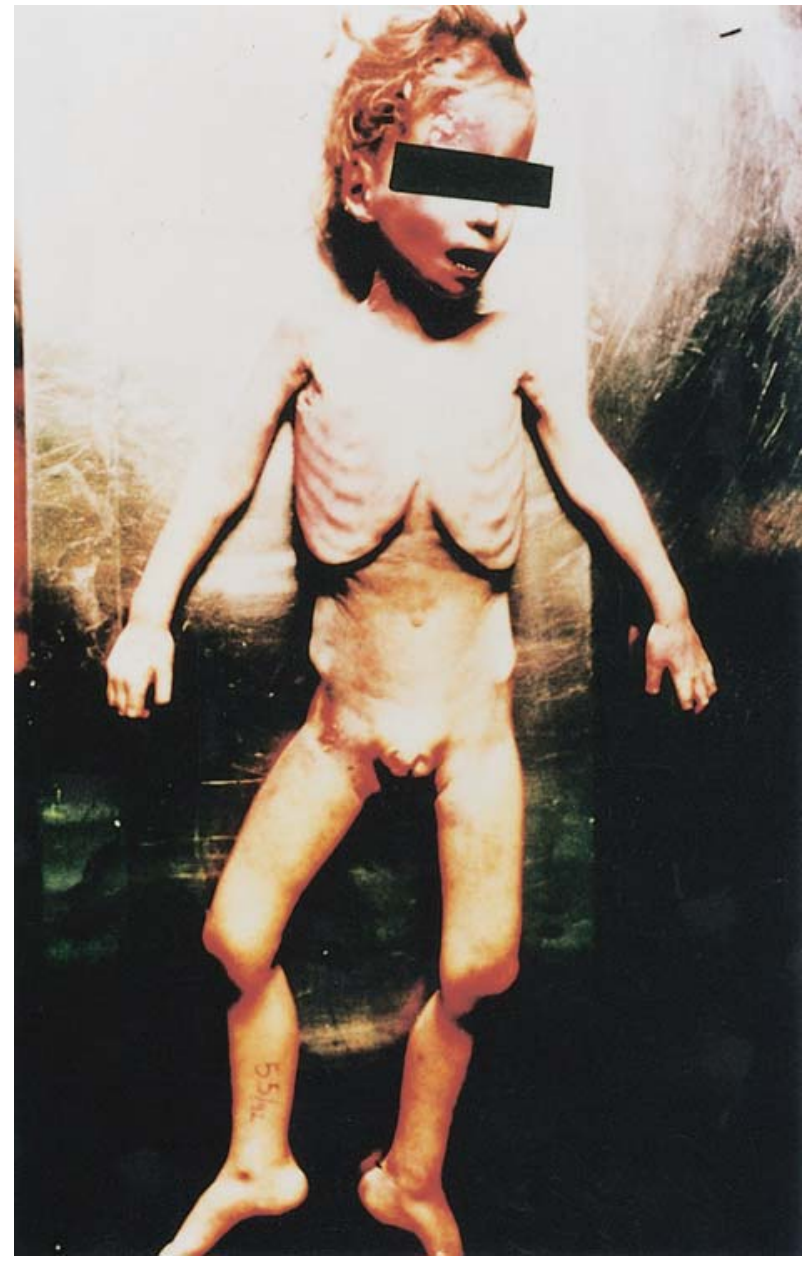

- Abb. 3.113. 2,5 Jahre alt gewordenes Kind. Todesursache: Verhungern in Kombination mit Exsikkose

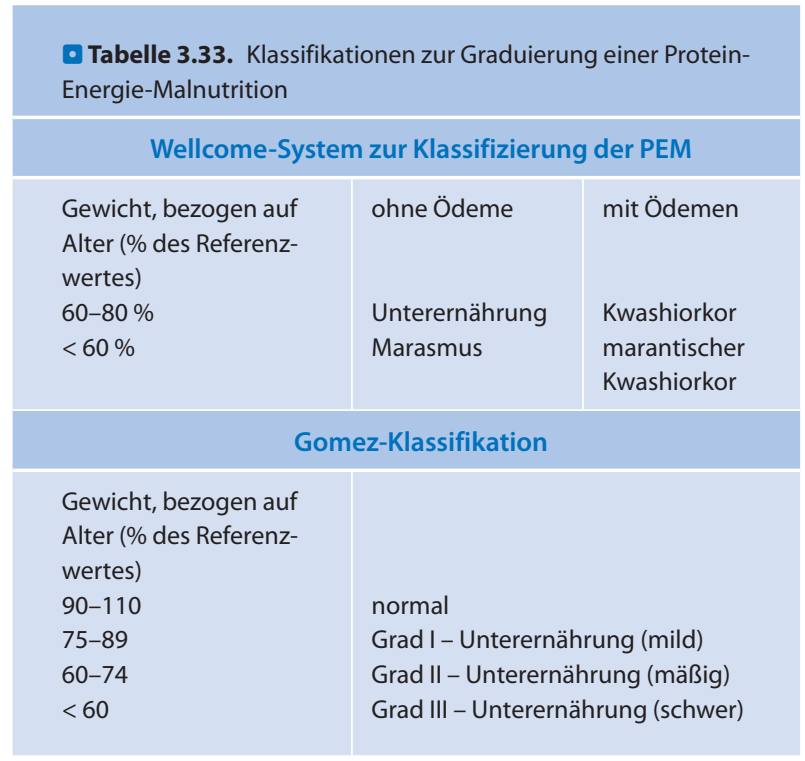

nen insbesondere die Waterlow-Klassifikation von rechtsmedizinischer Relevanz ist, da sich aus den anthropometrischen Daten unmittelbar eine Klassifizierung des Schweregrades der Unterernährung ergibt (-Tabelle 3.34). Entsprechend der Klassifikation von Waterlow, und damit gewonnener praktischer Erfahrungen, gilt das Wachstumsdefizit (»stunting«) als Maß für chronische Unterernährung (Retardierung), das Gewichtsdefizit (bezogen auf den Erwartungswert für die reale Körpergröße) als Maß für die akute Unterernährung (»wasting«). Mit beiden Kriterien werden also unterschiedliche Sachverhalte gemessen, was sich für die praktische Begutachtung als hilfreich erweist. Als weiterer Vorteil ergibt sich, dass das reale Gewicht nicht an altersspezifischen Referenzwerten, sondern am Erwartungswert für die reale Körpergröße - also altersunabhängig - beurteilt wird.

In foro wird immer wieder die Frage nach der Erkennbarkeit des Zustandes (der Unterernährung, der Lebensgefährlichkeit) einige Zeit vor Todeseintritt aufgeworfen. Wenn die Erkennbarkeit der Unterernährung einige Zeit (Tage bis wenige Wochen)

- Tabelle 3.34. Einschätzung des Ernährungszustandes nach der Waterlow-Klassifikation

Retardierung (chronisch)

\begin{tabular}{|l|l|l|l|l|}
\hline Grad & 0 (normal) & 1 (mild) & 2 (mäßig) & 3 (schwer) \\
\hline Größe in \% des altersbezogenen Erwartungswertes & $>95$ & $95-87,5$ & $87,5-80$ & $<80$ \\
\hline Unterernährung (akut) & & & 3 (schwer) \\
\hline Grad & 0 (normal) & 1 (mild) & 2 (mäßig) \\
\hline Gewicht in \% des Erwartungswertes für die aktuelle Größe & $>90$ & $90-80$ & $80-70$ & $<70$ \\
\hline
\end{tabular}


vor Todeseintritt zur Diskussion steht (etwa zur Beantwortung der Frage, ob die Eltern oder Mitarbeiter der Sozialbehörden ärztliche Hilfe hätten holen müssen), kann unter der Annahme vollständiger Nahrungskarenz und einer dadurch bedingten Gewichtsabnahme von $0,7-1 \%$ des Körpergewichtes pro Tag auf den in Frage kommenden Zeitpunkt extrapoliert werden. Das Körpergewicht in Prozent des auf die Körpergröße bezogenen Sollgewichtes veranschaulicht nach der Waterlow-Klassifikation den Grad der akuten Unterernährung. Da insbesondere bei chronischer Unterernährung in der Regel keine vollständige Nahrungskarenz vorlag, ist von niedrigeren Ausgangsgewichten für den in Frage stehenden Zeitpunkt auszugehen.

\subsection{Kindestötungen}

\section{B. Madea, R. Dettmeyer}

Im Vergleich zur Tätigkeit eines Gerichtsarztes vor 100 Jahren sind heute Fälle von Kindestötung oder Neugeborenensektionen zur Abgrenzung von Kindestötung, intrauterinem Fruchttod oder natürlichem Tod vergleichsweise selten. In der peinlichen Halsgerichtsordnung Kaiser Karls V. war die Kindestötung mit drakonischen Strafen belegt (zum Beispiel»lebendig Begraben«), später wurde sie gegenüber der vorsätzlichen Tötung privilegiert. $\S 217$ StGB in der früheren Fassung lautete:

\section{§ 217 Kindestötung}

»(1) Eine Mutter, welche ihr nichteheliches Kind in oder gleich nach der Geburt tötet, wird mit Freiheitsstrafe nicht unter drei Jahren bestraft.

(2) In minderschweren Fällen ist die Freiheitsstrafe von 6 Monaten bis zu 5 Jahren."

Mit dem am 01.04.1998 in Kraft getretenen 6. Strafrechtsreformgesetz ist der $\$ 217$ alter Fassung schließlich entfallen, da er in der forensischen Praxis kaum Anwendung fand, aufgrund der ausschließlichen Privilegierung von Müttern nichtehelicher Kinder verfassungsrechtlich problematisch war und vor allen Dingen die Privilegierung der Mütter nichtehelicher Kinder nicht mehr zeitgemäß erschien.

\section{(i) Infobox}

\section{Psychologie der Kindestötung}

Bei den Kindesmüttern, die ihr Neugeborenes in oder gleich nach der Geburt töten, handelt es sich meist um sehr junge Frauen, die entweder alleine oder noch im Elternhaus wohnen. In der Regel sind die Mütter zwischen 16 und 38 Jahre alt, wobei ca. $90 \% 25$ Jahre und jünger sind. Weniger als $20 \%$ sind verheiratet. Meist wird das Bestehen einer Schwanger$\nabla$ schaft über den ganzen Zeitraum hinweg geleugnet, abgestritten, negiert und sogar verdrängt. Selbst engste Familienangehörige oder der Freund wissen von einer bestehenden Schwangerschaft nichts. Einsetzende Geburtswehen treffen die Kindesmütter völlig unvorbereitet. Die Kindestöterin wird als passiv, infantil, unreif und gemütskalt beschrieben. Immer wieder sind jedoch auch Neugeborenentötungen bei älteren, verheirateten Frauen mit bereits mehreren Kindern zu beobachten.

\subsubsection{Rechtsmedizinische Untersuchung und Begutachtung}

Gegenstand rechtsmedizinischer Untersuchungen bei Verdacht auf Kindestötung ist in der Regel das Neugeborene als Objekt eines möglichen Verbrechens. An tatverdächtigen Frauen sind folgende Fragestellungen forensisch relevant:

- Diagnose des Wochenbettes beziehungsweise der kürzlich erfolgten Geburt und

- Zeiteingrenzung, wann die Geburt stattgefunden hat (von Bedeutung insbesondere in Relation zur Liegezeit des Kindes).

Untersuchungen an tatverdächtigen Frauen sollten regelhaft von einem Gynäkologen durchgeführt werden (Feststellung der Größe des Uterus mit Stand des Fundus uteri, Lage des Uterus und Weite von Portio und Cervix uteri, Zustand des Lochialsekretes, der Brüste und Mamillen). Bei der Obduktion eines Neugeborenen ist $\$ 90$ StPO zu beachten.

\section{$\S 90$ StPO Neugeborenes Kind}

\begin{abstract}
»Bei Öffnung der Leiche eines neugeborenen Kindes ist die Untersuchung insbesondere auch darauf zu richten, ob es nach oder während der Geburt gelebt hat und ob es reif oder wenigstens fähig gewesen ist, das Leben außerhalb des Mutterleibes fortzusetzen."
\end{abstract}

Die Fragestellungen bei Sektion eines Neugeborenen oder eines verdächtigen Abortes sowie die Reifezeichen sind in $\bullet$ Tabelle $3.35+3.36$ zusammengefasst. Zu klären ist, ob es sich um ein Neugeborenes handelt, ob es reif war und ob es gelebt hat. Schließlich ist die Erhebung bzw. der Ausschluss von Geburtsverletzungen von Bedeutung (spezielle Sektionstechnik mit Korbhenkelschnitt zum Ausschluss geburtstraumatischer Schädigungen des Gehirns). Nach Möglichkeit sollten Nabelschnur und Plazenta untersucht werden.

\section{Nachweis der Lebensfähigkeit}

Typische Reifezeichen des Neugeborenen sind:

- Körpergröße $\geq 48 \mathrm{~cm}$

- Körpergewicht $\geq 2.500 \mathrm{~g}$ 
- Tabelle 3.35. Fragestellungen bei Sektion eines Neugeborenen oder eines verdächtigen Abortes

\begin{tabular}{|c|c|c|c|c|}
\hline $\begin{array}{l}\text { Neugeboren- } \\
\text { heit }\end{array}$ & Reifezustand & Gelebthaben & Geburtsverletzungen & $\begin{array}{l}\text { nat. } \\
\text { Todesursache }\end{array}$ \\
\hline $\begin{array}{l}\text { Nicht gereinigt } \\
\text { oder gesäubert } \\
\text { von Blut, Kinds- } \\
\text { pech, Käse- } \\
\text { schmiere }\end{array}$ & $\begin{array}{l}\text { Körperlänge } \\
\text { Körpergewicht } \\
\text { Maße des Kopfes } \\
\text { Membrana pupillaris, } \\
\text { Ohren-, Nasenknor- } \\
\text { pel entwickelt? }\end{array}$ & $\begin{array}{l}\text { Thoraxform, Hautdesquamation, } \\
\text { Ikterus, Nabelstumpf-Demarkation } \\
\text { Lunge: Röntgenaufnahme, beat- } \\
\text { met, Schwimmprobe, Fremdinhalt } \\
\text { in Bronchien, Cave: Fäulnisgase, } \\
\text { Histologie aber nicht an Gefrier- } \\
\text { schnitten }\end{array}$ & $\begin{array}{l}\text { Sektionstechnik: Abziehen des } \\
\text { Periosts der Schädelknochen, Korb- } \\
\text { henkelschnitt, Untersuchung der } \\
\text { Halsweichteile in Blutleere } \\
\text { Caput succedaneum, Cephalhaema- } \\
\text { toma externa, interna }\end{array}$ & $\begin{array}{l}\text { Krankheiten: } \\
\text { Hydrops fetalis } \\
\text { universalis }\end{array}$ \\
\hline $\begin{array}{l}\text { Nabelschnur } \\
\text { ohne/mit vitalen } \\
\text { Demarkations- } \\
\text { zeichen am Haut- } \\
\text { nabel } \\
\text { ohne/mit Ver- } \\
\text { trocknungen } \\
\text { (Pseudodemar- } \\
\text { kation) } \\
\text { Höhe und Art der } \\
\text { Nabelschnurab- } \\
\text { bindung } \\
\text { Richtung der } \\
\text { Durchtrennung } \\
\text { (bei Vertrocknung } \\
\text { Wässerung!) }\end{array}$ & $\begin{array}{l}\text { Stelle des Nabels } \\
\text { Hoden deszendiert? } \\
\text { Schamlippengröße } \\
\text { Nagellänge } \\
\text { Lanugo } \\
\text { Reifezustand der in- } \\
\text { neren Organe (z.B. } \\
\text { Hirnwindungen; } \\
\text { Knochenkerne) } \\
\text { Gewicht der Plazenta } \\
\text { Länge der Nabel- } \\
\text { schnur }\end{array}$ & $\begin{array}{l}\text { Magen: Röntgenaufnahme, Gasblä- } \\
\text { hung Schwimmprobe, Cave: Fäunis- } \\
\text { gase, Nahrungsmittel, Fremdinhalt } \\
\text { Darmprobe: Rö-Aufnahme, Gasblä- } \\
\text { hung, Ausdehnung } \\
\text { Schwimmproben nur nach vorheri- } \\
\text { ger Unterbindung z.B. von Luftröhre } \\
\text { und Ösophagus bzw. Duodenum, in } \\
\text { situ Abbindung des Dünndarmes } \\
\text { im Abstand von } 20 \text { cm, Cave: Fäul- } \\
\text { nisgase } \\
\text { Kreisförmiges Umschneiden des } \\
\text { Afters zur Darstellung des ver- } \\
\text { schließenden Schleimpfropfes } \\
\text { Abgang von Kindspech, Ausmaß } \\
\text { der Entleerung von Kindspech } \\
\text { Paukenhöhlen: Gas, Blut, Fremdin- } \\
\text { halt } \\
\text { Harnblase: gefüllt/leer }\end{array}$ & $\begin{array}{l}\text { Verformung des Kopfes } \\
\text { Verschiebung der Schädelknochen } \\
\text { Schädelbrüche } \\
\text { Intrakranielle Blutungen } \\
\text { Tentoriumsrisse } \\
\text { Blutung in die Hirnkammern } \\
\text { Fontanelle eingesunken/vorge- } \\
\text { wölbt } \\
\text { Primäre oder sekundäre Nebennie- } \\
\text { reneinblutungen, subkapsuläre Le- } \\
\text { berblutungen, Leberrupturen (DD: } \\
\text { hypoxische Blutungen) } \\
\text { Dehnungsrisse d.Haut, z.B. der Leis- } \\
\text { tengegend nach innerer Wendung } \\
\text { Brüche der Extremitätenknochen } \\
\text { Probleme: Verletzung am Lebenden } \\
\text { oder am während der Geburtspas- } \\
\text { sage bereits verstorbenen Körper }\end{array}$ & $\begin{array}{l}\text { Lues congenita } \\
\text { Pneumonia alba } \\
\text { Osteochondritis } \\
\text { Feuersteinleber } \\
\text { Plazentainfarkte } \\
\text { Ablatio placen- } \\
\text { tae } \\
\text { Placenta praevia } \\
\text { Insertio vela- } \\
\text { mentosa } \\
\text { Retroplazenta- } \\
\text { res Hämatom } \\
\text { Mekonium im } \\
\text { Fruchtwasser }\end{array}$ \\
\hline
\end{tabular}

- Kopfumfang ca. $34-35 \mathrm{~cm}$

- Nabelschnurlänge ca. $50 \mathrm{~cm}$

- Plazentagewicht ca. $500 \mathrm{~g}$

- Fingernägel überragen die Fingerkuppen, Zehennagelränder überragen die Zehenkuppen

- Lanugohaare nur noch an den Schultern

- Deszensus der Hoden, Überdecken der kleinen durch die großen Schamlippen

- Schulterbreite $\geq 12,5 \mathrm{~cm}$

- Hüftbreite $\geq 9,5 \mathrm{~cm}$

- Felsenbeinknochenkern $\geq 9,5 \mathrm{~mm}$

- So genannter Beclard'scher Knochenkern (distale Femurepiphyse) $\geq 5 \mathrm{~mm}$ Durchmesser

Grundsätzlich besteht Lebensfähigkeit, wenn das Kind zum Zeitpunkt der Geburt eine Körpergröße über $35 \mathrm{~cm}$ aufweist, geburtsbedingte Todesursachen wie zum Beispiel intrakranielle Blutungen fehlen und keine schweren Missbildungen oder eine todesursächliche intrauterine Infektion vorhanden sind. Lebensunfähigkeit kann zum Beispiel durch Unreife bedingt sein. Die quantitative Erfassung des Reifegrades eines Neugeborenen berücksichtigt darüber hinaus zahlreiche weitere körperliche Merkmale, wie Hautbeschaffenheit, -farbe, Behaarung, Zustand des Genitale, plantare Hautfältelung.

\section{Nachweis des Neugeborenseins}

Das Neugeborene ist noch nicht von Blut und Käseschmiere gereinigt. Die Nabelschnur weist noch keine Demarkationszeichen am Hautnabel auf.

\section{Feststellung des Gelebthabens und der Lebensdauer}

Zum Nachweis des Gelebthabens wird seit mehr als 300 Jahren die Lungenschwimmprobe, der Nachweis der Lufthaltigkeit der Lungen, herangezogen. Bereits intrathorakal zeichnet sich eine beatmete Lunge dadurch aus, dass sie im Gegensatz zur nichtbeatmeten den Thorax vollständig ausfüllt. Die Oberfläche ist hellgrau-rot, mit unscharf begrenzten, eingesunkenen Atelektasebezirken. Die Konsistenz ist luftkissenartig, bei Betasten knistert die Lunge. Die gesamten Halsorgane mit Lungen werden exenteriert und in ein bereitgestelltes Gefäß mit Wasser gelegt. Schwimmen die Lungen obenauf und »halten « das Organpaket, ist die Lungenschwimmprobe positiv und weist auf eine kräftige Belüftung 
- Tabelle 3.36. Anthropometrische Daten in Abhängigkeit von der Gestationsdauer

\begin{tabular}{|c|c|c|c|c|c|}
\hline & \multicolumn{2}{|c|}{ Abortus u. Fehlgeburt } & \multirow{2}{*}{$\begin{array}{l}\text { Frühgeburt } \\
\text { 28.-38. Woche }\end{array}$} & \multirow[t]{2}{*}{ Geburt Ende der Schwangerschaft } & \multirow{2}{*}{$\begin{array}{l}\text { Übertragene } \\
\text { Schwangerschaft }\end{array}$} \\
\hline & 1.-16.Woche & 16.-28. Woche & & & \\
\hline
\end{tabular}

der Lungen hin (• Abb. 3.114). Anschließend werden die Lungen einzeln sowie Anteile aller Lungenlappen isoliert der Lungenschwimmprobe unterzogen. Damit kann gezeigt werden, dass, auch wenn anfangs eine Lungenschwimmprobe nicht eindeutig positiv verlaufen ist, einzelne Abschnitte der Lunge trotzdem belüftet und damit beatmet gewesen sein können. Nach Präparation der Halsorgane mit durchgeführter Lungenschwimmprobe wird der Magen-Darm-Trakt einer Schwimmprobe unterzogen und dazu in toto auf Wasser gelegt. Nicht belüftete Abschnitte sinken, belüftete Darmabschnitte schwimmen an der Oberfläche. Die Luftfüllung des Magen-Darm-Traktes gibt Hinweise auf die Zeitdauer des Gelebthabens (- Tabelle 3.37).

Bei der Magen-Darm-Schwimmprobe ergeben sich wie bei der Lungenschwimmprobe falsch-positive Ergebnisse bei Vorliegen von Fäulnis oder künstlicher Beatmung und falsch-negative Befunde bei Vorliegen einer Tötung vor dem ersten Atemzug oder Aspiration von Flüssigkeit.

In der Regel geht die Belüftung der Lungen dem Luftschlucken voraus (positive Lungen- bei negativer Magen-DarmSchwimmprobe), bei frustranen Atembewegungen, Behinderung der Ventilation kann Luft in den Magen, nicht aber in die Lungen gelangen, da zur Entfaltung der Lungen mehr inspiratorische Kraft notwendig ist als zum Verschlucken von Luft. Bei negativer Lungenschwimmprobe wird in derartigen Fällen die MagenDarm-Schwimmprobe das wesentliche Kriterium für das Gelebthaben des Kindes. Histologische Untersuchungen der Lungen sind in jedem Fall zum Nachweis des Gelebthabens, zum Gestationsalter (Alveolarstruktur) und auch zum Vorliegen einer Fruchtwasseraspiration durchzuführen.

\section{Todesursache}

Zur Klärung der Todesursache ist das gesamte differentialdiagnostische Spektrum natürlicher und gewaltsamer Todesursachen abzugrenzen. Bei vorliegenden Mazerationserscheinungen ist darüber hinaus an einen intrauterinen Fruchttod zu denken. Typische natürliche Todesursachen sind:

- Übertragung,

- Unreife,

- Missbildung innerer Organe,

- intrauterine Asphyxie, 
- Abb. 3.114. a Negative und $\mathbf{b}$ positive Lungenschwimmprobe

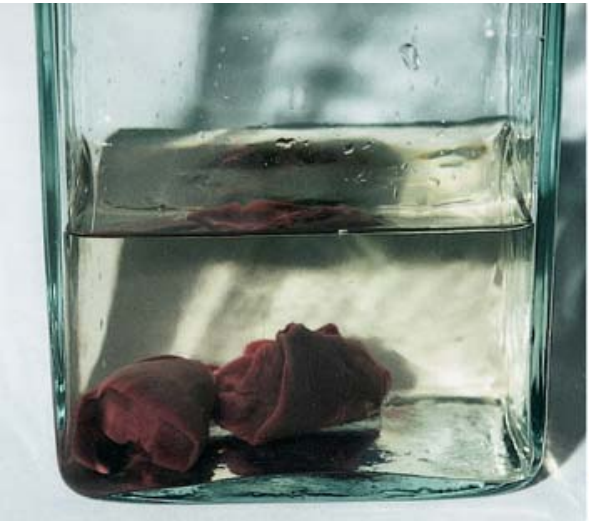

a

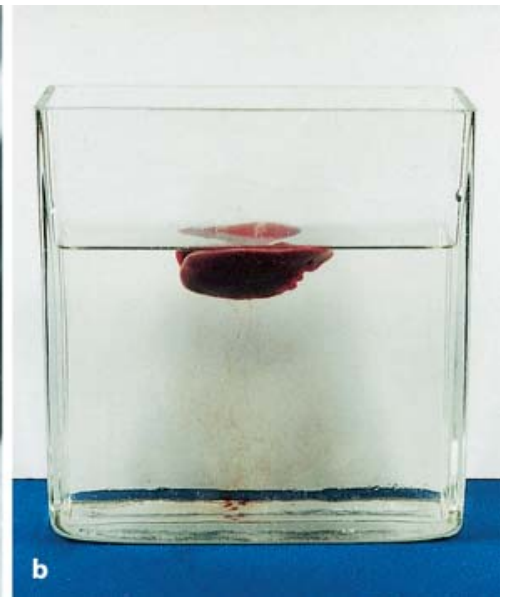

- Tabelle 3.37. Zeitdauer des Gelebthabens. (Nach Fritsch 1901 und Forster 1986)

Magen-Darm-Schwimmprobe

- Luft nur im Magen und oberen Dünndarm: wenige Minuten bis maximal 30 Minuten

- Luft im gesamten Dünndarm: ca. 6 Stunden

- Luft auch im gesamten Dickdarm: 12 Stunden

\section{Mekonium}

- Mekonium noch im gesamten Dickdarm: Lebensdauer unter 2 Tagen

- Mekonium nur noch in den Darmbuchten: Lebensdauer 2-3 (manchmal 5) Tage

Nabelschnurabfall

- Abfall nach ca. 4-5 (3-8) Tagen

- schon frühpostpartal beginnende progrediente demarkierende Entzündung

- Geburtstrauma und

- plazentogene Ursachen.

Bei gewaltsamer Tötung eines Neugeborenen stehen im Vordergrund Halskompression (Drosseln, Würgen), Verschluss der Atemöffnungen (auch um das Kind am Schreien zu hindern), stumpfe Gewalteinwirkung, Stich-/Schnittverletzungen und schließlich Unterkühlung, wenn das Neugeborene unversorgt abgelegt wird.

\section{(5) Fallbeispiel}

Kurz nach Mitternacht wird der ärztliche Notdienst vom Ehemann informiert, dass seine 33-jährige Frau - bereits Mutter eines 31/2jährigen Kindes - unter akuten kolikartigen Bauchschmerzen leide. Bei Eintreffen des Arztes saß die adipöse Frau auf der Toilet- te und äußerte, dass etwas abgegangen sei. Bei Nachschau fand sich in der Toilette ein noch mit der Mutter über die Nabelschnur in Verbindung stehendes Neugeborenes mit dem Kopf nach oben im Abflussrohr des Flachspülers, vollständig unter Wasser eingeklemmt. Unter Kraftaufwendung musste das Kind herausgezogen werden, kurz darauf wurde die Plazenta ausgestoßen. Die Betroffene gab an, von einer Schwangerschaft nichts bemerkt zu haben, auch sei ihre Periode regelmäßig erfolgt. Der $1.420 \mathrm{~g}$ schwere männliche Fet mit einer Scheitel-Fersenlänge von $40 \mathrm{~cm}$ wies Zeichen der Mazeration und Unreife auf. Lebenszeichen waren nicht vorhanden (Lungen- und Magen-Darm-Schwimmprobe negativ). Als Ursache des intrauterinen Fruchttodes konnte eine Plazentainsuffizienz festgestellt werden. Bei Inspektion der Plazenta fand sich in einer zweiten Fruchthöhle ein weiterer männlich Fet mit einer Scheitel-Steißlänge von $8 \mathrm{~cm}$, der bereits hochgradig autolytisch erweicht war (• Abb. 3.115). 


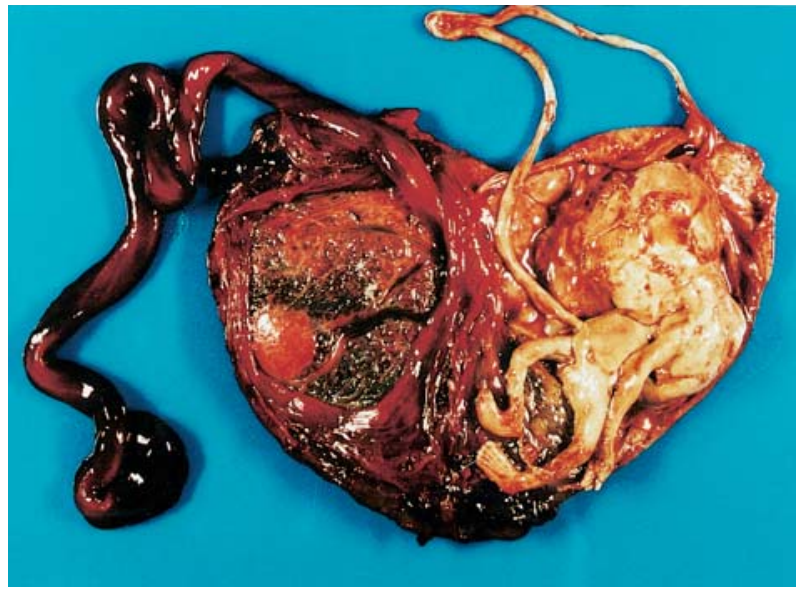

- Abb. 3.115. Plazentainsuffizienz als Verursacher eines intrauterinen Fruchttodes bei Zwillingsschwangerschaft. In der rechten Fruchthöhle hochgradig autolytischer Fet

\section{Einreden und Schutzbehauptungen}

Sturzgeburt. Am häufigsten wird angegeben, dass eine Sturzgeburt mit plötzlichem Stuhldrang und dann völlig überraschender Geburt des Kindes in die Toilettenschüssel stattgefunden habe. Eine Sturzgeburt als solche führt jedoch nicht zum Tode, da bei üblichen Stürzen von Kleinkindern bis zu einer Höhe von $150 \mathrm{~cm}$ in der Regel keine relevanten Verletzungen auftreten.

Nabelschnurzerreißung und Verblutungstod. Die durch eine Sturzgeburt eventuell hervorgerufene »Nabelschnurzerreißung « führt in der Regel deshalb nicht zum Tode des Kindes, da die Blutungen in Kürze spontan zum Stillstand kommen (Kontraktion der Umbilikalarterie, Blutumverteilung in den kleinen Kreislauf durch Entfaltung der Lungen, geringer Blutdruck der Nabelarterie). Allenfalls bei asphyktischen Kindern kann ein Verblutungstod diskutiert werden. Ein Zerreißen der Nabelschnur ist darüber hinaus nur in Ausnahmefällen anzunehmen, da bei den Presswehen reflexartig eine Hockstellung eingenommen wird, die die Höhe zwischen Geburtskanal und Boden vermindert.

Handlungsunfähigkeit der Kindesmutter. Eine echte Ohnmacht unter der Geburt wird nur nach erheblichem Blutverlust der Mutter (Hämoglobinmessung) bei der Geburt oder im Rahmen einer Eklampsie beziehungsweise Epilepsie beobachtet. Bei normalem Geburtsverlauf kommt es nicht zur Bewusstlosigkeit. Zur Beurteilung, ob eine Ohnmacht vorgelegen hat, ist auf die Art der Durchtrennung der Nabelschnur zu achten (glattrandig durchtrennt, durchgerissen).

\subsection{Abtreibung}

\author{
E. Lignitz
}

\section{Einleitung}

Der plötzliche oder unerwartete Tod in der (Früh-)Schwangerschaft erweckt zunächst aus forensischer Sicht stets den Verdacht auf eine Abtreibung. Das jedenfalls war die Maxime bis in die 70er-Jahre des vergangenen Jahrhunderts.

Eine moderne Gesetzgebung, die vor allem der Selbstbestimmung der Frau Rechnung trägt, hat zu einer vollständigen Veränderung der peripartalen Sterblichkeit geführt, diese weitgehend auf die natürlichen Risiken der Gravidität, der Entbindung und des Wochenbettes reduziert und die artifizielle (kriminelle) Abortsterblichkeit aus dem Todesursachengefüge gebärfähiger Frauen ausgerottet.

\section{Definition}

Abbrechen der Schwangerschaft ist (im strafrechtlichen Sinne) jede nicht auf bloße Nidationshemmung angelegte Einwirkung auf die Schwangere oder die Frucht, die final darauf gerichtet ist, das Absterben der noch lebenden Frucht im Mutterleib oder den Abgang der Frucht in nichtlebensfähigem Zustand herbeizuführen, und die diesen Erfolg erreicht. Der illegal induzierte Abort (syn.: Abtreibung) ist (im forensischen Sinne) die Herbeiführung einer nicht erlaubten, entgegen rechtlichen Bestimmungen durchgeführten Schwangerschaftsunterbrechung. Der legal induzierte Abort ist ein artifizieller Abort, der im Rahmen bestehender Gesetze erlaubt ist und medizinisch kontrolliert durchgeführt wird.

Die Ausstoßung bzw. Entfernung eines Feten aus der Gebärmutter bis zu einem Gewicht von $500 \mathrm{~g}$ wird als Fehlgeburt (Abort) bezeichnet. Die Ausstoßung eines lebenden oder toten Feten mit einem Gewicht über $500 \mathrm{~g}$ wird gemäß Personenstandsgesetz als Geburt oder Totgeburt bezeichnet.

\section{Wichtig}

Eine durch legalen Schwangerschaftsabbruch entfernte Frucht wird unabhängig vom Gewicht in den Personenstandsbüchern nicht beurkundet.

\section{Ätiologie des Abortes}

Wegen der zahlreichen medizinischen Ursachen darf eine vorzeitige Beendigung einer Gravidität nicht a priori kriminalisiert werden. Das Leitsymptom der Fehlgeburt ist die uterine Blutung, die mit Unterleibsschmerzen einhergehen kann. Einige Erscheinungsformen des Abortes haben praktisch nur klinische Bedeutung. Beim Abortus imminens (drohender Abort) bestehen leichte Blutungen, beim beginnenden Abort (Abortus incipiens) ist der Gebärmutterhals geöffnet und Teile der Fruchtanlage können sichtbar sein. Beim vollständigen bzw. unvollständigen Abort (Abortus completus/incompletus) bestehen stärkere Blutungen, und es wird ein Abgang von Gewebe nachgewiesen oder berich- 
tet. Der Gebärmutterhals kann weit oder schon wieder geschlossen sein. Wenn im Zusammenhang mit einem Abort Fieber auftritt, liegt ein febriler oder septischer Abort vor, der nicht selten im Zusammenhang mit einem artifiziellen (kriminellen) Abort steht.

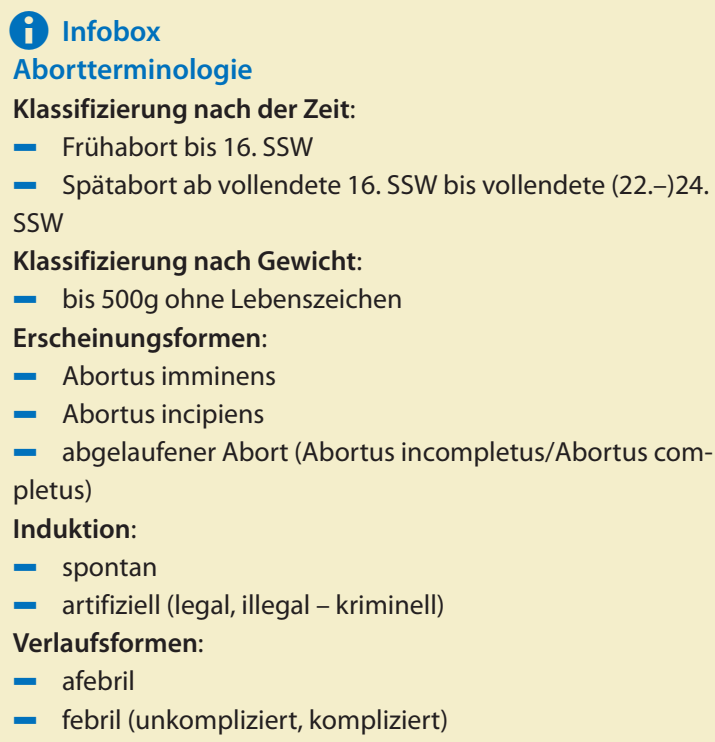

\section{Rechtsgrundlagen}

Das Grundgesetz verpflichtet den Staat in Art. 1, 2 II GG, das menschliche Leben, auch das ungeborene, zu schützen. Der Schutz der $\$ \$ 218$ ff. StGB umfasst den Zeitraum von der Einnistung des befruchteten Eies (Nidation) bis zum Beginn der Geburt.

\section{Wichtig}

Alle Fristen werden post conceptionem (p.c.) angegeben! Nimmt man die letzte Menstruation zur Grundlage, wird also die Zeit post menstruationem (p.m.) angegeben, sind maximal 14 Tage zusätzlich zu berechnen.

Der Gesetzgeber verbietet zum Schutze des ungeborenen Lebens grundsätzlich einen Schwangerschaftsabbruch ( $\$ 218$ StGB), benennt aber Ausnahmetatbestände für den Fall, dass von der Frau die Austragung der Schwangerschaft nicht erwartet werden kann. Im einzelnen ist das die

- medizinische Indikation (Gefahr für Leben oder Gesundheit der Mutter),

- kriminologische Indikation (Schwangerschaft als Folge eines Sexualdeliktes),

- Notlagenindikation (psychisch-sozialer Konflikt, der die Unzumutbarkeit der Schwangerschaftsbeendigung ernsthaft begründet und nunmehr auch die sog. embryopathische Indikation umfassen soll).
Ein Schwangerschaftsabbruch $(\$ 218)$ ist weiterhin ein Straftatbestand. Als besonders schwerer Fall gilt die Handlung gegen den Willen der Schwangeren und die leichtfertige Herbeiführung einer Gesundheitsschädigung oder der Todesgefahr für die Schwangere.

Der Schwangerschaftsabbruch ist indes nicht strafbar, wenn er unter bestimmten Bedingungen $(\$ 218 \mathrm{a}$, Abs. 1 - auf Verlangen und mit Einwilligung der Schwangeren, Nachweis einer Beratung, Einhaltung einer Frist von maximal 12 Wochen, Durchführung des Eingriffs durch einen Arzt) erfolgt. Der Abbruch der Schwangerschaft ist nicht rechtswidrig, wenn ( $\$ 218 \mathrm{a}$, Abs. 2) durch die Schwangerschaft eine Gefahr für das Leben oder eine schwerwiegende Beeinträchtigung des körperlichen und seelischen Gesundheitszustandes der Schwangeren gegeben ist. Eine derart indizierte Unterbrechung, die auch die »embryopathische Indikation « umfasst, ist zeitlich nicht limitiert; einer Pflichtberatung bedarf es nicht.

Ein Abbruch ohne schriftlich fixierte ärztliche Feststellung der gegebenen Voraussetzungen ist strafbar $(\$ 218 \mathrm{~b})$. Das ist auch so, wenn wider besseres Wissen unrichtige Feststellungen über die Voraussetzungen gem. $\$ 218$ a Abs. $2+3$ getroffen werden. Ebenso ist es strafbar, ohne Anhörung der Schwangeren, ohne umfangreiche medizinische Aufklärung und ohne erneute Untersuchung zur Feststellung der Schwangerschaftsdauer den Eingriff durchzuführen (\$218c). - Tabelle 3.38 fasst die rechtlichen Grundlagen, die Voraussetzungen und die ärztlichen Pflichten zusammen. Die Beratung der Schwangeren ( $\$ 219$ StGB) dient dem Schutz des ungeborenen Lebens und wird von einer anerkannten Schwangerschaftskonfliktberatungsstelle ergebnisoffen geführt und bescheinigt.

Die Strafbestimmungen ( $\$ 218$ ff StGB) zum Schwangerschaftsabbruch wurden nach jahrzehntelangem Ringen 1995 bundeseinheitlich geändert. Die Gesetzgebung hat einen Kompromiss gefunden, nach dem der Abbruch in der Frühphase der Schwangerschaft unter definierten Bedingungen nicht mehr (alte Bundesländer) bzw. nicht wieder (neue Bundesländer) unter Strafe gestellt ist, grundsätzlich aber eine rechtswidrige (nicht poenalisierte) Handlung ist.

\section{Wichtig}

Grundsätzlich ist ein Schwangerschaftsabbruch eine Tötungs-

handlung am Embryo und nicht Heilbehandlung an der

Schwangeren.

\section{Epidemiologie}

Abtreibungen haben über Jahrzehnte die peripartale mütterliche Sterblichkeit beherrscht und das forensische Sektionsgut geprägt. So wurden in Berlin (Charité) in 40 Jahren 243 mütterliche Sterbefälle untersucht, von denen 153 (63\%) auf kriminelle Aborte zurückzuführen waren (• Abb. 3.116). 6,5\% aller obduzierten Frauen bzw. $14 \%$ aller obduzierten Frauen im gebärfähigen Alter waren peripartale Sterbefälle.

Die gesetzliche Regelung der Schwangerschaftsunterbrechung hatte einen unverkennbar segensreichen Einfluss auf die 
- Tabelle 3.38. Legale Schwangerschaftsabbrüche: Indikationen, Voraussetzungen, Fristen und ärztliche Pflichten

\begin{tabular}{|c|c|c|c|c|}
\hline Indikation & $\begin{array}{l}\text { Rechtsgrundlage } \\
\text { gem. StGB }\end{array}$ & Fristen & Voraussetzungen & Ärztliche Pflichten \\
\hline Ohne Indikation & $\S 218 a, 1$ & 12 Wochen & $\begin{array}{l}\text { - Schwangere verlangt Eingriff } \\
\text { - Pflichtberatung gem. } \$ 219 \\
\text { - Bescheinigung } \\
\text { - Ärztl. Eingriff }\end{array}$ & $\begin{array}{l}\text { - Ärztliche Aufklärung } \\
\text { - Meldepflicht }\end{array}$ \\
\hline $\begin{array}{l}\text { Kriminologische } \\
\text { Indikation }\end{array}$ & $\S 218 \mathrm{a}, 3$ & 12 Wochen & $\begin{array}{l}\text { - Auskunftspflicht der Schwangeren } \\
\text { - Ohne Pflichtberatung gem. § } 219\end{array}$ & $\begin{array}{l}\text { - Prüfung der Kausalität (Sexual- } \\
\text { delikt und Schwangerschafts- } \\
\text { alter) »nach ärztlicher Erkenntnis« }\end{array}$ \\
\hline
\end{tabular}

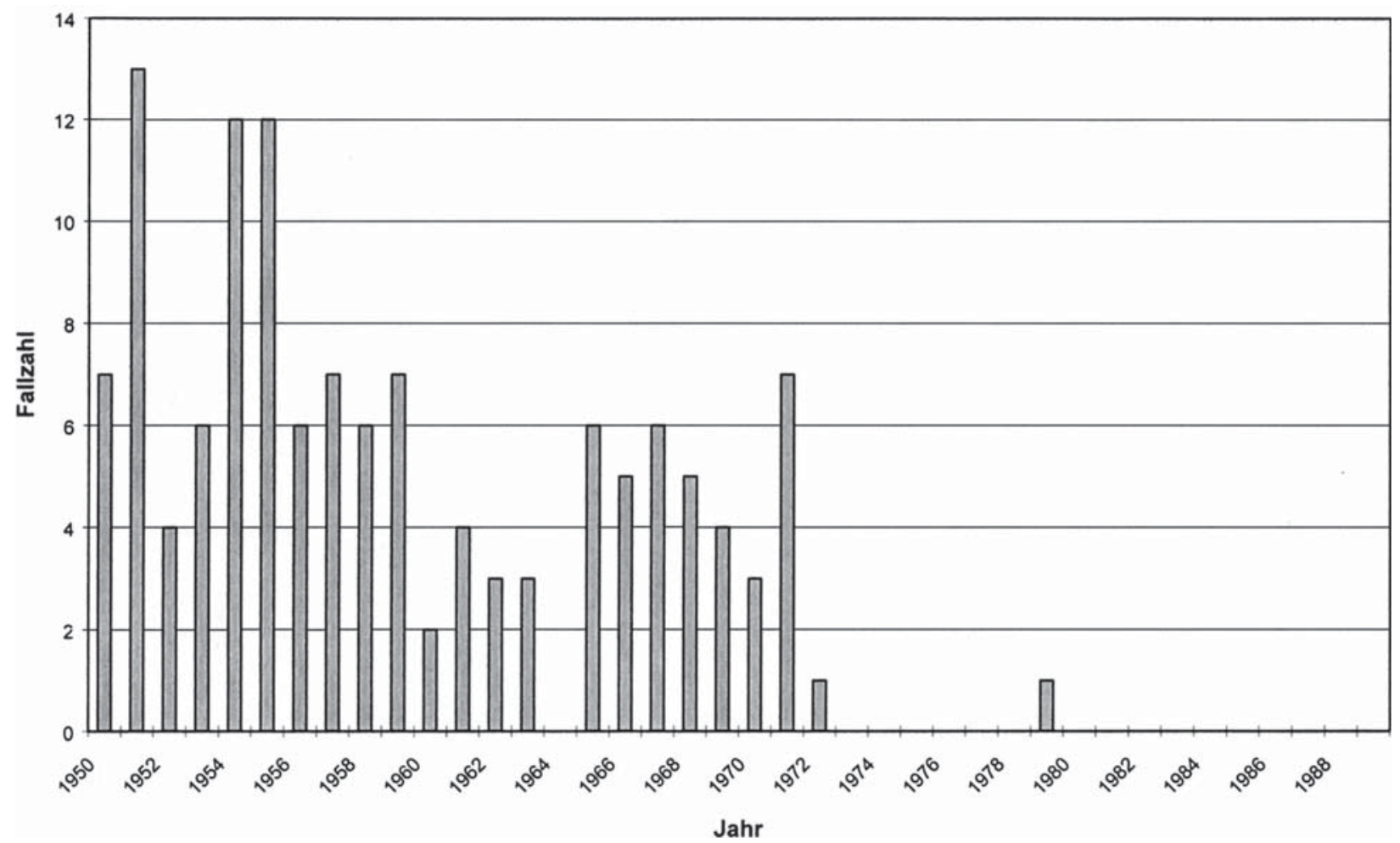

- Abb. 3.116. Entwicklung der Sterblichkeit durch kriminelle Aborte vor und nach der gesetzlichen Freigabe der Schwangerschaftsunterbrechung 1972 (Berlin/Charité)

mütterliche Sterblichkeit und führte zu einem Panoramawandel der peripartalen Mortalität, wie er nur selten in der Medizingeschichte vorgekommen ist. Die peripartalen Sterbefälle sanken innerhalb des Gesamtsektionsgutes von 2,5\% (1950) auf 0,1\% (1989). Diese Häufigkeitsabnahme mütterlicher Sterbefälle nach »Freigabe« (Legalisierung) der Schwangerschaftsunterbrechung war im Berliner Sektionsgut signifikant.

\section{Die legale Schwangerschaftsunterbrechung}

Die legalisierte Schwangerschaftsunterbrechung wird je nach Schwangerschaftsdauer einzeitig (bis zur 12. Woche nach der Empfängnis!) oder zweizeitig (nach der 12. Woche) vorgenommen. Die Fruchtentfernung erfolgt medikamentös oder instrumentell vorzugsweise durch Saugkürettage. Letztere gilt als weniger riskant. Nach der 12. Woche wird eine Spontanausstoßung 
des Schwangerschaftsproduktes induziert und das Uteruscavum nachgeräumt.

\section{( Wichtig}

Komplikationen des legalen Schwangerschaftsabbruchs sind Blutungen, Narkosezwischenfälle, Risse des Gebärmutterhalses, Perforationen der Gebärmutterhöhle und Infektionen.

Als Spätfolgen gelten Zunahme von Infertilität, Abort- und Frühgeborenenrate.

\section{Fallbeispiel}

Bei einer 32-jährigen Frau wurde im 3. Schwangerschaftsmonat eine legale Unterbrechung mittels Vakuumabsaugung (Exhaustion) durchgeführt, bei der die Frau an einer Luftembolie verstarb. Durch Verwechslung der Anschlüsse für Saug- und Druckstutzen am Gerät kam es statt einer Absaugung zu einer Lufteinblasung, die sofort tödlich war.

\section{Die illegale Schwangerschaftsunterbrechung (Abtreibung)}

Methoden illegaler Eingriffe zum Schwangerschaftsabbruch durch

\section{- mechanische Mittel:}

- Einführung von Gegenständen in die Gebärmutter. Medizinische Instrumente (Katheter, Sonden, Stifte, Kornzangen), aber auch Drähte, Strick- und Haarnadeln, selbst Fahrradspeichen werden in die Gebärmutterhöhle eingeführt und sollen die Fruchtblase verletzen oder die Frucht unmittelbar schädigen, Wehen auslösen und die Ausstoßung des Schwangerschaftsproduktes induzieren.

- Einspritzen von Flüssigkeiten in die Gebärmutter. Alle möglichen Formen von Spritzen und Ballons mit den unterschiedlichsten Lösungen werden verwendet, am häufigsten zweifellos Seifenlösungen, jedoch auch Alkohol, Kupfersulfat, Glukose, Kochsalz, Formalin, Kaliumpermanganat, Desinfektionsmittel oder auch nur Wasser.

- Maßnahmen an der Cervix uteri. Sie dienten vor allem einer Weitung des Gebärmutterhalses und erfolgten mit Laminar- oder Hegarstiften, Intrauterinpessaren, Obturatoren oder auch nur durch einen Finger.

- Mechanische und thermische Alterationen. Dazu zählen Massagen aller Art und Traumatisierungen wie wiederholtes Springen aus geringer Höhe, Schläge oder Tritte in den Bauch, ggf. in Kombination mit heißen Sitz- oder Vollbädern, um wehenauslösend zu wirken.

\section{innere Mittel:}

- Bei ihrer Anwendung spielt die individuelle Verträglichkeit und Abortbereitschaft eine entscheidende Rolle, ebenso Dosis-Wirkungs-Beziehungen. Das allgemeine Wirkungsprinzip ist eine Steigerung der Durchblutung der Gebärmutter, die Anregung der Wehentätigkeit oder die toxische Schädigung der Frucht. Zu Allgemeinintoxikationen führen u.a. Alkaloide wie Chinin und Mutterkorn.
Der Ideenreichtum im Einsatz von »bewährten« Mitteln ist unbegrenzt und kaum vollständig darzustellen. Problematisch bleibt immer der Nachweis des kausalen Zusammenhangs zwischen Eingriff und Abort, besonders wenn mehrere Mittel eingesetzt werden und deren Anteil am »Erfolg « erfasst werden soll.

\section{Todesursachen nach illegalem Abort}

Die Todesursachen nach artifiziellem (kriminellem) Abort sind nach der Häufigkeit folgende: Sepsis/Septikopyaemie, venöse Luftembolie, (Perforations-) Peritonitis, Seifenintoxikation, Gasbrandinfektion, Fett- und Seifenembolie, Verbluten und Fruchtwasserembolie.

Da Spülflüssigkeit und "Instrumente« bakteriell kontaminiert sind und zusätzlich manche Spülflüssigkeiten auf Blut und Gewebe toxisch wirken, führen Spülungen häufig zu Infektionen und Intoxikationen, z.B. zur toxischen Hämolyse. Übliche Folgeerscheinungen sind ein funktionelles Nierenversagen durch hämoglobinurische Nephrose oder bilaterale Nierenrindennekrosen, eng verknüpft mit disseminierter intravasaler Koagulopathie bei Endotoxinschock.

Der septische Abort ist die klassische Komplikation der illegalen Abtreibung. Die bakteriellen Allgemeininfektionen sind bei Eingriffen am Uterus meist mit Entzündungen des Endomyometriums verbunden, ebenso mit Thrombose und Thrombophlebitis, sodass auch (septische) Embolien vorkommen und nicht selten zum Tode führen. Als Erreger finden sich meist Staphylokokken und Streptokokken, seltener Gasbrand und Tetanus. Gramnegative Keime sind 20-mal häufiger als grampositive.

Luftembolien sind praktisch immer auf Spülungen zurückzuführen. Es wird nämlich regelmäßig übersehen, dass die sog. »Frauenduschen «(Gummiballons), mit denen die Spülflüssigkeit eingebracht wird, nie vollständig mit Flüssigkeit, sondern immer auch mit Luft gefüllt sind. Diese dringt dann in die geöffneten Blutgefäße ein und wird meist foudroyant, seltener protrahiert embolisiert.

\section{(3) Fallbeispiel}

Nachdem bei einer 25-jährigen Frau die Regel 2 Monate ausgeblieben war, ließ der Ehemann eine sfremde Frau« in die Wohnung kommen, die mittels eines wassergefüllten Ballons einen Abtreibungsversuch vornahm. Beim Einspritzen der Flüssigkeit stellten sich Übelkeit und Erbrechen ein. Nachdem die Schwangere sich aufgerichtet hatte, brach sie plötzlich zusammen und war tot. Todesursache Luftembolie.

Jeder zweizeitig verlaufende, inkomplette Abort innerhalb der ersten drei Schwangerschaftsmonate ist verdächtig, illegal verursacht worden zu sein. Das gilt auch für fieberhafte Aborte und bei Verweigerung und Verzögerung der ärztlichen Behandlung. Auch die Art der Komplikation hat hinweisende Bedeutung für die Illegalität des Eingriffs. 


\section{Nachweis des Abortes}

Zum Nachweis eines Abortes gehört die klinisch-gynäkologische und sonographische Untersuchung. Beweisend ist die histologische Untersuchung des Abortmaterials, des Kürettagematerials, bzw. im Todesfalle die Untersuchung der Gebärmutter. Vorherrschend ist eine nekrotische, eitrig-fibrinös entzündete Dezidua mit Blutungen und Thromben (Endometritis post abortum). Mehr oder weniger regressiv veränderte Plazentazotten

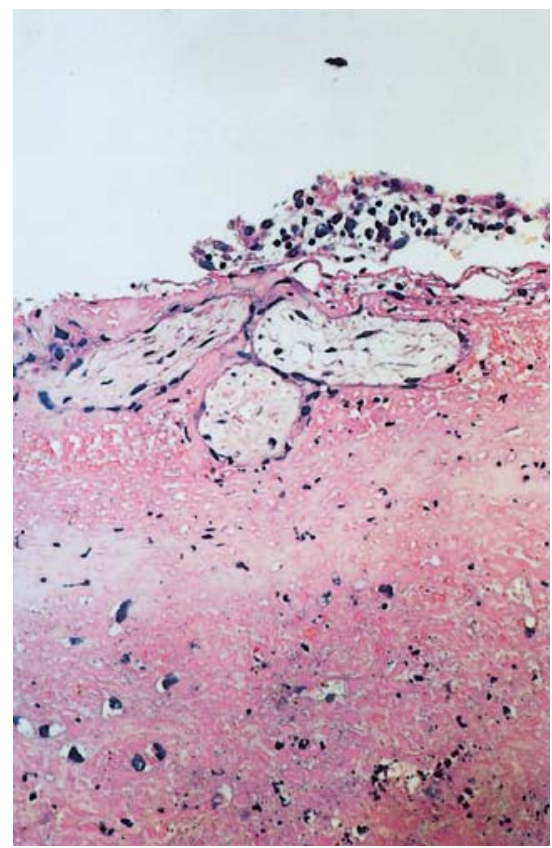

- Abb. 3.117. Abortmaterial mit regressiv veränderten Chorionzotten und nekrotisierter, entzündeter Dezidua

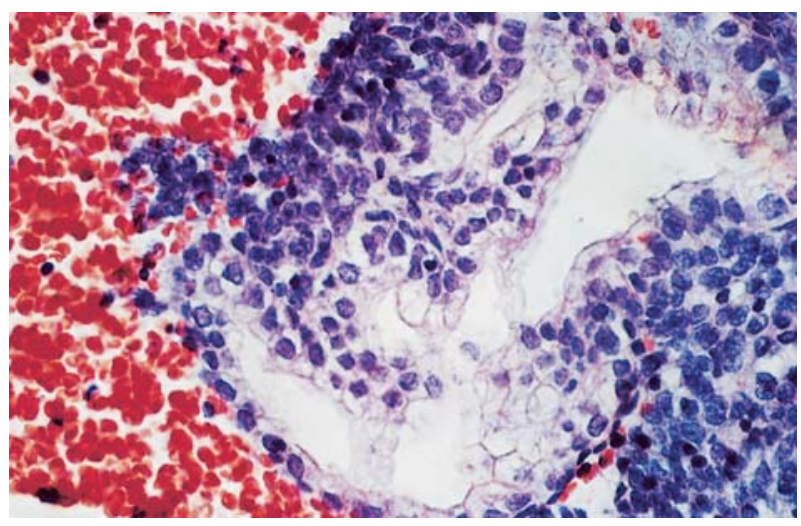

- Abb. 3.118. Arias-Stella-Phänomen mit verformten, depolarisierten und chromatindichten Kernen der endometrialen Drüsenepithelien bei typischer Zytoplasmaaufhellung; randständige Blutungen und Trophoblastzellverbände (sog. choriale Wanderzellen) beweisen die intrauterine Schwangerschaft (• Abb. 3.117). Auf eine besondere hormonelle Situation weist das Arias-Stella-Phänomen (•Abb. 3.118) hin, eine Überstimulation des Drüsenepithels mit unförmig vergrößerten, chromatindichten Kernen und Aufhellung des Zytoplasmas.

\subsection{Tödliche Unfälle bei autoerotischer (autosexueller) Betätigung}

\section{G. Geserick}

Für den Arzt (Leichenschauarzt) sind Kenntnisse über autoerotische Unfälle erforderlich, da eine nichtnatürliche Todesart vorliegt und unter Umständen die Abgrenzung zwischen einem Unfall und einer Tötung durch eigene oder fremde Hand schwierig sein kann. Da die Ereignisse sich im Verborgenen abspielen, gibt es in der Regel weder Zeugen für den Hergang noch Kenntnisse über die Neigungen des Verstorbenen im Verwandtschafts- oder Freundeskreis. Richtungweisend ist die Auffindungssituation.

\section{Rechtliche Grundlagen}

Der tödliche Ausgang eines selbstverschuldeten Unfalls (bzw. einer suizidalen Handlung) hat im deutschen Recht keine strafrechtlichen Konsequenzen. Für den Leichenschauarzt, der einen Todesfall als autoerotischen Unfall erkennt, besteht die Pflicht zur Meldung des nichtnatürlichen Todesfalls an die Polizei (nach den Leichenschaugesetzen der Bundesländer). Für den Betroffenen bzw. seine Hinterbliebenen können versicherungsrechtliche Folgen bedeutsam sein.

\section{Grundlagen}

\section{- Definition}

Es handelt sich um tödliche Unglücksfälle (»Betriebsunfälle«) als direkte Folge autoerotischer (besser autosexueller) Handlungen Einzelner zur sexuellen Stimulation oder Selbstbefriedigung ohne Sexualpartner. Das Ziel wird durch zentrale Erregung infolge Dämpfung kortikaler Kontrollfunktionen (z.B. Sauerstoffmangel, Narkotika) oder periphere Reizung, vor allem der erogenen Zonen (z.B. elektrisch, mechanisch), zu erreichen versucht. Schließlich kann auch die Schaffung einer Angst- oder Leidenssituation (Masochismus) angestrebt sein. Zusätzliche Komponenten können Fetischismus und Transvestitismus sein. Nach internationalen Klassifikationen lautet der Oberbegriff „Störung der sexuellen Präferenz« (ICD 10, WHO 1993) bzw. »Paraphilie« (DSM-IV, Am. Psychiatr. Ass. 1994). Plötzliche natürliche Todesfälle, die bei sexueller Erregung oder Betätigung eintreten (z.B. durch akuten Myokardinfarkt oder Hirnmassenblutung), werden von dem Begriff nicht erfasst. 
(i) Infobox

Epidemiologische Daten

Literaturberichte sind meist kasuistische Darstellungen oder Sammlungen von Todesfällen. Berichte über Lebende sind naturgemäß selten, für sie besteht eine große Dunkelziffer. Opfer sind ganz überwiegend Männer (Angaben aus den USA:Todesfälle pro Jahr >1.000 Männer: <20 Frauen). Geschätzte jährliche Häufigkeit etwa 1-2 pro 1 Mio. Einwohner. Betroffen können ab der Pubertät alle Alters- und Berufsgruppen sein. Nach Berichten der letzten Jahrzehnte dürften Strangulationsfälle am häufigsten sein. Seit den 50er-Jahren wird eine Zunahme der Erstickungsfälle in Plastikbeuteln beobachtet.

\section{Typische Auffindungssituation}

\section{(8) Wichtig}

Kein unüberlegtes Verändern der Auffindungssituation, wenn die Beurteilung kriminalistische oder rechtsmedizinische Erfahrung erfordert!

- Wohnung bzw. Raum sind von innen verschlossen, seltener Auffindung im Freien (den Blicken Fremder entzogen)

- Auffällige Bekleidung (z.B. weibliche Unterwäsche, Gummioder Lederkleidung)

- Entblößung von Unterkörper oder Genitalien, Zeichen der Ejakulation, Kondom oder andere Vorrichtungen zum Auffangen des Sperma

- Maßnahmen zur sexuellen Stimulation (durch Asphyxie, Elektrizität, Narkotika, mechanische Reizung)

- Sadomasochistische Utensilien wie Peitschen, Ketten, Stacheldraht, Fesseln

- Pornographische Literatur, Bilder, Videos u.a.

- Aufgestellte Spiegel

- Vorrichtungen zum Fotografieren oder Filmen der autoerotischen Maßnahmen

\section{Häufige Todesursachen und Befunde}

Strangulation. Durch Scheitern des Versuchs, mit »dosiertem Erhängen« zur sexuellen Befriedigung zu kommen, stirbt das Opfer im Strangwerkzeug hängend (manchmal auch stehend, sitzend oder sogar liegend). Es finden sich die äußeren und inneren Zeichen des Erhängens. Seltener werden auch Fälle mit Selbsterdrosseln beobachtet.

Ersticken. Durch Verlegung der Atemöffnungen: Nach Überziehen eines Plastikbeutels über den Kopf (mitunter am Hals durch Klebeband, Schnur o. Ä. abgedichtet) kommt es durch Verbrauch des Sauerstoffs und Anreicherung von Kohlendioxid zur Erstickung. Seltener werden auch die Atemöffnungen durch Klebeband oder Einwickeln in Gummi- oder Plastikfolien verschlossen. Äußere Erstickungsbefunde sind spärlich und uncharakteristisch.
Intoxikation. Durch berauschende Stoffe: Zur Rauscherzeugung und sexuellen Stimulation werden flüchtige Stoffe (z.B. Azeton, Alkohole, Benzin, Ether, Halogenkohlenwasserstoffe, früher auch Chloroform) inhaliert (»geschnüffelt«), die in Zubereitungen wie Lackverdünnern, Klebstoffen, Fleckenwasser oder Nagellackentferner haushaltsüblich und leicht erreichbar sind. Der Tod kann infolge Überdosierung durch Atemlähmung oder Herzrhythmusstörungen eintreten, bei Einatmung aus einem Plastikbeutel auch durch Ersticken. In einzelnen Fällen wurde auch Kohlenmonoxid inhaliert oder Kokain appliziert. Die Intoxikationen sind nur durch eine chemisch-toxikologische Laboranalyse nachzuweisen.

Tod durch elektrischen Strom. Durch Anbringen von elektrischen Leitern (als Elektroden werden Drähte, Klemmen, Metallfolien u.a. verwendet) an Genitalien oder erogenen Zonen (Brustwarzen, After) tritt ein tödlicher Stromfluss auf. Die Stromzuführung kann technisch äußerst simpel bis kompliziert sein. An den Kontaktstellen können Strommarken auftreten.

Tod durch mechanische Verletzung. Der autoerotische Unfalltod durch mechanische Verletzung, z.B. Messerstiche (s. Fallbeispiel; • Abb. 3.119a, b) ist selten. Äußere Wunden durch stechende oder schneidende Werkzeuge sind bei gründlicher Leichenschau nicht zu übersehen. Innere Verletzungen (z.B. eine Darmperforation nach Einführung von Instrumenten in das Rektum) werden erst nach klinischer Revision oder Obduktion erkannt. Die Einordnung in einen autoerotischen Unfall erfordert immer die Berücksichtigung der Gesamtsituation.

\section{(2) Fallbeispiel}

Ein 23-jähriger Mann wurde tot in seinem von innen verschlossenen Zimmer neben der Liege aufgefunden, das Schlüsselloch von innen abgedeckt. Der Leichnam war nur mit Damenunterwäsche (Büstenhalter und Slip) bekleidet und geschminkt. Auf der Liege fanden sich eine Abbildung (Frau in Unterwäsche) und ein aufgestellter Spiegel. Im Oberbauch des Leichnams mehrere frische Stichwunden. An der Fußseite des Bettes war eine selbstkonstruierte Stichvorrichtung angebaut, bei der an einem Brett fixierte Messer mit einem Seilzug über eine Rolle schrankenartig gehoben und gesenkt werden konnten (Fernbedienung mit Elektromotor!). Durch Riss des Seiles kam es zum tödlichen Unfall. Obduktionsbefund: Verbluten aus drei Stichwunden mit Perforation der Bauchwand, Verletzung von Dünndarm, Mesenterium und V. cava inferior. Zahlreiche Narben unterschiedlichen Alters an der äußeren und inneren Bauchwand als Zeichen wiederholter früherer Verletzungen. 
- Abb. 3.119. a Auffindungssituation eines verstorbenen jungen Mannes mit Stichverletzungen im Bauch; b Skizze zur Anordnung des Stichwerkzeugs

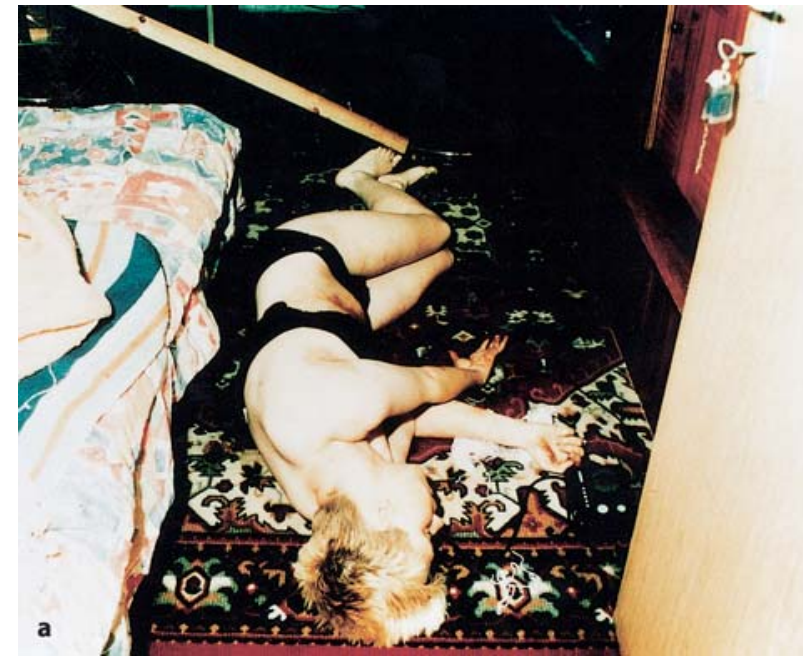

Schraubstockkonstruktion, gemeinsam mit Metallscheibe an obere Latte gespannt

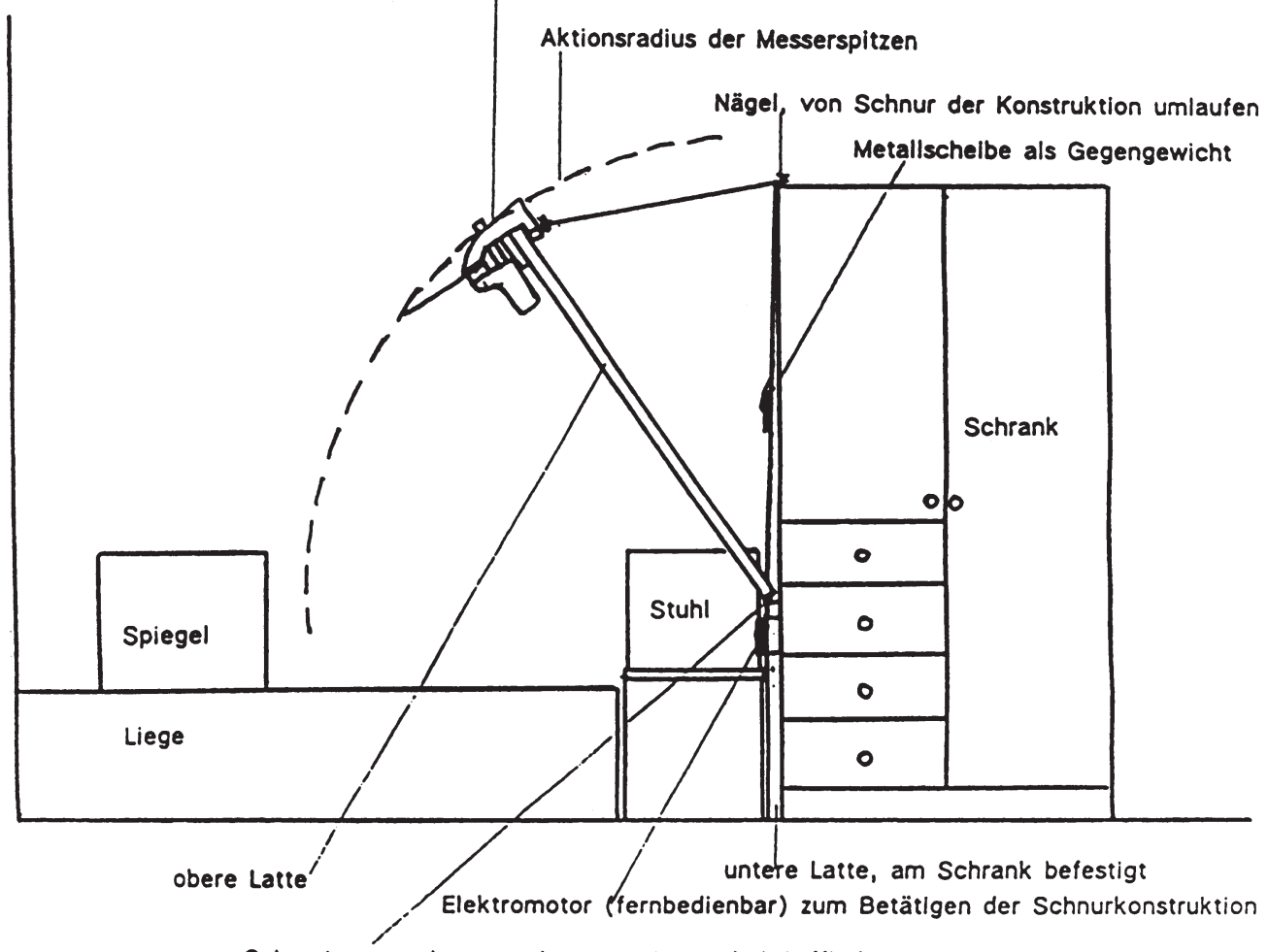

b

Scharnier $2 w$. oberer und unterer Latte, bei Auffindung ausgerissen 


\subsection{Tod in abnormer Körperposition - Physical restraint}

\section{P. Schmidt, B. Madea}

Unter diesem Oberbegriff werden zusammenfassend Todesfälle behandelt, bei denen das polizeiliche Ermittlungsergebnis beziehungsweise die Auffindesituation darauf hindeuten, dass durch die Körperhaltung bedingte pathophysiologisch-funktionelle Geschehensabläufe, insbesondere Beeinträchtigungen der Atembeziehungsweise Herz-Kreislauf-Funktion kausale Bedeutung für den Todeseintritt haben.

$\mathrm{Zu}$ den charakteristischen forensischen Fallkonstellationen gehören:

- haltungsbedingte Asphyxie (»positional asphyxia« oder "postural asphyxia «),

- Todesfälle bei der mechanischen Fixierung erregter Personen (»restraint asphyxiation in excited delirium «),

- Todesfälle in Krankenhäusern oder Pflegeeinrichtungen während mechanischer Fixierung,

- Tod in aufrechter Körperhaltung (z.B. Kletterunfälle im Gebirge),

- Kruzifikation und

- Tod in Kopftieflage (z.B. autoerotischer Unfall).

\section{Haltungsbedingte Asphyxie}

\section{- Definition}

Die Todesursache »haltungsbedingte Asphyxie» darf dann in Betracht gezogen werden, wenn der Verstorbene in einer Körperhaltung aufgefunden wird, die in plausibler Weise eine mechanische Beeinträchtigung der Atemfunktion begründet, und eine anderweitige Todesursache ausgeschlossen ist.

Diagnose. Die Diagnose stützt sich auf folgende Kriterien:

- Auffindung in einer Körperhaltung, aus der sich plausibel eine Beeinträchtigung der Atemfunktion ableiten lässt,

- fehlende Möglichkeit zur Selbstrettung (z.B. Intoxikation, körperliche oder geistige Behinderung),

- Ausschluss einer Atemwegsobstruktion aus innerer Ursache (z.B. Aspiration),

- Ausschluss einer Kohlenmonoxidvergiftung oder einer anderweitigen inhalatorischen Erstickung und

- morphologischer Ausschluss einer signifikanten Herzerkrankung.

Auffindesituation. $\mathrm{Zu}$ den charakteristischen Auffindesituationen, bei denen eine haltungsbedingte Erstickung in Betracht gezogen werden darf, gehören:

- umgebungsbedingte Hyperflexion des Halses mit Obstruktion der Atemwege,

- Bauchlage mit Aufliegen des Gesichtes auf einer Unterfläche und Verschluss von Nase und Mund,
- Aufliegen des Oberkörpers auf einer Kante mit Beeinträchtigung der Brustkorbexkursionen,

- aufrechte Sitzhaltung mit stark hyperflexiertem Hals und Aufliegen des Kinns auf dem Brustkorb und

- Kompression des Rumpfes durch Fixationsvorrichtungen bei »Rollstuhlunfällen«.

(D) Wichtig

Als Risikofaktoren für einen Tod durch haltungsbedingte Erstickung haben sich akuter Alkoholrausch, Alkoholismus, Betäubungsmittel- oder Medikamentenintoxikation, neurodegenerative Erkrankungen sowie körperliche und geistige Behinderungen identifizieren lassen.

\section{Rechtsmedizinische Untersuchungsbefunde}

Bei der Leichenschau finden sich in knapp der Hälfte der Fälle petechiale Stauungsblutaustritte in charakteristischer Lokalisation. Autoptisch zeigt sich in der Regel ein ausgeprägtes Lungenödem mit stark erhöhten Organgewichten. Eventuelle Verletzungen sollten sich unter einen Geschehensablauf subsumieren lassen, der plausibel mit der Auffindesituation vereinbar ist. Vorbestehende innere Erkrankungen dürfen vom Schweregrad her nicht als konkurrierende Todesursachen in Betracht kommen. Chemisch-toxikologische Untersuchungen auf Alkohol, Medikamente und Betäubungsmitteln führen zu negativen bzw. nicht konkurrierend todesursächlichen Befunden.

(D) Wichtig

Bei unspezifischen Sektionsbefunden, denen allenfalls Hinweischarakter zukommt, beruht die korrekte Diagnosestellung also entscheidend auf Einbeziehung und adäquater Bewertung der Todesumstände und der Auffindesituation.

\section{Todesfälle nach mechanischer Fixierung erregter Personen}

Vor allem im angloamerikanischen Schrifttum haben Todesfälle unter Fixation im Polizeigewahrsam Beachtung gefunden. Bei einer häufig angewandten Methode wird der Arrestant in Bauchlage verbracht und Handgelenke bzw. Fußgelenke werden auf dem Rücken aneinander gefesselt (»hog-tied prone position«). Betroffen sind vor allem stark erregte Delinquenten, die sich selbst und ihre Umwelt durch ausgeprägt aggressives Verhalten gefährden und Widerstandshandlungen gegen die Ermittlungsbeamten begehen. Die Ursachen für die starke Erregung können in psychiatrischen Grunderkrankungen oder einer akuten Alkohol- bzw. Rauschmittelbeeinflussung liegen.

Die integrierende rechtsmedizinische Bearbeitung ergibt folgendes Gesamtbild:

\section{- Vorgeschichte:}

- Aggressives, auch körperlich gewalttätiges Benehmen vor polizeilicher Fixation, heftige Gegenwehr gegen die Fesselung, 
- Minuten später einsetzende Bewegungslosigkeit, Bewusstlosigkeit und Todeseintritt.

- Postmortale Untersuchungsbefunde:

- durch die vorausgegangene körperliche Auseinandersetzung und Fixation erklärbare Verletzungsbefunde von nichttodesursächlichem Schweregrad,

- diskrete Petechien von Haut und serösen Häuten (fakultativ),

- vorbestehende innere Erkrankungen, die für sich genommen den Todeseintritt nicht begründen können,

- chemisch-toxikologische Untersuchungsbefunde, die als psychiatrisch indizierte Medikation zu werten sind oder einen rauschbedingten Erregungszustand erklären, nicht jedoch als konkurrierende Todesursache in Betracht kommen.

Pathophysiologie. Pathophysiologisch werden als Todesursache Herzrhythmusstörungen oder ein Atemstillstand angenommen, denen ein Missverhältnis zwischen Sauerstoffbedarf und -zufuhr zugrunde liegt. Hierzu tragen folgende Faktoren bei:

- katecholaminvermittelte Stressreaktion bei psychischer Erregung und Konfrontation mit der Polizei,

- motorische Hyperaktivität bei Widerstand gegen Polizei und Fesselung sowie

- Beeinträchtigung der Atemfunktion durch mechanische Behinderung der Exkursionen von Brustwand und Zwerchfell.

Experimentell konnte gesichert werden, dass Herzfrequenz und periphere Sauerstoffsättigung nach körperlicher Anstrengung wesentlich langsamer zum Ausgangsniveau zurückkehren, wenn die Versuchspersonen in »hog-tied prone position « fixiert sind. Der Todeseintritt kann ferner begünstigt werden durch Missbildungen von Brustwand und Brustorganen, toxische Depression des Atemzentrums und arrhythmogene Wirkung von Alkohol oder Medikamenten wie zum Beispiel Phenothiazinen.

\section{Plötzliche Todesfälle mechanisch fixierter Krankenhauspatienten}

In charakteristischer Weise werden die Patienten leblos in einer verrutschten Leibbandage neben dem Bett hängend oder auf dem Fußboden kniend aufgefunden. Daneben wurden auch Strangulationen im Schlupflochrand von Schutztüchern beschrieben, die primär als Hilfsmittel der Pflege bestimmt sind. Autoptisch finden sich fakultativ Prellungen oder Hämatome des Brustkorbes, die den Rückschluss auf eine Thoraxkompression gestatten, Strangmarken am Hals, die eine Halskompression belegen oder Stauungsblutaustritte in typischer Lokalisation. Vor allem bei krankheitsbedingter Reduktion des Allgemeinzustandes oder medikamentöser Dämpfung kann die tödliche Erstickung beschleunigt ablaufen, sodass lokale Verletzungsbefunde und systemische Zeichen der hämodynamischen Wirksamkeit nur sehr gering ausgeprägt sind. Der Todeseintritt in Folge der Thorax- kompression oder der Strangulation kann durch kardiale Vorerkrankungen, arrhythmogene oder atemdepressive Nebenwirkungen von Medikamenten oder Drogen und Immobilisationsstress begünstigt werden.

\section{Wichtig}

Häufige äußere Ursache für plötzliche Todesfälle mechanisch fixierter Patienten ist eine fehlerhafte Durchführung der Fixierung. Typische Fehler beim Anlegen einer Leibbandage bestehen im Weglassen eines Bettgitters und dem Verzicht auf die Leibbandagen-Seitenfixierung.

\section{Tod in aufrechter Körperposition}

Die forensischen Konzepte zum pathophysiologischen Geschehensablauf des Todeseintritts in aufrechter Körperposition wurden anhand von Einzelfallbeobachtungen tödlicher Unfälle junger Männer erarbeitet. Hierzu gehören zum Beispiel das Abgleiten und Steckenbleiben im engen Schacht eines Schornsteins mit über den Kopf geschlagenen Armen oder Kletterunfälle im Gebirge mit Einklemmen des freihängenden Unfallopfers im unter die Achsel hochgerutschten Brustgeschirr der Seilfixierung. Bei diesen Beobachtungen waren autoptisch weder todesursächliche Verletzungen noch vorbestehende innere Erkrankungen nachzuweisen. Feingeweblich zeigten sich disseminierte Sauerstoffmangelschäden von Herzmuskulatur und Leber. Auf dem Ausschlussweg wurde als Todesursache ein orthostatischer Kollaps angenommen. Durch die Körperhaltung der Verstorbenen bei Auffindung lassen sich eine Behinderung der Atemexkursionen mit verminderter exspiratorischer Sogwirkung und eine Beeinträchtigung der Muskelpumpe in den Beinen erklären. Dies führt zu einer Reduktion von zentralem Blutvolumen, venösem Rückstrom zum Herzen, Schlagvolumen und Herzzeitvolumen und letztendlich zur tödlichen »extrakoronarbedingten Hypoxie des Herzens .

\section{Kreuzigung}

Beim Tod am Kreuz tragen folgende Mechanismen zum Todeseintritt bei:

\section{- Dehydratation,}

- mechanische Erstickung: Durch das Hochziehen der Arme werden der Brustkorb und die Interkostalmuskulatur in extremer Inspirationsstellung fixiert, sodass die Ausatembewegungen ausschließlich durch das Zwerchfell getragen werden und

- hypovolämischer Schock: Bei Versuchen mit freiwilligen, jungen und gesunden Medizinstudenten, die mit den Armen an einer querliegenden Stange aufgehängt wurden, wobei kontinuierlich Röntgen- und EKG-Kontrollen durchgeführt und Herzfrequenz, Blutdruck und Vitalkapazität erfasst wurden, zeigte sich bereits nach 6 Minuten ein Absinken des Blutdrucks auf $70 \mathrm{~mm} \mathrm{Hg}$, und spätestens nach 12 Minuten entwickelte sich bei allen Versuchspersonen das Vollbild des orthostatischen Kollapses. 


\section{Tod in Kopftieflage}

$\mathrm{Zu}$ Todesfällen in Kopftieflage kommt es etwa im Rahmen autoerotischer Unfälle oder bei Übersteigen von Zäunen und Mauern, unfallmäßigem Abgleiten mit Fixation von Fuß oder Bein und bodenwärts hängendem Oberkörper und $\operatorname{Kopf}(\bullet$ Abb. 3.120).

Hypothesen zur Kreislaufdysregulation in Kopftieflage lassen sich aus der Kenntnis der physiologischen Regulationsmechanismen beim Übergang vom Liegen zum Stehen ableiten.

Dabei geraten Kopf, Hals und Brustkorb unter die hydrostatische Indifferenzebene, und der hydrostatische Druck in der »oberen « Körperhälfte nimmt zu. Dies bewirkt ein Versacken von Blut in den intrathorakalen Blutspeichern, in denen im Vergleich zu den unteren Gliedmaßen die Muskel-Venen-Pumpe unzureichend ausgebildet ist, und einen verminderten venösen Rückfluss zum Herzen (Sind die Atembewegungen durch Besonderheiten des Geschehensablaufes, z.B. bei autoerotischen Unfällen, zusätzlich eingeschränkt, wird dieser Effekt durch einen Wegfall der Saug-Druck-Pumpenwirkung der Atmung verstärkt). Ferner ist denkbar, dass der erhöhte statische Druck im Bereich des Karo-

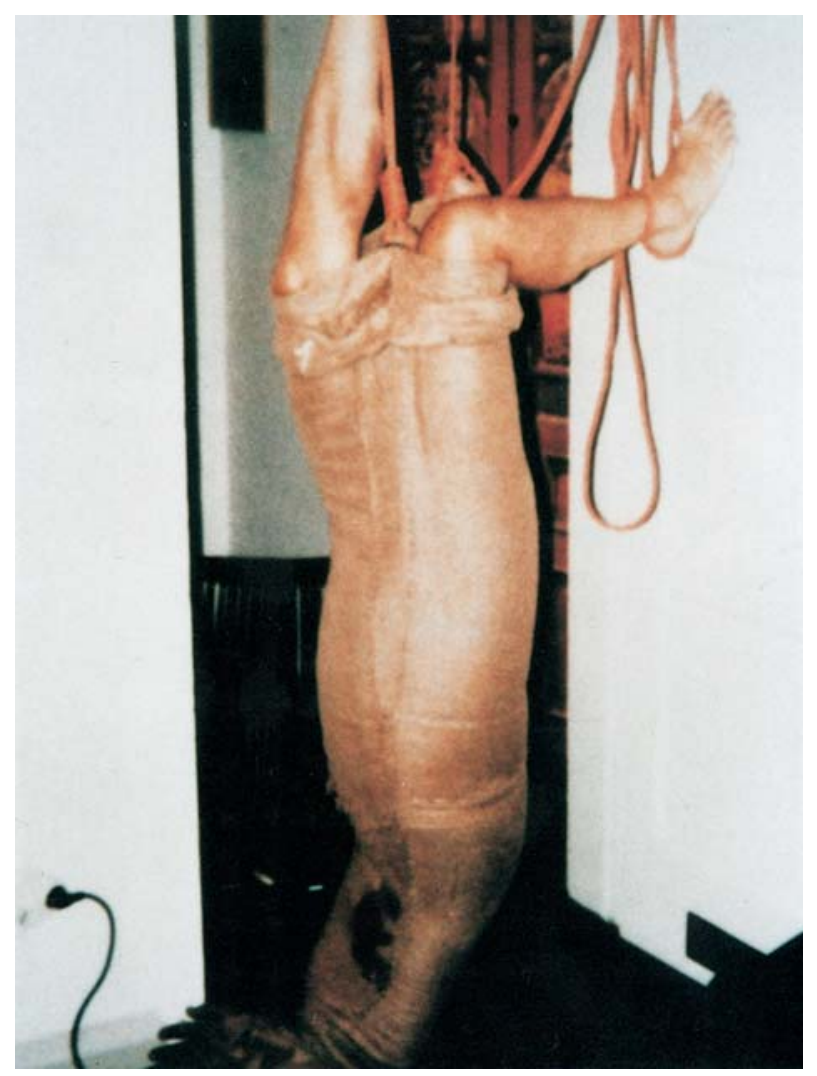

- Abb. 3.120. Autoerotischer Unfall mit Suspension in einem Sack in Kopftieflage. Todesursächlich war ein protrahiertes Kreislaufversagen und Ersticken (Behinderung der Atemexkursionen durch einen dem Thorax eng anliegenden Sack) mit stundenlanger Agonie tissinus reflektorisch zu einer Senkung von arteriellem Blutdruck und Herzfrequenz führt.

\section{(. Wichtig}

Allen Todesfällen in abnormer Körperposition ist gemeinsam, dass nach Ausschluss einer todesursächlichen Erkrankung, Verletzung oder Intoxikation aus Todesumständen und Auffindungssituation pathophysiologisch eine mechanische Erstickung oder ein Kreislaufkollaps abgeleitet werden.

Diese Todesfälle stellen ein Paradebeispiel dafür dar, dass die rechtsmedizinische Todesursachenklärung nicht in der isolierten Erhebung von morphologischen oder toxikologischen Befunden besteht, sondern vielmehr in der integrativen Zusammenschau medizinisch-naturwissenschaftlicher Untersuchungsbefunde mit dem Ergebnis der polizeilichen Ermittlungen und der Auffindesituation.

\section{Checkliste}

Diagnoseschema beim Tod in abnormer Körperposition Vorgeschichte:

- psychiatrische Erkrankung, Alkohol-, Medikamenten- oder Betäubungsmittelabhängigkeit?

- ausgeprägter Erregungszustand?

- körperliche Aktivität (Auseinandersetzung, Anstrengung, Erschöpfung)?

- Symptome einer Beeinträchtigung der Atemfunktion (Tachypnoe, Dyspnoe, gurgelnde oder stöhnende Atemgeräusche, Schnappatmung)?

\section{Auffindesituation:}

- Lassen sich aus der Auffindesituation plausibel eine mechanische Beeinträchtigung der Atmung oder eine Kreislaufdysregulation erklären?

- Sind bei der äußeren Leichenschau feststellbare Verletzungen mit der Auffindesituation vereinbar?

- Lässt sich aus der Auffindesituation ein Unfallgeschehen rekonstruieren oder ergeben sich Hinweise auf eine Beteiligung Dritter?

Pathomorphologische Untersuchungsbefunde:

- vorbestehende innere Erkrankungen oder Missbildungen, insbesondere solche des ZNS und des Herzens, die eine Beeinträchtigung der Atemfunktion, kardiale Arrhythmien oder orthostatische Regulationsstörungen begünstigen, ein Unfallgeschehen verursachen können, für sich genommen jedoch keine zwingende Todesursache darstellen,

- Verletzungen, die zu einem Unfallgeschehen beitragen können, in den rekonstruktiven Schlussfolgerungen aus der Auffindesituation eine adäquate Erklärung finden, jedoch keine todesursächliche Wertigkeit besitzen.

v 
Toxikologische Untersuchungsbefunde:

- Analysenergebnisse, die als Kausalfaktoren für einen Erregungszustand oder ein Unfallgeschehen in Betracht kommen, atemdepressive oder arrhythmogene Nebenwirkungen haben können, für sich genommen jedoch nicht zwingend den Tod erklären.

\subsection{Spezialfragen bei der Begutachtung nichtnatürlicher Todesfälle}

\section{E. Lignitz}

Im Zusammenhang mit der Untersuchung von Leichen ergeben sich regelmäßig besondere Fragestellungen, die je nach Ausgangslage und Ermittlungsergebnis bei der Abwägung der Bedeutung verschiedener pathomorphologischer Befunde für den Todeseintritt im Gutachten besprochen werden müssen. Teilweise sind spezielle Sektionstechniken erforderlich, sodass bereits vor Beginn der Sektion der Umfang der Fragen definiert beziehungsweise der Umfang der Untersuchungen eindeutig konzipiert sein muss.

\subsubsection{Unterscheidung vitaler und postmortaler Verletzungen}

Zur Feststellung vitaler Reaktionen wird auf das $>$ Kapitel 3.5 verwiesen. Zusammenfassend ist zu achten auf:

- Aspirationen (Einatmung von Fremdkörpern, Blut, Gewebe),

- Verschlucken (Fremdmaterialien: Ruß, Blut),

- Embolien (arteriell oder venös, Thromben, Gewebe, Luft) und

- Schockzeichen.

Die Unterscheidung von vitalen und postmortalen Verletzungen ist praktisch nur im Rahmen einer Obduktion oder durch histologische Untersuchungen möglich. Der Sonderfall der Wundaltersbestimmung wird ebenfalls in $>$ Kapitel 3.5 dargestellt.

\section{Postmortale Verletzungen}

\section{( Wichtig}

Nach Todeseintritt beigebrachte Verletzungen weisen im frühpostmortalen Intervall Befunde auf, die von vitaler Gewalteinwirkung kaum zu unterscheiden sind. Auf die Vermeidung derartiger Artefakte im Rahmen postmortaler Untersuchungen ist zu achten.

Vertrocknungen. Bei längerer Leichenliegezeit entstehen durch Flüssigkeitsverluste Hauteintrocknungen. Sie sind meist flächen- haft ausgebildet (z.B. am Skrotum). Hautvertrocknungen entstehen dort, wo Haut geschürft wurde. Vital entstandene Epithelverluste sind dabei mit Wundschorfen bedeckt. Umschriebene Hautschürfungen durch postmortale Einflüsse vertrocknen nach einiger Zeit ohne Schorfbildung. Bei der Präparation der tieferen Weichteilschichten sind keine weiteren Vitalitätshinweise wie z.B. Blutungen nachweisbar. Selbst vital gesetzte Würgemale oder Strangmarken sind gelegentlich einige Stunden nach dem Tode durch die Eintrocknung besser zu erkennen als unmittelbar nach dem Tode. Allein durch äußere Besichtigung ist jedoch insbesondere bei Strangmarken nicht zu entscheiden, ob sie vital oder postmortal entstanden sind. Wurden Oberhautabschürfungen durch Werkzeugeinwirkung im engen zeitlichen Zusammenhang mit dem Todeseintritt gesetzt, können deren Formmerkmale am Vertrocknungsbild identifiziert werden.

\subsubsection{Konkurrenz und Koinzidenz von Todesursachen}

\section{Konkurrenz von Todesursachen}

Treten zu einer vorbestehenden Erkrankung Verletzungen hinzu, so muss im Einzelfall entschieden werden, ob diesen eine (mit)todesursächliche Bedeutung zukommt oder ob die vorbestehende Erkrankung allein den Todeseintritt zur Folge hatte $(\diamond$ Kap. 2.1.3).

\section{(D) Wichtig}

Diese Beurteilung einer partialen- oder kumulativen Kausalität ist ohne Obduktion und eventuelle weitergehende Untersuchungen nicht möglich. Es handelt sich jeweils um Einzelfallentscheidungen unter Berücksichtigung aller Untersuchungsergebnisse einschließlich des Ermittlungsergebnisses.

Die unterschiedliche Gewichtung gleichartiger Teilbefunde sei am Beispiel hoher Blutalkoholkonzentrationen erläutert. Ist bei dem Opfer eines Gewaltdeliktes eine Blutalkoholkonzentration von 3\%o oder mehr nachweisbar, so ist diese bei gleichzeitigem Vorliegen schwerer Verletzungen (z.B. eines vital entstandenen Schädelhirntraumas) ein nichttodesursächlich relevanter $\mathrm{Ne}$ benbefund, während bei einer unverletzten Person die todesursächliche Relevanz anders zu beurteilen ist. Bei geringerem Verletzungsausmaß sollte eine abschließende Beurteilung nicht ohne histologische und chemisch-toxikologische Untersuchungen erfolgen.

\section{Koinzidenz von Todesursachen}

Gerade bei älteren Menschen ist das gleichzeitige Bestehen zweier oder mehrerer Krankheiten häufig. Die Entscheidung über die letztendlich dominierende todesursächliche Erkrankung ist hier zuweilen schwierig bis unmöglich. Gerade diese Kumulation pathologischer Organbefunde kann auch die kausale Beurteilung eventuell geringer Fremdeinwirkung erschweren. 


\section{(3) Fallbeispiel}

Eine 86-jährige Frau, die mit einer Bluthochdruckkrankheit, mehrfachen »Herzattacken« sowie transitorischen zerebralen Ischämien bereits verwirrt aufgefallen war, wurde tot auf dem Bett liegend aufgefunden. Das Gesicht ist mit einem Kissen bedeckt, ein Haarnetz leicht verschoben. Bei der gerichtlichen Leichenschau zeigen sich keinerlei gravierende Gewalteinwirkungen, jedoch wenige kleine Lid- und Bindehautblutungen. Bei der Sektion sind geringe oberflächliche Halsmuskelblutungen zu sehen. Trotz nachgewiesener zerebraler Insulte und hochgradiger Koronararteriensklerose mit kleineren Herzmuskelschwielen wurde angesichts der Auffindesituation der Verdacht des Erstickens unter weicher Bedeckung geäußert. Die Ermittlung der Polizei führt zu einem Jugendlichen aus dem Wohnhaus, der immer wieder Geld von der alten Frau borgte. Diesmal hatte er einen neuen Betrag vor Rückzahlung der alten Schulden verlangt. Die alte Frau lehnte das ab. Daraufhin drückte er die Frau auf ihr Bett und hielt bis zu ihrer körperlichen Erschlaffung das Kopfkissen fest auf ihr Gesicht. Als er keine Lebenszeichen mehr bemerkte, nahm er das Geld und verließ die Wohnung. Ohne sachkundige Leichenschau und vor allem ohne Sektion wäre der nichtnatürliche Tod unerkannt geblieben.

\section{Wichtig}

Wegen der schwierigen Unterscheidung von Koinzidenz und Konkurrenz von Erkrankung und Verletzung sollte der Leichenschauarzt im Zweifel bei der Leichenschau von einer ungeklärten Todesart ausgehen.

\subsubsection{Priorität von Verletzungen}

Mehrfachverletzungen. Liegen mehrere Verletzungen vor, kann die zeitliche Reihenfolge und die Wertigkeit in Bezug auf die Todesursache eine große Rolle spielen. Bei Beteiligung mehrerer Täter wird von diesen Feststellungen die rechtliche Bewertung im Strafverfahren abhängen.

\section{Dichtig}

Falls keine einzelne per se tödlich wirkende Verletzung vorliegt, sondern viele, von denen jede für sich nicht als unmittelbar tödlich angesehen wird, und andere konkurrierende Todesursachen ebenfalls nicht vorliegen, so ist man berechtigt anzunehmen, dass die Summe der Verletzungen den Tod herbeigeführt hat.

Das spielt insbesondere bei Tötungsdelikten durch Schlagen und Treten eine große Rolle. Keine Probleme bestehen, wenn Verletzungsfolgen einer Gewaltart zeitlich weit auseinander liegend zugefügt wurden (z.B. bei Kindesmisshandlung).

Verkehrsunfälle. Wurde ein Fußgänger, Rad- oder Motorradfahrer bei einem Verkehrsunfall getötet, sind verschiedene Fragestellungen zu beachten. Zur abschließenden Beurteilung ist in jedem Fall die Einbeziehung sämtlicher Ermittlungsergebnisse einschließlich eines technischen Gutachtens erforderlich. Hohe Priorität hat die Identifizierung der primären Kontaktstelle mit dem Unfallfahrzeug ( $>$ Kap. 3.3), da es rechtlich eine große Rolle spielen kann, ob eine stehende Person angefahren oder eine liegende Person überrollt wurde.

Schädelbrüche. Die Biomechanik der Schädelbrüche wird in Kapitel 3.3 ausführlich dargestellt. Die Bruchreihenfolge kann anhand der Puppe'schen Regel festgestellt werden ( $>$ dort).

Schussverletzungen. Je nach Schnelligkeit der Schussabgabe kann es schwierig bis unmöglich sein, eine Schussreihenfolge festzulegen. Bei primärer Verletzung größerer Gefäße können spätere Schüsse - auch bei relativ zeitnaher Verletzung - durch eine schwächer ausgeprägte Einblutung erkennbar sein.

Penetrierende Verletzungen. Das gilt auch für eine Vielzahl von Stichverletzungen. Allerdings hilft die histologische Untersuchung zur Wundaltersbestimmung dann weiter, wenn gleichartige Verletzungen mit einer Differenz von Stunden beigebracht worden sein sollten.

\section{Kombinationen unterschiedlicher Gewalteinwirkung}

Findet sich bei einer Strangulation ein deutliches Stauungssyndrom des Kopfes, so werden etwaige Platzwunden oder Schnitt- und Stichwunden am Kopf erst später gesetzt worden sein (z.B. durch so genannte Sicherheitsstiche). Sind sie zuerst entstanden und besteht somit am Kopf eine sog. Blutaustrittspforte, kann zwar tödlich stranguliert werden, sich jedoch keine intensive Stauung entwickeln, weil das Blut abfließen kann.

\subsubsection{Handlungsfähigkeit nach Verletzungen}

Bei einer Dislokation von Tat- und Fundort oder bei offensichtlicher Diskrepanz zwischen Aussagen von Täter und Opfer über den Ablauf einer tätlichen Auseinandersetzung taucht regelmäßig die Frage nach der Handlungsfähigkeit verletzter Personen auf. Der Gutachter wird die realistischen Handlungsmöglichkeiten eines Verletzen nach dem vorliegenden Verletzungsmuster und den ableitbaren Folgen einschätzen müssen.

\section{Definition}

Im Gegensatz zur juristischen Definition meint Handlungsfähigkeit hier die Fähigkeit zur bewussten, sinnvollen und zielgerichteten psychophysischen Reaktion eines Menschen nach einer Verletzung durch äußere Gewalt. Das ist vollkommen unabhängig vom Ausgang der erlittenen Schädigung. Der handlungsfähige Verletzte muss dabei nicht seine frühere geistige und körperliche Reaktionsfähigkeit voll entfalten können. Als sinn- und zielgerichtet gelten Hilferufe, Fluchtbewegungen, Ab- und Gegenwehr. Reflexartige Bewegungen zählen nicht dazu, auch wenn sie zu Positionsänderungen der verletzten Person geführt haben. 


\section{Beurteilungskriterien}

Wesentliches Kriterium ist die Verletzungsschwere. Personen, die an ihren Verletzungen noch am Ort des Geschehens versterben, werden in der Regel schnell handlungsunfähig gewesen sein.

Schädel-Hirn-Verletzungen. Bei stumpfer Traumatisierung des Kopfes, die zu einer Blutung im Schädelinnenraum führt, sind symptomfreie Intervalle bekannt, die unter Umständen einige Stunden betragen können. In dieser Zeit ist die Handlungsfähigkeit vielfach im vollen Umfang erhalten, ehe schwere Symptome (und damit Handlungsunfähigkeit) oder der Tod eintreten. Bei lokal begrenzten Hirnverletzungen durch Bolzenschussgeräte oder Stichwerkzeuge kann die Handlungsfähigkeit ggf. tagelang erhalten sein, ehe der Tod durch infektiöse Komplikationen eintritt ( $\triangleright$ Kap. 3.4). Selbst nach Hiebverletzungen und anderen Impressionstraumata des Gehirns sind Handlungen beschrieben worden. So muss bei einer Begutachtung geprüft werden, welche Hirnstrukturen verletzt wurden und welche Folgen sich daraus ableiten lassen bzw. ob die unverletzten Hirnstrukturen die mögliche Handlung zulassen. Verletzungen der motorischen Rinde erlauben posttraumatische Handlungen nicht.

Nervenverletzungen. Auch die Verletzung des Rückenmarks bedeutet nicht immer sofortige oder umfassende Handlungsunfähigkeit. Das neurologische Defizit entwickelt sich bei Wirbelsäulenfrakturen manchmal erst allmählich. Bewegungsunfähigkeit der Extremitäten tritt bei Abriss des entsprechenden Nervenplexus ein.

Extremitätenverletzungen. Auch die Lokalisation von Verletzungen an Gelenken und Gliedmaßen entscheidet z.B. über Bewegungs- und Ausweichfähigkeit sowie Fluchtmöglichkeit. Mit eingekeilten Hüftgelenkfrakturen ist Laufen noch möglich, pertrochantere Frakturen gestatten das nicht. Aktives Gehen ist bei Patellafrakturen und Quadrizepssehnenrissen unmöglich. Tibiakopffrakturen und verschiedene Fußwurzelknochenbrüche und -luxationen beheben jegliche statische Stabilität.

Halsverletzungen. Beim Erhängen wird Handlungsfähigkeit nicht in Betracht kommen. Es ist jedoch ein Fall berichtet worden, in dem nach Reißen der Schnur der Betroffene noch $200 \mathrm{~m}$ lief, leise sprechen konnte und erst nach 10-20 Minuten starb. Bei der Sektion fanden sich Intimaverletzungen beider Halsschlagadern und ein Abriss der Trachea.

Bei Stich- und Schnittverletzungen des Halses hängt der Ausgang und damit die zeitliche Begrenzung einer Handlungsfähigkeit von den Verletzungsfolgen ab. Bei Verletzungen großer Arterien ist kaum von einer wesentlichen Handlungsmöglichkeit zu sprechen. Andererseits sind bei einseitigen Arterienverletzungen immer wieder längere Überlebenszeiten und damit auch Handlungsmöglichkeiten berichtet worden, wenn infolge einer Kontraktion der Gefäßstümpfe die Blutung zunächst einmal sistiert.

Brustkorbverletzungen. Verletzungen der Aorta sind im Allgemeinen sofort oder zumindest sehr schnell tödlich. Kleinere Herzperforationen sind es dagegen nicht immer, besonders nicht, wenn das austretende Blut aus dem Herzbeutel in den Brustraum abfließen kann. Einseitige Schuss- und Stichverletzungen der Lun- ge bedeuten keineswegs sofortige Handlungsunfähigkeit. Nach vielfacher Beobachtung sind etwa $25 \%$ der Stichverletzten sofort handlungsunfähig, ca. $50 \%$ nach 5 Minuten und $25 \%$ brachen erst nach mehr als 5 Minuten handlungsunfähig zusammen.

Schussverletzungen. Auch von schussverletzten Personen sind erhebliche posttraumatische Leistungen berichtet worden. Anhand der anatomischen Lage des jeweiligen Schusskanals ist zu prüfen, ob eine Handlungsfähigkeit möglich war. Auch die Energieübertragung muss konkret nach Art von Waffe und Munition in Betracht gezogen werden. Schussverletzungen der Brücke und des verlängerten Markes bedeuten sofortigen Verlust von Bewusstsein und Handlungsfähigkeit. Eine Zerstörung des Halsmarkes bedeutet sofortige schlaffe Lähmung und würde eine Schussauslösung selbst dann verhindern, wenn der Finger schon am Abzug ist. Handlungsfähigkeit bei Verletzung anderweitiger lebenswichtiger Hirnzentren ist nicht anzunehmen. Bei Schussverletzungen von Gefäßen begrenzen die Schnelligkeit und das Ausmaß des Blutverlustes die Handlungsfähigkeit. Bei unkomplizierten Lungenschüssen bleibt die Handlungsfähigkeit lange erhalten.

Es gilt die Regel, dass die mechanische Wirksamkeit von Schussverletzungen mit der resultierenden totalen Wirksamkeit nicht identisch ist (s. unten: »Psychische Komponenten «). Auch die »umwerfende« Wirkung von Geschosstreffern geht mehr auf die Schmerzwirkung zurück als auf eine physikalisch definierte Aufhaltekraft. Von zwei Geschossen gilt aus Sicht der Polizei das als wirkungsvoller, das die größere Wundfläche und damit den größeren Schmerz auslöst. Von »militärischen « Geschossen ist unter dem Begriff des »Casualty Criterion « ein kinetischer Energiebetrag bekannt, der einen Soldaten außer Gefecht setzt. Sachkundige sehen die »Kugelfestigkeit« von Teilnehmern an kriegerischen Handlungen als eine Funktion der psychischen Verfassung an. Diese wird, falls keine lebenswichtigen Zentren sofort durch Schuss zerstört werden, die Handlungsfähigkeit des Getroffenen wesentlich bestimmen. Bei Polizeiaktionen, die eine Aufgabe des Gegners mittels Schusswaffengebrauch zum Ziel haben, ist zu beachten, dass der Gegner handlungsfähig und damit potentiell gefährlich bleibt.

\section{F Fallbeispiel}

In einer Silvesternacht kam es zwischen einem Mann und einer Frau, die sich erst Stunden zuvor in einer Kneipe kennen gelernt hatten, bei fortschreitender Alkoholisierung und offensichtlich im Zustand einer gewissen sexuellen Geneigtheit zu einer Tätlichkeit, bei der beide erhebliche Stichverletzungen erlitten. Während der Mann eine tiefe mediastinale Stichwunde tagelang überlebte und aussagefähig war, bis er an einer Mediastinitis starb, floh die Frau mit einer spritzenden arteriellen Blutung viele Meter aus der Wohnung. Der Fluchtweg war durch arkadenartige (blutdruckabhängige) Blutspritzspuren an den Wänden des Etagenflures gekennzeichnet. Die verletzte Frau klingelte schließlich an einer fremden Wohnung. Als geöffnet wurde, fiel sie vornüber, eine Hand an den blutenden Halswunden, äußerte gurgelnd Hilferufe und verstarb nach Eintreffen eines Notarztes. 


\section{Psychische Komponenten}

Die Willensstärke und die emotionale Situation des Verletzten beeinflusst die Handlungsfähigkeit bei nicht sofort tödlichen Verletzungen entscheidend mit. In der besonderen psychischen Anspannung (Ausnahmesituation) eines Kampfgeschehens wird eine Verletzung oft nicht einmal bemerkt, was relativ häufig bei perforierenden Verletzungen des Bauchraumes auftritt.

\section{Schlussfolgerung}

Handlungsfähigkeit nach Verletzungen der verschiedensten Art ist eine Tatsache und muss bei entsprechender Fragestellung oder bei objektiv gegebenen Fakten in Erwägung gezogen werden. Dogmatische Zeitbegrenzungen sind nicht angebracht. Es wird die sachkundige Beurteilung unter Beiziehung von bildgebenden diagnostischen Verfahren in der Klinik bzw. die neuropathologische Untersuchung in der Rechtsmedizin zur Beweismittelsicherung empfohlen, ggf. ergänzt durch die Konsultation von Neurologen und Neurochirurgen.

\subsubsection{Leichenbeseitigung und Leichenzerstückelung}

Definition
Leichenzerstückelungen sind typische postdeliktische Hand-
lungen zur Verschleierung einer Tat. Leichenbeseitigungen
dienen dem Verbergen des Opfers und Leichenverstümme-
lungen der Unkenntlichmachung eines Tatopfers.

Die Tathandlungen gehen meist Hand in Hand und verfolgen vorzugsweise die Absicht, ein Tötungsdelikt zu verbergen und eine Identifizierung zu erschweren. Gelegentlich sollen Individualmerkmale beseitigt werden.

\section{Epidemiologische Daten}

Leichenzerstückelungen sind seltene Ereignisse mit Häufungen in großstädtischen Ballungsgebieten. Gelegentlich gehören sie zur spezifischen Verbrechensausführung bei Serientätern. In der Bundesrepublik Deutschland ist mit ca. 10 Fällen im Jahr zu rechnen. Natürliche Leichenzersetzungen und akzidentelle Zerstückelungen werden ca. 10-mal häufiger beobachtet. Wegen des Ideenreichtums bei der Leichenbeseitigung ist ein gewisses Dunkelfeld anzunehmen. Kriminalistisch werden Tötungsdelikte mit Opferbeseitigung anfangs meist als Vermisstenmeldungen polizeibekannt. Täter sind überwiegend Männer. Wesentlich seltener treten Frauen als Täterinnen oder Helferinnen in Erscheinung. Bei den Opfern handelt es sich überwiegend um Frauen (etwa 2,5:1). Bei den aufgeklärten Fällen bestand überwiegend eine enge Täter-Opfer-Beziehung.

\section{Arten der Leichenzerstückelung}

\section{Definition}

Grundsätzlich werden natürliche, zufällige, nichtkriminelle und kriminelle Leichenzerstückelungen unterschieden.

Der natürliche Leichenzerfall ist ein Produkt von Fäulnis und Verwesung über Monate und Jahre. Er folgt anatomischen Gegebenheiten und ist somit leicht zu erkennen. Zufällige Leichenzerstückelungen ergeben sich durch massive mechanische Gewalteinwirkungen wie z.B. durch rotierende Schiffsschrauben. Zergliederungen menschlicher Körper sind häufig auch Folge von Eisenbahnüberfahrungen, Flugzeugabstürzen und Explosionen. Schließlich kann auch durch Tierfraß ein Leichnam erheblich entstellt und zerteilt werden.

\section{Wichtig}

Die Vitalität von sofort tödlichen, gewaltsamen Zergliederungen durch massive Gewalteinwirkungen der verschiedensten Art ist schwer festzustellen. Bei Eisenbahnüberfahrung ist stets die Frage »Verdeckungstat oder Suizid« zu prüfen.

Auch Leichenzerstückelungen und -beseitigungen ohne vorausgegangene Straftat sind bekannt. Sie haben unterschiedliche Motive, zumeist sind es ökonomische Zwänge (Einsparung der Transport- und Bestattungskosten) bzw. Ablenkmanöver von einem kriminellen Umfeld, z.B. der Rauschgiftszene, gelegentlich Nekrophilie und Aberglaube.

(D) Wichtig

Verwechslung von Präparaten des Anatomieunterrichtes und von operativ entfernten Organen, Organteilen und Gliedmaßen mit krimineller Leichenzerstückelung.

Gelegentlich kommen Zerstückelung und Leichenbeseitigung auch bei natürlichen Todesfällen oder zur Verschleierung eines anderweitig kriminellen Umfeldes (Rauschgiftszene!) oder zum Zwecke des Versicherungsbetruges vor.

Üblicherweise folgt die Einteilung nach morphologischen Gesichtspunkten in defensive und offensive Leichenzerstückelung (- Abb. $3.121+3.122)$. Neuerdings wird das Befundmuster kombiniert mit dem möglichen Motiv als Kriterium der Einteilung verwendet:

- Typ I (»defensive mutilation« auch »dismemberment«): Zur Erschwerung der Identifikation des Opfers, entspricht in der herkömmlichen Einteilung der defensiven Leichenzerstückelung.

- Typ II (»aggressive mutilation«): Entspricht der bisherigen offensiven Leichenzerstückelung, d.h. durch den Tötungsakt wird ein Exzess mit Zerstückelung häufig mit Verstümmelung des Gesichtes und der Genitalorgane ausgelöst und sofort angeschlossen.

- Typ III (»offensive mutilation «): Wird unterteilt: Die Tötungsmotivation ergibt sich entweder aus der Absicht zur anschließenden Ausführung sexueller Handlungen an toten Körpern 

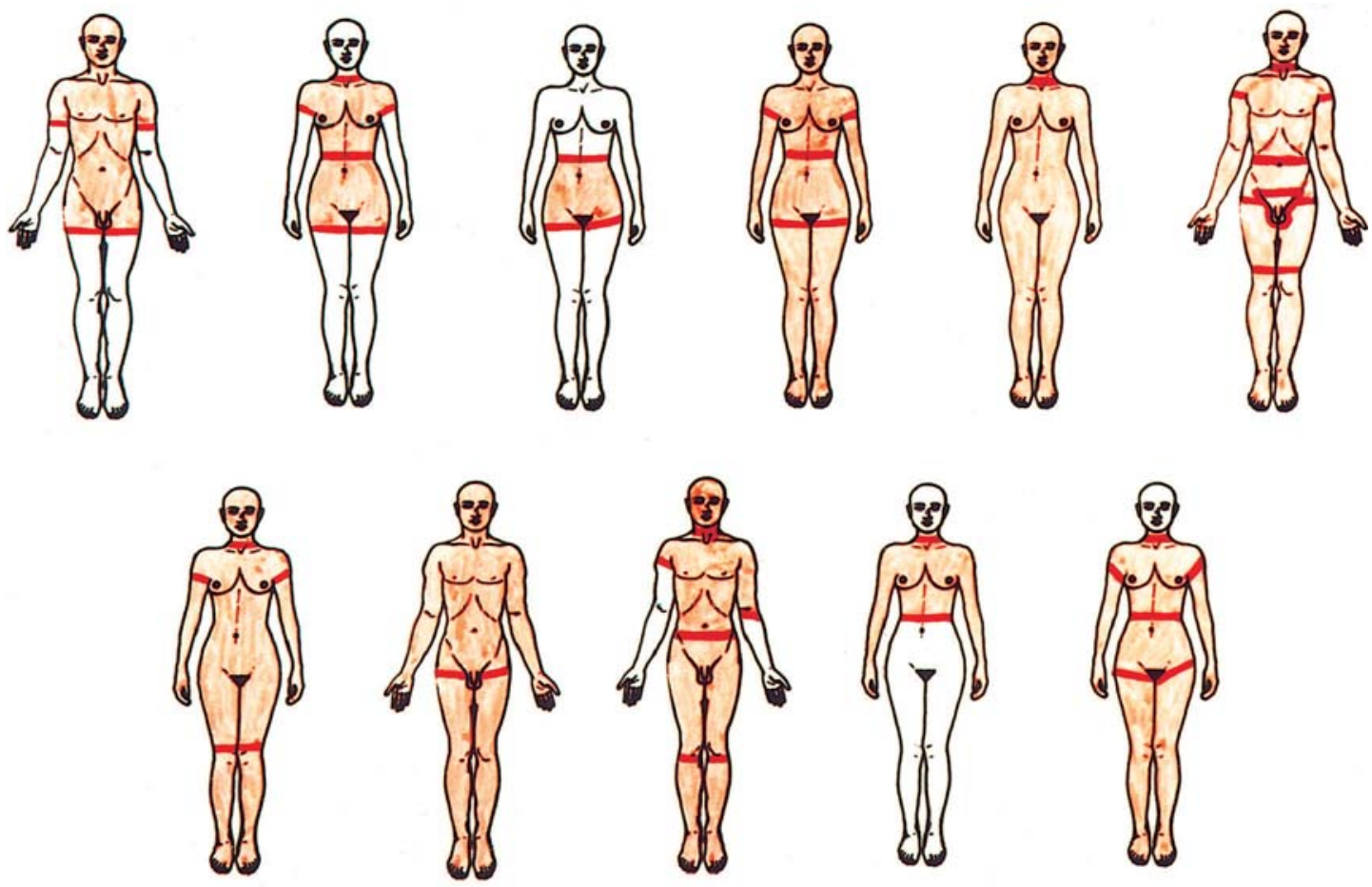

$\square$ bisher gefundene Leichenteile

bisher nicht gefundene Leichenteile

- Abb. 3.121. Befundmuster bei defensiver Leichenzerstückelung

mit vorausgehender oder nachfolgender Zerstückelung (Typ IIIa), oder infolge einer sexual-sadistischen Trieblage werden sexuelle Handlungen mit Zufügung von Schmerzen oder Verletzungen - letztendlich bis zur Tötung - ausgeführt und nach Todeseintritt fortgesetzt (Typ IIIb). Die Untergruppen des Typs III (Kannibalismus eingeschlossen) sind durch besondere Befundmuster gekennzeichnet.

- Typ IV (»necromanic mutilation «): An toten Körpern, hat den Zweck der Benutzung abgetrennter Körperteile als Fetisch, Symbol oder Trophäe.

\section{Befundmuster der defensiven Zerstückelung}

Bei der defensiven Zerstückelung dominiert eine überwiegend "methodische« Vorgehensweise, deren Umfang sich weitgehend nach den Möglichkeiten der Leichenbeseitigung richtet. Da mit der Absicht der Beseitigung von Leichenteilen meist auch eine Identifizierung des Opfers verhindert werden soll, ergänzen Folgen der Verstümmelung ebenfalls das Befundmuster. Zunächst imponiert die avitale Beschaffenheit aller Trennflächen und Exartikulationen. Meistens wird der Kopf der Leiche abgetrennt, in der Häufigkeit folgen die Abtrennung der Extremitäten, seltener eine Quer- oder Längsdurchtrennung des Rumpfes, was die Evis- zeration zur Folge hat. Überwiegend sind diese Körperzerteilungen einigermaßen systematisiert, indem die Abtrennung der Gliedmaßen in den Gelenken oder wenigstens deren Nähe erfolgt. Wenn es die Umstände erfordern, werden die Beine ein weiteres mal getrennt und auch die Oberarme von den Unterarmen separiert. Dazu kommt dann die Entfernung von Wiedererkennungsmalen. Immer wieder sieht man Schnittversuche, Zipfelbildungen von Haut und Weichteilen durch neu angesetzte Schnitte in paralleler Ausführung, Einkerbungen in Knochen und Knorpel, Anschnitte in Gelenkflächen. Dazu kommen Sägespuren oder splitternde Knochendurchtrennungen durch Hiebwerkzeuge.

\section{(D) Wichtig}

Rückschlüsse auf den Beruf des Täters aus der Art der Befunde ableiten zu wollen, stößt auf erhebliche Schwierigkeiten, ist meist nicht zuverlässig möglich und sollten nur mit größter Zurückhaltung gezogen werden. Die unbegründet sichere Annahme gewisser »Berufserfahrungen« des Täters kann die Ermittlungstätigkeit erheblich in die Irre führen. 

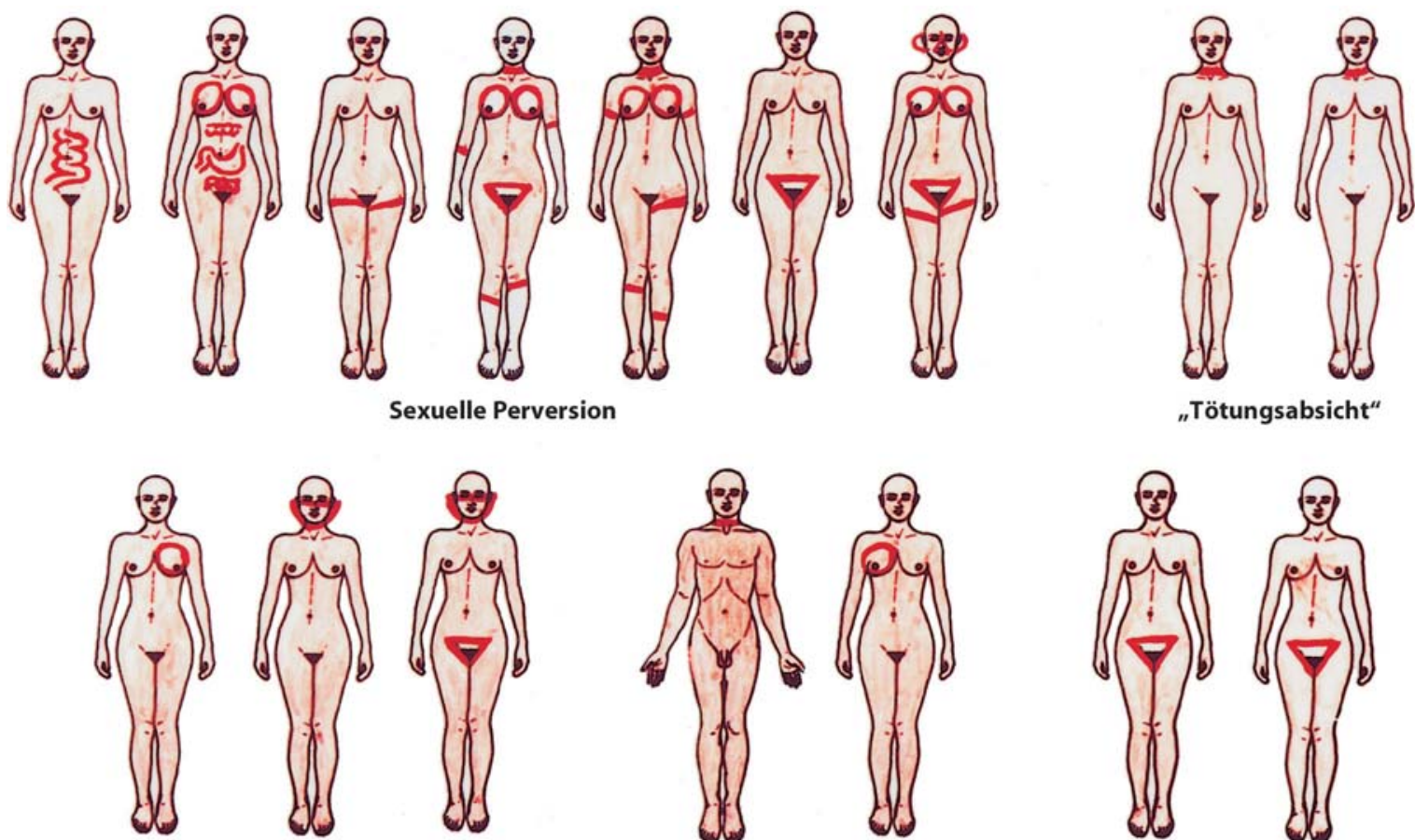

Psychose / Wahn

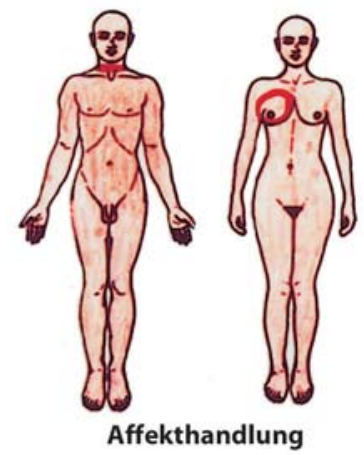

Affekthandlung
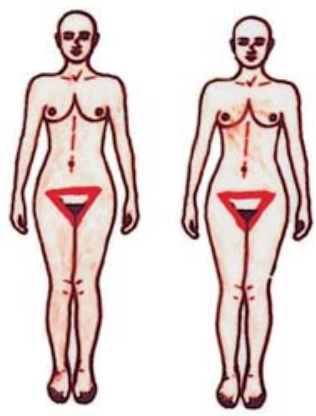

Nekrophilie bisher gefundene Leichenteile

bisher nicht gefundene Leichenteile

- Abb. 3.122. Befundmuster bei offensiver Leichenzerstückelung

\section{Befundmuster der offensiven Zerstückelung}

Im Gegensatz dazu ist bei den offensiven Zerstückelungen die unregelmäßige und völlig sinn- und zwecklose Verstümmelung der Leiche, die Verstreuung der Leichenteile in der Nähe des Tatortes ohne die Tendenz, sie völlig zu verbergen, und schließlich sogar die Mitnahme von Leichenteilen auffällig. Wenn sich die offensive Leichenzerstückelung aus einer sexualsadistischen Motivation oder sexuellen Perversionshandlung bzw. nur im Anschluss an einen Geschlechtsakt mit abrupter Änderung der Stimmungslage entwickelt, wird eine Abtrennung der Geschlechtsteile oder deren Verstümmelung mehr oder weniger das Befundmuster bestimmen.

\section{Todesursache}

Die Feststellung der Todesursache kann problematisch sein, wenn es sich um einen unvollständigen Leichenfund handelt (insbesondere bei fehlendem Kopf) oder wenn bei Strangulationen die Trennstelle des Kopfes vom Hals in der Ebene der Strangulation liegt.

\section{Instrumentarium}

Entsprechend der "phantasievollen « Vorgehensweise der Täter sind der Verwendung von Werkzeugen bei der Zerstückelung praktisch keine Grenzen gesetzt. Im Allgemeinen werden Messer, Äxte und Sägen unterschiedlicher Größe verwendet. Exotisch anmutende Gerätschaften wie Schredder, Papierschneidemaschine, Trennschleifer, Bajonett, Fleischhackmaschine und Vorschlaghammer wurden fallweise bekannt.

\section{Methoden der Beseitigung}

Leichenteile werden in Wohnungen, Möbelstücken, Hausböden und Kellern abgelegt, versteckt oder eingemauert, im Hausmüll entsorgt, in Reisetaschen und Müllsäcken transportiert, in Gewässern versenkt, in Flüsse geworfen, vergraben, einbetoniert, verstreut, in Tiefkühlschränken konserviert, verbrannt, mit Säuren vernichtet oder auch Tieren zum Fraß vorgeworfen. Alle Methoden sind mit dem Risiko der späteren Entdeckung durch Wiederauffinden, Auftauchen oder des Verbleibs von identifizierbaren Resten belastet. Insgesamt hat sich für das Ablegen von Leichenteilen der Begriff des Dumping eingebürgert. Für das Verbringen von Leichenteilen ins Ausland wurde der Begriff der »Transitleiche« geprägt. 


\section{F) Fallbeispiele}

Neben der befestigten Fahrbahn eines Parkplatzes der Autobahn Berliner Ring wurden Plastiksäcke und ein Karton mit Leichenteilen gefunden. Tat- und Fundort lagen, wie sich herausstellen sollte, in zwei verschiedenen politischen Hoheitsgebieten. Der Karton enthielt einen männlichen Rumpf, die verschiedenen Säcke je ein Bein, beide Arme und den Kopf. Die in 6 Teilen gefundene Leiche war vollständig. Körpergröße und Körpergewicht ließen sich zuverlässig rekonstruieren. Nach Erscheinungsbild, Haarfarbe und Gesichtszügen handelte es sich um einen Ausländer, dessen Herkunft auf der Achse Türkei, Iran, Afghanistan vermutet wurde. Die Arme waren im Schultergelenk und ein Bein im Hüftgelenk ohne Beschädigung der Gelenkflächen ausgelöst worden, am anderen Bein war der Hüftkopf grob und in Stufen durch ein Hiebwerkzeug abgetrennt. Alle Trennflächen korrespondierten gut miteinander und imponierten avital. Neben 3 Platzwunden der linken Stirnregion fanden sich als Todesursache 2 Schädelimpressionsfrakturen mit tiefer vitaler Hirnwunde. Es wurde ein hammerartiges Werkzeug vermutet und später als stumpfe Rückseite eines sog. Tomahawks ermittelt. Zahlreiche individuelle Besonderheiten wie dunkle Zahnbeläge, eine chronische Segmentpneumonie, unterschiedliche Fußlängen, Blutgruppenprägung, Zahnstatus und Lebensaltersschätzung (zwischen 30 und 40 Jahren) führten nicht zur Identifizierung, sondern ein Fingerabdruck. Danach handelte es sich um einen 33-jährigen afghanischen Staatsbürger, für den es keinen Vermisstenvorgang gab. In diesem Fall hatten die gemeinschaftlich handelnden Täter in einer sich krisenhaft zuspitzenden persönlichen Situation ein Tötungsdelikt begangen und zur Verdeckung die ehemaligen Grenzverhältnisse in Deutschland ausgenutzt (Transitleiche).

Nach vollzogenem Akt signalisierte eine junge Frau alsbald den Wunsch nach Fortsetzung des Beischlafs, wozu der Partner sogleich nicht in der Lage war. Daraufhin versuchte die Frau ihrem Partner aus Enttäuschung den Penis mit einem Messer abzusetzen. In einem Kampfgetümmel konnte der Mann das Messer an sich bringen; er tötete die Frau mit einem tiefen Halsschnitt und versuchte sofort im Anschluss ihr die Brüste abzusetzen und den Leib zu öffnen. Im Gegensatz zum obigen Fall bestand das sinnund regellose Befundmuster einer offensiven Zerstückelung, aus einem Tatgeschehen heraus entwickelten Bild. Bei der gerichtsärztlichen Untersuchung des Täters fand sich tatsächlich eine einzelne oberflächliche Schnittverletzung am Glied. Es folgte eine Verurteilung wegen Totschlags im Affekt.

\section{(i) Infobox}

Bei der Auffindung von Leichen und Leichenteilen, Skeletten und Skelettteilen ist die Polizei in jedem Falle zur Feststellung von Identität und Todesursache zu verständigen!

\subsubsection{Tötungsdelikte durch Tritte}

\section{Henn}

\section{F) Fallbeispiele}

Ein 37 Jahre alter Mann wurde morgens tot neben seinem Fahrrad liegend vor einem Stall aufgefunden, nachdem er am Abend zuvor in einer Gaststätte reichlich Alkohol genossen hatte. Zunächst deutete die Situation auf einen tödlichen Fahrradsturz hin. Die Leichenschau vor Ort durch einen Rechtsmediziner stellte die Weichen jedoch in eine andere Richtung, sodass eine gerichtliche Obduktion angeordnet wurde. Die Sektion ergab als Hauptbefund ein massives Bauchtrauma mit Hämaskos von 1400 ml infolge Mesenterialgefäßruptur. Das Nebeneinander von Rippenbrüchen, Nasenbeinbruch, Platzwunden am Hinterkopf sowie mehreren Hämatomen war mit einem Fahrradsturz nicht zwanglos zu erklären und wies auf eine mehrfache massive stumpfe Gewalteinwirkung (wahrscheinlich Tritte) hin. Die Blutalkoholkonzentration betrug 2,62\%o, die Urinalkoholkonzentration 4,08\%o. Nach 1 1 $\frac{2}{2}$ Jahre währender Ermittlungsarbeit wurden die Täter, 3 Jugendliche, gestellt, die einräumten, den Mann aus Langeweile und Lust auf Streit und weil er sich nie wehrte geschlagen und getreten zu haben.

Körpermisshandlung und Töten durch Treten kommt regional unterschiedlich häufig vor, wobei sozialen Faktoren eine erhebliche Rolle zukommt, wie die Auswertung der Protokolle von 446 Fällen in verschiedenen Regionen Deutschlands gezeigt hat.

Meist führen Streitigkeiten alkoholisierter Personen, die in ein Handgemenge übergegangen sind, final zu Tritten gegen einen bei der Auseinandersetzung zu Fall gekommenen. Blutalkoholkonzentrationen der Opfer von 3,00\%o und mehr sind dabei keine Seltenheit. (Bei den in Hamburg, Greifswald und Halle untersuchten Fällen lag die Durchschnitts-BAK bei 2,16\%o, die höchste BAK bei 4,5\%o!)

Daneben sind aber auch »Lust auf Streit « und die Ansicht, dass »Obdachlose und Landstreicher nicht in die Gesellschaft passen « und »Obdachlose dem Steuerzahler auf der Tasche liegen«, Gründe für eine tödlich endende Gewaltanwendung. Die Wahl des Opfers ist häufig ein »Zufallsprodukt «. Seltener sind die Fälle, bei denen gezielt ein Opfer aufgesucht und - manchmal über mehrere Tage hinweg - durch Schläge und Tritte misshandelt wird.

Die meist alkoholisierten Täter nehmen für sich in Anspruch, aufgrund der alkoholbedingten kognitiven Beeinträchtigung nicht mehr gewusst zu haben, was sie tun - also erheblich in ihrer Steuerungsfähigkeit eingeschränkt gewesen zu sein ( $>$ Kap. 6.4.1) - und wahllos auf das Opfer eingetreten zu haben. Diese Einlassung ist dann widerlegt, wenn sich die Verletzungen - wie in vielen Fällen - fast ausschließlich am Kopf befinden, da allein die Kopf-Körper-Relation »bei wahllosem Zutreten« ein anderes Verteilungsmuster der Verletzungen erwarten lässt. 


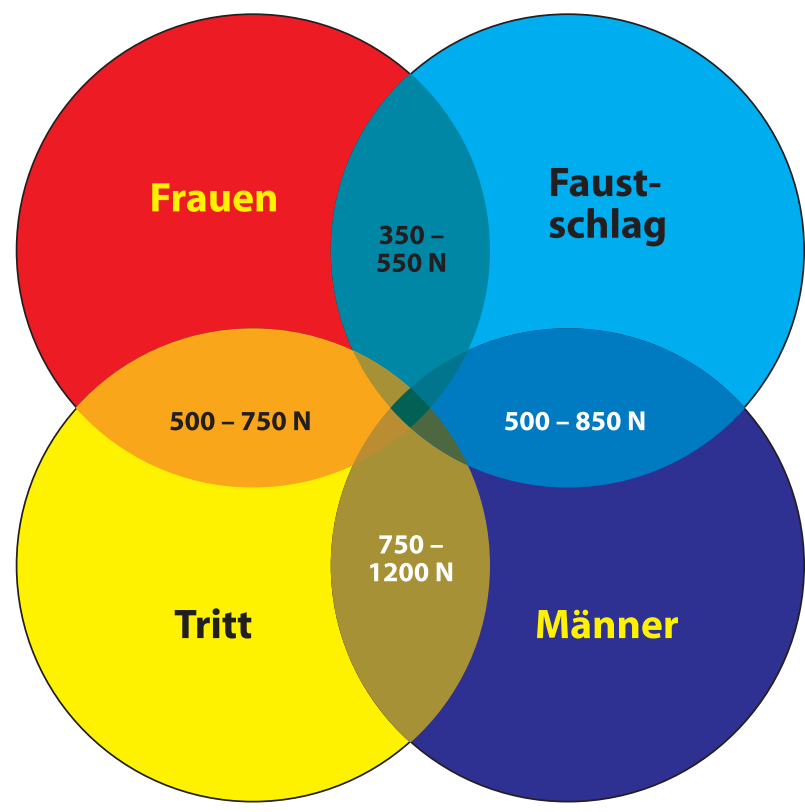

- Abb. 3.123. Tritt-Kräfte-Diagramm

Das Bagatellisieren der erwarteten Verletzungsschwere mit der Begründung, dass lediglich mit Turnschuhen getreten worden sei und nicht mit Stahlkappenschuhen (sog. Springerstiefel), ist aufgrund der Kraftentfaltung, die durch das Treten und nicht durch den Schuh bedingt wird, widerlegt. In Experimenten wurden durch Faustschläge Werte von 350-850 N, durch Tritte von 500-1200 N durch Männer und Frauen erreicht. Diese Ergebnisse wurden von den Autoren in einem Satz zusammengefasst: »Die kleinsten Messwerte für Tritt und die größten für den Faustschlag überschneiden sich bei beiden Geschlechtern ..." (• Abb. 3.123).

Die Ergebnisse der Auswertung von »Tritt-Todesfällen«, bei denen Täter festgenommen wurden, machten deutlich, dass häufig mit Turnschuhen getreten wurde. In einem Fall waren sogar Tritte mit »nackten« Füßen tödlich.

\section{Infobox}

Juristisch können sowohl Springerstiefel als auch Turnschuhe als gefährliches Werkzeug gelten. Die Bewertung ist dabei abhängig von der Begehungsart des Deliktes. Bei einem Tritt mit dem Springerstiefel auf den Zeh eines Geschädigten ist nicht vom Einsatz eines gefährlichen Werkzeuges auszugehen, wohingegen der Tritt mit dem Turnschuh gegen den Kopf eines Opfers diesen Tatbestand erfüllt ( $\triangleright$ Kap. 5.5.1).

\section{Verletzungslokalisationen und äußere Befundmuster}

Die äußeren Verletzungsmuster sind je nach betroffener Körperregion und abhängig von der Richtung der einwirkenden Gewalt sehr unterschiedlich ausgeprägt.

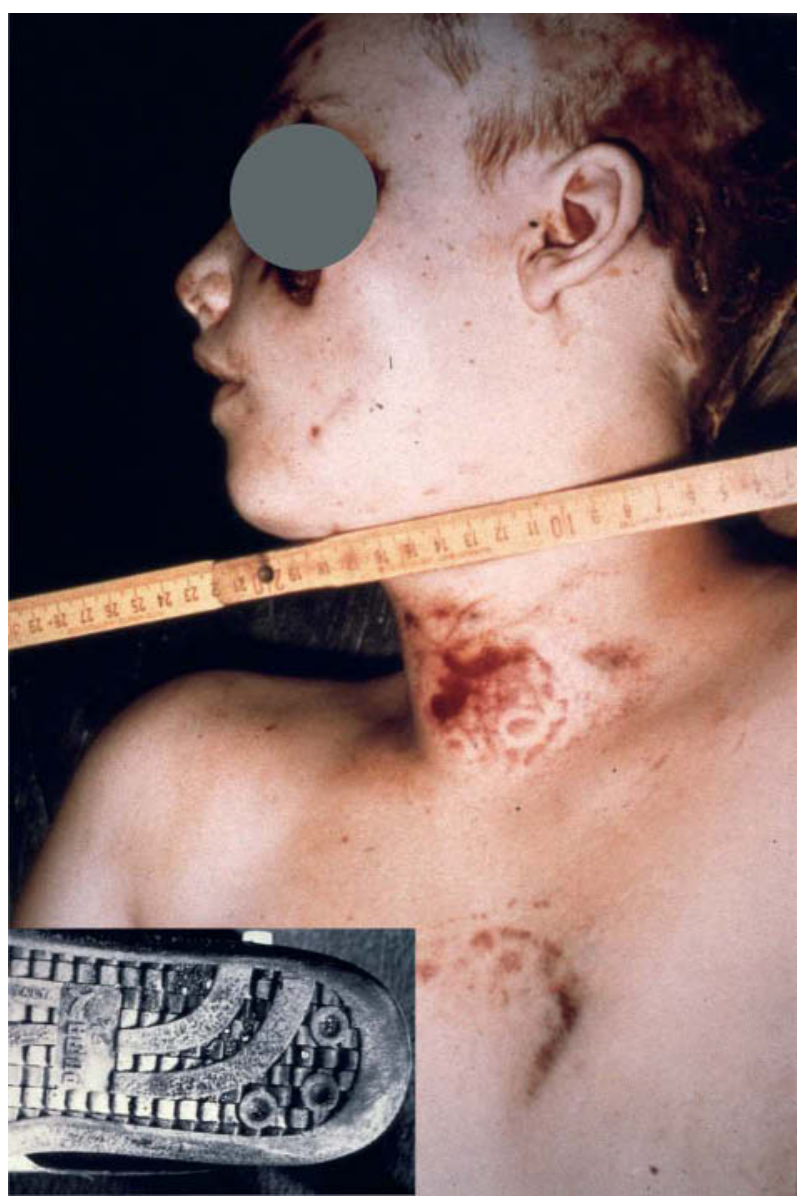

- Abb. 3.124. Sohlenabdruck am Hals und am Thorax

Bei orthograder stumpfer Gewalteinwirkung auf ungepolsterte Körperregionen (z.B. Stirn) wird das Tatwerkzeug durch Hautblutungen oft als »Negativabdruck « 1:1 abgebildet. Hierbei kommt der vom Schlag mit einem Stock oder einem stockähnlichen Gegenstand bekannte Mechanismus, der zu einer doppelt konturierten Verletzung führt, zum Tragen ( $\checkmark$ Kap. 5.2.1).

Bei Bedeckung einer Körperregion durch dünnschichtige Bekleidung kann innerhalb des Tatwerkzeugabdruckes das Muster der getragenen Textile erkennbar sein. Bei größerer Schichtdicke oder Wattierung der Kleidung tritt ein sog. Knautschzoneneffekt auf, der an der Haut oftmals nur undeutliche oder keine Spuren der äußeren Gewalteinwirkung erkennen lässt.

Ein »endogener « Knautschzoneneffekt tritt bei Polsterung durch Unterhautfettgewebe auf, sodass an durch Kleidung bedeckten und an fettreichen Körperpartien die am wenigsten richtungsweisenden Befunde zu erwarten sind (• Abb. 3.124).

Die inneren Verletzungen werden durch verschiedene Mechanismen bedingt. Zum einen ist es die direkte Gewalteinwirkung, die z.B. zu Unterhautfettgewebsblutungen, Knochenbrü- 


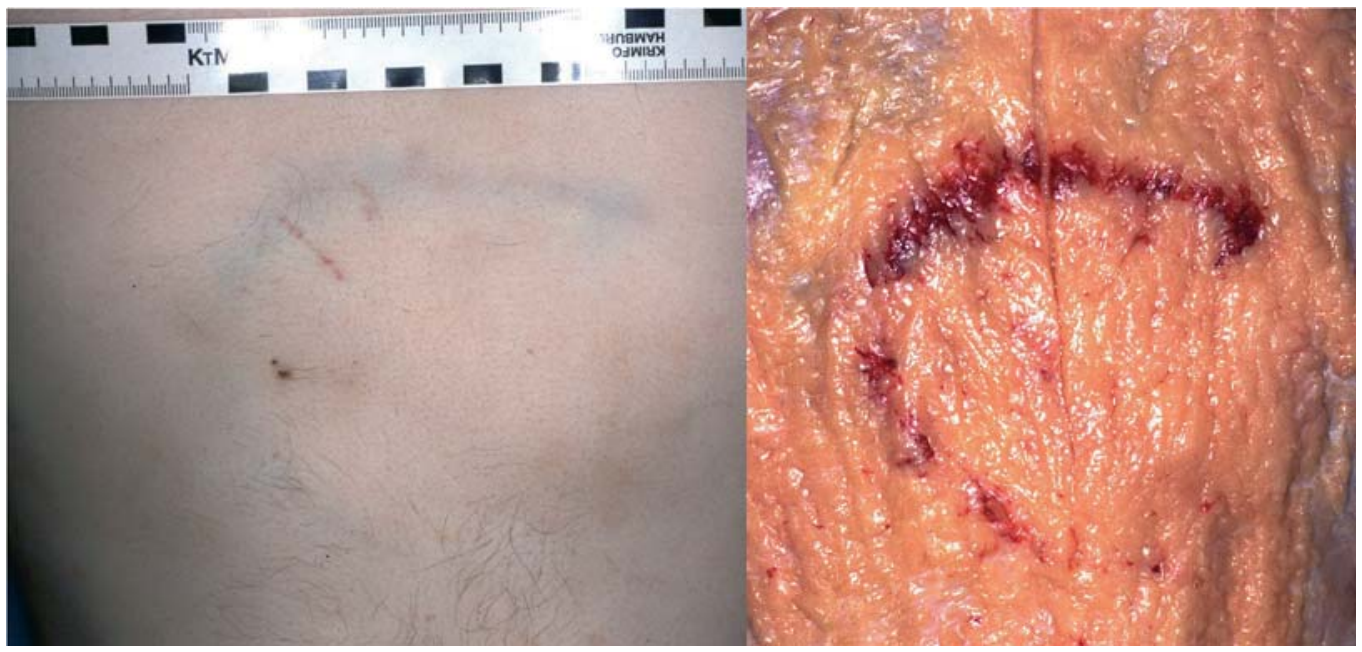

- Abb.3.125. Bogenförmiges Hämatom im Unterhautfettgewebe des Bauches nach Tritt mit einem Halbschuh

chen und Organzerreißungen führen kann. Zum anderen ist es die indirekte Gewalteinwirkung in Form eines Beschleunigungstraumas, die vor allem bei Kopf-/Gehirnverletzungen eine große Rolle spielt.

Tritte gegen den Kopf führen oft zu Knochenbrüchen, wobei besonders häufig Mittelgesichtsfrakturen (25\% der Fälle) zu nennen sind. Daneben geben Frakturen der Schädelbasis (12\%) und des Schädeldaches (10\%) deutliche Hinweise auf die Krafteinwirkung.

Die Beschleunigung, die der Kopf einer am Boden liegenden Person bei Tritten gegen den Kopf erfährt, beträgt im Mittel $56 \mathrm{~g}$ und übersteigt die Gehirnbeschleunigung, die ein Fußgänger beim Kopfaufprall auf eine Motorhaube eines PKW bei einer Kollisionsgeschwindigkeit von $36 \mathrm{~km} / \mathrm{h}$ erfährt. Die durch Tritte gegen den Kopf erreichten Maximalwerte von 103 g entsprechen etwa der Kopfbeschleunigung eines angegurteten Fahrers bei einem Frontalcrash mit $50 \mathrm{~km} / \mathrm{h}$. Die am Hals wirkenden Drehmomente liegen bei etwa 16,3-40 Nm (maximal $125 \mathrm{Nm}$ ). Die Kopfbeschleunigung und die gleichzeitige Rotation des Kopfes bedingen zum einen Coup- und Contrecoup-Verletzungen des Gehirns sowie Rotationstraumata.

Intrathorakale Organverletzungen füllen das Spektrum von beispielsweise geringfügigen Lungenkontusionen bis zu Herzrupturen aus.

Die Breite der intraabdominellen Verletzungen reicht von Einrissen der relativ ungeschützten Leberkapsel über zentrale Leberzertrümmerungen bis hin zu Kontusionen und Rupturen der relativ geschützt liegenden Nieren.

Vorbestehende Erkrankungen können durch Folgeschäden den Todeseintritt maßgeblich begünstigen wie z.B. ein Verbluten aus Riss-Quetsch-Wunden bei vorbestehenden Blutgerinnungsstörungen infolge alkoholtoxischer Leberzirrhose.

Auch bei höheren Blutalkoholkonzentrationen (insbesondere bei Werten über 3,50\%o) ist zu überlegen, ob eine Alkoholintoxi- kation maßgeblich zum Todeseintritt beigetragen hat oder sogar konkurrierend als Todesursache in Betracht kommt. Eine Alkoholintoxikation als Todesursache ist aber immer dann zu negieren, wenn allein die Verletzungen den Todeseintritt - unabhängig von einer Alkoholisierung des Opfers - erklären können.

\section{Befunddokumentation}

Orthograde Treffer auf ungepolsterte Körperregionen werden am einfachsten dokumentiert, indem man das Befundmuster auf Folie »durchzeichnet«. Hierdurch erhält man ein 1:1-Abbild des Befundes. Weiterhin sollte eine fotographische Dokumentation ohne und mit Maßstab erfolgen.

Bei gepolsterten Körperregionen erübrigt sich ein »Durchzeichnen « meist aufgrund fehlender oder nur schemenhafter Befunde. Eine Fotodokumentation sollte dennoch immer erfolgen.

\section{(8) Wichtig}

Bei Untersuchung Lebender sollten selbst bei äußerlich unauf-

fälligem Bild an die Möglichkeit von inneren Organverletzungen gedacht werden!

Bei Untersuchung Lebender kann eine Ultraschalluntersuchung der betroffenen Körperregionen hilfreich sein, um äußerlich nicht erkennbare Hämatome nachzuweisen. Die Befunde sind allerdings mit Zurückhaltung zu interpretieren, da das Alter der Hämatome mittels dieser Methode für enge Zeiträume nicht konkretisiert werden kann. Auch Verletzungen innerer Organe sollten bei äußerlich unauffälligem Bild auf jeden Fall in Betracht gezogen und per Sonographie abgeklärt werden.

Bei Sektion der Verstorbenen sind Haut- und Unterhautfettgewebe schichtweise zu präparieren, um möglicherweise nur diskret ausgebildete Fettgewebsblutungen, die aber durchaus Hinweis auf ein Tatwerkzeug geben können, zu erkennen (- Abb. 3.125). 
Oft lassen erst die akribisch dokumentierten einzelnen Verletzungen sowie ihr Gesamtbild einen Unfall wie im eingangs genannten Fallbeispiel als Todesursache ausschließen.

\section{Checkliste}

Es sind folgende Maßnahmen zu ergreifen bzw. Fragen zu prüfen:

- Fotografische Dokumentation des Fundortes!

- Tierisches oder menschliches Gewebe?

- Falls menschlich: männlich oder weiblich? Eine oder mehrere Leichen?

- Art der Zerstückelung (defensiv, offensiv, nichtkriminell)?

- Todesursache feststellbar? Hinweise auf Leichenliegezeit (Insekten)?

- Ergeben sich Hinweise auf einen oder mehrere Täter bzw. die Mitwirkung von Helfern?

- Sind Fund- und Tatort identisch?

- Gibt es Hinweise auf vorübergehende Tiefkühllagerung des Fundstückes?

- Vorsorgliche Präparation und Fotodokumentation der knöchernen Trennstellen!

- Fotografische Dokumentation der Weichteiltrennstellen!

- Feststellung und Dokumentation von Werkzeugspuren bei Tötung und Zerstückelung!

- Histologische Untersuchung der Weichteilverletzungen zum Nachweis vitaler Reaktionen!

- Toxikologische Untersuchung der Leichenfundstücke!

- Erfolgte bei unvollständigen Leichenfunden ein Abgleich mit Vermisstenvorgängen in anderen geographischen Regionen des Landes oder im Ausland?

- Asservierung, Untersuchung und Einstellung von DNAMerkmalen in die zentrale Datenbank beim Bundeskriminalamt (auch zur schnelleren Zusammenführung von Leichenteilfunden bei sukzessiver Ablage oder sequentieller Auffindung)!

\section{Literatur}

\section{zu Kap. 3.1}

Baumbach A, Lauterbach W, Albers J, Hartmann P (2005) Zivilprozessordnung mit Gerichtsverfassungsgesetz und anderen Nebengesetzen. 63. neubearb. Aufl. Beck, München

Eisenberg U (2006) Jugendgerichtsgesetz. 11. neubearb. Aufl. Beck, München

Fischer T (2006) Strafgesetzbuch und Nebengesetze. 53. neubearb. Aufl. Beck, München

Meyer-Goßner L (2005) Strafprozessordnung, Gerichtsverfassungsgesetz, Nebengesetze und ergänzende Bestimmungen. 48. neubearb. Aufl. Beck, München

\section{zu Kap. 3.2}

Eisenberg U (2005) Kriminologie. 6. neubearb. Aufl. Heymanns, Köln Berlin Bonn München

Hoffmann J, Musolff C (2000) Fallanalyse und Täterprofil. BKA Forschungsreihe Bd. 52. Bundeskriminalamt, Wiesbaden

Hoffmann J, Musolff C (2002) Täterprofile bei Gewaltverbrechen. Springer, Berlin Heidelberg New York

Kaiser G, Kerner HJ, Sack F, Schellhoss H (Hrsg) (1993) Kleines kriminologisches Wörterbuch. UTB 1.274, 3. neubearb. Aufl. Müller, Heidelberg

Rössner D, Jehle JM (Hrsg) (1999) Kriminalität, Prävention und Kontrolle. Neue Kriminologische Schriftenreihe 104. Kriminalistik Verlag, Heidelberg

Schneider HJ (1994) Kriminologie der Gewalt. Hirzel, Stuttgart Leipzig

Schwind HD (2005) Kriminologie. Eine praxisorientierte Einführung mit Beispielen. 15. neubearb. Aufl. Kriminalistik Verlag, Heidelberg

\section{zu Kap. 3.4}

Baron DN (1993) The chemical pathology of trauma. In: Mason JK (ed) The pathology of trauma. Arnold, London Boston Melbourne, pp 315323

Barton RN, Frayn KN, Little RA (1990) Trauma, burns and surgery. In: Cohen RD, Lewis B, Alberti KGMM, Denman AM (eds) The metabolic and molecular basis of acquired disease. Baillière Tindall, London

Border JR, Allgöwer M, Hansen ST Jr, Rüedi TP (Hrsg) (1990) Blunt multiple trauma: comprehensive pathophysiology and care. Marcel Dekker, New York

Clows GHA Jr (1988) Trauma, sepsis and shock: the physiological basis of therapy. Marcel Dekker, New York

Douglas AS (1993) Haemorrhage, haemostasis and thrombosis. In: Mason JK (ed) The pathology of trauma. Arnold, London Boston Melbourne, pp 324-339

Hechtman HB, Tedeschi LG (1977) Systemic response to trauma. In: Tedeschi CG, Eckert WG, Tedeschi LG (eds) Forensic medicine. A study in trauma and environmental hazards, Vol 1. WB Saunders Comp, Philadelphia London Toronto, pp 386-405

Madea B (1992) Hämorrhagische Gastroenterocolopathie als Folge einer Halsstichverletzung. Rechtsmedizin 2: 74-77

Madea B (2006) Die ärztliche Leichenschau. Rechtsgrundlagen - Praktische Durchführung - Problemlösungen. 2. Aufl. Springer, Berlin Heidelberg New York Tokio

Madea B, Mußhoff F (2006) Feststellung der Todesursache, insbesondere bei Intoxikationen: In: Pragst F, Aderjan R (Hrsg) GTFCh-Symposium 2005. Praxis der Forensischen Toxikologie, S. 423-446. Verlag Dr. Dieter Helm, Heppenheim

Madea B, Schmidt P (1995) Mitwirkung vorbestehender Erkrankungen bei Unfalltod. Z ges. Versicherungswiss. 33: 491-527

Sandritter W, Beneke G (1981) Allgemeine Pathologie. Schattauer, Stuttgart New York

Tedeschi LG, Hechtmann HB (1977) Posttraumatic embolism. In: EckertWG, Tedeschi LG (eds) Forensic medicine. A study in trauma and environmental hazards, Vol 1. WB Saunders Comp, Philadelphia London Totonto, pp 406-422

Zollinger HU (1982) Unfallursachen und Unfalltod im Alter. Zeitschr. Unfallmed. Berufskrankh. 75: 207-216 


\section{zu Kap. 3.5}

Betz P (1996) Collagen subtypes - markers for the healing of skin wounds. In: Oehmichen M, Kirchner H (Hrsg) The wound healing process: Forensic pathological aspects. Schmidt-Römhild, Lübeck, S. 247-256

Betz P (1996) Neue Methoden zur histologischen Altersbestimmung menschlicher Hautwunden. Schmidt-Römhild, Lübeck

Grellner W (2004) Zytokine und Adhäsionsmoleküle in der rechtsmedizinischen Vitalitäts- und Wundaltersbestimmung. Shaker, Aachen

Hausmann R (2002) Die Altersbestimmung von Hirnkontusionen bei gedecktem Schädel-Hirn-Trauma des Menschen. Schmidt-Römhild, Lübeck

Hunt TK, Hopf HW, Mueller RV (1997) Wound healing. In: Cooper GJ, Dudley HAF, Gann DS, Lilltle RA, Maynard RL (eds) Scientific foundations of trauma. Butterworth-Heinemann, London Boston Singapore Sydney Toronto Wellington, pp 530-550

Madea B, Grellner W (1996) Vitality and supravitality in forensic medicine. In: Oehmichen M, Kirchner H (Hrsg) The wound healing process: Forensic patholgical aspects. Schmidt-Römhild, Lübeck, S. 259-282

Madea B, Grellner W (2002) Vitale Reaktionen (Teil 1). Rechtsmedizin 12:378-394

Madea B, Grellner W (2003) Vitale Reaktionen (Teil 2). Rechtsmedizin 13:32-48

Oberholzer MJ (2001) Pathologie verstehen. Molekulare Grundlagen der allgemeinen Pathologie. Thieme, Stuttgart

Oehmichen M (1990) Die Wundheilung. Springer, Berlin Heidelberg New York Tokio

Oehmichen M, Kirchner H (Hrsg) (1996) The wound healing process: Forensic pathological aspects. Research in Legal Medicine, Vol 13. SchmidtRömhild, Lübeck

Oehmichen M, Raff G (1980) Timing of cortical contusions. Z Rechtsmed 84: 79-94

Thomas C (1992) Histopathologie. Lehrbuch und Atlas für die Kurse der allgemeinen und speziellen Pathologie. Schattauer, Stuttgart New York

Tutsch-Bauer E, Baur C, Tröger HD, Liebhardt E (1981) Untersuchungen zur Altersbestimmung an künstlich gesetzten Hämatomen. Beitr Gerichtl Med 39: 83-86

Wilmore DW, Stoner HB (1997) The wound organ. In: Cooper GJ, Dudley HAF, Gann DS, Lilltle RA, Maynard RL (eds) Scientific foundations of trauma. Butterworth-Heinemann, London Boston Singapore Sydney Toronto Wellington, pp 524-529

\section{zu Kap. 3.6.1}

Fischer H (1993) Einführung in die allgemeine und spezielle Traumatologie. Dr. Kovac, Hamburg

Fischer H, Kirkpatrick CJ (1991) A colour Atlas of Trauma Pathology. Wolfe, London

Fischer H, Spann W (1967) Pathologie des Trauma. Bergmann, München

Gülich H-A (1988) Biomechanische Belastungsgrenzen. Aktualisierte Literaturstudie zur Belastung des Menschen beim Aufprall. Bundesanstalt für Straßenwesen, Bereich Unfallforschung, Bergisch Gladbach

Janssen W (1977) Forensische Histologie. Schmidt-Römhild, Lübeck

Krauland W (1961) Über die Quellen des akuten und chronischen subduralen Hämatoms. Thieme, Stuttgart

Krauland W (1982) Verletzungen der intrakraniellen Schlagadern. Springer, Berlin Heidelberg New York

Mason JK (1993) The Pathology of Trauma. 2nd Ed. Arnold, London
Nahum AM, Melvin JW (1993) Accidental injury. Springer, Berlin Heidelberg New York Tokio

Sellier K, Unterharnscheid F (1963) Mechanik und Pathomorphologie der Hirnschäden nach stumpfer Gewalteinwirkung auf den Schädel. Hefte Unfallheilk. Springer, Berlin Göttingen Heidelberg

Unterharnscheid F (1963) Die gedeckten Schäden des Gehirns. Experimentelle Untersuchungen mit einmaliger, wiederholter und gehäufter stumpfer Gewalteinwirkung auf den Schädel. Spinger, Berlin Göttingen Heidelberg

Unterharnscheid F (1993) Pathologie des Nervensystems VI.A. Traumatologie von Hirn und Rückenmark. Traumatische Schäden des Gehirns (forensische Pathologie). In: Doerr W, Seifert G, Uehlinger E (Hrsg) Spezielle pathologische Anatomie. Springer, Berlin Heidelberg New York Tokio

Unterharnscheid F (1993) Pathologie des Nervensystems VI.B. Traumatologie von Hirn und Rückenmark. Traumatische Schäden des Gehirns (forensische Pathologie). In: Doerr W, Seifert G, Uehlinger E (Hrsg) Spezielle pathologische Anatomie. Springer, Berlin Heidelberg New York Tokio

Unterharnscheid F (1994) Pathologie des Nervensystems VI.C. Traumatologie von Hirn und Rückenmark. Traumatische Schäden des Gehirns (forensische Pathologie). In: Doerr W, Seifert G, Uehlinger E (Hrsg) Spezielle pathologische Anatomie. Springer, Berlin Heidelberg New York Tokio

Unterharnscheid F (1993) Pathologie des Nervensystems VII. Traumatologie von Hirn und Rückenmark. Traumatische Schäden von Hirn und Rückenmark (forensische Pathologie). In: DoerrW, Seifert G, Uehlinger E (Hrsg) Spezielle pathologische Anatomie. Springer, Berlin Heidelberg New York Tokio

Vogel H (1997) Gewalt im Röntgenbild. Befunde zu Krieg, Folter und Verbrechen. Ecomed, Landsberg

Voigt GE (1968) Die Biomechanik stumpfer Brustverletzungen besonders von Thorax, Aorta und Herz. Springer, Berlin Heidelberg New York

\section{zu Kap. 3.6.2}

Cooper GJ, Dudley HAF, Gann DS, Little RA, Maynard RL (eds) (1997) Scientific foundations of trauma. Butterworth-Heinemann, London Boston Singapore Sydney Toronto Wellington

Di Maio DJ, Di Maio VMJ (eds) (1989) Forensic pathology. Elsevier, New York Amsterdam Oxford

Driever F, Schmidt P, Madea B (2000) Zur Differenzierung zwischen Selbstund Fremdbeibringung von Halsschnittverletzungen. Arch Kriminol 205: 92-101

Driever F, Madea B (2003) Thoraxstich durch einen Grillspieß mit Verformung des Werkzeuges. Archiv Kriminol 211:174-180

Driever F, Madea B (2003) Tiefgreifende Halsschnittverletzung durch ein elektrisches Messer. Archiv Kriminol 212:104-109

König HG (1966) Spurentechnischer Vergleich realer und fingierter Überfälle. In: Saternus HG, Kernbach-Wighton G (Hrsg) Selbstbeschädigung. Schmidt-Römhild, Lübeck, 23-49

Madea B, Lignitz E, Weinke H (1993) Schädelverletzungen durch Schlag mit Glasflaschen. Arch Kriminol 192: 73-79

Madea B, Schmidt P (1993) Über ungewöhnliche suizidale Stichverletzungen. Arch Kriminol 192: 137-148

Mallach HJ (1987) Der Stellenwert der Luftembolie in der modernen Medizin. Springer, Berlin Heidelberg New York Tokio

Mason JK (ed) (1978) The pathology of violent injury. Arnold, London Boston Melbourne Auckland 
Pollak S, Ropohl D (1992) Befundmuster bei suizidalen Stichverletzungen des Halses. Arch Kriminol 190: 72-81

Saternus KS, Bessel G, Staak M (1983) Zur Frage der Handlungsfähigkeit bei penetrierender und stumpfer Bauchverletzung. Z Rechtsmed 91: 101-114

Saternus KS, Kernbach-Wighton G (Hrsg) (1996) Selbstbeschädigung, Forensische Bewertung und Therapiemöglichkeiten. Schmidt-Römhild, Lübeck

Watson AA (1993) Stabbing and other incisional wounds. In: Mason JK (ed) The pathology of trauma. Arnold, London Bosten Melbourne Auckland, 87-108

Werkgartner A (1940) Stichworte: Hiebverletzungen, Tod und Gesundheitsbeschädigung infolge Verletzung durch Hieb, Stichverletzungen, Tod und Gesundheitsbeschädigung infolge Verletzung durch Schnitt. In: Neureiter F. v., Pietrusky F, Schütt E (Hrsg) Handwörterbuch der gerichtlichen Medizin und naturwissenschaftlichen Kriminalistik. Springer, Berlin

\section{zu Kap. 3.7}

DiMaio VJM (1999) Gunshot wounds. Practical aspects of firearms, ballistics, and forensic techniques, $2^{\text {nd }}$ edn. CRC Press, Boca Raton London New York Washington

Dodd MJ (2006) Terminal ballistics. A text and atlas of gunshot wounds. CRC Press, Boca Raton

Faller-Marquardt M, Bohnert M, Pollak S (2004) Detachment of the periosteum and soot staining of its underside in contact shots to the cerebral cranium. Int J Legal Med 118: 343-347

Große Perdekamp M, Vennemann B, Mattern D, Serr A, Pollak S (2005) Tissue defect at the gunshot entrance wound: what happens to the skin? Int J Legal Med 119: 217-222

Heard BJ (2001) Handbook of firearms and ballistics. Examining and interpreting forensic evidence. Wiley \& Sons, Chichester

Hueske EE (2006) Practical analysis and reconstruction of shooting incidents. CRC Press, Boca Raton

Karger B (2001) Forensische Ballistik von Schussverletzungen. Rechtsmedizin 11: 104-119

Karger B (2004) Schussverletzungen. In: Brinkmann B, Madea B (Hrsg) Handbuch gerichtliche Medizin 1. Springer, Berlin Heidelberg New York Tokio, S 593-682

Kneubuehl BP (1994) Geschosse. Ballistik, Treffsicherheit, Wirkungsweise. Stocker-Schmid, Zürich

Kneubuehl BP (2004) Geschosse, Bd 2. Ballistik, Wirksamkeit, Messtechnik. Stocker-Schmid, Zürich

Lew EO, Dolinak D, Matshes EW (2005) Firearm injuries. In: Dolinak D, Matshes EW, Lew EO (eds) Forensic pathology. Principles and Practice. Elsevier Academic Press, Burlington San Diego London, pp 163-200

Pollak S, Saukko PJ (2000) Humane killing tools. In: Siegel JA, Saukko PJ, Knupfer GC (eds) Encyclopedia of forensic sciences. Academic Press, London, pp 939-944

Pollak S, Saukko PJ (2003) Atlas of forensic medicine (CD-ROM), ISBN 0-444-82597-5. Elsevier, Amsterdam

Rothschild MA (1999) Freiverkäufliche Schreckschusswaffen. Medizinische, rechtliche und kriminalistische Bewertung. Schmidt-Römhild, Lübeck

Sellier K (1982) Schusswaffen und Schusswirkungen I, 2. Aufl. SchmidtRömhild, Lübeck
Sellier K (1988) Schussentfernungsbestimmung, 2. Aufl. Schmidt-Römhild, Lübeck

Sellier K, Kneubuehl BP (2001) Wundballistik und ihre ballistischen Grundlagen, 2. Aufl. Springer, Berlin Heidelberg New York Tokio

\section{Danksagung: Die Schießversuche auf Simulanzien wurden von Herrn Dr. B. Kneubuehl (Thun/CH) durchgeführt.}

\section{zu Kap. 3.8.1+3.8.2}

Anmerkung: Die im Text zitierten, im Literaturverzeichnis aber nicht aufgeführten Beiträge finden sich in dem Buch »Brinkmann B, Püschel K: Ersticken«.

Brettel HF (1986) Ersticken infolge Strangulation. In: Forster B (Hrsg) Praxis der Rechtsmedizin. Thieme, Stuttgart New York, S. 121-143

Brinkmann B (1978) Zur Pathophysiologie und Pathomorphologie beim Tod durch Druckstauung. Z Rechtsmedizin 81: 79-96

Brinkmann B, Püschel K (1990) Ersticken. Fortschritte in der Beweisführung. Springer, Berlin Heidelberg New York Tokio

Camps FE (1976) Gradwohl's Legal Medicine. Wright and Sons, Bristol

DiMaio DJ, DiMaio VJM (1989) Forensic Pathology. Elsevier, New York Amsterdam Oxford

Härm T, Rajs J (1981) Types of injuries and interrelated conditions of victims and assailants in attempted homicidal strangulation. Forensic Sci Internat 18: 101-123

Knight B (1996) Forensic Pathology. Oxford University Press, New York

Mallach HJ, Oehmichen M (1982) Bolustod - Reflex oder Erstickung? Beitr Gerichtl Med 40: 474-485

Maxeiner H (1996) Vorkommen und Auswirkungen von Knebelungen bei Tötungshandlungen. Rechtsmedizin 6: 147-155

Maxeiner H (1997) Über Kopfstauung, Petechien und »Zyanose« beim Tod durch homizidale Halskompression. Rechtsmedizin 7: 37-44

Mueller B (1975) Erstickung. In: Mueller B (Hrsg) Gerichtliche Medizin, Bd 1. Springer, Berlin Heidelberg New York, S. 439-468

Polson CJ, Gee DJ, Knight B (1985) The Essentials of Forensic Medicine. Pergamon Press, Oxford

Ponsold A (1967) Erstickung im Besonderen. In: Ponsold A (Hrsg) Lehrbuch der gerichtlichen Medizin. Thieme, Stuttgart, S. 312-345

Prokop O, Göhler W (1976) Forensische Medizin. Fischer, Stuttgart, New York

Rossen R, Kabat H, Anderson JP (1943) Acute Arrest of Cerebral Circulation in Man. Arch Neurol Psychiat 50: 510-528

Saternus KS (1979) Die Verletzungen von Halswirbelsäule und Halsweichteilen. Hippokrates, Stuttgart

Vanezis P (1989) Pathology of Neck Injury. Butterworth-Heinemann, London Boston Singapore Sydney Toronto Wellington

\section{zu Kap. 3.8.3}

Bernett P, Haas W (1984) Ertrinken, Badetod und andere Zwischenfälle beim Schwimmsport. Fortsch Med 102: 752-754

Fletemeyer JR, Freas SJ (1999) Drowning - New Perspectives on Intervention and Prevention. CRC Press, Baco Raton London New York Washington

Hasibeder W, Friesenecker B, Mayr AJ (2003) Beinaheertrinken: Epidemiologie - Pathophysiologie - Therapie. Anaesthesiol Intensivmed Notfallmed Schmerzther 38: 333-340

Modell JH (1993) Drowning. N Engl J Med 328: 253-256

Pollanen MS (1998) Forensic diatomology and drowning. Elsevier, Amsterdam Lausanne 
Priemer F, Keil W, Kandolf R (1999) Hydrocution in a case of coxsackie virus infection. Int J Legal Med 112: 368-371

Prokop O (1975) Ertrinken und Tod im Wasser. In: Prokop O, Göhler W (Hrsg) Die Erstickung. Forensische Medizin, 3. Aufl. Volk und Gesundheit, Berlin, S. 120-139

Reh H (1969) Diagnostik des Ertrinkungstodes und Bestimmung der Wasserzeit. Triltsch, Düsseldorf

Schmidt P, Madea B (1995) Homicide in the bathtub. Forensic Sci Int 72, 135-146

Statistisches Bundesamt (2004) Todesursachenstatistik. Zweigstelle Bonn

\section{zu Kap. 3.9.1}

Allgöwer M, Siegrist J (1957) Verbrennungen. Pathophysiologie, Pathologie, Klinik, Therapie. Springer, Berlin Göttingen Heidelberg

Herndon (1997) Total Burn Care. Saunders Company, London Philadelphia Toronto Sydney Tokyo

Madea B (1992) Branddauer und Verkohlungsgrad einer Brandleiche. Arch Kriminol 189: 39-47

Madea B, Schmidt P (1999) Vitale, supravitale, postmortale Befunde bei Verbrennungen. In: Oehmichen M (Hrsg) Hyperthermie, Brand und Kohlenmonoxid. Research in Legal Medicine / Rechtsmedizinische Forschungsergebnisse, Vol. 21. Schmidt-Römhild, Lübeck

Madea B, Schmidt P, Banaschak S, Schyma C (2001) Tötung durch Verbrennen. Archiv Kriminol 208:1-9

Maxeiner H (1988) Umstände und Befunde bei 202 Brandtodesfällen. Beitr ger Med 46: 313-325

McDougal WS, Glade CL, Pruitt BA (1978) Manual of Burns. Springer, Berlin Heidelberg NewYork

Moritz AR (1947) Studies of thermal Injury III. The Pathology and Pathogenesis of Cutaneous Burns. An Experimental Study. Am J Path 23: 915-941

Moritz AR, Henriques FC (1947) Studies of thermal injury II. The relative importance of time and surface temperature in the causation of cutaneous burns. Am J Path 23: 695-720

Moritz AR, Henriques FC, McLean R (1945) The effects of inhaled heat on the air passages and lungs. Am J Pathol 21: 311-331

Oehmichen M (Hrsg) Hyperthermie, Brand und Kohlenmonoxyd. Research in Legal Medicine/Rechtsmedizinische Forschungsergebnisse. Vol. 21, Schmidt-Römhild, Lübeck

Panke TW, McLeod CG (1985) Pathology of thermal injury: a practical approach. Grune \& Stratton, Orlando San Diego New York

Price PH, Call DE, Hansen FL, Zerwick CJ (1953) Penetration of Heat in Thermal Burns. Surgical Forum 4: 433-438

\section{zu Kap. 3.9.2}

Braun-Falco O, Plewig G, Wolff HH (1996) Dermatologie und Venerologie. Springer, Berlin Heidelberg New York Tokio

Büchner F (1962) Allgemeine Pathologie. Urban \& Schwarzenberg, München Berlin

Bünte H (1996) Chirurgie. Urban \& Schwarzenberg, München Wien Baltimore

Burchardi H (Hrsg) (1993) Akute Notfälle. Pathophysiologie Diagnostik Erstbehandlung. 4. Aufl. Thieme, Stuttgart

Classen M, DiehI V, Kochsiek K (1991) Innere Medizin. Urban \& Schwarzenberg, München Wien Baltimore

Ellinger K, Oswald PM, Stange K (1998) Fachkundenachweis Rettungsdienst. 2. Aufl. Springer, Berlin Heidelberg New York Tokio
Gillner E, Waltz H (1971) Zur Symptomatik des Erfrierens. Kriminalistik. Forensische Wissenschaften 5, 179-185

Madea B, Oehmichen M (1989) Ungewöhnliche Befunde in einem Fall von Unterkühlung. Z Rechtsmedizin 102, 59-67

Madea B, Preuß J, Lignitz E (2003) Unterkühlung: Umstände, Morphologische Befunde und ihre Pathogenese. Rechtsmedizin 14:41-49

Madea B, Preuß J, Henn V, Lignitz E (2004) Morphological findings in fatal hypothermia and their pathogenetics. In: Oehmichen M (Hrsg) Hypothermia - clinical, pathomorphological and forensic features. SchmidtRömhild, Lübeck

Oehmichen M (Hrsg) (2004) Hypothermia - clinical, pathomorphological and forensic features. Schmidt-Römhild, Lübeck

Prokop O, Göhler W (1975) Forensische Medizin. 3. Aufl. Volk und Gesundheit, Berlin

Schmidt RF, Thews G (1997) Physiologie des Menschen. Springer, Berlin Heidelberg New York Tokio

\section{zu Kap. 3.10}

Anders S, Tsokos M, Püschel K (2002) Nachweis der Stromwirkung und des Stromweges im Körper. Rechtsmedizin 12: 1-9

Brinkmann K, Schaefer H (Hrsg) (1982) Der Elektrounfall. Springer, Berlin Heidelberg New York

Janssen W (1984) Forensic histopathology. Springer, Berlin Heidelberg New York Tokio, pp 261-274

Krause D (2004) Tod durch Elektrizität. In: Brinkmann B, Madea B (Hrsg) Handbuch gerichtliche Medizin 1. Springer, Berlin Heidelberg New York, S. 825-838

Pollak S (1980) Pathomorphologische Befundkonstellationen beim Tod durch hochgespannten elektrischen Strom. Arch Kriminol 165: 1-16

Pollak S, Saukko P (2003) Atlas of forensic medicine (CD-ROM), ISBN 0-444-82597-5, Elsevier, Amsterdam

Pollak S, Stellwag-Carion C, Binder R (1988) Zur Pathomorphologie tödlicher Blitzunfälle. In: Bauer G (Hrsg) Gerichtsmedizin - Festschrift für Wilhelm Holczabek. Deuticke, Wien, S. 139-153

Sellier K (1975) Schäden durch Elektrizität. In: Mueller B (Hrsg) Gerichtliche Medizin, 2. Aufl. Springer, Berlin Heidelberg New York

Wetli CV (1996) Keraunopathology - an analysis of 45 fatalities. Am J Forensic Med Pathol 17: 89-98

Zack F, Schniers E, Wegener R (2004) Blitzunfall. Rechtsmedizin 14: 396401

\section{zu Kap. 3.11}

Adelsberger L (1946) Medical observations in Auschwitz concentration camp. Lancet 2: 317-319

Giese W, Hörstebrock R (1962) Allgemeine Pathologie des exogenen quantitativen Nahrungsmangels. In: Büchner F, Letterer E, Roulet F (Hrsg) Handbuch der allgemeinen Pathologie. 11. Bd, Umwelt II, 1. Teil. Springer, Berlin Göttingen Heidelberg, S. 446-591

Gomez F, Galvan RR, Cravioto J, Frenk S (1955) Malnurition in infancy and childhood with special reference to Kwashiokor. Advanc Pediat 131169

Leiter LA, Marliss EB (1982) Survival during fasting may depend on fat as well as protein stores. JAMA 248: 2.306-2.307

Madea B (2005) Death as a result of starvation - diagnostic criteria. In: Tsokos M (ed) Forensic pathology reviews. Vol. 2. Humana Press, Totowa NJ, pp 1-23

Madea B, Michalk DV, Lignitz E (1994) Verhungern infolge Kindesvernachlässigung. Arch Kriminol 194: 29-38 
Meade JL, Brissie RM (1985) Infanticide by starvation: calculation of caloric deficit to determine degree of deprivation. J For Sci 30: 1.263-1.268

Missliwetz J, Mortinger H (1992) Tod durch Hypoglykämie nach Hungerzustand - Pathophysiologie versus Morphologie. Beitr Gerichtl Med 50: 319-323

Waterlow JC (1972) Classification and definition of protein-caloric malnutrition. Br Med J 2: 566-569

Waterlow JC (1973) Note on the assessment and classification of proteinenergy malnutrition in children. Lancet: 87-89

Waterlow JC, Buzina R, Keller W, Lane M, Nichaman MZ, Tanner JM (1977) The presentation and use of height and weight data for comparing the nutritional status of groups of children under the age of 10 years. Bulletin World Health Organisation 55 (4) 489-498

Wolff-Eisner A (1947) Über Mangelerkrankungen auf Grund von Beobachtungen im KonzentrationslagerTheresienstadt. Sauer-Morhard, Würzburg

\section{zu Kap. 3.12}

Dülmen R van (1991) Frauen vor Gericht. Kindsmord in der Frühen Neuzeit. Fischer, Mainz

Fritsch K (1901) Gerichtsärztliche Geburtshilfe. Enke, Stuttgart

Gerchow J (1957) Die ärztlich-forensische Beurteilung von Kindesmörderinnen. Ein Beitrag zum Problem der abartigen Erlebnisreaktion. Marhold, Halle

Gordon I, Shapiro HA (1982) Forensic Medicine. Churchill, Livingstone

Kosa F (1999) Die Knochen des Fetus und Neugeborenen. In: Leopold D (Hrsg) Identifikation unbekannter Toter. Schmidt-Römhild, Lübeck

Rauch E, Tutsch-Bauer E, Krupinski M (1997) § 217 StGB - Kindestötung, ein nicht mehr zeitgemäßer Paragraph? Forensische Aspekte zur geplanten Reform des § 217 StGB. Geburtsh Frauenheilk 57 (12) 699-702

Shapiro HA (1977) Microscopy of human fetal lung and the diagnosis of postnatal respration. In: Wecht C (ed) Legal medicine annual 1976. Appleton Century Crofts, New York

Stocker JT, Dehner LP (1992) Pediatric Pathology, 2 Vol, Lippincott Company, Philadelphia

Thomsen H, Bauermeister M, Wille R (1992) Zur Kindestötung unter der Geburt. Eine Verbundstudie über die Jahre 1980-1989. Rechtsmed 2: 135-142

Zink R, Reinhardt G (1969) Gerichtsmedizinische Untersuchungen zum Verhalten der Nabelschnur bei gewaltsamer Zerreißung. Dtsch Z ges gerichtl Med 66: 86-96

\section{zu Kap. 3.13}

Beller FK (1999) Schwangerschaftsabbruch. In: Schneider H, Husslein P, Schneider KTM (Hrsg) Geburtshilfe. Springer, Berlin Heidelberg New York Tokio, S. 41-50.

Förster H (1995) Entwicklung peripartaler Sterbefälle unter dem Einfluss der Legalisierung des Schwangerschaftsabbruches in der DDR. Eine retrospektive Analyse aus dem Gerichtsmedizinischen Institut der Charité zu Berlin im Zeitraum 1950-1989 unter besonderer Beachtung der Problematik illegaler Abortsterbefälle und Suizide. Med Diss Berlin (HU)

Hartmann G, Mueller B (1975) Schwangerschaftsabbruch, Abtreibung und Kindstötung. In: Mueller B (Hrsg) Gerichtliche Medizin, 2. Bd. Springer, Berlin Heidelberg New York, S. 1.124-1.211

Lignitz E, Förster H (1991) Analyse der Müttersterblichkeit in Berlin (Ost) im Zeitraum 1969-1987. Eine kritische Bestandsaufnahme aus der Retro- spektive. Medizinrecht - Psychopathologie - Rechtsmedizin. Springer, Berlin Heidelberg New York Tokio, S. 442-456

Prokop O, Göhler W (1975) Forensische Medizin, 3. Aufl. Volk und Gesundheit, Berlin

Surbek DV, Holzgreve W (2000) Pathologie der Frühschwangerschaft. In: Dietrich K (Hrsg) Gynäkologie und Geburtshilfe. Springer, Berlin Heidelberg New York Tokio, S. 365-381

\section{zu Kap. 3.14}

Beier K, Bosinski HA, Hartmann U, Loewit K (2001) Sexualmedizin. Urban und Fischer, München Jena

Byard RW, Hucker SJ, Hazelwood RR (1990) A comparison of typical death scene features in cases of fatal male and female autoerotic asphyxia with a review of the literature. Forens Sci Int 48: 113-121

Ross D, Chan TC (2006) Sudden deaths in custody. Humana Press, Totowa NJ

Dürwald W (1962) Zur Beurteilung autoerotischer Unfälle. Beitr gerichtl Med 22: 91-101

Krings H (1973) Autoerotische Unfälle. Medizinische Dissertation. Universität Köln

Naeve W, Wittram S (1977) Tödliche autoerotische Unfälle. Verlag Versicherungswirtschaft, Karlsruhe

Saternus KS, Kernbach-Wighton G (Hrsg) (2002) Fixierung erregter Personen. Todesfälle in Klinik und Gewahrsam. Research in Legal Medicine. Vol. 28. Schmidt-Römhild, Lübeck

Uva JL (1995) Review: Autoerotic asphyxiation in the United States. J Forens Sci 40(4) 574-581

Weimann W (1960) Tödliche Unglücksfälle bei autoerotischer Betätigung. In: Prokop O (Hrsg) Lehrbuch der gerichtlichen Medizin. Volk und Gesundheit, Berlin, S. 255-273

\section{zu Kap. 3.15}

Barbet P (1953) Die Passion Jesu Christi in der Sicht des Chirurgen. Badenia, Karlsruhe

Berg S, Rolle R, Seemann A (1981) Der Archäologie und der Tod. Archäologie und Gerichtsmedizin. Bucher, München Luzern

Madea B (1993) Death in a head-down-position. For. Sci. Int. 61, 119-132

Patscheider H (1961) Die Todesursache beim frei hängenden, am Rumpf suspendierten Menschen. Beitr gerichtl Med 21, 87-93

Reay D, Fligner CL, Stilwell A, Arnold J (1992) Positional asphyxia during law enforcement transportation. Am For Med Path 13, 90-97

Reay D, Howard JD, Fligner CL et al (1988) Effects of positional restraint on oxygen saturation and heart rate following exercise. Am For Med Path 8, 16-18

Du Chesne A, Brinkmann B (2004) Autoerotische Unfälle. In: Brinkmann B, Madea B (Hrsg) Handbuch gerichtliche Medizin. Springer, Berlin Heidelberg New York, S 794-796

Prokop O, Radam G (1987) Tödliche Unglücksfälle bei autorerotischer Betätigung. In: Atlas der gerichtlichen Medizin. Volk und Gesundheit, Berlin, S 581-605

\section{zu Kap. 3.16.1-3.16.4}

Dürwald W (1966) Gerichtsmedizinische Untersuchungen bei Verkehrsunfällen. Thieme, Leipzig

Fischer H, Spann W (1967) Pathologie des Trauma. Bergmann, München

Janssen W (1984) Forensic Histopathology. Springer, Berlin Heidelberg New York Tokyo 
Mattig W (2003) Handlungsfähigkeit. In: Brinkmann B, Madea B (Hrsg) Handbuch gerichtliche Medizin 1. Springer, Berlin Heidelberg New York S 547ff

Mueller B (1953) Gerichtliche Medizin. Springer, Berlin Göttingen Heidelberg

Mueller B (1975) Gerichtliche Medizin, Bd. 1. Springer, Berlin Heidelberg New York

Prokop O, Göhler W (1975) Forensische Medizin, 3. Aufl. Volk und Gesundheit, Berlin

Sellier K (1982) Schusswaffen und Schusswirkungen I, 2. Aufl. SchmidtRömhild, Lübeck

Sellier K (1977) Schusswaffen und Schusswirkungen Il, Schmidt-Römhild, Lübeck

Sellier K, Kneubuehl B (1992) Wundballistik. Springer, Berlin Heidelberg New York Tokio

\section{zu Kap. 3.16.5}

Bonte W (1975) Tool marks in bones and cartilage. J Forensic Sci 20, 315325

Püschel K, Koops E (1987) Zerstückelung und Verstümmelung. Arch Kriminol 180, 28-40, 88-100

Raijs J, Lundström M, Broberg M, Lidberg L, Lindquist O (1997) Criminal mutilation of the human body in Sweden - a thirty year medico-legal and forensic psychiatric study. J Forensic Sci 43, 563-580

Thorwald J (1964) Das Jahrhundert der Detektive, Bd 2. Droemer-Knauer, München Zürich

Weimann W, Prokop O (1963) Bildatlas für gerichtliche Medizin. Volk und Gesundheit, Berlin

Ziemke E (1918) Über die kriminelle Zerstückelung von Leichen und die Sicherstellung ihrer Identität. Vjschr gerichtl Med 56, 270-318

\section{zu Kap. 3.16.6}

Bodziak WJ (1990) Footwear impression evidence. Elsevier, New York Amsterdam London, pp 126-130

Böhm E (1987) Zur Morphologie und Biomechanik von Trittverletzungen. Beitr gerichtl Med 45:319-329

Böhm E, Schmidt BU (1987) Kriminelle und kinetische Energie bei Tötungshandlungen durch stumpfe Gewalt. Beitr gerichtl Med 45:331-338

Brüschweiler W, Braun M, Fuchser HJ, Dirnhofer R (1997) Photogrammetrische Auswertung von Haut- und Weichteilwunden sowie Knochenverletzungen zur Bestimmung des Tatwerkzeuges - grundlegende Aspekte. Rechtsmedizin 7:76-83

Graß H, Madea B, Schmidt P, Glenewinkel F (1996) Zur Phänomenologie des Tretens und Tottretens. Arch Kriminol 98:73-78

Henn V, Lignitz E, Philipp KP, Püschel K (2000) Zur Morphologie und Phänomenologie des Tottretens, Teil 1. Arch Krimonol 205:15-24

Henn V, Lignitz E, Philipp KP, Püschel K (2000) Zur Morphologie und Phänomenologie des Tottretens, Teil 2. Arch Krimonol 205:65-74

Reh H, Weiler G (1975) ZurTraumatologie des Tottretens. Beitr gerichtl Med 33:148-153

Schrader S (1933) Wunde und Werkzeug. Tödliche Schädelverletzung durch Fußtritte. Arch Kriminol 92:229-231

Steffen W (1989) Turnschuh als »gefährliches Werkzeug«. NJW 14:920921

Strauch H, Wirth I, Taymoorian U, Geserick G (2001) Kicking to death - forensic and criminological aspects. Forensic Science International 123:165-171

Tröndle H, Fischer T (2003) Beck'sche Kurzkommentare, Strafgesetzbuch und Nebentexte. 50. neubearb. Aufl. Beck, München 1395-1397 